University of Rhode Island

\title{
DigitalCommons@URI
}

Open Access Dissertations

2018

\section{Organocatalytic Pathway to the Creation of Polymeric Materials with Some Mechanistic Investigations}

Partha Datta

University of Rhode Island, partha_datta@uri.edu

Follow this and additional works at: http://digitalcommons.uri.edu/oa_diss

Terms of Use

All rights reserved under copyright.

\section{Recommended Citation}

Datta, Partha, "Organocatalytic Pathway to the Creation of Polymeric Materials with Some Mechanistic Investigations" (2018). Open Access Dissertations. Paper 712.

http://digitalcommons.uri.edu/oa_diss/712

This Dissertation is brought to you for free and open access by DigitalCommons@URI. It has been accepted for inclusion in Open Access Dissertations by an authorized administrator of DigitalCommons@URI. For more information, please contact digitalcommons@etal.uri.edu. 
ORGANOCATALYTIC PATHWAY TO THE CREATION OF POLYMERIC MATERIALS WITH SOME MECHANISTIC INVESTIGATIONS

BY

PARTHA DATTA

\begin{abstract}
A DISSERTATION SUBMITTED IN PARTIAL FULFILLMENT OF THE REQUIREMENTS FOR THE DEGREE OF DOCTOR OF PHILOSOPHY IN CHEMISTRY
\end{abstract}

UNIVERSITY OF RHODE ISLAND 2018 


\section{DOCTOR OF PHILOSOPHY DISSERTATION}

OF

Partha Datta

APPROVED:

Dissertation Committee

Major Professor

Matthew K. Kiesewetter

Brett Lucht

Alvin Bach II

Nasser H. Zawia

DEAN OF THE GRADUATE SCHOOL

UNIVERSITY OF RHODE ISLAND

2018 


\begin{abstract}
The overarching theme of my research work involves understanding the mechanistic aspects of dually activated hydrogen-bonding catalyst systems and applying that knowledge to synthesize polymers from some of the less explored monomers. This entailed a thorough approach to some of the already hypothesized mechanisms in the polymer community and building on that with additional perspective on catalytic interactions. The other aspect of my research encompassed the application of these H-bond mediated catalysts in controlled ring-opening polymerization (ROP) of sulfur-based lactones. This allowed the growth in monomer scope using these catalysts for the first time. H-bonding catalysis, particularly the ones involving ureas and thioureas, began about a decade or so ago. The tremendous rise in organocatalytic ring-opening polymerization has sparked a wide range of catalysts developments in the past few years. Due to their lower cost, reduced toxicity and greener approach, the field has been booming ever since its inception. The wide range of architectures in polymer production that were seemingly difficult previously were possible with great control and selectivity. Using a bifunctional catalytic species, either as one unit or two separate entities, monomer activation and chain propagation can be achieved for polymer production. The first chapter in this dissertation delineates on that growth of dual activation process in organocatalysis as a book chapter "Bifunctional and Supramolecular Organocatalysts for Polymerization" in Organic Catalysts for Polymerization. My contribution to this review work has primarily focused on Dual Catalysts, Rate Accelerated Dual Catalysis and Supramolecular Catalysts.

In the second chapter, we looked at the binding interaction that inherently is a determining factor in the dual activation process. We obtained binding constants between
\end{abstract}


the cocatalytic pair of thiourea and a set of bases which allowed us to comprehend the reason behind enhanced selectivity and reaction control. Finally, we applied this phenomenon to test its feasibility with a new, very active cocatalyst pair for a wellcontrolled ROP of some common cyclic esters. I was involved in the latter part of this study where I applied our binding interaction knowledge to test via ROP using a commercial base and thiourea.

As our understanding of the activation process grew, we determined that a higher order moiety of (thio)urea may prove to be an even better choice for increased rate and selectivity in polyester synthesis. It is with this notion that we developed a tris-urea motif for the monomeric activation of lactones, described in the third chapter. Although a rate acceleration is distinctly demonstrated using such a catalytic species, the molecular weight control or living behavior in ROP was never sacrificed along the way. My part in this study was only limited to the synthesis of this tris-urea catalyst with some initial reaction condition screening.

Carrying that knowledge of catalytic interaction with monomer from the initial studies, we delved into the investigation of equilibrium process of the ROP in the fourth chapter. We observed a catalyst dependence on the overall reaction process of lactonebased ROP where a change in reactant and product interaction with the thiourea can be observed. This results in a similar Gibbs free energy difference between monomer to catalyst and polymer to catalyst. As a result, a change in monomer concentration (recoverable) can be seen at the reaction equilibrium with a change in catalyst concentration. This work was mainly performed by me, except the final recovery of the monomer at equilibrium. 
After this point, the scope of monomers that can undergo this dual activation was broadened with some of the sulfur-based monomers. Since previous literature studies demonstrated poor control in ROP of such monomers with the assistance of metal-based catalysts, the use of H-bonding catalysts was deemed to be very appropriate. With that in mind, I performed the first-ever organocatalyzed ring-opening polymerization of a sulfurized lactone, $\varepsilon$-thionocaprolactone, shown in fifth chapter. Both reaction control and living nature allowed the possibility of copolymer production using this monomer under the same $\mathrm{H}$-bonding catalysis. A range of new polymeric materials were created at the end of this study.

From that initial sulfur-based monomer, the study was extended to some of the less explored thionated monomers in sixth chapter. The same H-bonding organocatalysis was implemented here as well for a broad range of larger lactones (macrolactones). Besides validating the mechanistic aspects of these polymerizations, thermodynamics and kinetics of reaction were also evaluated. As expected for macrolactones over 10 ring sizes, entropic contribution showed dominance over enthalpy which was the case for 9-membered lactones or below. Further material characterizations are currently undergoing to shed light on future applications of these polymers. My contribution to this study involved mainly the synthesis of 8-membered lactones ( $\zeta$-heptalactone, $\zeta$-thionoheptalactone), thiono-ethylene brassylate and optimization of reaction conditions for the polymerization of those monomers.

In the seventh chapter, I have included some of the other thionated monomer synthesis besides lactones and their preliminary ROP results. Though none of those monomers of amides and lactide functionality showed good prospect for organocatalyzed 
ROP, further growth in tuning the structure of the monomers may demonstrate a better way to synthesize polymers from such systems. Additionally, other applications of these sulfurbased polymers (i.e. newer copolymerizations, crosslinking ability) were reported for possible development in these materials in the future. This chapter fully encompasses all of these unfinished works that can be quite useful for a researcher to pick up at a later time.

The eighth chapter is quite different from the rest of the other chapters in this dissertation in that no organic catalysts were employed for the molecular transformation of styrene to stilbene. In fact, metal catalyst developed by Prof. Robert Grubbs was utilized for this transformation via cross-metathesis reaction. This was a manuscript for educational purpose of undergraduate laboratory setting where the ulitization of a well-known Nobel winning catalyst was used by students to form carbon-carbon bond from an olefinic motif. My input in this experiment was mainly to assist the co-authors of the manuscript to carry out the reaction properly in the undergraduate laboratory with students comprising mostly of chemistry major as well as formulate a report to aid in the writing portion of the journal publication. 


\section{ACKNOWLEDGMENTS}

I would like to express my sincerest gratitude to Professor Matthew Kiesewetter for guiding and advising me throughout my entire graduate school career, not just as a chemist, but also as a better human being. I am humbly thankful to Professor Brett Lucht, Professor Sze Yang, Professor Alvin Bach II, and Professor Bongsup Cho for reviewing my dissertation out of their busy schedules. I am particularly very grateful to Professor Alvin Bach II for teaching all that he has taught me over the years on NMR. I would also like to thank the Chemistry department at the University of Rhode Island for providing the necessary resources for the completion of this work and supporting my education with a Teaching Assistantship and Research Assistantship throughout my graduate school career. I am also thankful to University of Rhode Island Graduate School for providing me a Diversity Fellowship award in 2017-18.

Finally, I would like to thank my parents, Madhu S. Datta and Niva R. Datta, my brother, Shubhra S. Datta for all the support they have provided me throughout these past five years and before. I also would like to thank all my colleagues within the lab and outside for assisting me to be a better researcher over the last few years. In addition, I would like to give special shout out to Dr. Elizabeth Kiesewetter for helping me become adept at my synthetic and laboratory skills from when I started off research in my first year. As an avid follower of cricket, I take inspiration from some of the well-known athletes. In that regard, I would like to thank one such inspirational role model, Mahendra Singh Dhoni, for demonstrating how stresses can be turned into success with a calm, cool attitude at one's goals. 


\section{PREFACE}

This following dissertation is presented in manuscript format with eight manuscripts. The first chapter "Bifunctional and Supramolecular Organocatalysts for Polymerization" is a book chapter submitted for publication in Organic Catalysts for Polymerization under Royal Society of Chemistry. The second chapter "Cooperative Hydrogen-Bond Pairing in Organocatalytic Ring-Opening Polymerization" has been published in Macromolecules in 2014. The third chapter "Bis- and Tris-Urea H-Bond Donors for Ring-Opening Polymerization: Unprecedented Activity and Control from an Organocatalyst" has been published in ACS Macro Lett in 2016. The fourth chapter "Coupled Equilibria in H-Bond Donating Ring-Opening Polymerization: The Effective Catalyst-Determined Shift of a Polymerization Equilibrium" has been published in European Polymer Journal in 2017. The fifth chapter "Controlled Organocatalyzed RingOpening Polymerization of $\varepsilon$-Thionocaprolactone" has been published in Macromolecules in 2016. The sixth chapter "Organocatalytic Synthesis of Poly(thionolactone)s: New Materials Abilities from Sulfur-Containing Polylactones" has been prepared for submission to Macromolecules. The seventh chapter "Possibilities with Thionated Monomers and Beyond" contains some of the unfinished works in sulfur-containing monomer and polymerizations which could be useful for further developments in the future. The eighth chapter "Stilbene Synthesis by Olefin Metathesis Reaction" has been prepared for submission to Journal of Chemical Education. 


\section{TABLE OF CONTENTS}

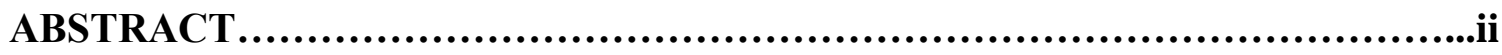

ACKNOWLEDGMENT ............................................................vi

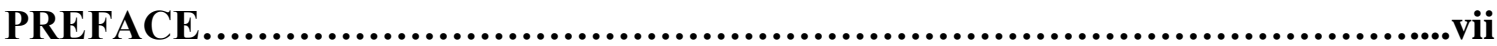

TABLE OF CONTENTS .........................................................iii

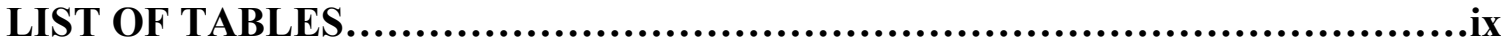



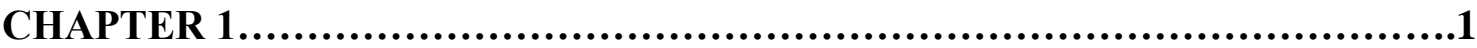

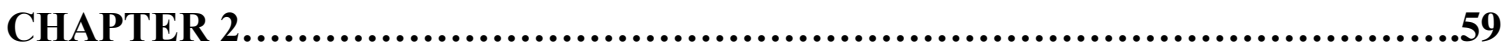

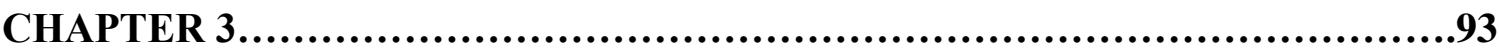

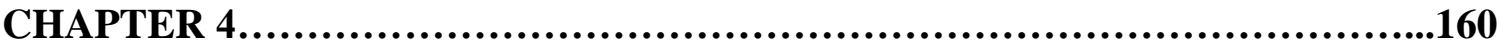

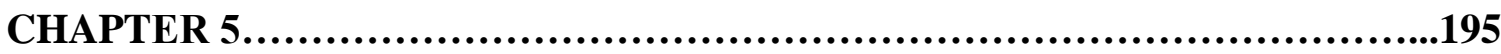

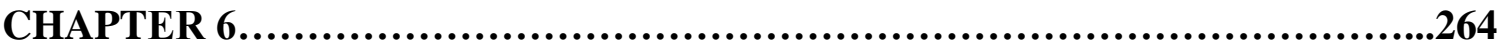








\section{LIST OF TABLES}

TABLE

PAGE

Table 2.1. Binding constants and observed rate constants for the bases studied.........90

Table 2.2. The 1/BEMP catalyzed ROP of cyclic monomers........................................91

Table 2.3. Thermodynamic Values of Binding between 1 and various bases .............92

Table 3.1. MTBD and bis- or tristhiourea Catalyzed ROP of VL and CL ................144

Table 3.2. Bis- and Tris-urea Cocatalyzed ROP of Lactones........................145

Table 3.3. Transesterification of Ethyl Acetate..................................146

Table 3.4. Low 3-O/MTBD Cocatalyst Loadings in the ROP of VL.................147

Table 3.5. Solvent Screen in the 3-O/MTBD Cocatalyzed ROP of VL..............148

Table 3.6. Post-polymerization Transesterification in 3-O/MTBD Cocatalyzed

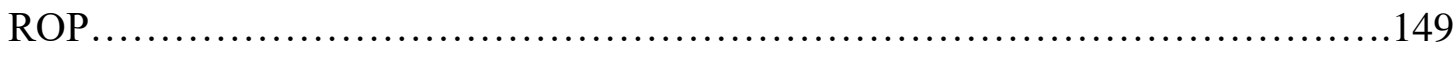

Table 5.1. ROP of tnCL with Base Catalysts.......................................238

Table 5.2. Thiourea Plus Base Cocatalyzed ROP of tnCL.........................239

Table 5.3. Copolymers of tnCL and VL with Varying Monomer Feeds..............240

Table 6.1. ROP of HL with urea base cocatalyst system..........................306

Table 6.2. ROP of tnHL with urea base cocatalyst system.........................307

Table 6.3. ROP of NL with (thio)urea base cocatalyst system.......................308

Table 6.4. ROP of tnNL with (thio)urea base cocatalyst system.....................309

Table 6.5. ROP of tnPDL with (thio)urea base cocatalyst system....................310

Table 6.6. ROP of tnEB with (thio)urea base cocatalyst system.......................311

Table 7.1. ROP Attempts of tnCLa with various catalysts...........................335

Table 7.2. ROP Attempts of L-tnLA with different catalysts.......................336 
Table 7.3. ABA Triblock Copolymers of tnCL and L-LA with Different Monomer

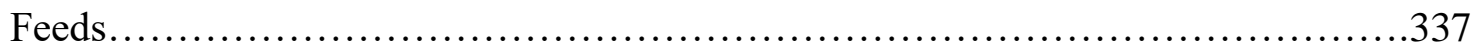




\section{LIST OF FIGURES}

FIGURE

PAGE

Figure 1.1. The Takemoto catalyst was the inspiration for the popular thiourea plus base catalyst system. Weaker base cocatalysts effect the ROP of lactide, while stronger bases open other monomers .47

Figure 1.2. Functionalizable monomers which undergo controlled ROP by $2 /$ base....48

Figure 1.3. Squaramide H-bond Donors for ROP of Lactide.......................49

Figure 1.4. Internal Lewis Acid Stabilized (Thio)ureas for ROP ...................50

Figure 1.5. Sulfonamide H-bonding Catalysts..............................51

Figure 1.6. Diphenyl Phosphate, Phosphoramidic and Imidodiphosphoric Acid Catalyzed

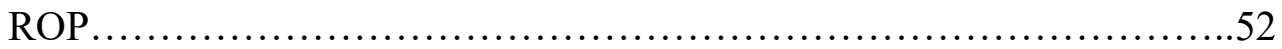

Figure 1.7. Phenol and Benzylic Alcohol H-bond Donors for ROP ................53

Figure 1.8. Azaphosphatrane H-bond Donor.................................54

Figure 1.9. Electrophilic Monomer Activation by Stable Cations...................55

Figure 1.10. Bronsted Acid and Base Cocatalysts for ROP ........................56

Figure 1.11. Ammonium Betaine Mediated ROP ................................57

Figure 1.12. Thiazoline and Oxazoline Bifunctional Catalysts....................58

Figure 2.1. For the ROP of VL, observed rate constant (kobs) vs $[\mathbf{1}]+[\mathrm{DBU}] \ldots \ldots . . .80$

Figure 2.2. The bases studied along with the respective binding curves to $1 \ldots \ldots \ldots . . .81$

Figure 2.3. Van't Hoff plots of binding between 1 and various bases................82

Figure 2.4. First order evolution of $[\mathrm{VL}]$ vs time when $[\mathbf{1}]=[\mathrm{DBU}]$ when $[\mathrm{VL}]=2 \mathrm{M}$,




Figure 2.5. First order evolution of $[\mathrm{VL}]$ vs time when $[\mathbf{1}]>[\mathrm{DBU}]=0.05 \mathrm{M}$ while $[\mathrm{VL}]$ $=2 \mathrm{M},[$ benzyl alcohol $]=0.04 \mathrm{M}$ in $\mathrm{C} 6 \mathrm{D} 6$ .84

Figure 2.6. First order evolution of [VL] vs time when [1] $=0.05 \mathrm{M}<[\mathrm{DBU}]$ while [VL] $=2 \mathrm{M},[$ benzyl alcohol $]=0.04 \mathrm{M}$ in $\mathrm{C} 6 \mathrm{D} 6$ .85

Figure 2.7. For the ROP of VL, observed rate constant vs $[\mathbf{1}]$ when $[\mathbf{1}]>[\mathrm{DBU}]=50$ mM. .86

Figure 2.8. For the ROP of VL, observed rate constant vs $[\mathrm{DBU}]^{-1}$ when $[\mathrm{DBU}]>[\mathbf{1}]=50$ $\mathrm{mM}$ 87

Figure 2.9. $M_{n}(G P C)$ and $M_{w} / M_{n}$ vs percent conversion for the $1 / B E M P$ catalyzed ROP of VL .88

Figure 2.10. First order evolution of [VL] vs time for the 1/BEMP catalyzed ROP of VL

Figure 3.1. Base and (thio)urea cocatalysts evaluated for ROP

Figure 3.2. $\mathrm{M}_{\mathrm{n}}$ vs conversion for the 2-S/MTBD catalyzed ROP of VL... .118 Figure 3.3. First order evolution of [VL] vs time for the 2-S/MTBD catalyzed ROP of VL 119

Figure 3.4. $\mathrm{M}_{\mathrm{n}}$ vs $[\mathrm{VL}]_{\mathrm{o}} /[\mathrm{I}]_{\mathrm{o}}$ for the 2-S/MTBD catalyzed ROP of VL 120

Figure 3.5. GPC traces of the polymer resulting from the 2-S/MTBD (5 mol\% each, $0.0499 \mathrm{mmol})$ cocatalyzed ROP and subsequent chain extension of VL (0.999 mmol, then $0.999 \mathrm{mmol}$ more) from 1-pyrenebutanol $(0.0199 \mathrm{mmol})$ in $\mathrm{C}_{6} \mathrm{D} 6(999 \mu \mathrm{L}) \ldots . .121$ Figure 3.6. Observed rate constant $\left(\mathrm{k}_{\mathrm{obs}}, \mathrm{min}^{-1}\right)$ vs $[\mathrm{MTBD}]$ in the 2-S/MTBD catalyzed

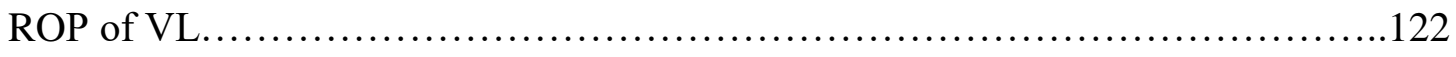
Figure 3.7. Observed rate constant $\left(k_{\mathrm{obs}}, \mathrm{h}^{-1}\right)$ vs $[\mathbf{2 - S}]$ in the 2-S/MTBD catalyzed ROP of 
Figure 3.8. $\mathrm{M}_{\mathrm{n}}$ vs conversion of VL for the 3-O/MTBD catalyzed ROP of VL......124 Figure 3.9. First order evolution of [VL] vs time for the 3-O/MTBD catalyzed ROP of VL 125

Figure 3.10. $\mathrm{M}_{\mathrm{n}}$ vs conversion for the 3-O/MTBD catalyzed ROP of CL...........126 Figure 3.11. First order evolution of [CL] vs time for the 3-O/MTBD catalyzed ROP of CL. 127

Figure 3.12. First order evolution of [CL] and [VL] vs time for the 3-O/MTBD catalyzed copolymerization of CL........................................... 128

Figure 3.13. GPC traces of the polymer resulting from the 3-O/MTBD $(1.67 \mathrm{~mol} \%$ each, $0.015 \mathrm{mmol})$ cocatalyzed ROP and subsequent chain extension of CL $(0.876 \mathrm{mmol}$, then $1.1723 \mathrm{mmol}$ more) from 1-pyrenebutanol $(0.035 \mathrm{mmol})$ in $\mathrm{C}_{6} \mathrm{D} 6$ $(219 \mu \mathrm{L})$ 129

Figure 3.14. Observed rate constant $\left(\mathrm{k}_{\mathrm{obs}}, \min ^{-1}\right)$ vs $[3-\mathrm{O}]$ in the 3-O/MTBD catalyzed

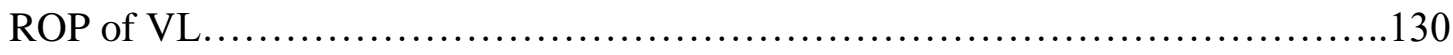

Figure 3.15. DFT B3LYP//6-31G** geometry optimized structures of 3-S.........131

Figure 3.16. DFT B3LYP//6-31G** geometry optimized structures of 3-O........132 Figure 3.17. MALDI-TOF of the PLA resulting from the 3-O/(tris[2(dimethylamino)ethyl]amine) catalyzed ROP of L-LA.

Figure 3.18. Downfield half of the ${ }^{H} \mathrm{H}$ NMR spectra (acetone + trace benzene-d6 (lock), $400 \mathrm{MHz}$ ) of (upper) 1-O, (middle) 2-O, and (lower) 3-O. The progressive downfield shift of the $\mathrm{NH}$ protons is indicative of increased (2-O vs 3-O) intramolecular Hbonding. 134 


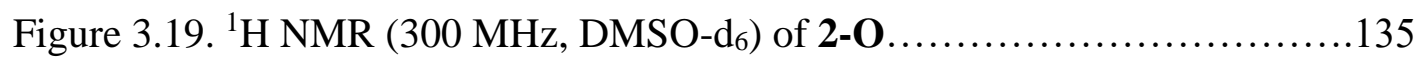

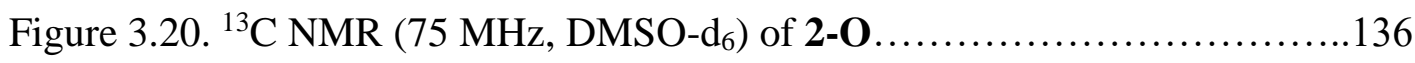

Figure 3.21. ${ }^{1} \mathrm{H}$ NMR (300 MHz, DMSO-d6) of 1-[3,5-bis(trifluoromethyl)phenyl

thiourea $]$-3-aminopropane............................................ 137

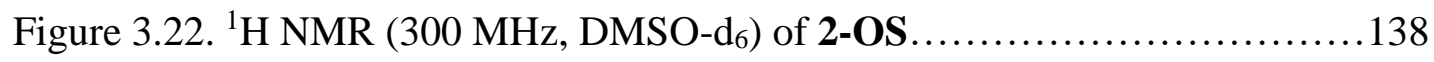

Figure 3.23. ${ }^{13} \mathrm{C}$ NMR $\left(75 \mathrm{MHz}\right.$, acetone- $\left.\mathrm{d}_{6}\right)$ of 2-OS ...........................

Figure 3.24. ${ }^{1} \mathrm{H}$ NMR (300 MHz, acetone- $\left.\mathrm{d}_{6}\right)$ of 3-O............................

Figure $3.25 .{ }^{13} \mathrm{C}$ NMR $\left(75 \mathrm{MHz}\right.$, acetone- $\left.\mathrm{d}_{6}\right)$ of 3-O.......................... 141

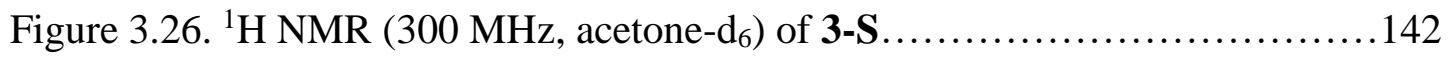

Figure 3.27. ${ }^{13} \mathrm{C}$ NMR (75 MHz, acetone-d6) of 3-S........................... 143

Figure 4.1. H-bond mediated ROP of VL................................ 180

Figure 4.2. Evolution of $[\mathrm{VL}]_{\mathrm{T}}$ vs time for ROPs with varied $[\mathbf{1}]_{\mathrm{EFF}} \ldots \ldots \ldots \ldots \ldots \ldots 1$

Figure 4.3. Total monomer concentration at equilibrium ([VL]T,eq) vs $[\mathbf{1}]_{\mathrm{EFF}} \ldots \ldots \ldots 182$

Figure 4.4. Concentration of VL vs time for the sequential addition of $\mathbf{1}$ to the ROP of

$\mathrm{VL}$ in progress....................................................... 183

Figure 4.5. Sequential addition of 2 to the ROP of VL........................ 184

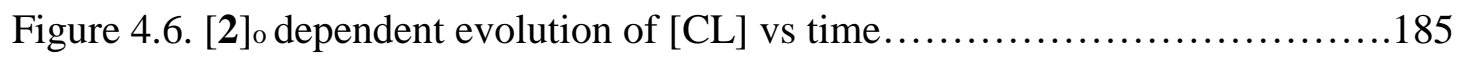

Figure 4.7. ${ }^{1} \mathrm{H}$ NMR spectra showing the polymerization of VL and subsequent

depolymerization of poly(valerolactone) upon the addition of $1 \ldots \ldots \ldots \ldots \ldots \ldots \ldots . \ldots 186$

Figure 4.8. Selected expanded ${ }^{1} \mathrm{H}$ NMR spectra of from the polymerization of VL and subsequent depolymerization of poly(valerolactone) upon the addition of $1 \ldots \ldots \ldots .187$

Figure 4.9. NMR tubes containing the top and bottom runs from Figure $4.2 \ldots \ldots . .188$

Figure 4.10. Reproduction of $\mathbf{1}$ addition experiment from Figure $4.3 \ldots \ldots \ldots \ldots \ldots . . .189$ 
Figure 4.11. [VL] $]_{\text {,eq }}$ Vs [catalysts $]_{\mathrm{o}}$ for the ROPs when the initial concentrations of cocatalysts are varied together......................................... 190

Figure 4.12. [VL] eq as a function of temperature............................ 191

Figure 4.13. Titration curve for the $\mathrm{BnOH} / \mathrm{DBU}$ binding in benzene-d $\mathrm{d}_{6} \ldots \ldots \ldots \ldots . .192$

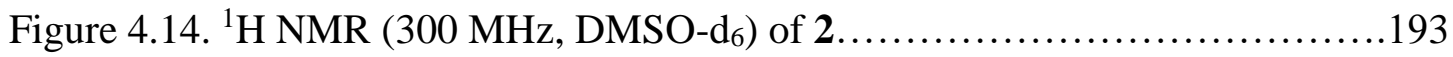

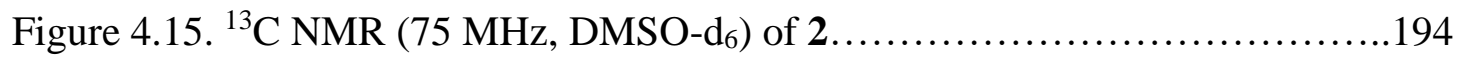

Figure 5.1. Evolution of $\mathrm{M}_{\mathrm{n}}$ vs conversion for the (upper) DBU catalyzed ROP of tnCL from benzyl alcohol..............................................219

Figure 5.2. Percent mass loss for PtnCL and copolymers with VL in acidic (0.25 M HCl), basic $(0.25 \mathrm{M} \mathrm{NaOH})$ and neutral (distilled water) conditions vs time. .220

Figure 5.3. MALDI-TOF of the PtnCL resulting from the 1/BEMP catalyzed ROP of

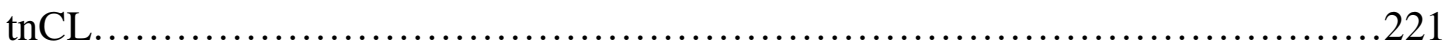

Figure 5.4. GPC trace of PtnCL resulting from the 1/BEMP catalyzed ROP of tnCL from pyrenebutanol...................................................222

Figure 5.5. GPC traces (UV) showing the evolution of the peak shape as a function of

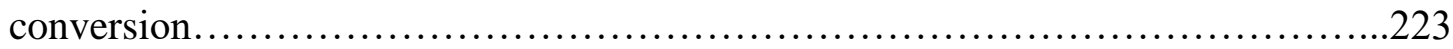

Figure 5.6. GPC traces (UV) of the polymer species resulting from allowing the ROP solution to stir with catalysts after full conversion (5h is full conversion)..........224 Figure 5.7. Temperature dependent equilibrium constant for the reversible ROP of tnCL catalyzed by TBD from benzyl alcohol .225

Figure 5.8. First order evolution of monomer vs time for the copolymerization of tnCL



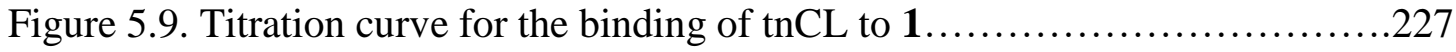


Figure 5.10. First order evolution of [tnCL] vs time in the 1/BEMP catalyzed ROP from benzyl alcohol...................................................228

Figure 5.11. The binding of ethyl acetate (or ethyl thionoacetate) to $\mathbf{1}$ is too low to be measured, and the binding of tnCL to 1 is: $\mathrm{Keq}=1.6$. .229 Figure 5.12. ${ }^{13} \mathrm{C} \mathrm{NMR}\left(75 \mathrm{MHz}, \mathrm{CDCl}_{3}\right)$ spectrum of the polymer resulting from the ROP of tnCL (2M, toluene) from benzyl alcohol (1 mol\%) catalyzed by DBU (5 mol\%) at room temperature. 230

Figure 5.13. ${ }^{13} \mathrm{C} \mathrm{NMR}\left(75 \mathrm{MHz}, \mathrm{CDCl}_{3}\right)$ spectrum of the polymer resulting from the ROP of tnCL (2M, toluene) from benzyl alcohol (1 mol\%) catalyzed by DBU (5 mol\%) at $100^{\circ} \mathrm{C}$ results in the formation of poly(thiono-co-thiocaprolactone) as evidenced by the two carbonyl resonances at $223 \mathrm{ppm}$ and $199 \mathrm{ppm}$ .231

Figure 5.14. ${ }^{13} \mathrm{C} \mathrm{NMR}\left(75 \mathrm{MHz}, \mathrm{CDCl}_{3}\right)$ spectrum of $\mathrm{P}(\mathrm{tnCL}-\mathrm{co}-\mathrm{VL})(50: 50) \ldots . . .232$ Figure 5.15. ${ }^{1} \mathrm{H}$ NMR $\left(300 \mathrm{MHz}, \mathrm{CDCl}_{3}\right)$ spectrum of $\mathrm{P}(\mathrm{tnCL}-\mathrm{co}-\mathrm{VL})(50: 50) \ldots . .233$ Figure 5.16. ${ }^{1} \mathrm{H}$ NMR $\left(300 \mathrm{MHz}, \mathrm{CDCl}_{3}\right)$ spectrum of tnCL....................234 Figure 5.17. ${ }^{13} \mathrm{C} \mathrm{NMR}\left(75 \mathrm{MHz}, \mathrm{CDCl}_{3}\right)$ spectrum of tnCL....................235 Figure 5.18. ${ }^{1} \mathrm{H}$ NMR $\left(300 \mathrm{MHz}, \mathrm{CDCl}_{3}\right)$ spectrum of PtnCL...................236 Figure 5.19. Calculated (DFT B3LYP//6-31G**) electrostatic potential of atoms in the

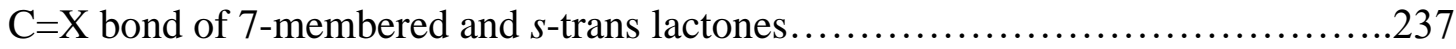
Figure 6.1. ${ }^{1} \mathrm{H}$ NMR $\left(400 \mathrm{MHz}, \mathrm{CDCl}_{3}\right)$ spectrum of tnEB ......................295 Figure $6.2 .{ }^{13} \mathrm{C}$ NMR $\left(100 \mathrm{MHz}, \mathrm{CDCl}_{3}\right)$ spectrum of $\operatorname{tnEB} \ldots \ldots \ldots \ldots \ldots \ldots \ldots \ldots . . \ldots 296$ Figure 6.3. ${ }^{13} \mathrm{C}\left(75 \mathrm{MHz}, \mathrm{CDCl}_{3}\right)$ spectrum of homopolymer of PtnHL $\left(2 \mathrm{M}, \mathrm{C}_{6} \mathrm{D}_{6}\right)$ initiated from benzyl alcohol (1 mol\%) catalyzed by TCC/BEMP (5 mol\% each), displaying almost no carbonyl peak but thiocarbonyl resonance at $224 \mathrm{ppm}$ 
Figure 6.4. ${ }^{13} \mathrm{C}\left(100 \mathrm{MHz}, \mathrm{CDCl}_{3}\right)$ spectrum of homopolymer of PtnNL (2M, acetone- $\left.d_{6}\right)$ initiated from benzyl alcohol (1 mol\%) catalyzed by TCC/BEMP (5 mol\% each), displaying almost no carbonyl peak but thiocarbonyl resonance at $224 \mathrm{ppm} . \ldots \ldots \ldots . .298$ Figure $6.5 .{ }^{13} \mathrm{C}\left(100 \mathrm{MHz}, \mathrm{CDCl}_{3}\right)$ spectrum of homopolymer of PtnPDL (5M, toluene) initiated from benzyl alcohol (1 mol\%) catalyzed by TCC/BEMP (5 mol\% each), displaying almost no carbonyl peak but thiocarbonyl resonance at 224 ppm 299

Figure 6.6. ${ }^{13} \mathrm{C}\left(75 \mathrm{MHz}, \mathrm{CDCl}_{3}\right)$ spectrum of homopolymer of PtnEB (2M, toluene) initiated from benzyl alcohol ( $1 \mathrm{~mol} \%)$ catalyzed by TCC/BEMP ( $5 \mathrm{~mol} \%$ each) at $80^{\circ} \mathrm{C}$, displaying almost no carbonyl peak but thiocarbonyl resonance at 224 ppm .300

Figure 6.7. Temperature dependent equilibrium constant for the reversible ROP of tnHL (1M, C6 6 6) catalyzed by TBD (5 mol\%) from benzyl alcohol (1 mol\%).......301

Figure 6.8. Evolution of $\mathrm{Mn}$ vs conversion for the (a) TCC/MTBD (5 mol\% each) catalyzed ROP of HL (2M in $\left.\mathrm{C}_{6} \mathrm{D}_{6}\right)$ initiated from benzyl alcohol (1 mol\%); (b) TCC/MTBD (5 mol\% each) catalyzed ROP of tnHL (2M in $\mathrm{C}_{6} \mathrm{H} 6$ ) initiated from benzyl alcohol (1 mol\%) .302

Figure 6.9. First order evolution of (a) $[\mathrm{HL}]$ and (b) [tnHL] vs time in the TCC/MTBD catalyzed ROP from benzyl alcohol. 303

Figure 6.10. Evolution of $\mathrm{Mn}$ vs conversion for the (a) TCC/BEMP (5 mol\% each) catalyzed ROP of NL (2M in acetone- $\left.d_{6}\right)$ initiated from benzyl alcohol (1 mol\%); (b) TCC/BEMP ( $5 \mathrm{~mol} \%$ each) catalyzed ROP of tnNL ( $2 \mathrm{M}$ in acetone- $\left.d_{6}\right)$ initiated from benzyl alcohol (1 mol\%) .304 
Figure 6.11. First order evolution of (a) [NL] and (b) [tnNL] vs time in the TCC/BEMP catalyzed ROP from benzyl alcohol................................305

Figure 7.1. ${ }^{1} \mathrm{H}$ NMR (400 MHz, $\left.\mathrm{CDCl}_{3}\right)$ of L-tnLA monomer.....................332

Figure 7.2. ${ }^{13} \mathrm{C}$ NMR $\left(100 \mathrm{MHz}, \mathrm{CDCl}_{3}\right)$ of L-tnLA monomer.....................333

Figure 7.3. ${ }^{13} \mathrm{C} \mathrm{NMR}\left(100 \mathrm{MHz}, \mathrm{CDCl}_{3}\right)$ spectrum of $\mathrm{P}($ LLA-co-tnCL-co-LLA)

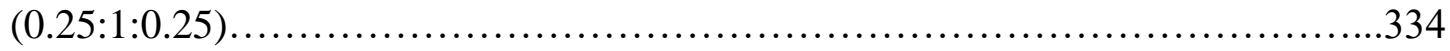

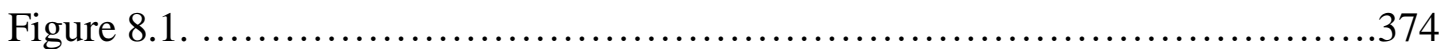




\title{
MANUSCRIPT - I
}

\author{
Submitted for publication in Organic Catalysts for Polymerization
}

Bifunctional and Supramolecular Organocatalysts for Polymerization

Kurt V. Fastnacht, Partha P. Datta and Matthew K. Kiesewetter

Chemistry, University of Rhode Island, Kingston, RI, USA

Corresponding Author: Matthew Kiesewetter, Ph.D.

Chemistry

University of Rhode Island

140 Flagg Road

Kingston, RI, 02881, USA

Email address: mkiesewetter@chm.uri.edu 


\begin{abstract}
Bimolecular, H-bond mediated catalysts for ROP — thiourea or urea plus base, squaramides, and protic acid/base pairs, among others - are unified in a conceptual approach of applying a mild Lewis acid plus mild Lewis base to effect ROP. The bimolecular, and other supramolecular catalysts for ROP, produce among the best-defined materials available via synthetic chemistry through a delicately balanced series of competing chemical reactions by interacting with substrate at an energy of $<4 \mathrm{kcal} / \mathrm{mol}$. These catalysts are among the most controlled available for ROP. Part of this arises from the modular, highly-tunable nature of dual catalysts, which effect extremely controlled ROP of a host of cyclic monomers. The broader field of organocatalytic polymerization is a bridge between the disparate worlds of materials chemist (ease of use) and synthetic polymer chemist (mechanistic interest). The cooperative and collegial nature of the organocatalysis for ROP community has facilitated the synergistic evolution of new mechanism to new abilities - in monomer scope, polymer architecture and level of reaction control.
\end{abstract}




\section{INTRODUCTION}

The catalysts in this chapter conduct polymerization via non-nucleophilic, H-bond mediated pathways. These catalysts include thiourea or urea plus base, squaramides, and protic acid/base pairs — which are unified in a conceptual approach of applying a mild Lewis acid plus Lewis base to effect ring-opening polymerization (ROP) — as well as other supramolecular catalysis. This class of catalyst produces among the best-defined materials available via synthetic chemistry through a delicately balanced series of competing chemical reactions by interacting with substrate at an energy of $<4 \mathrm{kcal} / \mathrm{mol} .^{1,2}$ Indeed, the multitude of simultaneous chemical reactions in a typical supramolecular polymerization is as much awe-inspiring as it is difficult to comprehend, and changing any one factor $(\mathrm{H}-$ bond donor, H-bond acceptor, reagent, solvent, temperature, etc.) impacts all the interactions in solution. The polymerization catalysis community has been building an understanding of these systems incrementally over the last decade, and our understanding and abilities in rate, selectivity, diversity of polymer architectures available and reaction control continue to evolve.

The purview of the catalysts in this chapter is ring-opening polymerization (ROP), especially of cyclic esters and carbonates. Conceptually, the catalysts in this chapter are ideally suited to effect highly controlled polymerizations. Catalysts for the ROP of lactones and carbonates effect polymerization by 1) activating the chain-end, 2) activating the monomer, or 3) activating both. By separating the roles of monomer and chain-end activation into discrete functions, the dual catalysts can be separately tuned to effect enchainment and thus minimize side reactions. Conceptually, a dual catalyst consists of 
both a hydrogen bond donor (HBD) (e.g. urea or thiourea) for monomer activation and a hydrogen bond acceptor (HBA) (e.g. tertiary amines) for chain-end activation. Such dual catalysts may be a single molecule, but in common practice, bimolecular cocatalysts are employed to activate monomer and initiator alcohol/chain end separately, Scheme 1.1.

The fountainhead of dual catalysis is undoubtedly the 2005 manuscript and its follow-up from Hedrick and Waymouth. ${ }^{3,4}$ The roots of organocatalysis reach back more than 100 years to synthesis of quinine alkaloids, ${ }^{5}$ and, in fact, organocatalysts were among the earliest catalysts for the synthesis of polyesters. ${ }^{6}$ The renaissance of organocatalysis circa 2000 saw the application of supramolecular catalysts for small molecule synthesis. ${ }^{7}$ However, it was the veritable Johnny Appleseeds of organocatalytic polymerization that disclosed supramolecular catalysts for ROP along their continuing journey of discovery and subsequently nurtured field such that it now encompasses many branches of questioning by several research groups. ${ }^{4}$ The first supramolecular catalyst for ROP (the Takemoto catalyst, 1, Figure 1.1) was adapted from the work of Takemoto, who used chiral H-bonding catalysts for asymmetric Michael reactions. ${ }^{8}$ The thiourea/amine base catalyst 1 was introduced into the polymerization community for the organocatalytic ROP of lactide. ${ }^{4}$ The inspired (and somewhat miraculous) step of separating the roles of HBD and HBA into discrete cocatalysts facilitated modulation of the individual cocatalysts leading to the ROP of other monomers and launched a field, Figure 1.1.3,4

The class of organic molecules that effects catalysis via supramolecular interactions are among the most controlled catalysts available for ROP. Part of this is due to the modular, highly-tunable nature of dual catalysts, which effect extremely controlled ROP (PDI $\left.=Ð=D_{m}=M_{w} / M_{n}<1.1\right)$ of a host of different cyclic monomers. ${ }^{9}, 10$ Most of the 
research in the field of dual catalysis for organic polymerizations has been dedicated to the ROP of cyclic esters and carbonates; however, other monomers will be mentioned. Dual catalysts effect living polymerizations, which is a type of chain growth polymerization that proceeds without chain-transfer or termination. ${ }^{11}$ This is ultimately a kinetic distinction, and it is often said that a polymerization exhibits the characteristics of a 'living' polymerization: molecular weights $\left(M_{n}\right)$ are predictable from $[\mathrm{M}]_{\mathrm{o}} /[\mathrm{I}]_{\mathrm{o}}$, linear evolution of $M_{n}$ with conversion, first order consumption of monomer and narrow weight distributions $\left(M_{w} / M_{n}\right){ }^{11}$ In practice, these conditions arise when a polymerization has a fast initiation rate relative to propagation rate and few to no side reactions. We shall refrain from pointing out when a catalyst (system) exhibits the characteristics of a 'living' polymerization, and rather point out when it is either especially well-controlled or exhibits low levels of control. Several, thorough reviews have been conducted in the wider field, ${ }^{12-21}$ but not with quite the level of focus that the current platform provides. Hence, we will attempt to emphasize the virtues and deficits of the various catalysts, especially as they contrast to other organic catalysts for polymerization. 


\section{DUAL CATALYSTS}

The dual catalysts for polymerization are a logical mechanistic conclusion of early organocatalysts for ROP, and H-bond mediated (supramolecular) polymerization mechanisms have been implicated for catalysts in a host of architectures. ${ }^{2,22-24}$ For example, the pyridine bases 4-(dimethylamino)pyridine (DMAP) and 4pyrrolidinopyridine (PPY) have been proposed to effect the zwitterionic ROP of lactones. $^{25-28}$ However, subsequent mechanistic studies suggest that the nucleophilic and H-bonding pathways are both accessible with the hydrogen-bonded pathway being energetically favorable. ${ }^{29-32}$ An alcohol-activated mechanism of enchainment has been proposed for the phosphazene bases (e.g. $\mathrm{P}_{1}-\mathrm{Bu}, \mathrm{P}_{2}-t \mathrm{Bu}, t-\mathrm{BuP}_{4}, \mathrm{BEMP}$ in Figure 1.1), which have been shown to effect the ROP of lactones in the presence of alcohols. ${ }^{24,33-36} \mathrm{~A}$ similar pathway can be envisaged for the guanidine and amidine bases, 7-methyl-1,5,7triazabicyclo[4.4.0]dec-5-ene (MTBD) and 1,8-diazabicyclo[5.4.0]undec-7-ene (DBU). ${ }^{2,23}$ The dual catalysis conceptual approach of separately activating the monomer and propagating chain end arises from these early organocatalysts which often suffered from low activity or reaction control. ${ }^{4,22,23}$ By separately activating both reactive species, greater specificity and control can be achieved.

Thiourea H-bond Donors

As with many organocatalysts for polymerization, thiourea/base mediated ROP has its roots in small molecule transformations where Jacobsen et al. had shown that an array of ureas and thioureas were effective catalysts for Mannich, Strecker, Pictet-Spengler, and hydrophosphonylation reactions, ${ }^{37-44}$ among others. ${ }^{7}$ Indeed, the parent dual catalyst, 1, 
for ROP was used by Takemoto et al. for enantioselective aza-Henry and Michael additions. ${ }^{8,45,46}$ In the seminal polymerization work, $\mathbf{1}$ was shown to effect the ROP of lactide with, at the time, remarkably living behavior. ${ }^{4}$ Incredibly, failure to quench the reaction after full conversion to polymer did not result in broadening of molecular weight distribution, signifying very minimal transesterification, and minimal racemization was observed. ${ }^{4}$ When the HBD and HBA roles of 1 were divided into separate HBD (2) and HBA ( $N, N$-dimethylcyclohexylamine) molecules, a field of research was born, Figure 1.1. Polylactide formation was only successful when both 2 and $N, N$-dimethylcyclohexylamine were applied simultaneously, and a range of non-H-bonding solvents were found to facilitate ROP (e.g. chloroform, dichloromethane and toluene), while THF and DMF failed. ${ }^{4}$ A host of alkylamine cocatalysts (with 2) has been shown to be effective for the ROP of lactide. ${ }^{3,47}$ Strong bases - MTBD, DBU and later BEMP - are effective cocatalysts with 2 for the ROP of other monomers: $\delta$-valerolactone (VL), $\varepsilon$-caprolactone $(\mathrm{CL})$, trimethylene carbonate (TMC), MTC and others, Figures 1.1 and 1.2.,48 The stronger bases will effect a less-controlled ROP of lactide in the absence of thiourea, but thiourea plus strong base is necessary to open other lactones and carbonates with reasonable rates. ${ }^{2}$ The ROP of $\beta$-butyrolactone (BL) is not easily performed with most organocatalysts. ${ }^{2,49} \mathrm{~A}$ common red herring in the ROP literature will attribute unexplainable and otherwise 'spooky' observations to ring strain. Indeed, it is often observed for organocatalytic ROP that enchainment rates $\left(\mathrm{k}_{\mathrm{LA}}>\mathrm{k}_{\mathrm{VL}}>\mathrm{k}_{\mathrm{CL}}>\mathrm{k}_{\mathrm{BL}}\right)^{50,51}$ have no correlation to ring strain as measured by equilibrium monomer concentration, $[\mathrm{M}]_{\mathrm{eq}}$ : $[\mathrm{VL}]_{\mathrm{eq}}\left(\right.$ low strain) $\gg[\mathrm{CL}]_{\mathrm{eq}} \sim$ $[\mathrm{LA}]_{\mathrm{eq}} \gg>[\mathrm{BL}]_{\mathrm{eq}}$ (high strain)..$^{50,51}$ 
The origin of the high selectivity for monomer is thought to arise from selective binding of thiourea to monomer versus polymer. The binding constants of lactones (s-cis esters) and open $s$-trans esters to 2 were measured by ${ }^{1} \mathrm{H}$ NMR titration. ${ }^{2}$ The $s$-trans ester (ethyl acetate) exhibited minimal binding while binding constants of $\mathrm{K}_{\mathrm{eq}} \sim 40$ were observed between VL or CL and thioureas. ${ }^{2}$ Thiourea H-bond donors have subsequently been shown to bind much more strongly to base cocatalyst, where the nature of the cocatalyst binding constant is a better indicator of co-catalytic activity than monomer binding. ${ }^{48,52-54}$ The cocatalyst binding can be inhibitory to catalysis under the proper circumstances. ${ }^{48,52-55}$ However, the rapid, reversible and promiscuous binding of thiourea to several reagents in solution appears to reduce the overall order of the transformation $\left(\right.$ Rate $\left.=\mathrm{k}[\mathrm{M}][\mathrm{I}]_{\mathrm{o}}[\text { cocatalysts }]_{\mathrm{o}}\right),{ }^{48,53,54}$ and the notion of thiourea as an entropy trap prior to enchainment has been repeatedly reinforced. ${ }^{56,57}$ Indeed, our understanding of the multitude of interrelated interactions that occur during a (thio)urea/base mediated ROP continues to unfold. ${ }^{58-60}$ The theme of competitive binding repeats throughout the literature, including the amide and indole H-bond donor catalysts applied to the ROP of LA which are structurally reminiscent to (thio)ureas. ${ }^{52,61,62}$ The major take-away message is that the high selectivity of H-bonding catalysts appears to rise from two sources, 1) selective binding of thiourea to monomer versus polymer, and 2$)$ strong binding $\left(\mathrm{K}_{\mathrm{eq}}=100\right.$ - 4,200) of thiourea to base cocatalysts which reduces their relative affinity to other reagents and can become an inhibitory interaction. ${ }^{48,52}$ The high selectivity for $s$-cis esters and carbonates has been used to great effect for the generation of classes of functionalizable monomers, Figure 1.2. ${ }^{63-68}$

Thiourea-mediated Stereoselective ROP 
The stereoselective ROP of $r a c$-lactide is an attractive method for the generation of polylactides (PLAs) with highly regular or novel stereosequences, and the modular scaffold and rich diversity of chiral thiourea H-bond donors has proved an enticing target for several groups. The ROP of rac- or meso-lactide to generate highly tactic PLA has been well documented. ${ }^{69-71}$ Briefly, stereoselective enchainment of the chiral monomer onto the chiral chain end can occur via control rendered by 1) the propagating chain end, 2) a chiral catalyst or 3) a mixed mechanism. ${ }^{69,72,73}$ For the ROP of rac-LA, a high probability of propagating with retention of stereochemistry $\left(P_{m}=\right.$ probability of meso enchainment $)$ will result in a highly isotactic PLA. ${ }^{3,69}$ Waymouth and Hedrick reported the $(\boldsymbol{R}, \boldsymbol{R})-\mathbf{1}$ mediated ROP of $r a c$-lactide to proceed with modest selectivity $\left(P_{m}=0.76\right)$; however, $2 /(-)$-sparteine catalyzed ROP of $r a c$-LA rendered similar selectivity $\left(P_{m}=0.77\right){ }^{3}$ The polymers did not display a melting point, suggesting low stereoregularity. ${ }^{3}$ Exceeding these $P_{m}$ values has become a benchmark of sorts for the stereoselective ROP of rac-lactide by H-bonding catalysts. Despite its successes, (-)-sparteine itself fell out of favor as an organocatalyst when it became scarce circa 2010, but a replacement base, benzyl bispidine, was disclosed which renders similar reaction rates and selectivity in the ROP of $r a c$-lactide with $2, P_{m}=$ $0.74 .{ }^{47,74}$

Recent research into photoresponsive azobenzene-based thiourea, $\mathbf{3}$, for the ROP of rac-lactide suggests a conceptual approach to switchable organocatalysts for ROP. ${ }^{75,76}$ Catalysts that are switchable by external stimuli (i.e. redox pathways, lights, coordination chemistry etc. $)^{76-94}$ offer an attractive route to advanced catalyst structures and, presumably, polymer architectures. Thiourea $\mathbf{3}$ is based on the classic photoswitchable azobenzene moiety, Scheme 1.3. The trans-3 isomer contains an open active site for 
coordination of lactide by $\mathrm{H}$-bonding whereas cis-3 is blocked by intramolecular $\mathrm{H}-$ bonding to the nitro group. The 3/PMDETA (Scheme 1.3) cocatalyzed ROP of rac-LA proceeded with moderate isoselectivities $\left(P_{\mathrm{m}} \sim 0.74\right)$ at room temperature. ${ }^{75}$ The ROP was proposed to proceed from the trans-isomer, presumably via a chain-end control mechanism. ${ }^{3,75}$ We make the safe prediction that switchable organic catalysts for ROP will play an important role in the next decade. ${ }^{76,91}$

A thiourea with pendant cinchona alkaloid, 5 in Scheme 1.4, provided the first example of isotactic-rich, stereogradient PLA via kinetic resolution polymerization with organocatalysts. The bifunctional 4 (internal nitrogen base) effected the ROP of rac-LA to generate isotactic-rich PLA, $P_{m}=0.69 .{ }^{95}$ No transesterification was observed in MALDI-TOF MS, and almost no epimerization was observed. Polymerization experiments, isolation of residual monomer and analysis by chiral HPLC suggest that the stereoselectivity in the 4-catalyzed polymerization of rac-LA arises from the kinetic resolution by the catalyst/initiator to produce enantioenriched (stereogradient) PLAs. This motif was later incorporated into a thiourea/BINAM-containing organocatalyst, 5 (Scheme 1.4), for the kinetic resolution ROP of lactide. ${ }^{96}$ This stereoselective ROP scheme arguably the current gold standard - used an epimerization catalyst to transform meso- to rac-LA which 5 was able to enchain to isotactic poly(l-lactide) with high selectivity, $\mathrm{k}_{S} / \mathrm{k}_{\mathrm{R}}$ $=53 .{ }^{96}$ Not surprisingly, solvent (and other reaction conditions) dramatically perturb the selectivity. ${ }^{96}$ It should also be noted that structurally similar H-bond donors failed to produce ROP with appreciable rates or selectivities, ${ }^{95,96}$ which highlights a challenge of stereoselective, organocatalytic ROP. Indeed, a significant amount of inspiring ground work exists upon which to build highly successful stereoselective catalysts for ROP, and 
the field could proceed along this trial and error pathway. However, more fundamental information that might provide a solid mechanistic basis for a path forward may save a tremendous amount of effort.

Squaramides

The squaramide H-bond donor scaffold has been used to great success in small molecule catalysis ${ }^{97}$ and may represent an underexplored opportunity for polymer synthesis. Guo et al. examined squaramides for the ROP of l-lactide in dichloromethane at room temperature, initiated from benzyl alcohol. ${ }^{98}$ Squaramide $\mathbf{6}$ was unable to effect polymerization alone but was active with tertiary amine, (-)-sparteine, cocatalyst, Figure 1.3. H-bond donor 6 plus sparteine exhibits similar activity for ROP of lactide versus thiourea $\mathbf{2}$, and squaramides with no electron withdrawing substituents saw less conversion than their electron-deficient counterparts. ${ }^{98}$ A slate of bifunctional squaramide catalysts, 7, was also evaluated for ROP, Figure 1.3.99,100 The bifunctional catalyst 7-Me displayed reduced activity versus pentyl groups on the amine motif 7 , which was the only one of the examined structures to achieve full conversion in $24 \mathrm{~h} .{ }^{99}$ No epimerization was observed during polymerization. A classic $\mathrm{H}$-bond mediated mechanism of enchainment was corroborated by NMR titration studies. ${ }^{99}$ The H-bonding ability of squaramides is perturbed versus that of thioureas, ${ }^{99}$ but they have approximately the same acidity (Schreiner's thiourea (8) $\mathrm{p} K_{\mathrm{a}}=8.5 ; 6 \mathrm{p} K_{\mathrm{a}}=8.4$; both in DMSO). ${ }^{101,102}$ The altered structures possessing minimally altered $\mathrm{pK}_{\mathrm{a}}$ may have unseen implications for nascent imidate-mediated ROP, see below. 


\section{RATE-ACCELERATED DUAL CATALYSIS}

From the very early days of the field, thiourea/base cocatalysts exhibited remarkably controlled ROP, so remarkable that the poor activity and productivity of the catalysts could be justified. However, with the application of $N$-heterocyclic carbene (NHC) and TBD organocatalysts to ROP, it became very clear that organocatalysts could possess activity to rival that of metal catalysts. ${ }^{16,23,49}$ The dream of combining the rate of NHCs or TBD with the high selectivity of thiourea/base systems became an alluring research goal for several groups. One route that can be envisaged uses internal Lewis acids to stabilize the (thio)urea as it binds to monomer. The challenge became finding synthetically accessible (thio)ureas with Lewis acids that are compatible with ROP.

Internal Lewis Acid Enhanced H-Bond Donors

A urea H-bond donating catalyst with an internal boronate ester, 9, displayed enhanced activity versus its parent urea, 10 (Figure 1.4). HBD 9 was applied with sparteine cocatalyst for the ROP of LA at room temperature $\left(\mathrm{k}_{2} / \mathrm{k}_{9} \sim 1\right) .{ }^{103}$ Importantly, the ROP of LA with 9/sparteine showed good control and maintained a narrow molecular weight distribution $\left(M_{w} / M_{n} \sim 1.18\right)$ for days after the reaction had finished (initial $M_{w} / M_{\mathrm{n}} \sim 1.16$ ), indicating minor transesterification. This motif is an extreme example of the internal $\mathrm{H}$ bond stabilization that is thought to be present in all (thio)ureas bearing electron deficient aryl rings. ${ }^{104}$

Multi (thio)urea Catalysts

Mechanistic studies on 2 /base cocatalyzed ROP led to the development of highly

effective bis- and tris-(thio)urea H-bond donors. ${ }^{53,105}$ In general, urea HBDs are more active than thioureas, and tris-donors are more active than bis- which are more active than 
mono-; although tris-thiourea (14) is markedly inactive, Scheme $1.5 .^{53,105}$ These general trends hold for most monomers that have been examined, but the rate accelerations are most dramatic for the slower monomers (i.e. CL). ${ }^{53,105}$ Just as with 2, weak alkylamine base cocatalysts are required for the ROP of lactide with $\mathbf{1 1 - 1 5},{ }^{4,47,53}$ but strong base cocatalysts are required for $\mathrm{VL}, \mathrm{CL}$ and carbonate monomers. ${ }^{2,105}$ For the trisurea (15)/BEMP cocatalyzed ROP of CL, a 500 times increase in rate is observed versus 2/BEMP, and the reaction is more controlled. ${ }^{48,105}$ A typical (thio)urea/base cocatalyzed ROP is run $\sim 2 \mathrm{M}$ monomer and displays good control for $M_{n}$ from $[\mathrm{M}]_{\mathrm{o}} /[]_{\mathrm{o}}=\sim 20$ $500,,^{2,53,105}$ although enhanced (vs 2) weight control is observed for $\mathbf{1 3}$ and $\mathbf{1 5}$ at higher $[\mathrm{M}]_{\mathrm{o}} /[\mathrm{I}]_{\mathrm{o}}{ }^{105}$ The comparisons above are controlled for mol percent (thio)urea moiety in the ROP; typical catalyst loadings are $5 \mathrm{~mol} \%$ mono-(thio)urea/base; $2.5 \mathrm{~mol} \%$ bisdonor/base; 1.67 mol\% tris-donor/base..$^{2,105}$

An activated-(thio)urea mechanism is proposed for multi-H-bond donor mediated ROP in non-polar solvent, but urea H-bond donors remain highly-active in polar solvent. Kinetic studies on the several systems in benzene- $d_{6}$ reveal the (thio)urea ROPs to be first order in monomer, initiator, and cocatalysts, suggesting one mono-/bis-/tris-H-bond donor acting at one monomer in the transition state. ${ }^{48,53,54,105} \mathrm{H}$-bonds are electrostatic in nature and have low directionality, ${ }^{106}$ which allows for the possibility of multi-(thio)ureas directly activating monomer in a multi-activation mechanism. Computational models suggest that tristhiourea $\mathbf{1 4}$ is C3 symmetric (all H-bonded), ${ }^{105}$ and an analogue of $\mathbf{1 5}$ with n-propyl (versus ethyl) linking arms is highly inactive for ROP, ${ }^{107}$ suggesting that the (thio)urea moieties prefer to bind to themselves. These experiments, along with computational studies, suggest an activated-(thio)urea mechanism is operative in non-polar solvent. ${ }^{105}$ 
Traditional H-bonding catalysts (e.g. 2/base) become very inactive in polar solvent, which limits their utility. ${ }^{3}$ The urea HBDs, however, remain highly active in polar solvents (e.g. acetone and THF). ${ }^{105,108}$ Recent, and still-evolving, studies suggest that a different mechanism involving urea anions is operative in polar solvent. ${ }^{58-60}$

Urea and Thiourea Anions

The deprotonation of urea or thiourea with strong bases (alkoxides or metal hydrides) has been shown to produce the corresponding urea anion or thiourea anion (also: imidate or thioimidate) which are incredibly active for the ROP of lactones. ${ }^{59,60}$ An active catalyst system generated by the treatment of urea 17 with potassium methoxide (KOMe) in $\mathrm{THF}$ results in the extremely active ROP of 1-lactide at room temperature, Scheme 1.6. ${ }^{59,60}$ The same ROP with KOMe alone slowed almost 200 times while broadening $M_{w} / M_{n}(2.22$ versus 1.06$)$, and the $17 / \mathrm{KOMe}$ cocatalyst system is $\sim 25$ times more active than thiourea anion motif. ${ }^{59,60}$ Polymerizations with VL and CL were also completed within seconds. ${ }^{59}$ An ROP with similar activity can be achieved by a urea (e.g. 16) plus strong organic base (e.g. MTBD, DBU, BEMP) cocatalyzed ROP. ${ }^{108}$ The latter method may be operationally simpler, and urea plus organic base cocatalyzed ROP may be more controlled, especially post polymerization. ${ }^{108}$ The rates of the two methods appear to be very similar and mark a departure from early H-bond mediated ROP: seconds instead of hours or days! Remarkably, the ROPs remain highly controlled.

The urea/base cocatalyst systems operate by a different mechanism than classic $\mathrm{H}$ bond mediated ROP. For the urea/alkali base cocatalyzed ROP, the proton transfer to form the 'hyperactive' (thio)imidate is largely irreversible. Hence, more acidic (thio)ureas are thought to generate more basic (thio)imidates, resulting in faster catalysis. Indeed, there is 
a negative linear correlation between $\ln (\mathrm{kp})$ against number of $\mathrm{CF}_{3}$ substituents, ${ }^{59,108}$ and Schreiner et al. reported a linear reduction in $\mathrm{p} K_{\mathrm{a}}$ with number of $\mathrm{CF}_{3}$ substituents on the diaryl ureas and thioureas in DMSO. ${ }^{102,109}$ This mechanism is reminiscent of a bifunctional TBD-mediated ROP of lactones, ${ }^{23,59}$ where the imidate can serve as both H-bond donor and acceptor. This same mechanism is believed to be operative for bis- and tris-urea H-bond donors in polar solvent as well. ${ }^{48,53,105,108}$

An antibacterial compound, triclocarban (TCC, Scheme 1.6), was shown to be a very effective H-bond donating catalyst for the ROP of lactones when used with organic base cocatalysts. ${ }^{108}$ It was proposed that this compound effects ROP through the same mechanism as other urea/strong base mediated polymerizations, and TCC/BEMP displays the same approximate rate and control behavior as trisurea (15)/BEMP, although the trisurea is more active $\left(\mathrm{k}_{15} / \mathrm{k}_{\mathrm{TCC}} \sim 4, \mathrm{VL}\right) .{ }^{105,108}$ We anticipate that the movement towards readily available reagents will prompt wider adoption of organocatalysts and facilitate new applications; the success of TBD may be due, at least in part, to its commercial availability. To demonstrate this point, TCC/base cocatalyzed ROP was applied to the solvent-free polymerization of several lactones, which was previously limited due to 1) the presumed inactivity of urea HBDs in polar (monomer) solvent, and 2) the large amounts of catalyst required for neat conditions. ${ }^{58}$ Solvent-free ROP catalyzed by TCC/base allowed for the one-pot synthesis of di- and tri-block copolymers, and TCC/alkylamines were effective for the solvent-free ROP of LA, ${ }^{58}$ a longstanding challenge. ${ }^{110}$ The reactions remained highly controlled and 'living' in nature despite solidifying prior to full conversion. 


\section{NON-(THIO)UREA LEWIS ACID/BASE CATALYSIS}

Sulfonamides, Phosphoric and Phosphoramide H-bond Donor/Acceptors

A selection of mono- and bis-sulfonamide HBDs which have been applied with base cocatalysts for the ROP of LA are shown in Figure 1.5. The 18/DMAP cocatalysts produced the most rapid ROP of LA of the HBDs examined, and it was well-controlled. ${ }^{111}$ Structurally similar catalysts, $\mathbf{1 9}$ and $\mathbf{2 0}$, were less active, and no monosulfonamide/base cocatalyzed ROPs of LA have been shown to reach full conversion in $24 \mathrm{~h}$. Neither mononor bis-sulfonamides promoted the ring opening of LA in the absence of an amine cocatalyst. For the monosulfonamides, it was suggested that low catalyst activity might arise from reduced H-bond donation versus the bis donors. ${ }^{111}$ This account is consistent with observations for the mono-, bis- and tris-(thio)urea H-bond donors. ${ }^{105}$

Phosphoric and phosphoramidic acids, the weak acidity of which contrasts with strong acids used for electrophilic monomer activated $\mathrm{ROP},{ }^{13}$ can act as bifunctional organocatalysts for ROP. ${ }^{112-117}$ Diphenyl phosphate (21), phosphoramidic (22) and imidodiphosphoric (23) acids were used for the ROP of cyclic esters and carbonates, Figure 1.6. Catalysts $\mathbf{2 1}$ and $\mathbf{2 2}$ were found to be active towards the ROP of CL, yielding conversion to polymer in 5.5 and $1.5 \mathrm{~h}$, respectively. ${ }^{112}$ Catalyst $\mathbf{2 3}$ is also active for the ROP of VL, CL or TMC monomers, albeit sluggish. ${ }^{114-116}$ The reactions are wellcontrolled $\left(M_{w} / M_{n}<1.2\right)$. Binding studies between catalyst and monomer or benzyl alcohol (initiator) suggest H-bonding, which have previously been observed with these catalyst motifs (e.g. $\mathrm{P}=\mathrm{O}$ and P-NH). ${ }^{118}$ Computational studies on $\mathbf{2 1}$ and $\mathbf{2 2}$ indicate the possibility of bifunctional activation. ${ }^{112}$ Solvent screens performed on $\mathbf{2 2}$ and $\mathbf{2 3}$ (ROP of TMC) show dramatic slowing of reaction rate in THF (versus $\mathrm{CH}_{2} \mathrm{Cl}_{2}$ or toluene), corroborating an $\mathrm{H}$ - 
bond mediated mechanism. These systems are part of the vast underpinning of mechanistic studies that have propelled this field forward, and these systems are advantageous in their synthetic modularity and highly controlled nature. This work has roots in the methyl sulfonic acid and triflic acid catalyzed ROP of lactones, which have been proposed to operate through both electrophilic monomer activated and bifunctional H-bond activated mechanisms. ${ }^{113}$

\section{Phenol and Benzyl Alcohol H-bond Donors}

Considering their efficacy for the ROP of several monomers, electron deficient alcoholic H-bond donors may constitute an underdeveloped class of $\mathrm{H}$-bond donating catalyst. Bibal et al. evaluated certain $o-, m-, p$ - substituted phenols $\mathbf{2 4}$ for their catalytic activity towards the ROP of LA (Figure 1.7). ${ }^{119}$ Full conversion of lactide initiated from 4-biphenylmethanol (a fluorescent alcohol) was observed in $24 \mathrm{~h}$ for all phenol/sparteine cocatalyst systems except for $o$ - and $p$-OMe-phenol, and the fastest reaction rates were produced from phenols with electron withdrawing groups. MALDI-TOF MS indicated the presence of polymer chains initiated from phenols, an inherent liability with using alcoholic catalysts for organocatalytic ROP of esters and carbonates. Bis-donor catalysts $(\mathbf{2 4}, o$ diphenol and $m$-diphenol; Figure 1.7) plus DBU cocatalyst are effective for the ROP of VL from 4-biphenylmethanol. ${ }^{120}$ The electron rich diols gave high conversions while the electron poor H-bond donors had lower conversions. Strong binding between cocatalysts has been shown to be inhibitory under some circumstances. ${ }^{48,52}$ However, Hedrick et al. suggested that steric bulk surrounding the catalytic alcohol would limit initiation from catalyst, producing more controlled reactions (Figure 1.7). ${ }^{121}$ The hexafluoroalcohol (26, $\mathrm{R}=\mathrm{H}$ ) plus sparteine cocatalyzed ROP of LA initiated from benzyl alcohol resulted in full 
conversion of monomer in $23 \mathrm{~h}$, but the bulky $\mathrm{H}$-bond donor $26\left(\mathrm{R}=\mathrm{CF}_{3}\right)$ showed no conversion, which may be due to its high acidity $\left(\mathrm{pK}_{\mathrm{a}}{ }^{\mathrm{DMSO}}\left(\mathrm{CF}_{3}\right)_{3} \mathrm{COH}=10.7\right)^{122}$. In a rare display by H-bond mediated ROP, even $\beta$-BL was polymerized by 25 $\left(\mathrm{R}=\right.$ methacryloyl)/sparteine to $71 \%$ conv. in $138 \mathrm{~h}^{121}$

Experimental and computational data suggest the H-bond mediated ROP is mechanistically similar to those previously described. Only minimal binding between phenol and VL was observed, but this important observation reinforces early conclusions that weak binding between catalysts and monomer is not vital to catalysis. ${ }^{48}$ Rather, a larger picture approach considering all reagent bindings, especially cocatalyst bindings, must be considered. ${ }^{15,48,52}$ However, binding measurements on the more effective H-bond donors, 25 ( $\mathrm{R}=$ methacryloyl) and $26(\mathrm{R}=\mathrm{Me})$ indicate H-bonding to VL.

Certainly, structural modulation of the established thiourea and urea scaffolds will continue to offer new catalysts - especially if mechanistic advances like the urea anions continue to appear. These changes may occur through the application of these catalysts in new roles. For examples, thioureas have recently been applied as additives in the strong acid mediated ROP of lactones. Guo et al. found that thioureas when added to a trifluoroacetic acid (TFA) catalyzed ROP of VL or CL increased the reaction rate by up to 3 times in an electrophilic monomer activation mechanism; the $M_{w} / M_{n}$ was reduced and higher conversions were achieved than with TFA alone. ${ }^{123,124}$ However, the drastic departures from the conventional offer a good chance for truly new and exciting developments. The azaphosphatrane (27) cocatalyzed (with sparteine) ROP of cyclic esters is the perfect example, Figure 1.8. ${ }^{125}$ These structures suggest a new catalytic handle to 
provide monomer activation with attenuated cocatalyst binding. ${ }^{125,126}$ Further, they are highly modular and have multiple sites available for optimization. ${ }^{125}$

\section{Electrostatic Monomer Activation by Cations}

H-bonds - a very poor name for the phenomenon - require no orbital overlap and are a type of electrostatic interaction. ${ }^{106}$ Bibal et al. have demonstrated electrostatic activation of monomer by cationic species along with base cocatalysts to effect the ROP of LA, VL and CL; both tertiary alkyl ammonium salts and alkali metal cations encapsulated in crown ethers have been successfully applied, Figure $1.9 .^{127}$ The fastest ROP rates for LA were observed with [15-c-5]Na and sparteine, where full conversion was achieved in $2 \mathrm{~h}$. However, full conversions of LA and VL to polymer were achieved for all cocatalyst systems within $24 \mathrm{~h}$ (sparteine for LA; DBU for VL and CL). As usual, the ROP of CL was the slowest, achieving only $53 \%$ conversion in $120 \mathrm{~h}$ with [15-c-5]Na/sparteine. For the ammonium salt mediated ROPs, exchanging $\mathrm{NTf}_{2}$ for a BARF counterion (Figure 1.9) resulted in a slight increase in reaction rate for all catalytic systems, which is likely attributed to the increased solubility of BARF versus $\mathrm{NTf}_{2 .}{ }^{127}$ The ammonium species do not polymerize cyclic esters in the absence of a base cocatalyst, which suggests that the native counter-anion is insufficient for alcohol activation. DFT calculations reinforce activation of monomer by the electrophilic portions of the alkylammonium (i.e. the methyl groups) and activation of alcohol end group by base cocatalyst, Figure 1.9. ${ }^{127}$ Further exploration of this interesting class of catalysts may provide new reactivity and synthetic possibilities. 


\section{BRONSTED ACID/BASE PAIRS}

The accepted mechanism for the dual organocatalytic ROP of cyclic esters relies on two factors when promoting polymerization: the activation of monomer and initiator/chain end with a Lewis acid (HBD) and Lewis base (HBA), respectively. One can imagine employing a protic acid in place of a thiourea (e.g.) which would result in proton transfer to base cocatalyst, generating a new cocatalyst system where the activation of monomer may occur by base- $\mathrm{H}^{+}$and activation of chain end may occur by acid-. Indeed, the previously discussed 'hyperactive' urea anions may operate by this mode when a strong organic base (e.g. BEMP) is employed. ${ }^{58,108}$ Practically, catalysts of this type are employed by reacting organic bases - many of which are themselves organic catalysts for ROP - with a protic acid to form an acid/base pair. One representative pair, DBU plus benzoic acid (Figure 1.10), was derived serendipitously by incompletely quenching a DBU-catalyzed ROP of lactide.

Benzoic acid, which is widely used to quench organic catalysts by protonating amine bases, ${ }^{2}$ forms an active ROP cocatalyst when mixed 1:1 with DBU. ${ }^{128}$ Hedrick et al. found that a 1:1 ratio of DBU to benzoic acid produced well controlled PLA $\left(M_{w} / M_{n} \sim\right.$ 1.06) to full conversion in $24 \mathrm{~h}$. When the ratio [benzoic acid]/[DBU] increased to 1.5 and 2, the polymerization rate decreased and stopped, respectively. At lower than 1 equivalence of acid (to DBU), the reaction was faster and less controlled due to free

DBU. ${ }^{2,129}$ Molecular modeling of the acid/base pair with LA and methanol suggests a catalytic ion pair where $\mathrm{DBU}-\mathrm{H}^{+}$activates monomer and the benzoate anion $\left(\mathrm{BA}^{-}\right)$ activates chain end. The acid/base pairs of $\mathrm{DBU}$ with $\mathrm{HCl}$, acetic acid $(\mathrm{AcOH})$ or $p$ toluenesulfonic acid (TsOH) were also evaluated for catalytic activity. No catalytic activity 
was found after $48 \mathrm{~h}$ using $\mathrm{HCl}$. However, the resonance stabilized $\mathrm{AcO}^{-}$and $\mathrm{TsO}^{-}$anions both were able to polymerize LA with DBU- $\mathrm{H}^{+}$cocatalyst, providing controlled molecular weights and narrow $M_{w} / M_{n}{ }^{128}$ On a superficial level, these results provide a clear rationale for using two equivalents of benzoic acid with respect to base to quench an ROP (co)mediated by organic bases.

Several conjugate acid/base pairs have also been applied for organocatalytic ROP. $^{130}$ An exemplary pair consisting of 1 eq. DMAP and 1 eq. DMAP $\cdot H X(X=C l$, MSA, TfOH) was used as a catalyst for the ROP of LA in solution, and it exhibited augmented rates versus DMAP alone. The conjugate pair with triflate counterion was found to be the most active catalyst, although full conversion to polymer was not achieved in $24 \mathrm{~h}$. The ideal ratio of DMAP to DMAP•HX is $1: 1$. The same group of conjugate acid/base pairs were also evaluated for the ROP of LA, VL and CL in bulk conditions at $100^{\circ} \mathrm{C}{ }^{131}$ For LA, the same trend was found in the bulk as was found in solution, with the conjugate pair DMAP/DMAP-H ${ }^{+} / \mathrm{TfO}^{-}$system having the highest rate and full conversion in $1 \mathrm{~h}$. DMAP/DMAP- $\mathrm{H}^{+} / \mathrm{TfO}^{-}$was the only catalyst system effective for the ROP of VL and CL, but full conversions were not achieved within $24 \mathrm{~h}$. VL and CL were not as controlled as LA, giving $M_{w} / M_{n}>1.3$, for reactions with degree of polymerization (DP) 100. For all ROPs, side reactions that are likely to broaden $M_{w} / M_{n}$ often occur at long reactions times. As with many acid mediated ROP, water impurities complicated mechanistic analysis. Several other advancements on this theme have been explored by applying known H-bond acceptors with acids for ROP. ${ }^{132-136}$ Conceptually interesting, increased synthetic effort may be able to transition this scheme from concept to practice. 


\section{SUPRAMOLECULAR CATALYSTS}

Betaines

Narrow polydispersity and high molecular weights are possible with ammonium betaine catalysts. Coulembier et al. demonstrated that ammonium betaines, used as bifunctional organic catalysts, H-bond with initiating/propagating alcohols at the phenoxide, Figure 1.11. ${ }^{137}$ ROP of l-lactide was performed with $m$ (trimethylammonio)phenolate betaine (27) producing a living and controlled polymerization, with minimal transesterification and high isotacticity. ${ }^{137,138}$ Faster rates are seen in chloroform versus THF, which was taken to suggest that the ionic catalyst acts via a H-bonding mechanism. ${ }^{137}$ Computational studies suggest that strong interactions are seen between 1-pyrenemethanol and the phenolate anion of $m$-betaine (relative to the other isomers), which is consistent with the rapid ROP with $m$-betaine versus the $p$ - and $o$ isomers. ${ }^{137}$

\section{Amino-Oxazoline}

The structures of amino-oxazolines and thiazolines are analogous to that of TBD. An initial screening of the thiazoline catalyzed ROP of LA determined that thiazolines with electron withdrawing groups resulted in reduced ROP activity and produced atactic PLA. ${ }^{139}$ Amino-thiazolines with electron donating alkyl groups are more active, and amino-thiazoline with cyclohexyl groups demonstrated the fastest rates for ROP of LA, Figure 1.12; however, this catalyst is much less active than the 'parent' TBD catalyst. ${ }^{139}$ Elevated temperatures indicated little to no rate enhancement, which could arise from weaker supramolecular interactions during the enchainment transition state. ${ }^{1} \mathrm{H}$ NMR binding experiments demonstrate the more electron-deficient compounds have stronger interactions with cyclic esters and conversely have weaker interactions with initiating 
alcohol. These experiments corroborate the presumption that both the H-bond accepting and donating sites are necessary for effective catalysis. ${ }^{139}$ These catalysts are notable because they are mechanistically similar to TBD but far more modular synthetically. With the rising interest in specialized catalyst architectures, these motifs may prove highly useful.

\section{Cyclodextrins}

Cyclodextrins (CDs) have garnered interest due to their selective inclusion properties and reactivities, ${ }^{140-142}$ and they constitute an example of extremely mild supramolecular catalyst for ROP. ${ }^{143,144}$ The ability of CDs to catalyze the hydrolysis of polyesters in water was thought to proceed via a polymer inclusion complex with CDs. ${ }^{141}$ In the absence of water, CDs catalyze the ROP of lactone monomers. ${ }^{141}$ Further, CDs can create selective inclusion complexes with some lactones where the size of a CD can promote or suppress the transesterification of lactones. The inclusion of lactones in the hydrophobic CD cavity is believed to be the driving force to yield polyesters, ${ }^{140}$ and the existence of hydrophobic, catalytic pockets has been proposed for other organocatalysts for ROP. ${ }^{56,105,140}$ Accordingly, the ROPs catalyzed by the CD with a smaller cavity (i.e $\alpha$ $\mathrm{CD}$ in Scheme 1.7) produce the highest yields of $\beta$-butyrolactone $(\beta-\mathrm{BL})$ under solventfree conditions at $100^{\circ} \mathrm{C}$, while the larger lactones, VL and CL, experience higher yields with the larger $\gamma$-CD (Scheme 1.7). ${ }^{140}$ Solvent-free copolymerizations of VL and LA were also performed. ${ }^{140}$

Mechanistic studies suggest that ROP is initiated from the CD and that the lactone/CD inclusion complex is vital to catalysis. When ROP is attempted using an acylated CD (no free hydroxyls), no conversion to polylactone is observed, which suggests 
that CDs are covalently attached to the polylactone chain end in a normal CD-catalyzed ROP. ${ }^{140}$ Further, suppression of the ROP of VL was noted with a $\beta$-CD/adamantane inclusion complex catalyst system. The adamantane guest is strongly inserted in the $\beta$-CD cavity, which excludes VL, suggesting that lactone/CD inclusion complexes are essential for ROP. ${ }^{140}$ The mechanistic picture that emerges suggests that, initially, a complex is formed between lactone and $\mathrm{CD}$ at a ratio of 1:1, and a hydroxyl group at the $\mathrm{C}_{2}$-position attacks the monomer to begin enchainment. Further development of these or similar extremely mild catalysts for ROP could provide new and exciting methods of ultracontrolled ROP. 


\section{CONCLUSION}

The narrative of this chapter can be summarized by following the circular evolution of dual catalysts away from and back towards the popular organocatalyst, TBD. When the TBD catalyzed ROP of lactones was disclosed in $2006,{ }^{23}$ it was the perfect storm of a successful catalyst. It is easy to use, readily available, highly active and exhibits decent selectivity for monomer and control $\left(M_{w} / M_{n} \sim 1.2\right)$. While TBD was originally proposed to operate via a nucleophilic mechanism of enchainment, an $\mathrm{H}$-bond mediated, bifunctional, mechanism was also envisaged. ${ }^{23}$ This mechanism has been much debated, and it is not entirely certain which mechanism is operative and when. ${ }^{32,145,146}$ Conceptually, a thiourea/base mediated ROP can be viewed as separating the H-bond donating and accepting roles of TBD into separate cocatalyst moieties. This approach, while highlytunable and beneficial for the reasons described above, required sacrificing reaction rate. The various efforts to increase reaction rate without sacrificing control (serendipitously?) brought the community back to an active catalyst which bears a strong structural resemblance to TBD, urea plus strong base mediated ROP. Far from ending up in the same place, the numerous studies that brought us 'full circle' have greatly enriched our understanding of how these catalysts operate and have largely mitigated the activity versus selectivity problem of organocatalytic ROP, Scheme 1.8. By no means is this story complete, and as of January 2018 our mechanistic understanding of nascent urea/strong base mediated ROP is still evolving. Indeed, the broader field of organocatalytic polymerization is a bridge between the disparate worlds of materials chemist (ease of use) and synthetic polymer chemist (mechanistic interest). We assert that the cooperative and 
collegial nature of our community has facilitated the synergistic evolution of new mechanism to new abilities - in monomer scope, polymer architecture and level of reaction control. We hope that this will continue. 


\section{LIST OF REFERENCES}

(1) Anslyn, E. V.; Dougherty, D. A. In Modern Physical Organic Chemistry; 2006; pp $162-194$.

(2) Lohmeijer, B. G. G.; Pratt, R. C.; Leibfarth, F.; Logan, J. W.; Long, D. A.; Dove, A. P.; Nederberg, F.; Choi, J.; Wade, C.; Waymouth, R. M.; Hedrick, J. L. Macromolecules 2006, 39 (25), 8574-8583.

(3) Pratt, R. C.; Lohmeijer, B. G. G.; Long, D. A.; Lundberg, P. N. P.; Dove, A. P.; Li, H.; Wade, C. G.; Waymouth, R. M.; Hedrick, J. L. Macromolecules 2006, 39 (23), 7863-7871.

(4) Dove, A. P.; Pratt, R. C.; Lohmeijer, B. G. G.; Waymouth, R. M.; Hedrick, J. L.; V, S. U. J. Am. Chem. Soc. 2005, 127, 13798-13799.

(5) Bredig, G.; Fiske, P. S. Biochem. Z. 1912, 46, 7.

(6) Flory, P. J. J. Am. Chem. Soc. 1940, 62 (9), 2261-2264.

(7) Doyle, A. G.; Jacobsen, E. N. Chem. Rev. 2007, 107 (12), 5713-5743.

(8) Okino, T.; Hoashi, Y.; Takemoto, Y. J. Am. Chem. Soc. 2003, 125 (42), $12672-$ 12673.

(9) $Ð$, like PDI (polydispersity index), is sufficiently incoherent to the novice, yet $Ð$ features the added deficit of being nearly impossible to google.

(10) Stepto, R. F. T. Pure Appl. Chem. 2009, 81 (2), 351-353.

(11) Odian, G. In Principles of Polymerization; New York, 1991; pp 532-603.

(12) Kiesewetter, M. K.; Shin, E. J.; Hedrick, J. L.; Waymouth, R. M. Macromolecules 2010, 43 (5), 2093-2107.

(13) Kamber, N. E.; Jeong, W.; Waymouth, R. M.; Pratt, R. C.; Lohmeijer, B. G. G.; 
Hedrick, J. L. Chem. Rev. 2007, 107 (12), 5813-5840.

(14) Dove, A. P. In Handbook of Ring-Opening Polymerization; Dubois, P., Coulembier, O., Raquez, J.-M., Eds.; Wiley-VCH: Federal Republic of Germany, 2009; pp 357-378.

(15) Thomas, C.; Bibal, B. Green Chem. 2014, 16 (4), 1687-1699.

(16) Dove, A. P. ACS Macro Lett. 2012, 1 (12), 1409-1412.

(17) Zhang, X.; Fevre, M.; Jones, G. O.; Waymouth, R. M. Chem. Rev. 2017, acs.chemrev.7b00329.

(18) Byers, J. A.; Biernesser, A. B.; Delle Chiaie, K. R.; Kaur, A.; Kehl, J. A. In Synthesis, Structure and Properties of Poly(lactic acid); Di Lorenzo, M. L., Androsch, R., Eds.; Springer International Publishing: Cham, 2018; pp 67-118.

(19) Grubbs, R. B.; Grubbs, R. H. Macromolecules 2017, No. X, acs.macromol.7b01440.

(20) Hu, S.; Zhao, J.; Zhang, G.; Schlaad, H. Prog. Polym. Sci. 2017, 74, 34-77.

(21) Song, Q.-L.; Hu, S.-Y.; Zhao, J.-P.; Zhang, G.-Z. Chinese J. Polym. Sci. 581-601 Chinese J. Polym. Sci. 2017, 35 (5), 581-601.

(22) Jeong, W.; Shin, E. J.; Culkin, D. A.; Hedrick, J. L.; Waymouth, R. M. J. Am. Chem. Soc. 2009, 131 (13), 4884-4891.

(23) Pratt, R. C.; Lohmeijer, B. G. G.; Long, D. A.; Waymouth, R. M.; Hedrick, J. L. J. Am. Chem. Soc. 2006, 128 (14), 4556-4557.

(24) Zhang, L.; Nederberg, F.; Pratt, R. C.; Waymouth, R. M.; Hedrick, J. L.; Wade, C. G. Macromolecules 2007, 40 (12), 4154-4158.

(25) Nederberg, F.; Connor, E. F.; Glausser, T.; Hedrick, J. L. Chem. Commun. 2001, 
No. 20, 2066-2067.

(26) Nederberg, F.; Connor, E. F.; Möller, M.; Glauser, T.; Hedrick, J. L. Angew. Chem. Int. Ed. Engl. 2001, 40, 2712-2715.

(27) Kricheldorf, H. R.; Lomadze, N.; Schwarz, G. Macromolecules 2008, 41 (21), 7812-7816.

(28) Kricheldorf, H. R.; Lossow, C. Von; Schwarz, G. J. Polym. Sci. Part A Polym. Chem. 2006, 44 (15), 4680-4695.

(29) Bonduelle, C.; Martín-Vaca, B.; Cossío, F. P. P.; Bourissou, D. Chem. - A Eur. J. 2008, 14 (17), 5304-5312.

(30) Chuma, A.; Horn, H. W.; Swope, W. C.; Pratt, R. C.; Zhang, L.; Lohmeijer, B. G. G.; Wade, C. G.; Waymouth, R. M.; Hedrick, J. L.; Rice, J. E. J. Am. Chem. Soc. 2008, 130 (21), 6749-6754.

(31) Lai, C.-L. L.; Lee, H. M.; Hu, C.-H. H. Tetrahedron Lett. 2005, 46 (37), 62656270.

(32) Simón, L.; Goodman, J. M. J. Org. Chem. 2007, 72 (25), 9656-9662.

(33) Schwesinger, R.; Schlemper, H. Angew. Chemie Int. Ed. English 1987, 26 (11), $1167-1169$.

(34) Molenberg, A.; Möller, M. Macromol. Rapid Commun. 1995, 16 (6), 449-453.

(35) Kaljurand, I.; Kütt, A.; Sooväli, L.; Rodima, T.; Mäemets, V.; Leito, I.; Koppel, I. A. J. Org. Chem. 2005, 70 (3), 1019-1028.

(36) Schlaad, H.; Kukula, H.; Rudloff, J.; Below, I. Macromolecules 2001, 34 (13), $4302-4304$.

(37) Sigman, M. S.; Vachal, P.; Jacobsen, E. N. Angew. Chemie - Int. Ed. 2000, 39 (7), 
$1279-1281$.

(38) Vachal, P.; Jacobsen, E. N. Org. Lett. 2000, 2 (6), 867-870.

(39) Vachal, P.; Jacobsen, E. N. J. Am. Chem. Soc. 2002, 124 (34), 10012-10014.

(40) Wenzel, A. G.; Jacobsen, E. N. J. Am. Chem. Soc. 2002, 124 (44), 12964-12965.

(41) Taylor, M. S.; Jacobsen, E. N. J. Am. Chem. Soc. 2004, 126 (34), 10558-10559.

(42) Yoon, T. P.; Jacobsen, E. N. Angew. Chemie - Int. Ed. 2005, 44 (3), 466-468.

(43) Joly, G. D.; Jacobsen, E. N. J. Am. Chem. Soc. 2004, 126 (13), 4102-4103.

(44) Fuerst, D. E.; Jacobsen, E. N. J. Am. Chem. Soc. 2005, 127 (25), 8964-8965.

(45) Okino, T.; Nakamura, S.; Furukawa, T.; Takemoto, Y. Org. Lett. 2004, 6 (4), 625627.

(46) Hoashi, Y.; Okino, T.; Takemoto, Y. Angew. Chemie - Int. Ed. 2005, 44 (26), 4032-4035.

(47) Coady, D. J.; Engler, A. C.; Horn, H. W.; Bajjuri, K. M.; Fukushima, K.; Jones, G. O.; Nelson, A.; Rice, J. E.; Hedrick, J. L. ACS Macro Lett. 2012, 1 (1), 19-22.

(48) Kazakov, O. I.; Datta, P. P.; Isajani, M.; Kiesewetter, E. T.; Kiesewetter, M. K. Macromolecules 2014, 47, 7463-7468.

(49) Jeong, W.; Hedrick, J. L.; Waymouth, R. M. J. Am. Chem. Soc. 2007, 129 (27), $8414-8415$.

(50) Duda, A.; Kowalski, A. In Handbook of Ring-Opening Polymerization; Dubois, P., Coulembier, O., Raquez, J.-M., Eds.; Wiley-VCH Verlag GmbH \& Co. KGaA, 2009; pp 1-52.

(51) Olsén, P.; Odelius, K.; Albertsson, A. C. Biomacromolecules 2016, 17 (3), 699 709. 
(52) Koeller, S.; Kadota, J.; Peruch, F.; Deffieux, A.; Pinaud, N.; Pianet, I.; Massip, S.;

Léger, J.-M.; Desvergne, J.-P.; Bibal, B. Chem. Eur. J. 2010, 16 (14), 4196-4205.

(53) Spink, S. S.; Kazakov, O. I.; Kiesewetter, E. T.; Kiesewetter, M. K.

Macromolecules 2015, 48 (17), 6127-6131.

(54) Kazakov, O. I.; Kiesewetter, M. K. Macromolecules 2015, 48 (17), 6121-6126.

(55) Thomas, C.; Peruch, F.; Bibal, B. RSC Adv. 2012, 2 (33), 12851.

(56) Datta, P. P.; Pothupitiya, J. U.; Kiesewetter, E. T.; Kiesewetter, M. K. Eur. Polym. J. 2017, 95 (May), 671-677.

(57) Lippert, K. M.; Hof, K.; Gerbig, D.; Ley, D.; Hausmann, H.; Guenther, S.;

Schreiner, P. R. European J. Org. Chem. 2012, 2012 (30), 5919-5927.

(58) Pothupitiya, J. U.; Dharmaratne, N. U.; Jouaneh, T. M. M.; Fastnacht, K. V.;

Coderre, D. N.; Kiesewetter, M. K. Macromolecules 2017, 50 (22), 8948-8954.

(59) Lin, B.; Waymouth, R. M. J. Am. Chem. Soc. 2017, 139 (4), 1645-1652.

(60) Zhang, X.; Jones, G. O.; Hedrick, J. L.; Waymouth, R. M. Nat. Chem. 2016, 8 (11), 1047-1053.

(61) Koeller, S.; Thomas, C.; Peruch, F.; Deffieux, A.; Massip, S.; Léger, J. M.;

Desvergne, J. P.; Milet, A.; Bibal, B. Chemistry 2014, 20 (10), 2849-2859.

(62) Koeller, S.; Kadota, J.; Deffieux, A.; Peruch, F.; Massip, S.; Léger, J. M.;

Desvergne, J. P.; Bibal, B. J. Am. Chem. Soc. 2009, 131 (42), 15088-15089.

(63) Blake, T. R.; Waymouth, R. M. J. Am. Chem. Soc. 2014, 136 (26), 9252-9255.

(64) Engler, A. C.; Chan, J. M. W.; Coady, D. J.; O’Brien, J. M.; Sardon, H.; Nelson, A.; Sanders, D. P.; Yang, Y. Y.; Hedrick, J. L. Macromolecules 2013, 46 (4), $1283-1290$. 
(65) Chang, Y. A.; Rudenko, A. E.; Waymouth, R. M. ACS Macro Lett. 2016, 5 (10), 1162-1166.

(66) Venkataraman, S.; Ng, V. W. L.; Coady, D. J.; Horn, H. W.; Jones, G. O.; Fung, T. S.; Sardon, H.; Waymouth, R. M.; Hedrick, J. L.; Yang, Y. Y. J. Am. Chem. Soc. 2015, 137 (43), 13851-13860.

(67) McKinlay, C. J.; Waymouth, R. M.; Wender, P. A. J. Am. Chem. Soc. 2016, 138 (10), 3510-3517.

(68) Sanders, D. P.; Fukushima, K.; Coady, D. J.; Nelson, A.; Fujiwara, M.; Yasumoto, M.; Hedrick, J. L. J. Am. Chem. Soc. 2010, 132 (42), 14724-14726.

(69) Ovitt, T. M.; Coates, G. W. J. Am. Chem. Soc. 2002, 124 (7), 1316-1326.

(70) Chamberlain, B. M.; Cheng, M.; Moore, D. R.; Ovitt, T. M.; Lobkovsky, E. B.; Coates, G. W. J. Am. Chem. Soc. 2001, 123 (14), 3229-3238.

(71) Bakewell, C.; Cao, T. P. A.; Long, N.; Le Goff, X. F.; Auffrant, A.; Williams, C. K. J. Am. Chem. Soc. 2012, 134 (51), 20577-20580.

(72) Zell, M. T.; Padden, B. E.; Paterick, A. J.; Thakur, K. A. M.; Kean, R. T.; Hillmyer, M. A.; Munson, E. J. Macromolecules 2002, 35, 7700-7707.

(73) Thakur, K. a M.; Kean, R. T.; Hall, E. S.; Kolstad, J. J.; Lindgren, T. a; Doscotch, M. a; Siepmann, J. I.; Munson, E. J. Macromolecules 1997, 30 (8), 2422-2428.

(74) Todd, R.; Rubio, G.; Hall, D. J.; Tempelaar, S.; Dove, A. P. Chem. Sci. 2013, 4 (3).

(75) Dai, Z.; Cui, Y.; Chen, C.; Wu, J. Chem. Commun. 2016, 52 (57), 8826-8829.

(76) Guillaume, S. M.; Kirillov, E.; Sarazin, Y.; Carpentier, J.-F. Chem. - A Eur. J. 2015, 21 (22), 7988-8003. 
(77) Cacciapaglia, R.; Di Stefano, S.; Mandolini, L. J. Am. Chem. Soc. 2003, 125 (8), $2224-2227$.

(78) Beswick, J.; Blanco, V.; De Bo, G.; Leigh, D. A.; Lewandowska, U.;

Lewandowski, B.; Mishiro, K. Chem. Sci. 2015, 6 (1), 140-143.

(79) Wang, J.; Feringa, B. L. Science (80-. ). 2011, 331 (6023), 1429-1432.

(80) Sud, D.; Norsten, T. B.; Branda, N. R. Angew. Chemie Int. Ed. 2005, 44 (13), 2019-2021.

(81) Neilson, B. M.; Bielawski, C. W. J. Am. Chem. Soc. 2012, 134 (30), 12693-12699.

(82) Samanta, M.; Siva Rama Krishna, V.; Bandyopadhyay, S. Chem. Commun. 2014, 50 (73), 10577-10579.

(83) Imahori, T.; Yamaguchi, R.; Kurihara, S. Chem. - A Eur. J. 2012, 18 (35), 1080210807.

(84) Gregson, C. K. A.; Gibson, V. C.; Long, N. J.; Marshall, E. L.; Oxford, P. J.; White, A. J. P. J. Am. Chem. Soc. 2006, 128 (23), 7410-7411.

(85) Broderick, E. M.; Guo, N.; Wu, T.; Vogel, C. S.; Xu, C.; Sutter, J.; Miller, J. T.; Meyer, K.; Cantat, T.; Diaconescu, P. L. Chem. Commun. 2011, 47 (35), $9897-$ 9899.

(86) Yoon, H. J.; Kuwabara, J.; Kim, J.-H.; Mirkin, C. A. Science (80-. ). 2010, 330 (6000), 66-69.

(87) Broderick, E. M.; Guo, N.; Vogel, C. S.; Xu, C.; Sutter, J.; Miller, J. T.; Meyer, K.; Mehrkhodavandi, P.; Diaconescu, P. L. J. Am. Chem. Soc. 2011, 133 (24), 92789281.

(88) Romain, C.; Williams, C. K. Angew. Chemie Int. Ed. 2014, 53 (6), 1607-1610. 
(89) Sohtome, Y.; Tanaka, S.; Takada, K.; Yamaguchi, T.; Nagasawa, K. Angew. Chemie Int. Ed. 2010, 49 (48), 9254-9257.

(90) Piermattei, A.; Karthikeyan, S.; Sijbesma, R. P. Nat. Chem. 2009, 1, 133-137.

(91) Coulembier, O.; Moins, S.; Todd, R.; Dubois, P. Macromolecules 2014, 47 (2), 486-491.

(92) Tian, X.; Cassani, C.; Liu, Y.; Moran, A.; Urakawa, A.; Galzerano, P.; Arceo, E.; Melchiorre, P. J. Am. Chem. Soc. 2011, 133 (44), 17934-17941.

(93) Blanco, V.; Leigh, D. A.; Marcos, V.; Morales-Serna, J. A.; Nussbaumer, A. L. J. Am. Chem. Soc. 2014, 136 (13), 4905-4908.

(94) Broderick, E. M.; Thuy-Boun, P. S.; Guo, N.; Vogel, C. S.; Sutter, J.; Miller, J. T.; Meyer, K.; Diaconescu, P. L. Inorg. Chem. 2011, 50 (7), 2870-2877.

(95) Miyake, G. M.; Chen, E. Y. X. Macromolecules 2011, 44 (11), 4116-4124.

(96) Zhu, J.-B.; Chen, E. Y.-X. J. Am. Chem. Soc. 2015, 137 (39), 12506-12509.

(97) Banik, S. M.; Levina, A.; Hyde, A. M.; Jacobsen, E. N. Science (80-. ). 2017, 358 (6364), 761-764.

(98) Liu, J.; Chen, C.; Li, Z.; Wu, W.; Zhi, X.; Zhang, Q.; Wu, H.; Wang, X.; Cui, S.; Guo, K. Polym. Chem. 2015, 6 (20), 3754-3757.

(99) Rostami, A.; Sadeh, E.; Ahmadi, S. J. Polym. Sci. Part A Polym. Chem. 2017, 55, 2483-2493.

(100) Liu, J.; Xu, J.; Li, Z.; Xu, S.; Wang, X.; Wang, H.; Guo, T.; Gao, Y.; Zhang, L.; Guo, K. Polym. Chem. 2017, 8, 7054-7068.

(101) Ni, X.; Li, X.; Wang, Z.; Cheng, J. P. Org. Lett. 2014, 16 (6), 1786-1789.

(102) Jakab, G.; Tancon, C.; Zhang, Z.; Lippert, K. M.; Schreiner, P. R. Org. Lett. 2012, 
$14(7), 1724-1727$.

(103) Xu, S.; Sun, H.; Liu, J.; Xu, J.; Pan, X.; Dong, H.; Liu, Y.; Li, Z.; Guo, K. Polym. Chem. 2016, 7 (44), 6843-6853.

(104) Wittkopp, A.; Schreiner, P. R. Chem. - A Eur. J. 2003, 9 (2), 407-414.

(105) Fastnacht, K. V.; Spink, S. S.; Dharmaratne, N. U.; Pothupitiya, J. U.; Datta, P. P.; Kiesewetter, E. T.; Kiesewetter, M. K. ACS Macro Lett. 2016, 5 (8), 982-986.

(106) Anslyn, E. V.; Dougherty, D. A. In Modern Physical Organic Chemistry; University Science, 2006; pp 145-222.

(107) Fastnacht, K. V.; Dharmaratne, N. U.; Kiesewetter, M. K. Unpubl. results.

(108) Dharmaratne, N. U.; Pothupitiya, J. U.; Bannin, T. J.; Kazakov, O. I.; Kiesewetter, M. K. ACS Macro Lett. 2017, 6 (4), 421-425.

(109) Blain, M.; Yau, H.; Jean-Gérard, L.; Auvergne, R.; Benazet, D.; Schreiner, P. R.; Caillol, S.; Andrioletti, B. ChemSusChem 2016, 9 (16), 2269-2272.

(110) Mezzasalma, L.; Dove, A. P.; Coulembier, O. Eur. Polym. J. 2017, 95 (May), 628634.

(111) Alba, A.; Schopp, A.; De Sousa Delgado, A.-P.; Cherif-Cheikh, R.; Martí-n-Vaca, B.; Bourissou, D. J. Polym. Sci. Part A Polym. Chem. 2010, 48 (4), 959-965.

(112) Delcroix, D.; Couffin, A.; Susperregui, N.; Navarro, C.; Maron, L.; Martin-Vaca, B.; Bourissou, D. Polym. Chem. 2011, 2 (10), 2249-2256.

(113) Susperregui, N.; Delcroix, D.; Martin-Vaca, B.; Bourissou, D.; Maron, L. J. Org. Chem. 2010, 75 (19), 6581-6587.

(114) Kan, S.; Jin, Y.; He, X.; Chen, J.; Wu, H.; Ouyang, P.; Guo, K.; Li, Z. Polym. Chem. 2013, 4 (21), 5432. 
(115) He, X.; Ji, Y.; Jin, Y.; Kan, S.; Xia, H.; Chen, J.; Liang, B.; Wu, H.; Guo, K.; Li, Z. J. Polym. Sci. Part A Polym. Chem. 2014, 52 (7), 1009-1019.

(116) Chen, J.; Kan, S.; Xia, H.; Zhou, F.; Chen, X.; Jiang, X.; Guo, K.; Li, Z. Polym. (United Kingdom) 2013, 54 (16), 4177-4182.

(117) Macdonald, E. K.; Shaver, M. P. Eur. Polym. J. 2017, 95 (April), 702-710.

(118) Akiyama, T. Chem. Rev. 2007, 107 (12), 5744-5758.

(119) Thomas, C.; Peruch, F.; Deffieux, A.; Milet, A.; Desvergne, J. P.; Bibal, B. Adv. Synth. Catal. 2011, 353 (7), 1049-1054.

(120) Thomas, C.; Peruch, F.; Bibal, B. All Res. J. Chem. 2012, 3, 7-11.

(121) Coulembier, O.; Sanders, D. P.; Nelson, A.; Hollenbeck, A. N.; Horn, H. W.; Rice, J. E.; Fujiwara, M.; Dubois, P.; Hedrick, J. L. Angew. Chemie - Int. Ed. 2009, 48 (28), 5170-5173.

(122) Taft, R. W.; Bordwell, F. G. Acc. Chem. Res. 1988, 21 (12), 463-469.

(123) Li, X.; Zhang, Q.; Li, Z.; Wang, X.; Liu, J.; Cui, S.; Xu, S.; Zhao, C.; Chen, C.; Guo, K. Polym. (United Kingdom) 2016, 84, 293-303.

(124) Li, X.; Zhang, Q.; Li, Z.; Xu, S.; Zhao, C.; Chen, C.; Zhi, X.; Wang, H.; Zhu, N.; Guo, K. Polym. Chem. 2016, 7 (7), 1368-1374.

(125) Zhang, D.; Jardel, D.; Peruch, F.; Calin, N.; Dufaud, V.; Dutasta, J. P.; Martinez, A.; Bibal, B. European J. Org. Chem. 2016, 2016 (8), 1619-1624.

(126) Sun, H.; Xu, S.; Li, Z.; Xu, J.; Liu, J.; Wang, X.; Wang, H.; Dong, H.; Liu, Y.; Guo, K. Polym. Chem. 2017, 1, 5570-5579.

(127) Thomas, C.; Milet, A.; Peruch, F.; Bibal, B. Polym. Chem. 2013, 4 (12), 34913498. 
(128) Coady, D. J.; Fukushima, K.; Horn, H. W.; Rice, J. E.; Hedrick, J. L. Chem. Commun. 2011, 47 (47), 3105-3107.

(129) Brown, H. A.; De Crisci, A. G.; Hedrick, J. L.; Waymouth, R. M. ACS Macro Lett. 2012, 1 (9), 1113-1115.

(130) Kadota, J.; Pavlović, D.; Desvergne, J. P.; Bibal, B.; Peruch, F.; Deffieux, A. Macromolecules 2010, 43 (21), 8874-8879.

(131) Kadota, J.; Pavlović, D.; Hirano, H.; Okada, A.; Agari, Y.; Bibal, B.; Deffieux, A.; Peruch, F. RSC Adv. 2014, 4 (28), 14725.

(132) Gontard, G.; Amgoune, A.; Bourissou, D. J. Polym. Sci. Part A Polym. Chem. 2016, 54 (20), 3253-3256.

(133) Wang, X.; Cui, S.; Li, Z.; Kan, S.; Zhang, Q.; Zhao, C.; Wu, H.; Liu, J.; Wu, W.; Guo, K. Polym. Chem. 2014, 5 (20), 6051-6059.

(134) Coulembier, O.; Josse, T.; Guillerm, B.; Gerbaux, P.; Dubois, P. Chem. Commun. 2012, 48 (95), 11695.

(135) Zhi, X.; Liu, J.; Li, Z.; Wang, H.; Wang, X.; Cui, S.; Chen, C.; Zhao, C.; Li, X.; Guo, K. Polym. Chem. 2016, 7 (2), 339-349.

(136) Chen, S.; Liu, Y.; Li, Z.; Wang, X.; Dong, H.; Sun, H.; Yang, K.; Gebru, H.; Guo, K. Eur. Polym. J. 2017, 97 (November), 389-396.

(137) Guillerm, B.; Lemaur, V.; Cornil, J.; Lazzaroni, R.; Dubois, P.; Coulembier, O. Chem. Commun. (Camb). 2014, 50 (70), 10098-10101.

(138) Tsutsumi, Y.; Yamakawa, K.; Yoshida, M.; Ema, T.; Sakai, T. Org. Lett. 2010, 12 (24), 5728-5731.

(139) Becker, J. M.; Tempelaar, S.; Stanford, M. J.; Pounder, R. J.; Covington, J. A.; 
Dove, A. P. Chem. - A Eur. J. 2010, 16 (20), 6099-6105.

(140) Harada, A.; Osaki, M.; Takashima, Y.; Yamaguchi, H. Acc. Chem. Res. 2008, 41 (9), 1143-1152.

(141) Takashima, Y.; Osaki, M.; Harada, A. J. Am. Chem. Soc. 2004, 126 (42), 1358813589.

(142) Osaki, M.; Takashima, Y.; Yamaguchi, H.; Harada, A. Macromolecules 2007, 40 (9), 3154-3158.

(143) Glockner, P.; Ritter, H. Macromol. Chem. Phys. 2000, 201 (17), 2455-2457.

(144) Takayanagi, M.; Ito, S.; Matsumoto, K.; Nagaoka, M. J. Phys. Chem. B 2016, 120 (29), 7174-7181.

(145) Kiesewetter, M. K.; Scholten, M. D.; Kirn, N.; Weber, R. L.; Hedrick, J. L.;

Waymouth, R. M. J. Org. Chem. 2009, 74 (24), 9490-9496.

(146) Horn, H. W.; Jones, G. O.; Wei, D. S.; Fukushima, K.; Lecuyer, J. M.; Coady, D. J.; Hedrick, J. L.; Rice, J. E. J. Phys. Chem. A 2012, 116 (51), 12389-12398. 
$\mathrm{n}$

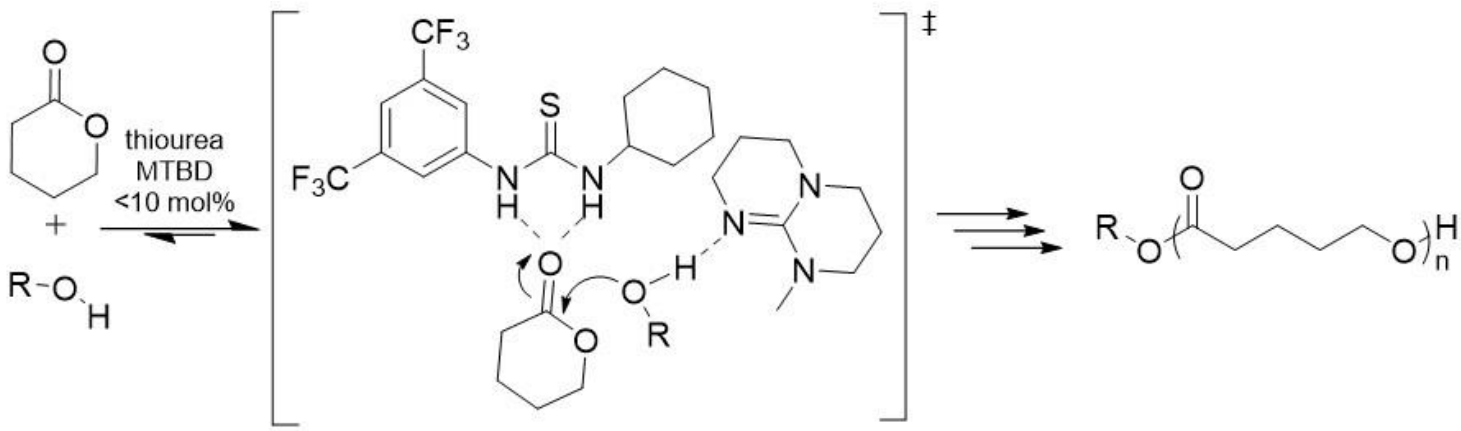

Scheme 1.1. Dual catalyst (bimolecular) mediated ROP of $\delta$-valerolactone. Thiourea and MTBD are exemplary H-bond donors (HBDs) and H-bond acceptors (HBAs), respectively. 




Scheme 1.2. DMAP catalyzed ROP of lactide has been proposed to proceed via nucleophilic (upper) and H-bond mediated (lower) pathways. 


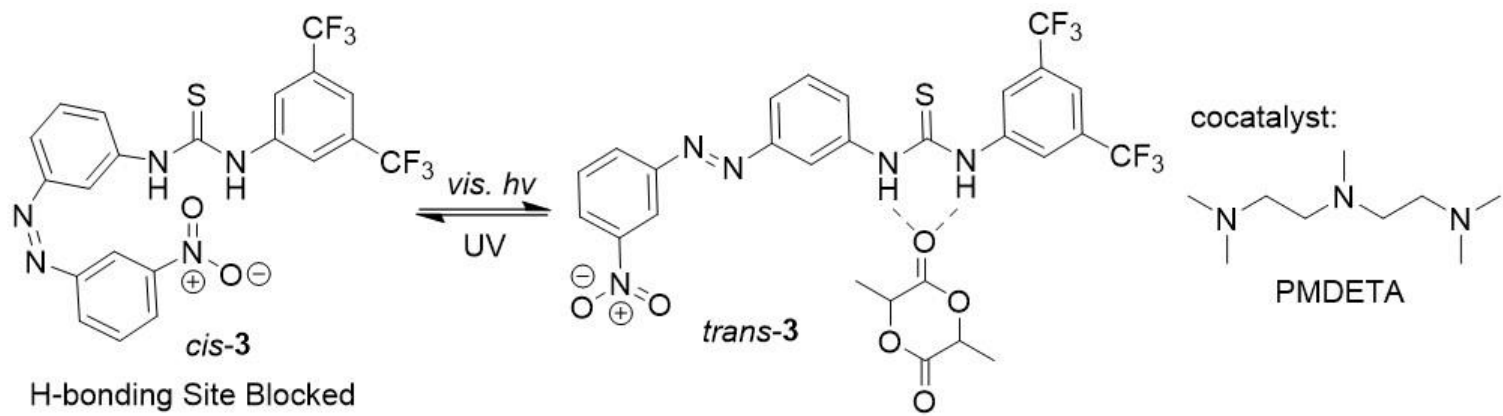

Scheme 1.3. Azobenzene-based Switchable Thiourea. 

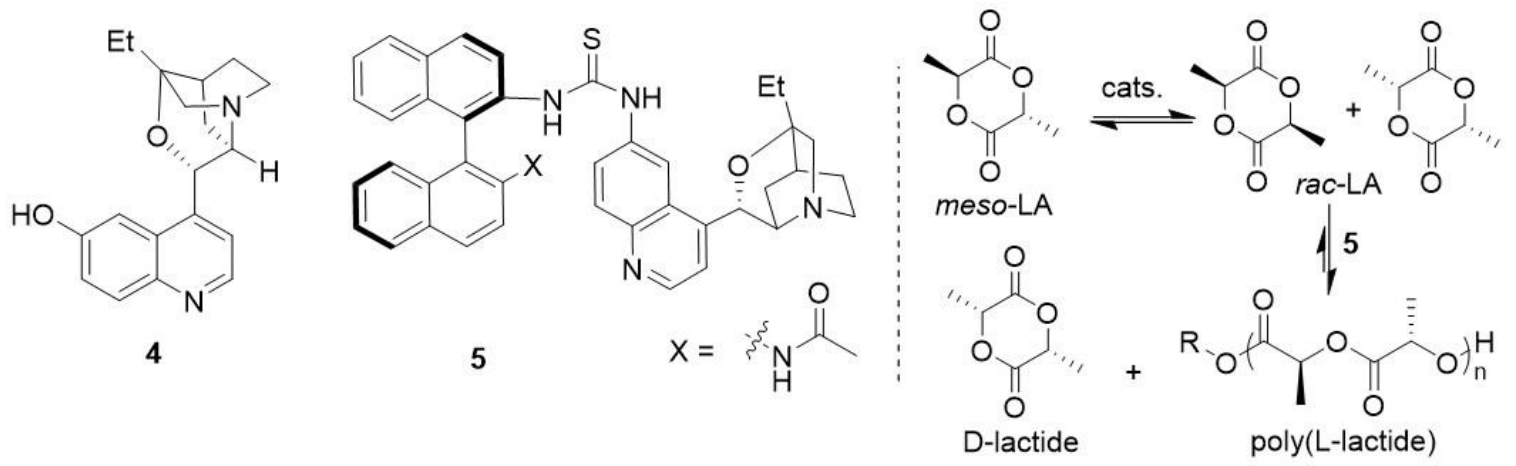

Scheme 1.4. Cinchona Alkaloid-based H-bond Donors for the Stereoselective ROP of rac-Lactide. 
<smiles>O=C(Nc1cc(C(F)(F)F)cc(C(F)(F)F)c1)NC1CCCCC1</smiles><smiles>[X]C(NCCCNC([X])Nc1cc(C(F)(F)F)cc(C(F)(F)F)c1)Nc1cc(C(F)(F)F)cc(C(F)(F)F)c1</smiles>

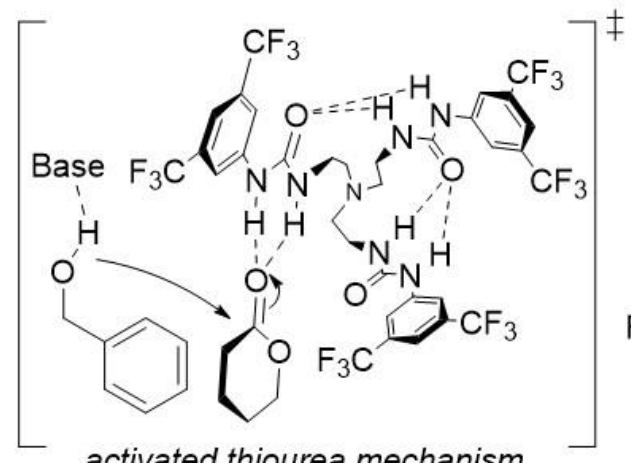

12: $X=S$

13: $X=0$<smiles>[13CH3]</smiles><smiles>[X][X]#[Y4]([Y4])=O</smiles>

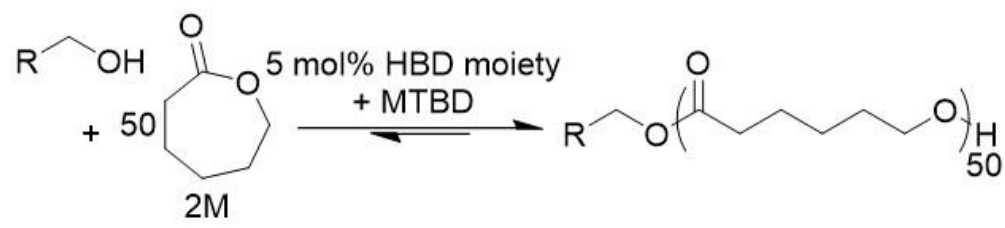

2: $45 \mathrm{~h}, M_{w} / M_{n}=1.09$

12: $10 \mathrm{~h}, M_{W} / M_{n}=1.11$

14: $42 \mathrm{~h}, M_{w} / M_{n}=1.07$

15: $26 \mathrm{~min}, M_{w} / M_{n}=1.05$

15/BEMP: $6 \mathrm{~min}, M_{w} / M_{n}=1.04$

Scheme 1.5. Multi-(thio)urea H-bond Donors for ROP. 


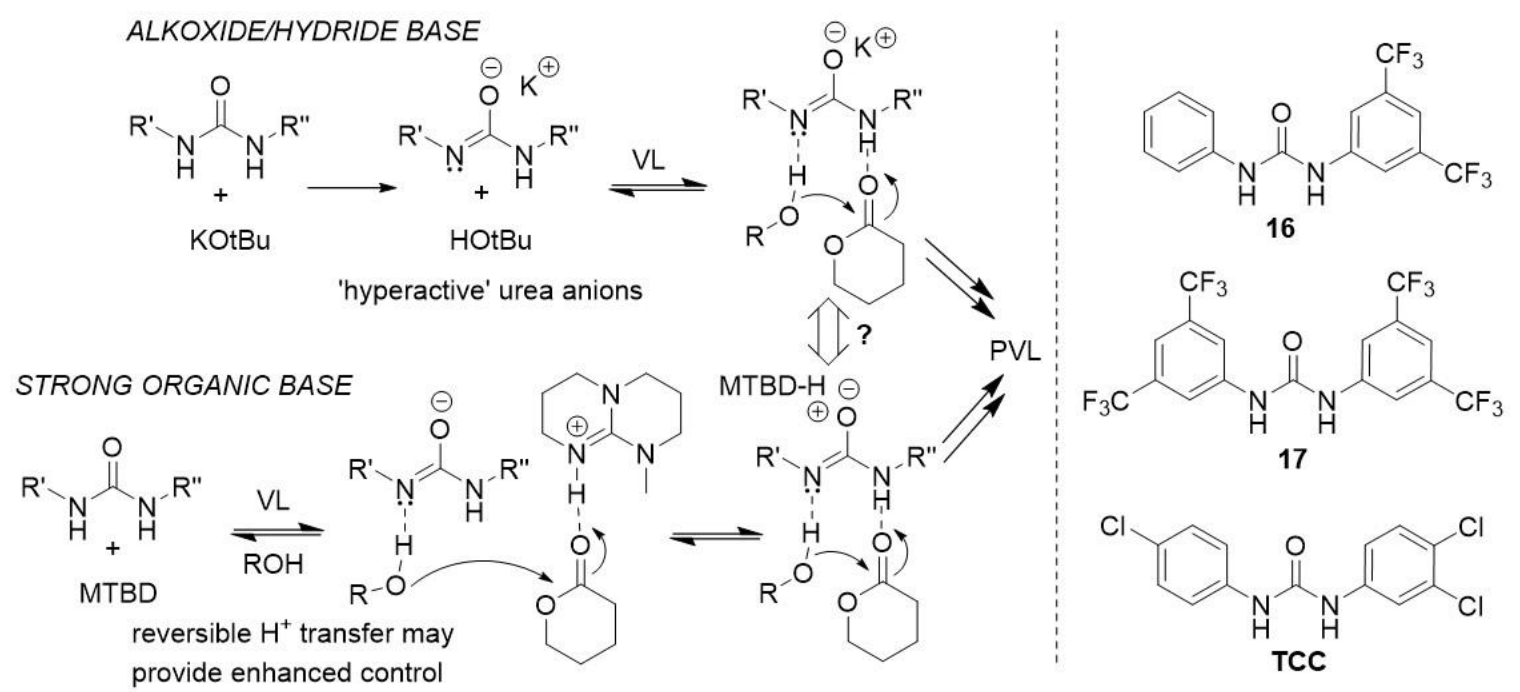

Scheme 1.6. Urea Anion Mediated ROP. 

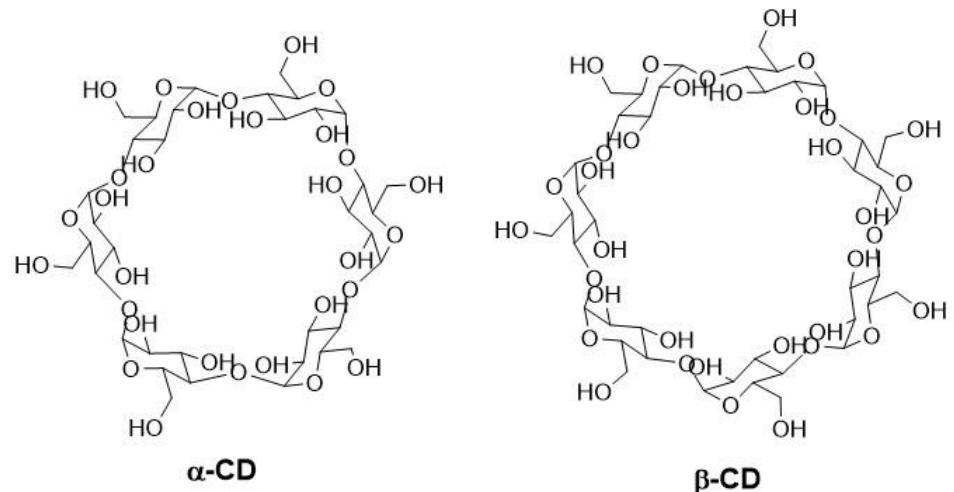

$\beta-C D$

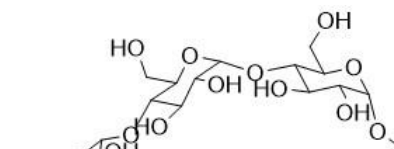

Ho

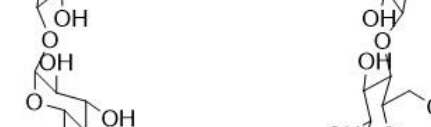

$\mathrm{HO}$

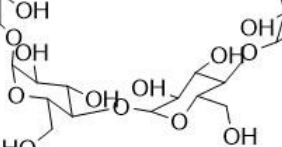

FAVORABLY BINDS:

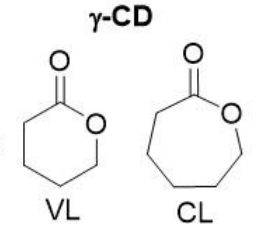



NO POLYMERIZATION



POLYMERIZATION FROM CD

Scheme 1.7. Cyclodextrin Promoted ROP of Lactones. 


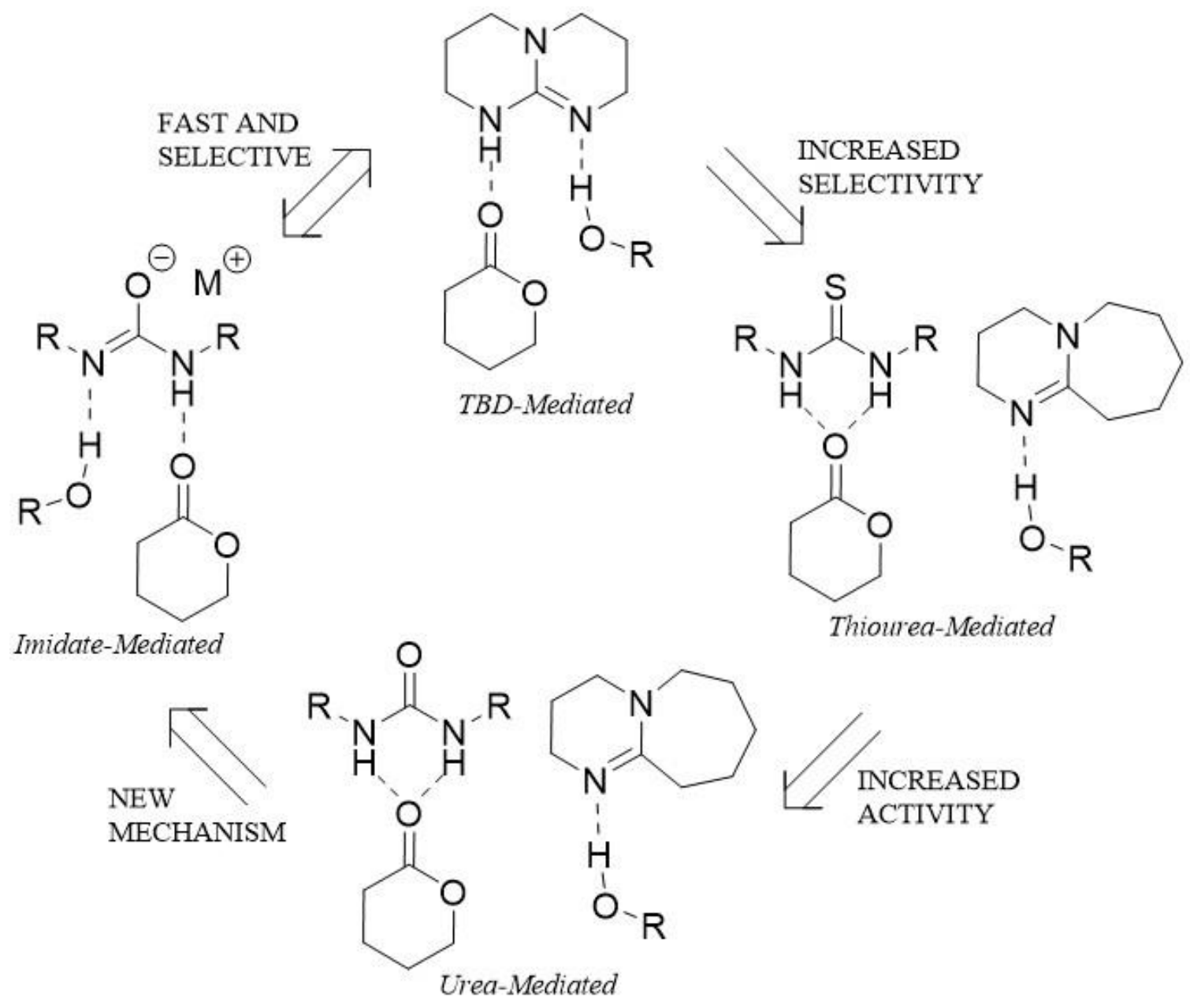

Scheme 1.8. Evolution of Dual Catalysts for ROP. 
<smiles>CN(C)[C@H]1CCCC[C@H]1NC(=S)Nc1cc(C(F)(F)F)cc(C(F)(F)F)c1</smiles>

(Takemoto catalyst)<smiles>CC1OC(=O)C(C)OC1=O</smiles>

LA

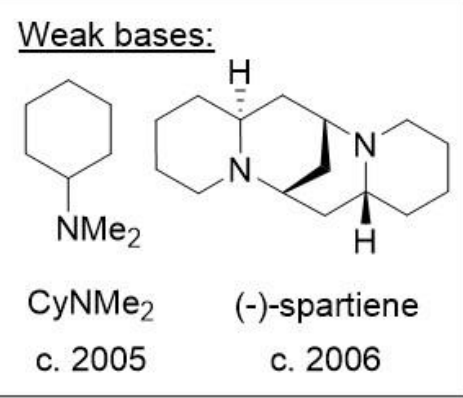

c. 2005

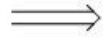

$\mathrm{H}_{2} \mathrm{H}_{3}$

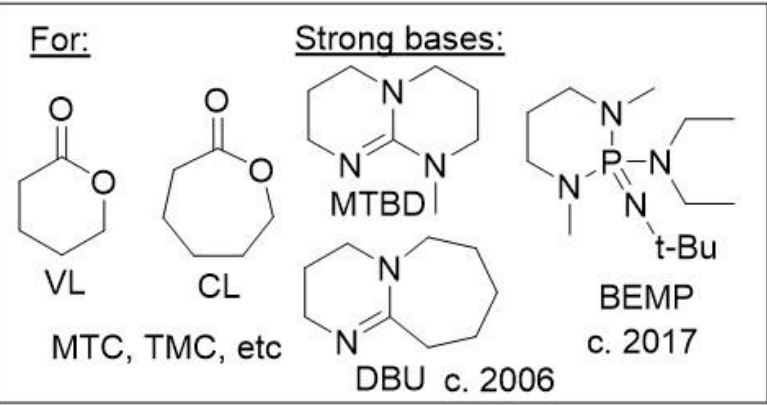

Figure 1.1. The Takemoto catalyst was the inspiration for the popular thiourea plus base catalyst system. Weaker base cocatalysts effect the ROP of lactide, while stronger bases open other monomers. 

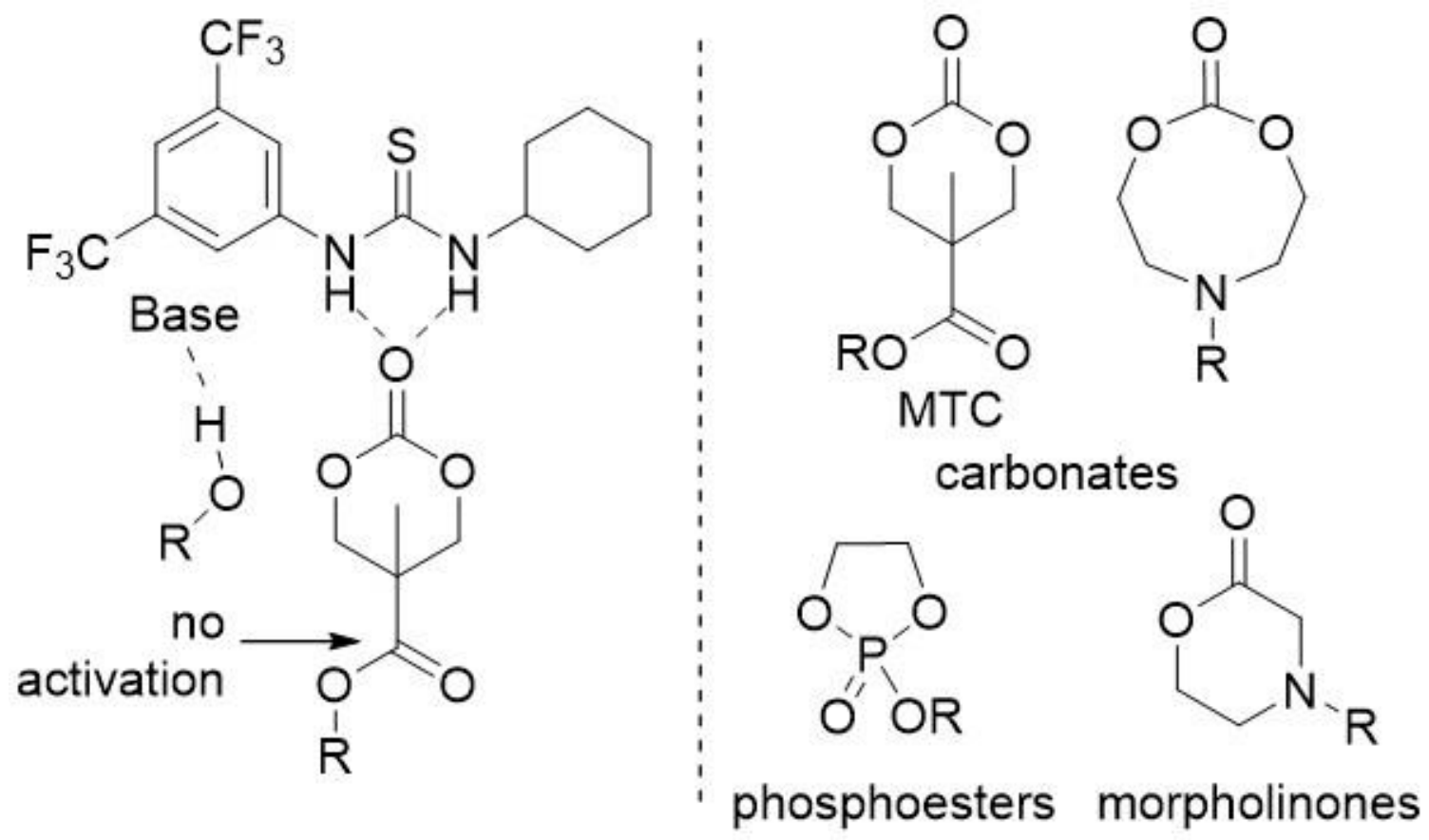

Figure 1.2. Functionalizable monomers which undergo controlled ROP by $2 /$ base. 


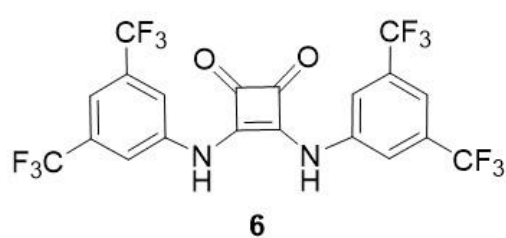

6

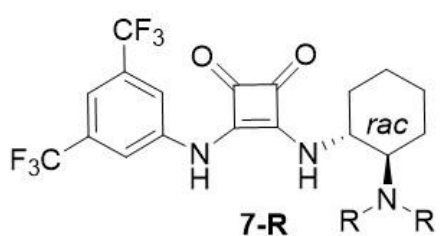

7-R $\mathrm{R}^{-\mathrm{N}} \mathrm{R}$

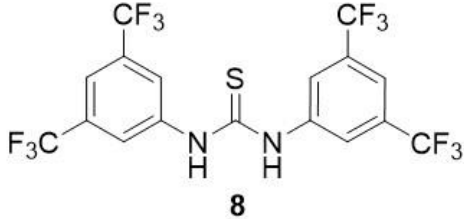

(Schreiner thiourea)

Figure 1.3. Squaramide H-bond Donors for ROP of Lactide. 




internal Lewis acid

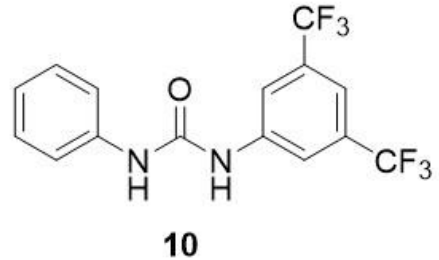

10

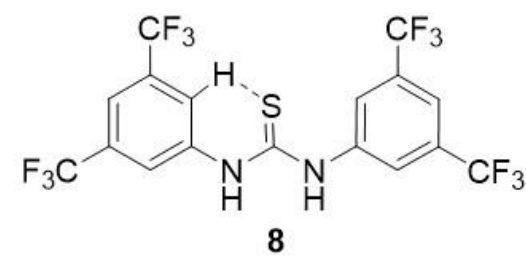

$\mathrm{S}$ to $\mathrm{o}-\mathrm{H}$ interaction

Figure 1.4. Internal Lewis Acid Stabilized (Thio)ureas for ROP. 

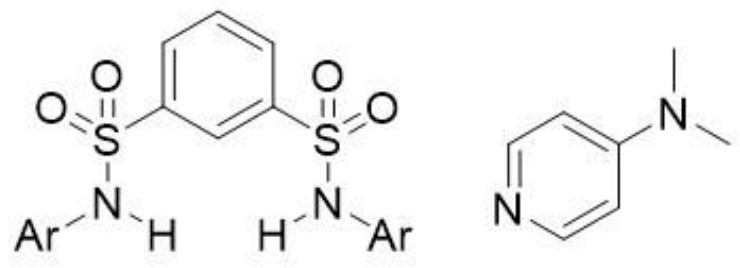

18: $\mathrm{Ar}=$ Phenyl DMAP

19: $\mathrm{Ar}=3,5$-bis $\left(\mathrm{CF}_{3}\right)$ phenyl

20: $\mathrm{Ar}=$ mesityl

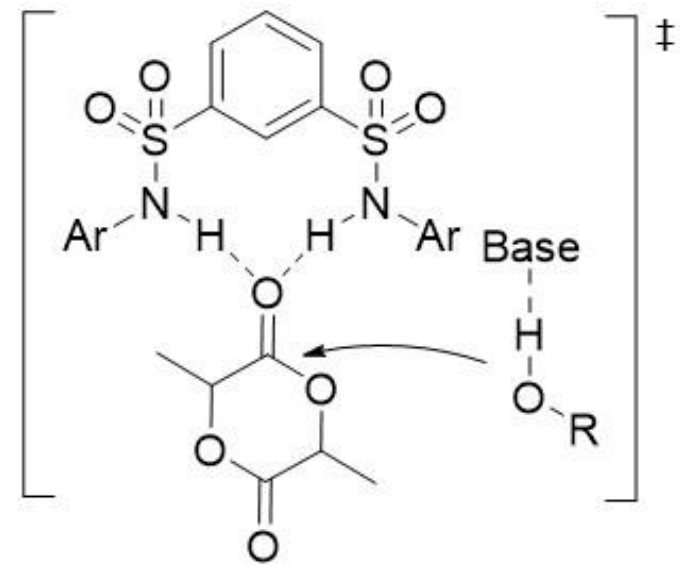

Figure 1.5. Sulfonamide H-bonding Catalysts. 

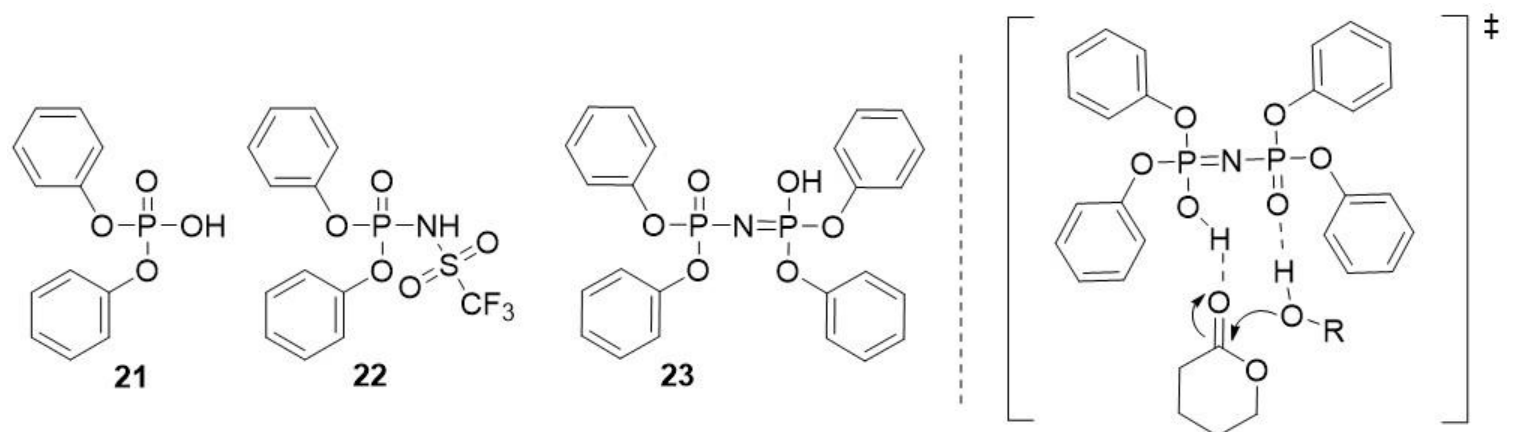

Figure 1.6. Diphenyl Phosphate, Phosphoramidic and Imidodiphosphoric Acid Catalyzed ROP. 


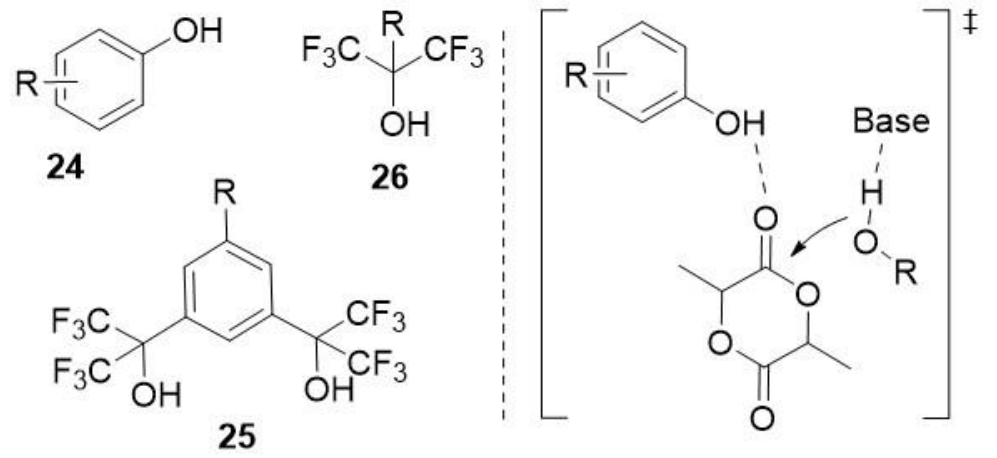

Figure 1.7. Phenol and Benzylic Alcohol H-bond Donors for ROP. 

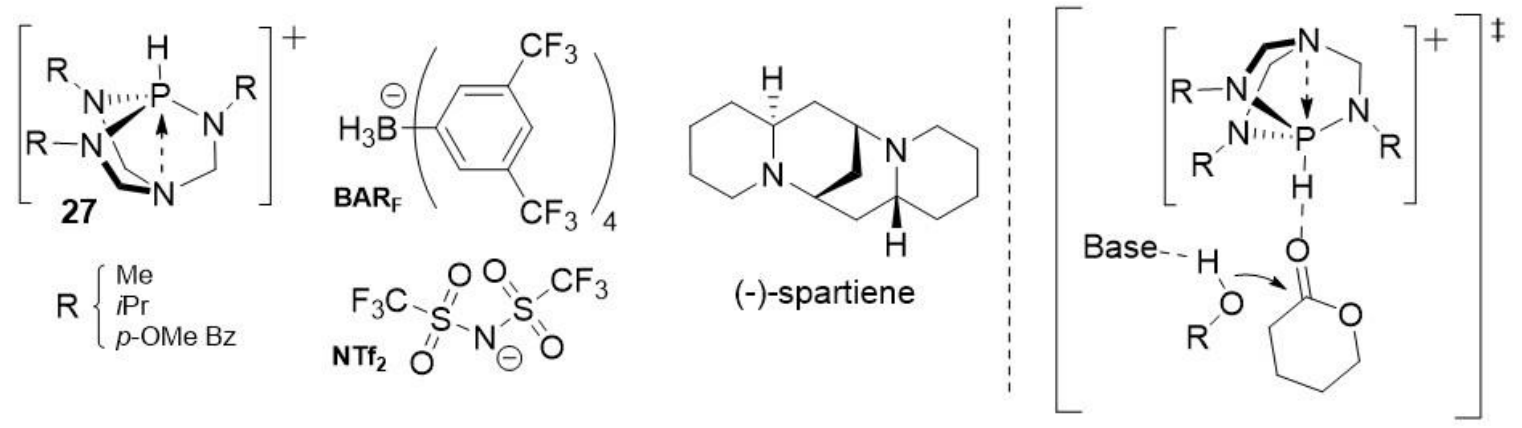

Figure 1.8. Azaphosphatrane H-bond Donor. 




Figure 1.9. Electrophilic Monomer Activation by Stable Cations. 


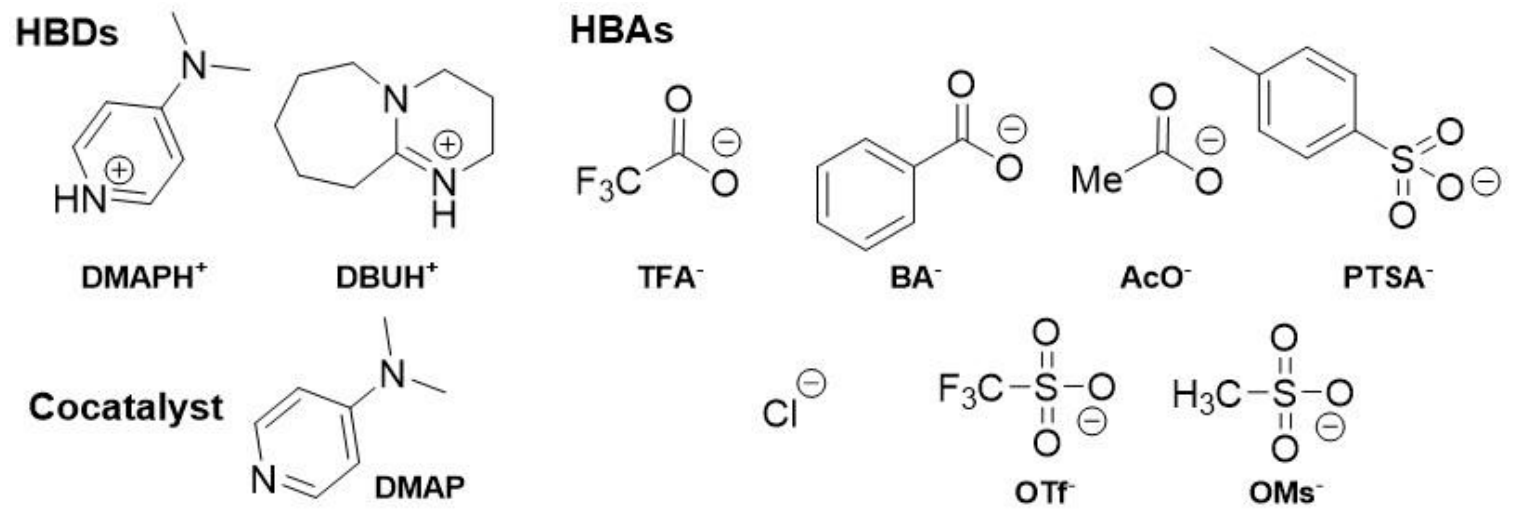

Figure 1.10. Bronsted Acid and Base Cocatalysts for ROP. 


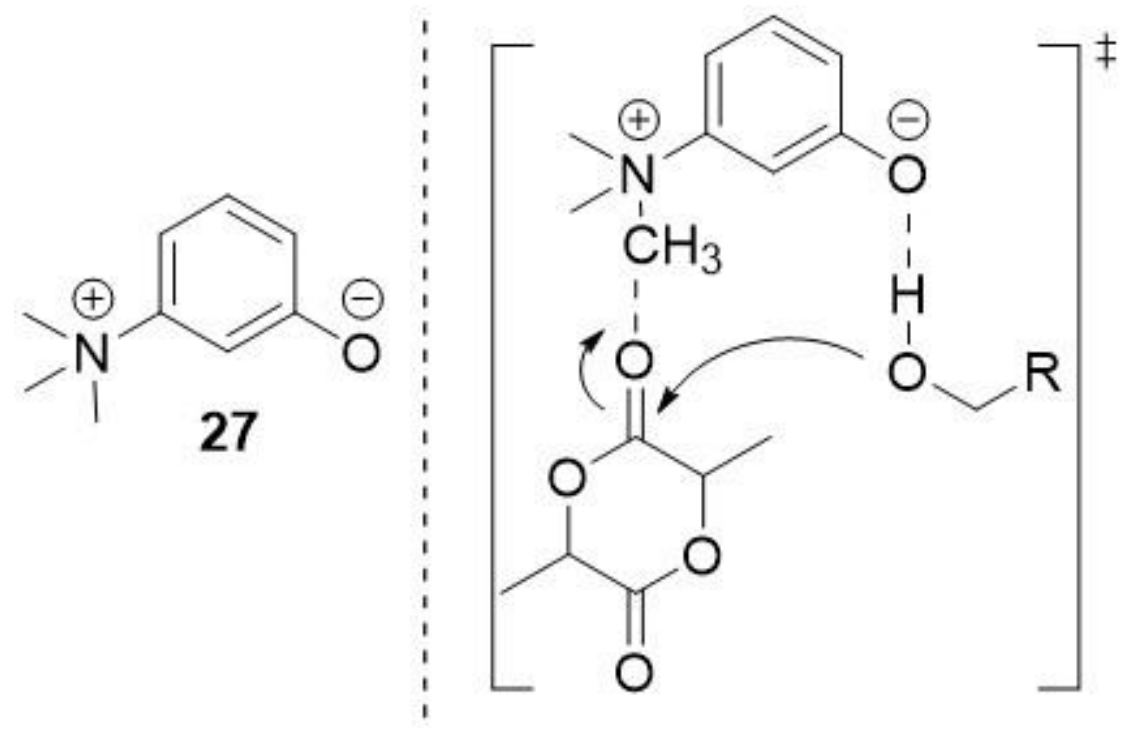

Figure 1.11. Ammonium Betaine Mediated ROP. 


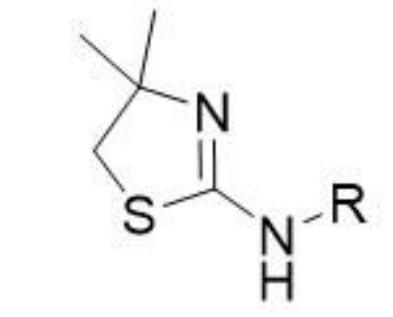

amino-thiazoline

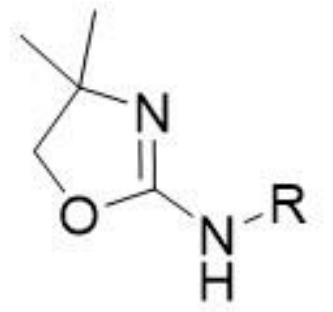

amino-oxazoline

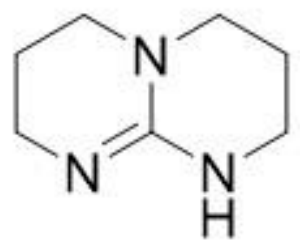

TBD

Figure 1.12. Thiazoline and Oxazoline Bifunctional Catalysts. 


\title{
MANUSCRIPT - II
}

\author{
Published in Macromolecules
}

\section{Cooperative Hydrogen-Bond Pairing in Organocatalytic Ring-Opening Polymerization}

Oleg I. Kazakov, Partha P. Datta, Meghedi Isajani, Elizabeth T. Kiesewetter and Matthew K. Kiesewetter

Chemistry, University of Rhode Island, Kingston, RI, USA

Corresponding Author: $\quad$ Matthew Kiesewetter, Ph.D.

Chemistry

University of Rhode Island

140 Flagg Road

Kingston, RI, 02881, USA

Email address: mkiesewetter@chm.uri.edu 


\begin{abstract}
Thiourea (TU)/amine base cocatalysts are commonly employed for well-controlled, highly active "living" organocatalytic ring-opening polymerizations (ROPs) of cyclic esters and carbonates. In this work, several of the most active cocatalyst pairs are shown by ${ }^{1} \mathrm{H}$ NMR binding studies to be highly associated in solution, dominating all other known noncovalent catalyst/reagent interactions during ROP. One strongly binding catalyst pair behaves kinetically as a unimolecular catalyst species. The high selectivity and activity exhibited by these ROP organocatalysts are attributed to the strong binding between the two cocatalysts, and the predictive utility of these binding parameters is applied for the discovery of a new, highly active cocatalyst pair.
\end{abstract}




\section{INTRODUCTION}

The multitude of polymer architectures and constructs that can be generated via organocatalytic ring-opening polymerization (ROP) is largely driven by the precise level of reaction control engendered by the catalysts. ${ }^{1-4}$ The asymmetrical thiourea, $\mathbf{1}$ in Scheme 2.1, is believed to selectively activate cyclic esters and carbonates for ROP (Eq. 2.1 ); ${ }^{5}$ it is conveniently synthesized, highly active, and has become a preferred hydrogen bond donor for ROP. ${ }^{5-11}$ A more varied slate of base cocatalysts (H-bond acceptors) is used to activate the initiating/propagating alcohol for nucleophilic attack (Eq. 2.2) $)^{5,7,9}$ and stronger bases are generally more active as cocatalysts for ROP. ${ }^{12}$ The imine bases, particularly 1,8 diazabicyclo[5.4.0] undec-7-ene (DBU in Scheme 2.1), have found common implementation in ROP. ${ }^{1,2,4,5,8,13}$ The preponderance of experimental ${ }^{5,11,14,15}$ and computational $^{14,16,17}$ evidence suggests that bimolecular hydrogen bond activation of lactone and initiating/propagating alcohol facilitates the rapid ROP of lactone monomers exhibited by 1/DBU (Scheme 2.1). ${ }^{4,5,18}$ The exact balance of interactions that must exist for a "living" ROP to occur is impressive, ${ }^{6}$ and deep mechanistic insights into the robust and diverse set of H-bonding ROP organocatalysts will be the driving force for the development of the improved catalysts which precede new materials. In the following, we present evidence that $\mathbf{1}$ and amine base cocatalysts are highly associated in solution and that this binding is productive rather than inhibitory toward the high activity and selectivity of these 1/amine base systems. This increased mechanistic understanding is applied to the discovery of a new cocatalyst pair for ROP. 


\section{EXPERIMENTAL SECTION}

\section{General Considerations}

All manipulations were performed in an MBRAUN stainless steel glovebox equipped with a gas purification system under a nitrogen atmosphere. All chemicals were purchased from Fisher Scientific and used as received unless stated otherwise. Toluene and THF were dried on an Innovated Technologies solvent purification system with alumina columns and nitrogen working gas. Benzene- $\mathrm{d}_{6}$ was supplied by Cambridge Isotope Laboratories and distilled from $\mathrm{CaH}_{2}$ under a nitrogen atmosphere. $\delta$-Valerolactone (VL; 99\%) and $\varepsilon$-caprolactone (CL; 99\%) were distilled from $\mathrm{CaH}_{2}$ under high vacuum. Benzyl alcohol was distilled from $\mathrm{CaH}_{2}$ under high vacuum. L-Lactide was supplied by Acros Organics and recrystallized from dry toluene prior to use. 1-[3,5-Bis(trifluoromethyl)phenyl]-3-cyclohexylthiourea (1) was synthesized and purified according to literature procedures. ${ }^{5}$ 1,8-Diazabicyclo[5.4.0]undec-7-ene (DBU) and 7-methyl-1,5,7triazabicyclo[4.4.0]dec-5-ene (MTBD) were purchased from TCI. NMR experiments were performed on a Bruker Avance $300 \mathrm{MHz}$ spectrometer. Size exclusion chromatography (SEC) was performed at $40^{\circ} \mathrm{C}$ in dichloromethane (DCM) using an Agilent Infinity GPC system equipped with three Agilent PLGel columns $7.5 \mathrm{~mm} \times 300 \mathrm{~mm}(5 \mu \mathrm{m}$, pore sizes:

103, 104, and $105 \AA$ ). Molecular weight and $\mathrm{M}_{\mathrm{w}} / \mathrm{M}_{\mathrm{n}}$ were determined versus PS standards (500 g/mol-3150 kg/mol; Polymer Laboratories).

Determination of Binding Constant by the Dilution Method

A stock solution containing $1(2.8 \mathrm{mg}, 0.0075 \mathrm{mmol})$ and DBU (0.0011 mL, 0.0075 mmol) was prepared in deuterated benzene $(1.5 \mathrm{~mL})$. This solution was distributed to $6-10$ 
NMR tubes, and each NMR tube was diluted with benzene- $\mathrm{d}_{6}$ to give final concentrations ranging from 5 to $0.313 \mathrm{mM} .{ }^{1} \mathrm{H}$ NMR spectra (referenced to residual benzene-H) were acquired for each tube at multiple temperatures, and the chemical shift of the ortho-protons of 1 was noted. The $\mathrm{K}_{\mathrm{eq}}$ values were determined from the linearized (Lineweaver-Burke) forms of the binding equations (see Equations Used for Binding Studies below), which are a powerful means of accurately measuring binding constants with fewer samples (versus curve fitting). ${ }^{19}$ The binding constant for each $\mathbf{1} /$ base pair was determined at elevated temperatures $(303-323 \mathrm{~K})$. The enthalpy and entropy of binding were determined by plotting $\ln \mathrm{K}_{\mathrm{eq}}$ versus 1/T to conduct a Van't Hoff analysis, and error was determined from linear regression at the $95 \%$ confidence interval.

Example Determination of $\mathrm{k}_{\mathrm{obs}}$

In a glovebox under a nitrogen atmosphere, one vial (baked at $140^{\circ} \mathrm{C}$ overnight) was loaded with a stir bar and $\delta$-valerolactone (VL) $(0.0927 \mathrm{~mL}, 1.00 \mathrm{mmol})$. A second dried vial was loaded with benzyl alcohol $(0.0021 \mathrm{~mL}, 0.020 \mathrm{mmol}), 1$ (18.5 mg, 0.050 mmol), and DBU (0.0075 mL, $0.050 \mathrm{mmol}) .200 \mu \mathrm{L}$ of deuterated benzene was added to the first vial, and $300 \mu \mathrm{L}$ of deuterated benzene was added to the second vial. The solutions were stirred until homogeneous. The reaction was started by transferring the solution of VL into the vial containing catalyst solution and stirred to mix before transferring to an NMR tube. The change in the concentration of the monomer was monitored by ${ }^{1} \mathrm{H}$ NMR. Rate constants were extracted from a plot of $\ln \left([\mathrm{VL}]_{0} /[\mathrm{VL}]\right)$ versus time; the reaction is linear on this plot to $3+$ half-lives. The slope of this plot is $\mathrm{k}_{\mathrm{obs}}$, and the error was determined by propagation of NMR integration error at $\pm 5 \%$. Only [1] and [DBU] were varied between individual kinetic runs. 


\section{Example Ring-Opening Polymerization}

In a typical polymerization, $\mathrm{VL}(0.100 \mathrm{~g}, 0.999 \mathrm{mmol})$ was added to a $20 \mathrm{~mL}$ glass vial containing a stir bar, both of which were baked at $140^{\circ} \mathrm{C}$ overnight. In another dried $20 \mathrm{~mL}$ glass vial with stir bar, 1 (0.0185 g, $0.499 \mathrm{mmol})$, BEMP (14.45 $\mu \mathrm{L}, 0.499 \mathrm{mmol})$, and 1-pyrenebutanol $\left(9.96 \mu \mathrm{mol}\right.$ ) were added. Solvent (for $\mathrm{C}_{6} \mathrm{D}_{6} 0.4744 \mathrm{~g}, 2 \mathrm{M}$ in VL) was added to both vials to bring the total mass of solvent to the desired level, approximately equal portions of solvent per vial. After stirring for $5 \mathrm{~min}$, the VL solution was transferred via pipet to the vial containing catalysts and initiator. To quench the reaction, benzoic acid ( 2 mol equiv to base) was added. The vial was removed from the glovebox, and the polymer solution was treated with hexanes to precipitate the polymer. The hexanes supernatant was decanted, and the polymer removed of volatiles under reduced pressure. Yield, 90\%; $\mathrm{M}_{\mathrm{w}} / \mathrm{M}_{\mathrm{n}}=1.03 ; \mathrm{M}_{\mathrm{n}(\mathrm{GPC})}=16800 .{ }^{1} \mathrm{H}$ NMR $\left(\mathrm{C}_{6} \mathrm{D}_{6}\right) \delta: 7.22-7.17(2 \mathrm{H}, \mathrm{d}$, benzyl aryls), 7.13-7.05 (3H, m, benzyl aryls), 4.97 (2H, s, benzylic), $3.91\left(193 \mathrm{H}, \mathrm{t},-\mathrm{C}(\mathrm{O}) \mathrm{OCH}_{2}-\right), 2.04$ $\left(193 \mathrm{H}, \mathrm{t},-\mathrm{CH}_{2} \mathrm{C}(\mathrm{O}) \mathrm{O}^{-}\right), 1.58-1.30\left(386 \mathrm{H}, \mathrm{m}, \mathrm{C}(\mathrm{O}) \mathrm{CH}_{2} \mathrm{CH}_{2} \mathrm{CH}_{2} \mathrm{CH}_{2} \mathrm{O}-\right)$.

Equations Used for Binding Studies

For dilution: $\quad \Delta \delta /[$ base $]=-2 \mathrm{~K}_{\mathrm{eq}} \Delta \delta+\mathrm{K}_{\mathrm{eq}} \delta \mathrm{c}$

For titration: $\quad \Delta \delta /[$ base $]=-\mathrm{K}_{\mathrm{eq}} \Delta \delta+\mathrm{K}_{\mathrm{eq}} \delta \mathrm{c}$

Where $\Delta \delta$ is the difference between the chemical shift of the observed orthoprotons in the TU-Base mixture and of pure TU; $\delta c$ is the chemical shift of the orthoprotons of TU in the complex, TU-Base; $\mathrm{K}_{\mathrm{eq}}$ is the binding constant between $\mathbf{1}$ and a Base. The determination of binding constants from the slope of the linear (Lineweaver-Burke) forms of the binding equation (above) has several benefits over fitting the binding curve. ${ }^{20}$ It should be noted that the linearized form of the binding equations are rigorously true and 
can be derived from the equilibrium expression using simple algebra. ${ }^{21}$ Very accurate data can be obtained with fewer data points (versus curve fitting) because experimental errors from inaccurate concentration are attenuated in the linearized form. For this method, the accuracy of $\mathrm{K}_{\mathrm{eq}}$ versus number of data points has been tested in the literature and shown to be highly accurate with 5 data points. ${ }^{22}$ These studies even omitted the plateau of the binding curve,${ }^{22}$ which was never the case in our studies. Further, computationally fitting the binding curve introduces indeterminable error from the fitting approximations. Error in the slope of the linear form $\left(\mathrm{K}_{\mathrm{eq}}\right)$ is solely determined by the scatter in data (from residual error in concentration), and the error in $\mathrm{K}_{\mathrm{eq}}$ is exactly the error in the slope of the line, which can be determined from linear regression. ${ }^{21}$ 


\section{RESULTS AND DISCUSSION}

\section{Chemical Kinetics}

Kinetic studies were undertaken to help elucidate the roles of $\mathbf{1}$ and DBU in the ROP of $\delta$-valerolactone (VL). While holding the concentration of VL (2 M, $1.00 \mathrm{mmol})$ and benzyl alcohol $(0.04 \mathrm{M}, 0.020 \mathrm{mmol})$ constant in $\mathrm{C}_{6} \mathrm{D}_{6}$, the concentrations of 1 and DBU were varied from $[\mathbf{1}]=[\mathrm{DBU}]=0.05$ to $0.20 \mathrm{M}$ (see Figure 2.4). The resulting plot (Figure 2.1) of observed rate constant, $\mathrm{k}_{\mathrm{obs}}$, versus $([\mathbf{1}]+[\mathrm{DBU}])$, where $[\mathbf{1}]=[\mathrm{DBU}]$, is linear, which describes an ROP reaction that is first order in cocatalysts: Rate $=\mathrm{k}_{\mathrm{obs}}[\mathrm{VL}]$, where $\mathrm{k}_{\mathrm{obs}}=\mathrm{k}_{\mathrm{P}}([1]+[\mathrm{DBU}])\left[\right.$ benzyl alcohol], and $\mathrm{k}_{\mathrm{P}}$ is the polymerization rate constant. This observation is in contrast to a previous report which assumed for purposes of kinetic fitting that rate is proportional to both [1] and [base] (i.e., $\mathrm{k}_{\mathrm{obs}}=\mathrm{k}_{\mathrm{P}}[\mathbf{1}][$ base][benzyl alcohol] $).{ }^{5}$ The ROP rate being proportional to ([1] + [DBU]) suggests a cocatalyst system that behaves as a discrete catalyst species, yet the role of the individual cocatalyst moieties is unclear.

Kinetic studies were also undertaken when $[\mathbf{1}] \neq[\mathrm{DBU}]$. For the case where $\mathbf{1}$ is in excess, the observed rate constant is insensitive to [1] (within error) for the concentration range examined (see Figure 2.5). The thiourea, 1, is known to self-bind at high concentrations, ${ }^{6}$ and any increased monomer activation may be attenuated by catalyst selfinhibition (due to $\mathbf{1 \cdot 1}$ ) at $[\mathbf{1}]>0.2 \mathrm{M}$. In the case of $[\mathrm{DBU}]>[\mathbf{1}]$, the data describe a reaction that is inverse first order in $[\mathrm{DBU}]$ for the entire concentration range examined $(100 \mathrm{mM}<[\mathrm{DBU}]<400 \mathrm{mM} ;[\mathbf{1}]=50 \mathrm{mM})$ (see Figure 2.6). The fact that both cocatalysts must be present for ROP to occur suggests that DBU facilitates catalysis. However, the 
empirical rate dependences upon [1] and [DBU] imply an inhibitory role for DBU which would occur upon a strong binding interaction between $\mathbf{1}$ and DBU.

\section{Cocatalyst Binding}

Inhibitory interactions by amine base cocatalysts upon $\mathbf{1}$ have been suggested by other researchers to decrease ROP rate. ${ }^{6}$ In an illuminating study of several cocatalysts, it was found via ${ }^{1} \mathrm{H}$ NMR binding studies that $\mathbf{1}$ and sparteine, an erstwhile favorite catalyst pair for the ROP of lactide, ${ }^{10}$ exhibit a moderate binding constant of $\mathrm{K}_{\mathrm{eq}}\left(\mathrm{CDCl}_{3}\right)=6 \pm 1$. $^{6,23}$ This magnitude of binding constant was not thought to be inhibitory to catalysis, but the same study ascribed the reduced activity of some more strongly binding cocatalysts to an undesirable $\mathrm{H}$-bond equilibrium that reduces the effective concentration of catalyst through self-inhibition. ${ }^{6,8}$ The potent H-bonding ability of $\mathrm{DBU}^{24}$ and high activity of $\mathbf{1} / \mathrm{DBU}$ for ROP belie this concept.

A ${ }^{1} \mathrm{H}$ NMR binding study ${ }^{25}$ conducted in our laboratory by serial dilution of a 1:1 mixture of DBU and 1 (from 5 to $0.125 \mathrm{mM}$ ) reveals a strong $\mathbf{1} \cdot \mathrm{DBU}$ binding constant of $\mathrm{K}_{\mathrm{eq}}=4200 \pm 170$ (Eq. 2.3) (see Table 2.3). Such strong interactions have previously been posited (vide infra) between Coulombically tethered cocatalysts, ${ }^{15}$ and strong cocatalyst binding is not necessarily inhibitory to ROP. All binding processes are reversible and rapid on the NMR time scale, and the ROP is determined by the approach to the equilibrium monomer concentration, $[\mathrm{VL}]_{\mathrm{eq}}$. The strong 1.DBU binding constant may simply act in concert with other known interactions (1.VL and DBU·benzyl alcohol; Eqs. 1 and 2) to hold all reagents in close proximity during a rapid exchange of binding partners, thereby accelerating the reaction. ${ }^{26}$ However, the kinetic data suggest that the strong binding could serve to make a distinct catalytic species. ${ }^{27}$ The binding and kinetic data collectively 
describe a reaction process where highly self-associated cocatalysts can be cooperatively interrupted by VL and alcohol to result in a reaction turnover (Scheme 2.2).

The selectivity of $\mathbf{1} / \mathrm{DBU}$ for monomer in the ROP of VL can be rationalized by the magnitude of the 1.DBU binding constant. This selectivity has previously been attributed to the preference of $\mathbf{1}$ to bind to s-cis esters (monomers) versus s-trans esters (polymer backbone) ${ }^{5}$ however, some $\mathbf{1} /$ amine base combinations result in almost zero transesterification of the resultant polymer after $4 \mathrm{~h}^{28}$ The very dependence of postpolymerization transesterification upon the identity of the base cocatalyst suggests that factors other than the 1-ester binding constants control ROP selectivity. Indeed, the identity of the base cocatalyst dominates the equilibria which describe the ability of ethyl acetate (a surrogate for polymer, which exhibits a small but nonzero binding to $\mathbf{1})^{5}$ to interrupt the 1. DBU pair (Eq. 2.4) versus that of VL (Eq. 2.5). These values $\left(\mathrm{K}_{\mathrm{eq}}=0.003 \mathrm{vs} \mathrm{K}_{\mathrm{eq}}=0.13\right.$, respectively), which can be found through thermodynamic sums, could account for the high selectivity of the ROP reaction. Further, altering the base cocatalyst would be expected to drastically alter the cocatalyst selectivity for monomer, as empirically observed. ${ }^{1-4,28}$

Our study was continued on a variety of base cocatalysts (with 1) for ROP, and a relationship between cocatalyst binding and ROP activity was discovered. Binding constants to $\mathbf{1}$ in $\mathrm{C}_{6} \mathrm{D}_{6}$ were measured by either the dilution or titration method ${ }^{19-21,29}$ for bases previously evaluated as cocatalysts in the ROP literature: DBU, MTBD (7-methyl1,5,7-triazabicyclo[4.4.0]dec-5-ene), pyridine, proton sponge $\quad(1,8$ bis(dimethylamino)naphthalene), and DMAP (4-(dimethylamino)pyridine). The $\mathrm{k}_{\mathrm{obs}}$ values were also measured for each of these bases (see Table 2.3) in the $\mathbf{1}(0.1 \mathrm{M}, 0.050$ $\mathrm{mmol})$ and base $(0.1 \mathrm{M}, 0.050 \mathrm{mmol})$ catalyzed ROP of cyclic ester monomers (2 M, 1.00 
mmol) from benzyl alcohol $(0.04 \mathrm{M}, 0.020 \mathrm{mmol})$; the results of these experiments are shown in Table 2.1. In general, a strong 1-base binding constant is associated with rapid ROP, and weakly binding cocatalysts exhibit very low or zero ROP activity.

In the low binding constant regime, $\mathrm{K}_{\mathrm{eq}}$ correlates with polymerization rate, and cocatalyst binding constant appears to be a better predictor of catalytic activity than does $\mathrm{pK}_{\mathrm{a}}$. The $\mathrm{k}_{\mathrm{obs}}$ for the systems that exhibited weak binding ( 1 with DMAP, pyridine, or proton sponge) were measured for the $1 /$ base catalyzed ROP of L-lactide (LA) (Table 2.1) as they are not active for the ROP of VL. Of these cocatalysts, only 1/DMAP exhibits ROP activity: $\mathrm{k}_{\mathrm{obs}}(\mathrm{LA})=4.1 \times 10-3 \mathrm{~min}^{-1}$. Both $\mathbf{1} /$ pyridine and $\mathbf{1} /$ proton sponge are inactive for the ROP of LA, but $\mathbf{1}$. pyridine displays weak binding (1-pyridine $\left.\mathrm{K}_{\mathrm{eq}}=9 \pm 1\right)$ whereas 1.proton sponge exhibits none. The binding constant observed for 1.DMAP was the strongest of the three $\left(\mathbf{1} \cdot \mathrm{DMAP} \mathrm{K}_{\mathrm{eq}}=170 \pm 30\right)$. A pK $\mathrm{a}_{\mathrm{a}}$ explanation of ROP activity is unsuccessful for the case of DMAP vs proton sponge (in acetonitrile: $\mathrm{DMAP}-\mathrm{H}^{+} \mathrm{pK}_{\mathrm{a}}=$ $18.2{ }^{30}$ proton sponge- $\left.\mathrm{H}^{+} \mathrm{pK}_{\mathrm{a}}=18.7\right),{ }^{31,32}$ yet their ROP activities correlate well with the strength of their binding to $\mathbf{1}$. For the $\mathbf{1}$ /pyridine system, its moderate binding constant yet lack of ROP activity could indicate that ROP is only feasible when cocatalyst binding becomes competitive with $1 \cdot$ lactone binding $\left(1 \cdot \mathrm{VL} \mathrm{K}_{\mathrm{eq}}\left(\mathrm{C}_{6} \mathrm{D}_{6}\right)=44 ;{ }^{5} \mathbf{1} \cdot \mathrm{LA} \mathrm{K}_{\mathrm{eq}}\left(\mathrm{CDCl}_{3}\right)=\right.$ $2)^{6}$ such that the cocatalysts are closely associated in solution.

The binding constant between 1 and DBU was the strongest measured, but this catalyst pair is not the most active of those examined for the ROP of VL. 1/MTBD exhibited a faster rate for the ROP of VL than 1/DBU, which is reasonably predicted by $\mathrm{pK}_{\mathrm{a}}: \mathrm{MTBD}^{-\mathrm{H}_{+}} \mathrm{pK}_{\mathrm{a}}{ }^{\mathrm{MeCN}}=25 \cdot 4 ;^{32} \mathrm{DBU}-\mathrm{H}^{+} \mathrm{pK}_{\mathrm{a}}^{\mathrm{MeCN}}=24.3 .^{32}$ As Bibal et al. noted, strong cocatalyst binding is anticipated to be inhibitory to $\mathrm{ROP},{ }^{6,7}$ and one interpretation of the 
1/DBU vs 1/MTBD reactions is that ROP activity ( $\mathrm{k}_{\mathrm{obs}}$ ) becomes attenuated due to catalyst inhibition if the cocatalyst binding constant becomes too large, $1500<\mathrm{K}_{\mathrm{eq}}<4200$.

\section{BEMP/1 Catalyzed ROP}

One of the most powerful applications of reaction mechanism elucidation is in the discovery of new catalyst species, and we sought to ply our increased understanding of 1/base catalyzed ROP to this end. While this work was ongoing, Dixon et al. reported the ROP of VL by a phosphazene-inspired bifunctional TU-iminophosphorane catalyst, 2 in Eq. 2.6. ${ }^{33}$ The bifunctional catalyst 2 exhibits "living" ROP behavior, the usual relative monomer reactivity $\left(\mathrm{k}_{\mathrm{LA}}>\mathrm{kVL}_{\mathrm{VL}} \gg \mathrm{k}_{\mathrm{CL}}\right)$, and good selectivity for monomer. ${ }^{33}$ While the application of phosphazene bases like BEMP (2-tert-butylimino-2-diethylamino-1,3dimethylperhydro-1,3,2-diazaphosphorine) to the ROP of LA is known, ${ }^{34}$ this superbase is not active for the ROP of VL except in neat monomer where reaction control is poor (2 days, $93 \%$ conversion, $\left.\mathrm{M}_{\mathrm{w}} / \mathrm{M}_{\mathrm{n}}=1.23\right) .{ }^{35}$

The binding constant of BEMP and 1 was measured in $\mathrm{C}_{6} \mathrm{D}_{6}, \mathrm{~K}_{\mathrm{eq}}=1200 \pm 40$. Within the set of $\mathrm{K}_{\mathrm{eq}} \mathrm{vs} \mathrm{k}_{\mathrm{obs}}$ data, the strength of the 1-BEMP binding constant suggests its VL ROP activity should be similar to that of 1/MTBD. Indeed, the observed rate constant for the 1/BEMP catalyzed ROP of VL $\left(\mathrm{k}_{\mathrm{obs}}(\mathrm{VL})=17.8 \times 10^{-3} \mathrm{~min}^{-1}\right)$ is slightly less than that of $1 / \mathrm{MTBD}$, as would be expected by the 1 . BEMP $\mathrm{K}_{\text {eq }}$ value. This result would not be anticipated by a $\mathrm{pK}_{\mathrm{a}}$ argument: $\mathrm{BEMP}-\mathrm{H}^{+} \mathrm{pK}_{\mathrm{a}}^{\mathrm{MeCN}}=27.6{ }^{36} \mathrm{MTBD}^{\mathrm{M}} \mathrm{H}^{+} \mathrm{pK}_{\mathrm{a}}{ }^{\mathrm{MeCN}}=25.4 .^{32}$ Further studies show that 1/BEMP is active for the ROP of VL, $\varepsilon$-caprolactone (CL), and trimethylene carbonate (TMC) but is inactive for $\beta$-butyrolactone (BL) (Table 2.2). The 1/BEMP catalyzed ROP of VL from 1-pyrenebutanol exhibits the characteristics of a "living" ROP: linear evolution of $\mathrm{M}_{\mathrm{n}}$ with conversion (see Figure 2.9), evidence of end- 
group fidelity (overlapping RI and UV signals by GPC), and $\mathrm{M}_{\mathrm{n}}$ that is predictable by $[\mathrm{M}]_{\mathrm{o}} /[\mathrm{I}]_{\mathrm{o}}$. The evidence of H-bonding for both BEMP-to-alcohol ${ }^{35}$ and 1-to-VL ${ }^{5}$ taken with these experimental observations suggests an H-bond mediated "living" ROP of VL. The ROP activity (for VL) of the cocatalyst systems 1/BEMP, 1/DBU, and 1/MTBD is only slightly attenuated in THF. 


\section{CONCLUSION}

For the organocatalytic ROP cocatalysts examined, the magnitude of the cocatalyst binding constant has been shown to be proportional to the ROP rate. For the bases studied, cocatalyst binding constant is a far better predictor of catalytic activity than $\mathrm{pK}_{\mathrm{a}}$. The strongly binding 1/DBU system behaves kinetically as a unimolecular catalyst species, and it could be representative of a hydrogen-bonding analogue of so-called "cooperative ion pairing" in asymmetric organocatalysis. ${ }^{27} \mathrm{We}$ agree with the conclusion of Bibal et al. that TU/amine base binding can be inhibitory to $\mathrm{ROP}^{6,7}$ but submit that (1) the phenomenon is much more general than first proposed, (2) the magnitude of the interaction may be a good predictor of cocatalyst activity, and (3) the point at which cocatalyst binding becomes counterproductive to catalysis is significantly higher than once believed. As organocatalysis strives to mimic the awe-inspiring catalytic abilities of nature, it is important to fully understand the catalytic systems being employed. As it would happen, the roles of $\mathbf{1}$ and DBU in the ROP of VL are not very dissimilar from those of enzyme and cofactor. Further mechanistic studies are ongoing; such studies have already revealed one new catalyst system for ROP (1/BEMP), and they are expected to yield dividends in the form of more new catalyst systems. 


\section{LIST OF REFERENCES}

(1) Dove, A. P. ACS Macro Lett. 2012, 1 (12), 1409-1412.

(2) Bourissou, D.; Moebs-Sanchez, S.; Martín-Vaca, B. Comptes Rendus Chim. 2007, 10 (9), 775-794.

(3) Kamber, N. E.; Jeong, W.; Waymouth, R. M.; Pratt, R. C.; Lohmeijer, B. G. G.;

Hedrick, J. L. Chem. Rev. 2007, 107 (12), 5813-5840.

(4) Kiesewetter, M. K.; Shin, E. J.; Hedrick, J. L.; Waymouth, R. M. Macromolecules 2010, 43 (5), 2093-2107.

(5) Bas G. G. Lohmeijer Frank Leibfarth, John W. Logan, R. C. P.; David A. Long Fredrik Nederberg, Jeongsoo Choi, A. P. D.; Charles Wade and James L. Hedrick, R. M. W.; Lohmeijer, B. G. G.; Pratt, R. C.; Leibfarth, F.; Logan, J. W.; Long, D. A.; Dove, A. P.; Nederberg, F.; Choi, J.; Wade, C.; Waymouth, R. M.; Hedrick, J. L. Macromolecules 2006, 39 (25), 8574-8583.

(6) Koeller, S.; Kadota, J.; Peruch, F.; Deffieux, A.; Pinaud, N.; Pianet, I.; Massip, S.; Léger, J. M.; Desvergne, J. P.; Bibal, B. Chem. - A Eur. J. 2010, 16 (14), 41964205.

(7) Thomas, C.; Bibal, B. Green Chem. 2014, 16 (4), 1687-1699.

(8) Thomas, C.; Peruch, F.; Bibal, B. RSC Adv. 2012, 2 (33), 12851.

(9) Lippert, K. M.; Hof, K.; Gerbig, D.; Ley, D.; Hausmann, H.; Guenther, S.; Schreiner, P. R. European J. Org. Chem. 2012, 2012 (30), 5919-5927.

(10) Todd, R.; Rubio, G.; Hall, D. J.; Tempelaar, S.; Dove, A. P. Chem. Sci. 2013, 4 (3), 1092-1097.

(11) Dove, A. P.; Pratt, R. C.; Lohmeijer, B. G. G.; Waymouth, R. M.; Hedrick, J. L. J. 
Am. Chem. Soc. 2005, 127 (40), 13798-13799.

(12) Pratt, R. C.; Lohmeijer, B. G. G.; Long, D. A.; Lundberg, P. N. P.; Dove, A. P.; Li, H.; Wade, C. G.; Waymouth, R. M.; Hedrick, J. L. Macromolecules 2006, 39 (23), $7863-7871$.

(13) Misaka, H.; Kakuchi, R.; Zhang, C.; Sakai, R.; Satoh, T.; Kakuchi, T. Macromolecules 2009, 42 (14), 5091-5096.

(14) Zhang, L.; Pratt, R. C.; Nederberg, F.; Horn, H. W.; Rice, J. E.; Waymouth, R. M.; Wade, C. G.; Hedrick, J. L. Macromolecules 2010, 43 (3), 1660-1664.

(15) Coady, D. J.; Fukushima, K.; Horn, H. W.; Rice, J. E.; Hedrick, J. L. Chem. Commun. 2011, 47 (11), 3105-3107.

(16) Horn, H. W.; Jones, G. O.; Wei, D. S.; Fukushima, K.; Lecuyer, J. M.; Coady, D. J.; Hedrick, J. L.; Rice, J. E. J. Phys. Chem. A 2012, 116 (51), 12389-12398.

(17) Coady, D. J.; Horn, H. W.; Jones, G. O.; Sardon, H.; Engler, A. C.; Waymouth, R. M.; Rice, J. E.; Yang, Y. Y.; Hedrick, J. L. ACS Macro Lett. 2013, 2 (4), 306-312.

(18) Becker, J. M.; Tempelaar, S.; Stanford, M. J.; Pounder, R. J.; Covington, J. A.; Dove, A. P. Chem. - A Eur. J. 2010, 16 (20), 6099-6105.

(19) Horman, I.; Dreux, B. Anal. Chem. 1983, 55 (8), 1219-1221.

(20) Deranleau, D. A. J. Am. Chem. Soc. 1969, 91 (15), 4044-4049.

(21) Peters, S. J.; Stevenson, C. D. J. Chem. Educ. 2004, 81 (5), 715.

(22) Horman, I.; Dreux, B. Anal. Chem. 1983, 55 (8), 1219-1221.

(23) The units of binding constant in this chapter are $\mathrm{M}^{-1}$. The chemical convention of unitless $K_{e q}$ is used throughout.

(24) Laurence, C.; Brameld, K. A.; Graton, J.; Le Questel, J.-Y.; Renault, E. J. Med. 
Chem. 2009, 52 (14), 4073-4086.

(25) The ortho-protons were monitored for all studies as the NH protons of $\mathbf{1}$ are not always observed. The large chemical shifts of the NH protons upon binding and rapid exchange rates broaden those resonances into the baseline.

(26) Rebek, J. Angew. Chemie Int. Ed. 2005, 44 (14), 2068-2078.

(27) Briere, J.-F.; Oudeyer, S.; Dalla, V.; Levacher, V. Chem. Soc. Rev. 2012, 41 (5), $1696-1707$.

(28) Coady, D. J.; Engler, A. C.; Horn, H. W.; Bajjuri, K. M.; Fukushima, K.; Jones, G. O.; Nelson, A.; Rice, J. E.; Hedrick, J. L. ACS Macro Lett. 2012, 1 (1), 19-22.

(29) Kelly, T. R.; Kim, M. H. J. Am. Chem. Soc. 1994, 116 (16), 7072-7080.

(30) Augustin-Nowacka, D.; Chmurzyñski, L. Anal. Chim. Acta 1999, 381 (2), 215220.

(31) Koppel, I. A.; Koppel, J. B.; Pihl, V. O. Org. React. 1987, 24 (4), 387-398.

(32) Kaljurand, I.; Kütt, A.; Sooväli, L.; Rodima, T.; Mäemets, V.; Leito, I.; Koppel, I. A. J. Org. Chem. 2005, 70 (3), 1019-1028.

(33) Goldys, A. M.; Dixon, D. J. Macromolecules 2014, 47 (4), 1277-1284.

(34) Zhang, L.; Nederberg, F.; Messman, J. M.; Pratt, R. C.; Hedrick, J. L.; Wade, C. G. J. Am. Chem. Soc. 2007, 129 (42), 12610-12611.

(35) Zhang, L.; Nederberg, F.; Pratt, R. C.; Waymouth, R. M.; Hedrick, J. L.; Wade, C. G. Macromolecules 2007, 40 (12), 4154-4158.

(36) Schwesinger, R.; Schlempep, H.; Hasenfratz, C.; Willaredt, J.; Dambacher, T.; Breuer, T.; Ottaway, C.; Fletschinger, M.; Boele, J.; Fritz, H.; Putzas, D.; Rotter, H. W.; Bordwell, F. G.; Satish, A. V.; Ji, G.; Peters, E.; Peters, K.; Schnering, H. 
G. Von; Walz, L. Liebigs Ann. 1996, 1055-1081. 




(Eq. 2.1)

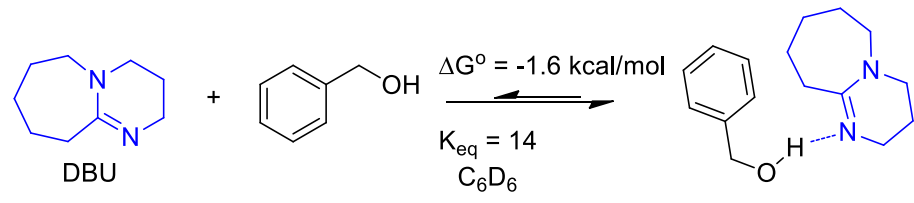

(Eq. 2.2)

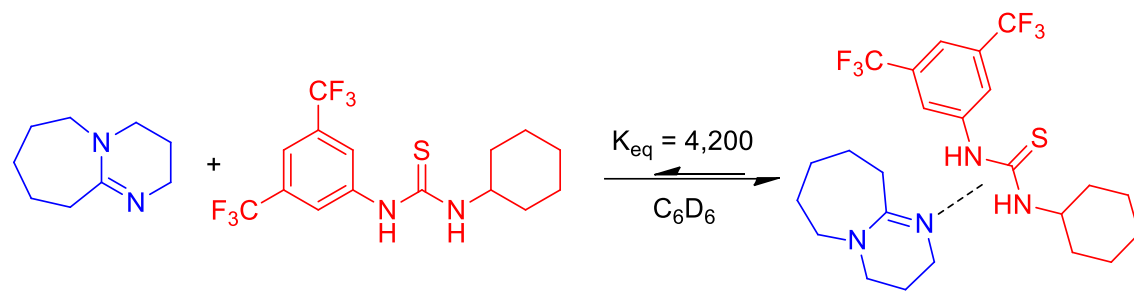

(Eq. 2.3)

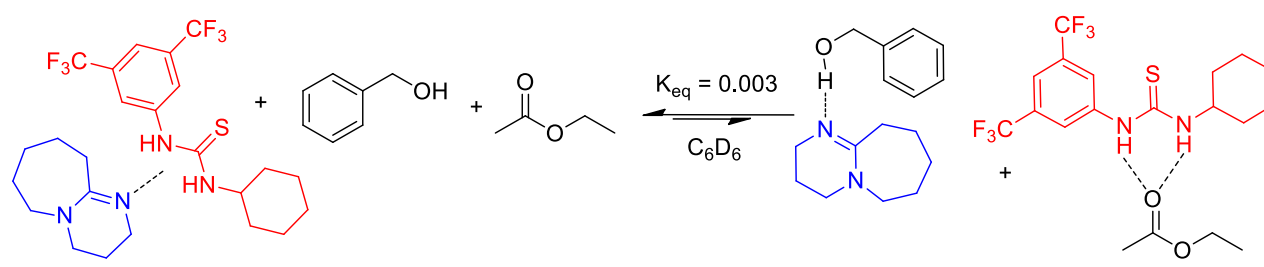

(Eq. 2.4)

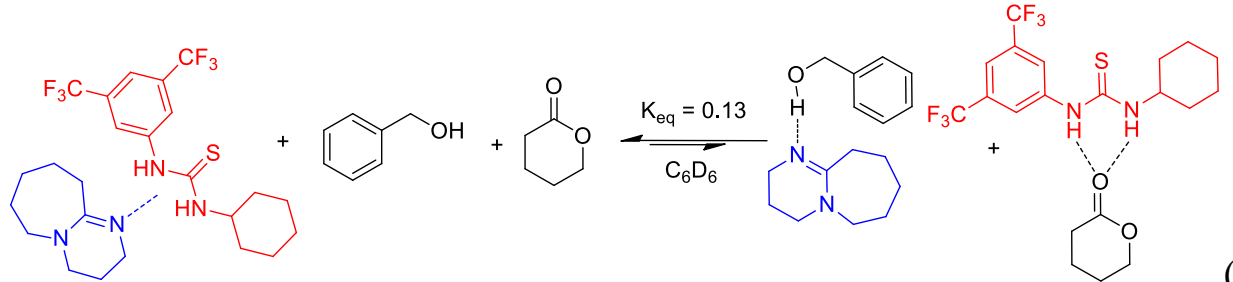

(Eq. 2.5)<smiles>COc1ccc(P(=NCCNC(=S)Nc2cc(OC)cc(C(F)(F)F)c2)(c2ccc(OC)cc2)c2ccc(C(F)(F)F)cc2)cc1</smiles>

(Eq. 2.6) 


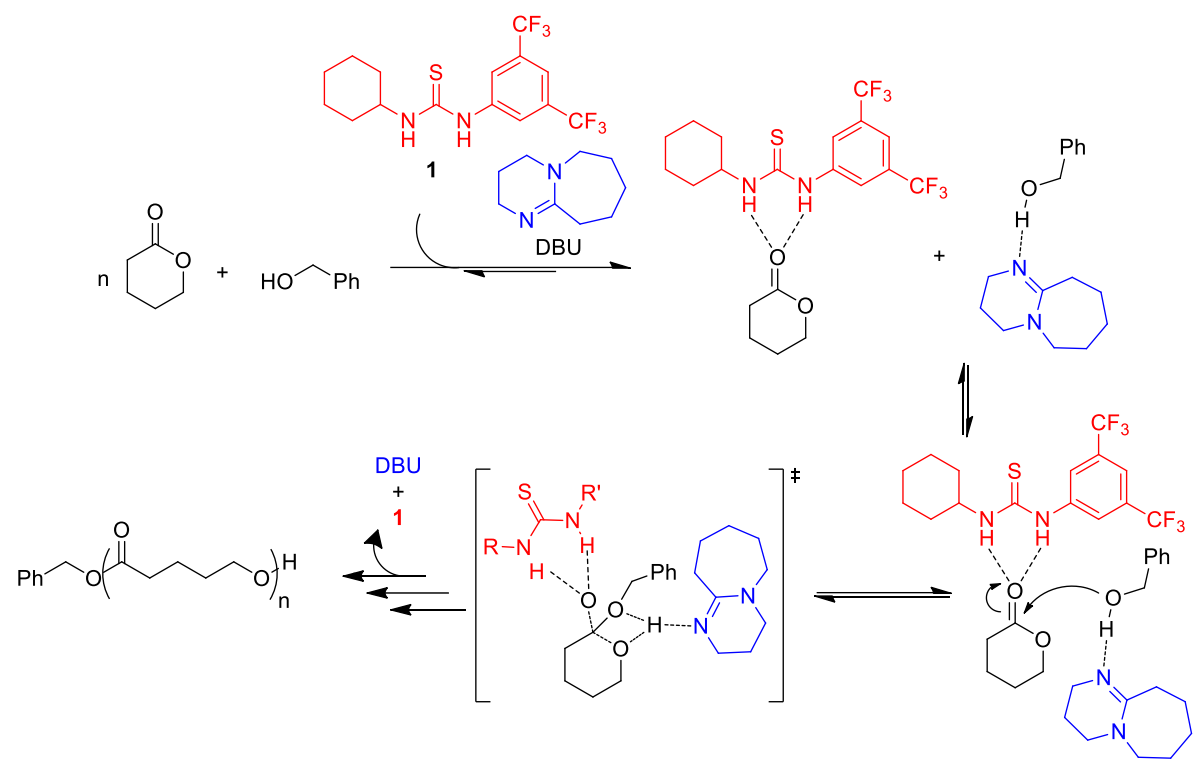

Scheme 2.1. H-bonding mechanism for the ROP of $\delta$-valerolactone 


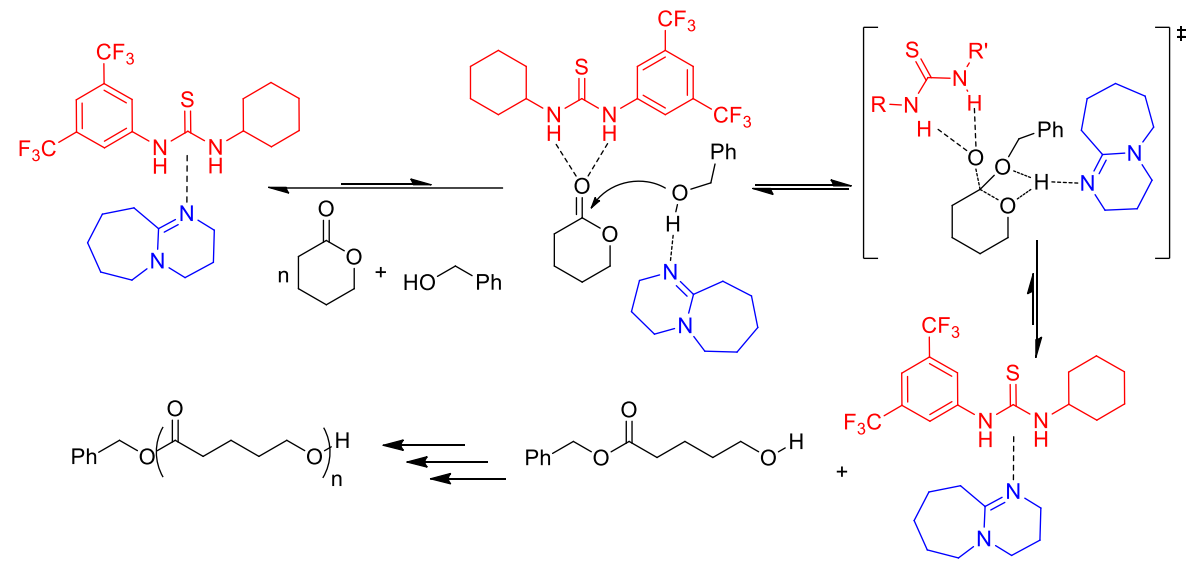

Scheme 2.2. Proposed Co-catalyst Binding Mechanism for the ROP of VL 


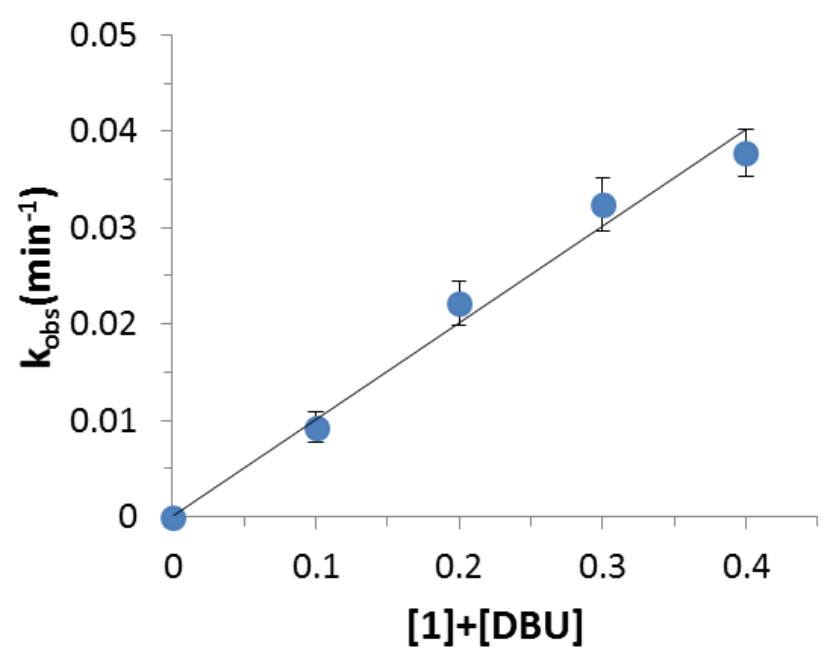

Figure 2.1. For the ROP of VL, observed rate constant ( $\left.\mathrm{k}_{\mathrm{obs}}\right)$ vs $[\mathbf{1}]+[\mathrm{DBU}]$. Conditions: VL (2M, $100 \mathrm{mg})$; benzyl alcohol 50:1 in $\mathrm{C}_{6} \mathrm{D}_{6}$. Rate $=\mathrm{k}_{\mathrm{obs}}[\mathrm{VL}]$; where $\mathrm{k}_{\mathrm{obs}}=\mathrm{k}_{\mathrm{p}}$ $([\mathbf{1}]+[\mathrm{DBU}])$ [benzyl alcohol] 


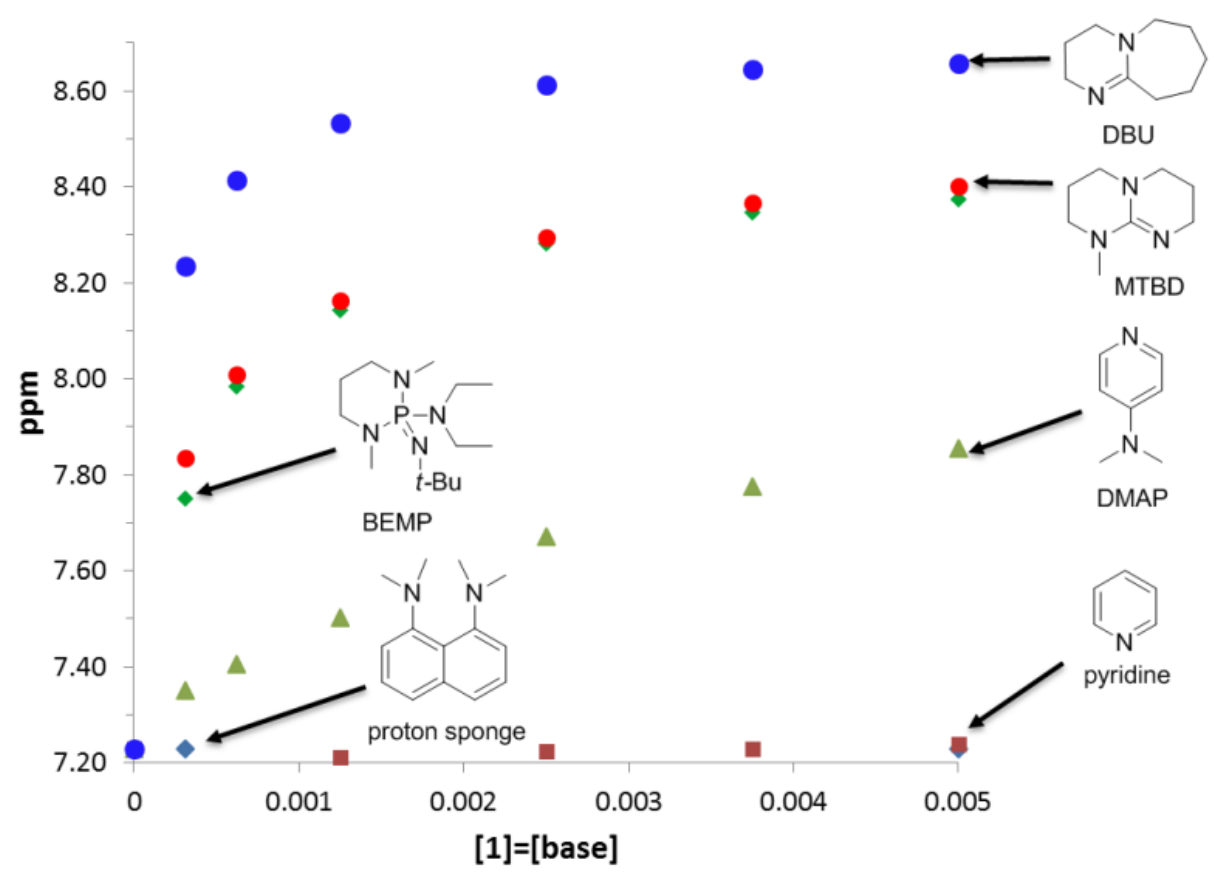

Figure 2.2. The bases studied along with the respective binding curves to 1 . 


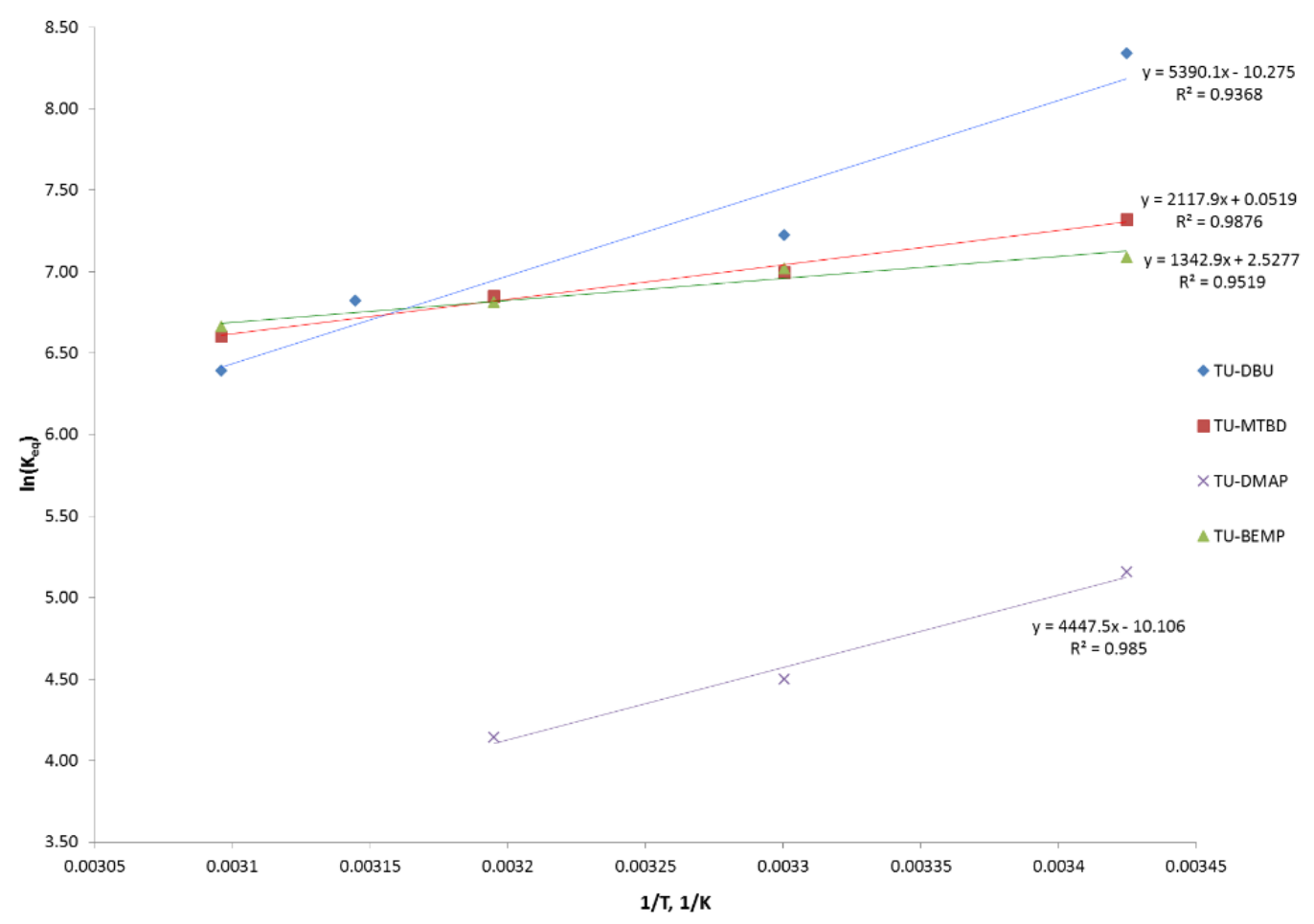

Figure 2.3. Van't Hoff plots of binding between $\mathbf{1}$ and various bases. 


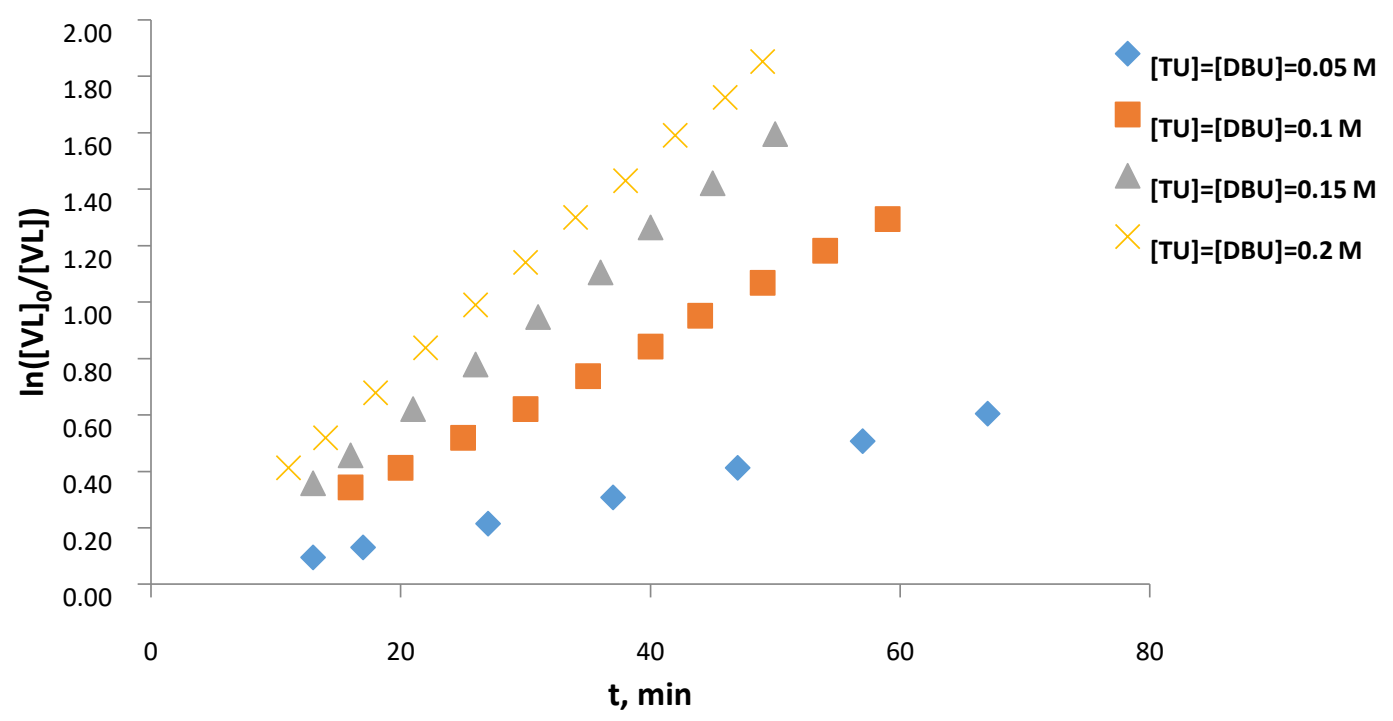

Figure 2.4. First order evolution of $[\mathrm{VL}]$ vs time when $[1]=[\mathrm{DBU}]$ when $[\mathrm{VL}]=2 \mathrm{M}$, [benzyl alcohol] $=0.04 \mathrm{M}$ in $\mathrm{C}_{6} \mathrm{D}_{6}$. 


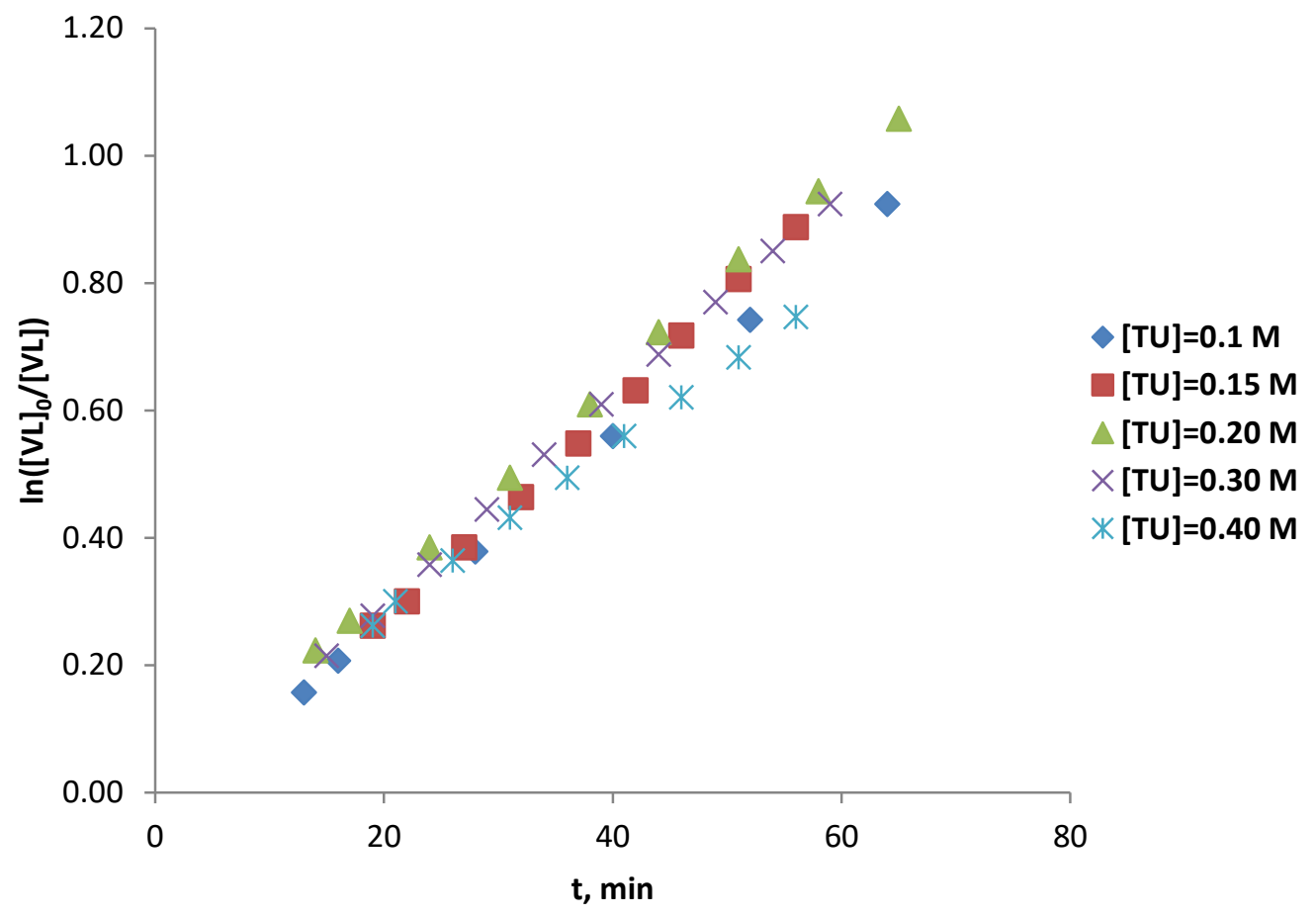

Figure 2.5. First order evolution of [VL] vs time when [1] $>$ [DBU] $=0.05 \mathrm{M}$ while [VL] $=2 \mathrm{M}$, [benzyl alcohol $]=0.04 \mathrm{M}$ in $\mathrm{C}_{6} \mathrm{D}_{6}$. 


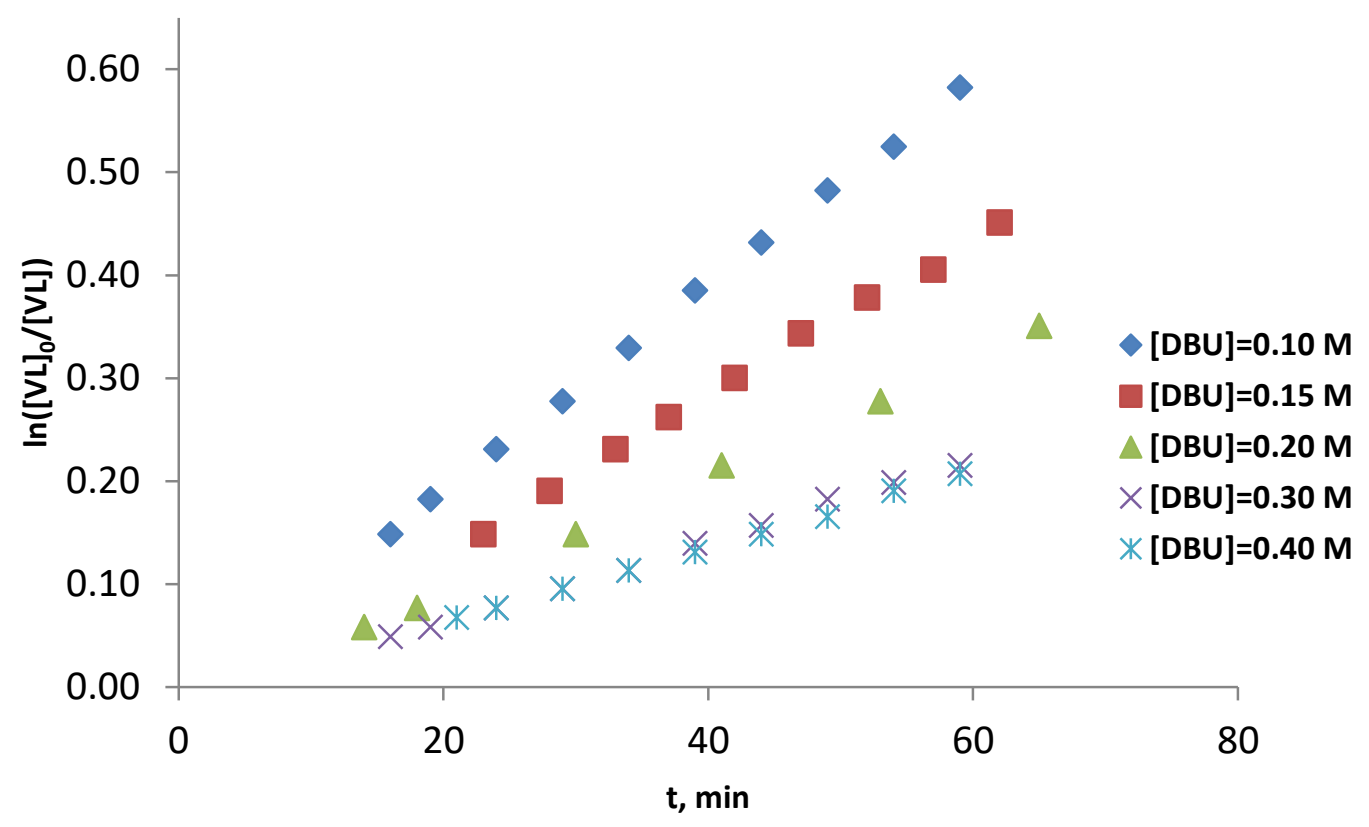

Figure 2.6. First order evolution of [VL] vs time when $[1]=0.05 \mathrm{M}<[\mathrm{DBU}]$ while $[\mathrm{VL}]$ $=2 \mathrm{M}$, [benzyl alcohol $]=0.04 \mathrm{M}$ in $\mathrm{C}_{6} \mathrm{D}_{6}$. 


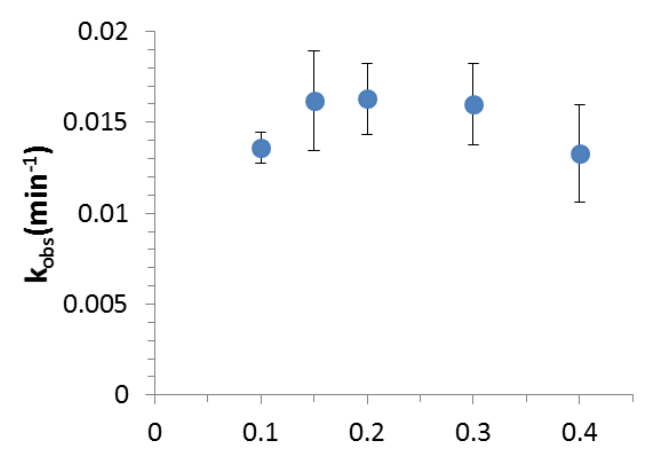

[1]

Figure 2.7. For the ROP of VL, observed rate constant vs $[\mathbf{1}]$ when $[\mathbf{1}]>[\mathrm{DBU}]=50 \mathrm{mM}$. Conditions: VL (2M, $100 \mathrm{mg}$ ); benzyl alcohol 50:1 in $\mathrm{C}_{6} \mathrm{D}_{6}$. Rate $=\mathrm{k}_{\mathrm{obs}}$ [VL]; where $\mathrm{k}_{\mathrm{obs}}$ $=\mathrm{k}_{\mathrm{p}}[\mathbf{1}+\mathrm{DBU}]$ [benzyl alcohol]. 


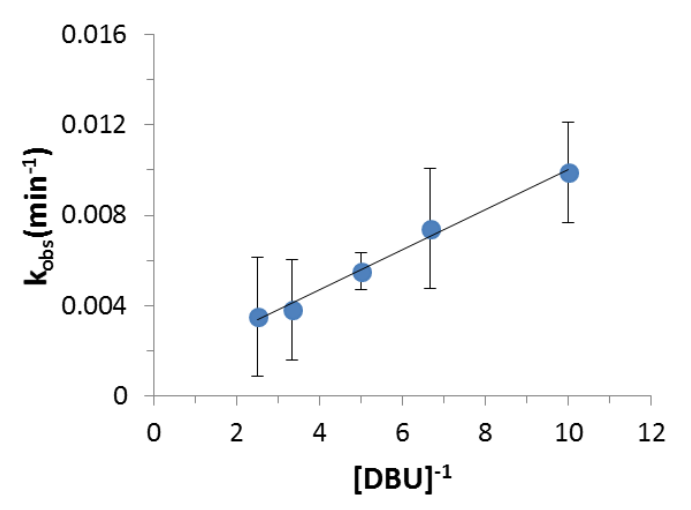

Figure 2.8. For the ROP of VL, observed rate constant vs $[\mathrm{DBU}]^{-1}$ when $[\mathrm{DBU}]>[\mathbf{1}]=50$ mM. Conditions: VL (2M, $100 \mathrm{mg})$; benzyl alcohol 50:1 in $\mathrm{C}_{6} \mathrm{D}_{6}$. Rate $=\mathrm{k}_{\text {obs }}$ [VL]; where $\mathrm{k}_{\mathrm{obs}}=\mathrm{k}_{\mathrm{p}}[\mathbf{1}+\mathrm{DBU}][$ benzyl alcohol $]$. 




Figure 2.9. $M_{n}(G P C)$ and $M_{w} / M_{n}$ vs percent conversion for the $1 / B E M P$ catalyzed ROP of VL. Reaction conditions: VL (2 M, 100 mg): 1: BEMP: benzyl alcohol :: 100: 5: 5: 1 in toluene. 


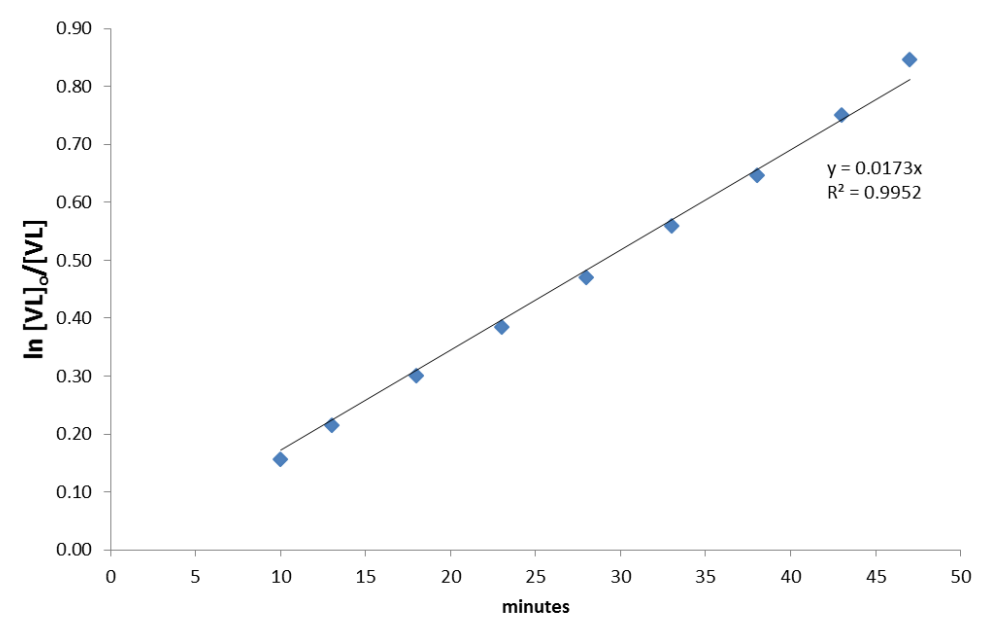

Figure 2.10. First order evolution of [VL] vs time for the 1/BEMP catalyzed ROP of VL. Conditions: VL (2M, $100 \mathrm{mg})$ : 1: BEMP: benzyl alcohol :: 100: 5: 5: 2 in $\mathrm{C}_{6} \mathrm{D}_{6}$. 


\begin{tabular}{|l|l|l|}
\hline base & $\mathbf{K}_{\mathbf{e q}}{ }^{\mathbf{a}}$ & $\mathbf{k o b s}^{\mathbf{b}} \mathbf{x} \mathbf{1 0}^{-3}, \mathbf{m i n}^{-1}$ \\
\hline proton sponge & 0 & $0^{\mathrm{c}}$ \\
\hline pyridine & $9 \pm 1$ & $0^{\mathrm{c}}$ \\
\hline DMAP & $170 \pm 30$ & $4.1 \pm 0.2^{\mathrm{c}}$ \\
\hline BEMP & $1,200 \pm 40$ & $17.8 \pm 0.3$ \\
\hline MTBD & $1,500 \pm 100$ & $20.0 \pm 0.1$ \\
\hline DBU & $4,200 \pm 170$ & $16.2 \pm 0.1$ \\
\hline
\end{tabular}

Table 2.1. Binding constants and observed rate constants for the bases studied.

a) Binding constant (at $292 \mathrm{~K}$ ) for base $+\mathbf{1}$ in equilibrium with $\mathbf{1} \bullet$ base as measured with NMR titration/dilution experiments. b) Observed rate constant, $k_{\mathrm{obs}}$, for the $1 / \mathrm{base}$ catalyzed ROP of VL from benzyl alcohol. Conditions VL:base:1:benzyl alcohol :: 100 (100 mg, 2M):5:5:2 in $\mathrm{C}_{6} \mathrm{D}_{6}$. c) Observed rate constant (at 100 hours) for the ROP of LA, same experimental conditions as b. 


\begin{tabular}{|c|c|c|c|c|c|}
\hline monomer & {$[\mathbf{M}]_{\mathbf{0}} /[\mathbf{I}]_{\mathbf{0}}$} & time (h) & \% conv. & $\mathbf{M}_{\mathbf{n}}(\mathbf{G P C})$ & $\mathbf{M}_{\mathbf{w}} / \mathbf{M}_{\mathbf{n}}$ \\
\hline $\mathrm{BL}^{\mathrm{b}}$ & 100 & 48 & 0 & -- & -- \\
\hline $\mathrm{VL}$ & 50 & 0.75 & 88 & 6,200 & 1.05 \\
\hline $\mathrm{VL}$ & 100 & 2 & 92 & 14,600 & 1.03 \\
\hline $\mathrm{VL}$ & 200 & 3 & 83 & 32,200 & 1.01 \\
\hline $\mathrm{VL}$ & 500 & 5 & 98 & 92,600 & 1.01 \\
\hline $\mathrm{CL}^{\mathrm{b}}$ & 50 & 42 & 98 & 8,900 & 1.03 \\
\hline $\mathrm{CL}^{\mathrm{b}}$ & 100 & 75 & 94 & 17,000 & 1.02 \\
\hline $\mathrm{TMC}^{\mathrm{b}}$ & 50 & 0.2 & 99 & 2,800 & 1.07 \\
\hline $\mathrm{TMC}^{\mathrm{b}}$ & 100 & 0.3 & 97 & 7,600 & 1.03 \\
\hline
\end{tabular}

Table 2.2. The 1/BEMP catalyzed ROP of cyclic monomers

a) Reaction conditions: monomer (2M, $100 \mathrm{mg}$ ), pyrenebutanol, $5 \mathrm{~mol} \%$ BEMP and 5 mol\% 1. Reactions conducted in dry toluene in a glove box $\left(\mathrm{N}_{2}\right)$ and quenched at the given time by the addition of two mol equivalents of benzoic acid to BEMP. b) Reactions performed in $\mathrm{C}_{6} \mathrm{D}_{6}$. 


\begin{tabular}{|l|l|l|l|l|l|l|}
\hline Value Base & $\begin{array}{l}\text { Proton } \\
\text { Sponge }\end{array}$ & Pyridine & DMAP & BEMP & MTBD & DBU \\
\hline $\mathrm{K}_{\mathrm{eq}}$ (at 292K) & 0 & 9 & $170 \pm 30$ & $1,200 \pm 40$ & $1,500 \pm 100$ & $4,200 \pm 170$ \\
\hline $\begin{array}{l}\Delta \mathrm{H}^{\mathrm{o}} \\
(\mathrm{kcal} / \mathrm{mol})\end{array}$ & -- & -- & $-8.8 \pm 1.1$ & $-2.7 \pm 0.4$ & $-4.2 \pm 0.3$ & $-10.7 \pm 2.0$ \\
\hline $\begin{array}{l}\Delta \mathrm{S}^{\mathrm{o}} \\
(\mathrm{cal} / \mathrm{mol} \cdot \mathrm{K})\end{array}$ & -- & -- & $-20.1 \pm 3.6$ & $5.0 \pm 1.4$ & $0.1 \pm 1.1$ & $-20.4 \pm 6.4$ \\
\hline
\end{tabular}

Table 2.3. Thermodynamic Values of Binding between 1 and various bases. 


\section{MANUSCRIPT - III}

Published in ACS Macro Letters

Bis- and Tris-Urea H-Bond Donors for Ring-Opening Polymerization: Unprecedented Activity and Control from an Organocatalyst

Kurt V. Fastnacht, Samuel S. Spink, Nayanthara U. Dharmaratne, Jinal U. Pothupitiya, Partha P. Datta, Elizabeth T. Kiesewetter and Matthew K. Kiesewetter Chemistry, University of Rhode Island, Kingston, RI, USA

Corresponding Author: $\quad$ Matthew Kiesewetter, Ph.D.

Chemistry

University of Rhode Island

140 Flagg Road

Kingston, RI, 02881, USA

Email address: mkiesewetter@chm.uri.edu 


\begin{abstract}
A new class of H-bond donating ureas was developed for the ring-opening polymerization (ROP) of lactone monomers, and they exhibit dramatic rate acceleration versus previous $\mathrm{H}$-bond mediated polymerization catalysts. The most active of these new catalysts, a tris-urea H-bond donor, is among the most active organocatalysts known for ROP, yet it retains the high selectivity of H-bond mediated organocatalysts. The urea cocatalyst, along with an H-bond accepting base, exhibits the characteristics of a "living" ROP, is highly active, in one case, accelerating a reaction from days to minutes, and remains active at low catalyst loadings. The rate acceleration exhibited by this $\mathrm{H}$-bond donor occurs for all base cocatalysts examined. A mechanism of action is proposed, and the new catalysts are shown to accelerate small molecule transesterifications versus currently known monothiourea catalysts. It is no longer necessary to choose between a highly active or highly selective organocatalyst for ROP.
\end{abstract}




\section{INTRODUCTION}

The H-bonding catalysts for ring-opening polymerization (ROP) stand out among the highly controlled polymerization methods for their ability to tolerate functional groups while precisely controlling molecular weight and polydispersity. ${ }^{1-7} \mathrm{H}$-bond donating cocatalysts are believed to effect a "living" ROP via dual activation of monomer by a Hbond donor, usually a thiourea (TU), and activation of alcohol chain end by base cocatalyst. ${ }^{8,9}$ The exquisite and remarkable combination of rate and selectivity present in other fields (e.g., olefin polymerization catalysis) $)^{10,11}$ has yet to be paralleled in organocatalytic ROP, especially H-bond mediated transformations. The development of organocatalysts for polymerization has largely proceeded along divergent pathways toward highly selective $\mathrm{e}^{1,9,12-15}$ or highly active ${ }^{16-19}$ catalysts. Indeed, the low activity of organocatalysts for ROP has been specifically identified as a shortcoming of the field, whereas highly active metal-containing catalysts for ROP are well-known. ${ }^{20,21}$ We recently disclosed a bisthiourea (bisTU) H-bond donating cocatalyst, 2-S in Figure 3.1, for the ROP of L-lactide (LA), which displayed enhanced catalytic activity (over monoTU), but no reduction in reaction control. ${ }^{22}$ During the process of extending the utility of this system to other lactone monomers, we developed a trisurea (trisU, 3-O in Figure 3.1) H-bond donor featuring remarkable activity for the ROP of lactones. Not only does this cocatalyst demonstrate the utility of the under-explored urea motif (c.f. thiourea) of H-bond donors, but when applied with a H-bond accepting cocatalyst, it is the most active ROP organocatalyst known, and one whose enhanced rate does not come at the expense of reaction control, Scheme 3.1 . 


\section{EXPERIMENTAL SECTION}

\section{General Considerations}

All manipulations were performed in an MBRAUN stainless steel glovebox equipped with a gas purification system or using Schlenk technique under a nitrogen atmosphere. All chemicals were purchased from Fischer Scientific and used as received unless stated otherwise. Tetrahydrofuran and dichloromethane were dried on an Innovative Technologies solvent purification system with alumina columns and nitrogen working gas. Benzene- $\mathrm{d}_{6}$ and chloroform- $\mathrm{d}$ were purchased from Cambridge Isotope Laboratories and distilled from $\mathrm{CaH}_{2}$ under a nitrogen atmosphere. $\delta$-valerolactone (VL; 99\%), $\varepsilon^{-}$ caprolactone $(\mathrm{CL} ; 99 \%)$ and benzyl alcohol were distilled from $\mathrm{CaH}_{2}$ under reduced

pressure. 1,3-diaminopropane, 3,5-bis(trifluoromethyl)phenyl isocyanate and cyclohexylamine were purchased from Acros Organics. 3,5-bis(trifluoromethyl)phenyl isothiocyanate was purchased from Oakwood Products. 7-methyl-1,5,7triazabicyclo[4.4.0]dec-5-ene (MTBD) was purchased from TCI. Tris(2-aminoethyl)amine was purchased from Alpha Aesar. The H-bond donors 1-S, 1-O and 2-S were prepared according to published procedures. ${ }^{23-25}$ NMR experiments were performed on Bruker Avance III $300 \mathrm{MHz}$ or $400 \mathrm{MHz}$ spectrometers. Size exclusion chromatography (SEC) was performed at $40{ }^{\circ} \mathrm{C}$ using dichloromethane eluent on an Agilent Infinity GPC system equipped with three Agilent PLGel columns $7.5 \mathrm{~mm} \times 300 \mathrm{~mm}(5 \mu \mathrm{m}$, pore sizes: 103, $104,105 \AA$ ). $M_{n}$ and $M_{w} / M_{n}$ were determined versus PS standards (500 g/mol-3150 kg/mol, Polymer Laboratories). Water and acetonitrile were all Optima HPLC grade solvents from Fisher Chemical (Fair Lawn, NJ, USA). 
Mass spectrometry was performed using a Thermo Electron (San Jose, CA, USA) LTQ Orbitrap XL mass spectrometer affixed with either an atmospheric-pressure chemical ionization (APCI) or electrospray ionization (ESI) interface, positive ions were produced and introduced into the instrument. Tune conditions for infusion experiments $(10 \mu \mathrm{L} / \mathrm{min}$ flow, sample concentration $<20 \mu \mathrm{g} / \mathrm{mL}$ in $50 / 50 \mathrm{v} / \mathrm{v}$ water/acetonitrile) were as follows: ionspray voltage, $5000 \mathrm{~V}$; capillary temperature, $275^{\circ} \mathrm{C}$; sheath gas $\left(\mathrm{N}_{2}\right.$, arbitrary units), 8; auxiliary gas ( $\mathrm{N}_{2}$, arbitrary units), 0 ; capillary voltage, $35 \mathrm{~V}$; and tube lens, $110 \mathrm{~V}$. Prior to analysis, the instrument was calibrated for positive ions using Pierce LTQ ESI positive ion calibration solution (lot \#PC197784). Ion trap experiments used $\mathrm{N}_{2}$ as a collision gas with normalized collision energies (NCE) between $10-25 \mathrm{eV}$ for multistage fragmentation. High-energy collision (HCD) experiments were performed with $\mathrm{He}$ as the collision gas with a NCE of $25 \mathrm{eV}$.

\section{Computational Details}

The Spartan '14 package for Windows 7 was used for all computations. Computed structures were geometry optimized at the B3LYP/6-31G* level of theory. Reported energies were calculated in $\mathrm{CH}_{2} \mathrm{Cl}_{2}$ solvent and were calculated at the B3LYP/6-31G** level of theory from the DFT-optimized structures. Energies, structures and coordinates are given below.

Synthesis of 1-[3,5-bis(trifluoromethyl)phenyl thiourea]-3-aminopropane

A dried $50 \mathrm{~mL}$ Schlenk flask was charged with a stir bar, dichloromethane (15.0 $\mathrm{mL}$ ) and 1,3-diaminopropane (0.45 $\mathrm{mL}, 5.40 \mathrm{mmol}) .3,5$-bis(trifluoromethyl)phenyl isothiocyanate $(1.00 \mathrm{~mL}, 5.495 \mathrm{mmol})$ was added dropwise to the round bottom flask. The solution was stirred for 24 hours, and the solvent was removed under reduced pressure. 
The resulting solid was purified via silica gel column chromatography with 90:10 dichloromethane:methanol mobile phase. Yield: 21\%. ${ }^{1} \mathrm{H}$ NMR (300 MHz, DMSO) spectrum below. Product was carried on without full characterization. ${ }^{1} \mathrm{H}$ NMR $(300 \mathrm{MHz}$, $\left.\mathrm{C}_{2} \mathrm{D}_{6} \mathrm{OS}\right) \delta 1.6(\mathrm{p}, J=6,2 \mathrm{H}) 2.65(\mathrm{t}, J=6,2 \mathrm{H}) 3.54(\mathrm{br}, 2 \mathrm{H}) 7.69(\mathrm{~s}, 1 \mathrm{H}) 8.23(\mathrm{~s}, 2 \mathrm{H})$.

Synthesis of 2-OS

1-[3,5-bis(trifluoromethyl)phenyl thiourea]-3-aminopropane $\quad(100.8 \quad \mathrm{mg}$, $0.292 \mathrm{mmol}$ ) was added to a dried $10 \mathrm{~mL}$ Schlenk flask containing dichloromethane (1 $\mathrm{mL}), \quad 3,5$-bis(trifluoromethyl)phenyl isocyanate $(74.0 \mathrm{~mL}, 0.290 \mathrm{mmol})$. Product precipitated from solution and was isolated by decanting the solvent. Solid was recrystallized from dichloromethane and dried under high vacuum overnight. Yield: 70\%. HRMS m/z calcd $\left(\mathrm{C}_{21} \mathrm{H}_{16} \mathrm{~F}_{12} \mathrm{~N}_{4} \mathrm{OS}+\mathrm{H}^{+}\right)$601.0926, found 601.0893. ${ }^{1} \mathrm{H} \mathrm{NMR}(300 \mathrm{MHz}$, DMSO-d $\left._{6}\right) \delta 1.74(\mathrm{p}, J=6,2 \mathrm{H}) 3.19(\mathrm{q}, J=6,2 \mathrm{H}) 3.55(\mathrm{br}, 2 \mathrm{H}) 6.75(\mathrm{t}, J=6,1 \mathrm{H}) 7.53$ $(\mathrm{s}, 1 \mathrm{H}) 7.73(\mathrm{~s}, 1 \mathrm{H}) 8.08(\mathrm{~s}, 2 \mathrm{H}) 8.24(\mathrm{~s}, 2 \mathrm{H}) 9.33(\mathrm{~s}, 1 \mathrm{H}) 10.15(\mathrm{~s}, 1 \mathrm{H}) .{ }^{13} \mathrm{C}$ NMR $(75$ MHz, acetone-d6) $\delta 29.0,36.8,41.4,113.0,115.7,116.8,121.1,121.5,123.0$ (q), 124.8, $130.2(\mathrm{q}), 141.5,142.2,154.5,180.1$.

Synthesis of 2-0

A dried $10 \mathrm{~mL}$ Schlenk flask was charged with a stir bar, dichloromethane (7 $\mathrm{mL}), 1,3$-diaminopropane (35.9 $\mu \mathrm{L}, 0.43 \mathrm{mmol}) .3,5$-bis(trifluoromethyl)phenyl isocyanate $(148.6 \mu \mathrm{L}, 0.86 \mathrm{mmol})$ was added dropwise to the round bottom flask. The resulting slurry was stirred for $1 \mathrm{hr}$, filtered and washed with cold dichloromethane. Yield: 97\%. HRMS $\mathrm{m} / \mathrm{z}$ calcd $\left(\mathrm{C}_{21} \mathrm{H}_{16} \mathrm{~F}_{12} \mathrm{~N}_{4} \mathrm{O}_{2}+\mathrm{H}^{+}\right)$585.1154, found 585.1100. ${ }^{1} \mathrm{H}$ NMR (300 MHz, DMSOd6) $\delta 1.68(\mathrm{p}, J=6 \mathrm{~Hz}, 2 \mathrm{H}) 3.22(\mathrm{q}, J=6 \mathrm{~Hz}, 4 \mathrm{H}) 6.59(\mathrm{t}, J=6,2 \mathrm{H}) 7.58(\mathrm{~s}, 2 \mathrm{H}) 8.14(\mathrm{~s}$, 
4H) $9.39(\mathrm{~s}, 2 \mathrm{H}) .{ }^{13} \mathrm{C}$ NMR (75 MHz, DMSO-d $) \delta 30.3,36.6,113.3,117.1,123.3$ (q), 130.5 (q), 142.6, 154.9.

Synthesis of 3-S

A dried $100 \mathrm{~mL}$ Schlenk flask was charged with a stir bar, tetrahydrofuran $(50 \mathrm{~mL}), \operatorname{tris}(2$-aminoethyl) amine $(1.05 \mathrm{~mL}, 6.84 \mathrm{mmol}), 3,5$-bis(triflouromethyl)phenyl isocyanate $(3.90 \mathrm{~mL}, 21.20 \mathrm{mmol})$. The solution was left to stir for $24 \mathrm{hrs}$ and the solvent was subsequently removed in vacuo. The resulting solid product was purified using a silica gel column with a 90:10 hexanes:ethyl acetate mobile phase. Product was removed of volatiles under high vacuum overnight. Yield: $87 \%$. HRMS m/z calcd $\left(\mathrm{C}_{33} \mathrm{H}_{27} \mathrm{~F}_{18} \mathrm{~N}_{7} \mathrm{~S}_{3}+\right.$ $\left.\mathrm{H}_{+}\right) 960.1275$, found 960.1262. ${ }^{1} \mathrm{H} \mathrm{NMR}(300 \mathrm{MHz}$, acetone-d 6$) \delta 2.82(\mathrm{t}, J=6,6 \mathrm{H}) 3.68$ $(\mathrm{m}, 6 \mathrm{H}) 7.44(\mathrm{~s}, 3 \mathrm{H}) 7.71(\mathrm{br}, 2 \mathrm{H}) 8.04(\mathrm{~s}, 6 \mathrm{H}) 9.40(\mathrm{br}, 2 \mathrm{H}) .{ }^{13} \mathrm{C}$ NMR $(75 \mathrm{MHz}$, acetone$\left.\mathrm{d}_{6}\right) \delta 43.7,53.7,117.6,123.3,124.2(\mathrm{q}), 131.8(\mathrm{q}), 142.5,182.1$.

Synthesis of 3-O

A dried $100 \mathrm{~mL}$ Schlenk flask was charged with a stir bar, tetrahydrofuran $(50 \mathrm{~mL}), \operatorname{tris}(2$-aminoethyl) amine $(1.03 \mathrm{~mL}, 6.84 \mathrm{mmol}), 3,5$-bis(triflouromethyl)phenyl isocyanate $(3.6 \mathrm{~mL}, 21.20 \mathrm{mmol})$. The solution was stirred for $24 \mathrm{hrs}$. The solvent was removed in vacuo. Resulting solid was purified using a silica gel column with a 96:4 dichloromethane:methanol mobile phase. Yield: 88\%. HRMS m/z calcd $\left(\mathrm{C}_{33} \mathrm{H}_{27} \mathrm{~F}_{18} \mathrm{~N}_{7} \mathrm{O}_{3}+\right.$ $\left.\mathrm{H}_{+}\right)$912.1961, found 912.1933. ${ }^{1} \mathrm{H}$ NMR $\left(300 \mathrm{MHz}\right.$, acetone-d $\left.{ }_{6}\right) \delta 2.58(\mathrm{t}, J=3,6 \mathrm{H}) 3.21$ $(\mathrm{m}, 6 \mathrm{H}) 6.32(\mathrm{~m}, 2 \mathrm{H}) 7.29(\mathrm{~s}, 3 \mathrm{H}) 7.86(\mathrm{~s}, 6 \mathrm{H}) 8.58(\mathrm{~s}, 2 \mathrm{H}) .{ }^{13} \mathrm{C}$ NMR $(75 \mathrm{MHz}$, acetoned6) $\delta 39.3,55.8,114.9,118.3,124.4(q), 132.3(q), 143.3,156.3$.

Example VL Polymerization Experiment 
A $7 \mathrm{~mL}$ vial was charged with 3-O $(15.2 \mathrm{mg}, 0.0167 \mathrm{mmol})$, MTBD $(2.4 \mu \mathrm{L}, 0.0167$ mmol), benzyl alcohol $(2.08 \mu \mathrm{L}, 0.01999 \mathrm{mmol})$ and $\mathrm{C}_{6} \mathrm{D}_{6}(250 \mu \mathrm{L})$. In a second $7 \mathrm{~mL}$ vial, $\mathrm{VL}(0.100 \mathrm{~g}, 0.999 \mathrm{mmol})$ was dissolved in $\mathrm{C}_{6} \mathrm{D} 6(249 \mu \mathrm{L})$. The contents of the second vial were transferred to the first via pipette and stirred until homogenous, approximately $1 \mathrm{~min}$. The contents were transferred to an NMR tube via pipette, and the reaction was monitored by ${ }^{1} \mathrm{H}$ NMR. The reaction was quenched using benzoic acid $(4.06 \mathrm{mg}, 0.0333 \mathrm{mmol})$. Polymer was precipitated with the addition of hexanes. Supernatant was decanted and solid PVL was dried in vacuo. Yield: $89 \%, \mathrm{M}_{\mathrm{n}}=7,500, \mathrm{M}_{\mathrm{w}} / \mathrm{M}_{\mathrm{n}}=1.07$.

For Chain Extension Experiment

A $7 \mathrm{~mL}$ vial was loaded with $\mathbf{3 - O} \quad(13.3 \mathrm{mg}, \quad 0.015 \mathrm{mmol})$, MTBD (2.2mg, 0.015mmol), 1-pyrenebutanol $(9.6 \mathrm{mg}, 0.035 \mathrm{mmol})$, and $\mathrm{C}_{6} \mathrm{D}_{6}(219 \mu \mathrm{L})$. In a second $7 \mathrm{~mL}$ vial, $\mathrm{CL}(100 \mathrm{mg}, 0.876 \mathrm{mmol})$ and $\mathrm{C}_{6} \mathrm{D}_{6}(219 \mu \mathrm{L})$ were loaded. The contents of the second vial were added to the first and stirred. After $15 \mathrm{~min}$, a $150 \mu \mathrm{L}$ aliquot was taken from the reaction vial, quenched with benzoic acid $(1.2 \mathrm{mg}, 0.010 \mathrm{mmol})$, and additional CL (197.3mg, 1.723mmol) was added to the reaction vial. After another $50 \mathrm{~min}$, a second aliquot was quenched with benzoic acid $(1.2 \mathrm{mg}, 0.010 \mathrm{mmol})$. Samples from both the first and second aliquots were then transferred to NMR tubes and conversion was determined via ${ }^{1} \mathrm{H}$ NMR analysis. The remainder of the aliquots was precipitated with the addition of hexane, and the supernatants were decanted. Each solid PCL sample was dried in vacuo, and GPC analysis was performed.

Example Copolymerization Experiment

A $7 \mathrm{~mL}$ vial was charged with 3-O $(15.2 \mathrm{mg}, 0.0167 \mathrm{mmol})$, MTBD $(2.4 \mu \mathrm{L}, 0.0167$ mmol), benzyl alcohol (1.04 $\mu \mathrm{L}, 0.00999 \mathrm{mmol})$ and $\mathrm{C}_{6} \mathrm{D}_{6}(250 \mu \mathrm{L})$. In a second $7 \mathrm{~mL}$ vial, 
VL (0.100 g, $0.999 \mathrm{mmol})$ and CL (0.144 g, $0.999 \mathrm{mmol})$ were dissolved in $\mathrm{C}_{6} \mathrm{D}_{6}(249 \mu \mathrm{L})$. The contents of vial 2 were transferred to the first via pipette and stirred until homogenous, approximately $5 \mathrm{sec}$. The contents were transferred to an NMR tube via pipette, and the reaction was monitored by ${ }^{1} \mathrm{H}$ NMR. The reaction was quenched using benzoic acid (4.06 $\mathrm{mg}, 0.0333 \mathrm{mmol})$. Polymer was precipitated with the addition of hexanes. Supernatant was decanted and solid polymer was dried in vacuo, 91\% yield (196 mg), $M_{n}=21,400$; $\mathrm{M}_{\mathrm{w}} / \mathrm{M}_{\mathrm{n}}=1.21$

Example ROP of Lactide

L-lactide $(72 \mathrm{mg}, 0.5 \mathrm{mmol})$ and $o$-dichlorobenzene $(0.5 \mathrm{~mL})$ were added into a 7 $\mathrm{mL}$ vial and stirred until a homogenous solution was obtained. To a second $7 \mathrm{~mL}$ vial, benzyl alcohol $(2.163 \mathrm{mg}, 0.02 \mathrm{mmol}), \mathrm{Me}_{6} \mathrm{TREN}(0.008 \mathrm{mmol})$ and 3-O $(0.008 \mathrm{mmol})$ were added. Contents from the first vial were transferred into vial 2 via Pasteur pipette. The contents were mixed and transferred to an NMR tube. Reaction progression was monitored by ${ }^{1} \mathrm{H}$ NMR. After 30 min, the reaction had reached 55\% conversion and was quenched with benzoic acid. The reaction was removed of volatiles and treated with hexanes/isopropanol (1:1) to dissolve monomer. The residual polymer was subjected to dialysis in DCM against methanol. Yield: $38 \mathrm{mg}, 52 \% ; \mathrm{M}_{\mathrm{n}}=2,700 ; \mathrm{M}_{\mathrm{w}} / \mathrm{M}_{\mathrm{n}}=1.11$.

Example Transesterification Experiment

Ethyl acetate $(100 \mathrm{mg} .1 .14 \mathrm{mmol}), \mathbf{1 - S}(0.057 \mathrm{mmol})$ and $\mathrm{C}_{6} \mathrm{D}_{6}(0.22 \mathrm{~mL})$ were added to a $7 \mathrm{ml}$ glass vial. To a second $7 \mathrm{~mL}$ glass vial, benzyl alcohol (122.7 $\mathrm{mg}, 1.14$ mmol $)$, MTBD $(0.057 \mathrm{mmol})$ and $\mathrm{C}_{6} \mathrm{D}_{6}(0.22 \mathrm{~mL})$ were added. The contents of vial 2 were transferred via Pasteur pipette to vial 1, and the solution was stirred until homogeneous (1 
min). The solution was transferred to an NMR tube, and reaction progression was monitored by ${ }^{1} \mathrm{H}$ NMR. 


\section{RESULTS AND DISCUSSION}

The effects of bisTU on the ROP of $\delta$-valerolactone (VL) and $\varepsilon$-caprolactone (CL)

were evaluated, and the rate acceleration in the presence of $\mathbf{2 - S}$ versus 1-S is general to both lactone monomers. For the ROP of either VL or CL (2 M, $100 \mathrm{mg}$ ) from benzyl alcohol in $\mathrm{C}_{6} \mathrm{D}_{6}$, the application of 2 -S/MTBD (2.5 mol \% each) produces a rate acceleration over the traditional monothiourea (1-S/MTBD $5 \mathrm{~mol} \%$ each) that is not associated with loss of reaction control, Table 3.1. The reactions retain the characteristics of "living" polymerizations, exhibiting a linear evolution of $M_{n}$ versus conversion, first order consumption of monomer, $\mathrm{M}_{\mathrm{n}}$ that is predictable by $[\mathrm{M}]_{\mathrm{o}} /[\mathrm{I}]_{\mathrm{o}}$ and a living chain end that is susceptible to chain extension, see Figures 3.2-7. The imine base, DBU, and phosphazene base, BEMP, are also effective cocatalysts for the ROP of lactones (with 2S), but the reaction is more active with MTBD cocatalyst, Table 3.1.

ROP involving 2-S is suggested to proceed through an activated-TU mechanism, whereby one TU moiety activates the other, which in turn activates the monomer. The ROPs of VL and CL are first order in the consumption of monomer (Figure 3.3 and 3.10), which suggests one bisTU (2-S) molecule activating one monomer in the transition state. This is consistent with previous suggestions that H-bond-mediated ROP operates via dual activation of monomer by $\mathbf{1}$ and of alcohol chain end by base. ${ }^{1}$ Because $\mathrm{H}$-bonds require no orbital overlap and are electrostatic in nature, ${ }^{26}$ we cannot rule out a dual-thiourea activated mechanism, Eq. 1. However, computational studies for the activation of lactones by 2-S suggest an activated-TU mechanism is preferred over a dual-thiourea activation 
mechanism, Eq. 1; this assertion is also supported by the 2-S/alkylamine cocatalyzed ROP of lactide. $^{22,27}$

The series of thiourea H-bond donating catalysts was extended to a trisTU H-bond donor, 3-S, but this catalyst exhibits significantly reduced activity versus 1-S or 2-S in the TU/base cocatalyzed ROP of lactones, Table 3.1. This suggests that simply adding TU moieties does not result in faster ROP. Geometry optimized DFT computations suggest that a stable conformation of $\mathbf{3 - S}$ is the C3 symmetric structure, see Figure 3.15 and 3.16. This calculated structure features a cyclic arrangement of the three TU moieties, each serving as a H-bond donor and a H-bond acceptor to each of the adjacent TU moieties with H-bond lengths of $2.61 \pm 0.07 \AA$. We hypothesize that the added stability due to the three intramolecular H-bonds attenuates the activity of 3-S (vs 2-S). In contrast, the intramolecular H-bond activation in 2-S leaves a TU moiety available for catalysis. Additive effects from multiple TU moieties are found in nature, ${ }^{28}$ and such constructs have been observed to be beneficial to catalysis, ${ }^{22,29,30}$ although not universally so. ${ }^{24,31}$ Interested in extending the suite of $\mathrm{H}$-bond-mediated catalysts, we noted that changing the $\mathrm{C}=\mathrm{S}$ to the shorter $\mathrm{C}=\mathrm{O}$ bond would be expected to disrupt the intramolecular H-bond network, freeing one urea moiety for catalysis. The trisurea H-bond donor (3-O) is predicted by DFT calculations to have much longer average H-bond lengths versus 3-S, $2.92 \pm 0.81 \AA$.

The application of the tris $U$ catalyst 3-O in combination with organic bases effects the fastest organocatalytic ROP of lactones that has been reported, yet the reaction remains highly controlled..$^{3,17-21}$ The 3-O/MTBD (1.67 mol \% each) catalyzed ROP of VL ( $2 \mathrm{M}$, $100 \mathrm{mg}$ ) from benzyl alcohol ( $2 \mathrm{~mol} \%)$ proceeds to full conversion in $3 \mathrm{~min}$, Table 3.2. The comparable reactions with 2-S/MTBD (2.5 mol \% each) or 1-S/MTBD (5 mol \% each) 
achieve full conversion in $102 \mathrm{~min}$ or $2 \mathrm{~h}$, respectively. The rate acceleration for the ROP of $\mathrm{CL}$ with 3-O/MTBD is even more remarkable; this reaction achieves full conversion in 26 min. This constitutes a marked rate acceleration versus 2-S or 1-S with MTBD, which achieves full conversion in 10 or $45 \mathrm{~h}$, respectively, and the polydispersities for the 3O/MTBD catalyzed ROP of VL or CL remain less than $M_{w} / M_{n}=1.07$, Table 3.2. The 3O mediated ROPs of both monomers are highly controlled, exhibiting the characteristics of "living" polymerizations, (see Figures 3.10 and 3.11). Initiation of a CL ROP from 1pyrenebutanol produces PCL with overlapping refractive index and UV traces in the GPC, suggesting end-group fidelity; the "living" alcohol chain end is susceptible to chain extension by repeated additions of monomer, (see Figure 3.13). The 3-O/MTBD cocatalysts remain active at low concentration; full conversion for the ROP of VL ( $2 \mathrm{M}$, $\left.\mathrm{C}_{6} \mathrm{D}_{6}\right)$ from benzyl alcohol $\left([\mathrm{M}]_{\mathrm{o}} /[\mathrm{I}]_{\mathrm{o}}=50\right)$ was achieved in $5 \mathrm{~h}$ at $0.25 \mathrm{~mol} \% \mathbf{3 - O} / \mathrm{MTBD}$ loading, (see Table 3.4).

The efficacy of 3-O/base cocatalysts for the ROP of other ester and carbonate monomers was evaluated. The 3-O/MTBD $(1.67 \mathrm{~mol} \%)$ cocatalysts are effective for the ROP of trimethylene carbonate (TMC). This reaction (100 mg TMC, $1 \mathrm{M}$ in $\mathrm{CH}_{2} \mathrm{Cl}_{2}$ ) reaches $97 \%$ conversion in $1 \min \left(\mathrm{M}_{\mathrm{n}}=9000 ; \mathrm{M}_{\mathrm{w}} / \mathrm{M}_{\mathrm{n}}=1.05 ;[\mathrm{M}]_{\mathrm{o}} /[\mathrm{I}]_{\mathrm{o}}=50\right)$, which is more active than the 1-S/DBU catalyzed ROP of TMC. ${ }^{5}$ For the ROP of LA, 3-O (with tris[2-(dimethylamino)ethyl]amine) exhibits a solvent incompatibility with LA and PLA, resulting in the precipitation of polymer or catalyst prior to full conversion (see Figure 3.17). The best conversion was achieved in o-dichlorobenzene, $55 \%$ in $30 \min \left(\mathrm{M}_{\mathrm{n}}=2700\right.$; $\mathrm{M}_{\mathrm{w}} / \mathrm{M}_{\mathrm{n}}=1.11 ;[\mathrm{M}]_{\mathrm{o}} /[]_{\mathrm{o}}=25 ; 52 \%$ yield). This is less active than our previously reported catalyst, 2-S, which reaches full conversion in minutes. ${ }^{22}$ MALDI analysis of the PLA 
resulting from the ROP of LA shows only minor transesterification $(\mathrm{m} / \mathrm{z}= \pm 72 \mathrm{n}$; see Figure 3.17). A copolymerization of VL and CL was conducted with 3-O/MTBD. As determined by ${ }^{1} \mathrm{H}$ NMR, the consumption of VL is almost complete prior to the incorporation of $\mathrm{CL}$ units, suggesting the formation of a gradient-copolymer (see Figure 3.12 and Experimental Section; $\mathrm{M}_{\mathrm{n}}=21400 ; \mathrm{M}_{\mathrm{w}} / \mathrm{M}_{\mathrm{n}}=1.29 ; 91 \%$ yield). The H-bond donor 3-O with MTBD is not active for the ROP of $\beta$-butyrolactone, which is consistent with other H-bonding ROP catalysts. $^{8}$

It is proposed here that 3-O/MTBD cocatalyzed ROP occurs via an activated-urea mechanism, whereby a single 3-O activates a lactone and MTBD activates an alcohol chain end through H-bonding, Scheme 3.2. A plot of observed rate constant ( $\left.\mathrm{k}_{\mathrm{obs}}\right)$ versus [3-O] for the ROP of VL from benzyl alcohol suggests that the ideal stoichiometry of the 3O/MTBD catalyzed reaction is 1:1 (see Figure 3.14). Further, the 3-O/MTBD cocatalyzed ROP of VL is first order in monomer (see Figure 3.9), which suggests that a single 3-O molecule acting at one monomer is present in the transition state. This is consistent with previous reports that suggest that H-bond donors featuring multiple (thio)urea moieties activate one reagent prior to the TU-reagent complex undergoing further chemistry, ${ }^{22,32}$ and it is also consistent with a report of a urea-thiourea H-bond donating catalyst, which was proposed to be operative via an activated-(thio)urea mechanism. ${ }^{28}$ Indeed, ${ }^{1} \mathrm{H}$ NMR spectra (in acetone) of 1-O, 2-O, and 3-O show a progressive downfield shift of the $\mathrm{N}-\mathrm{H}$ protons, which can be interpreted to arise from stronger intramolecular H-bonding in 3-O and 2-O versus 1-O. A multiurea activated mechanism (e.g., Eq. 1), which is reminiscent of a solvophobic pocket, cannot be ruled out. However, the marked inefficacy toward ROP of 3-S, which is geometrically able to adopt a conformation featuring strong intramolecular 
H-bonds (see Figure 3.15 and 3.16), suggests that the activated-urea mechanism is the more robust proposal.

Among catalysts for the ROP of lactones, the 3-O/base cocatalysts stand out due to the extremely rapid rate that they exhibit at room temperature. For comparison, we conducted the ROP of CL (2 M) from benzyl alcohol (1 mol \%) with the bifunctional catalyst TBD, Table 3.2. The guanidine base, TBD (Figure 3.1), has been regarded as one of the most active organocatalysts available for the ROP of lactones. ${ }^{16}$ The TBD catalyzed ROP of CL from benzyl alcohol (Table 3.2, entry 12) proceeds to 93\% conversion in 140 $\min \left(M_{w} / M_{n}=1.37\right)$, whereas the same ROP with 3-O/MTBD (Table 3.2, entry 8) achieves $97 \%$ conversion in $26 \min \left(M_{w} / M_{n}=1.05\right)$.

In small molecule transformations, urea H-bond donating catalysts have been observed to possess similar activity to their heavy chalcogen counterparts. ${ }^{33}$ The development of urea and thiourea H-bond donating catalysts continued apace until the turn of the millennium when several reports emerged that extolled the operational (e.g., increased solubility) $)^{34,35}$ and synthetic (e.g., higher yields and enantioselectivities) $)^{35-37}$ benefits of thioureas over ureas. In our estimation, the ubiquity of the thiourea motif in Hbond mediated transformations may be more due to the coincidental timing of these reports than any general superiority of thioureas over urea H-bonding catalysts. Indeed, ureas are more polar than thioureas and should be expected to be better H-bond activators, ${ }^{33}$ and in some catalysis applications, urea catalysts are clearly superior. ${ }^{38,39}$ The late Margaret Etter may have presaged our observation of 3-O as an effective H-bond donating catalyst in her characterization of aryl ureas featuring meta-electron withdrawing groups by noting that urea carbonyls are good H-bond acceptors. ${ }^{38}$ 
The urea versions of $\mathbf{2}$ and $\mathbf{1}$ were synthesized and evaluated for their efficacy in the ROP of VL ( $2 \mathrm{M}, 100 \mathrm{mg}, 1$ equiv) from benzyl alcohol ( $2 \mathrm{~mol} \%)$ in $\mathrm{C}_{6} \mathrm{D}_{6}$. In general, all $\mathrm{n}-\mathrm{O}(\mathrm{n}=1,2$, or 3$)$ catalysts were more active than the corresponding n-S H-bond donors, Tables 3.1 and 3.2. For the 2-X $(\mathrm{X}=\mathrm{O}, \mathrm{S}$, or $\mathrm{OS}) \mathrm{H}$-bond donors, the rate of ROP increases with the progressive substitution of $O$ (vs $S$ ) and $M_{w} / M_{n}$ remains low. These results suggest the increased utility of ureas versus thioureas for H-bond-mediated ROP. All reported urea catalysts are soluble under the desired reaction conditions with the exception of 2-O, which requires an extra equivalent of MTBD to become homogeneous in $\mathrm{C}_{6} \mathrm{D}_{6}{ }^{40} \mathrm{~A}$ plot of the observed rate constant $\left(\mathrm{k}_{\mathrm{obs}}\right)$ versus [MTBD] for the ROP of CL from benzyl alcohol increases linearly under conditions $[\mathrm{MTBD}] \leq[\mathbf{2 - S}]$, but becomes zero order in $[\mathrm{MTBD}]$ when $[\mathrm{MTBD}]>[\mathbf{2}-\mathrm{S}]$, (see Figure 3.7). This suggests that the proper stoichiometry of the 2-S/MTBD catalyzed reaction is 1:1. The catalysts (1-3 with MTBD) are all operative in $\mathrm{CH}_{2} \mathrm{Cl}_{2}, \mathrm{CHCl}_{3}$, and THF albeit with slightly reduced reaction rates or $\mathrm{M}_{\mathrm{w}} / \mathrm{M}_{\mathrm{n}}$ (see Table 3.5).

Preliminary studies suggest that these catalysts exhibit the same reactivity trends in small molecule transesterification and, hence, may have general applicability beyond ROP. The transesterification of ethyl acetate (1.6 M) with benzyl alcohol (1.6 M) was conducted in $\mathrm{C}_{6} \mathrm{D}_{6}$. Observed rate constants ( $\mathrm{k}_{\mathrm{obs}}$ ) at early reaction time were measured for each $\mathrm{H}$ bond donor/MTBD cocatalyzed transesterification. These rate constants show the same trends in catalyst activity that were observed for the ROP reactions: 3-O is the most rapid catalyst and it is 1-2 orders of magnitude more rapid than 1-S, (see Table 3.3). This suggests a general role for the increased activation of esters by urea H-bond donors (vs 
thioureas), yet the slower rates for the transesterification of s-trans (vs s-cis) esters accounts for the low rate of transesterification postpolymerization, (see Table 3.6). 


\section{CONCLUSION}

Urea H-bond donors in combination with base cocatalysts have been shown to be highly effective for the ROP of lactones. Despite being among the most rapid organocatalysts for ROP, the 3-O/MTBD cocatalyzed ROPs of VL and CL are among the most controlled polymerizations, exhibiting the characteristics of "living" polymerizations and producing polymers with narrow $\mathrm{M}_{\mathrm{w}} / \mathrm{M}_{\mathrm{n}}$. The source of the rate acceleration versus mono- and bisurea H-bond donors is proposed to arise from successively increased intramolecular $\mathrm{H}$-bond activation with each additional urea moiety. The reintroduction of the urea motif of H-bond donors to the lexicon of organocatalytic (ROP) chemistry provides a rich diversity of catalyst scaffolds to explore in mono-, bis-, tris-, and poly-H-

bond donors. Previous to the discovery of trisurea cocatalyzed ROP, one was forced to choose between a highly active or highly selective organocatalyst; this age is over. 


\section{LIST OF REFERENCES}

(1) Dove, A. P.; Pratt, R. C.; Lohmeijer, B. G. G.; Waymouth, R. M.; Hedrick, J. L. J. Am. Chem. Soc. 2005, 127 (40), 13798-13799.

(2) Kamber, N. E.; Jeong, W.; Waymouth, R. M.; Pratt, R. C.; Lohmeijer, B. G. G.; Hedrick, J. L. Chem. Rev. 2007, 107 (12).

(3) Kiesewetter, M. K.; Shin, E. J.; Hedrick, J. L.; Waymouth, R. M. Macromolecules 2010, 43 (5), 2093-2107.

(4) McKinlay, C. J.; Waymouth, R. M.; Wender, P. A. J. Am. Chem. Soc. 2016, 138 (10), 3510-3517.

(5) Pratt, R. C.; Nederberg, F.; Waymouth, R. M.; Hedrick, J. L. Chem. Commun. 2008, No. 1, 114-116.

(6) Sanders, D. P.; Fukushima, K.; Coady, D. J.; Nelson, A.; Fujiwara, M.; Yasumoto, M.; Hedrick, J. L. J. Am. Chem. Soc. 2010, 132 (42), 14724-14726.

(7) Blake, T. R.; Waymouth, R. M. J. Am. Chem. Soc. 2014, 136 (26), 9252-9255.

(8) Bas G. G. Lohmeijer Frank Leibfarth, John W. Logan, R. C. P.; David A. Long Fredrik Nederberg, Jeongsoo Choi, A. P. D.; Charles Wade and James L. Hedrick, R. M. W.; Lohmeijer, B. G. G.; Pratt, R. C.; Leibfarth, F.; Logan, J. W.; Long, D. A.; Dove, A. P.; Nederberg, F.; Choi, J.; Wade, C.; Waymouth, R. M.; Hedrick, J. L. Macromolecules 2006, 39 (25), 8574-8583.

(9) Pratt, R. C.; Lohmeijer, B. G. G.; Long, D. A.; Lundberg, P. N. P.; Dove, A. P.; Li, H.; Wade, C. G.; Waymouth, R. M.; Hedrick, J. L. Macromolecules 2006, 39 (23), 7863-7871.

(10) Kiesewetter, E. T.; Randoll, S.; Radlauer, M.; Waymouth, R. M. J. Am. Chem. Soc. 2010, 132 (16), 5566-5567.

(11) Cohen, A.; Kopilov, J.; Lamberti, M.; Venditto, V.; Kol, M. Macromolecules 2010, 43 (4), 1689-1691.

(12) Miyake, G. M.; Chen, E. Y. X. Macromolecules 2011, 44 (11), 4116-4124.

(13) Zhu, J.-B.; Chen, E. Y.-X. J. Am. Chem. Soc. 2015, 137 (39), 12506-12509.

(14) Delcroix, D.; Martín-Vaca, B.; Bourissou, D.; Navarro, C. Macromolecules 2010, 43 (21), 8828-8835.

(15) Makiguchi, K.; Kikuchi, S.; Satoh, T.; Kakuchi, T. J. Polym. Sci. Part A Polym. Chem. 2013, 51 (11), 2455-2463.

(16) Pratt, R. C.; Lohmeijer, B. G. G.; Long, D. A.; Waymouth, R. M.; Hedrick, J. L. J. Am. Chem. Soc. 2006, 128 (14), 4556-4557.

(17) Jeong, W.; Shin, E. J.; Culkin, D. A.; Hedrick, J. L.; Waymouth, R. M. J. Am. Chem. Soc. 2009, 131 (13), 4884-4891. 
(18) Naumann, S.; Scholten, P. B. V; Wilson, J. A.; Dove, A. P. J. Am. Chem. Soc. 2015, 137 (45), 14439-14445.

(19) Li, X.; Zhang, Q.; Li, Z.; Xu, S.; Zhao, C.; Chen, C.; Zhi, X.; Wang, H.; Zhu, N.; Guo, K. Polym. Chem. 2016, 7 (7), 1368-1374.

(20) Guillaume, S. M.; Kirillov, E.; Sarazin, Y.; Carpentier, J.-F. Chem. - A Eur. J. 2015, 21 (22), 7988-8003.

(21) Du, G.; Wei, Y.; Zhang, W.; Dong, Y.; Lin, Z.; He, H.; Zhang, S.; Li, X. Dalt. Trans. 2013, 42 (4), 1278-1286.

(22) Spink, S. S.; Kazakov, O. I.; Kiesewetter, E. T.; Kiesewetter, M. K. Macromolecules 2015, 48 (17), 6127-6131.

(23) Bas G. G. Lohmeijer Frank Leibfarth, John W. Logan, R. C. P.; David A. Long Fredrik Nederberg, Jeongsoo Choi, A. P. D.; Charles Wade and James L. Hedrick, R. M. W.; Lohmeijer, B. G. G.; Pratt, R. C.; Leibfarth, F.; Logan, J. W.; Long, D. A.; Dove, A. P.; Nederberg, F.; Choi, J.; Wade, C.; Waymouth, R. M.; Hedrick, J. L. Macromolecules 2006, 39 (25), 8574-8583.

(24) Bertucci, M. A.; Lee, S. J.; Gagne, M. R. Chem. Commun. 2013, 49 (20), 20552057.

(25) Tripathi, C. B.; Mukherjee, S. J. Org. Chem. 2012, 77 (3), 1592-1598.

(26) Anslyn, E. V.; Dougherty, D. A. In Modern Physical Organic Chemistry; University Science, 2006; pp 145-222.

(27) Kazakov, O. I.; Kiesewetter, M. K. Macromolecules 2015, 48 (17), 6121-6126.

(28) Jones, C. R.; Dan Pantoş, G.; Morrison, A. J.; Smith, M. D. Angew. Chemie Int. Ed. 2009, 48 (40), 7391-7394.

(29) Shi, Y.; Lin, A.; Mao, H.; Mao, Z.; Li, W.; Hu, H.; Zhu, C.; Cheng, Y. Chem. -A Eur. J. 2013, 19 (6), 1914-1918.

(30) Sohtome, Y.; Tanatani, A.; Hashimoto, Y.; Nagasawa, K. Tetrahedron Lett. 2004, 45 (29), 5589-5592.

(31) Li, X.; Deng, H.; Luo, S.; Cheng, J.-P. European J. Org. Chem. 2008, 2008 (25), 4350-4356.

(32) Breugst, M.; Houk, K. N. J. Org. Chem. 2014, 79 (13), 6302-6309.

(33) Doyle, A. G.; Jacobsen, E. N. Chem. Rev. 2007, 107 (12), 5713-5743.

(34) Curran, D. P.; Kuo, L. H. Tetrahedron Lett. 1995, 36 (37), 6647-6650.

(35) Wittkopp, A.; Schreiner, P. R. Chem. - A Eur. J. 2003, 9 (2), 407-414.

(36) Wenzel, A. G.; Jacobsen, E. N. J. Am. Chem. Soc. 2002, 124 (44), 12964-12965.

(37) Vachal, P.; Jacobsen, E. N. J. Am. Chem. Soc. 2002, 124 (34), 10012-10014. 
(38) Etter, M. C.; Panunto, T. W. J. Am. Chem. Soc. 1988, 110 (17), 5896-5897.

(39) Berkessel, A.; Cleemann, F.; Mukherjee, S.; Mueller, T. N.; Lex, J. Angew. Chemie - Int. Ed. 2005, 44 (5), 807-811.

(40) Kazakov, O. I.; Datta, P. P.; Isajani, M.; Kiesewetter, E. T.; Kiesewetter, M. K. Macromolecules 2014, 47, 7463-7468. 



(Eq. 3.1) 


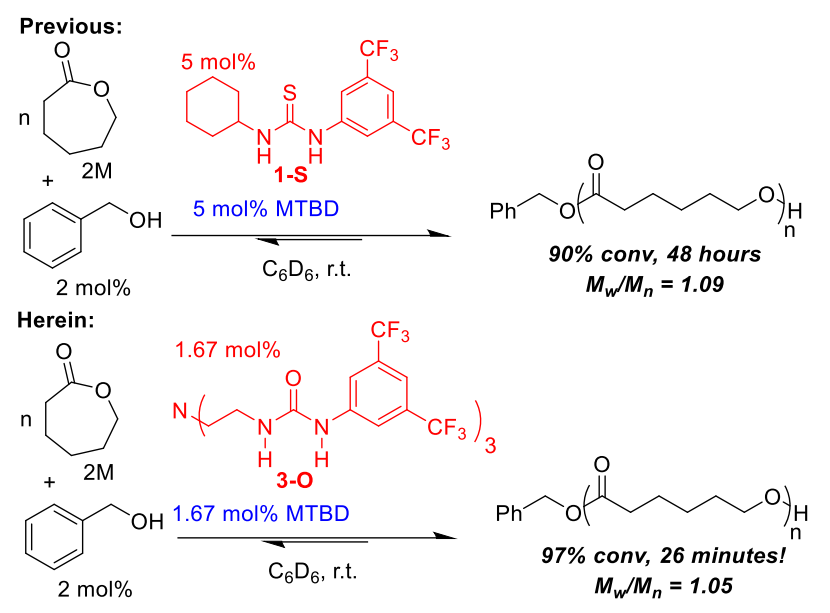

Scheme 3.1. Highly Active and Highly Selective H-bond Donor 3-O. 


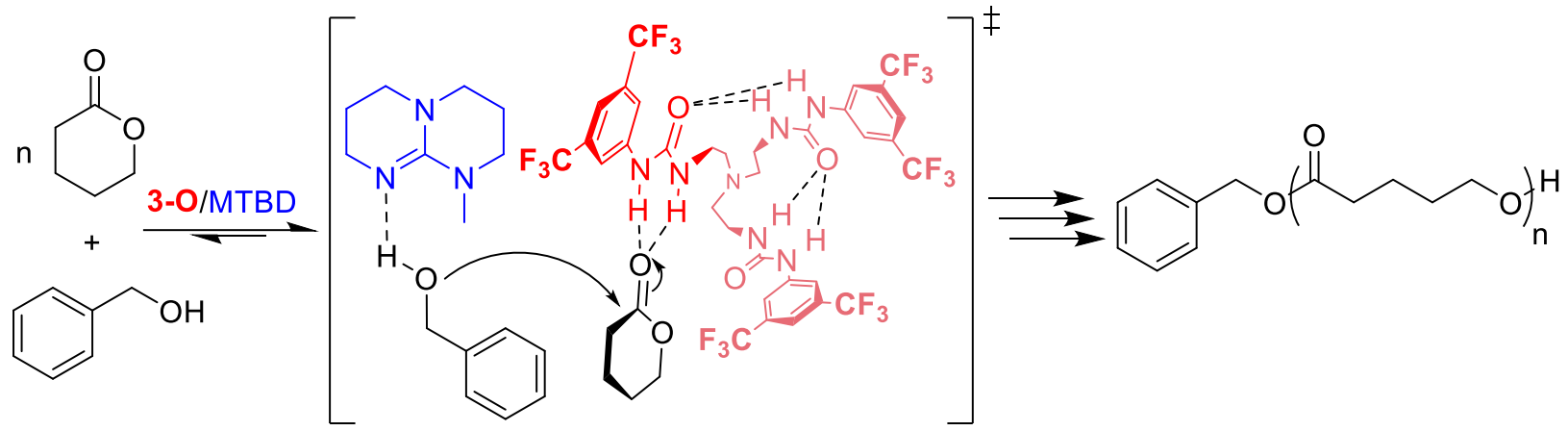

Scheme 3.2. Proposed Mechanism for 3-O/MTBD Catalyzed ROP. 




Figure 3.1. Base and (thio)urea cocatalysts evaluated for ROP. 




Figure 3.2. $\mathrm{M}_{\mathrm{n}}$ vs conversion for the 2-S/MTBD catalyzed ROP of VL. Conditions: VL (2.994 mmol, 1 equiv, $1 \mathrm{M}$ in $\mathrm{C}_{6} \mathrm{D}_{6}$ ), benzyl alcohol (2 mol\%, $0.0598 \mathrm{mmol}$ ), MTBD (5 mol\%, $0.1497 \mathrm{mmol}$ ) and 2-S (5 mol\%, $0.1496 \mathrm{mmol})$. (blue is $\mathrm{M}_{\mathrm{n}}$, red is $\mathrm{M}_{\mathrm{w}} / \mathrm{M}_{\mathrm{n}}$ ) 


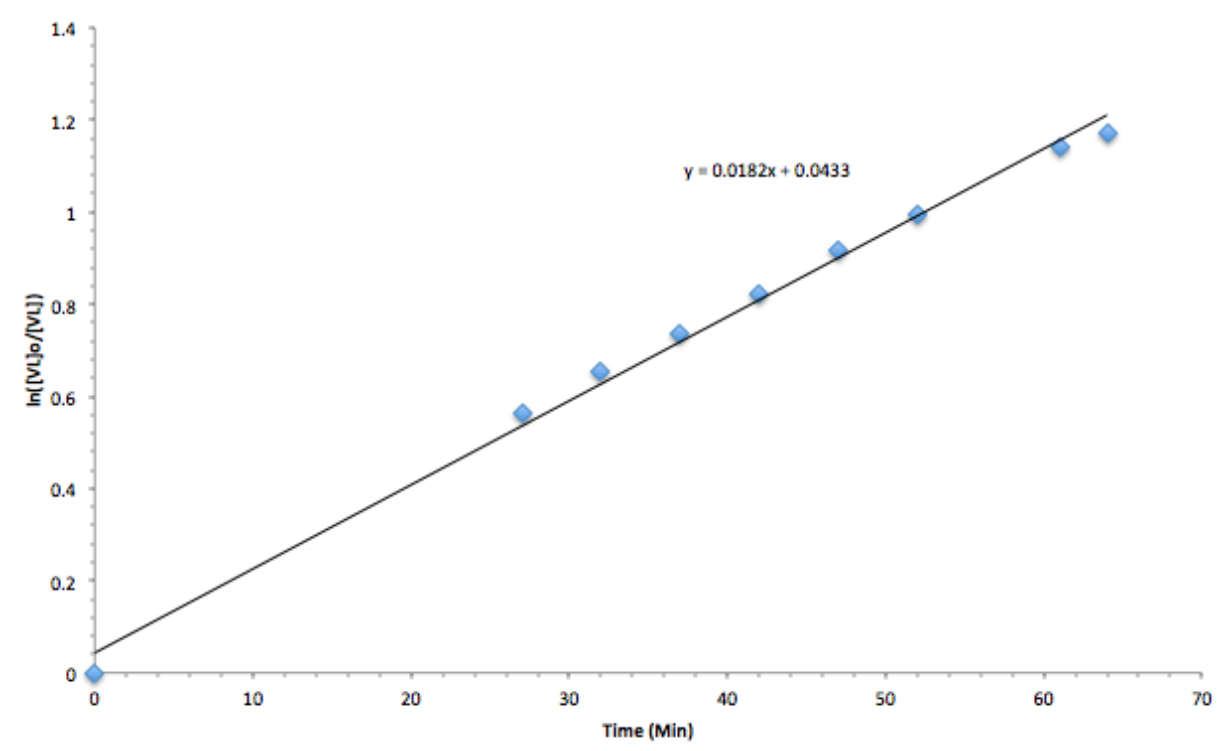

Figure 3.3. First order evolution of [VL] vs time for the 2-S/MTBD catalyzed ROP of VL.

Conditions: VL (0.999 mmol, 1 equiv, $2 \mathrm{M}$ in $\left.\mathrm{C}_{6} \mathrm{D}_{6}\right)$, benzyl alcohol (2.0 mol\%, 0.0199 mmol), MTBD (5.0 mol\%, $0.0499 \mathrm{mmol})$ and 2-S (5.0 mol\%, $0.0499 \mathrm{mmol})$. 


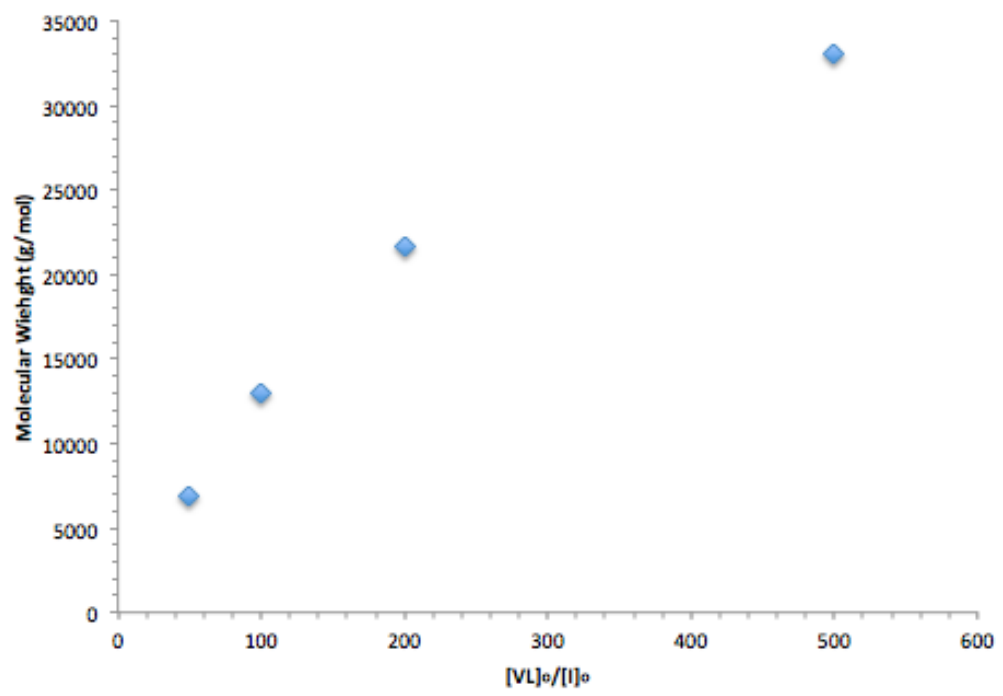

Figure 3.4. $\mathrm{M}_{\mathrm{n}}$ vs $[\mathrm{VL}]_{\mathrm{o}} /[\mathrm{I}]_{\mathrm{o}}$ for the 2-S/MTBD catalyzed ROP of VL. Conditions: VL (0.999 mmol, 1 equiv, $1 \mathrm{M}$ in $\mathrm{C}_{6} \mathrm{D}_{6}$ ), benzyl alcohol (2 mol\%, $\left.0.0199 \mathrm{mmol}\right)$, MTBD (5.0 mol\%, $0.0499 \mathrm{mmol})$ and 2-S (5.0 mol\%, $0.0499 \mathrm{mmol})$. 


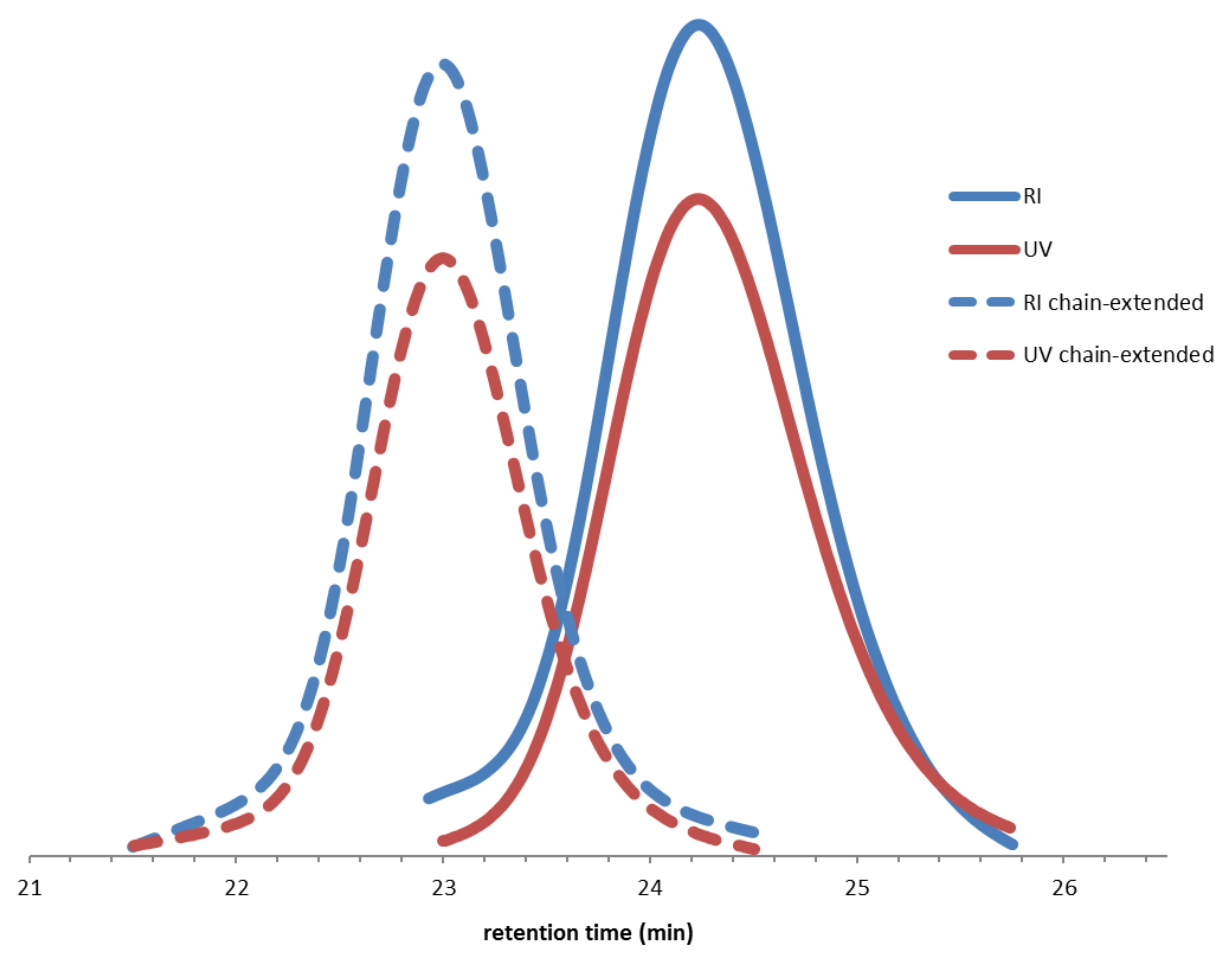

Figure 3.5. GPC traces of the polymer resulting from the 2-S/MTBD (5 mol\% each, 0.0499 mmol) cocatalyzed ROP and subsequent chain extension of VL (0.999 mmol, then 0.999 mmol more) from 1-pyrenebutanol $(0.0199 \mathrm{mmol})$ in $\mathrm{C}_{6} \mathrm{D}_{6}(999 \mu \mathrm{L})$. 


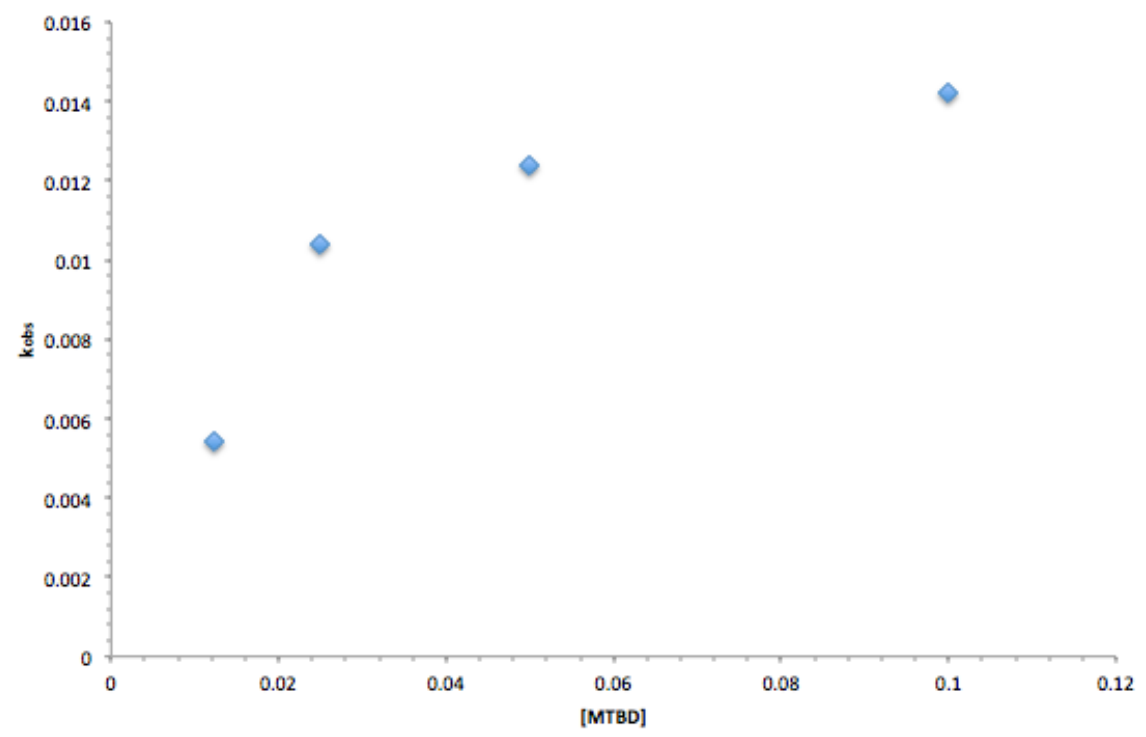

Figure 3.6. Observed rate constant $\left(\mathrm{k}_{\mathrm{obs}}, \mathrm{min}^{-1}\right)$ vs $[\mathrm{MTBD}]$ in the 2-S/MTBD catalyzed ROP of VL. Conditions: VL (0.999 mmol, 1 equiv, $1 \mathrm{M}$ in $\left.\mathrm{C}_{6} \mathrm{D}_{6}\right)$, benzyl alcohol (2 mol\%, $0.0199 \mathrm{mmol}), \mathrm{MTBD}(2.5 \mathrm{~mol} \%, 0.025 \mathrm{M})$. 


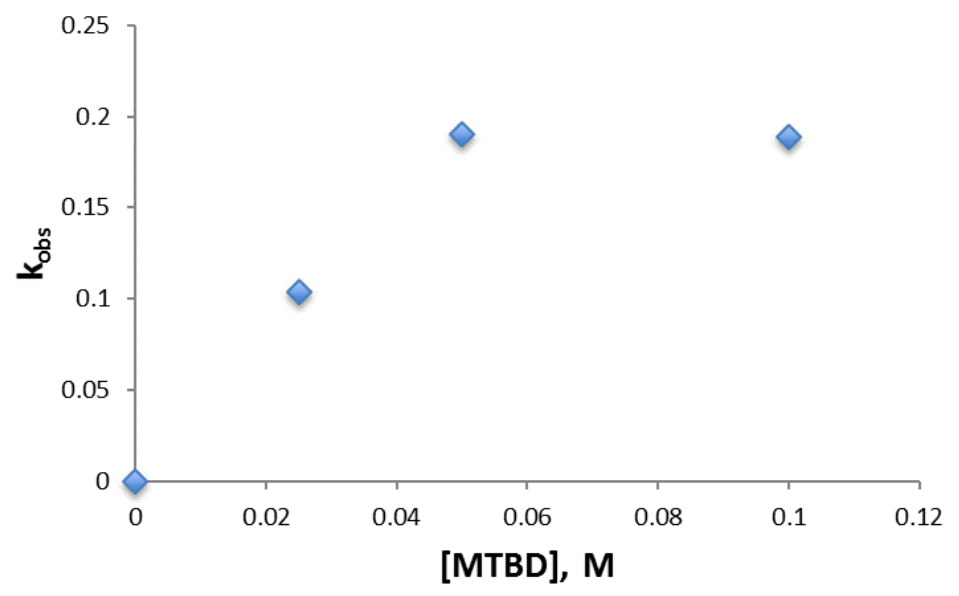

Figure 3.7. Observed rate constant $\left(\mathrm{k}_{\mathrm{obs}}, \mathrm{h}^{-1}\right)$ vs $[\mathbf{2 - S}]$ in the 2-S/MTBD catalyzed ROP of CL. Conditions: CL (0.999 mmol, 1 equiv, $2 \mathrm{M}$ in $\mathrm{C}_{6} \mathrm{D}_{6}$ ), benzyl alcohol (2 mol\%, 0.0199 mmol), 2-S (0.05M). 


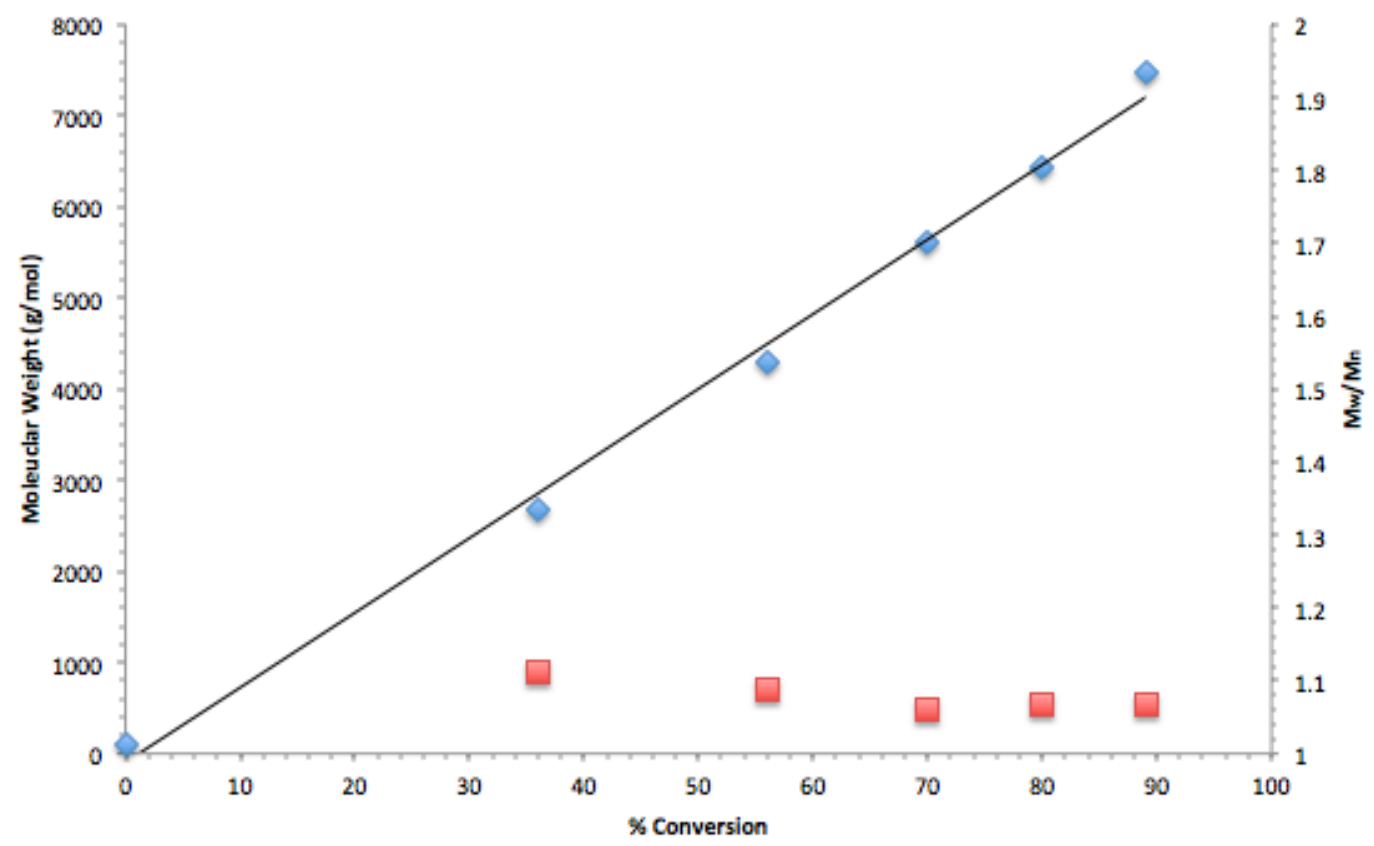

Figure 3.8. $\mathrm{M}_{\mathrm{n}}$ vs conversion of VL for the 3-O/MTBD catalyzed ROP of VL. Conditions: VL (0.999 mmol, 1 equiv, 2M in $\left.\mathrm{C}_{6} \mathrm{D}_{6}\right)$, benzyl alcohol (1mol\%, $\left.0.0199 \mathrm{mmol}\right)$, MTBD (1.67 mol\%, $0.0166 \mathrm{mmol})$ and 3-O (1.67 mol\%, $0.0166 \mathrm{mmol})$. (blue is $\mathrm{M}_{\mathrm{n}}$, red is $\mathrm{M}_{\mathrm{w}} / \mathrm{M}_{\mathrm{n}}$ ) 


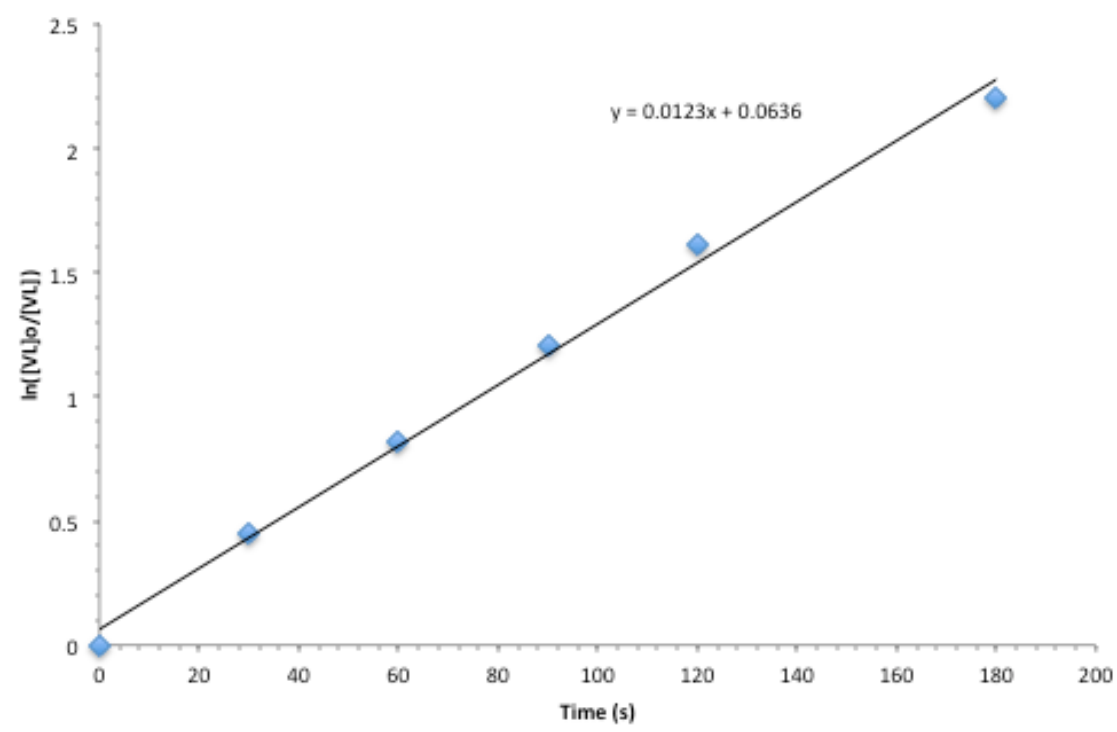

Figure 3.9. First order evolution of [VL] vs time for the 3-O/MTBD catalyzed ROP of VL.

Conditions: VL (0.999 mmol, 1 equiv, $2 \mathrm{M}$ in $\mathrm{C}_{6} \mathrm{D}_{6}$ ), benzyl alcohol (2 mol\%, 0.0199 mmol), MTBD (1.67 mol\%, $0.0166 \mathrm{mmol})$ and 3-O (1.67 mol\%, $0.0166 \mathrm{mmol})$. 


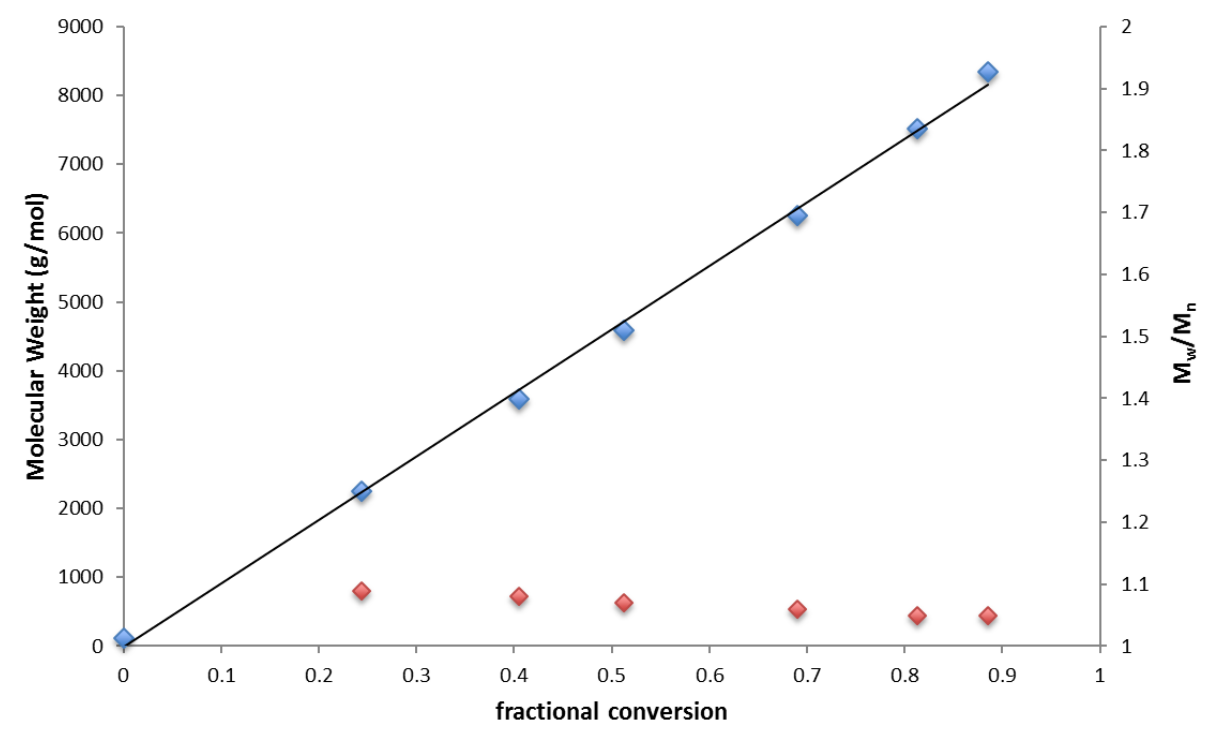

Figure 3.10. $\mathrm{M}_{\mathrm{n}}$ vs conversion for the 3-O/MTBD catalyzed ROP of CL. Conditions: CL (1.752 mmol, 1 equiv, $2 \mathrm{M}$ in $\mathrm{C}_{6} \mathrm{D}_{6}$ ), benzyl alcohol (2 mol\%, $\left.0.035 \mathrm{mmol}\right)$, MTBD (1.67 mol\%, $0.029 \mathrm{mmol}$ ) and 3-O $(1.67 \mathrm{~mol} \%, 0.029 \mathrm{mmol})$. (blue is $\mathrm{M}_{\mathrm{n}}$, red is $\mathrm{M}_{\mathrm{w}} / \mathrm{M}_{\mathrm{n}}$ ) 




Figure 3.11. First order evolution of [CL] vs time for the 3-O/MTBD catalyzed ROP of CL. Conditions: CL (1.752 mmol, 1 equiv, $2 \mathrm{M}$ in $\left.\mathrm{C}_{6} \mathrm{D}_{6}\right)$, benzyl alcohol (2 mol\%, 0.035 mmol), MTBD (1.67 mol\%, $0.029 \mathrm{mmol}$ ) and 3-O (1.67 mol\%, $0.029 \mathrm{mmol}$ ). 


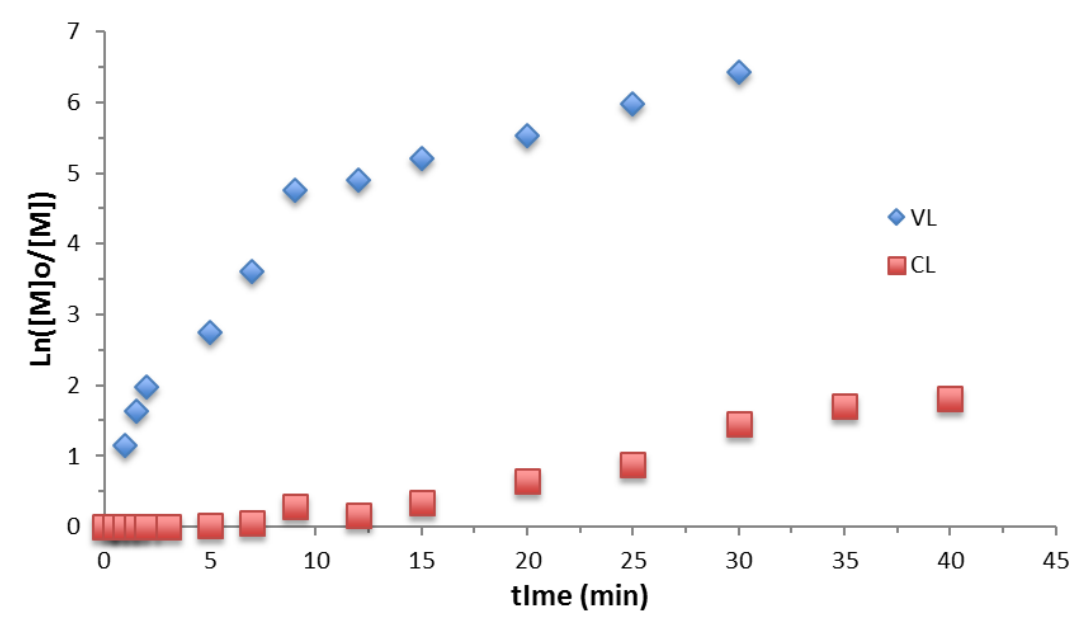

Figure 3.12. First order evolution of [CL] and [VL] vs time for the 3-O/MTBD catalyzed copolymerization of CL. Conditions: CL (1.752 mmol, 1 equiv, $2 \mathrm{M}$ in $\left.\mathrm{C}_{6} \mathrm{D}_{6}\right)$, benzyl alcohol (2 mol\%, $0.035 \mathrm{mmol})$, MTBD (1.67 mol\%, $0.029 \mathrm{mmol})$ and 3-O (1.67 $\mathrm{mol} \%$, $0.029 \mathrm{mmol})$. 


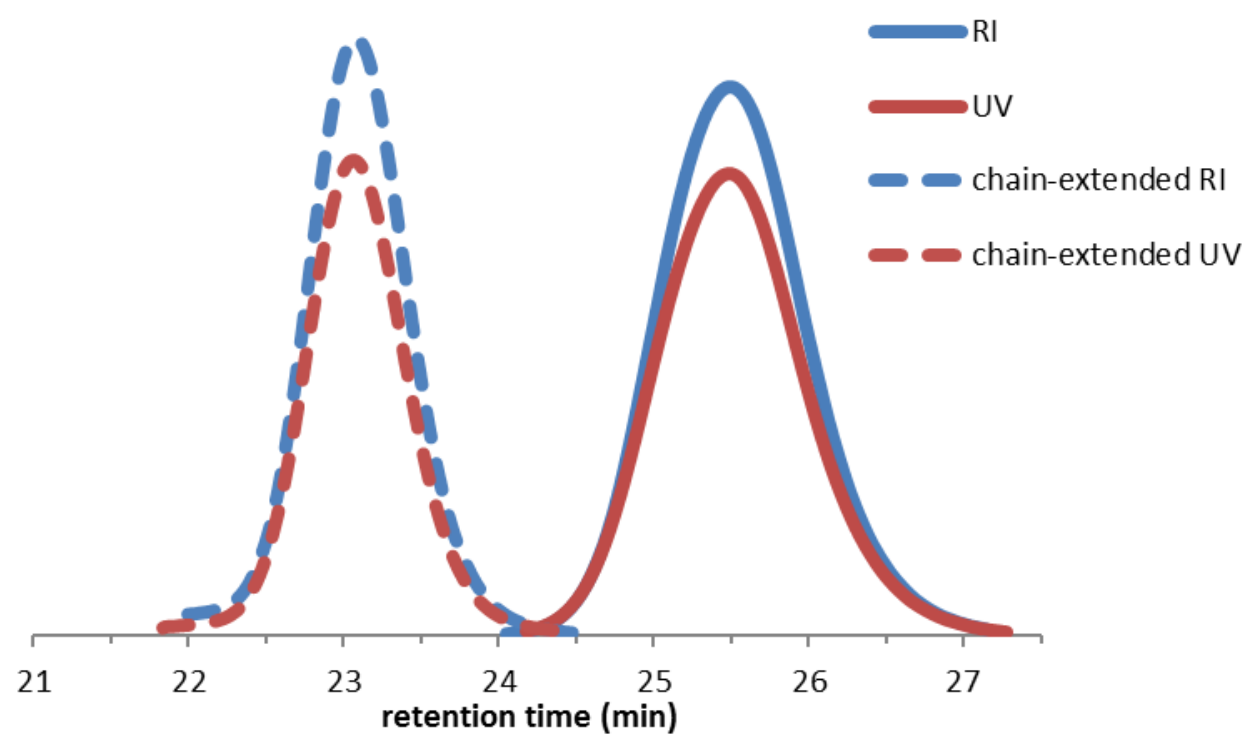

Figure 3.13. GPC traces of the polymer resulting from the 3-O/MTBD $(1.67 \mathrm{~mol} \%$ each, $0.015 \mathrm{mmol})$ cocatalyzed ROP and subsequent chain extension of CL ( $0.876 \mathrm{mmol}$, then $1.1723 \mathrm{mmol}$ more) from 1-pyrenebutanol $(0.035 \mathrm{mmol})$ in $\mathrm{C}_{6} \mathrm{D}_{6}(219 \mu \mathrm{L})$. 




Figure 3.14. Observed rate constant $\left(\mathrm{k}_{\mathrm{obs}}, \mathrm{min}^{-1}\right)$ vs [3-O] in the 3-O/MTBD catalyzed ROP of VL. Conditions: VL (0.999 mmol, 1 equiv, $0.5 \mathrm{M}$ in $\left.\mathrm{C}_{6} \mathrm{D}_{6}\right)$, benzyl alcohol (2 mol\%, $0.0199 \mathrm{mmol}), \operatorname{MTBD}(1.67 \mathrm{~mol} \%, 0.0166 \mathrm{mmol}, 0.008 \mathrm{M})$. 




Figure 3.15. DFT B3LYP//6-31G** geometry optimized structures of 3-S. 




Figure 3.16. DFT B3LYP//6-31G** geometry optimized structures of 3-O. 




Figure 3.17. MALDI-TOF of the PLA resulting from the 3-O/(tris[2(dimethylamino)ethyl]amine) catalyzed ROP of L-LA. 

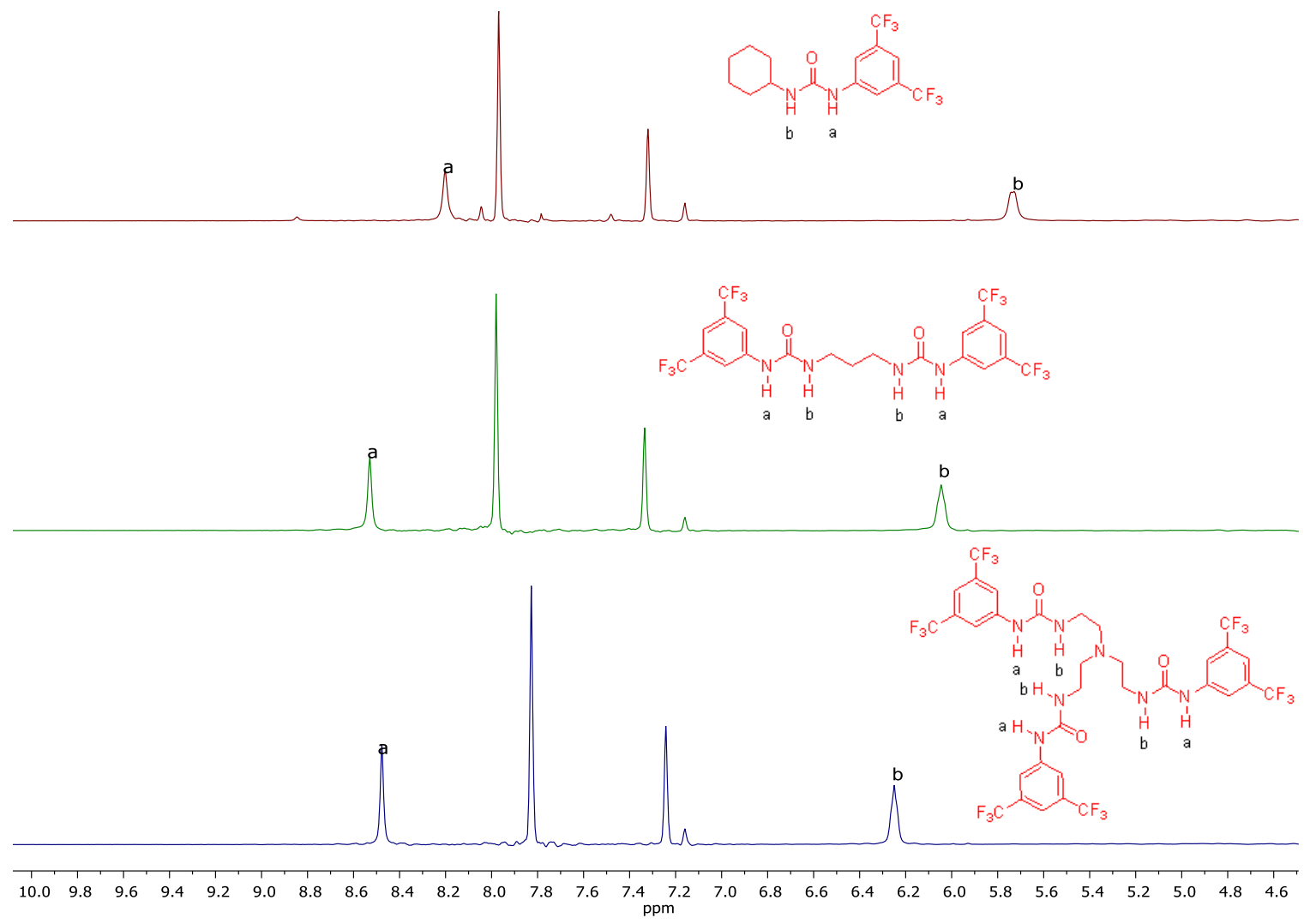

Figure 3.18. Downfield half of the ${ }^{1} \mathrm{H}$ NMR spectra (acetone + trace benzene- $\mathrm{d}_{6}$ (lock), $400 \mathrm{MHz}$ ) of (upper) 1-O, (middle) 2-O, and (lower) 3-O. The progressive downfield shift of the NH protons is indicative of increased (2-O vs 3-O) intramolecular H-bonding. 


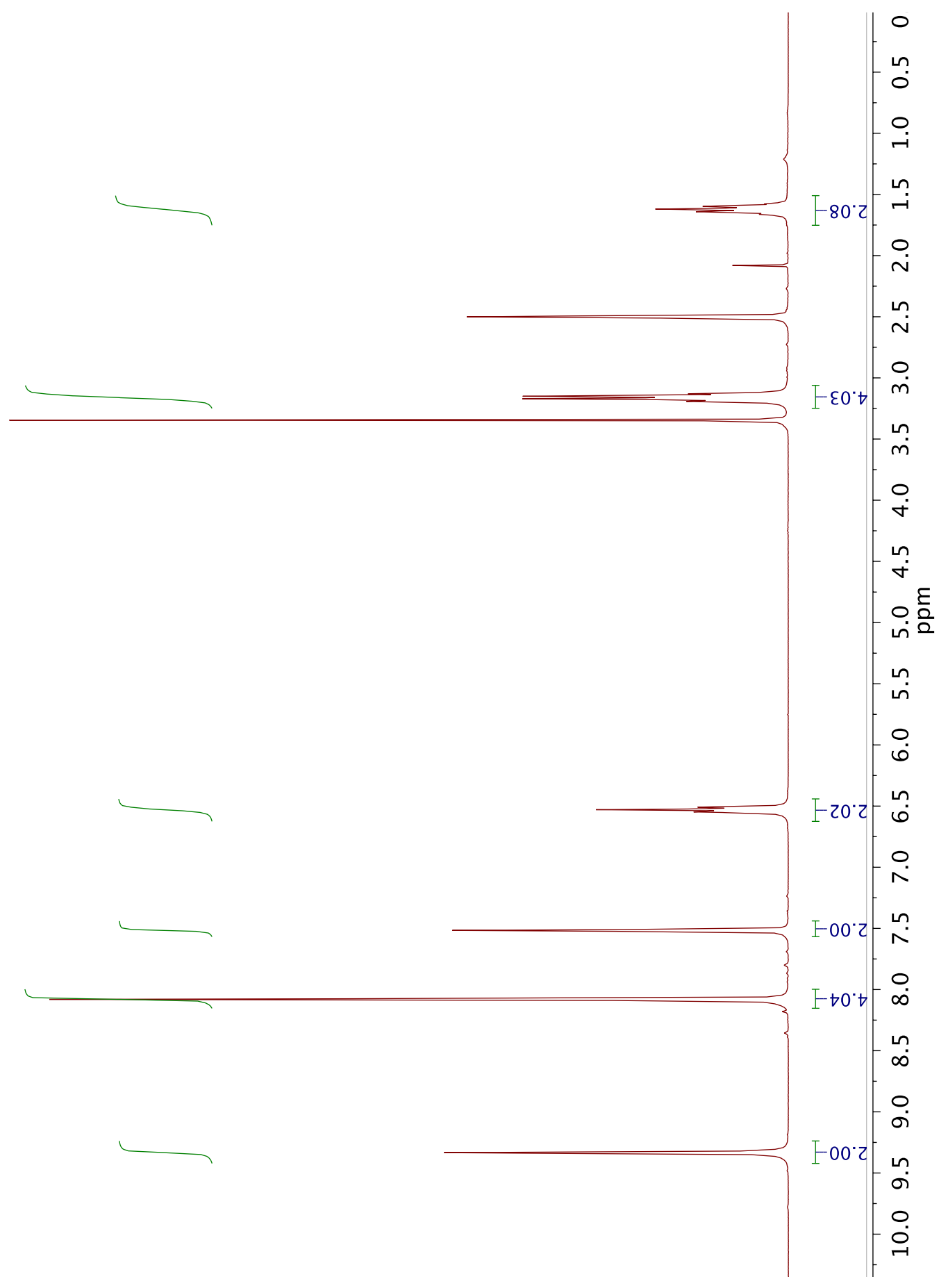

Figure 3.19. ${ }^{1} \mathrm{H}$ NMR (300 MHz, DMSO-d 6 ) of 2-O. 


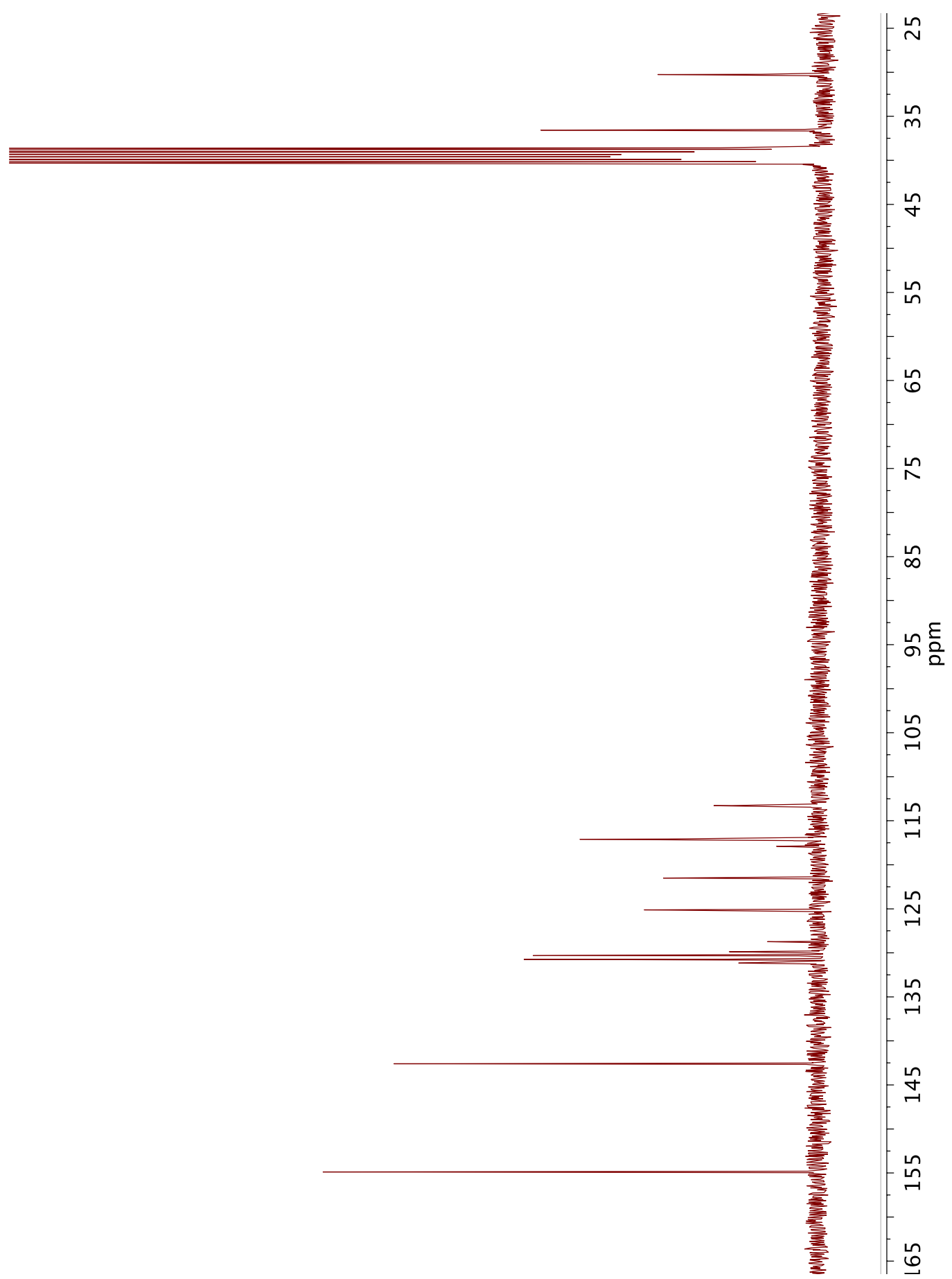

Figure 3.20. ${ }^{13} \mathrm{C}$ NMR (75 MHz, DMSO-d 6 ) of 2-O. 


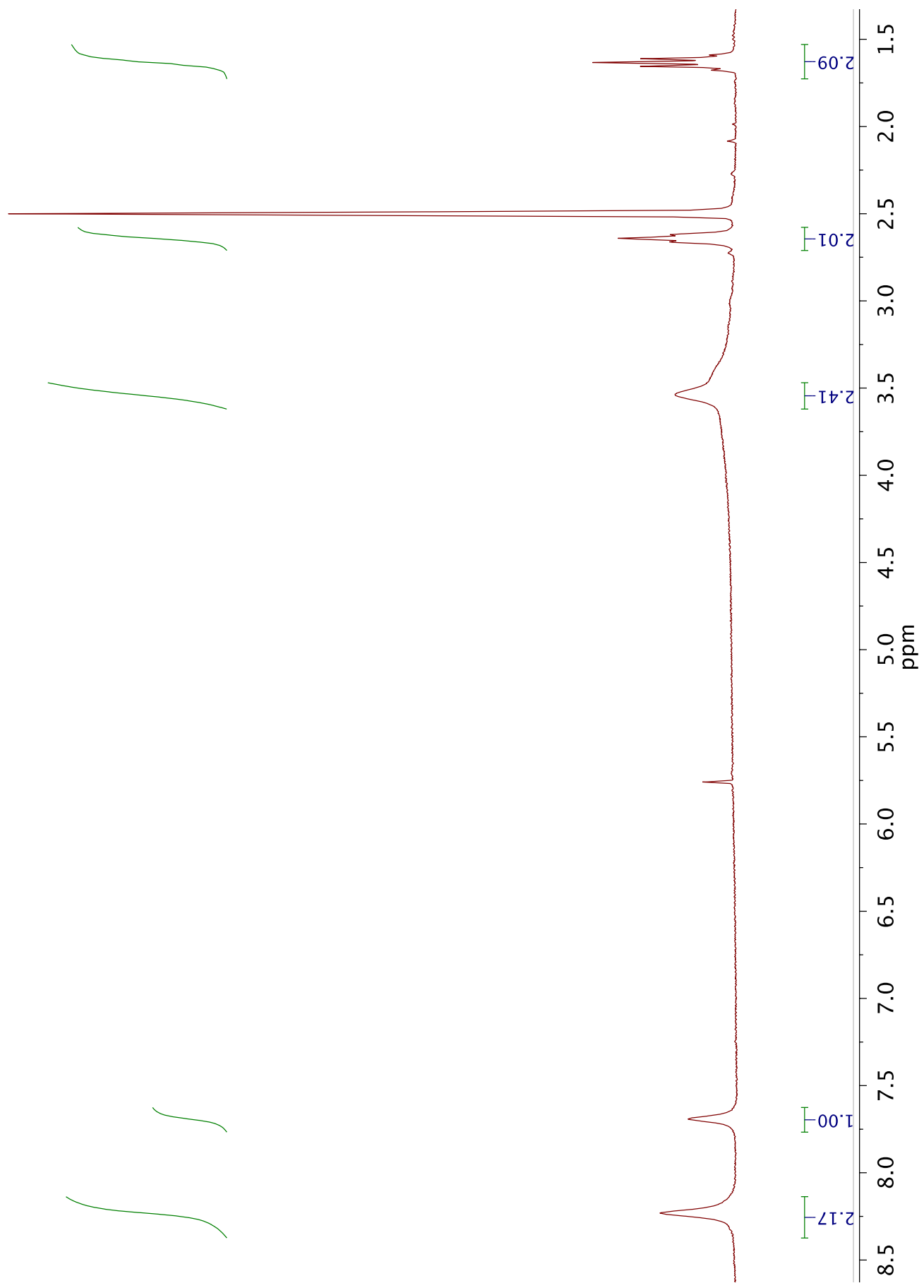

Figure 3.21. ${ }^{1} \mathrm{H}$ NMR (300 MHz, DMSO- $\mathrm{d}_{6}$ ) of 1-[3,5-bis(trifluoromethyl)phenyl thiourea]-3-aminopropane. 


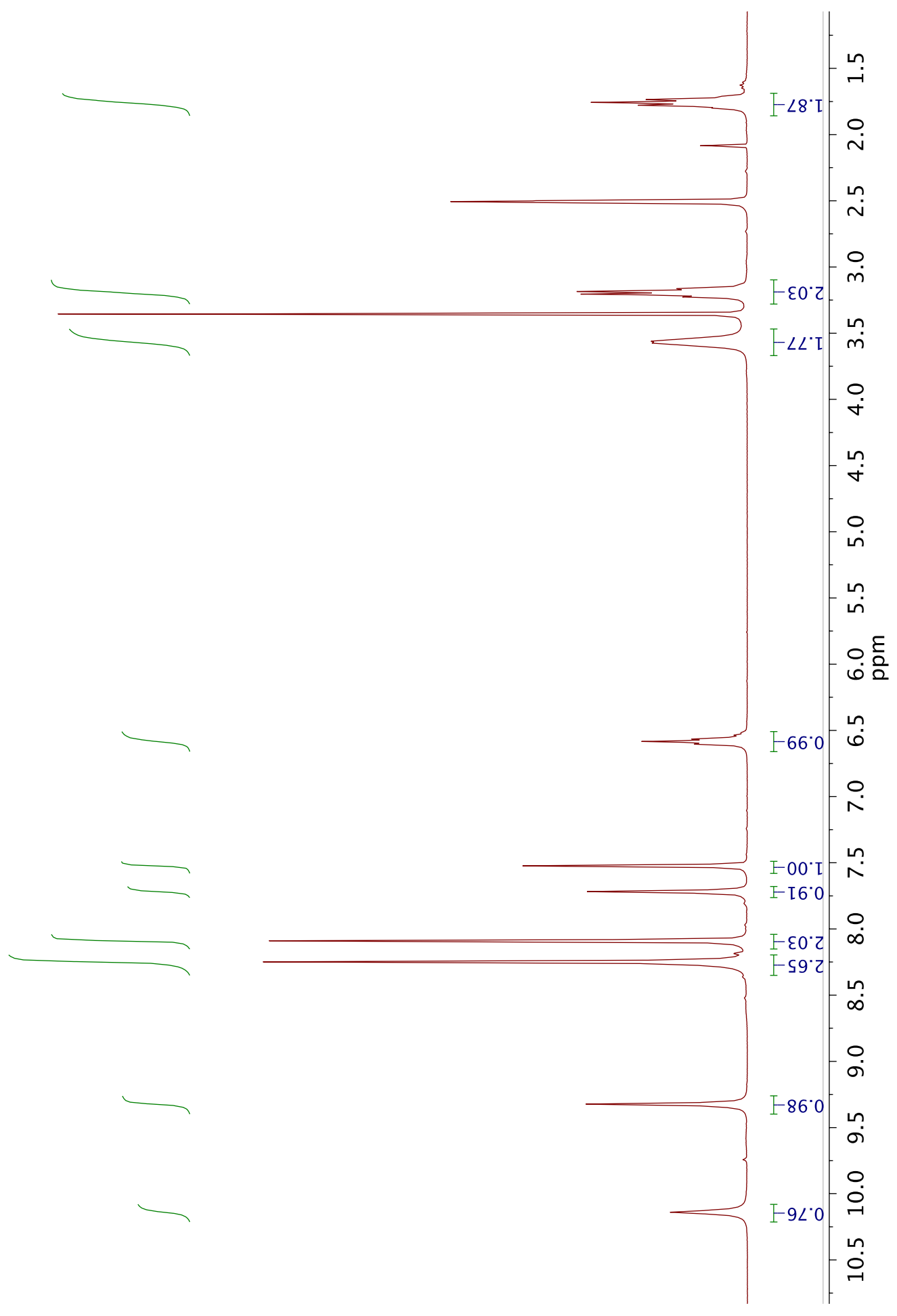

Figure 3.22. ${ }^{1} \mathrm{H}$ NMR (300 MHz, DMSO-d 6 ) of 2-OS. 


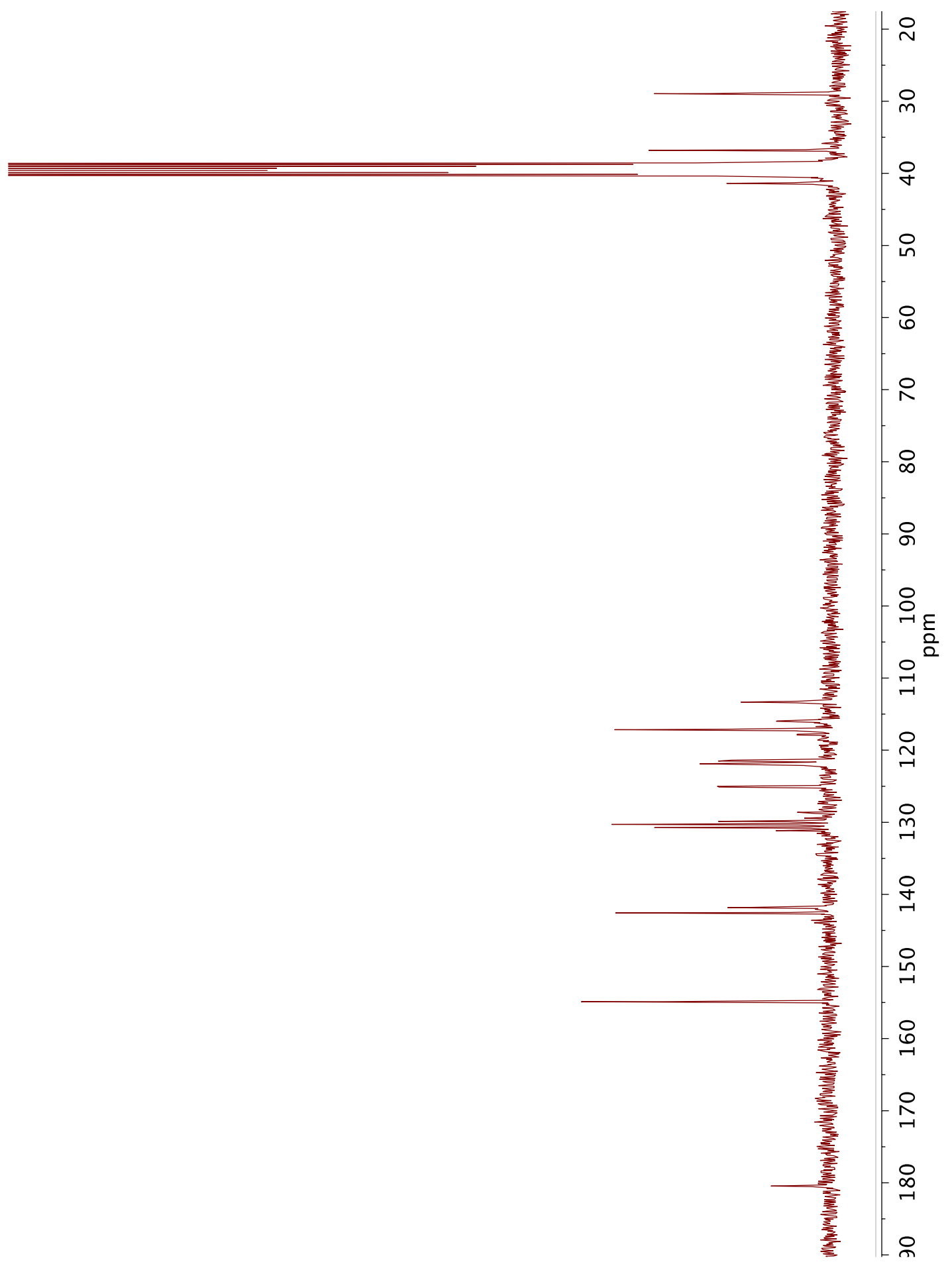

Figure 3.23. ${ }^{13} \mathrm{C}$ NMR (75 MHz, acetone-d 6 ) of 2-OS. 




Figure 3.24. ${ }^{1} \mathrm{H}$ NMR (300 MHz, acetone- $\left.\mathrm{d}_{6}\right)$ of 3-O. 


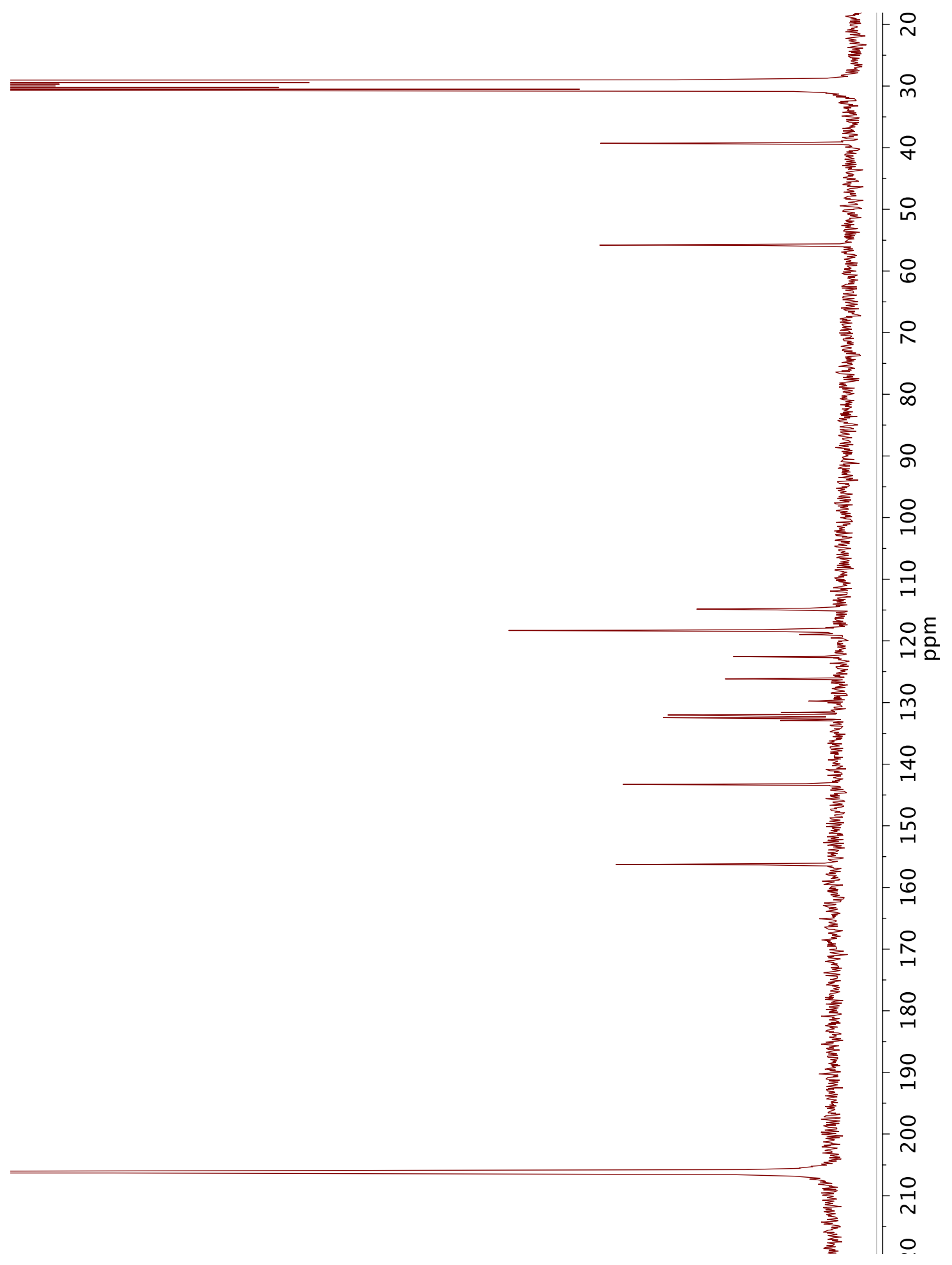

Figure 3.25. ${ }^{13} \mathrm{C}$ NMR (75 MHz, acetone- $\mathrm{d}_{6}$ ) of 3-O. 


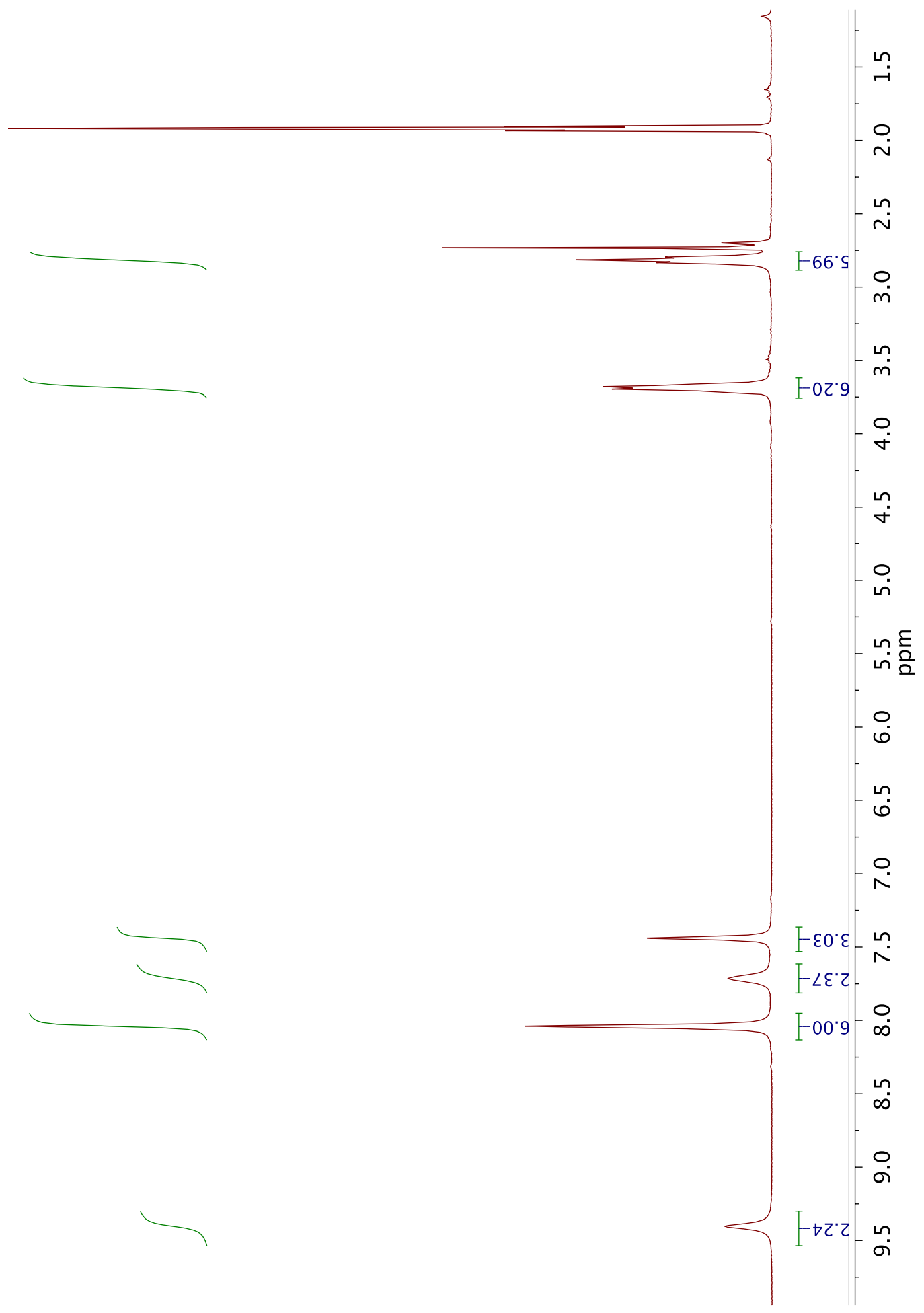

Figure 3.26. ${ }^{1} \mathrm{H}$ NMR (300 MHz, acetone- $\left.\mathrm{d}_{6}\right)$ of 3-S. 


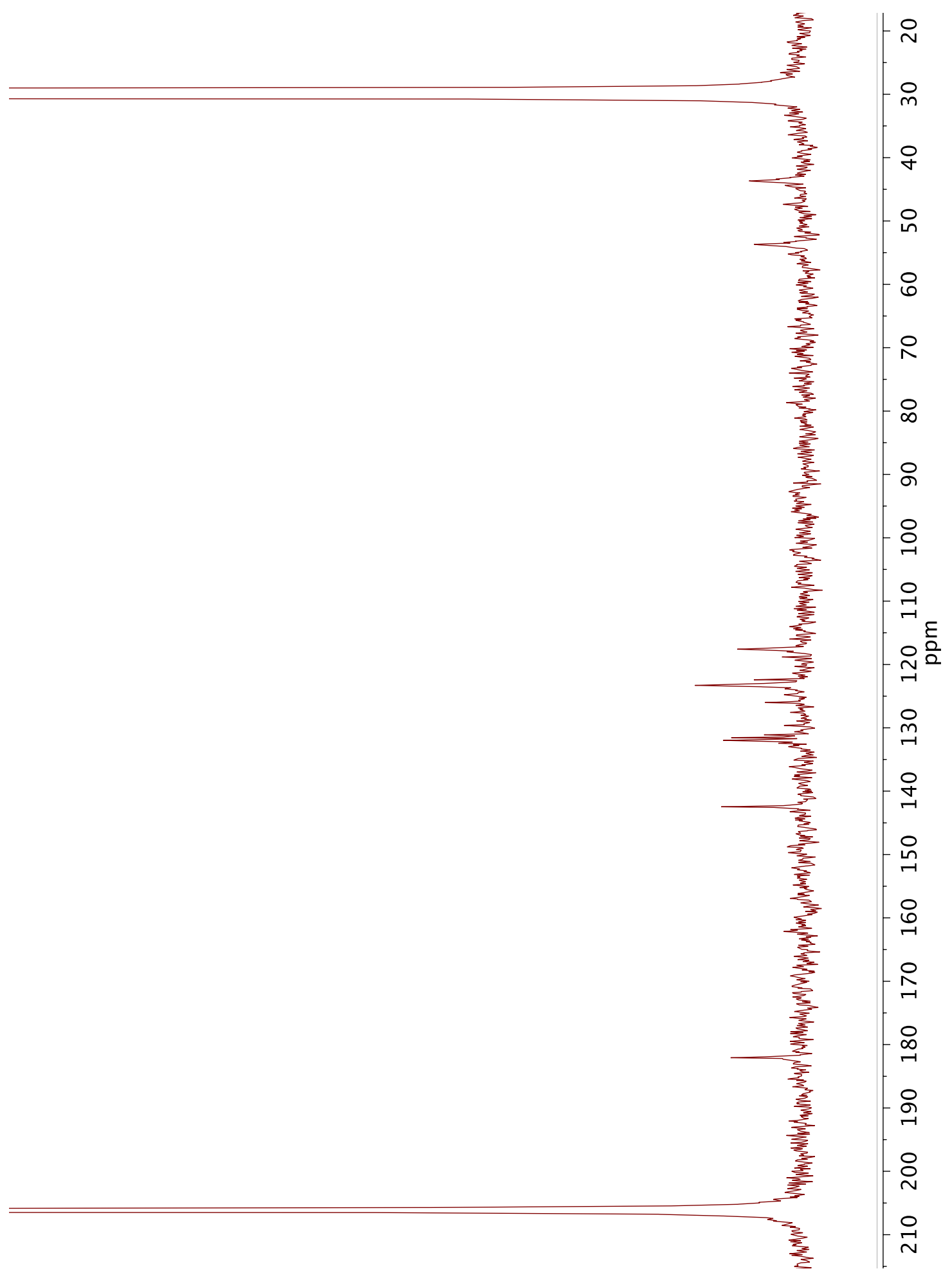

Figure 3.27. ${ }^{13} \mathrm{C}$ NMR (75 MHz, acetone- $\mathrm{d}_{6}$ ) of 3-S. 


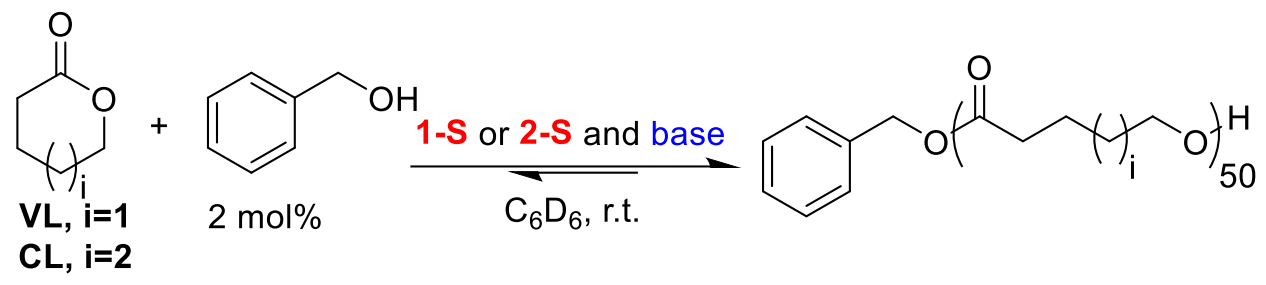

1 equiv. (2M)

\begin{tabular}{|c|c|c|c|c|c|c|c|}
\hline entry & monomer & $\begin{array}{c}\mathrm{TU} \\
(\mathrm{mol} \%)\end{array}$ & $\begin{array}{c}\text { Base } \\
(\mathrm{mol} \%)\end{array}$ & $\begin{array}{l}\text { time } \\
(\mathrm{min})\end{array}$ & $\begin{array}{c}\text { conv. }^{\mathrm{b}} \\
(\%)\end{array}$ & $\begin{array}{c}\mathrm{M}_{\mathrm{n}}^{\mathrm{c}} \\
(\mathrm{g} / \mathrm{mol})\end{array}$ & $\mathrm{M}_{\mathrm{w}} / \mathrm{M}_{\mathrm{n}}^{\mathrm{c}}$ \\
\hline 1 & $\mathrm{VL}$ & 1-S (5\%) & $\begin{array}{c}\text { MTBD } \\
(5 \%)\end{array}$ & 110 & 94 & 8,300 & 1.06 \\
\hline 2 & & 2-S $(2.5 \%)$ & $\begin{array}{l}\text { MTBD } \\
(2.5 \%)\end{array}$ & 80 & 90 & 6,800 & 1.07 \\
\hline 3 & & $\mathbf{2 - S}(2.5 \%)$ & $\begin{array}{l}\text { BEMP } \\
(2.5 \%)\end{array}$ & 84 & 91 & 8,900 & 1.06 \\
\hline 4 & & 2-S $(2.5 \%)$ & $\begin{array}{c}\text { DBU } \\
(2.5 \%)\end{array}$ & 90 & 86 & 8,400 & 1.05 \\
\hline 5 & & 3-S (1.67\%) & $\begin{array}{c}\text { MTBD } \\
(1.67 \%)\end{array}$ & 230 & 90 & 7600 & 1.06 \\
\hline 6 & $\mathrm{CL}$ & 1-S (5\%) & $\begin{array}{c}\text { MTBD } \\
(5 \%)\end{array}$ & $45 \mathrm{~h}$ & 90 & 7,200 & 1.09 \\
\hline 7 & & $\mathbf{2 - S}(2.5 \%)$ & $\begin{array}{l}\text { MTBD } \\
(2.5 \%)\end{array}$ & $10 \mathrm{~h}$ & 89 & 7,200 & 1.11 \\
\hline 8 & & 3-S $(1.67 \%)$ & $\begin{array}{l}\text { MTBD } \\
(1.67 \%)\end{array}$ & $42 \mathrm{~h}$ & 55 & 6,100 & 1.07 \\
\hline
\end{tabular}

Table 3.1. MTBD and bis- or tristhiourea Catalyzed ROP of VL and CL. ${ }^{a}$

a) Reaction conditions: VL or CL (1.0 mmol, 1 equiv, $2 \mathrm{M})$, benzyl alcohol (2 mol\%), $\mathrm{C}_{6} \mathrm{D}_{6}$.

b) monomer conversion was determined via ${ }^{1} H$ NMR. c) $M_{n}$ and $M_{w} / M_{n}$ were determined by $\mathrm{GPC}\left(\mathrm{CH}_{2} \mathrm{Cl}_{2}\right)$ vs polystyrene standards. 
<smiles>CC(C)(CCC(=O)OCC(C)(C)COc1ccccc1)COCc1ccccc1</smiles>

\begin{tabular}{|c|c|c|c|c|c|c|c|}
\hline entry & monomer & $\begin{array}{l}\text { TU or U } \\
(\mathrm{mol} \%)\end{array}$ & {$[\mathrm{M}]_{\mathrm{o}} /[\mathrm{I}]_{\mathrm{o}}$} & $\begin{array}{l}\text { time } \\
(\min )\end{array}$ & $\begin{array}{l}\text { conv. } \\
{ }^{\mathrm{b}}(\%)\end{array}$ & $\begin{array}{c}\mathrm{M}_{\mathrm{n}}^{\mathrm{c}} \\
(\mathrm{g} / \mathrm{mol})\end{array}$ & $\mathrm{M}_{\mathrm{w}} / \mathrm{M}_{\mathrm{n}}^{\mathrm{c}}$ \\
\hline 1 & VL & 1-O (5\%) & 50 & 70 & 90 & 6100 & 1.08 \\
\hline 2 & & $2-\operatorname{OS}(2.5 \%)$ & 50 & 88 & 90 & 8100 & 1.07 \\
\hline 3 & & 2-O $(2.5 \%)^{d}$ & 50 & 34 & 90 & 8000 & 1.07 \\
\hline 4 & & 3-O $(1.67 \%)$ & 50 & 3 & 89 & 7500 & 1.07 \\
\hline 5 & & & 100 & 6 & 90 & 15000 & 1.04 \\
\hline 6 & & & 200 & 10 & 92 & 28600 & 1.02 \\
\hline 7 & & & 500 & 16 & 92 & 41500 & 1.02 \\
\hline 8 & $\mathrm{CL}$ & 3-O (1.67\%) & 50 & 26 & 97 & 7900 & 1.05 \\
\hline 9 & & & 100 & 57 & 94 & 18500 & 1.02 \\
\hline 10 & & & 200 & 116 & 94 & 30700 & 1.03 \\
\hline 11 & & & 500 & 166 & 93 & 58600 & 1.03 \\
\hline $12^{\mathrm{e}}$ & & TBD $(1.67 \%)$ & 50 & 140 & 93 & 10400 & 1.37 \\
\hline
\end{tabular}

Table 3.2. Bis- and Tris-urea Cocatalyzed ROP of Lactones. ${ }^{a}$

a) Reaction conditions: VL or CL (1.0 mmol, 1 equiv, $2 \mathrm{M}$ ), urea or thiourea (given mol\%), MTBD (mol\% matched to $\mathrm{H}$-bond donor). b) Monomer conversion monitored via ${ }^{1} \mathrm{H}$ NMR. c) $M_{n}$ and $M_{w} / M_{n}$ were determined by $\mathrm{GPC}\left(\mathrm{CH}_{2} \mathrm{Cl}_{2}\right)$ vs polystyrene standards. d) 2-O (2.5 mol\%) and MTBD (5 mol\%) cocatalysts. e) no (thio)urea or MTBD cocatalysts were used in this run. 


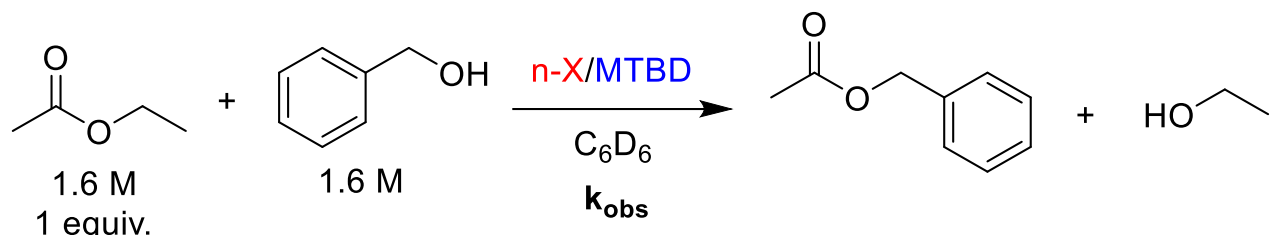

\begin{tabular}{cccc} 
entry & $\begin{array}{c}\mathrm{TU} \text { or U } \\
(\mathrm{mol} \%)\end{array}$ & $\begin{array}{c}\mathrm{k}_{\text {obs }} \\
(1 / \mathrm{min})^{\mathrm{a}}\end{array}$ & $\begin{array}{c}{[\mathrm{EA}]_{\mathrm{eq}}} \\
(\mathrm{M})^{\mathrm{b}}\end{array}$ \\
\hline 1 & $\mathbf{1 - S}(5 \%)$ & 0.00080 & 1.08 \\
2 & $\mathbf{1 - O}(5 \%)$ & 0.00357 & 0.88 \\
3 & $\mathbf{2 - S}(2.5 \%)$ & 0.00055 & 0.99 \\
4 & $\mathbf{2 - O}(2.5 \%)$ & 0.00410 & 0.99 \\
5 & $\mathbf{3 - S}(1.67 \%)$ & 0.00061 & 1.19 \\
6 & $\mathbf{3 - O}(1.67 \%)$ & 0.00211 & 0.89
\end{tabular}

Table 3.3. Transesterification of Ethyl Acetate.

a) Observed rate constant for the first order disappearance of [EA] vs time. Rate constant was extracted from the linear portion of the data, up to $\sim 20 \%$ conversion. b)

Concentration of ethyl acetate remaining at equilibrium. 


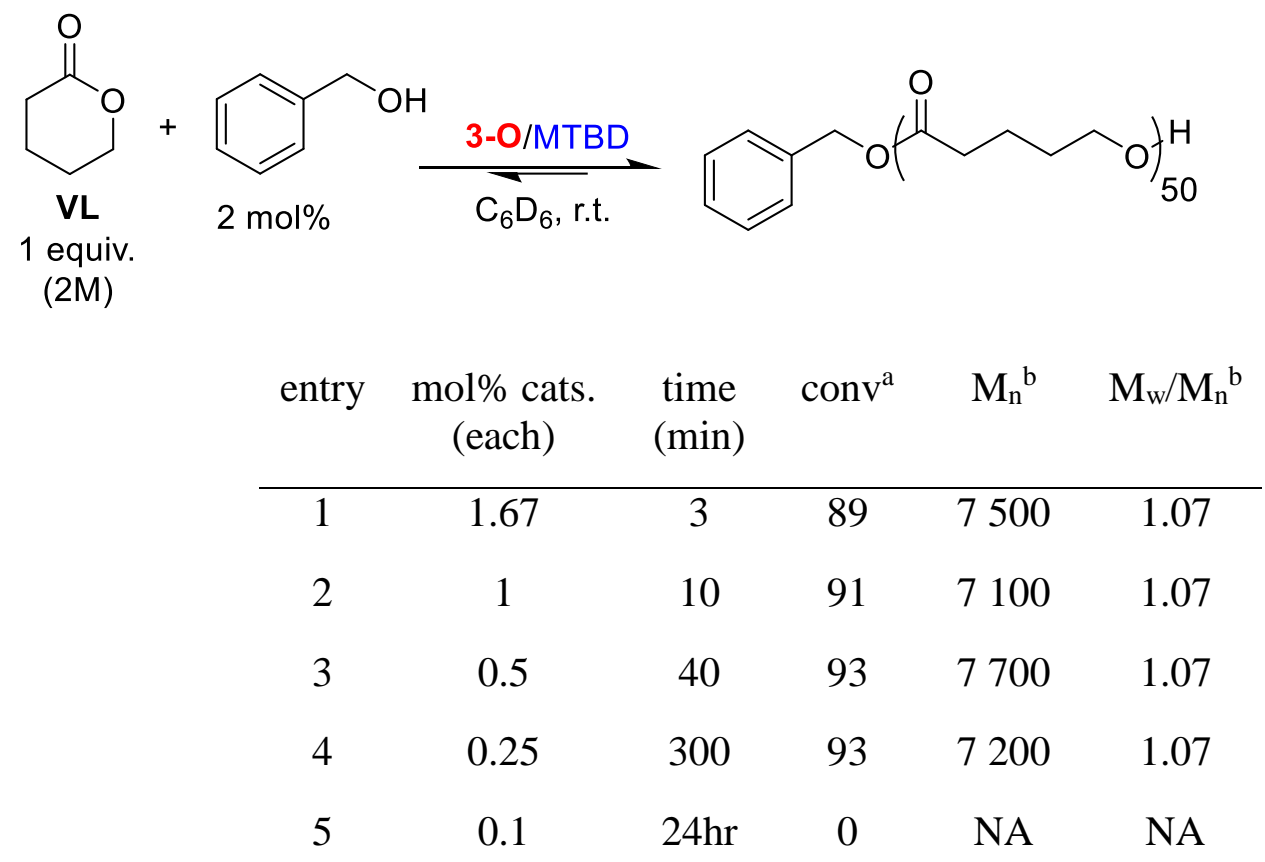

Table 3.4. Low 3-O/MTBD Cocatalyst Loadings in the ROP of VL.

Reaction conditions: VL(0.998 mmol, 1 equiv, 2M), $\mathrm{C}_{6} \mathrm{D}_{6}$ and benzyl alcohol (2 mol\%).

a) Monomer conversion was monitored via ${ }^{1} H$ NMR. b) $M_{n}$ and $M_{w} / M_{n}$ were determined by $\mathrm{GPC}\left(\mathrm{CH}_{2} \mathrm{Cl}_{2}\right)$ vs polystyrene standards. 


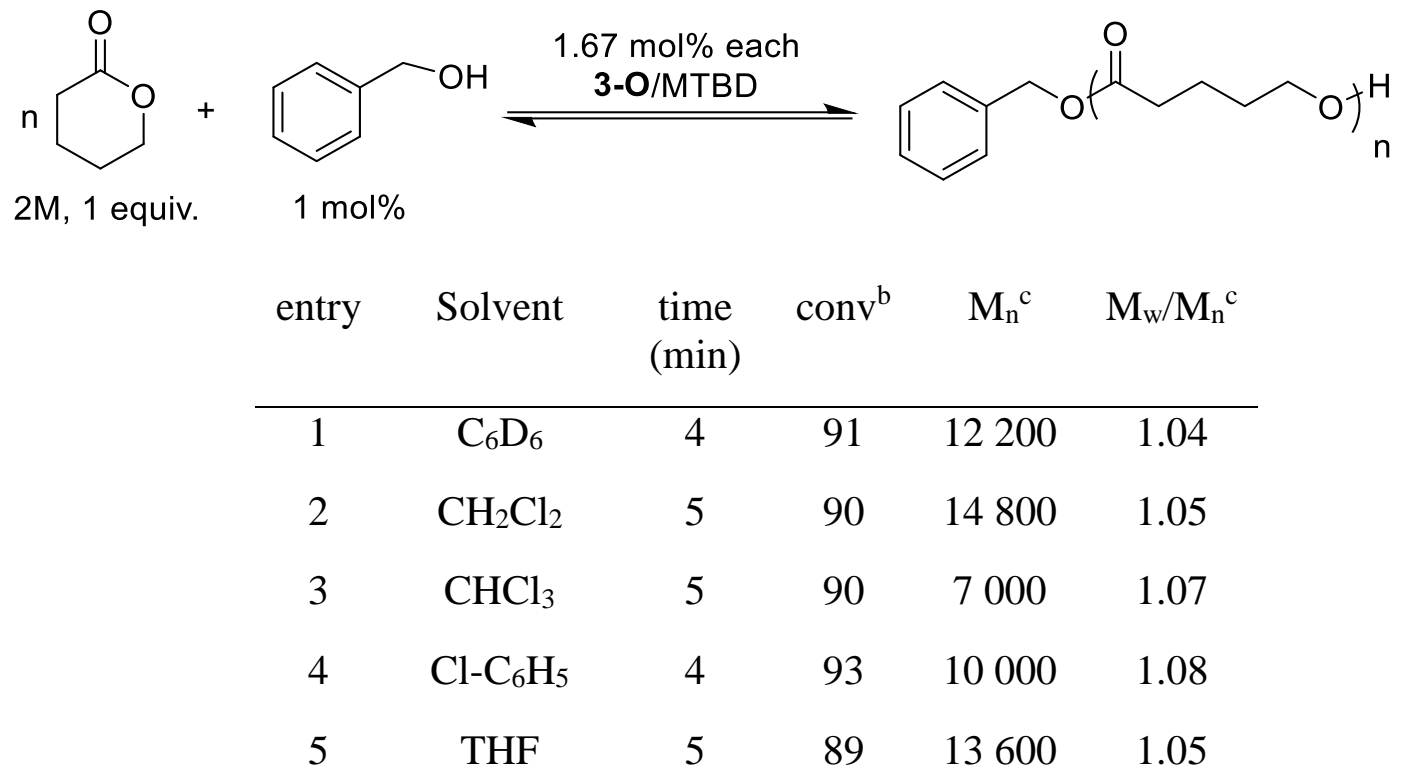

Table 3.5. Solvent Screen in the 3-O/MTBD Cocatalyzed ROP of VL. ${ }^{\text {a }}$

a) Reaction conditions: VL (0.998 mmol, 1 equiv, 2M), 1 mol\% benzyl alcohol, b) monomer conversion was monitored via ${ }^{1} \mathrm{H}$ NMR. c) $\mathrm{M}_{\mathrm{n}}$ and $\mathrm{M}_{\mathrm{w}} / \mathrm{M}_{\mathrm{n}}$ were determined by GPC. 


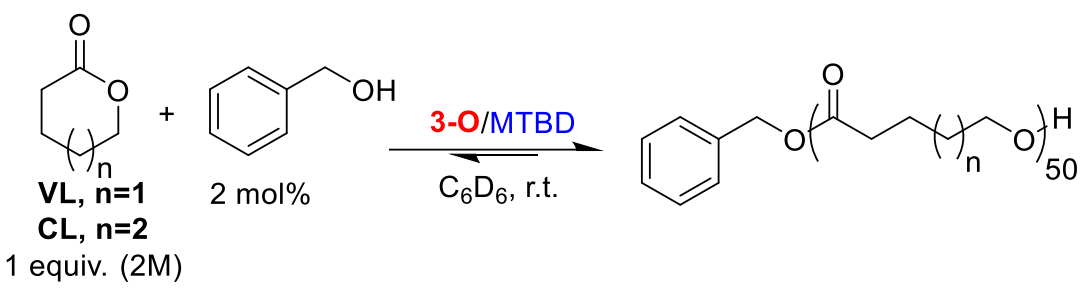

\begin{tabular}{cccccc} 
entry & monomer & $\begin{array}{c}\text { time } \\
(\mathrm{min})\end{array}$ & conv $^{\mathrm{b}}$ & $\mathrm{M}_{\mathrm{n}}{ }^{\mathrm{c}}$ & $\mathrm{M}_{\mathrm{w}} / \mathrm{M}_{\mathrm{n}}{ }^{\mathrm{c}}$ \\
\hline 1 & $\mathrm{VL}$ & 3 & 93 & 6200 & 1.10 \\
2 & $\mathrm{VL}$ & 6 & 93 & 6300 & 1.12 \\
3 & $\mathrm{VL}$ & 60 & 94 & 6600 & 1.21 \\
4 & $\mathrm{CL}$ & 25 & 91 & 9000 & 1.04 \\
5 & $\mathrm{CL}$ & 60 & 98 & 10000 & 1.05 \\
6 & $\mathrm{CL}$ & 120 & 99 & 10000 & 1.09
\end{tabular}

Table 3.6. Post-polymerization Transesterification in 3-O/MTBD Cocatalyzed ROP. ${ }^{a}$

a) Reaction conditions: VL (0.998 mmol, 1 equiv, 2M), 2 mol\% benzyl alcohol, b) monomer conversion was monitored via ${ }^{1} \mathrm{H} N \mathrm{NM}$. c) $\mathrm{M}_{\mathrm{n}}$ and $\mathrm{M}_{\mathrm{w}} / \mathrm{M}_{\mathrm{n}}$ were determined by GPC. 


\section{Computational Data}

\section{Dual-thiourea activiation in DCM}



Job type: Single point.

Method: RB3LYP

Basis set: 6-31G**

Number of shells: 258

Number of basis functions: 818

Multiplicity: 1

Solvation: dichloromethane [SM8]

Free Energy of Solvation : $\quad-111.5381226 \mathrm{~kJ} / \mathrm{mol}$

SCF total energy: -3369.3171898 hartrees

SPARTAN '14 Properties Program: (Win/64b)

Use of molecular symmetry disabled

Release 1.1 .8

\begin{tabular}{rccc}
\multicolumn{5}{c}{ Cartesian Coordinates (Angstroms) } \\
Atom & X & Y & Z \\
----- & - & & \\
1 C C1 & 3.0236320 & 1.8782697 & -1.5793812 \\
2 S S1 & 2.4865855 & 3.2691914 & -2.3431680 \\
3 N N1 & 4.1615953 & 1.8195168 & -0.8199347 \\
4 H H4 & 4.3807088 & 0.9334757 & -0.3754557 \\
5 N N2 & 2.4147858 & 0.6395117 & -1.6440053 \\
6 H H3 & 2.8892309 & -0.0770555 & -1.1048418
\end{tabular}




$\begin{array}{lccc}\text { 7 C C2 } & 1.1325107 & 0.2212543 & -2.0380878 \\ \text { 8 C C4 } & -1.4114202 & -0.8709745 & -2.5894579 \\ \text { 9 C C3 } & 0.9092347 & -1.1643141 & -1.9771559 \\ \text { 10 C C6 } & 0.0704432 & 1.0587976 & -2.4057200 \\ \text { 11 C C5 } & -1.1820850 & 0.5015117 & -2.6687443 \\ \text { 12 C C7 } & -0.3459752 & -1.7004420 & -2.2407846 \\ \text { 13 H H6 } & 1.7244618 & -1.8277314 & -1.7058464 \\ \text { 14 H H7 } & 0.2227144 & 2.1268062 & -2.4722793 \\ \text { 15 H H10 } & -2.3930742 & -1.2817497 & -2.7895526 \\ \text { 16 C C10 } & 4.9143846 & 2.9692703 & -0.3332953 \\ \text { 17 H H11 } & 4.5344106 & 3.8436800 & -0.8652759 \\ \text { 18 H H14 } & 5.9699149 & 2.8446241 & -0.6074915 \\ \text { 19 C C12 } & 3.4027906 & 3.4954232 & 1.7271696 \\ \text { 20 H H15 } & 2.9363627 & 4.2861218 & 1.1280701 \\ \text { 21 H H18 } & 3.4772296 & 3.8782160 & 2.7517105 \\ \text { 22 N N3 } & 2.5369424 & 2.3192087 & 1.7215030 \\ \text { 23 H H20 } & 2.9203006 & 1.4691364 & 1.3284096 \\ \text { 24 C C13 } & 1.2067155 & 2.3544009 & 1.9901580 \\ \text { 25 N N4 } & 0.6109505 & 1.1232290 & 1.7699492 \\ \text { 26 H H22 } & 1.2548396 & 0.3613405 & 1.5797141 \\ \text { 27 C C14 } & -0.7331750 & 0.7225280 & 1.7506543 \\ \text { 28 C C15 } & -3.3546714 & -0.3089651 & 1.5817556 \\ \text { 29 C C16 } & -1.8288593 & 1.5927093 & 1.6175975 \\ \text { 30 C C17 } & -0.9686276 & -0.6575333 & 1.7958613 \\ \text { 31 C C18 } & -2.2638136 & -1.1643464 & 1.7001179 \\ \text { 32 C C19 } & -3.1155194 & 1.0670046 & 1.5420799 \\ \text { 33 H H21 } & -1.6699138 & 2.6600289 & 1.5790835 \\ \text { 34 H H23 } & -0.1285243 & -1.3372930 & 1.8897131 \\ \text { 35 H H26 } & -4.3634555 & -0.6988767 & 1.5185011 \\ \text { 36 S S2 } & 0.4404338 & 3.7350223 & 2.5568843 \\ \text { 37 C C9 } & 4.8032256 & 3.1640599 & 1.1907223 \\ \text { 38 H H5 } & 5.2007974 & 2.2820671 & 1.7151565 \\ \text { 39 H H66 } & 5.4672919 & 3.9953370 & 1.4584577 \\ \text { 40 C C8 } & -2.3348816 & 1.4323499 & -2.9566642 \\ \text { 41 C C11 } & -0.5287562 & -3.1927444 & -2.1923754 \\ \text { 42 C C20 } & -2.4486148 & -2.6540574 & 1.7596442 \\ \text { 43 C C21 } & -4.2949114 & 1.9896479 & 1.3633098 \\ \text { 44 F F1 } & -1.5855148 & -3.2933792 & 0.9172092 \\ \text { 45 F F2 } & -3.6909519 & -3.0390037 & 1.4192127 \\ \text { 46 F F3 } & -2.1937480 & -3.1488662 & 2.9941791 \\ \text { 57 F F4 } & -5.2867990 & 1.6844871 & 2.2341956 \\ \text { 57 C C22 } & 3.2789749 & -1.9494030 & 0.9874045 \\ \text { 58 O O2 } & 2.0981603 & -2.3565783 & 1.4442692 \\ \text { 59 C C23 } & 4.3678470 & -2.9558988 & 0.6627870\end{array}$




$\begin{array}{lccc}60 \mathrm{H} \mathrm{H} 1 & 5.3107962 & -2.5327712 & 1.0237134 \\ 61 \mathrm{C} \mathrm{C} 25 & 1.7615096 & -3.7600728 & 1.7091266 \\ 62 \mathrm{H} \mathrm{H} 2 & 1.8528809 & -3.8888231 & 2.7924874 \\ 63 \mathrm{C} \mathrm{C} 24 & 4.1149410 & -4.3742363 & 1.1861986 \\ 64 \mathrm{C} \mathrm{C26} & 2.6466391 & -4.7344629 & 0.9547784 \\ 65 \text { H H9 } & 4.4431817 & -2.9637701 & -0.4338844 \\ 66 \text { H H12 } & 0.7120346 & -3.8367938 & 1.4267382 \\ 67 \text { H H16 } & 4.7842542 & -5.0791288 & 0.6833204 \\ 68 \text { H H17 } & 4.3433540 & -4.4264428 & 2.2584168 \\ 69 \text { H H19 } & 2.4193356 & -5.7464532 & 1.3082089 \\ 70 \text { H H24 } & 2.4038772 & -4.7036553 & -0.1143083\end{array}$

$\underline{\text { Activated-TU plus VL in DCM }}$

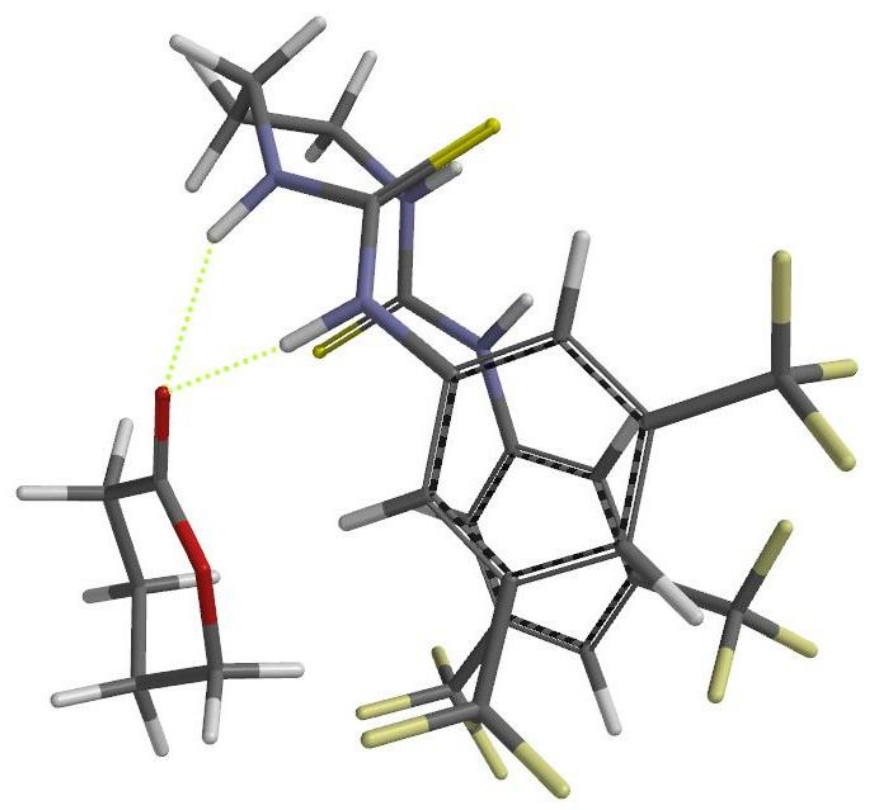

Job type: Single point.

Method: RB3LYP

Basis set: 6-31G**

Number of shells: 258

Number of basis functions: 818

Multiplicity: 1

Solvation: dichloromethane [SM8]

Free Energy of Solvation : $\quad-77.8518861 \mathrm{~kJ} / \mathrm{mol}$

SCF total energy: -3369.3245007 hartrees

SPARTAN '14 Properties Program: (Win/64b)

Release 1.1 .8

Use of molecular symmetry disabled

\begin{tabular}{rrrrr}
\multicolumn{5}{c}{ Cartesian Coordinates (Angstroms) } \\
Atom & X & Y & Z & \\
---------- & \\
& & & & \\
1 C C1 & 0.6186602 & 3.4506147 & -0.4942311 \\
2 S S1 & -0.1839783 & 4.1767400 & 0.8241190
\end{tabular}




\begin{tabular}{|c|c|c|c|}
\hline $3 \mathrm{~N} \mathrm{N1}$ & 1.9063132 & 3.7081440 & -0.8145935 \\
\hline $4 \mathrm{H} \mathrm{H} 4$ & 2.3364448 & 3.0985513 & -1.5079477 \\
\hline $5 \mathrm{~N} \mathrm{N2}$ & 0.0531547 & 2.5251892 & -1.3346437 \\
\hline $6 \mathrm{H} \mathrm{H} 3$ & 0.7188179 & 2.0849533 & -1.9741357 \\
\hline 7 C C2 & -1.1547538 & 1.8052874 & -1.2340656 \\
\hline $8 C \mathrm{C} 4$ & -3.4580090 & 0.1851075 & -1.1642309 \\
\hline 9 C C3 & -1.1316466 & 0.5028274 & -1.7487208 \\
\hline 10 C C6 & -2.3542935 & 2.3143430 & -0.7172609 \\
\hline 11 C C5 & -3.4821723 & 1.4924413 & -0.6759791 \\
\hline 12 C C7 & -2.2707223 & -0.2955969 & -1.7112994 \\
\hline $13 \mathrm{H} \mathrm{H} 6$ & -0.2138590 & 0.1114105 & -2.1743285 \\
\hline $14 \mathrm{H} \mathrm{H} 7$ & -2.4085778 & 3.3304717 & -0.3544325 \\
\hline $15 \mathrm{H} \mathrm{H} 10$ & -4.3392201 & -0.4410508 & -1.1138825 \\
\hline 16 C C10 & 2.8027843 & 4.6914441 & -0.2147555 \\
\hline $17 \mathrm{H} \mathrm{H} 11$ & 2.2071707 & 5.3416025 & 0.4302030 \\
\hline $18 \mathrm{H} \mathrm{H} 14$ & 3.1967392 & 5.3095899 & -1.0312663 \\
\hline 19 C C12 & 3.6869285 & 3.6006471 & 1.9812784 \\
\hline $20 \mathrm{H} \mathrm{H} 15$ & 3.4926792 & 4.4774002 & 2.6093305 \\
\hline $21 \mathrm{H} \mathrm{H} 18$ & 4.5555740 & 3.0769033 & 2.3864867 \\
\hline $22 \mathrm{~N} \mathrm{~N} 3$ & 2.5249512 & 2.7335360 & 2.1290191 \\
\hline $23 \mathrm{H} \mathrm{H} 20$ & 1.6271786 & 3.2234830 & 2.1204578 \\
\hline 24 C C13 & 2.5094968 & 1.3974966 & 1.9057752 \\
\hline $25 \mathrm{~N} \mathrm{~N} 4$ & 1.2256902 & 0.8893739 & 1.9753434 \\
\hline $26 \mathrm{H} \mathrm{H} 22$ & 0.5039979 & 1.5998622 & 2.0720812 \\
\hline 27 C C14 & 0.6984366 & -0.4058478 & 1.8631176 \\
\hline 28 C C15 & -0.5781053 & -2.9147839 & 1.6314277 \\
\hline 29 C C16 & 1.4548726 & -1.5782301 & 1.7537946 \\
\hline 30 C C17 & -0.7080836 & -0.5063312 & 1.8791934 \\
\hline 31 C C18 & -1.3281747 & -1.7434602 & 1.7746406 \\
\hline 32 C C19 & 0.8068657 & -2.8102748 & 1.6216030 \\
\hline $33 \mathrm{H}$ H21 & 2.5337814 & -1.5241645 & 1.7735517 \\
\hline $34 \mathrm{H} \mathrm{H} 23$ & -1.3157391 & 0.3883894 & 1.9709278 \\
\hline $35 \mathrm{H} \mathrm{H} 26$ & -1.0674423 & -3.8761542 & 1.5331906 \\
\hline 36 S S2 & 3.9137885 & 0.5130018 & 1.5863986 \\
\hline 37 C C9 & 3.9823780 & 4.0499365 & 0.5409167 \\
\hline $38 \mathrm{H} \mathrm{H} 5$ & 4.3622406 & 3.1976526 & -0.0342216 \\
\hline $39 \mathrm{H} \mathrm{H} 66$ & 4.7977458 & 4.7836087 & 0.5904183 \\
\hline $40 \mathrm{CC} 8$ & -4.7350832 & 2.0133800 & -0.0202682 \\
\hline 41 C C11 & -2.1500066 & -1.7036538 & -2.2285185 \\
\hline 42 C C20 & -2.8297569 & -1.8589628 & 1.8102446 \\
\hline 43 C C21 & 1.6636565 & -4.0277290 & 1.4065496 \\
\hline $44 \mathrm{~F} \mathrm{F1}$ & -3.4379221 & -0.6675959 & 1.9789275 \\
\hline 45 F F2 & -3.2341444 & -2.6701329 & 2.8116355 \\
\hline 46 F F3 & -3.3103570 & -2.3975544 & 0.6612719 \\
\hline 47 F F4 & 2.2021271 & -4.0279014 & 0.1506788 \\
\hline 48 F F5 & 0.9720086 & -5.1765982 & 1.5310462 \\
\hline 49 F F6 & 2.7028943 & -4.0758489 & 2.2624221 \\
\hline 50 F F7 & -4.7381778 & 1.7499162 & 1.3069433 \\
\hline $51 \mathrm{~F} \mathrm{F8}$ & -5.8465881 & 1.4468122 & -0.5385132 \\
\hline 52 F F9 & -4.8560965 & 3.3509353 & -0.1561754 \\
\hline 53 F F10 & -3.3293734 & -2.3455705 & -2.2770265 \\
\hline 54 F F11 & -1.6167016 & -1.7291299 & -3.4757367 \\
\hline 12 & -1.3143128 & -2.4466695 & -1.4544742 \\
\hline
\end{tabular}




$\begin{array}{llll}56 \text { C C27 } & 3.2831624 & -2.6893528 & -2.7877261 \\ \text { 57 C C26 } & 4.2042838 & -1.8989580 & -1.8562757 \\ 58 \text { C C23 } & 1.8607816 & -2.1796104 & -2.6467576 \\ 59 \text { H H1 } & 1.4433504 & -2.4372747 & -1.6707839 \\ 60 \text { O O3 } & 1.7549042 & -0.7255085 & -2.7677807 \\ 61 \text { C C24 } & 4.1439872 & -0.4106729 & -2.2166165 \\ 62 \text { H H2 } & 4.5853662 & 0.2219220 & -1.4415431 \\ 63 \text { C C25 } & 2.7476791 & 0.1196308 & -2.4682591 \\ 64 \text { O O4 } & 2.4921707 & 1.3173809 & -2.4670203 \\ 65 \text { H H19 } & 4.7032344 & -0.2167170 & -3.1438153 \\ 66 \text { H H24 } & 1.1890437 & -2.5577159 & -3.4195913 \\ 67 \text { H H25 } & 3.8858802 & -2.0439632 & -0.8171413 \\ 68 \text { H H27 } & 5.2374791 & -2.2545185 & -1.9257839 \\ 69 \text { H H29 } & 3.6188117 & -2.5973989 & -3.8292343 \\ \text { 70 H H30 } & 3.2857759 & -3.7555044 & -2.5347410\end{array}$

\section{$\underline{\text { 3-S vacuum }}$}

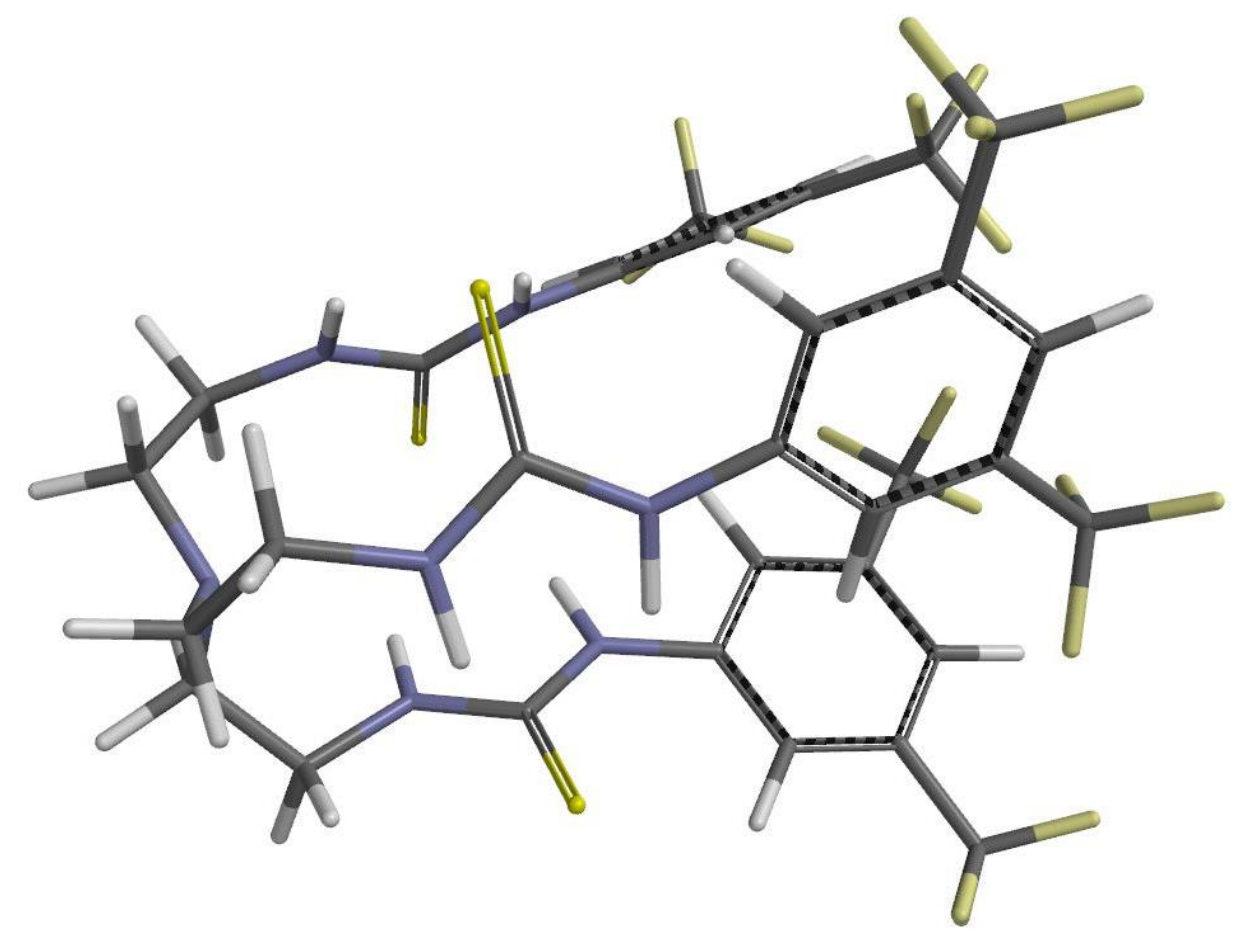

Job type: Single point.

Method: RB3LYP

Basis set: $6-31 G^{* *}$

Number of shells: 328

Number of basis functions: 1062

Multiplicity: 1

SCF total energy: $\quad-4648.8994977$ hartrees

\begin{tabular}{cccc} 
& \multicolumn{3}{c}{ Cartesian Coordinates (Angstroms) } \\
Atom & $X$ & $Y$ & Z \\
- & -
\end{tabular}




$\begin{array}{lccc}\text { 1 C C2 } & 2.7640909 & 1.5991396 & -1.8183489 \\ \text { 2 S S1 } & 2.7485580 & 3.1262167 & -1.0447103 \\ \text { 3 N N1 } & 3.9056042 & 0.9614619 & -2.1515710 \\ \text { 4 H H5 } & 3.8171067 & 0.0156768 & -2.5219318 \\ \text { 5 N N2 } & 1.6401143 & 0.8812915 & -2.1324001 \\ \text { 6 H H6 } & 1.7976801 & -0.1118807 & -2.3241979 \\ \text { 7 C C3 } & 0.3013170 & 1.2854719 & -2.3201684 \\ \text { 8 C C4 } & -2.3985949 & 1.8898648 & -2.8274305 \\ \text { 9 C C5 } & -0.6568049 & 0.2633989 & -2.3905650 \\ \text { 10 C C6 } & -0.0979427 & 2.6145578 & -2.5214042 \\ \text { 11 C C7 } & -1.4411826 & 2.8999161 & -2.7585829 \\ \text { 12 C C8 } & -1.9912618 & 0.5700573 & -2.6483517 \\ \text { 13 H H7 } & -0.3550712 & -0.7708386 & -2.2578626 \\ \text { 14 H H8 } & 0.6245689 & 3.4167849 & -2.4885809 \\ \text { 15 H H10 } & -3.4400894 & 2.1260593 & -3.0079652 \\ \text { 16 C C10 } & 5.2607492 & 1.4663694 & -1.9949113 \\ \text { 17 H H11 } & 5.2004357 & 2.3893175 & -1.4161725 \\ \text { 18 H H14 } & 5.6613762 & 1.7333310 & -2.9831061 \\ \text { 19 C C12 } & 5.2614840 & 1.0846459 & 2.2224675 \\ \text { 20 H H15 } & 5.6552614 & 1.8030174 & 2.9553352 \\ \text { 21 H H18 } & 5.2238331 & 0.1128850 & 2.7169096 \\ \text { 22 N N3 } & 3.8972486 & 1.4512082 & 1.8763081 \\ \text { 23 H H20 } & 3.7898009 & 2.2354648 & 1.2335711 \\ \text { 24 C C13 } & 2.7688079 & 0.8382435 & 2.2910562 \\ \text { 25 N N4 } & 1.6323822 & 1.4610792 & 1.8454412 \\ \text { 26 H H22 } & 1.7761138 & 2.1258750 & 1.0805754 \\ \text { 27 C C14 } & 0.2952149 & 1.3955875 & 2.2898815 \\ \text { 28 C C15 } & -2.4076716 & 1.4772893 & 3.0662460 \\ \text { 29 C C16 } & -0.1001634 & 0.8644173 & 3.5247431 \\ \text { 30 C C17 } & -0.6697046 & 1.9795466 & 1.4542447 \\ \text { 31 C C18 } & -2.0048731 & 2.0203529 & 1.8476473 \\ \text { 32 C C19 } & -1.4452920 & 0.9000425 & 3.8901120 \\ \text { 33 H H21 } & 0.6266397 & 0.4189908 & 4.1874706 \\ \text { 34 H H23 } & -0.3707644 & 2.4090036 & 0.5031227 \\ \text { 35 H H26 } & -3.4498786 & 1.4932500 & 3.3590253 \\ \text { 50 F F8 } & -2.0241180 & 4.5658245 & -4.3285172 \\ \text { 51 F F9 } & -0.9633317 & 5.2130042 & -2.5444791 \\ \text { 52 F F10 } & -4.0540158 & -0.2298086 & -3.4835079 \\ \text { 53 F F11 } & -2.4869431 & -1.6987577 & -3.1065017\end{array}$




\begin{tabular}{lccc} 
54 F F12 & -3.5312931 & -0.7659805 & -1.4445504 \\
55 H H2 & 7.2278878 & 0.8305944 & -1.4034350 \\
56 N N5 & 5.8050047 & 0.0710587 & 0.0077225 \\
57 C C1 & 5.3031898 & -2.3644845 & -0.2073505 \\
58 H H12 & 5.7187878 & -3.3500893 & 0.0462233 \\
59 N N6 & 3.9444816 & -2.2719799 & 0.3039059 \\
60 H H13 & 3.8481195 & -2.1103761 & 1.3062523 \\
61 C C23 & 2.8079859 & -2.3397588 & -0.4200681 \\
62 N N7 & 1.6771503 & -2.2902165 & 0.3516485 \\
63 H H16 & 1.8200060 & -1.9728928 & 1.3139804 \\
64 C C24 & 0.3476586 & -2.6736662 & 0.0707355 \\
65 C C25 & -2.3355466 & -3.4584339 & -0.2512243 \\
66 C C26 & -0.0128164 & -3.5441791 & -0.9645950 \\
67 C C27 & -0.6411242 & -2.2147546 & 0.9557636 \\
68 C C28 & -1.9660771 & -2.6107041 & 0.7933034 \\
69 C C29 & -1.3494205 & -3.9144412 & -1.1196988 \\
70 H H17 & 0.7365180 & -3.9304652 & -1.6412965 \\
71 H H19 & -0.3679088 & -1.5593844 & 1.7769814 \\
72 H H24 & -3.3673969 & -3.7561716 & -0.3826470 \\
73 S S3 & 2.8039171 & -2.4388198 & -2.1286046 \\
74 C C30 & -3.0225496 & -2.0715427 & 1.7241143 \\
75 C C31 & -1.6964523 & -4.8350935 & -2.2620423 \\
76 F F13 & -3.4712995 & -0.8605647 & 1.3225534 \\
77 F F14 & -2.5472708 & -1.9197359 & 2.9816806 \\
78 F F15 & -4.0938615 & -2.8882522 & 1.7904528 \\
79 F F16 & -3.0010486 & -5.1748762 & -2.2628214 \\
80 F F17 & -0.9777024 & -5.9799826 & -2.2020364 \\
81 F F18 & -1.4211603 & -4.2665814 & -3.4560206 \\
82 H H25 & 5.2463778 & -2.3130804 & -1.2958413 \\
83 C C32 & 6.2160596 & -1.2822943 & 0.3756853 \\
84 H H9 & 7.2550846 & -1.5046887 & 0.0680968 \\
85 H H27 & 6.2005151 & -1.3631277 & 1.4678909 \\
86 C C33 & 6.1849094 & 1.0733869 & 1.0009875 \\
87 H H1 & 6.1513581 & 2.0605765 & 0.5272716 \\
88 H H33 & 7.2243617 & 0.9408821 & 1.3561298 \\
& & & \\
\hline
\end{tabular}

\section{$\underline{\text { 3-O in vacuum }}$}




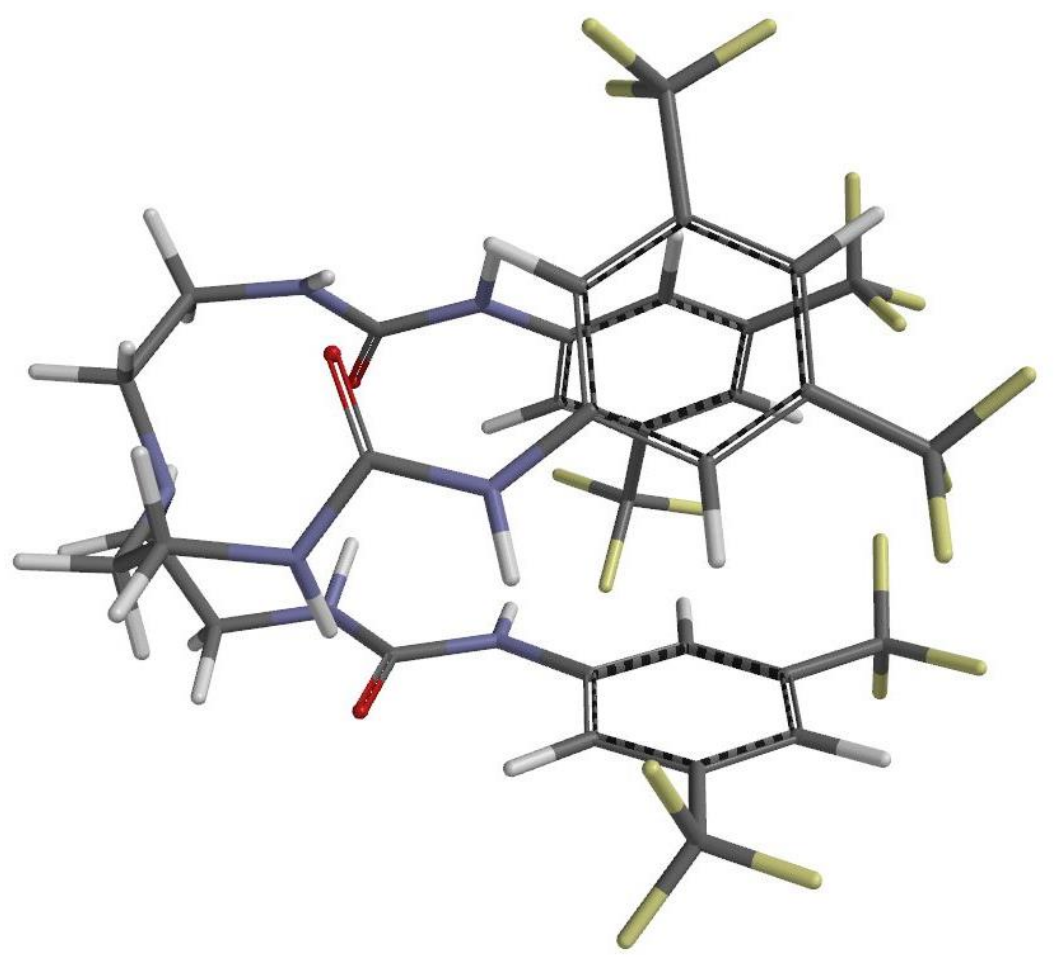

Job type: Single point.

Method: RB3LYP

Basis set: $6-31 G^{* *}$

Number of shells: 325

Number of basis functions: 1050

Multiplicity: 1

SCF total energy: $-\mathbf{3 6 8 0 . 0 5 6 2 3 1 1}$ hartrees

\begin{tabular}{lrcc}
\multicolumn{5}{c}{ Cartesian Coordinates (Angstroms) } \\
Atom & X & Y & Z \\
------------ \\
1 C C2 & -2.8191769 & -1.4459723 & 1.9139004 \\
2 N N1 & -3.6837952 & -2.1887576 & 1.1546312 \\
3 H H5 & -3.3678728 & -2.4709288 & 0.2289292 \\
4 N N2 & -1.4891232 & -1.8088459 & 1.7201386 \\
5 H H6 & -1.3189721 & -2.6347168 & 1.1595125 \\
6 C C3 & -0.3503171 & -1.2225691 & 2.2807562 \\
7 C C4 & 2.0493416 & -0.0862620 & 3.2500984 \\
8 C C5 & 0.8981553 & -1.7653417 & 1.9324703 \\
9 C C6 & -0.3880865 & -0.1375919 & 3.1660749 \\
10 C C7 & 0.8077738 & 0.4196093 & 3.6248772 \\
11 C C8 & 2.0751581 & -1.2017548 & 2.4093743 \\
12 H H7 & 0.9430649 & -2.6421333 & 1.2986243 \\
13 H H8 & -1.3417631 & 0.2382549 & 3.5069152 \\
14 H H10 & 2.9675834 & 0.3607257 & 3.6085817 \\
15 C C10 & -5.1238809 & -2.0446915 & 1.3102056 \\
16 H H11 & -5.2929735 & -1.5776720 & 2.2812241
\end{tabular}




$\begin{array}{lccc}\text { 17 H H14 } & -5.5727231 & -3.0456003 & 1.3512156 \\ \text { 18 C C12 } & -5.0034839 & 2.3026999 & 1.1529605 \\ \text { 19 H H15 } & -5.4141876 & 2.8510338 & 2.0107121 \\ \text { 20 H H18 } & -5.1638710 & 2.9229996 & 0.2706689 \\ \text { 21 N N3 } & -3.5626078 & 2.1839246 & 1.3289192 \\ \text { 22 H H20 } & -3.2552643 & 1.5176639 & 2.0341170 \\ \text { 23 C C13 } & -2.7075480 & 2.4561068 & 0.2947921 \\ \text { 24 N N4 } & -1.3695918 & 2.4426817 & 0.6796475 \\ \text { 25 H H22 } & -1.1808298 & 2.3856234 & 1.6730415 \\ \text { 26 C C14 } & -0.2468647 & 2.6493585 & -0.1300865 \\ \text { 27 C C15 } & 2.1192833 & 2.9594669 & -1.6481134 \\ \text { 28 C C16 } & -0.3128602 & 2.8382121 & -1.5177245 \\ \text { 29 C C17 } & 1.0130801 & 2.6622575 & 0.4901460 \\ \text { 30 C C18 } & 2.1732307 & 2.8145091 & -0.2614320 \\ \text { 31 C C19 } & 0.8652741 & 2.9766362 & -2.2530596 \\ \text { 32 H H21 } & -1.2765799 & 2.9083830 & -1.9996946 \\ \text { 33 H H23 } & 1.0841378 & 2.5679058 & 1.5672095 \\ \text { 34 H H26 } & 3.0246836 & 3.0602805 & -2.2319525 \\ \text { 35 C C9 } & -5.8077784 & -1.2796189 & 0.1690927 \\ \text { 36 H H66 } & -5.6264522 & -1.8391170 & -0.7522703 \\ \text { 37 C C11 } & 0.7017128 & 1.6374689 & 4.4992039 \\ \text { 38 C C20 } & 3.3929018 & -1.7795935 & 1.9584829 \\ \text { 39 C C21 } & 3.4964690 & 2.7661755 & 0.4591288 \\ \text { 40 C C22 } & 0.7286742 & 3.0647507 & -3.7472031 \\ \text { 41 F F1 } & 3.7883104 & 1.5106720 & 0.8698746 \\ \text { 42 F F2 } & 3.4783570 & 3.5405298 & 1.5685110 \\ \text { 43 F F3 } & 4.5149046 & 3.1852040 & -0.3163414 \\ \text { 44 F F4 } & -0.1933562 & 3.9728184 & -4.1229471 \\ \text { 45 F F5 } & 0.3041618 & 1.8626279 & -4.2545865 \\ \text { 46 F F6 } & 1.8827161 & 3.3625955 & -4.3652558 \\ \text { 47 F F7 } & 1.8768286 & 2.0056603 & 5.0334528 \\ \text { 48 F F8 } & -0.1816778 & 1.4698727 & 5.5017425 \\ \text { 49 F F9 } & 0.2438951 & 2.7088936 & 3.7708375 \\ \text { 50 F F10 } & 4.3632951 & -1.5832125 & 2.8730045 \\ \text { 51 F F11 } & 3.3009087 & -3.1113309 & 1.7348205 \\ \text { 52 F F12 } & 3.8139235 & -1.2205603 & 0.8016466 \\ \text { 53 H H2 } & -6.9020118 & -1.3084428 & 0.3481495 \\ \text { 54 N N5 } & -5.3242305 & 0.0878459 & -0.0254913 \\ \text { 55 C C1 } & -4.9805141 & -0.0027500 & -2.5265910 \\ \text { 66 C C28 } & 2.1546345 & -1.6471006 & -2.0585374 \\ \text { 67 C C29 } & 0.7775112 & -3.4885720 & -1.4103902 \\ \text { 68 H H17 } & -1.3550647 & -3.2014190 & -1.5908365 \\ \text { 69 H H19 } & 1.1224511 & 0.1238865 & -2.6959210\end{array}$




$\begin{array}{lccc}\text { 70 H H24 } & 2.9366803 & -3.5290567 & -1.3426359 \\ \text { 71 C C30 } & 3.5015073 & -1.0197297 & -2.3155295 \\ \text { 72 C C31 } & 0.5869700 & -4.8638394 & -0.8351782 \\ \text { 73 F F13 } & 3.6211695 & 0.1677586 & -1.6766378 \\ \text { 74 F F14 } & 3.6770187 & -0.7671852 & -3.6332394 \\ \text { 75 F F15 } & 4.5179740 & -1.8043213 & -1.9139979 \\ \text { 76 F F16 } & 1.7373992 & -5.5432264 & -0.7016732 \\ \text { 77 F F17 } & -0.2571635 & -5.6138996 & -1.5707294 \\ \text { 78 F F18 } & 0.0265986 & -4.7772247 & 0.4138683 \\ \text { 79 H H25 } & -5.1789666 & -1.0708885 & -2.6262966 \\ \text { 80 C C32 } & -5.7176408 & 0.6174481 & -1.3316820 \\ \text { 81 H H9 } & -6.8064816 & 0.5068331 & -1.5117772 \\ \text { 82 H H27 } & -5.5059394 & 1.6890298 & -1.3407401 \\ \text { 83 C C33 } & -5.7494111 & 0.9645796 & 1.0671436 \\ \text { 84 H H1 } & -5.5772680 & 0.4377785 & 2.0084475 \\ \text { 85 H H33 } & -6.8350147 & 1.1876081 & 1.0191279 \\ \text { 86 O O1 } & -3.1162551 & -2.0179532 & -1.8707390 \\ \text { 87 0 O2 } & -3.1675940 & -0.5810756 & 2.7256196 \\ \text { 88 0 O3 } & -3.0718983 & 2.7369773 & -0.8530852\end{array}$




\author{
MANUSCRIPT - IV \\ Published in European Polymer Journal
}

\title{
Coupled Equilibria in H-Bond Donating Ring-Opening Polymerization: The Effective Catalyst-Determined Shift of a Polymerization Equilibrium
}

Partha P. Datta, Jinal U. Pothupitiya, Elizabeth T. Kiesewetter and Matthew K. Kiesewetter Chemistry, University of Rhode Island, Kingston, RI, USA

Corresponding Author: Matthew Kiesewetter, Ph.D.

Chemistry

University of Rhode Island

140 Flagg Road

Kingston, RI, 02881, USA

Email address: mkiesewetter@chm.uri.edu 


\begin{abstract}
In the classic view of catalysis, a catalyst cannot alter the thermodynamicallydetermined endpoint of a reversible reaction. This conclusion is predicated on the assumption that the catalyst does not perturb the energy of product or reactant or does so to an equal extent. In the $\mathrm{H}$-bond mediated ring-opening polymerization (ROP) of lactone monomers, the strength of the interactions of thiourea with product and reactant are not equal, and the magnitudes of these interactions are of similar energy to the free energy of reaction. The total monomer concentration at equilibrium in the thiourea/base cocatalyzed ROP of lactones is shown to be a function of the initial concentration of thiourea. Because the binding of thiourea to monomer and the polymerization reaction itself are both reversible, the application of varying amounts of thiourea catalyst directly alters the total amount of monomer in the reaction solution at equilibrium, which can be recovered at the end of the reaction.
\end{abstract}




\section{INTRODUCTION}

The class of H-bond mediated catalysts for ROP, commonly a thiourea H-bond donor and one of a host of $\mathrm{H}$-bond accepting base cocatalysts, rank among the most highly controlled polymerization techniques. ${ }^{1-3}$ Catalysts of this class have been applied for the synthesis of well-defined and highly functionalized materials. ${ }^{4-6}$ The recent development of rapid catalysts for $\mathrm{H}$-bond mediated ROP promises to extend the utility of these systems, ${ }^{3,7-10}$ yet our understanding of the modes of action of these catalysts remains incomplete. Catalyst systems consisting of thiourea/base are believed to be operative via the H-bond activation of lactone monomer by thiourea and of initiating/propagating chain end by base (e.g. DBU in Figure 4.1). ${ }^{11-13}$ This mechanism is corroborated by ${ }^{1} \mathrm{H}$ NMR titration studies whereby lactones can be shown to H-bond to thiourea 1 (Figure 4.1), and base is observed to H-bond to benzyl alcohol, Eqs. (1) and (2). ${ }^{13}$ Presumably, these ground state interactions persist in the transition state, giving rise to catalysis and allowing the ROP to reach equilibrium. The high selectivity exhibited by $\mathbf{1}$ for polymerization vs transesterification is thought to arise from the selective binding of thiourea to monomer (scis ester) vs polymer ( $\mathrm{s}$-trans esters); the binding of $\mathbf{1}$ to ethyl acetate (an s-trans ester) is too small to be measured by ${ }^{1} \mathrm{H}$ NMR titration. ${ }^{13}$ In the ROP of $\delta$-valerolactone (VL), the free energy of binding of 1 to $\mathrm{VL}\left(\mathrm{K}_{\mathrm{eq}}=39 \text {, or } \Delta \mathrm{G}^{\mathrm{o}}=-2.2 \mathrm{kcal} / \mathrm{mol}, 300 \mathrm{~K}\right)^{13}$ is larger than the free energy of ROP, $\Delta \mathrm{G}^{\mathrm{o}}=-1.05$ to $-1.44 \mathrm{kcal} / \mathrm{mol} .{ }^{14}$ This relatively stronger binding of $\mathbf{1}$ to monomer vs ethyl acetate (polymer) effects a change in the relative energy of the reactant and product in the ROP reaction, producing an apparent change in the ROP equilibrium by the thiourea catalyst, 1 . 


\section{EXPERIMENTAL SECTION}

\section{General Considerations}

All the polymerizations were conducted in an MBRAUN stainless steel glovebox with gas purification system under a nitrogen atmosphere. All chemicals were purchased from Fisher Scientific and used as received unless stated otherwise. All glassware and stir bars were flame dried under nitrogen or baked at $140^{\circ} \mathrm{C}$ overnight prior to the introduction of reagents. Benzene- $\mathrm{d}_{6}$ was purchased from Cambridge Isotope Laboratories and distilled from $\mathrm{CaH}_{2}$ under nitrogen atmosphere. $\delta$-Valerolactone (VL; 99\%) and $\varepsilon$-caprolactone (CL) were distilled from $\mathrm{CaH}_{2}$ under high vacuum. THF was purified on an Innovative Technologies solvent purification system. Benzyl alcohol was distilled from $\mathrm{CaH}_{2}$ under high vacuum. 1-(3,5-bis(trifluoromethyl)phenyl)-3-cyclohexylthiourea (1) was synthesized and purified according to literature procedure. ${ }^{15} 1,8$ Diazabicyclo[5.4.0] undec-7-ene (DBU) was purchased from TCI and used as received. NMR experiments were performed on a Bruker Avance $300 \mathrm{MHz}$ or $400 \mathrm{MHz}$ spectrometer. Mass spectrometry data was collected using a Thermo Electron (San Jose, CA, USA) LTQ Orbitrap XL Mass Spectrometer coupled with either an electrospray ionization (ESI) or an atmospheric-pressure chemical ionization (APCI) interface, yielding positive ions which were subsequently introduced into the instrument. For the infusion experiments, the tune conditions $(10 \mu \mathrm{L} / \mathrm{min}$ flow, sample concentration $<20 \mu \mathrm{g} / \mathrm{mL}$ in $50 / 50 \mathrm{v} / \mathrm{v}$ water/acetonitrile) were: ionspray voltage, $5000 \mathrm{~V}$; capillary temperature $275^{\circ} \mathrm{C}$; sheath gas $\left(\mathrm{N}_{2}\right.$, arbitrary units), 8 ; auxiliary gas $\left(\mathrm{N}_{2}\right.$, arbitrary units), 0 ; capillary voltage, $35 \mathrm{~V}$; and tube lens, $110 \mathrm{~V}$. The instrument was calibrated for positive ions using Pierce 
LTQ ESI positive ion calibration solution (Lot \# PC197784) before any analysis. For the ion trap experiments, $\mathrm{N}_{2}$ was used as a collision gas with normalized collision energies (NCE) between 10-25 eV for multistage fragmentation. Performance of high-energy collision (HCD) experiments were conducted with He as collision gas with NCE of $25 \mathrm{eV}$. Synthesis of 2

A dried $100 \mathrm{~mL}$ Schlenk flask was charged with a stir bar under nitrogen. Dry tetrahydrofuran $(25 \mathrm{~mL})$, 2-methoxyethylamine (13 mmol, $1.2 \mathrm{~mL})$ and 3,5bis(trifluoromethyl)phenyl isothiocyanate $(13 \mathrm{mmol}, 2.5 \mathrm{~mL})$ were added via syringe. The solution was stirred for 24 hours and subsequently removed of solvent under reduced pressure. The resulting solid product was purified via a silica gel column with $1 \%$ methanol in dichloromethane. Yield: $1.37 \mathrm{~g}, 60 \%$. HRMS m/z calcd $\left(\mathrm{C}_{12} \mathrm{H}_{12} \mathrm{~F}_{6} \mathrm{~N}_{2} \mathrm{OS}+\mathrm{H}^{+}\right) 347.0647$, found 347.0648; NMR spectra below.

\section{Example Ring-Opening Polymerization}

In a typical polymerization, VL $(0.100 \mathrm{~g}, 0.999 \mathrm{mmol})$ was added to a $7 \mathrm{~mL}$ scintillation vial containing a stir bar. In another $7 \mathrm{~mL}$ scintillation vial with stir bar, $\mathbf{1}$ (0.0185 g, $0.0499 \mathrm{mmol})$, DBU $(7.47 \mu \mathrm{L}, 0.0499 \mathrm{mmol})$, and benzyl alcohol $(9.99 \mu \mathrm{mol})$ were added. $\mathrm{C}_{6} \mathrm{D}_{6}(0.4744 \mathrm{~g}, 0.499 \mathrm{~mL})$ was divided equally between the vials. After stirring for $2 \mathrm{~min}$, the VL solution was transferred via pipette to the vial containing catalysts and initiator. The entire solution was then moved to an NMR tube via pipette. Reaction progress was monitored by ${ }^{1} \mathrm{H}$ NMR.

Depolymerization Procedure

In air, $1(0.0370 \mathrm{~g}, 0.199 \mathrm{mmol})$ was added to the NMR tube containing the reaction solution. The NMR tube was capped and shaken until the solution was homogeneous. 
Reaction progress was monitored by ${ }^{1} \mathrm{H}$ NMR spectra. This process was repeated with a second addition of $\mathbf{1}(0.0462 \mathrm{~g}, 0.249 \mathrm{mmol})$.

Monomer Isolation

A typical polymerization reaction was carried out as described above, conditions: VL (202 mg, $2.0175 \mathrm{mmol}), 1$ (37.0 mg, $0.10 \mathrm{mmol})$, DBU (15.2 mg, $0.10 \mathrm{mmol})$, benzyl alcohol $(2.2 \mathrm{mg}, 0.020 \mathrm{~mol})$ and $\mathrm{C}_{6} \mathrm{D}_{6}(949 \mathrm{mg}, 999 \mu \mathrm{L})$. The reaction was stirred overnight, and conversion determined via ${ }^{1} \mathrm{H}$ NMR, 94\%. Then, 2 (345.6 mg, $\left.1.0 \mathrm{mmol}\right)$ was added to the reaction solution. Ten hours after the addition of $\mathbf{2}$, the reaction was quenched with benzoic acid $(14.6 \mathrm{mg}, 0.120 \mathrm{mmol})$, and analyzed via ${ }^{1} \mathrm{H}$ NMR to determine VL conversion to polymer, $81 \%$. The reaction contents were transferred to a dry $25 \mathrm{~mL}$ round bottom flask. The flask was removed of volatiles via rotary evaporation, maintaining the water bath at room temperature. The monomer was isolated via Kugelrohr distillation: high vacuum (25-30 mTorr) for 2 hours at room temperature, 2 hours at $40^{\circ} \mathrm{C}$ with the receiving flask cooled to $-78^{\circ} \mathrm{C}$. Characterization matches commercially available material, Yield: $29.1 \mathrm{mg} ; 77 \%$.

Binding study of Benzyl alcohol (BnOH) to 1,8-Diazabicyclo[5.4.0]undec-7-ene (DBU) by titration method

Stock solutions of DBU and benzyl alcohol were prepared in $\mathrm{C}_{6} \mathrm{D}_{6}$. In NMR tubes, varying amounts of each stock solution and neat $\mathrm{C}_{6} \mathrm{D}_{6}$ were added to each tube such that the total volume was $500 \mu \mathrm{L}$. The concentration of benzyl alcohol was kept constant at 1 $\mathrm{mM}$ and DBU was varied from 0 to $150 \mathrm{mM}$. A ${ }^{1} \mathrm{H}$ NMR spectrum of each tube was acquired at $300 \mathrm{~K}$, and the chemical shift of the methylene proton of the $\mathrm{BnOH}\left(-\mathrm{CH}_{2}-\right)$ was monitored, referencing each spectrum to residual benzene- $\mathrm{H}$. The binding constant 
between $\mathrm{BnOH}$ and DBU was then obtained using the curve fitting method, ${ }^{16-18}$ which matched the value determined from the Lineweaver-Burke method; ${ }^{19,20} 75 \pm 3$, binding curve below.

Dependence of $[\mathrm{VL}]_{\mathrm{eq}}$ upon temperature

VL $(0.100 \mathrm{~g}, 0.999 \mathrm{mmol})$ was added to a $7 \mathrm{~mL}$ scintillation vial containing a stir bar. A second $7 \mathrm{~mL}$ scintillation vial with stir bar was charged with: 1 (0.0185 g, 0.0499 mmol), DBU (7.47 $\mu \mathrm{L}, 0.0499 \mathrm{mmol})$, and benzyl alcohol $(9.99 \mu \mathrm{mol}) . \mathrm{C}_{6} \mathrm{D}_{6}(0.4744 \mathrm{~g}$, $0.499 \mathrm{~mL}$ ) was evenly divided between the vials. After stirring for $2 \mathrm{~min}$, the VL solution was transferred via pipette to the vial containing catalysts and stirred to mix. The solution was transferred into an NMR tube via pipette. Reaction equilibrium was monitored vs temperature by variable temperature ${ }^{1} \mathrm{H}$ NMR. Data were acquired upon heating and cooling to confirm measurement. Heating/cooling data are within error, and the heating data is shown in Figure 4.12. 


\section{RESULTS AND DISCUSSION}

The total concentration of monomer remaining at equilibrium in the 1/DBU catalyzed ROP of VL from benzyl alcohol in $\mathrm{C}_{6} \mathrm{D}_{6}$ is a function of the initial concentration of $\mathbf{1}$. The progress of these ROPs was monitored by ${ }^{1} \mathrm{H}$ NMR until reaction progress halted, and the total monomer concentration at equilibrium ([VL $\left.]_{\mathrm{T}, \mathrm{q}}\right)$ was noted, Figure 4.2. Because 1/VL binding is rapid and reversible, only $[\mathrm{VL}]_{\mathrm{T}}$ is measurable by ${ }^{1} \mathrm{H} N M R\left([\mathrm{VL}]_{\mathrm{T}}\right.$ $=[\mathrm{VL}]+[\mathrm{VL} \cdot \mathbf{1}])$. The $[\mathrm{VL}]_{\mathrm{T}, \text { eq }}$ is altered when $[\mathbf{1}]_{\mathrm{o}}$ is varied in excess of the cocatalyst $[D B U]_{\mathrm{o}}$. This latter observation is consistent with the previously observed prominent binding between cocatalysts, $\mathrm{K}_{\mathrm{eq}}=4200$ for Eq. $(4.3)(300 \mathrm{~K}) .{ }^{21}$ This strong binding suggests that $\mathbf{1}$ will primarily be associated with DBU until $[\mathbf{1}]_{\mathrm{o}}=[\mathrm{DBU}]_{\mathrm{o}}$, and any $\mathbf{1}$ in excess of $[\mathrm{DBU}]_{0}$ will be available to bind to monomer and is the effective concentration of $\mathbf{1}\left([\mathbf{1}]_{\mathrm{EFF}}=[\mathbf{1}]_{\mathrm{o}}-[\mathrm{DBU}]_{\mathrm{o}}\right)$. The $[\mathrm{VL}]_{\mathrm{T}, \mathrm{eq}}$ increases linearly with increasing $[\mathbf{1}]_{\mathrm{EFF}}$, Figure 4.2. Reactions were controlled for temperature ( $300 \mathrm{~K}$, unless stated otherwise), pressure, concentration of reagents, and [VL]T was monitored vs an internal standard $\left(\mathrm{C}_{6} \mathrm{H}_{6}\right)$. The solution volumes do not measurably change during the polymerization, see Figure 4.9. The observed variation in $[\mathrm{VL}]_{\mathrm{T}, \mathrm{eq}}$ cannot be due to minor temperature variations within the NMR probe; the temperature dependent change in $[\mathrm{VL}]_{\mathrm{eq}}$ does not vary to the observed extremes over narrow temperature windows, (see Figure 4.12). The 1/DBU catalyzed ROP has previously been shown to display first order evolution of [VL] vs time, a linear evolution of $M_{n}$ vs conversion and predictable $M_{n}$ (by $[M]_{0} /\left[[]_{0}\right.$ ), characteristics of a 'living' polymerization. ${ }^{1,13}$ Increasing the concentration of the cocatalysts together alters $[\mathrm{VL}]_{\mathrm{T}, \mathrm{eq}}$ to a lesser extent than increasing $[\mathbf{1}]_{\mathrm{EFF}}$, see Figure 4.11. 
In the envisaged scheme, the thiourea is explicitly added to the polymerization equilibrium by showing a reversible binding of lactone (M) to 1 (Eq. (4.4)) which competes with the enchainment of the monomer in the normal ROP equilibrium (Eq. (4.5)). Eq. (4.4) is microscopic reverse of the normal binding equilibrium between $\mathrm{M}$ and $\mathbf{1}$. The polymer chain is shown in Eq. 4.4 and 4.1 in Eq. (4.5) for mass balance in the total process, and just like the normal ROP equilibrium expression, the concentration of polymer (=[initiator $]_{0}$ ) is thermodynamically irrelevant so long as $\left[\mathrm{M}_{n}{ }^{*}\right]=\left[\mathrm{M}_{\mathrm{n}}+\mathbf{1}^{*}\right]{ }^{14}$ This scheme describes the roles of thiourea in ROP as being analogous to both inhibitor and catalyst in enzyme kinetics.

The effect of $\mathbf{1}$ upon an ROP equilibrium can be quantified by considering the known equilibria between a lactone ( $\mathrm{M}$ in Eqs. (4.4) and (4.5)), polymer chain and $\mathbf{1}$. The equilibrium expression for the total reaction is given in Eq. (4.6). The substitution of the thiourea mass balance Eq. (4.7) into Eq. (4.6) followed by rearranging gives Eq. (4.8) (assuming $\left[\mathrm{M}_{\mathrm{n}}{ }^{*}\right]=\left[\mathrm{M}_{\mathrm{n}}+\mathbf{1}^{*}\right]$, see full derivation below), which takes a linear form and describes the influence of $[\mathbf{1}]_{\mathrm{EFF}}$ upon $[\mathrm{M}]_{\mathrm{T}, \mathrm{eq}}$. In the $\mathbf{1}$ mediated ROP of VL as described by Eq. (4.8) $(\mathrm{M}=\mathrm{VL})$, the total amount of monomer remaining at thermodynamic equilibrium $\left([\mathrm{M}]_{\mathrm{T}, \mathrm{eq}}\right)$ is perturbed from the nominal ROP equilibrium $\left([\mathrm{M}]_{\mathrm{eq}}\right.$, the intercept of Eq. (4.8)) to an extent that is directly proportional to the effective concentration of $\mathbf{1}$ $\left([1]_{\mathrm{EFF}}\right)$. As a check on the validity of this analysis, $[\mathrm{VL}]_{\mathrm{eq}}$ can be determined from the $\mathrm{y}-$ intercept in Figure 4.2: $[\mathrm{VL}]_{\mathrm{eq}}=0.052 \mathrm{M}$. This value of $[\mathrm{VL}]_{\mathrm{eq}}$ is consistent with previous reports, ${ }^{14}$ and it is the inverse of the equilibrium constant for the ROP reaction, $\mathrm{K}_{\text {eq } 5}=$ $1 /[\mathrm{VL}]_{\mathrm{eq}}=19.1\left(\Delta \mathrm{G}^{\circ}{ }_{5}=-1.76 \pm 0.30 \mathrm{kcal} / \mathrm{mol}, 300 \mathrm{~K}\right)$, which is not affected by the changing $[\mathbf{1}]_{\mathrm{EFF}}$. The equilibrium constant for the total reaction, $\mathrm{K}_{\mathrm{eqT}}$, is the enchainment 
equilibrium in the presence of $\mathbf{1}$, and it is determined from the slope from Figure 4.3: $\mathrm{K}_{\text {eqT }}$ $=0.57\left(\Delta \mathrm{G}^{\circ} \mathrm{T}=0.34 \pm 0.01 \mathrm{kcal} / \mathrm{mol}, 300 \mathrm{~K}\right)$. The difference between $\mathrm{K}_{\text {eq } 5}$ and $\mathrm{KT}, \Delta \Delta \mathrm{G}^{\circ}$ $=2.1 \pm 0.3 \mathrm{kcal} / \mathrm{mol}$, represents binding energy of the monomer to $\mathbf{1}\left(\mathbf{1} / \mathrm{K}_{\text {eq }}\right)$, and this is in agreement with the independently measured value, $\mathbf{1} / \mathrm{K}_{\mathrm{eq} 4}=39\left(\Delta \mathrm{G}^{\circ}{ }_{4}=2.2 \mathrm{kcal} / \mathrm{mol}, 300\right.$ $\mathrm{K}) \cdot{ }^{13}$

A change in the location of the M. 1 species on the reaction coordinate does not alter the conclusions, only the description, of the phenomenon being observed. In the energy surface described above, the non-ROP role of thiourea is akin to that of inhibitor in enzyme kinetics, where excess thiourea disfavors the formation of product (polymer). An equally valid and equivalent (see Thiourea as catalyst interpretation below) interpretation envisages the role of thiourea as purely catalyst, where the formation of $\mathrm{M} \cdot \mathbf{1}$ occurs in a step intermediate to free monomer/thiourea and polymer formation, Eqs. (4.9) and (4.10). The energy surfaces described by Eqs. (4.4) and (4.5) or Eq. (4.9) and (4.10) are very shallow, with the largest gap $\sim 2 \mathrm{kcal} / \mathrm{mol}$, hence the system has free movement between the entire surface at room temperature. Indeed, re-deriving an equation to describe the influence of $[1]_{\mathrm{EFF}}$ upon $[\mathrm{M}]_{\mathrm{eq}}$ (c.f. Eq. (4.8)) based on the thiourea as catalyst interpretation (Eqs. (4.9) and (4.10)) produces the same Eq. (4.8) describing the influence of [1 $]_{\text {EFF }}$ upon ROP equilibrium (see Thiourea as catalyst interpretation below). This latter scheme qualitatively describes the role of (thio)urea in ROP as a thermodynamic trap for monomer prior to the endergonic enchainment of 1-bound VL. This conceptual framework describes classic views of enzyme-substrate interactions and is consistent with the existence of a 'Goldilocks' H-bond donor featuring a monomer/thiourea binding constant that is 'just right'. ${ }^{22}$ Regardless, H-bond donors have the ability to alter ROP thermodynamics. We do 
not wish to suggest the application of large concentrations of H-bond donor in common practice, but rather seek to understand the observed effects so that improved catalysts for (de)polymerization might be generated.

An enzyme-induced 'equilibrium shift' has been observed for reactions taking place in enzymatic active sites versus those in free solution. ${ }^{23}$ To our knowledge, such an effect has not been so clearly and controllably observed in homogeneous catalysis outside enzymatic systems. Because classic Michaelis-Menten kinetics consider an irreversible reaction, discussions regarding the energetic implications of the binding of substrate to enzyme have been largely considered with respect to the ramifications of enzyme/substrate adducts upon catalysis. ${ }^{24,25}$ In the biomimetic H-bond mediated ROP of lactones, the catalytic step is reversible, and the binding of $\mathbf{1}$ to monomer impacts the reaction thermodynamics too.

The addition of $\mathbf{1}$ to an ROP at equilibrium results in the generation of more monomer due to depolymerization, Figure 4.4. The 1/DBU cocatalyzed ROP of VL from benzyl alcohol in $\mathrm{C}_{6} \mathrm{D}_{6}$ was monitored by ${ }^{1} \mathrm{H}$ NMR and was allowed to reach equilibrium at which point additional 1 was added to the NMR tube. The reaction progress was observed to reverse, establishing a new, increased, $[\mathrm{VL}]_{\mathrm{T}, \mathrm{eq}}$, Figure 4.4. This process was repeated by the addition of another aliquot of $\mathbf{1}$. The same effect is observed if the experiment is repeated on separately prepared and isolated polyvalerolactone. The addition of $\mathbf{1}$ to the reaction does alter the solution volume but not significantly so, see Figure 4.10. Elevated temperatures have previously been employed to favor depolymerization and monomer recovery, ${ }^{26,27}$ and organic catalysts have been applied for the depolymerization 
of poly(ethylene terephthalate) with excess nucleophile, ${ }^{28-30}$ but we believe the current process is distinct from these observations.

The equilibrium perturbation by thiourea upon lactone monomers is not limited to 1 and VL. A new thiourea cocatalyst was synthesized, 2 in Figure 4.4, that exhibited much greater solubility in $\mathrm{C}_{6} \mathrm{D}_{6}$ (versus $\mathbf{1}$ ). The application of progressively increased amounts of 2 to the ROP of VL at equilibrium allowed for the depolymerization of this reaction to $[\mathrm{VL}]_{\mathrm{T}, \mathrm{eq}}=0.98 \mathrm{M}$. This is greater than the solubility-limited depolymerization of PVL with 1, $[\mathrm{VL}]_{\mathrm{T}, \mathrm{eq} \max }=0.67 \mathrm{M}$. Analysis of the polymer over the course of the depolymerization experiment (see Figure 4.4) suggests a linear de-evolution of $M_{n}$, and $M_{w} / M_{n}$ remains narrow throughout the reaction. The precise effects of the depolymerization upon the polymer are the subject of future investigation. Quenching a partially reverted ROP allows for the isolation of the depolymerized monomer. The 1/DBU catalyzed ROP of VL ( $2 \mathrm{M}$, $202 \mathrm{mg}$ ) from benzyl alcohol was depolymerized to the extent possible by the application of 2. The monomer was recovered from the reaction mixture after quenching and Kugelrohr distillation, (29.1 mg; 77\% yield, see Experimental Section). The new thiourea, 2, was also applied to control the endpoint in the ROP of $\varepsilon$-caprolactone (CL), Figure 4.5. The reduced efficacy of 2 in perturbing $[\mathrm{M}]_{\mathrm{T}, \mathrm{eq}}$ in the ROP of CL vs $\mathrm{VL}$ may be attributed to the increased ring strain of $\mathrm{CL}$ vs VL (i.e. reduced $[\mathrm{CL}]_{\mathrm{eq}}$ vs $[\mathrm{VL}]_{\mathrm{eq}}$ under normal conditions). 


\section{CONCLUSION}

Nominally, the end point of an ROP is thermodynamically determined by a function of monomer ring strain and is described by the equilibrium monomer concentration $\left([\mathrm{M}]_{\mathrm{eq}}\right.$ $\left.=1 / \mathrm{K}_{\mathrm{eq}}\right)$. The addition of thiourea to the ROP of VL or CL does not change $[\mathrm{M}]_{\mathrm{eq}}$ from that of an ROP in the absence of H-bond donor, hence the classic definition of 'catalyst' applies to thioureas. However, the H-bond donating ability of thiourea favors the depolymerization reaction to provide a lactone binding partner to thiourea. The rapid and reversible binding of thiourea to VL allows for monomer isolation and the effective thiourea-determined shift of a chemical equilibrium. At the very least, the effects of thiourea upon ROP represent a cautionary tale of superimposed equilibria, but perhaps H-bond donors can be applied to drive thermodynamic control with tandem catalysis or be applied to the chemical recycling of polymers. 


\section{LIST OF REFERENCES}

(1) Kiesewetter, M. K.; Shin, E. J.; Hedrick, J. L.; Waymouth, R. M. Macromolecules 2010, 43 (5), 2093-2107.

(2) Kamber, N. E.; Jeong, W.; Waymouth, R. M.; Pratt, R. C.; Lohmeijer, B. G. G.; Hedrick, J. L. Chem. Rev. 2007, 107 (12).

(3) Guillaume, S. M.; Kirillov, E.; Sarazin, Y.; Carpentier, J.-F. Chem. - A Eur. J. 2015, 21 (22), 7988-8003.

(4) McKinlay, C. J.; Waymouth, R. M.; Wender, P. A. J. Am. Chem. Soc. 2016, 138 (10), 3510-3517.

(5) Geihe, E. I.; Cooley, C. B.; Simon, J. R.; Kiesewetter, M. K.; Edward, J. A.; Hickerson, R. P.; Kaspar, R. L.; Hedrick, J. L.; Waymouth, R. M.; Wender, P. A. Proc. Natl. Acad. Sci. 2012, 109 (33), 13171-13176.

(6) Cooley, C. B.; Trantow, B. M.; Nederberg, F.; Kiesewetter, M. K.; Hedrick, J. L.; Waymouth, R. M.; Wender, P. A. J. Am. Chem. Soc. 2009, 131 (45), 1640116403.

(7) Zhang, X.; Jones, G. O.; Hedrick, J. L.; Waymouth, R. M. Nat Chem 2016, 8 (11), $1047-1053$.

(8) Fastnacht, K. V.; Spink, S. S.; Dharmaratne, N. U.; Pothupitiya, J. U.; Datta, P. P.; Kiesewetter, E. T.; Kiesewetter, M. K. ACS Macro Lett. 2016, 5 (8), 982-986.

(9) Lin, B.; Waymouth, R. M. J. Am. Chem. Soc. 2017, 139 (4), 1645-1652.

(10) Dharmaratne, N. U.; Pothupitiya, J. U.; Bannin, T. J.; Kazakov, O. I.; Kiesewetter, M. K. ACS Macro Lett. 2017, 6 (4), 421-425.

(11) Dove, A. P.; Pratt, R. C.; Lohmeijer, B. G. G.; Waymouth, R. M.; Hedrick, J. L. J. 
Am. Chem. Soc. 2005, 127 (40), 13798-13799.

(12) Pratt, R. C.; Lohmeijer, B. G. G.; Long, D. A.; Lundberg, P. N. P.; Dove, A. P.; Li, H.; Wade, C. G.; Waymouth, R. M.; Hedrick, J. L. Macromolecules 2006, 39 (23), $7863-7871$.

(13) Bas G. G. Lohmeijer Frank Leibfarth, John W. Logan, R. C. P.; David A. Long Fredrik Nederberg, Jeongsoo Choi, A. P. D.; Charles Wade and James L. Hedrick, R. M. W.; Lohmeijer, B. G. G.; Pratt, R. C.; Leibfarth, F.; Logan, J. W.; Long, D. A.; Dove, A. P.; Nederberg, F.; Choi, J.; Wade, C.; Waymouth, R. M.; Hedrick, J. L. Macromolecules 2006, 39 (25), 8574-8583.

(14) Duda, A.; Kowalski, A. In Handbook of Ring-Opening Polymerization; Dubois, P., Coulembier, O., Raquez, J.-M., Eds.; Wiley-VCH Verlag GmbH \& Co. KGaA, 2009; pp 1-52.

(15) Pratt, R. C.; Lohmeijer, B. G. G.; Long, D. A.; Lundberg, P. N. P.; Dove, A. P.; Li, H.; Wade, C. G.; Waymouth, R. M.; Hedrick, J. L. Macromolecules 2006, 39 (23), $7863-7871$.

(16) Webb, J. E. A.; Crossley, M. J.; Turner, P.; Thordarson, P. J. Am. Chem. Soc. 2007, 129 (22), 7155-7162.

(17) Thordarson, P. Chem. Soc. Rev. 2011, 40 (3), 1305-1323.

(18) Deranleau, D. A. J. Am. Chem. Soc. 1969, 91 (15), 4044-4049.

(19) Horman, I.; Dreux, B. Anal. Chem. 1983, 55 (8), 1219-1221.

(20) Peters, S. J.; Stevenson, C. D. J. Chem. Educ. 2004, 81 (5), 715.

(21) Kazakov, O. I.; Datta, P. P.; Isajani, M.; Kiesewetter, E. T.; Kiesewetter, M. K. Macromolecules 2014, 47, 7463-7468. 
(22) Lippert, K. M.; Hof, K.; Gerbig, D.; Ley, D.; Hausmann, H.; Guenther, S.;

Schreiner, P. R. European J. Org. Chem. 2012, 2012 (30), 5919-5927.

(23) Venning, J. D.; Jackson, J. B. Biochem. J. 1999, 341 (Pt 2), 329-337.

(24) Anslyn, E. V.; Dougherty, D. A. In Modern Physical Organic Chemistry; University Science, 2006; pp 145-222.

(25) Anslyn, E. V.; Dougherty, D. A. In Modern Physical Organic Chemistry; University Science, 2006; pp 490-530.

(26) Schneiderman, D. K.; Vanderlaan, M. E.; Mannion, A. M.; Panthani, T. R.; Batiste, D. C.; Wang, J. Z.; Bates, F. S.; Macosko, C. W.; Hillmyer, M. A. ACS Macro Lett. 2016, 5 (4), 515-518.

(27) Olsén, P.; Undin, J.; Odelius, K.; Keul, H.; Albertsson, A.-C. Biomacromolecules 2016, 17 (12), 3995-4002.

(28) Fukushima, K.; Coulembier, O.; Lecuyer, J. M.; Almegren, H. A.; Alabdulrahman, A. M.; Alsewailem, F. D.; Mcneil, M. A.; Dubois, P.; Waymouth, R. M.; Horn, H. W.; Rice, J. E.; Hedrick, J. L. J. Polym. Sci. Part A Polym. Chem. 2011, 49 (5), $1273-1281$.

(29) Fukushima, K.; Lecuyer, J. M.; Wei, D. S.; Horn, H. W.; Jones, G. O.; Al-Megren, H. A.; Alabdulrahman, A. M.; Alsewailem, F. D.; McNeil, M. A.; Rice, J. E.; Hedrick, J. L. Polym. Chem. 2013, 4 (5), 1610-1616.

(30) Gardea, F.; Garcia, J. M.; Boday, D. J.; Bajjuri, K. M.; Naraghi, M.; Hedrick, J. L. Macromol. Chem. Phys. 2014, 215 (22), 2260-2267. 




(Eq. 4.1)

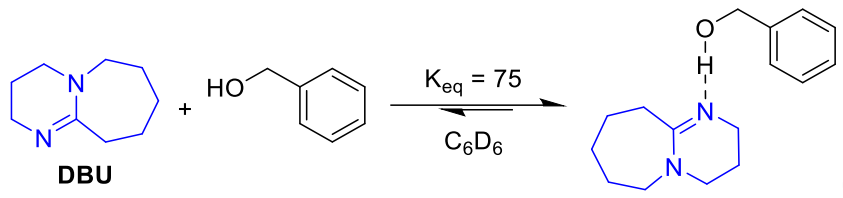

(Eq. 4.2)

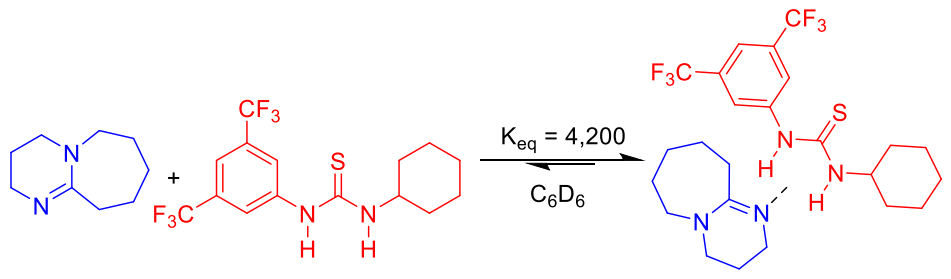

(Eq. 4.3)

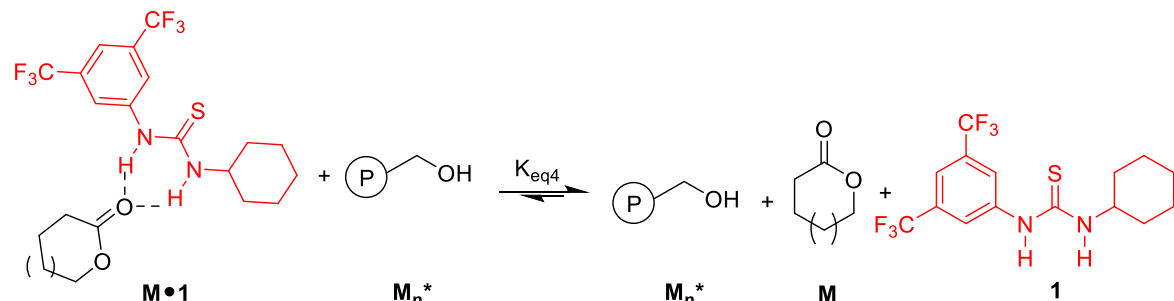

$M \cdot 1 \quad M_{n}{ }^{*} \quad M_{n}{ }^{*}$

(Eq. 4.4)



(Eq. 4.5)

$K_{e q T}=K_{e q 4} \bullet K_{e q 5}=\frac{[1]_{e q}}{[M \bullet 1]_{e q}}$

(Eq. 4.6)

$[\mathbf{1}]_{O}=[\mathbf{1}]_{e q}+[M \cdot \mathbf{1}]_{e q}+[D B U]_{O}$

(Eq. 4.7) 


$$
[M]_{T, e q}=\frac{1}{1+K_{e q T}}[\mathbf{1}]_{E F F}+[M]_{e q}
$$

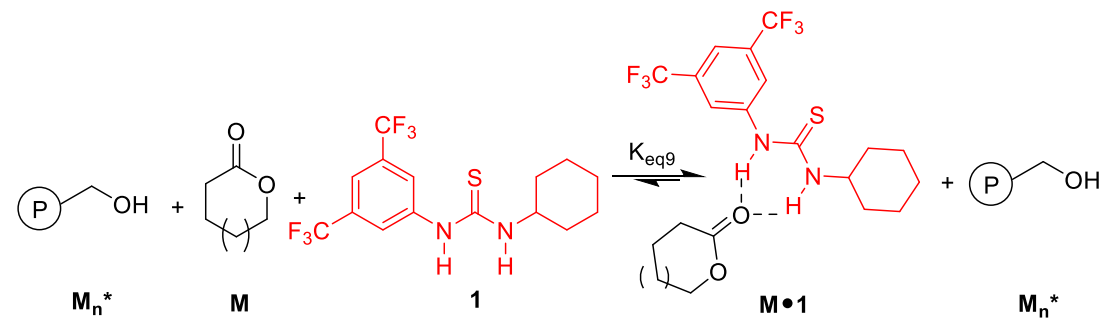

$\mathrm{M}_{\mathrm{n}}{ }^{*}$

M

1

$M \bullet 1$

$\mathbf{M}_{\mathrm{n}}{ }^{*} \quad$ (Eq. 4.9)
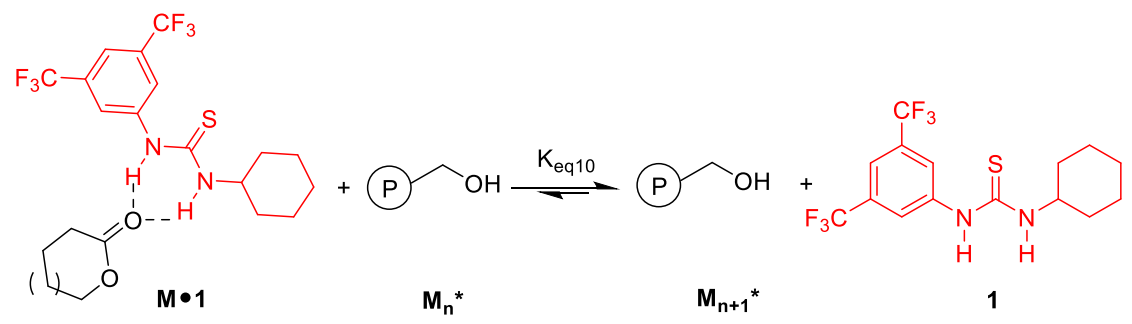

(Eq. 4.10) 
Derivation of Eq. 8:

$$
\begin{gathered}
M \bullet \mathbf{1}+M_{n}^{*} \leftrightharpoons M_{n}^{*}+M+\mathbf{1} \\
M_{n}^{*}+M+\mathbf{1} \leftrightharpoons M_{n+1}^{*}+\mathbf{1} \\
{[M]_{T, e q}=[M]_{e q}+[M \bullet \mathbf{1}]_{e q}} \\
K_{e q T}=K_{e q 4} \bullet K_{e q 5}=\frac{[\mathbf{1}] e q}{[M \cdot \mathbf{1}]_{e q}} \\
{[\mathbf{1}]_{O}=[\mathbf{1}]_{e q}+[M \cdot \mathbf{1}]_{e q}+[D B U]_{O}} \\
K_{e q 4}=\frac{[\mathbf{1}]_{e q}[M]_{e q}}{[M \bullet \mathbf{1}]_{e q}}
\end{gathered}
$$

Insert (7) into (6) and rearrange to get:

$$
K_{e q T}[M \bullet \mathbf{1}]_{e q}=[\mathbf{1}]_{O}-[M \bullet \mathbf{1}]_{e q}-[D B U]_{O}
$$

The effective concentration of $\mathbf{1},[\mathbf{1}]_{E F F}$, is defined to be that in excess of DBU:

$$
\begin{gathered}
{[\mathbf{1}]_{E F F}=[\mathbf{1}]_{O}-[D B U]_{O} \quad(\mathrm{~S} 4)} \\
\text { Insert eq. S4 into eq. S3: } \\
K_{e q T}[M \bullet \mathbf{1}]_{e q}=[\mathbf{1}]_{E F F}-[M \bullet \mathbf{1}]_{e q} \\
{[M \bullet \mathbf{1}]_{e q}\left(K_{e q T}+1\right)=[\mathbf{1}]_{E F F}} \\
\left([M]_{T, e q}-[M]_{e q}\right)\left(K_{e q T}+1\right)=[\mathbf{1}]_{E F F} \\
{[M]_{T, e q} K_{e q T}+[M]_{T, e q}-[M]_{e q}-[M]_{e q} K_{e q T}=[\mathbf{1}]_{E F F}} \\
{[M]_{T, e q}\left(1+K_{e q T}\right)=[\mathbf{1}]_{E F F}+[M]_{e q}\left(1+K_{e q T}\right)} \\
{[M]_{T, e q}=\frac{1}{1+K_{e q T}}[\mathbf{1}]_{E F F}+[M]_{e q}}
\end{gathered}
$$


Thiourea as catalyst interpretation:

$$
\begin{gathered}
M_{n}^{*}+M+\mathbf{1} \leftrightharpoons M \cdot \mathbf{1}+M_{n}^{*} \\
M \bullet \mathbf{1}+M_{n}^{*} \leftrightharpoons M_{n}^{*}+\mathbf{1} \\
K_{e q 9}=\frac{[M \bullet \mathbf{1}]_{e q}}{[\mathbf{1}]_{e q}[M]_{e q}} \\
K_{e q T}=K_{e q 9} \cdot K_{e q 10}=\frac{1}{[M]_{e q}}
\end{gathered}
$$

Insert definition of $\mathrm{K}_{\mathrm{eq} 10}$ into (S11) to get:

$$
K_{e q T}=\frac{K_{e q 9}[\mathbf{1}]_{e q}}{[M \bullet 1]_{e q}}
$$

Rearrange and insert eq. 7 into eq. S12 and rearrange to get:

$$
\begin{gathered}
{[M \bullet \mathbf{1}]_{e q}=\frac{K_{e q 9}}{K_{e q T}}\left([\mathbf{1}]_{O}-[M \bullet \mathbf{1}]_{e q}-[D B U]_{O}\right)} \\
\text { Insert eq. S4 into eq. S13: } \\
{[M \bullet \mathbf{1}]_{e q}+\frac{1}{K_{e q 10}}[M \bullet \mathbf{1}]_{e q}=\frac{1}{K_{e q 10}}[\mathbf{1}]_{E F F}} \\
{[M \bullet \mathbf{1}]_{e q}\left(1+\frac{1}{K_{e q 10}}\right)=\frac{1}{K_{e q 10}}[\mathbf{1}]_{E F F}} \\
\left([M]_{T, e q}-[M]_{e q}\right)\left(1+\frac{1}{K_{e q 10}}\right)=\frac{1}{K_{e q 10}}[\mathbf{1}]_{E F F} \\
{[M]_{T, e q}-[M]_{e q}+\frac{[M]_{T, e q}}{K_{e q 10}}-\frac{[M]_{e q}}{K_{e q 10}}=\frac{1}{K_{e q 10}}[\mathbf{1}]_{E F F}} \\
{[M]_{T, e q}\left(1+\frac{1}{K_{e q 10}}\right)=\frac{1}{K_{e q 10}}[\mathbf{1}]_{E F F}+[M]_{e q}\left(1+\frac{1}{K_{e q 10}}\right)} \\
{[M]_{T, e q}\left(K_{e q 10}+1\right)=[\mathbf{1}]_{E F F}+[M]_{e q}\left(K_{e q 10}+1\right)} \\
{[M]_{T, e q}=\frac{1}{1+K_{e q 10}}[\mathbf{1}]_{E F F}+[M]_{e q}}
\end{gathered}
$$






Figure 4.1. H-bond mediated ROP of VL. 
$\mathrm{n}$
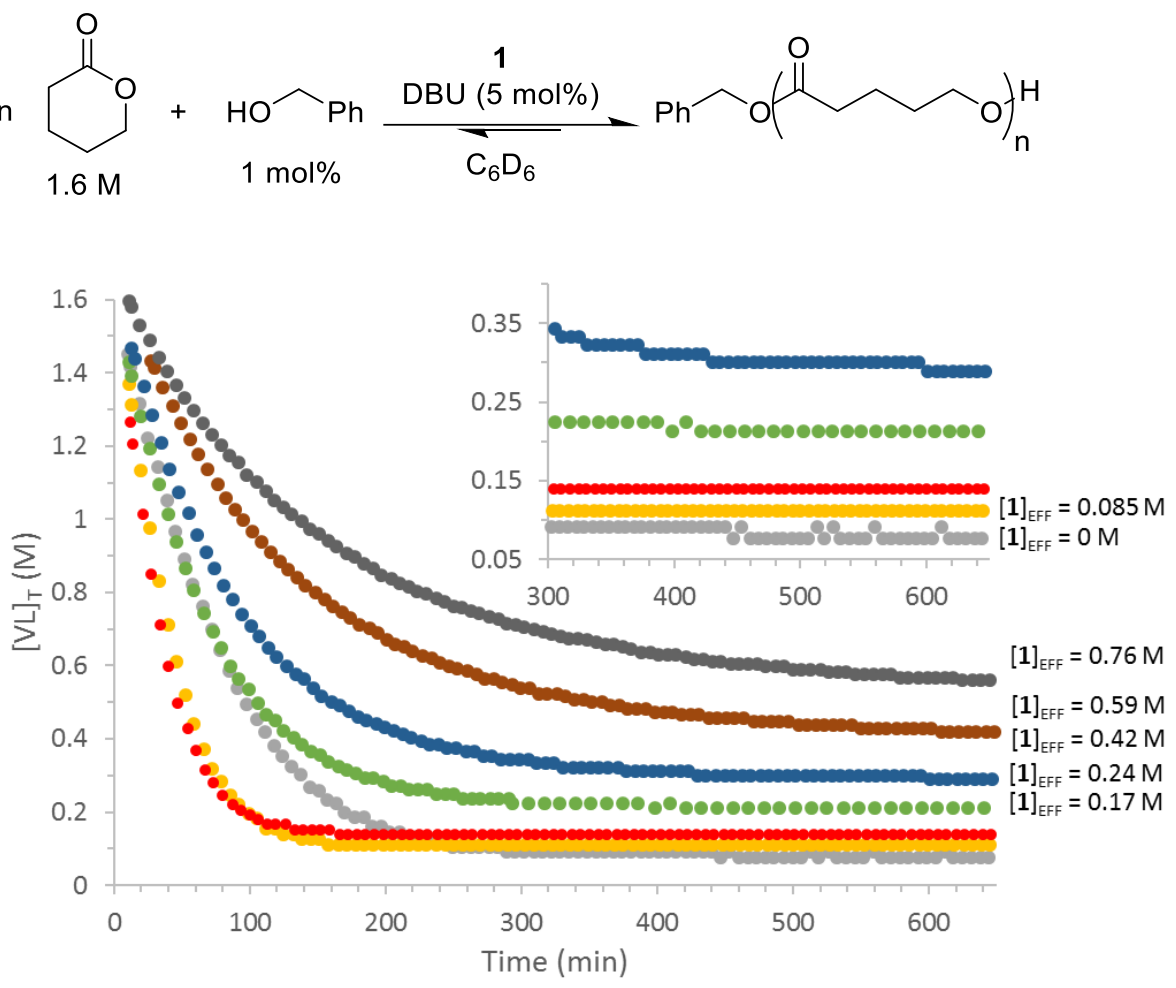

Figure 4.2. Evolution of $[\mathrm{VL}]_{\mathrm{T}}$ vs time for ROPs with varied [1 $]_{\mathrm{EFF}}$. Reaction conditions:

VL (100 mg, 1.63 M), DBU (0.082 M), benzyl alcohol $(0.016 \mathrm{M})$ in $\mathrm{C}_{6} \mathrm{D}_{6}$ with the given $[\mathbf{1}]_{\mathrm{EFF}}=[\mathbf{1}]_{\mathrm{o}}-[\mathrm{DBU}]_{\mathrm{o}}$ 


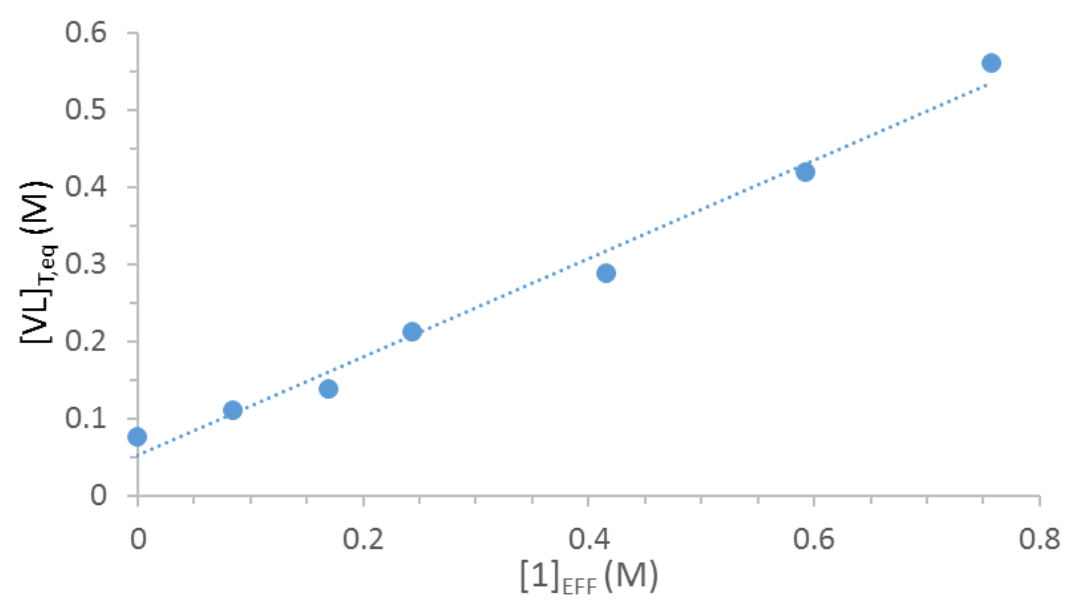

Figure 4.3. Total monomer concentration at equilibrium ([VL $\left.]_{\mathrm{T}, \mathrm{eq}}\right)$ vs $[\mathbf{1}]_{\mathrm{EFF}}$ for the reactions given above; slope $=0.636$, intercept $=0.0524, \mathrm{R}^{2}=0.985$. 


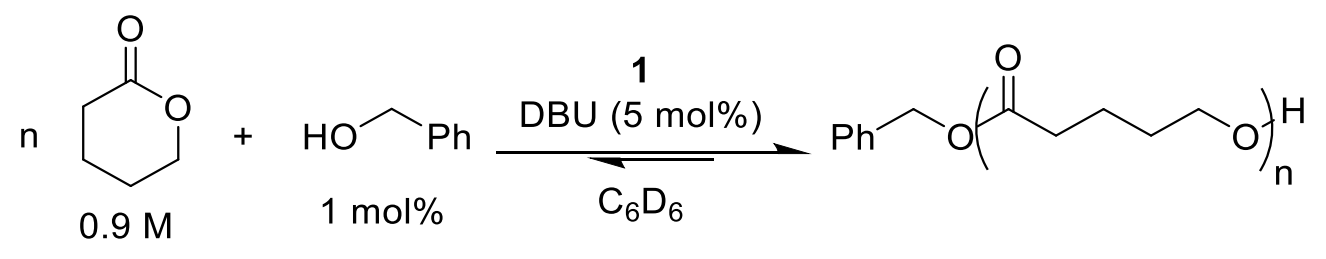

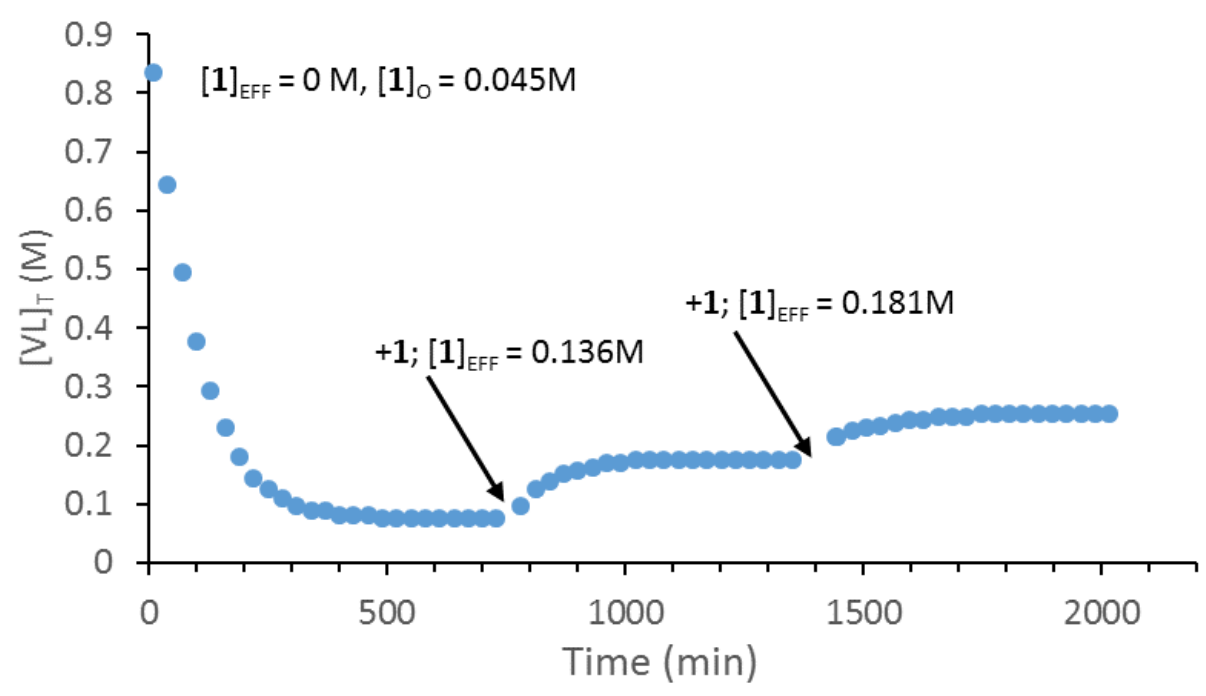

Figure 4.4. Concentration of VL vs time for the sequential addition of $\mathbf{1}$ to the ROP of VL in progress. Conditions: VL (100 mg, 0.9 M), DBU (0.045 M), benzyl alcohol (0.009 $\mathrm{M})$ in $\mathrm{C}_{6} \mathrm{D}_{6}$ with the given $[\mathbf{1}]_{\mathrm{EFF}}=[\mathbf{1}]_{\mathrm{o}}-[\mathrm{DBU}]_{\mathrm{o}}$. 
$\mathrm{n}$
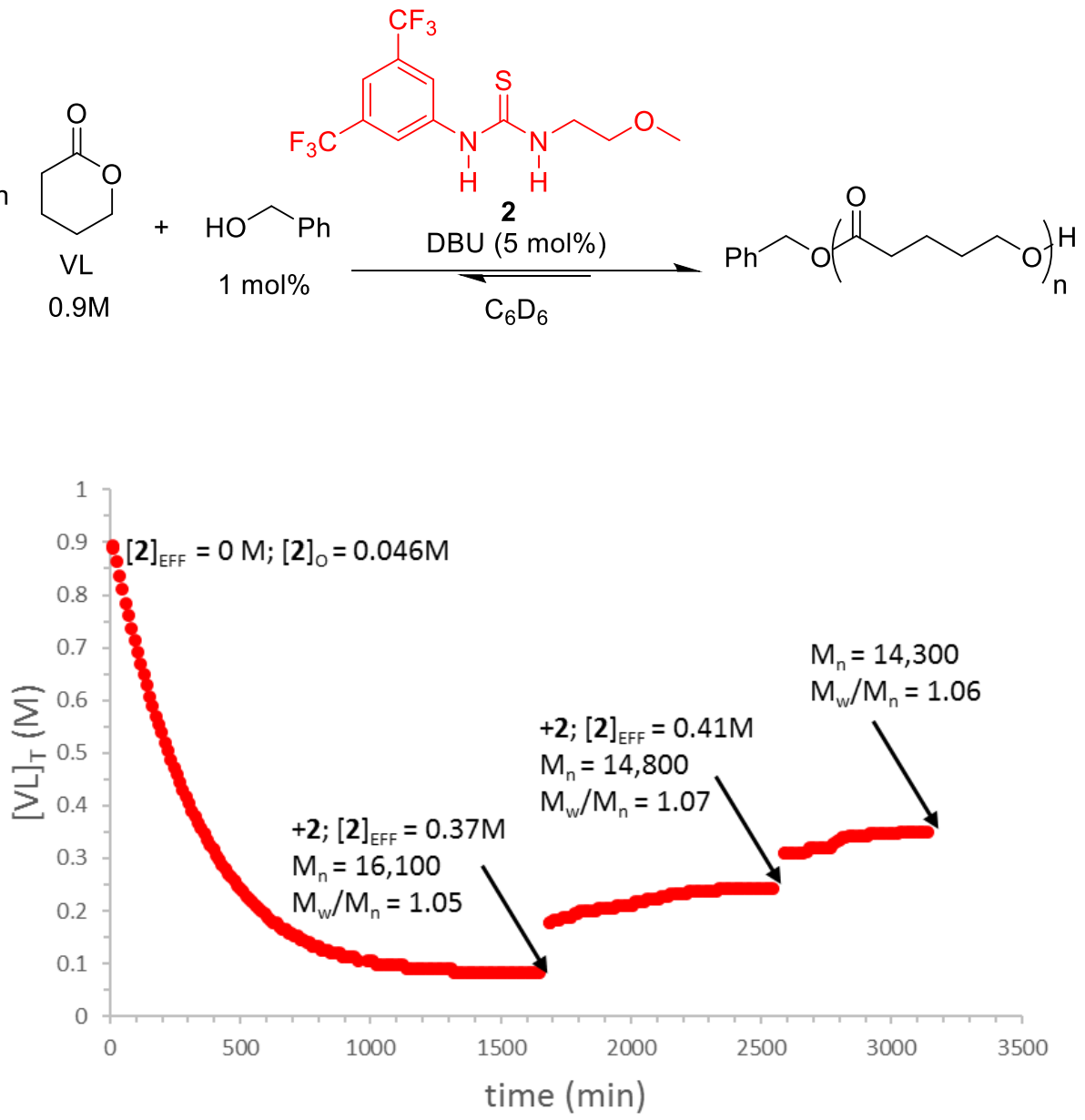

Figure 4.5. Sequential addition of 2 to the ROP of VL. Conditions: VL (100 mg, $0.91 \mathrm{M}$ ), DBU $(0.046 \mathrm{M})$, benzyl alcohol $(0.009 \mathrm{M})$ in $\mathrm{C}_{6} \mathrm{D}_{6}$ with the given $[\mathbf{1}]_{\mathrm{EFF}}=[\mathbf{1}]_{\mathrm{o}}-[\mathrm{DBU}]_{\mathrm{o}}$. 
$\mathrm{n}$
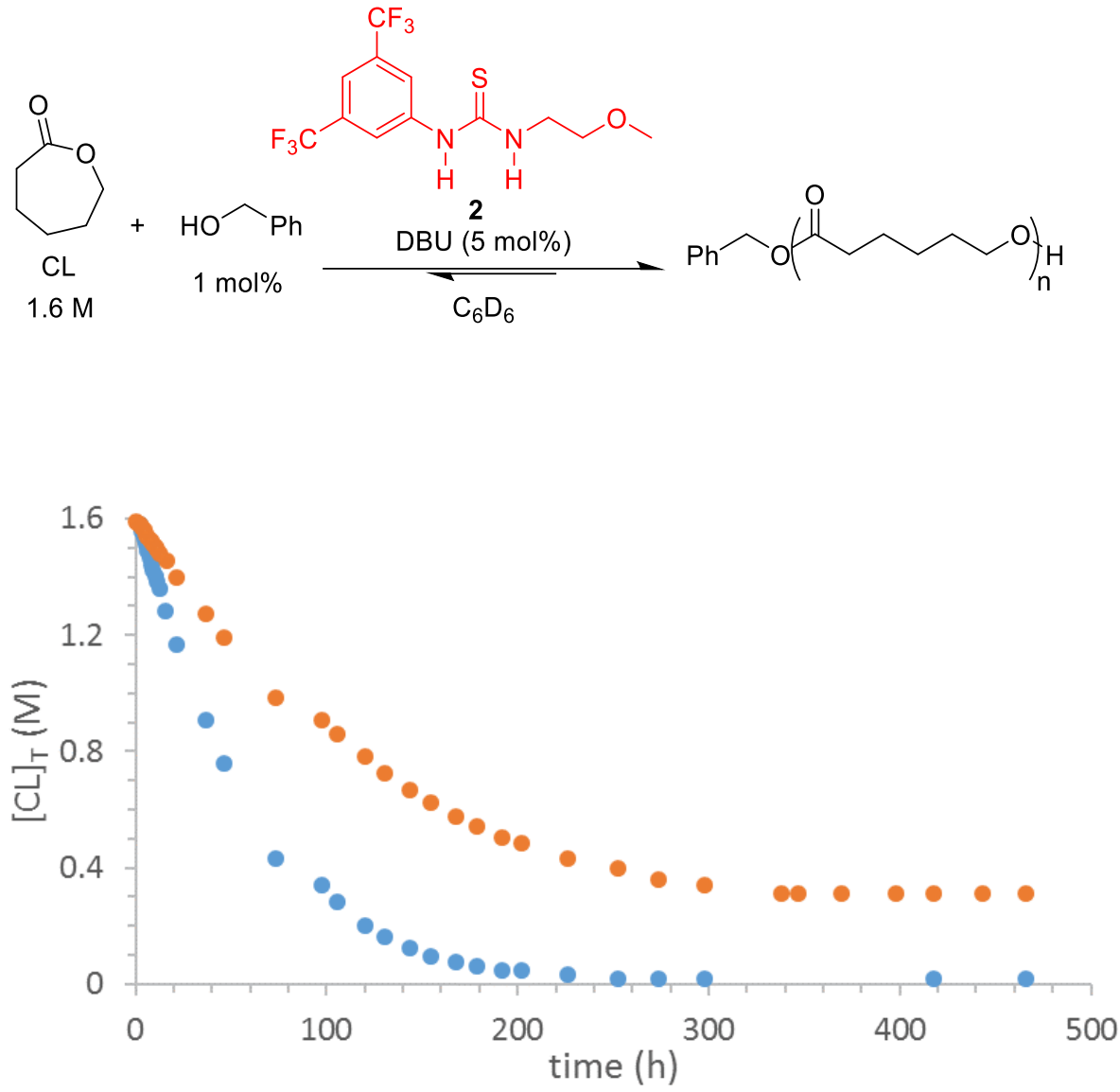

Figure 4.6. $[2]_{\mathrm{o}}$ dependent evolution of $[\mathrm{CL}]$ vs time. Conditions: $[\mathrm{CL}]_{\mathrm{o}}=1.6 \mathrm{M}(100$ $\mathrm{mg}) ;[\mathrm{DBU}]_{\mathrm{o}}=0.08 \mathrm{M} ;[\text { benzyl alcohol }]_{\mathrm{o}}=0.016 \mathrm{M}$; in $\mathrm{C}_{6} \mathrm{D}_{6} \cdot(\bullet)[2]_{\mathrm{o}}=0.80 \mathrm{M} ;(\bullet)[2]_{\mathrm{o}}$ $=0.41 \mathrm{M}$. 




Figure 4.7. ${ }^{1} \mathrm{H}$ NMR spectra showing the polymerization of VL and subsequent depolymerization of poly(valerolactone) upon the addition of $\mathbf{1}$. Reaction conditions: VL (0.499 mmol, 1 equiv, $1 \mathrm{M}$ in $\left.\mathrm{C}_{6} \mathrm{D}_{6}\right)$, benzyl alcohol (1 mol\%, $\left.4.99 \mu \mathrm{mol}\right)$, DBU (5 mol\%, $0.025 \mathrm{mmol})$ and 1 (5 mol\%, $0.025 \mathrm{mmol})$. After reaching equilibrium, aliquots of $\mathbf{1}$ were added (20 mol\%, $0.0999 \mathrm{mmol}$ and $25 \mathrm{~mol} \%, 0.125 \mathrm{mmol})$ at $781 \mathrm{~min}$ and 1441 min, respectively. 




Figure 4.8. Selected expanded ${ }^{1} \mathrm{H}$ NMR spectra of from the polymerization of VL and subsequent depolymerization of poly(valerolactone) upon the addition of $\mathbf{1}$. Reaction conditions: VL (0.499 mmol, 1 equiv, $1 \mathrm{M}$ in $\left.\mathrm{C}_{6} \mathrm{D}_{6}\right)$, benzyl alcohol $(1 \mathrm{~mol} \%, 4.99 \mu \mathrm{mol})$, DBU (5 mol\%, $0.025 \mathrm{mmol})$ and $1(5 \mathrm{~mol} \%, 0.025 \mathrm{mmol})$. After reaching equilibrium, aliquots of 1 were added (20 mol\%, $0.0999 \mathrm{mmol}$ and $25 \mathrm{~mol} \%, 0.125 \mathrm{mmol}$ ) at $781 \mathrm{~min}$ and 1441 min, respectively. 




Figure 4.9. NMR tubes containing the top and bottom runs from Figure 4.2 (left and right tube, respectively). (left) Start of the reaction. (right) At equilibrium. See Figure 4.2 for conditions. 




Figure 4.10. Reproduction of $\mathbf{1}$ addition experiment from Figure 4.3. Left tube is after all 1 additions, right tube is the starting conditions. See Figure 4.3 for conditions. 




Figure 4.11. [VL $]_{\text {T,eq }}$ vs [catalysts $]_{\mathrm{o}}$ for the ROPs when the initial concentrations of cocatalysts are varied together. Conditions: $2 \mathrm{M} \mathrm{VL}, 0.02 \mathrm{M}$ benzyl alcohol in $\mathrm{C}_{6} \mathrm{D}_{6}$. 


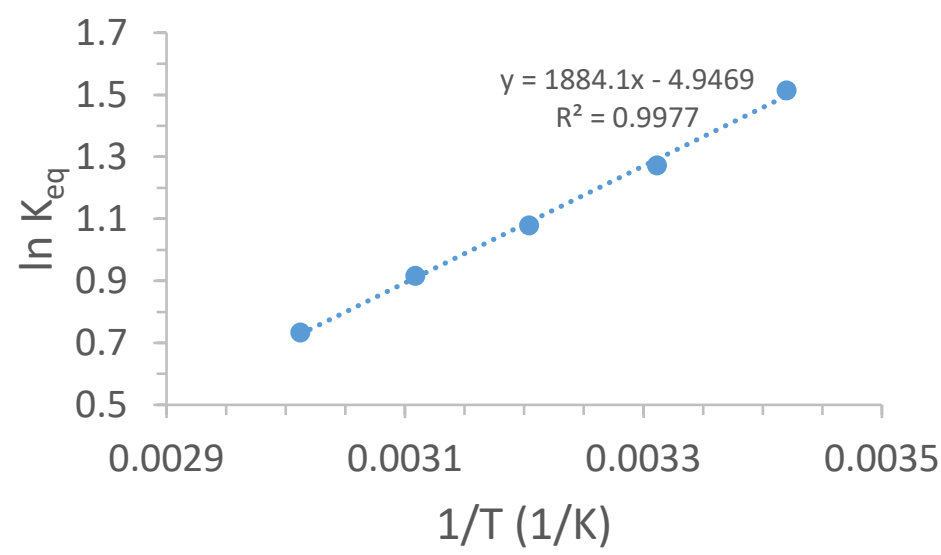

Figure 4.12. [VL $]_{\mathrm{eq}}$ as a function of temperature. Conditions: VL $(0.100 \mathrm{~g}, 0.999 \mathrm{mmol}$, $2 \mathrm{M}$ in $\left.\mathrm{C}_{6} \mathrm{D}_{6}\right), 1(0.0740 \mathrm{~g}, 0.199 \mathrm{mmol}), \mathrm{DBU}(7.47 \mu \mathrm{L}, 0.0499 \mathrm{mmol})$, and benzyl alcohol $(9.99 \mu \mathrm{mol})$. 


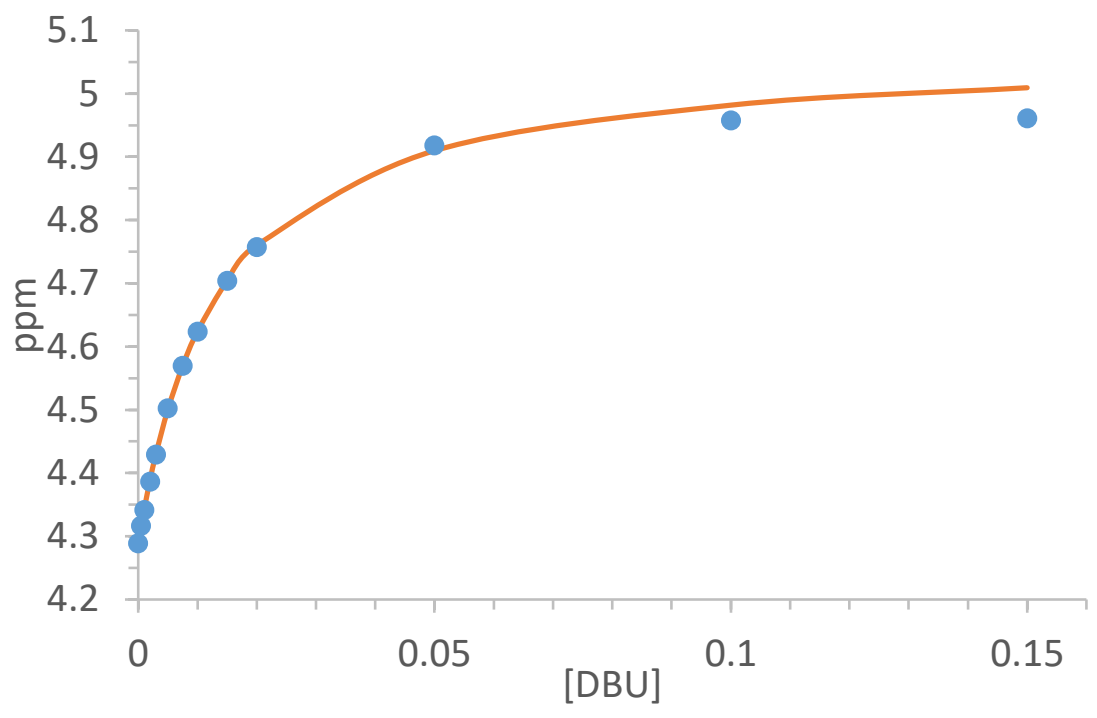

Figure 4.13. Titration curve for the $\mathrm{BnOH} / \mathrm{DBU}$ binding in benzene- $\mathrm{d}_{6}$. Chemical shift of the benzylic protons vs [DBU]; solid line is the fit from the binding equation. 


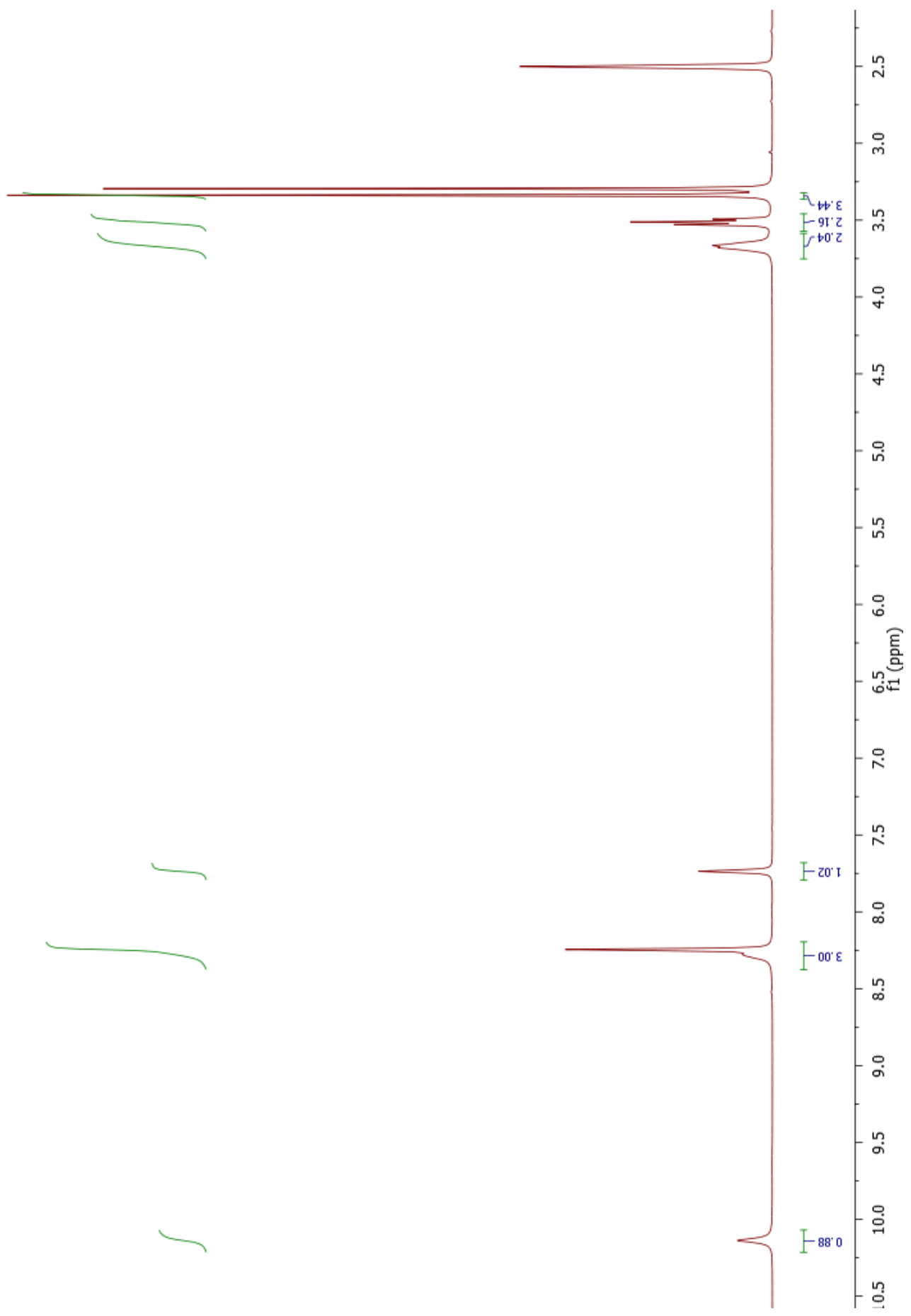

Figure 4.14. ${ }^{1} \mathrm{H}$ NMR (300 MHz, DMSO-d 6 ) of 2. 


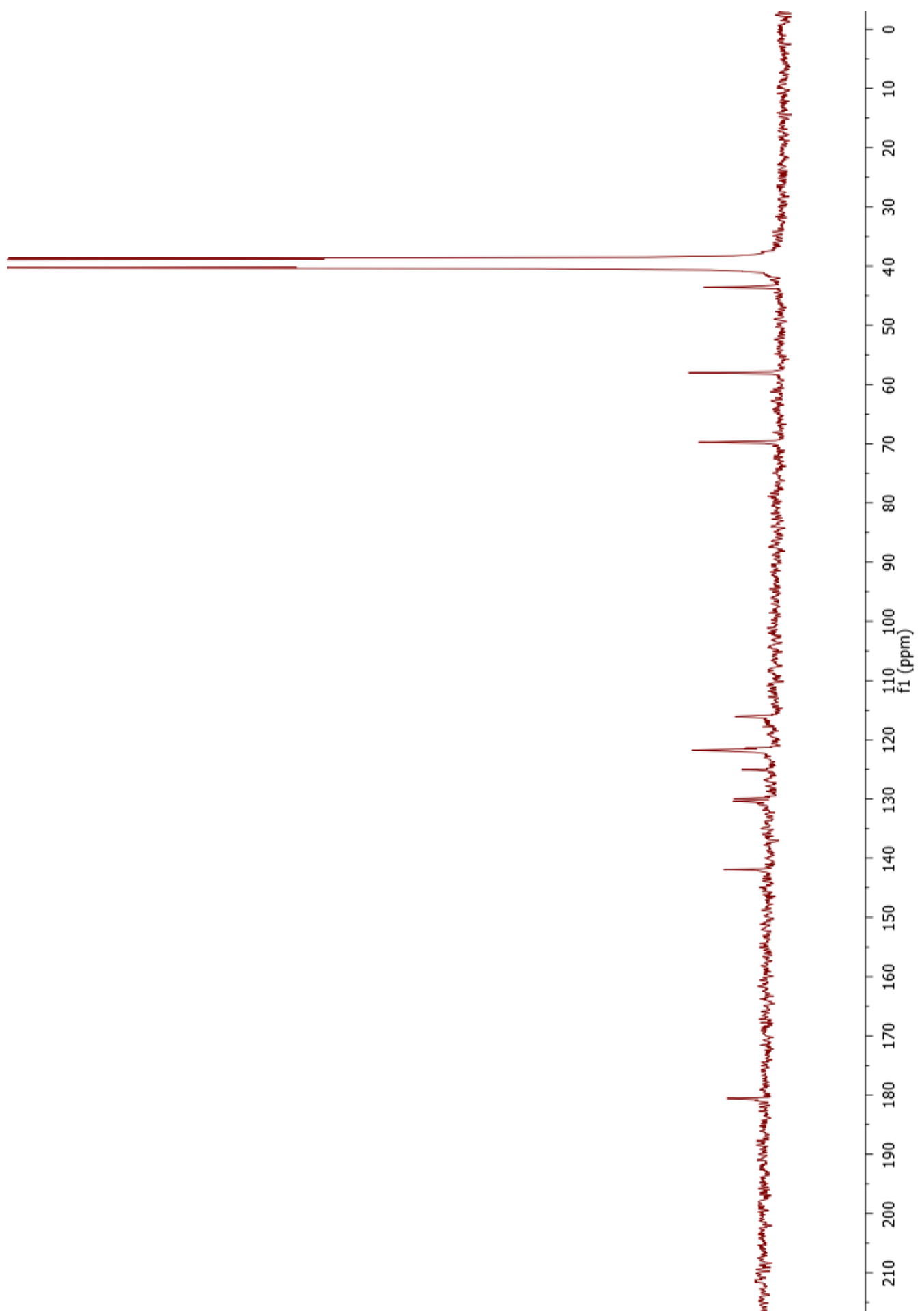

Figure 4.15. ${ }^{13} \mathrm{C}$ NMR (75 MHz, DMSO-d 6 ) of 2. 


\title{
MANUSCRIPT - V
}

\author{
Published in Macromolecules
}

\section{Controlled Organocatalytic Ring-Opening Polymerization of $\varepsilon$-Thionocaprolactone}

Partha P. Datta, and Matthew K. Kiesewetter

Chemistry, University of Rhode Island, Kingston, RI, USA

Corresponding Author: $\quad$ Matthew Kiesewetter, Ph.D.

Chemistry

University of Rhode Island

140 Flagg Road

Kingston, RI, 02881, USA

Email address: mkiesewetter@chm.uri.edu 


\begin{abstract}
For the first time, the controlled ring-opening polymerization (ROP) of $\varepsilon$ thionocaprolactone (tnCL) is conducted. The organocatalytic ROP of tnCL occurs without carbonyl scrambling, leading to homopoly( $\varepsilon$-thionocaprolactone) (PtnCL). The ROP by base catalysts alone is proposed to proceed via a nucleophilic mechanism, while the addition of an H-bond donating thiourea (TU) is shown to provide excellent reaction control. The increased reaction control provided by the TU occurs in the virtual absence of binding between tnCL and TU, and a mechanistic account for this observation is discussed. The monomer ring strain is measured and found to be similar to $\delta$-valerolactone (VL). Copolymers with VL are synthesized, and the resulting analysis of the copolymer materials properties provides the only known physical characterizations of poly(thio(no)ester-coester)s.
\end{abstract}




\section{INTRODUCTION}

Over the past decade, developments in organocatalytic ring-opening polymerization (ROP) have demonstrated the remarkable ability of these catalysts to generate well-defined and highly functionalized polyesters and other polymers. ${ }^{1-3}$ The Hbond-mediated organocatalysts, which are a paragon of highly controlled polymerization techniques, stand out in their ability to generate precisely tailored materials. ${ }^{4-6}$ These catalysts are believed to operate by H-bond-mediated activation of monomer by thiourea and of growing polymer chain by base. ${ }^{7,8}$ Given the remarkable control of these polymerization systems and the mild nature of their reactivity, the paucity of polymer backbone linkages which have been explored is surprising. The mild reactivity of organocatalysts for ROP perfectly position them for the generation of new polymer backbones and, hence, new materials and applications.

Our group recently disclosed the ROP of an S-substituted lactone, $\varepsilon$ thiocaprolactone (tCL). ${ }^{9}$ The ROP of this monomer was postulated to proceed through a classic transesterification mechanism mediated by $\mathrm{H}$-bond activation of thioester by thiourea and thiol end group by base. ${ }^{9}$ The other S-substituted caprolactone, $\varepsilon$ thionocaprolactone (tnCL, Scheme 5.1), has been the subject of only two published reports. ${ }^{10,11}$ Under cationic polymerization conditions, the ROP of tnCL proceeds with quantitative inversion of substitution at the thionoester to generate the same poly(thiocaprolactone) previously reported by Overberger and our group. ${ }^{9,12-14}$ The anionic ROP of tnCL from alkyllithium reagents retains the S-carbonyl substitution, but reaction control suffers, and this method does not allow for $\mathrm{M}_{\mathrm{n}}$ control, copolymerization, or end 
group selection. ${ }^{10}$ Partial inversion of substitution occurs with weak nucleophiles, resulting in a mixed polymer backbone. Polymerization conditions which result in inversion of S/O substitution are postulated to operate via an $\mathrm{S}_{\mathrm{N}} 2$ propagation (Scheme 5.1). ${ }^{10}$ If the chain end/monomer activation mechanism of H-bond mediated ROP of tCL is correct, we reasoned that organocatalytic ROP of tnCL should allow for the retention of the S/O substitution and controlled-generation of homopoly(thionocaprolactone). 


\section{EXPERIMENTAL SECTION}

\section{General Considerations}

All chemicals were used as received unless stated otherwise. $\mathrm{P}_{4} \mathrm{~S}_{10}$, hexamethyldisiloxane (HMDO), and 2-tert-butylimino-2-diethylamino-1,3dimethylperhydro-1,3,2-diazaphosphorine (BEMP) were supplied by Acros Organics. Acetonitrile, potassium carbonate, magnesium sulfate, benzyl alcohol, benzoic acid, ethyl acetate, and hexane were purchased from Fisher Scientific. $\varepsilon$-Caprolactone (CL) and $\delta$ valerolactone (VL) were supplied by Alfa Aesar and distilled from $\mathrm{CaH}_{2}$ under high vacuum. Benzene- $\mathrm{d}_{6}$ was supplied by Cambridge Isotope Laboratories and distilled from $\mathrm{CaH}_{2}$ under a nitrogen atmosphere. Benzyl alcohol was distilled from $\mathrm{CaH}_{2}$ under high vacuum. 1 [3,5-bis(trifluoromethyl)phenyl]-3-cyclohexylthiourea was synthesized and purified according to a literature procedure. ${ }^{7}$ 1,8-Diazabicyclo[5.4.0]undec-7-ene (DBU), 7-methyl- 1,5,7-triazabicyclo[4.4.0]dec-5-ene (MTBD), and 1,5,7-triazabicyclo[4.4.0]dec5-ene (TBD) were purchased from TCI. All polymerization reactions were performed in an MBRAUN stainless steel glovebox equipped with a gas purification system under a nitrogen atmosphere using glass vials and stir bars which were baked overnight at $140^{\circ} \mathrm{C}$. NMR experiments were performed on a Bruker Avance $300 \mathrm{MHz}$ (proton) spectrometer. The chemical shifts for proton $\left({ }^{1} \mathrm{H}\right)$ and carbon $\left({ }^{13} \mathrm{C}\right)$ NMR were recorded in parts per million (ppm) relative to a residual solvent. Size exclusion chromatography (SEC) was performed at $30^{\circ} \mathrm{C}$ in dichloromethane (DCM) using an Agilent Infinity GPC system equipped with three Agilent PLGel columns $7.5 \mathrm{~mm} \times 300 \mathrm{~mm}(5 \mu \mathrm{m}$ pore sizes: 103, 104, and $105 \AA$ ). Molecular weight and $\mathrm{M}_{\mathrm{w}} / \mathrm{M}_{\mathrm{n}}$ were determined versus polystyrene standards 
(500 g/mol-3150 kg/mol; Polymer Laboratories). Differential scanning calorimetry (DSC) curves were obtained on a DSC Q100 (TA Instruments) under $\mathrm{N}_{2}$ calibrated with an indium standard. The heating and cooling curves of DSC were run under a nitrogen atmosphere at a heating rate of $\pm 10{ }^{\circ} \mathrm{C} / \mathrm{min}$ in a $40 \mu \mathrm{L}$ aluminum crucible. Thermogravimetric analysis (TGA) was performed using a TGA Q500 from TA Instruments under a $\mathrm{N}_{2}$ atmosphere at a heating rate of $20^{\circ} \mathrm{C} / \mathrm{min}$ from 25 to $600^{\circ} \mathrm{C}$. MALDI-TOF MS analysis was performed at the University of Akron: reflectron mode with trans-2-[3-(4-tert-butylphenyl)-2-methyl2-propenylidene]-malononitrile (DCTB) matrix with sodium trifluoroacetate (NaTFA) salt.

\section{Example Synthesis of $\varepsilon$-Thionocaprolactone (TnCL)}

In a dried $100 \mathrm{~mL}$ round-bottom flask with stir bar, $\mathrm{P}_{4} \mathrm{~S}_{10}(1.95 \mathrm{~g}, 4.38 \mathrm{mmol}), \varepsilon-$ caprolactone (1.94 mL, $17.52 \mathrm{mmol}), \mathrm{HMDO}(6.18 \mathrm{~mL}, 29.08 \mathrm{mmol})$, and acetonitrile $(17.5 \mathrm{~mL})$ were added. The solution was refluxed for $1 \mathrm{~h}$ while stirring, and the reaction was cooled to room temperature with stirring. The flask was then placed in an ice-water bath, and aqueous $\mathrm{K}_{2} \mathrm{CO}_{3}$ solution (1.26 mL of $5.3 \mathrm{M}$ solution per mmol of $\left.\mathrm{P}_{4} \mathrm{~S}_{10}\right)$ was added followed by distilled water ( $1 \mathrm{~mL}$ per mmol of $\left.\mathrm{P}_{4} \mathrm{~S}_{10}\right)$ with stirring. Once cooled to room temperature, organics were extracted with dichloromethane and washed with brine. The organics were dried over $\mathrm{MgSO}_{4}$, filtered, and removed of volatiles to obtain crude product. The crude product was purified in two stages by silica gel flash chromatography: 75:25 toluene:hexanes mobile phase and a second column with 95:5 dichloromethane:hexanes mobile phase. Kugelrohr vacuum distillation at $80^{\circ} \mathrm{C}$ and 200 mTorr pressure yielded tnCL, a pure yellow oil which was transferred to the glovebox. Yield: $1.7 \mathrm{~g}, 75 \%$. Characterization matched the literature. ${ }^{15}$ 


\section{Example Ring-Opening Polymerization}

In a typical polymerization, tnCL $(0.100 \mathrm{~g}, 0.768 \mathrm{mmol})$ was added to a $20 \mathrm{~mL}$ glass vial with a stir bar. In another $20 \mathrm{~mL}$ glass vial with stir bar, $1(0.0142 \mathrm{~g}, 0.0384$ mmol), BEMP $(11.1 \mu \mathrm{L}, 0.0384 \mathrm{mmol})$, and benzyl alcohol $(1.6 \mu \mathrm{L}, 15.4 \mu \mathrm{mol})$ were added. Solvent (for $\mathrm{C}_{6} \mathrm{D}_{6}, 0.38 \mathrm{~mL}, 2 \mathrm{M}$ in tnCL) was divided equally between the two vials. After 2 min of stirring, the tnCL solution was transferred with a pipet to the other vial consisting of initiator and catalysts. The solution was then transferred via pipet to a NMR tube and taken out of the glovebox. Reaction conversion was monitored by ${ }^{1} \mathrm{H}$ NMR, and the reaction was quenched with benzoic acid ( 2 mol equiv to base) after desired conversion had been achieved. The polymer was treated with hexanes to precipitate the polymer. After decanting the hexanes supernatant, the polymer was removed of volatiles under reduced pressure. Yield 85\%; $\mathrm{M}_{\mathrm{w}} / \mathrm{M}_{\mathrm{n}}=1.11 ; \mathrm{M}_{\mathrm{n}(\mathrm{GPC})}=8200 ; \mathrm{M}_{\mathrm{n}(\mathrm{NMR})}=5200$. For $[\mathrm{M}]_{0} /[\mathrm{I}]_{0}=200, \mathrm{M}_{\mathrm{w}} / \mathrm{M}_{\mathrm{n}}=1.10 ; \mathrm{M}_{\mathrm{n}(\mathrm{GPC})}=20900 ; \mathrm{M}_{\mathrm{n}(\mathrm{NMR})}=15800 .{ }^{1} \mathrm{H}$ and ${ }^{13} \mathrm{C} \mathrm{NMR}$ spectra (see Figures 5.16 and 5.17) show resonances consistent with assignment of the polymer as quantitatively thionoester repeat unit, with the characteristic thiocarbonyl carbon peak at $224 \mathrm{ppm}$ in the ${ }^{13} \mathrm{C}$ spectrum. ${ }^{1} \mathrm{H}$ NMR $\left(300 \mathrm{MHz}, \mathrm{CDCl}_{3}\right) \delta: 7.38(5 \mathrm{H}$, aromatic); 5.48 (2H, benzylic); $4.43\left(78 \mathrm{H}, \mathrm{R}_{-}-\mathrm{CH}_{2}-\mathrm{R}^{\prime}\right.$ of PtnCL); $2.73\left(80 \mathrm{H}, \mathrm{R}^{-} \mathrm{CH}_{2}-\mathrm{R}^{\prime}\right.$ of tnCL); 1.80 (159 H, R-CH $-\mathrm{R}^{\prime}$ of tnCL); $1.46\left(88 \mathrm{H}, \mathrm{R}_{-} \mathrm{CH}_{2}-\mathrm{R}^{\prime}\right.$ of PtnCL). ${ }^{13} \mathrm{C}$ NMR (75 $\mathrm{MHz}, \mathrm{CDCl}_{3}$ ) (see Figure 5.17) $\delta: 224$ (R-C=S-R'); 128 (benzyl aromatics); 72 (1C, Ar$\left.\mathrm{CH}_{2}-\mathrm{OR}\right) ; 62$ (benzylic $\left.\mathrm{CH}_{2}\right) ; 47\left(\mathrm{R}-\mathrm{CH}_{2}-\mathrm{O}\right) ; 28$ (2C, R-C= $\left.\mathrm{SCH}_{2}-\mathrm{CH}_{2}-\mathrm{R}^{\prime}\right) ; 25$ (R-C=S$\left.\mathrm{CH}_{2}-\mathrm{CH}_{2}-\mathrm{CH}_{2}-\mathrm{R}^{\prime}\right)$.

Example Copolymerization of $\varepsilon$-Thionocaprolactone with $\delta$-Valerolactone 
VL (100 mg, $1 \mathrm{mmol})$ and tnCL (130 mg, $1 \mathrm{mmol})$ were added to a $20 \mathrm{~mL}$ glass vial with a stir bar. In another $20 \mathrm{~mL}$ glass vial with stir bar, $1(0.05 \mathrm{mmol}), \mathrm{BEMP}(0.05$ $\mathrm{mmol})$, and benzyl alcohol $(0.01 \mathrm{mmol})$ were added. Solvent (for $\mathrm{C}_{6} \mathrm{D}_{6}, 0.5 \mathrm{~mL}$ ) was added in equal proportion to both the vials. The monomer solution was transferred via pipet to the vial containing initiator after few minutes of stirring. The solution was then transferred to a NMR tube which was immediately taken out of the glovebox. Reaction progress was monitored by ${ }^{1} \mathrm{H} \mathrm{NMR}$, and the reaction was quenched with benzoic acid ( 2 mol equiv to base). The polymer was treated with hexanes to precipitate the polymer, the supernatant was decanted, and polymer was subjected to reduced pressure to remove volatiles. The polymer samples were dissolved in methylene chloride and dialyzed against methanol over $48 \mathrm{~h}$, changing the methanol solution after $24 \mathrm{~h}$. Yield 90\%; $\mathrm{M}_{\mathrm{w}} / \mathrm{M}_{\mathrm{n}}=1.25 ; \mathrm{M}_{\mathrm{n}(\mathrm{GPC})}=29$ 800. ${ }^{1} \mathrm{H}$ NMR (300 MHz, $\mathrm{C}_{6} \mathrm{D}_{6}$ ) (see Figure 5.15) $\delta: 7.30$ (5H, aromatic); $5.08(2 \mathrm{H}$, benzylic); 4.40 (164H, R-CH $-\mathrm{R}^{\prime}$ PVL); 4.04 (176H, R-CH $-\mathrm{CH}_{2}^{\prime}$ PtnCL); 2.69 (164H, R$\left.\mathrm{CH}_{2}-\mathrm{R}^{\prime} \mathrm{PVL}\right) ; 2.32$ (176H, R-CH $\left.-\mathrm{R}^{\prime} \mathrm{PtnCL}\right) ; 1.85-1.53$ (m, 680H, $\mathrm{R}^{-\mathrm{CH}_{2}-\mathrm{R}^{\prime} \mathrm{PtnCL} \text { and }}$ PVL); 1.40 (176H, R-CH2-R' PtnCL). ${ }^{13} \mathrm{C}$ NMR (75 MHz, $\mathrm{CDCl}_{3}$ ) (see Figure 5.14) $\delta: 224$ (1C, RC=S-R'); $173\left(1 \mathrm{C}, \mathrm{R}-\mathrm{C}=\mathrm{O}-\mathrm{R}^{\prime}\right) ; 128\left(6 \mathrm{C}\right.$, aromatic C's); 72.15 and $71.78\left(\varepsilon-\mathrm{CH}_{2}\right.$, tnCL-tnCL and tnCL-VL); 64.21 and $63.90\left(\delta-\mathrm{CH}_{2}, \mathrm{VL}-\mathrm{VL}\right.$, and tnCL-VL); $53.5(\alpha-$ $\left.\mathrm{CH}_{2}, \mathrm{VL}\right) ; 46.6\left(\alpha-\mathrm{CH}_{2}, \mathrm{tnCL}\right) ; 33.7\left(\gamma-\mathrm{CH}_{2}, \mathrm{VL}\right) ; 28.32,28.07,27.80,27.57\left(\gamma-\mathrm{CH}_{2}\right.$ and $\delta-\mathrm{CH}_{2}$, tnCL-tnCL and tnCL-VL); $25.2\left(\beta-\mathrm{CH}_{2}, \operatorname{tnCL}\right) ; 21.4\left(\beta-\mathrm{CH}_{2}, \mathrm{VL}\right)$.

Determination of Binding Constant $\left(\mathrm{K}_{\mathrm{eq}}\right)$ between $\mathrm{TnCL}$ and $\mathbf{1}$

The binding constant $\left(\mathrm{K}_{\mathrm{eq}}\right)$ between $\mathbf{1}$ and tnCL was determined in benzene- $\mathrm{d}_{6}$ by the titration method and curve fitting as previously described. ${ }^{20-22}$ The $\mathrm{K}_{\mathrm{eq}}$ values were determined by fitting the binding curve to the quadratic form of the binding equation with 
$\mathrm{K}_{\mathrm{eq}}$ and $\Delta \delta$ as variables: $\delta_{\mathrm{obs}}=\delta_{\mathrm{H}}-\left(\Delta \delta / 2[\mathrm{H}]_{0}\right)\left\{[\mathrm{H}]_{0}+[\mathrm{G}]_{0}+1 / \mathrm{K}-\left(\left([\mathrm{H}]_{0}+[\mathrm{G}]_{0}+1 / \mathrm{K}\right)^{2}\right.\right.$ $\left.\left.-4[\mathrm{H}]_{0}[\mathrm{G}]_{0}\right)^{1 / 2}\right\} ; \Delta \delta$ is the difference in the chemical shift of host and complex, $\delta_{\mathrm{obs}}$ is the observed chemical shift of the TU in the presence of monomer, and $\delta_{\mathrm{H}}$ is the chemical shift of free $\mathrm{TU}$ in the absence of monomer.

Determination of Thermodynamic Parameters

A sample of tnCL (100 mg, $0.77 \mathrm{mmol})$, TBD (5.3 mg, $0.038 \mathrm{mmol})$, and benzyl alcohol $(0.80 \mu \mathrm{L}, 0.0077 \mathrm{mmol})$ in $\mathrm{C}_{6} \mathrm{D}_{6}(2 \mathrm{M}$ in monomer $)$ was heated in a variable temperature NMR probe, and ${ }^{1} \mathrm{H}$ NMR spectra were acquired at temperatures from 290 to 330 K. Data points were taken in duplicate, during heating and cooling. The heating and cooling $[\mathrm{M}]_{\mathrm{eq}}$ values were within error; heating values are shown in Figure 5.7. The thermodynamic values of tnCL ROP were determined from a Van't Hoff plot of the data, and error was calculated from linear regression at 95\% confidence interval (see Figure 5.7).

Polymer Hydrolysis

Polymer samples (approximately $10 \mathrm{mg}$ each) were loaded into empty $20 \mathrm{~mL}$ scintillation vials. The polymers were then dissolved in dichloromethane to evenly distribute the polymer on the bottom of the vial, and the dichloromethane was subsequently removed under vacuum. Each vial was charged with $10 \mathrm{~mL}$ of aqueous $0.25 \mathrm{M} \mathrm{HCl}$, aqueous $0.25 \mathrm{M} \mathrm{NaOH}$ solution, or distilled water. Each hydrolysis medium was tested in quadruplicate. All vials were shaken on a rotary shaker for the duration of the study. To take a data point, the solutions were removed via syringe, and the polymer samples were rinsed with minimal distilled water $(\sim 1 \mathrm{~mL})$. After removing the distilled water via syringe, the vials were put in a vacuum oven overnight $\left(60^{\circ} \mathrm{C}, 30 \mathrm{in} . \mathrm{Hg}\right.$ vacuum $)$. The vials were 
cooled and weighed. Percent mass loss is given by $\left(\right.$ mass $_{0}-$ mass $\left._{\mathrm{i}}\right) / \mathrm{mass}_{0}$. The same steps were repeated over a three week period at different intervals.

\section{Computational Methods}

Computational experiments using Endo's methods ${ }^{10}$ were performed in Spartan '14

(Windows 7). Structures were geometry optimized at the DFT B3LYP/6-31G* level of theory in the gas phase. Energies and electrostatic charges in toluene solvent were calculated as single point energies (DFT B3LYP/6-31G**) from the DFT-optimized structures. Energies, electrostatic charges, computed structures, and coordinates of optimized structures are given in Figure 5.19. 


\section{RESULTS AND DISCUSSION}

\section{Organic Base Catalyzed ROP}

The organocatalytic room temperature $\mathrm{ROP}$ of tnCL initiated from alcohol or thiol initiators proceeds with retention of the $\mathrm{S} / \mathrm{O}$ substitution. The $\varepsilon$-thionocaprolactone (tnCL) is generated from $\varepsilon$-caprolactone $(\mathrm{CL})$ via a one-step reaction with $\mathrm{P}_{4} \mathrm{~S}_{10}$ (see Experimental Section); the reaction is workable on at least a $2 \mathrm{~g}$ scale (75\% yield). ${ }^{16,17}$ The application of DBU (5 mol \%; Table 5.1) to a $\mathrm{C}_{6} \mathrm{D}_{6}$ solution of tnCL (2 M) and octadecylthiol (1 mol $\%$ ) results in $90 \%$ conversion to polymer in $25 \mathrm{~h} .{ }^{13} \mathrm{C}$ NMR analysis of isolated tnCL suggests quantitative retention of the thiono-substitution, forming poly( $(\varepsilon-$ thionocaprolactone) (PtnCL) (see Figure 5.12). The room temperature application of DBU for the ROP of tnCL from benzyl alcohol results in a linear evolution of $\mathrm{M}_{\mathrm{n}}$ versus time and an initially narrow $\mathrm{M}_{\mathrm{w}} / \mathrm{M}_{\mathrm{n}}$ that broadens throughout the ROP (Figure 5.1). The guanidine bases, MTBD and TBD, were also applied for the ROP of tnCL. MTBD exhibited a similar activity in the ROP of tnCL to that of DBU whereas TBD effected a faster but less controlled ROP, resulting in erosion of $\mathrm{M}_{\mathrm{w}} / \mathrm{M}_{\mathrm{n}}$ (Table 5.1).

PtnCL was previously only available through the application of alkyllithiums at elevated temperatures which resulted in the uncontrolled ROP of tnCL. ${ }^{10}$ Endo's ROP of tnCL initiated from DBU at elevated temperatures was more controlled than the

alkyllithium ROP but resulted in scrambling of the S/O substitution (Scheme 5.1). ${ }^{13} \mathrm{C}$ NMR analysis of the polymer resulting from the repetition of our DBU-catalyzed ROP experiment at high temperature ( 1 equiv of tnCL, $5 \mathrm{~mol} \% \mathrm{DBU}$, toluene, $100^{\circ} \mathrm{C}$ ) in the presence of an alcohol initiator reveals that S/O scrambling does occur (see Figure 5.13), 
which suggests that the thiono/thio switching observed by Endo ${ }^{10}$ is simply due to heating the reaction solution.

The application of the phosphazene base, BEMP (Table 5.1), to a $\mathrm{C}_{6} \mathrm{D}_{6}$ solution of tnCL and benzyl alcohol does not result in ROP. The observation that the considerably more basic (vs DBU or MTBD) but non-nucleophilic BEMP $\left(\mathrm{BEMP}-\mathrm{H}+\right.$; $\mathrm{pK}_{\mathrm{a}}^{\mathrm{MeCN}}=$ 27.6) ${ }^{18}$ does not effect ROP suggests a nucleophilic mode of action for DBU (DBU-H+; $\left.\mathrm{pK}_{\mathrm{a}}{ }^{\mathrm{MeCN}}=24.3\right)^{19}$ and MTBD $\left(\mathrm{MTBD}-\mathrm{H}+; \mathrm{pK}_{\mathrm{a}}^{\mathrm{MeCN}}=25.4\right)^{19}$ in the ROP of tnCL (Scheme 5.2). DBU and MTBD have previously been suggested to operate as nucleophiles in ROP. ${ }^{20}$ Conducting the DBU catalyzed ROP of tnCL from alcoholic initiators (either benzyl alcohol or 1-pyrenebutanol) results in minimally altered $\mathrm{M}_{\mathrm{w}} / \mathrm{M}_{\mathrm{n}}$ compared to when initiated from octadecylthiol (Table 5.1). This suggests no reduction in the "living" character of the ROP due to slower initiation from alcohols vs thiols.

Organic Base and Thiourea Catalyzed ROP

The presence of thiourea 1 (Table 5.2) has a distinct impact upon the base cocatalyzed ROP of tnCL. The 1/DBU (5 mol \% each) cocatalyzed ROP of tnCL from octadecylthiol in $\mathrm{C}_{6} \mathrm{D}_{6}$ lowers the reaction time and $\mathrm{M}_{\mathrm{w}} / \mathrm{M}_{\mathrm{n}}$ versus the ROP with DBU alone. A similar effect is observed for MTBD (Table 5.2). The most striking results are observed with BEMP, which exhibits no activity in the absence of 1 , but the 1/BEMP (5 mol \% each) catalyzed ROP of tnCL ( $2 \mathrm{M}, 1$ equiv) from benzyl alcohol (1 mol \%) achieves full conversion in $5 \mathrm{~h}$. The reaction is highly controlled, exhibiting the characteristics of a "living" polymerization: linear evolution of $M_{n}$ vs conversion, narrow $M_{w} / M_{n}(=1.10)$ (Figure 5.1), first-order evolution of [tnCL] vs time (see Figure 5.10), and a $M_{n}$ that is predictable from $[\mathrm{M}]_{0} /[\mathrm{I}]_{0}$ (Table 5.2), although polymers begin to become insoluble in 
benzene at elevated molecular weight $\left(\mathrm{M}_{\mathrm{n}} \geq 22000\right)$. The ROP reaction proceeds in methylene chloride and chloroform but experiences reduced reaction control. Despite the narrow $\mathrm{M}_{\mathrm{w}} / \mathrm{M}_{\mathrm{n}}$, the GPC traces taken throughout the polymerization show the gradual growth of a high molecular weight tail, resulting in slight erosion of $\mathrm{M}_{\mathrm{w}} / \mathrm{M}_{\mathrm{n}}$ (see Figure 5.1 and Figure 5.4-6). ${ }^{13} \mathrm{C}$ NMR analysis of the isolated polymer confirms the quantitative retention of thiono-ester moieties, and the MALDI-TOF mass spectrum is consistent with linear benzyl alcohol-terminated PtnCL (see Figure 5.3). When initiated from 1pyrenebutanol, the refractive index and UV GPC traces of PtnCL overlap, including the high molecular weight tail (see Figure 5.4-6), suggesting end group fidelity. When allowed to stir past full conversion, the high weight tail on the GPC trace grows in prominence and eventually merges with the "main" polymer peak (see Figure 5.4-6). The high molecular weight tail in the GPC trace arises from an unknown postpolymerization reaction.

\section{Role of Thiourea in ROP}

The presence of the thiocarbonyl in tnCL was expected to perturb the ability of $\mathrm{H}$ bond donors to activate the monomer for ROP, yet the addition of thiourea $\mathbf{1}$ to the ROP solution clearly affects the course of the reaction. An NMR titration study ${ }^{21-23}$ was conducted in $\mathrm{C}_{6} \mathrm{D}_{6}$ to determine the binding constant between $\mathbf{1}$ and tnCL, $\mathrm{K}_{\mathrm{eq}}=1.6 \pm 0.2$ (in Eq. 5.1). The comparable binding between CL and TU was measured to be $K_{\text {eq }}=42 \pm$ 5.7 and a similarly dramatic perturbation from this latter strong binding value was previously measured for $\mathrm{tCL}, \mathrm{K}_{\mathrm{eq}}=2.7 \pm 0.5 .{ }^{9}$ The remarkable ability of $\mathbf{1}$ to activate thCL and $\mathrm{tCL}$ toward ROP despite the weak binding exhibited by $\mathbf{1}$ toward these monomers suggests an incongruity in the approximation of "magnitude of binding" as "extent of activation". 
The clear effects of $\mathbf{1}$ upon the ROP of tnCL in the absence of strong binding suggests that 1 plays a mechanistic role that cannot be fully understood by the magnitude of a binding constant between $\mathbf{1}$ and monomer. Because $\mathbf{1}$ does not measurably bind to ethyl acetate (a surrogate for open polymer), ${ }^{8}$ an approximation of the kinetic bias of $\mathbf{1}$ for polymerization vs depolymerization (or transesterification) is at most the magnitude of the $1 /$ monomer binding constant, $\mathrm{K}_{\mathrm{eq}}=1.6$, or $\Delta \Delta \mathrm{G}^{\ddagger}=0.27 \mathrm{kcal} / \mathrm{mol}$ (see Figure 5.11). This would only be true if $\mathbf{1}$ activates s-cis and s-trans esters equally. Indeed, DFT (B3LYP/6$31 \mathrm{G}^{* *}$ ) calculations suggest that $\mathbf{1}$ is equally effective at the activation of (i.e., increasing the electophilicity of ${ }^{10} \mathrm{CL}$, tnCL, tCL, methyl thionoacetate, and methyl acetate. For these compounds, both the electrostatic charges at carbon $(\mathrm{C}=\mathrm{X})$ and polarity of the $\mathrm{C}=\mathrm{X}$ bond increase by $\sim 5-10 \%$ upon the binding of 1 (see Figure 5.19). The effects rendered by 1 upon ROP must then be due to interactions in the transition state that are not adequately reflected in the magnitude of the binding of $\mathbf{1}$ to monomer (vs polymer) in the reactants/products. The increased reaction control provided by 1 during the ROP of tnCL could arise from the suppression of transesterification events due to prominent secondary interactions (e.g., 1 to base cocatalyst). ${ }^{5,23}$ These results suggest that despite minimal binding to tnCL (or tCL), the H-bond mediated ROP of tnCL is operative by dual activation of monomer by $\mathbf{1}$ and of chain end by base (Scheme 5.3).

Thionocaprolactone vs Other Monomers

The terminal conversion of the DBU catalyzed ROP of tnCL from alcohol initiators showed a strong temperature dependence; we sought to measure the thermodynamics of ROP to energetically place tnCL among other cyclic (thio)lactone monomers. The equilibrium monomer concentration, $[\mathrm{tnCL}]_{\mathrm{eq}}$, of a TBD catalyzed ROP of tnCL from 
benzyl alcohol in $\mathrm{C}_{6} \mathrm{D}_{6}$ was monitored as a function of temperature (see Experimental Section). The resulting Van't Hoff plot allowed for the extraction of the thermodynamic parameters of ROP: ${ }^{24} \Delta \mathrm{H}^{\circ}=-5.79 \pm 0.32 \mathrm{kcal} / \mathrm{mol}(298 \mathrm{~K}) ; \Delta \mathrm{S}^{\circ}=-13.5 \pm 1.0 \mathrm{cal} /(\mathrm{mol}$ $\mathrm{K}) ;[\mathrm{tnCL}]_{\mathrm{eq}}=0.05 \mathrm{M}$ at $300 \mathrm{~K}$, and $\mathrm{T}_{\text {ceiling }}=156^{\circ} \mathrm{C}$ (see Figure 5.7). These values suggest that tnCL is most energetically similar to $\mathrm{VL}:{ }^{24} \mathrm{~T}_{\text {ceiling }}=149^{\circ} \mathrm{C}$. For comparison, the ceiling temperatures ( $\mathrm{T}_{\text {ceiling }}$ ) of $\mathrm{CL}$ and tCL are $\mathrm{T}_{\text {ceiling }}(\mathrm{CL})^{24}=261^{\circ} \mathrm{C}$ and $\mathrm{T}_{\text {ceiling }}(\mathrm{tCL})^{9}=7000^{\circ} \mathrm{C}$. The low ceiling temperature of tnCL accounts for the low monomer conversions which are observed when the ROP of tnCL is attempted at elevated temperatures. ${ }^{10}$ Kinetically, tnCL is more reactive than VL. VL will not undergo ROP in the presence of MTBD or DBU alone (no 1), and the increased reactivity of tnCL (vs VL) is attributed to the increased electophilicity of thionoesters (vs esters). In contrast, the thioester, tCL, was observed to exhibit behavior that is both more and less reactive than VL. ${ }^{9}$

Copolymerization with $\delta$-Valerolactone

The observation of similar ROP thermodynamics for tnCL and VL suggests that random copolymerizations of these two monomers are possible. When 1/BEMP (5 mol \% each) is added to a mixture of VL ( $1 \mathrm{M}, 0.5$ equiv), tnCL ( $1 \mathrm{M}, 0.5$ equiv) and benzyl alcohol (1 mol \%) in $\mathrm{C}_{6} \mathrm{D}_{6}$, both monomers are observed to undergo ROP at approximately equal rates in a first-order evolution of [monomer]s vs time plot $\left(\mathrm{k}_{\mathrm{tnCL}} / \mathrm{k}_{\mathrm{VL}}=1.07\right)$, suggesting random copolymer formation (see Figure 5.8). ${ }^{13} \mathrm{C}$ NMR analysis of the copolymer confirms random monomer incorporation as evidenced by the equal intensities of the well resolved tnCL-tnCL vs tnCL-VL resonances (72.15 vs $71.78 \mathrm{ppm}$ ) (see Figure 5.14). The monomer feed can be adjusted to higher or lower $\mathrm{VL} / \mathrm{tnCL}$ ratios to give gradient copolymers. ${ }^{13} \mathrm{C}$ NMR analyses also confirm the retention of $\mathrm{C}=\mathrm{S}$ substitution in 
the copolymers (see Figure 5.14). Whereas PtnCL is an oil at room temperature for all molecular weights examined in our lab $(<20 \mathrm{~kg} / \mathrm{mol})$, copolymers of tnCL and VL with greater than $70 \% \mathrm{VL}$ are solid at room temperature. The materials properties $\left(\mathrm{T}_{\mathrm{m}}, \mathrm{T}_{\mathrm{c}}\right.$, and $\left.\mathrm{T}_{\mathrm{deg}}\right)$ of $\mathrm{P}(\mathrm{tnCL}-\mathrm{co}-\mathrm{VL})$ with varying tnCL content were analyzed by differential scanning calorimetry (DSC) and thermal gravimetric analysis (TGA, under $\mathrm{N}_{2}$ ). Polymers with increasing tnCL content show predictably reduced $\mathrm{T}_{\mathrm{m}}, \mathrm{T}_{\mathrm{c}}$, and $\mathrm{T}_{\mathrm{deg}}$ (Table 5.3). The hydrolytic stability of copolymers was measured under basic, acidic, and neutral conditions by established methods ${ }^{25}$ (Figure 5.2). Increased tnCL content in copolymers with VL is associated with reduced hydrolytic stability under basic conditions, increased stability toward hydrolysis under acidic conditions, and minimally altered stability in neutral water. These observations are consistent with general trends of thio(no)ester stability. ${ }^{12}$ To our knowledge, these are the only known characterizations of poly(thionoester-co-ester)s. 


\section{CONCLUSION}

The organocatalytic ROP of tnCL exhibits the characteristics of a "living" polymerization, particularly in the presence of an $\mathrm{H}$-bond donating thiourea, $\mathbf{1}$. The marked effect of $\mathbf{1}$ upon the course of the ROP is notable because it occurs in the absence of a strong binding between the H-bond donor and monomer. The increase in reaction rate and reaction control for the ROP of tnCL in the presence of $\mathbf{1}$ cannot be accounted for by the traditional model of selectivity in the differential ability of $\mathbf{1}$ to bind to monomer or polymer, and further studies are required to elucidate the source of selectivity, presumably due to interactions in the transition state. Copolymers of tnCL with VL are to our knowledge the first reported and characterized copolymers of S-lactone and lactone

monomers. The incorporation of tnCL to construct random (statistical), gradient, or block copolymers with traditional esters offers a unique and convenient method for tuning materials properties for custom tailored applications; the multitude of possible copolymers provides a wealth of research opportunities. 


\section{LIST OF REFERENCES}

(1) Dove, A. P. ACS Macro Lett. 2012, 1 (12), 1409-1412.

(2) Kiesewetter, M. K.; Shin, E. J.; Hedrick, J. L.; Waymouth, R. M. Macromolecules 2010, 43 (5), 2093-2107.

(3) Kamber, N. E.; Jeong, W.; Waymouth, R. M.; Pratt, R. C.; Lohmeijer, B. G. G.;

Hedrick, J. L. Chem. Rev. 2007, 107 (12).

(4) Cooley, C. B.; Trantow, B. M.; Nederberg, F.; Kiesewetter, M. K.; Hedrick, J. L.;

Waymouth, R. M.; Wender, P. A. J. Am. Chem. Soc. 2009, 131 (45), 1640116403.

(5) Kazakov, O. I.; Datta, P. P.; Isajani, M.; Kiesewetter, E. T.; Kiesewetter, M. K. Macromolecules 2014, 47, 7463-7468.

(6) Koeller, S.; Kadota, J.; Peruch, F.; Deffieux, A.; Pinaud, N.; Pianet, I.; Massip, S.; Léger, J. M.; Desvergne, J. P.; Bibal, B. Chem. - A Eur. J. 2010, 16 (14), 41964205.

(7) Bas G. G. Lohmeijer Frank Leibfarth, John W. Logan, R. C. P.; David A. Long Fredrik Nederberg, Jeongsoo Choi, A. P. D.; Charles Wade and James L. Hedrick, R. M. W.; Lohmeijer, B. G. G.; Pratt, R. C.; Leibfarth, F.; Logan, J. W.; Long, D. A.; Dove, A. P.; Nederberg, F.; Choi, J.; Wade, C.; Waymouth, R. M.; Hedrick, J. L. Macromolecules 2006, 39 (25), 8574-8583.

(8) Pratt, R. C.; Lohmeijer, B. G. G.; Long, D. A.; Lundberg, P. N. P.; Dove, A. P.; Li, H.; Wade, C. G.; Waymouth, R. M.; Hedrick, J. L. Macromolecules 2006, 39 (23), $7863-7871$.

(9) Bannin, T. J.; Kiesewetter, M. K. Macromolecules 2015, 48 (16), 5481-5486. 
(10) Sanda, F.; Jirakanjana, D.; Hitomi, M.; Endo, T. Macromolecules 1999, 32, 80108014.

(11) Sanda, F.; Jirakanjana, D.; Hitomi, M.; Endo, T. J. Polym. Sci. Part A Polym. Chem. 2000, 38 (22), 4057-4061.

(12) Kricheldorf, H. R.; Schwarz, G. J. Macromol. Sci. Part A 2007, 44 (6), 625-649.

(13) Overberger, C. G.; Weise, J. K. J. Am. Chem. Soc. 1968, 90 (13), 3533-3537.

(14) Overberger, C. G.; Weise, J. K. J. Am. Chem. Soc. 1968, 90 (13), 3538-3543.

(15) Boge, A.; Voss, J. Chem. Ber. 1990, 123, 1733-1737.

(16) Curphey, T. J. Tetrahedron Lett. 2002, 43 (3), 371-373.

(17) Curphey, T. J. J. Org. Chem. 2002, 67 (18), 6461-6473.

(18) Schwesinger, R.; Schlempep, H.; Hasenfratz, C.; Willaredt, J.; Dambacher, T.; Breuer, T.; Ottaway, C.; Fletschinger, M.; Boele, J.; Fritz, H.; Putzas, D.; Rotter, H. W.; Bordwell, F. G.; Satish, A. V.; Ji, G.; Peters, E.; Peters, K.; Schnering, H. G. Von; Walz, L. Liebigs Ann. 1996, 1055-1081.

(19) Kaljurand, I.; Kütt, A.; Sooväli, L.; Rodima, T.; Mäemets, V.; Leito, I.; Koppel, I. A. J. Org. Chem. 2005, 70 (3), 1019-1028.

(20) Brown, H. A.; De Crisci, A. G.; Hedrick, J. L.; Waymouth, R. M. ACS Macro Lett. 2012, 1 (9), 1113-1115.

(21) Thordarson, P. Chem. Soc. Rev. 2011, 40 (3), 1305-1323.

(22) Webb, J. E. A.; Crossley, M. J.; Turner, P.; Thordarson, P. J. Am. Chem. Soc. 2007, 129 (22), 7155-7162.

(23) Kazakov, O. I.; Kiesewetter, M. K. Macromolecules 2015, 48 (17), 6121-6126.

(24) Duda, A.; Kowalski, A. In Handbook of Ring-Opening Polymerization; Dubois, P., 
Coulembier, O., Raquez, J.-M., Eds.; Wiley-VCH Verlag GmbH \& Co. KGaA, 2009; pp 1-52.

(25) Shearouse, W. C.; Lillie, L. M.; Reineke, T. M.; Tolman, W. B. ACS Macro Lett. 2015, 4 (3), 284-288. 


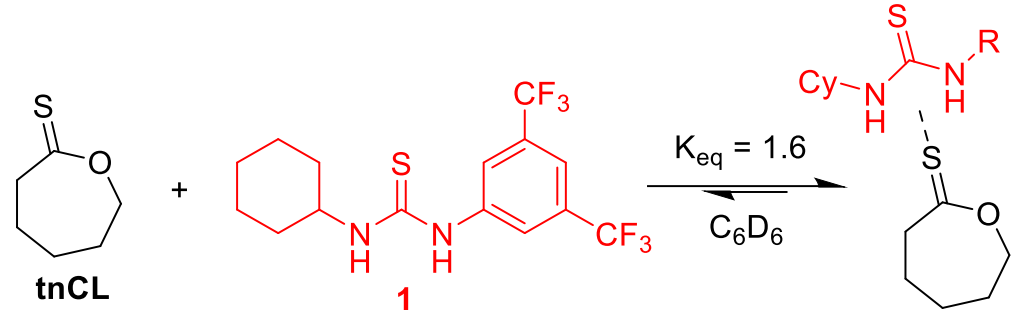

(Eq. 5.1) 


\section{CATIONIC ROP}

$\mathrm{n}$

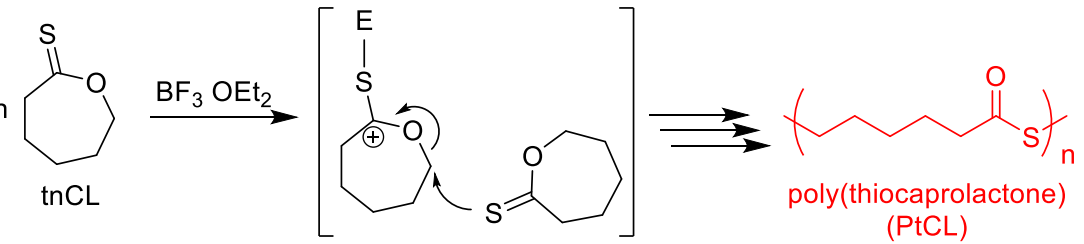

$\underline{\text { ANIONIC ROP }}$

n

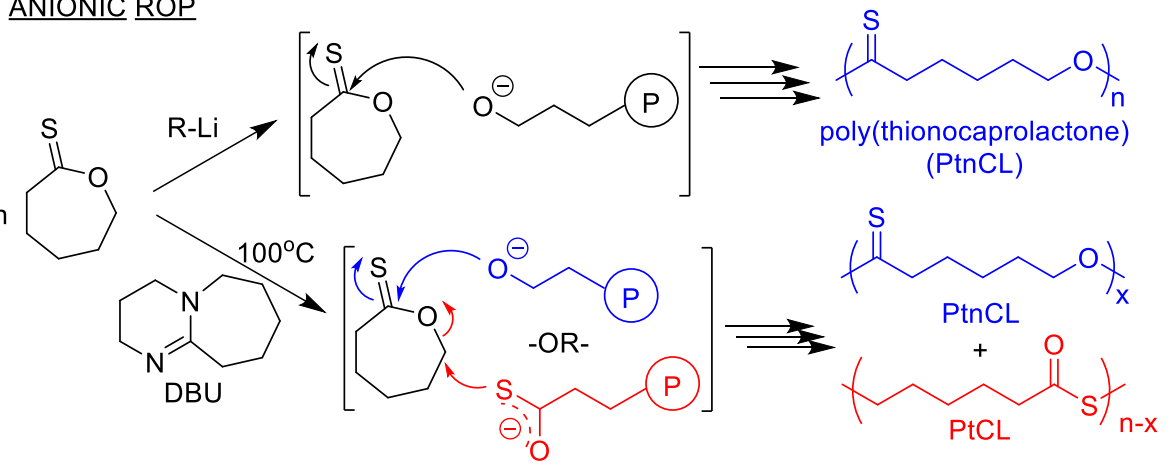

Scheme 5.1. Endo's anionic and cationic ROPs of tnCL have been shown to proceed with S/O scrambling. 




Scheme 5.2. Proposed mechanism for the DBU catalyzed ROP of tnCL. 




Scheme 5.3. Proposed mechanism for the H-bond mediated ROP of tnCL. 

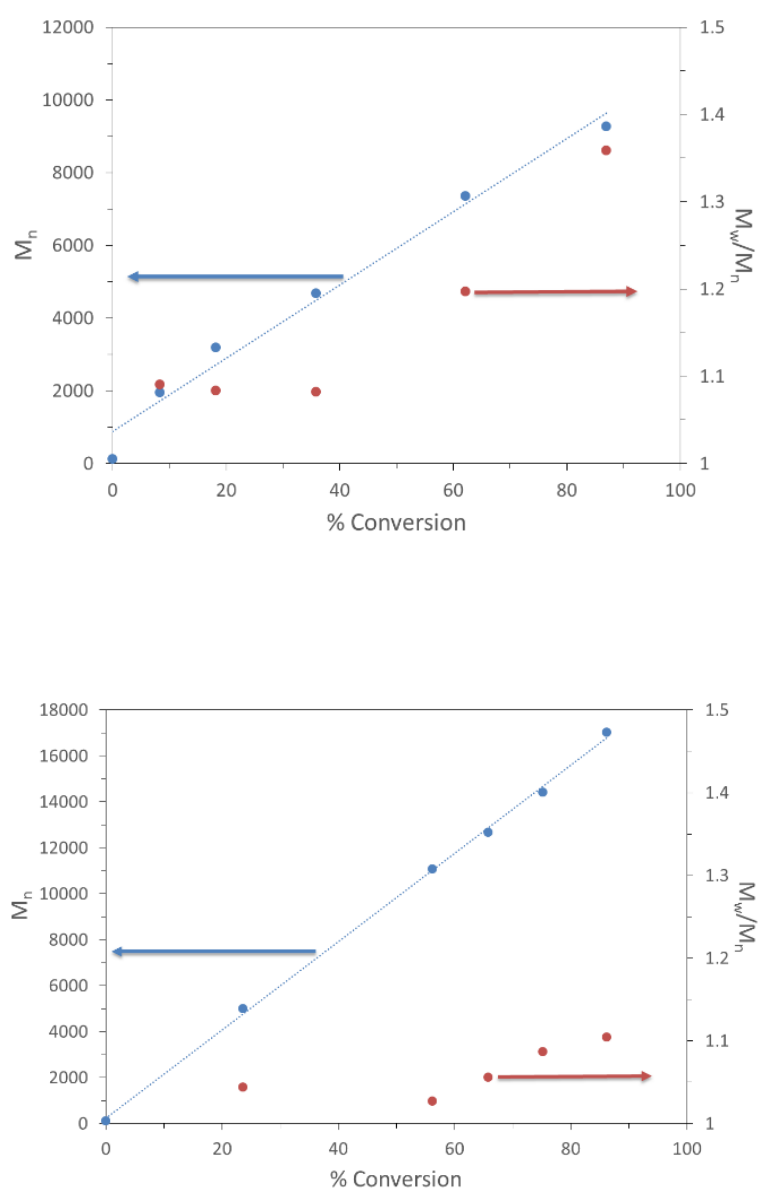

Figure 5.1. Evolution of $\mathrm{M}_{\mathrm{n}}$ vs conversion for the (upper) DBU catalyzed ROP of tnCL from benzyl alcohol; (lower) 1/BEMP ( 5 mol\% each) catalyzed ROP of tnCL (2M, 100 mg, 1 equiv.) from benzyl alcohol (1 mol\%). 

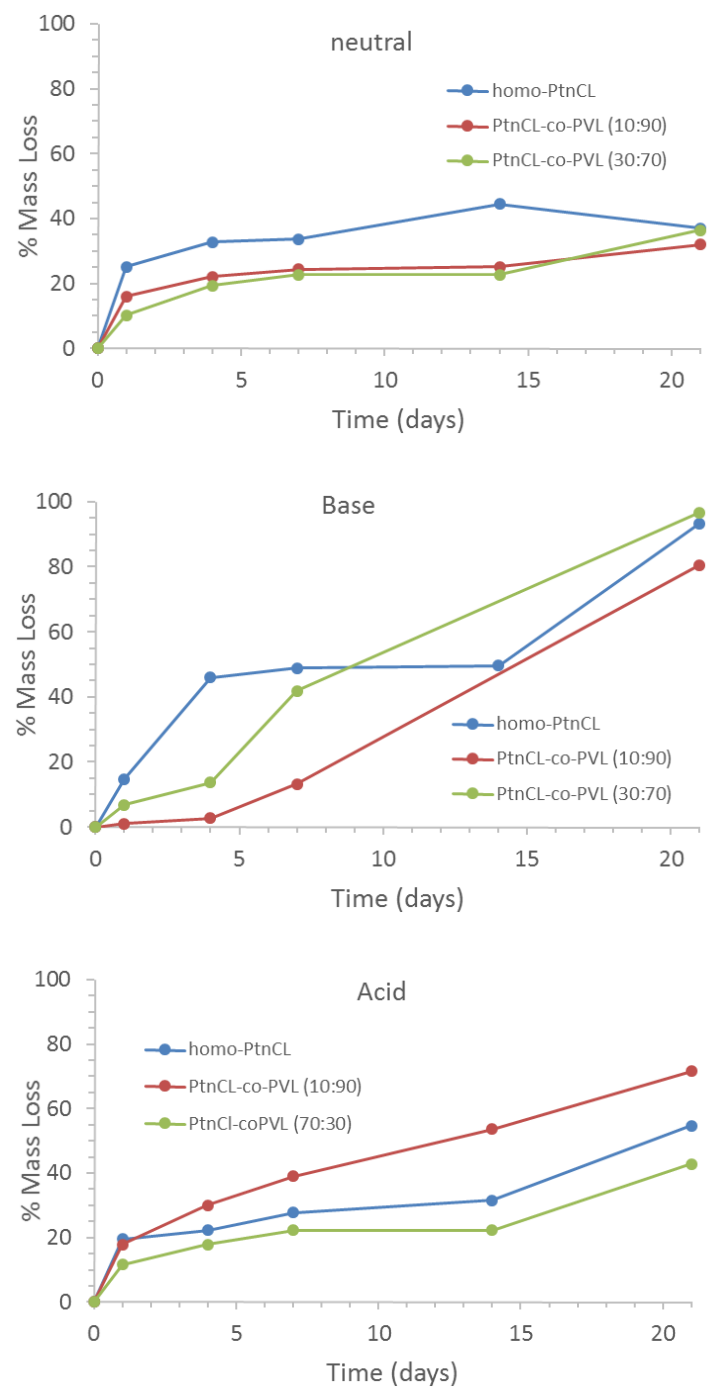

Figure 5.2. Percent mass loss for PtnCL and copolymers with VL in acidic (0.25 $\mathrm{M} \mathrm{HCl})$, basic $(0.25 \mathrm{M} \mathrm{NaOH})$ and neutral (distilled water) conditions vs time. The error from multiple measurements is $\pm 5 \%$. 


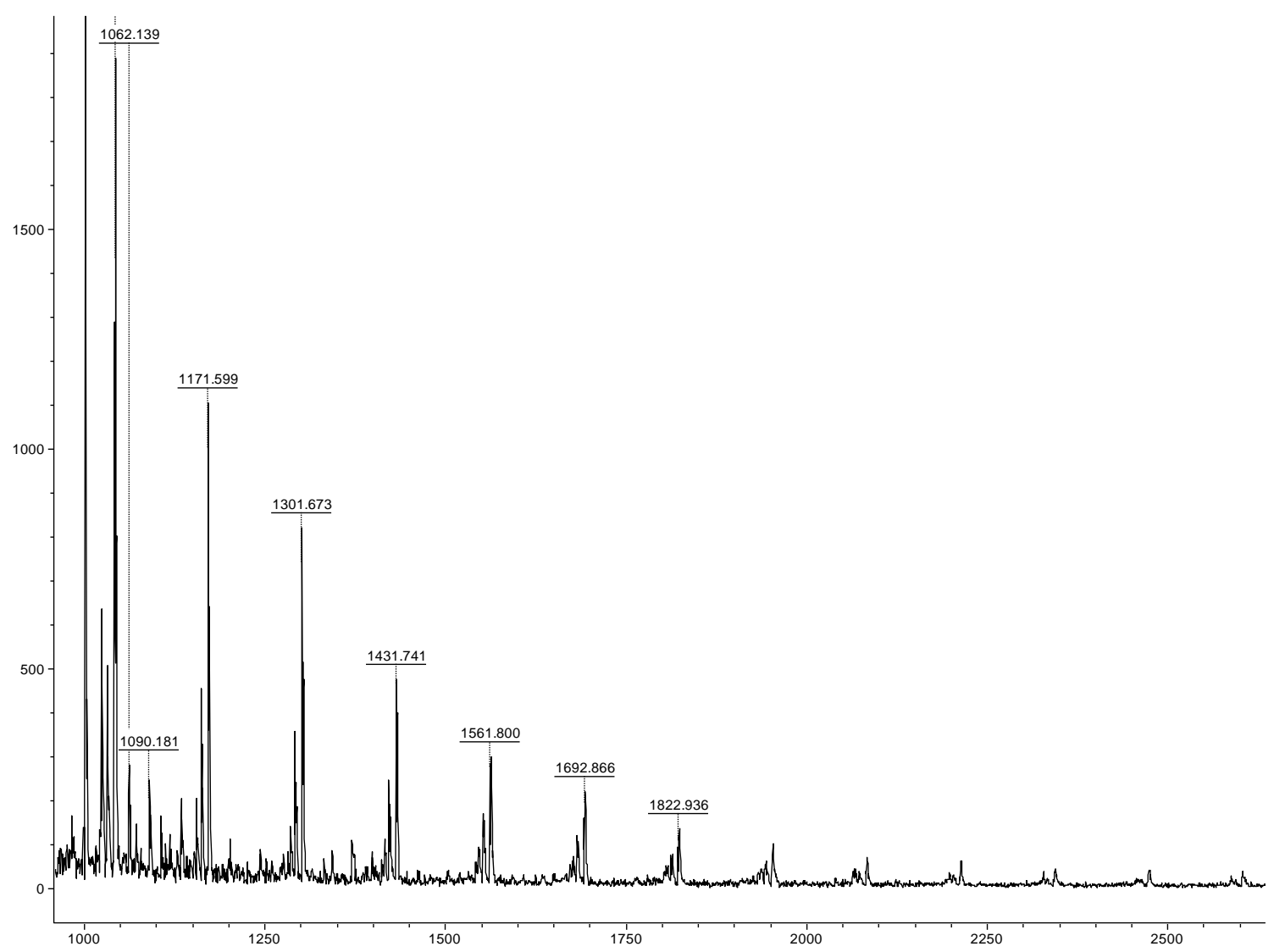

Figure 5.3. MALDI-TOF of the PtnCL resulting from the 1/BEMP catalyzed ROP of tnCL. Minor peaks could not be identified, but they are not consistent with $\mathrm{H}+, \mathrm{Li}+, \mathrm{Na}+$ or $\mathrm{K}+$ adducts of cyclic or linear PtnCL with benzyl alcohol or BEMP end groups. 


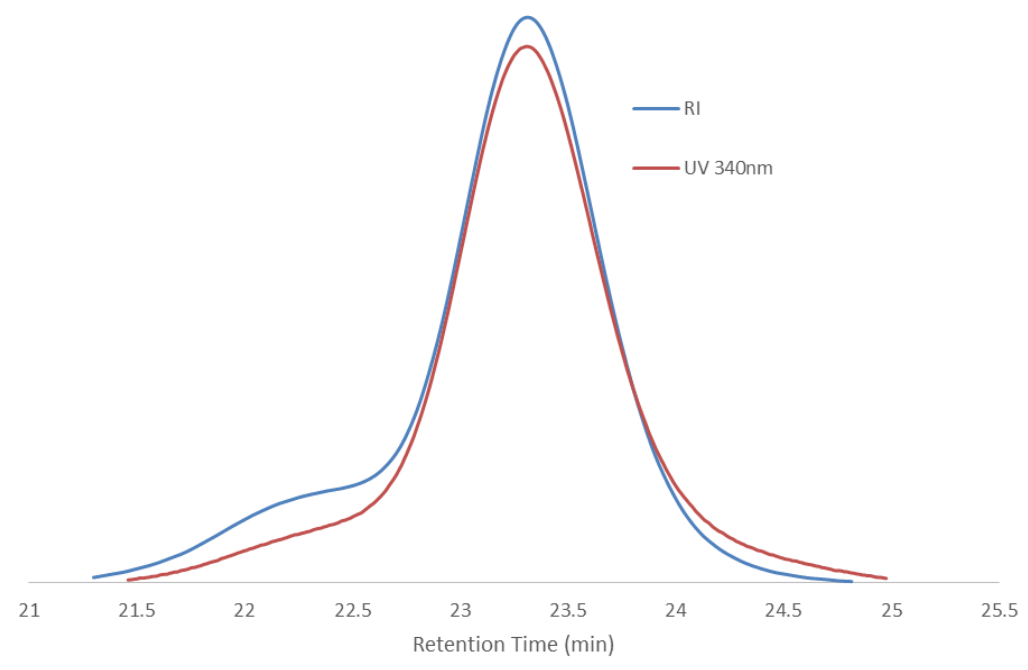

Figure 5.4. GPC trace of PtnCL resulting from the 1/BEMP catalyzed ROP of tnCL from pyrene butanol. The high weight tail grows in late in the ROP 




Figure 5.5. GPC traces (UV) showing the evolution of the peak shape as a function of conversion 




Figure 5.6. GPC traces (UV) of the polymer species resulting from allowing the ROP solution to stir with catalysts after full conversion (5h is full conversion). 


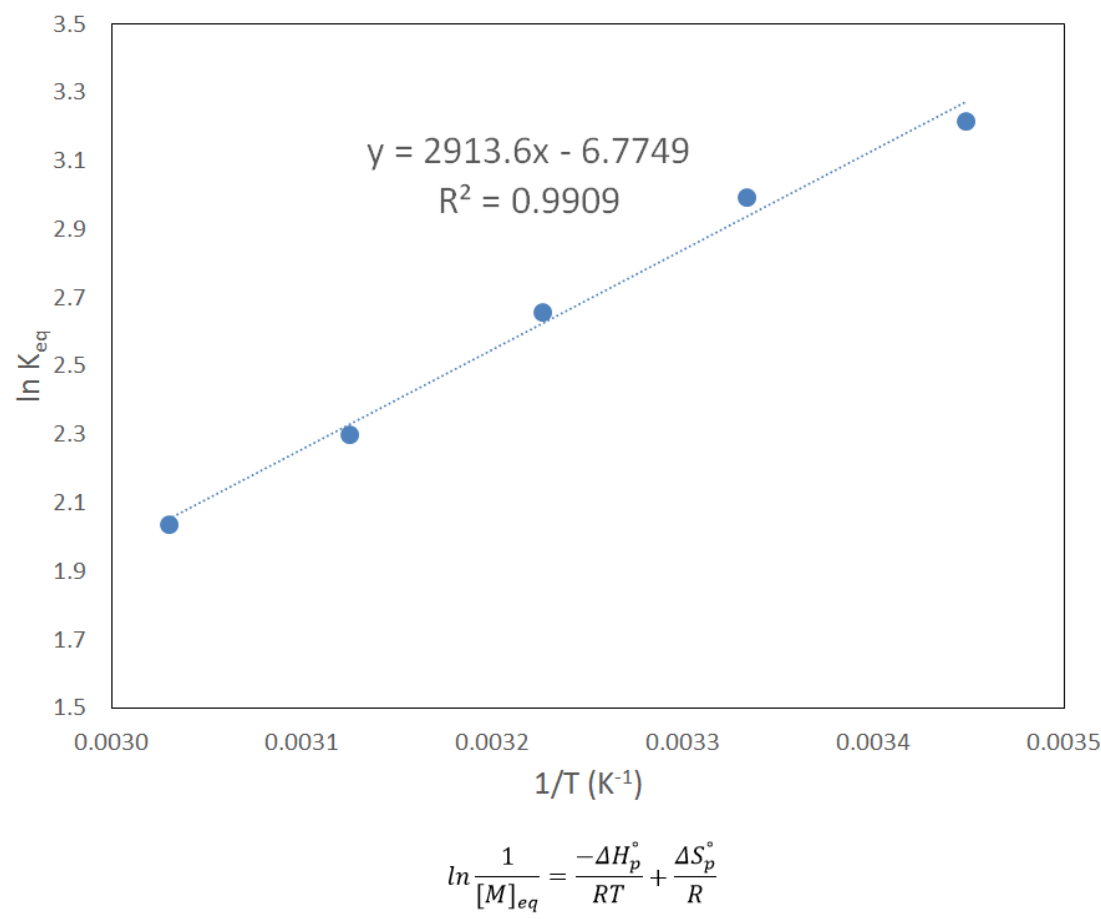

Figure 5.7. Temperature dependent equilibrium constant for the reversible ROP of tnCL catalyzed by TBD from benzyl alcohol. 


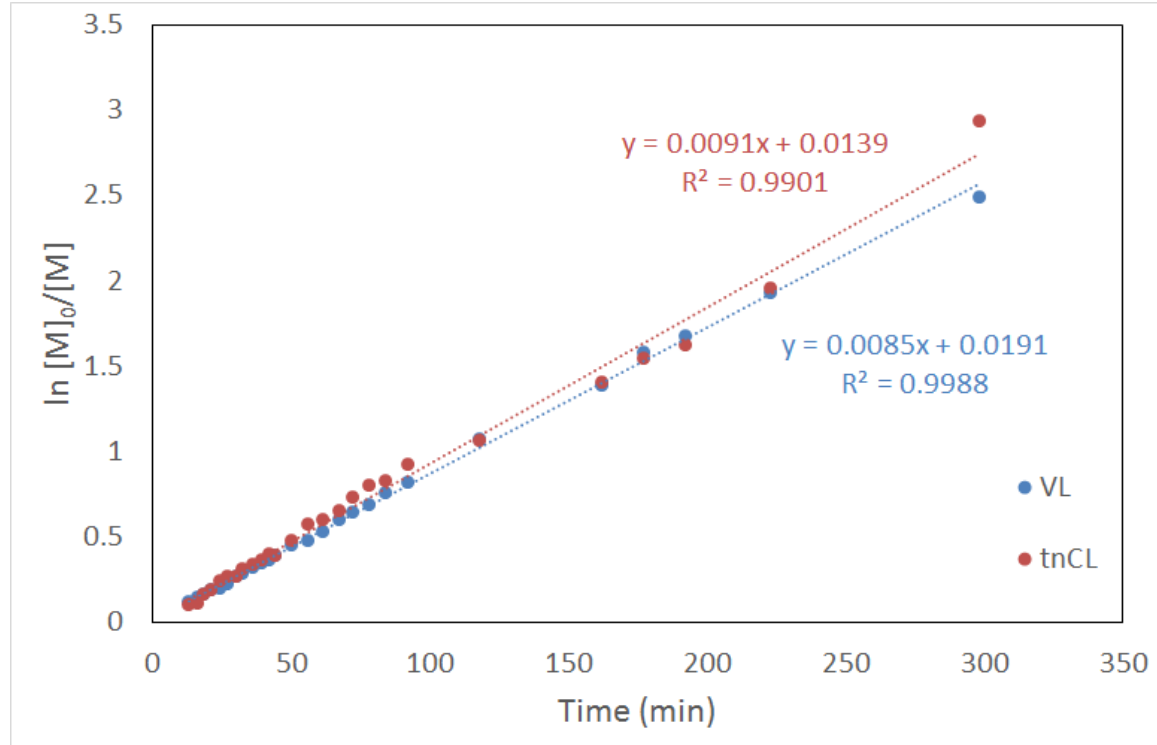

Figure 5.8. First order evolution of monomer vs time for the copolymerization of tnCL and VL. Reaction conditions tnCL (1M, $100 \mathrm{mg})$, VL (1M, $100 \mathrm{mg}), 1 \mathrm{~mol} \%$ benzyl alcohol, 5 mol\% BEMP, 5 mol\% 1 in $\mathrm{C}_{6} \mathrm{D}_{6}$. 


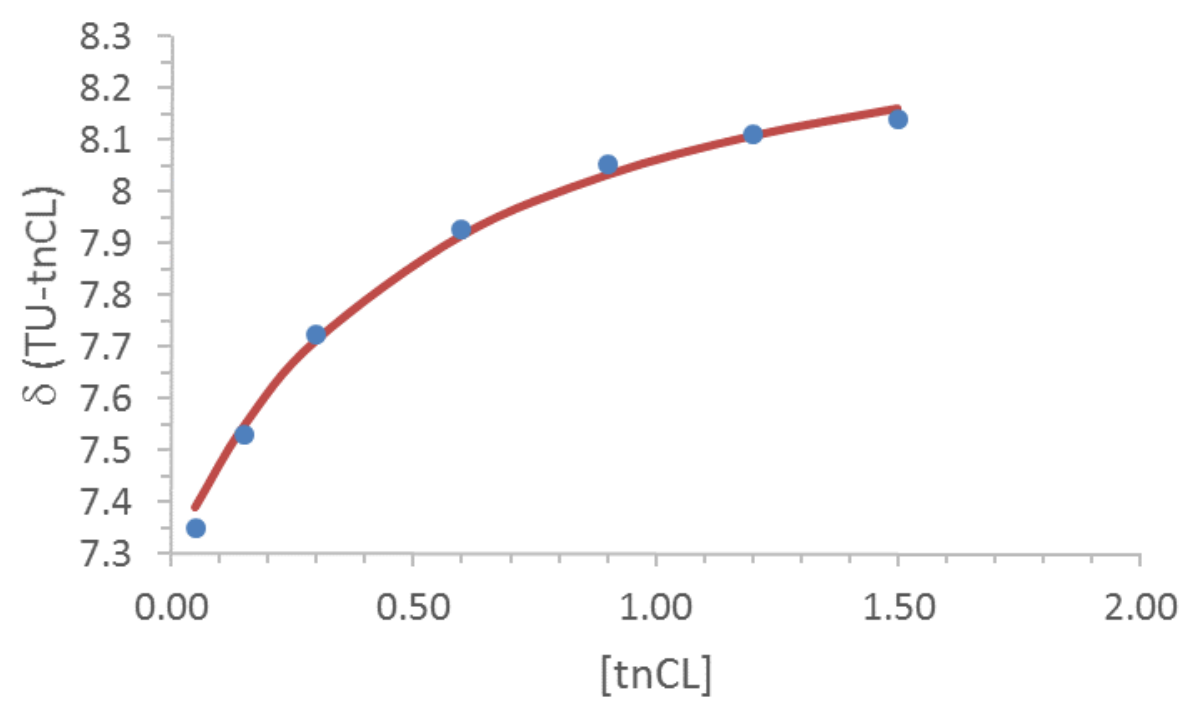

Figure 5.9. Titration curve for the binding of tnCL to $\mathbf{1}$. Observed chemical shift of $\mathbf{1}(o-$ protons, $5 \mathrm{mM}$ ) vs [tnCL] in $\mathrm{C}_{6} \mathrm{D}_{6}$. Solid line is the fit from the quadratic binding equation. 


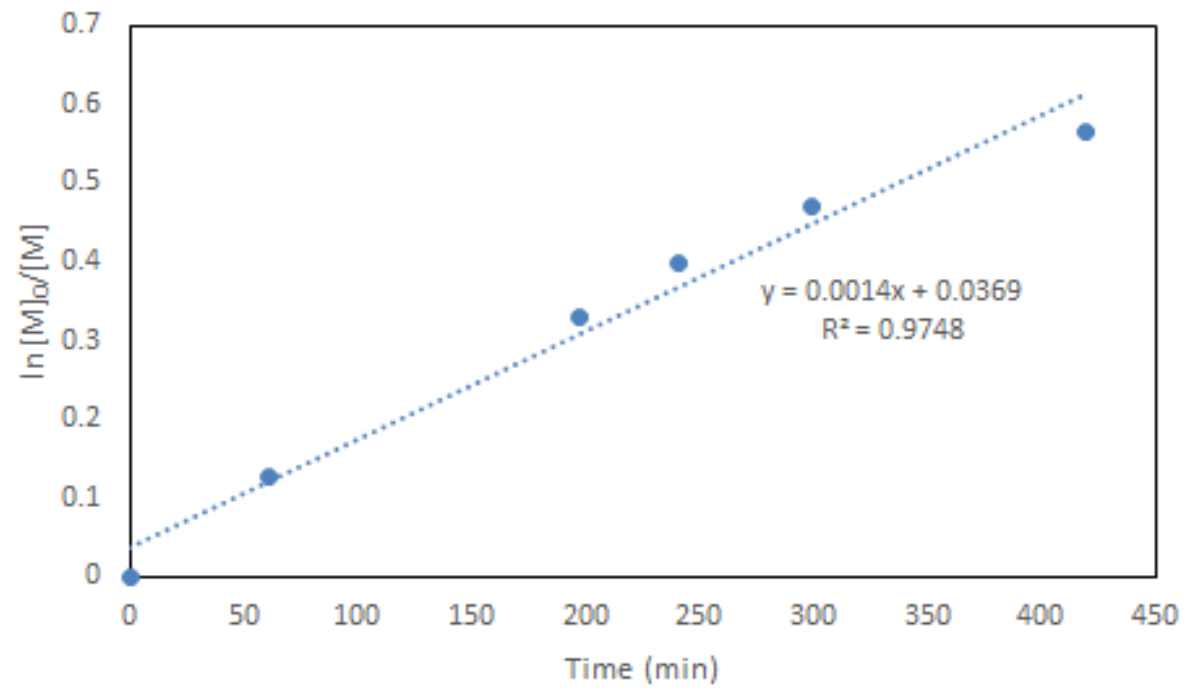

Figure 5.10. First order evolution of [tnCL] vs time in the 1/BEMP catalyzed ROP from benzyl alcohol. 

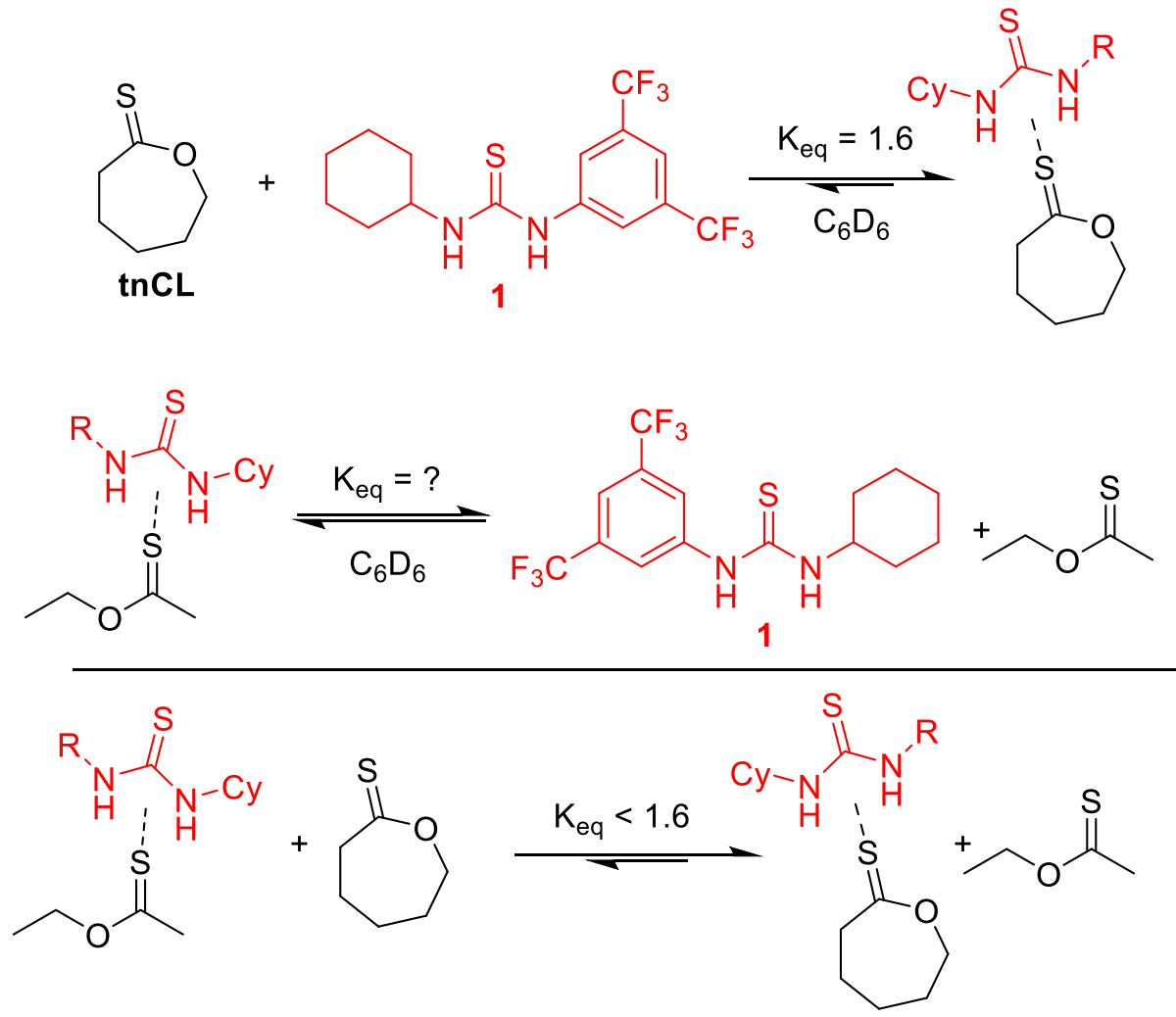

Figure 5.11. The binding of ethyl acetate (or ethyl thionoacetate) to $\mathbf{1}$ is too low to be measured, and the binding of tnCL to 1 is: $\mathrm{K}_{\mathrm{eq}}=1.6$. Because the binding constant of $\mathbf{1}$ to ethyl thionoacetate is greater than unity, the selectivity of $\mathbf{1}$ for tnCL must be: $\mathrm{K}_{\mathrm{eq}}(\mathrm{sel}$.) $\leq 1.6$, or $\Delta \Delta \mathrm{G}^{\neq}<0.27 \mathrm{kcal} / \mathrm{mol}$ if the selectivity at the reagents were to be translated to the transition state. This incongruity suggests other modes of action of $\mathbf{1}$ upon the reaction that are unique to the transition state. 


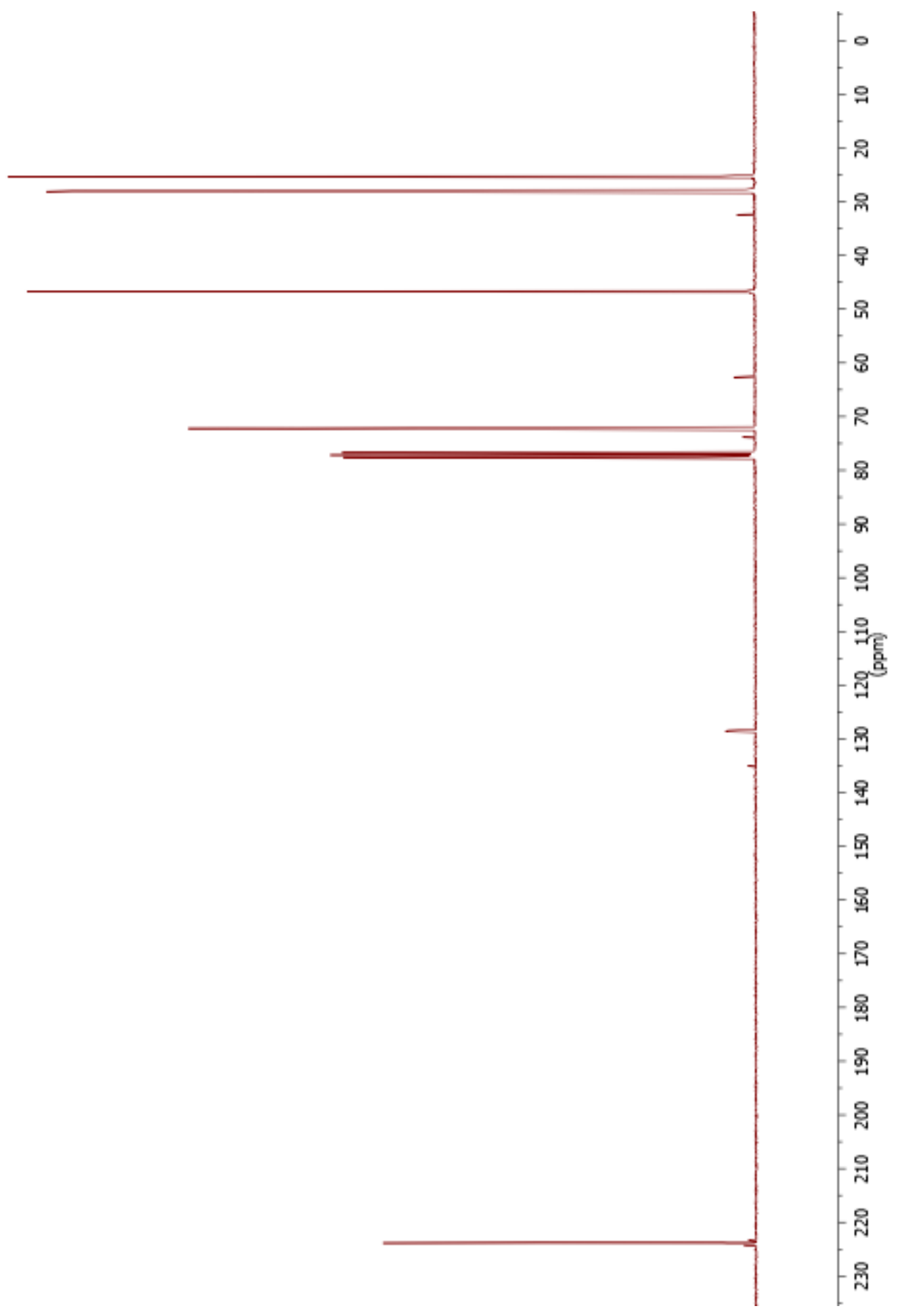

Figure 5.12. ${ }^{13} \mathrm{C} \mathrm{NMR}\left(75 \mathrm{MHz}, \mathrm{CDCl}_{3}\right)$ spectrum of the polymer resulting from the ROP of tnCL (2M, toluene) from benzyl alcohol (1 mol\%) catalyzed by DBU (5 mol\%) at room temperature. The formation of poly(thionocaprolactone) as evidenced by the carbonyl resonance at $223 \mathrm{ppm}$. 


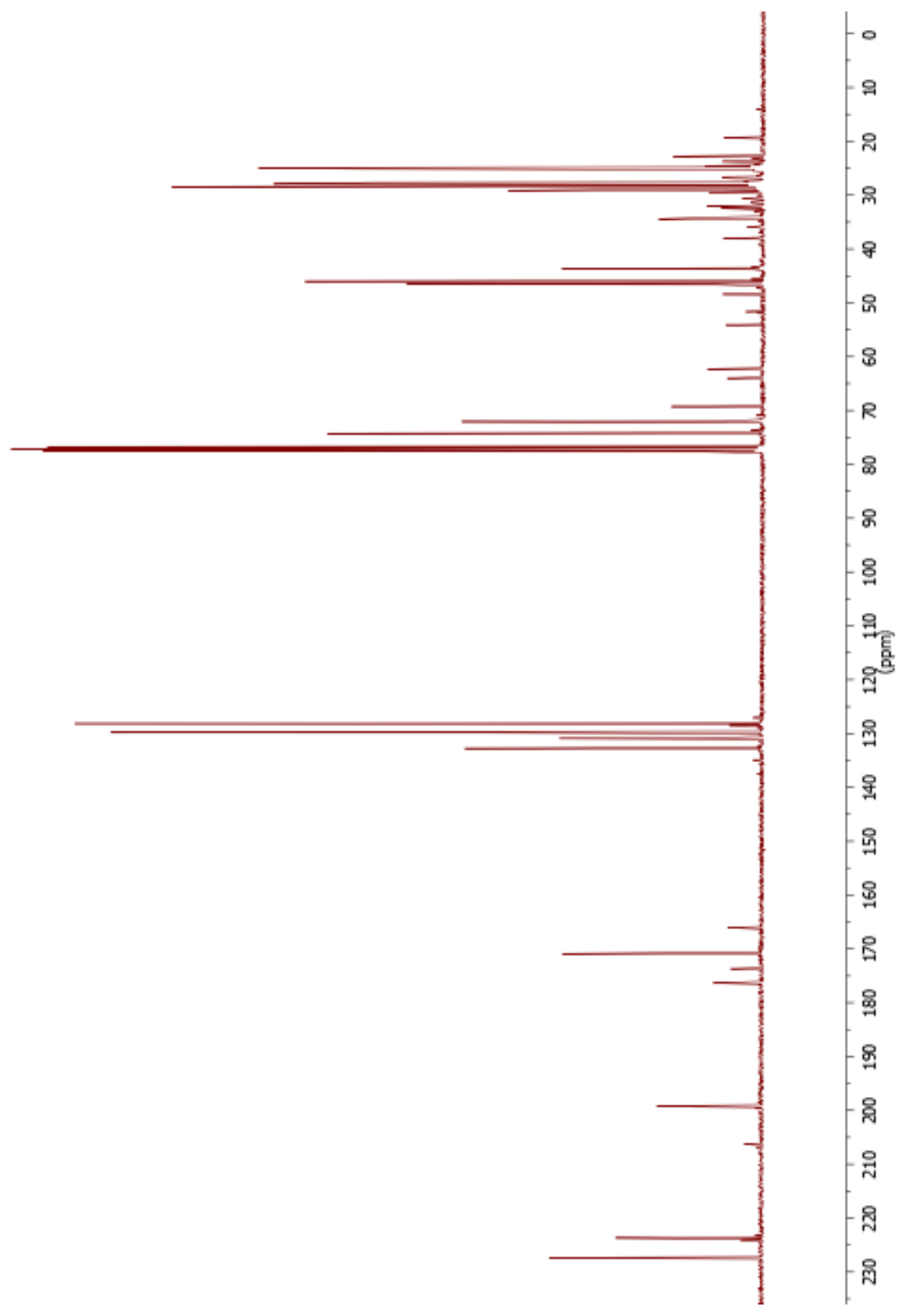

Figure 5.13. ${ }^{13} \mathrm{C} \mathrm{NMR}\left(75 \mathrm{MHz}, \mathrm{CDCl}_{3}\right)$ spectrum of the polymer resulting from the ROP of tnCL (2M, toluene) from benzyl alcohol (1 mol\%) catalyzed by DBU (5 mol\%) at $100^{\circ} \mathrm{C}$ results in the formation of poly(thiono-co-thiocaprolactone) as evidenced by the two carbonyl resonances at $223 \mathrm{ppm}$ and $199 \mathrm{ppm}$. The most downfield resonance is due to unconverted monomer. 


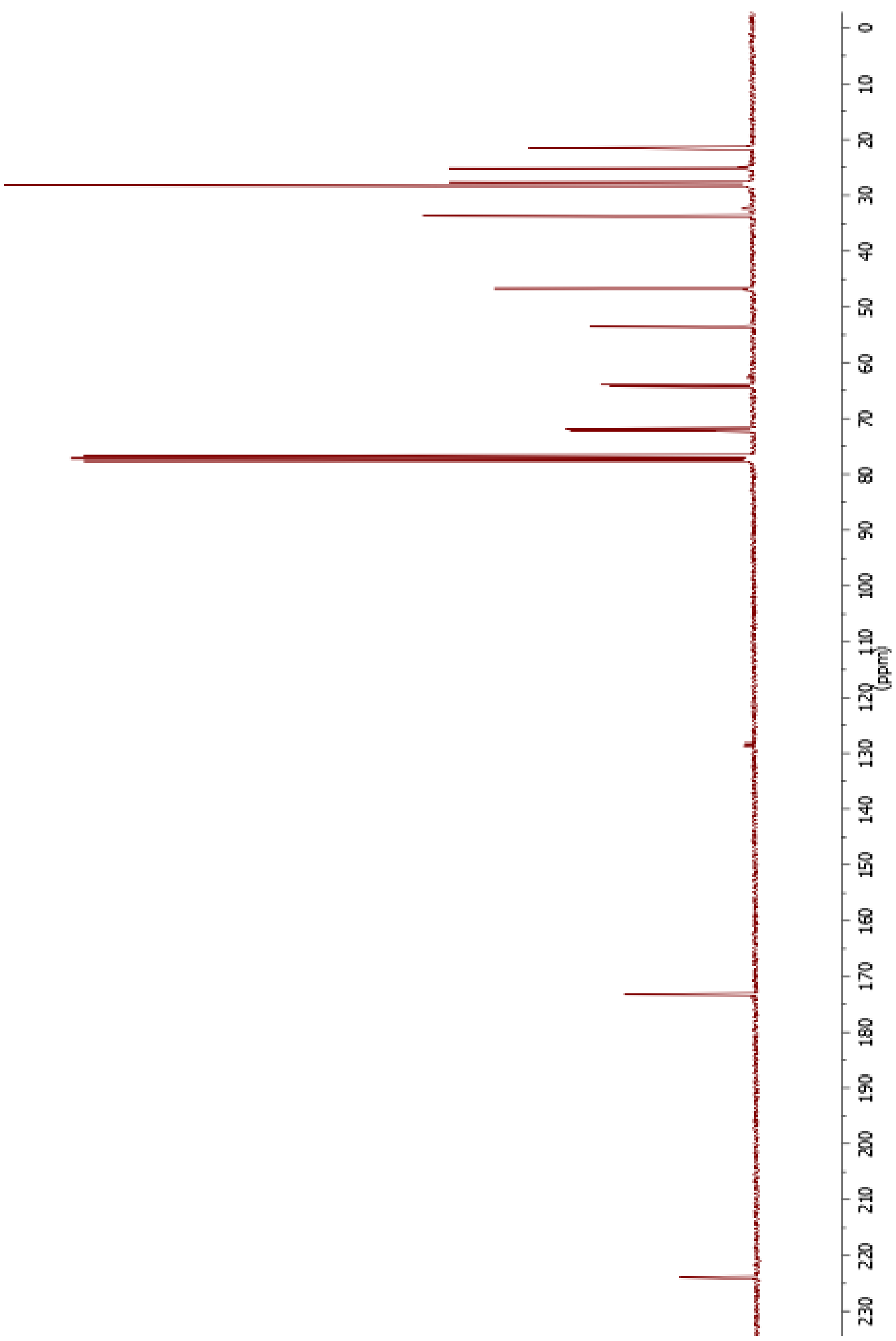

Figure 5.14. ${ }^{13} \mathrm{C}$ NMR (75 MHz, $\mathrm{CDCl}_{3}$ ) spectrum of $\mathrm{P}($ tnCL-co-VL) (50:50). 


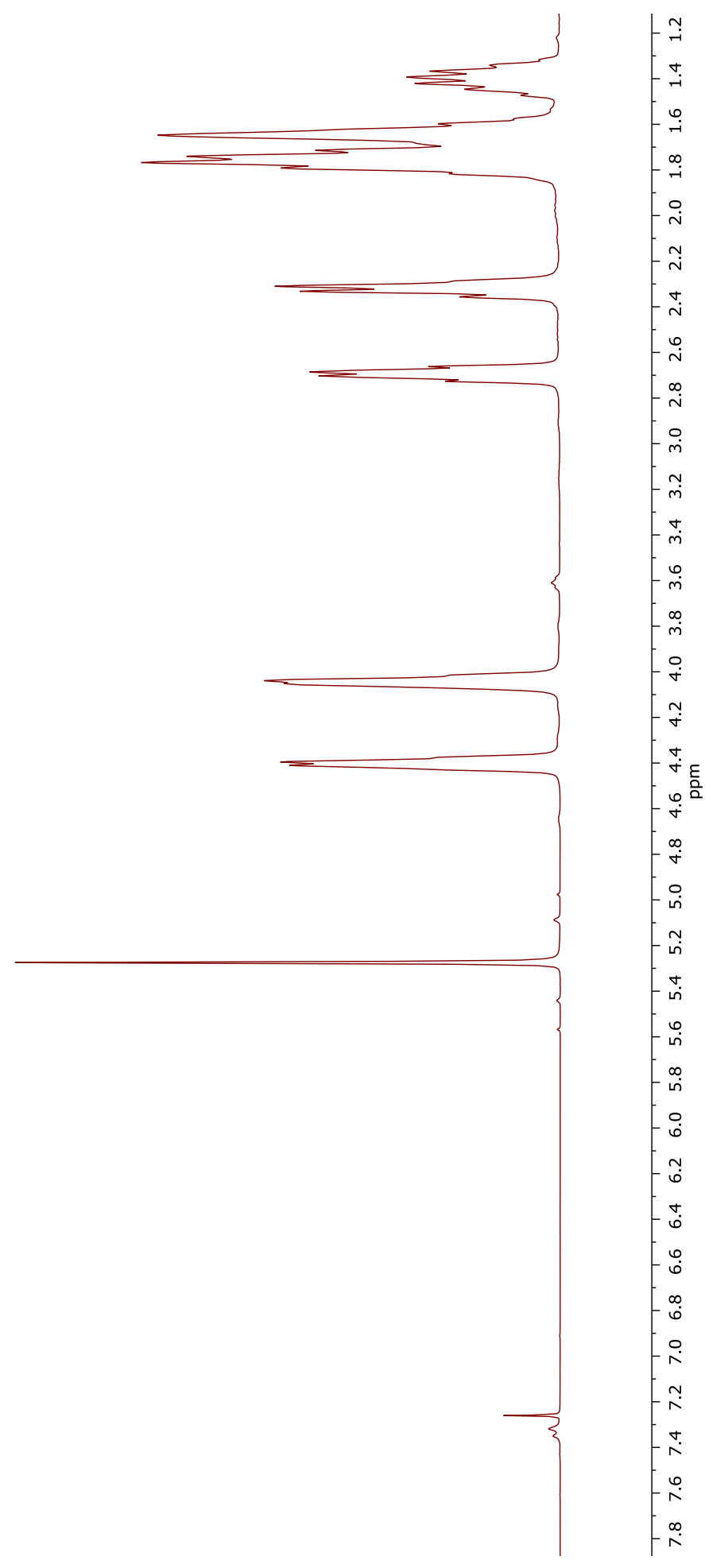

Figure 5.15. ${ }^{1} \mathrm{H}$ NMR (300 $\left.\mathrm{MHz}, \mathrm{CDCl}_{3}\right)$ spectrum of P(tnCL-co-VL) (50:50). 


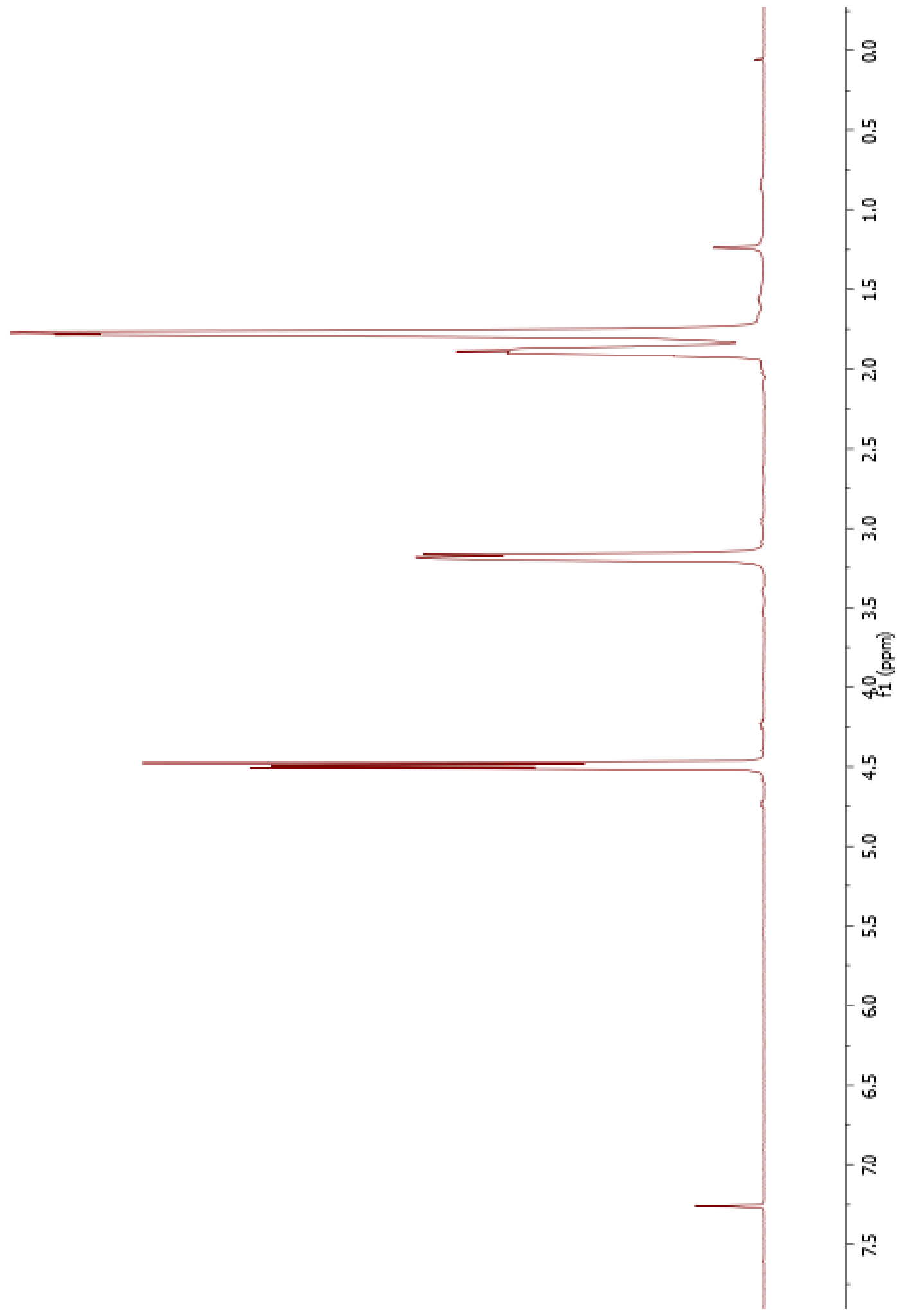

Figure 5.16. ${ }^{1} \mathrm{H} \mathrm{NMR}\left(300 \mathrm{MHz}, \mathrm{CDCl}_{3}\right)$ spectrum of tnCL. 


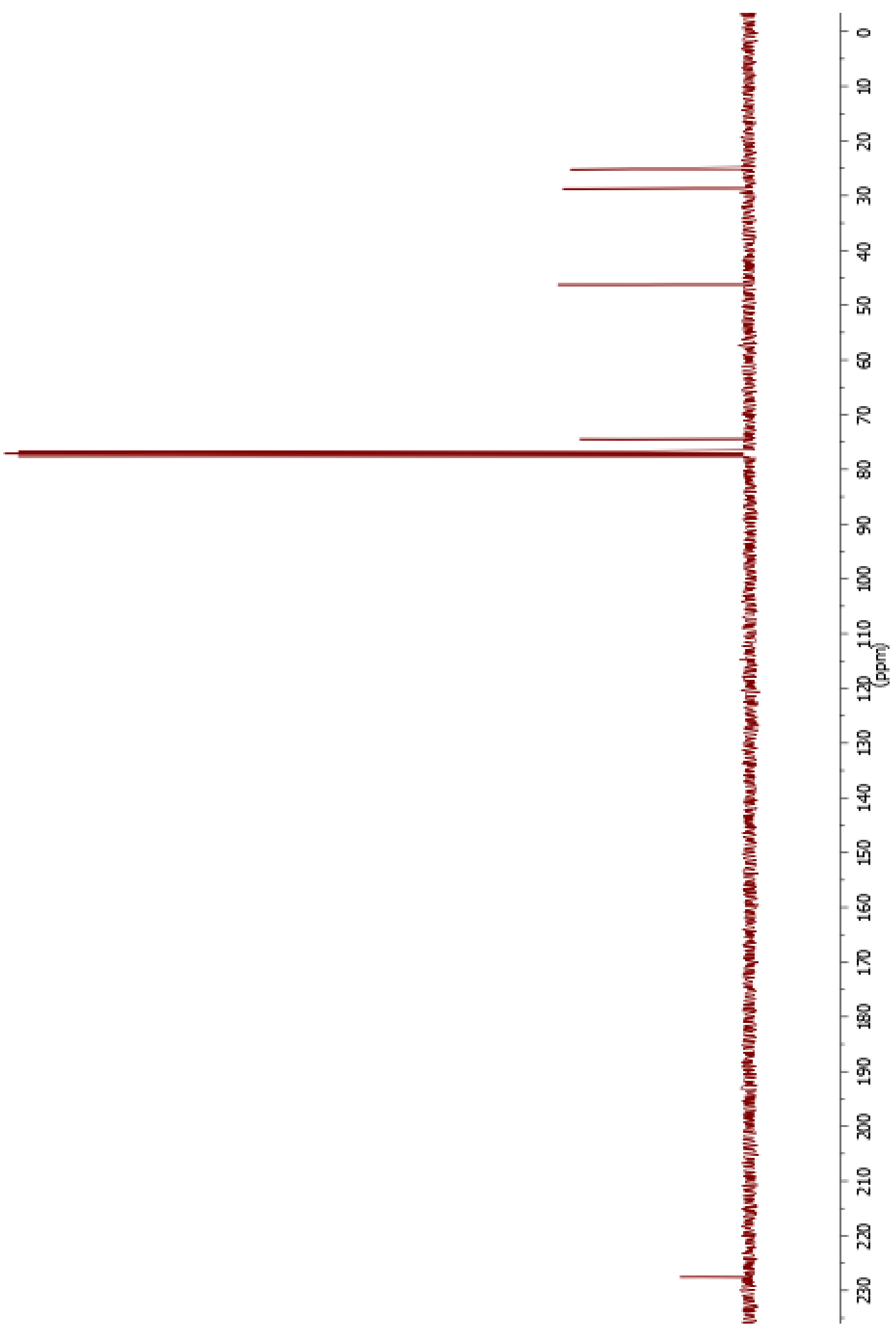

Figure 5.17. ${ }^{13} \mathrm{C} \mathrm{NMR}\left(75 \mathrm{MHz}, \mathrm{CDCl}_{3}\right)$ spectrum of tnCL. 


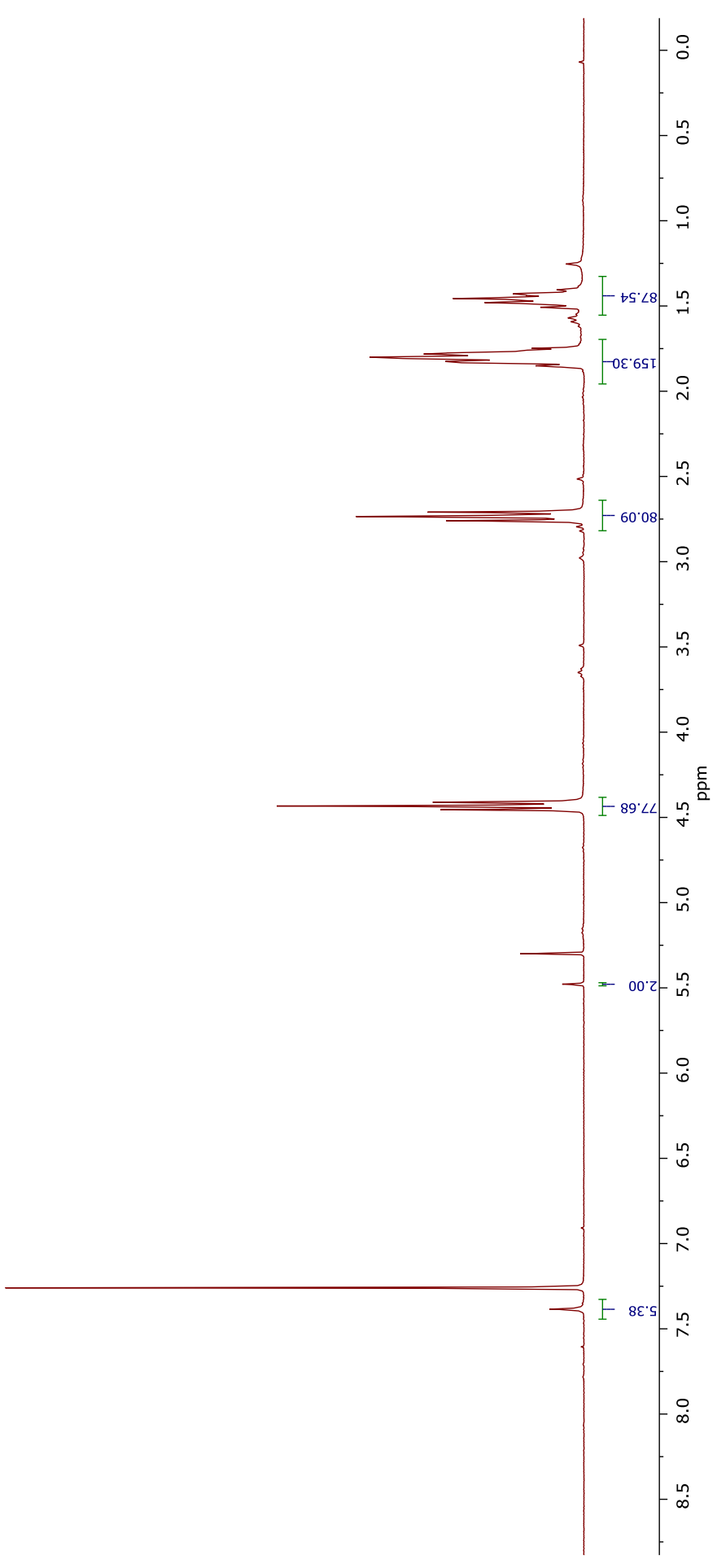

Figure 5.18. ${ }^{1} \mathrm{H}$ NMR $\left(300 \mathrm{MHz}, \mathrm{CDCl}_{3}\right)$ spectrum of PtnCL. 


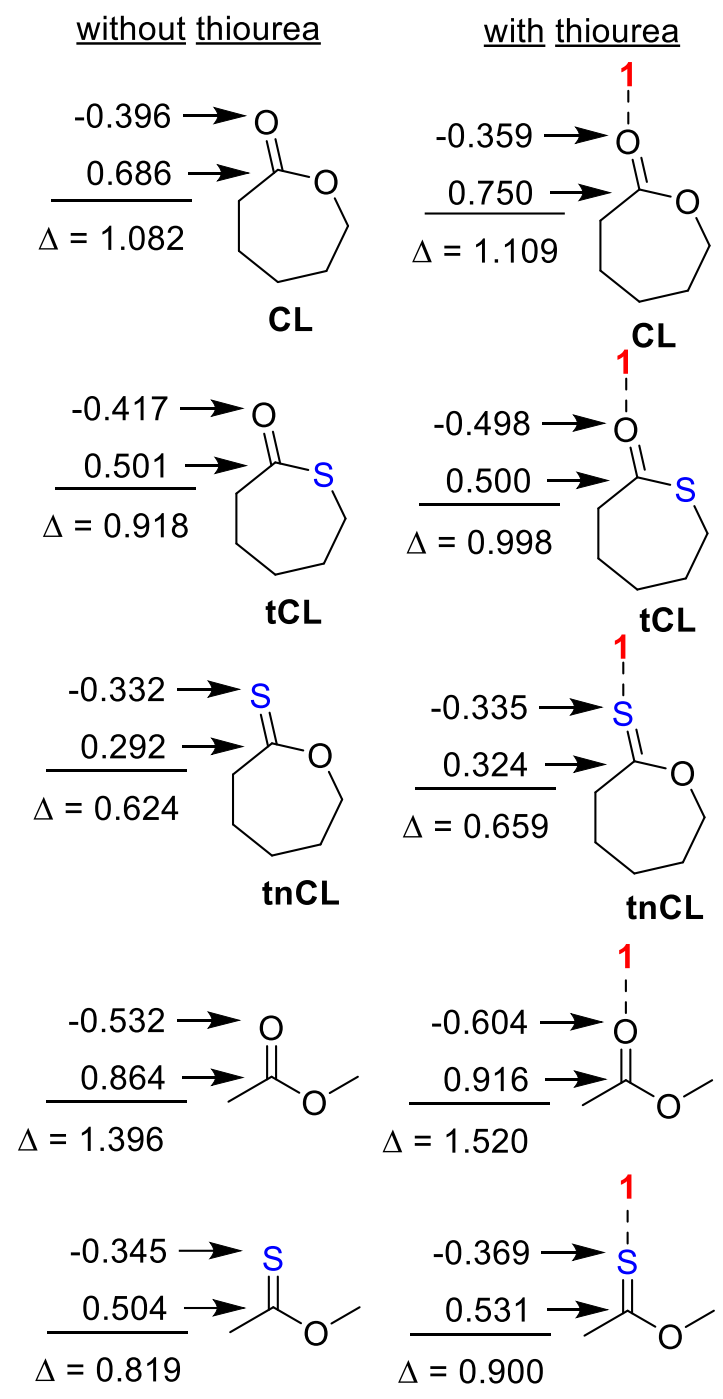

Figure 5.19. Calculated (DFT B3LYP//6-31G**) electrostatic potential of atoms in the $\mathrm{C}=\mathrm{X}$ bond of 7-membered and $s$-trans lactones. 


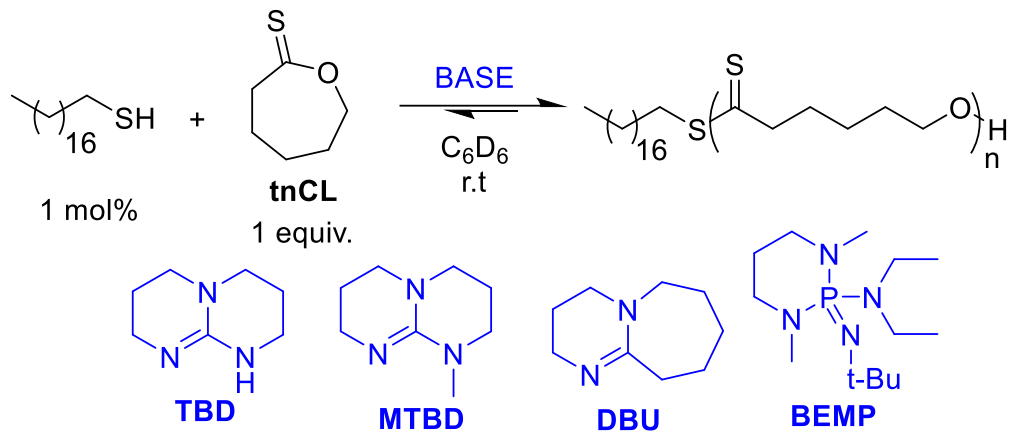

\begin{tabular}{|c|c|c|c|c|c|}
\hline entry & Base & conv. $^{\mathrm{b}}(\%)$ & time $(\mathrm{h})$ & $\mathrm{M}_{\mathrm{n}}{ }^{\mathrm{c}}(\mathrm{g} / \mathrm{mol})$ & $\mathrm{M}_{\mathrm{w}} / \mathrm{M}_{\mathrm{n}}{ }^{\mathrm{c}}$ \\
\hline $1^{\mathrm{d}}$ & TBD & $>99$ & 0.33 & 17,700 & 1.41 \\
\hline 2 & DBU & 89 & 25 & 17,200 & 1.30 \\
\hline 3 & MTBD & 90 & 23 & 14,100 & 1.24 \\
\hline 4 & BEMP & 0 & 25 & -- & -- \\
\hline $5^{\mathrm{e}}$ & DBU & 98 & 20 & 12,800 & 1.37 \\
\hline $6^{\mathrm{f}}$ & DBU & 98 & 19 & 19,700 & 1.20 \\
\hline
\end{tabular}

Table 5.1. ROP of tnCL with Base Catalysts. ${ }^{a}$

a) Reaction conditions: $2 \mathrm{M}(0.77 \mathrm{mmol}, 1$ equiv) tnCL, $1 \mathrm{~mol} \%$ octadecylthiol, $5 \mathrm{~mol} \%$ base, and $\mathrm{C}_{6} \mathrm{D}_{6}$. b) Conversion to polymer obtained by ${ }^{1} \mathrm{H}$ NMR. c) Determined by GPC $\left(\mathrm{CH}_{2} \mathrm{Cl}_{2}\right)$ vs polystyrene standards. d) 1 mol\% TBD. e) Initiation was from benzyl alcohol (1 mol\%). f) initiation was from 1-pyrenebutanol (1 mol\%). 


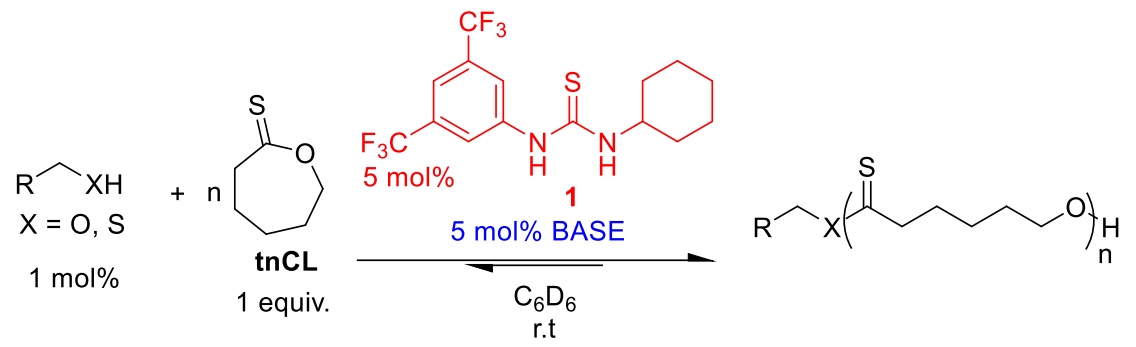

\begin{tabular}{|c|c|c|c|c|c|c|}
\hline entry & initiator & Base & conv. $^{\mathrm{b}}(\%)$ & time $(\mathrm{h})^{\mathrm{M}}$ & $\mathrm{M}_{\mathrm{n}}{ }^{\mathrm{c}}(\mathrm{g} / \mathrm{mol})$ & $\mathrm{M}_{\mathrm{w}} / \mathrm{M}_{\mathrm{n}}{ }^{\mathrm{c}}$ \\
\hline 1 & octadecylthiol & DBU & 93 & 17 & 22,500 & 1.14 \\
\hline 2 & octadecylthiol & MTBD & 92 & 19 & 14,800 & 1.16 \\
\hline 3 & octadecylthiol & BEMP & 84 & 5 & 14,600 & 1.25 \\
\hline 4 & 1-pyrenebutanol & BEMP & 89 & 6 & 15,200 & 1.11 \\
\hline 5 & benzyl alcohol & BEMP & 91 & 5 & 12,000 & 1.14 \\
\hline $6^{\text {d }}$ & benzyl alcohol & BEMP & 89 & 4.5 & 8,200 & 1.11 \\
\hline $7^{\text {e }}$ & benzyl alcohol & BEMP & 90 & 7 & 20,900 & 1.10 \\
\hline
\end{tabular}

Table 5.2. Thiourea Plus Base Cocatalyzed ROP of tnCL. ${ }^{a}$

a) Reaction conditions: $2 \mathrm{M}\left(0.77 \mathrm{mmol}, 1\right.$ equiv) tnCL, $1 \mathrm{~mol} \%$ octadecylthiol, $\mathrm{C}_{6} \mathrm{D}_{6}$ and given amount of catalyst. b) Conversion to polymer obtained by ${ }^{1} \mathrm{H}$ NMR. c) Determined by GPC $\left(\mathrm{CH}_{2} \mathrm{Cl}_{2}\right)$ vs polystyrene standards. d) 2 mol\% benzyl alcohol, $[\mathrm{M}]_{\mathrm{o}} /[\mathrm{I}]_{\mathrm{o}}=50$. e $)$ $0.5 \mathrm{~mol} \%$ benzyl alcohol, $[\mathrm{M}]_{\mathrm{o}} /[\mathrm{I}]_{\mathrm{o}}=200$. 


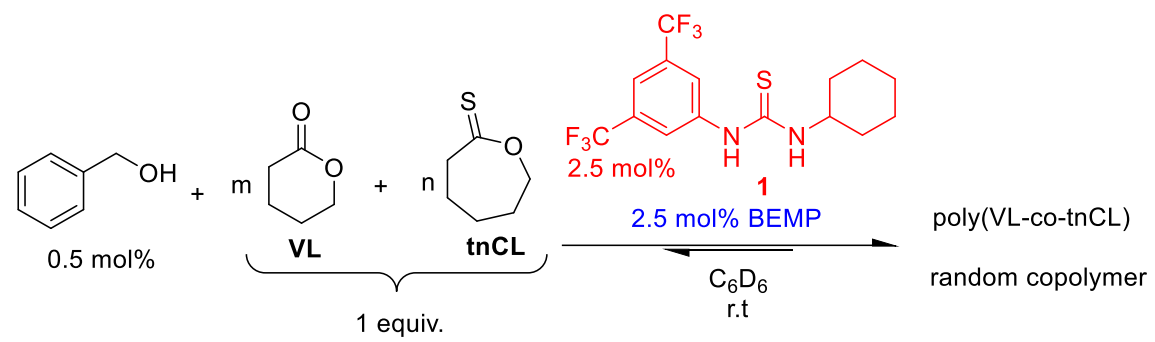

\begin{tabular}{|c|c|c|c|c|c|c|c|c|c|}
\hline entry & $\begin{array}{c}\text { tnCL } \\
(\% \text { feed })\end{array}$ & $\begin{array}{c}\text { VL } \\
(\% \text { feed })\end{array}$ & $\begin{array}{c}\text { time } \\
(\mathrm{h})\end{array}$ & $\begin{array}{c}\% \\
\text { conv. }^{\mathrm{b}}\end{array}$ & $\mathrm{M}_{\mathrm{n}}{ }^{\mathrm{c}}$ & $\mathrm{M}_{\mathrm{w}} / \mathrm{M}_{\mathrm{n}}{ }^{\mathrm{c}}$ & $\begin{array}{c}\mathrm{T}_{\mathrm{m}} \\
\left({ }^{\circ} \mathrm{C}\right)^{\mathrm{d}}\end{array}$ & $\begin{array}{c}\mathrm{T}_{\mathrm{c}} \\
\left({ }^{\circ} \mathrm{C}\right)^{\mathrm{d}}\end{array}$ & $\begin{array}{c}\mathrm{T}_{\text {deg }} \\
\left({ }^{\circ} \mathrm{C}\right)^{\mathrm{e}}\end{array}$ \\
\hline 1 & 0 & 100 & 5 & $0: 93$ & 12,300 & 1.06 & 53 & 27 & 380 \\
\hline 2 & 5 & 95 & 4 & $56: 90$ & 19,600 & 1.02 & 49 & 22 & 440 \\
\hline 3 & 10 & 90 & 5 & $73: 93$ & 19,200 & 1.02 & 43 & 22 & 360 \\
\hline 4 & 20 & 80 & 4 & $56: 90$ & 19,200 & 1.03 & 40 & 8 & 340 \\
\hline 5 & 30 & 70 & 5 & $79: 96$ & 18,200 & 1.05 & 31 & -8 & 320 \\
\hline 6 & 50 & 50 & 5 & $95: 92$ & 29,800 & 1.25 & 18 & n/a & 310 \\
\hline $7^{\text {f }}$ & 100 & 0 & 7 & $89: 0$ & 20,900 & 1.10 & 9 & n/a & 260 \\
\hline
\end{tabular}

Table 5.3. Copolymers of tnCL and VL with Varying Monomer Feeds. ${ }^{\mathrm{a}}$

a) Polymerization conditions: 4M ([VL] + [tnCL]) (2 mmol total), $2.5 \mathrm{~mol} \%$ 1/BEMP (each), $0.5 \mathrm{~mol} \%$ benzyl alcohol in $\mathrm{C}_{6} \mathrm{D}_{6}$. b) Percent conversion to polymer obtained by ${ }^{1} \mathrm{H}$ NMR. c) Determined by GPC $\left(\mathrm{CH}_{2} \mathrm{Cl}_{2}\right)$ vs polystyrene standards. d) Determined by DSC $\left(\mathrm{N}_{2}\right)$; no $\mathrm{T}_{\mathrm{g}}$ were observed $>-70^{\circ} \mathrm{C}$, the limit of our DSC. e) Determined by TGA $\left(\mathrm{N}_{2}\right)$. f) Polymerization conditions: tnCL (2M, $\left.1 \mathrm{mmol}\right), 5$ mol\% 1/BEMP (each), $1 \mathrm{~mol} \%$ benzyl alcohol in $\mathrm{C}_{6} \mathrm{D}_{6} . \mathrm{n} / \mathrm{a}=$ not observed above $-70^{\circ} \mathrm{C}$, the limit of our DSC. 


\section{Computational Output}

methyl thionoacetate

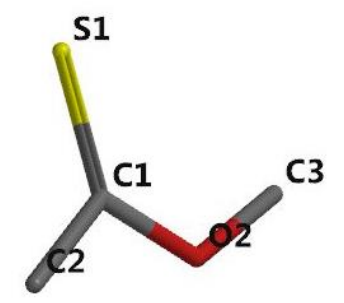

SPARTAN '14 Quantum Mechanics Driver: (Win/64b)

Release 1.1 .8

Method: RB3LYP

Basis set: $6-31 \mathrm{G}^{* *}$

Number of shells: 39

Number of basis functions: 109

Multiplicity: 1

Solvation: toluene [SM8]

Free Energy of Solvation : $\quad-22.1872126 \mathrm{~kJ} / \mathrm{mol}$

SCF total energy: $\quad-591.3523054$ hartrees

\begin{tabular}{rccc}
\multicolumn{5}{c}{ Cartesian Coordinates (Angstroms) } \\
Atom & X & Y & Z \\
- & \multicolumn{1}{c}{-------} \\
1 C C1 & 0.3532451 & 0.2893493 & 0.0004509 \\
2 C C2 & 1.4111241 & 1.3623631 & -0.0001518 \\
3 H H1 & 1.2929082 & 1.9980110 & -0.8861288 \\
4 H H4 & 2.4069332 & 0.9220162 & 0.0054894 \\
5 H H5 & 1.2861987 & 2.0067716 & 0.8784620 \\
6 O O2 & -0.8568645 & 0.8567950 & 0.0004491 \\
7 C C3 & -2.0251905 & 0.0225348 & -0.0002794 \\
8 H H2 & -2.0385119 & -0.6114545 & -0.8907101 \\
9 H H6 & -2.8678241 & 0.7151978 & -0.0001000 \\
10 H H7 & -2.0388805 & -0.6124678 & 0.8893784 \\
11 S S1 & 0.6486888 & -1.3323698 & -0.0000064
\end{tabular}

Atomic Charges:

Electrostatic Mulliken Natural

$\begin{array}{cllll}1 \mathrm{C} 1 & : & +0.504 & +0.207 & +0.231 \\ 2 \mathrm{C} 2 & : & -0.831 & -0.346 & -0.750 \\ 3 \mathrm{H} 1 & : & +0.253 & +0.156 & +0.268 \\ 4 \mathrm{H} 4 & : & +0.220 & +0.146 & +0.265 \\ 5 \mathrm{H} 5 & : & +0.252 & +0.156 & +0.268 \\ 6 \mathrm{O} 2 & : & -0.175 & -0.399 & -0.497 \\ 7 \mathrm{C} 3 & : & -0.409 & -0.097 & -0.340 \\ 8 \mathrm{H} 2 & : & +0.164 & +0.148 & +0.232 \\ 9 \mathrm{H} 6 & : & +0.204 & +0.143 & +0.236 \\ 10 \mathrm{H} 7 & : & +0.164 & +0.148 & +0.232\end{array}$


$11 \mathrm{~S} 1 \quad: \quad-0.345 \quad-0.264 \quad-0.147$ 
methyl thionoacetate + TU

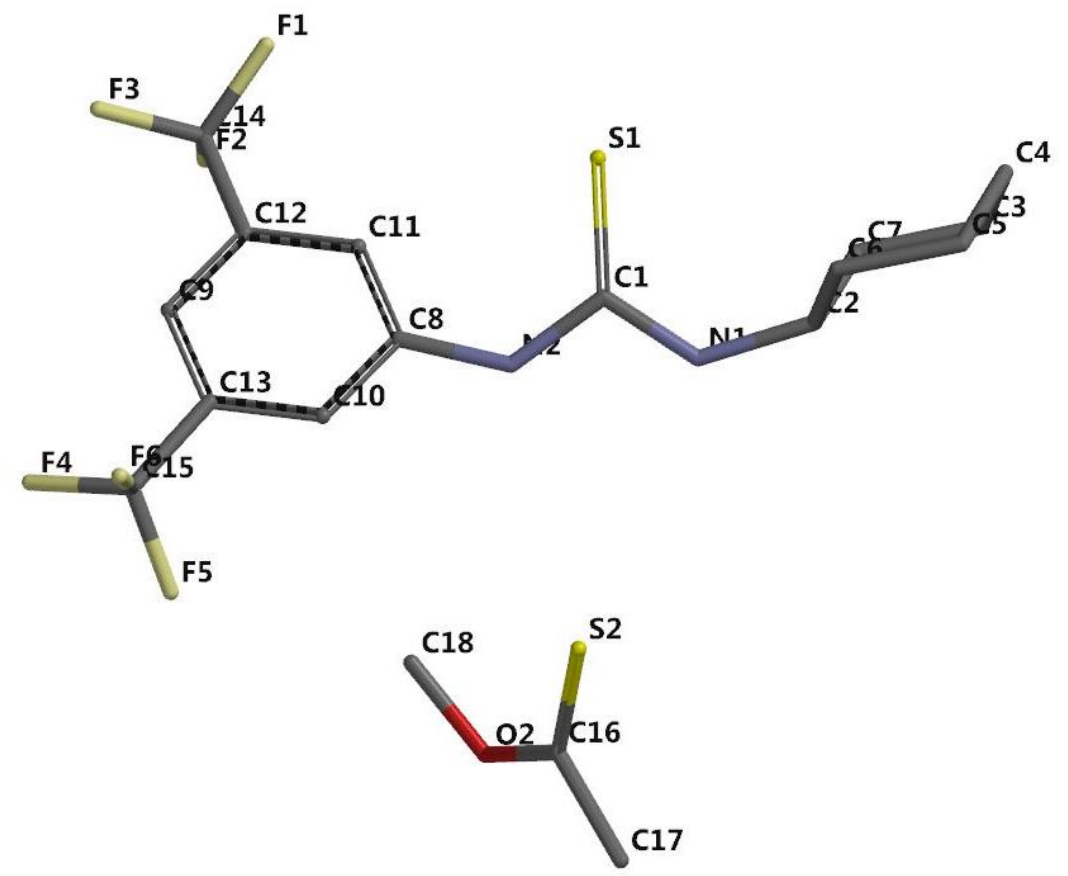

SPARTAN '14 Quantum Mechanics Driver: (Win/64b) Release 1.1.8

Method: RB3LYP

Basis set: $6-31 \mathrm{G}^{* *}$

Number of shells: 184

Number of basis functions: 553

Multiplicity: 1

Solvation: toluene [SM8]

Free Energy of Solvation : $\quad-60.5273905 \mathrm{~kJ} / \mathrm{mol}$

SCF total energy: -2279.4107961 hartrees

\begin{tabular}{ccccc}
\multicolumn{5}{c}{ Cartesian Coordinates (Angstroms) } \\
Atom & X & Y & Z \\
----- & --------- & \\
& & & \\
1 C C1 & 1.6758054 & 0.7905501 & 0.2103707 \\
2 S S1 & 1.7699850 & 2.4419889 & 0.5046662 \\
3 N N1 & 2.7382559 & -0.0349675 & 0.0191621 \\
4 H H3 & 2.5193857 & -0.9981657 & -0.2073029 \\
5 N N2 & 0.4840883 & 0.0813901 & 0.1525672 \\
6 H H2 & 0.5678196 & -0.9297880 & 0.2370184 \\
7 C C2 & 4.1838885 & 0.2584425 & 0.0771436 \\
8 C C3 & 6.1895278 & 1.3718173 & -0.9885321 \\
9 C C5 & 6.1896003 & 0.8582718 & 1.4927664 \\
10 C C4 & 6.6737079 & 1.8207331 & 0.3979827 \\
11 C C6 & 4.6606139 & 0.7008589 & 1.4716566 \\
12 C C7 & 4.6604995 & 1.2150088 & -1.0315947 \\
13 H H6 & 6.6647803 & 0.4118277 & -1.2430128 \\
14 H H10 & 6.6644235 & -0.1241215 & 1.3470772
\end{tabular}




$\begin{array}{lccc}\text { 15 H H7 } & 6.2899819 & 2.8293220 & 0.6073953 \\ \text { 16 H H11 } & 4.1834070 & 1.6511181 & 1.7308705 \\ \text { 17 H H1 } & 4.1762285 & 2.1869592 & -0.8982809 \\ \text { 18 H H5 } & 4.6420614 & -0.7202707 & -0.1233279 \\ \text { 19 H H8 } & 6.5076315 & 2.0879485 & -1.7562528 \\ \text { 20 H H9 } & 6.5081149 & 1.2121958 & 2.4810613 \\ \text { 21 H H12 } & 7.7688003 & 1.8908696 & 0.4126010 \\ \text { 22 H H13 } & 4.3383632 & -0.0379416 & 2.2161543 \\ \text { 23 H H14 } & 4.3409411 & 0.8259188 & -2.0069730 \\ \text { 24 C C8 } & -0.8491312 & 0.5166385 & 0.1899527 \\ \text { 25 C C9 } & -3.6030408 & 1.1251961 & 0.1782265 \\ \text { 26 C C10 } & -1.7898436 & -0.3961179 & 0.6880128 \\ \text { 27 C C11 } & -1.2980363 & 1.7468134 & -0.3128201 \\ \text { 28 C C12 } & -2.6616350 & 2.0375334 & -0.3011851 \\ \text { 29 C C13 } & -3.1508168 & -0.0996071 & 0.6644521 \\ \text { 30 H H4 } & -1.4505552 & -1.3473527 & 1.0874682 \\ \text { 31 H H15 } & -0.5893373 & 2.4645677 & -0.7001976 \\ \text { 32 H H18 } & -4.6591973 & 1.3634615 & 0.1705037 \\ \text { 33 C C14 } & -3.1276858 & 3.3368377 & -0.9073428 \\ \text { 34 C C15 } & -4.1141617 & -1.1166440 & 1.2125417 \\ \text { 35 F F1 } & -2.2546555 & 4.3385751 & -0.6827686 \\ \text { 36 F F2 } & -3.2693917 & 3.2301861 & -2.2504660 \\ \text { 37 F F3 } & -4.3268251 & 3.7194112 & -0.4152879 \\ \text { 38 F F4 } & -5.3814297 & -0.8881624 & 0.8146866 \\ \text { 39 F F5 } & -3.7852105 & -2.3723859 & 0.8036362 \\ \text { 40 F F6 } & -4.1134408 & -1.1424304 & 2.5625236 \\ \text { 41 C C16 } & 0.5795845 & -4.5345904 & -0.8423796 \\ \text { 42 O O2 } & -0.3952656 & -4.3628831 & -1.7213449 \\ \text { 43 C C17 } & 1.2148748 & -5.8862389 & -1.0311069 \\ \text { 44 H H19 } & 0.4928772 & -6.6656509 & -0.7574391 \\ \text { 45 C C18 } & -1.1993618 & -3.1672036 & -1.7105905 \\ \text { 46 H H21 } & -0.5656089 & -2.2818215 & -1.7978401 \\ \text { 47 H H26 } & -1.7878523 & -3.1162621 & -0.7918646 \\ \text { 48 H H28 } & 1.4680383 & -6.0347472 & -2.0867945 \\ \text { 49 H H29 } & 2.1052470 & -5.9873100 & -0.4122837 \\ \text { 50 H H30 } & -1.8519641 & -3.2616327 & -2.5782532 \\ \text { 51 S S2 } & 1.0452882 & -3.4593363 & 0.3329644\end{array}$

Atomic Charges:

Electrostatic Mulliken Natural

$\begin{array}{lllll}1 \mathrm{C} 1 & : & +0.350 & +0.376 & +0.277 \\ 2 \mathrm{~S} 1 & : & -0.375 & -0.319 & -0.264 \\ 3 \mathrm{~N} 1 & : & -0.430 & -0.536 & -0.617 \\ 4 \mathrm{H} 3 & : & +0.291 & +0.286 & +0.426 \\ 5 \mathrm{~N} 2 & : & -0.466 & -0.652 & -0.626 \\ 6 \mathrm{H} 2 & : & +0.313 & +0.285 & +0.427 \\ 7 \mathrm{C} 2 & : & +0.222 & +0.071 & -0.060 \\ 8 \mathrm{C} 3 & : & -0.141 & -0.182 & -0.466 \\ 9 \mathrm{C} 5 & : & -0.201 & -0.182 & -0.466\end{array}$




\begin{tabular}{|c|c|c|c|c|}
\hline & & & 176 & \\
\hline & & & -0.165 & \\
\hline & & & -0.162 & \\
\hline & & & +0.089 & \\
\hline & & +0 . & +0.089 & \\
\hline & & & & \\
\hline 11 & & & & \\
\hline & & +0 & & \\
\hline & & & & \\
\hline 18 & & +0 & &  \\
\hline 9 & & & & \\
\hline $\mathrm{H} 12$ & & & 88 & $8+$ \\
\hline & & & & $8+$ \\
\hline $\mathrm{H}$ & & & 87 & $7+$ \\
\hline 8 & & +0 & +0 & + \\
\hline & & -0 & & \\
\hline & & & & \\
\hline & & & -0.073 & -0 \\
\hline & & & & 0 \\
\hline & & & & 50 \\
\hline 4 & & +0 & +0 & +0.2 \\
\hline & & + & 35 & $5+0$. \\
\hline & & & & $1+0$. \\
\hline & & & 38 & 8 \\
\hline & & +0 & 91 & $1+1$. \\
\hline & & -0 & $6-$ & \\
\hline & & & 33 & \\
\hline 2 & & & 272 & \\
\hline 4 & & 4 & 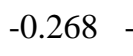 & \\
\hline & & & -0.275 & -0 . \\
\hline & & -0.1 & -0.259 & -0.360 \\
\hline & & & +0.223 & +0.2 \\
\hline $\mathrm{O} 2$ & & & -0.388 & -0.4 \\
\hline & & & -0.353 & -0 . \\
\hline & & & +0.169 & $9+0.27$ \\
\hline & & & -0.115 & -0.34 \\
\hline  & & +0.132 & +0.155 & $5+0.23$ \\
\hline $\mathrm{H}$ & & & +0.146 & $6+0.2$ \\
\hline & & & & $3+0.27$ \\
\hline $\mathrm{H} 29$ & & +0.220 & +0.152 & $2+0.26$ \\
\hline & & +0.202 & +0.159 & $9+0.246$ \\
\hline & & & -0.272 & -0.199 \\
\hline
\end{tabular}


methyl acetate

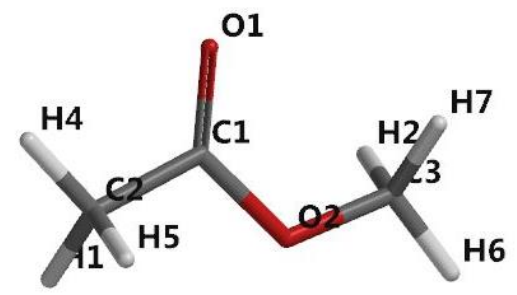

Method: RB3LYP

Basis set: 6-31G**

Number of shells: 38

Number of basis functions: 105

Multiplicity: 1

Solvation: toluene [SM8]

Free Energy of Solvation : $\quad-12.7057187 \mathrm{~kJ} / \mathrm{mol}$

SCF total energy: $\quad-268.4015947$ hartrees

\begin{tabular}{|c|c|c|c|}
\hline \multirow[b]{2}{*}{ Atom } & \multicolumn{3}{|c|}{ Cartesian Coordinates (Angstroms) } \\
\hline & $\mathrm{X}$ & $\mathrm{Z}$ & \\
\hline $1 \mathrm{O} \mathrm{O} 1$ & -0.2846776 & -1.3762272 & -0.0001264 \\
\hline $2 \mathrm{C} \mathrm{C} 1$ & -0.4609993 & -0.1779412 & -0.0024056 \\
\hline $3 \mathrm{C} \mathrm{C} 2$ & -1.8012517 & 0.5173882 & 0.0006146 \\
\hline $4 \mathrm{H} \mathrm{H} 1$ & -1.9132642 & 1.1058138 & 0.9161049 \\
\hline $5 \mathrm{H} \mathrm{H} 4$ & -2.5949307 & -0.2261949 & -0.0594131 \\
\hline $6 \mathrm{H} \mathrm{H} 5$ & -1.8702228 & 1.2107086 & -0.8425385 \\
\hline $7 \mathrm{O} \mathrm{O} 2$ & 0.5487682 & 0.7248714 & -0.0026729 \\
\hline $8 \mathrm{C} \mathrm{C} 3$ & 1.8714313 & 0.1631529 & 0.0017315 \\
\hline $9 \mathrm{H} \mathrm{H} 2$ & 2.0280054 & -0.4493535 & 0.8932729 \\
\hline $0 \mathrm{H} \mathrm{H} 6$ & 2.5529681 & 1.0133969 & -0.0022095 \\
\hline $1 \mathrm{H} \mathrm{H} 7$ & 2.0296374 & -0.4591225 & -0.8824658 \\
\hline
\end{tabular}

Atomic Charges:

Electrostatic Mulliken Natural

$\begin{array}{lllll}1 \mathrm{O} 1 & : & -0.532 & -0.469 & -0.607 \\ 2 \mathrm{C} 1 & : & +0.864 & +0.552 & +0.824 \\ 3 \mathrm{C} 2 & : & -0.915 & -0.380 & -0.790 \\ 4 \mathrm{H} 1 & : & +0.251 & +0.154 & +0.266 \\ 5 \mathrm{H} 4 & : & +0.248 & +0.140 & +0.260 \\ 6 \mathrm{H} 5 & : & +0.249 & +0.152 & +0.265 \\ 7 \mathrm{O} 2 & : & -0.312 & -0.452 & -0.556 \\ 8 \mathrm{C} 3 & : & -0.364 & -0.088 & -0.337 \\ 9 \mathrm{H} 2 & : & +0.162 & +0.129 & +0.222 \\ 10 \mathrm{H} 6 & : & +0.186 & +0.132 & +0.231 \\ 11 \mathrm{H} 7 & : & +0.162 & +0.129 & +0.222\end{array}$


methyl acetate + TU



Method: RB3LYP

Basis set: 6-31G**

Number of shells: 183

Number of basis functions: 549

Multiplicity: 1

Solvation: toluene [SM8]

Free Energy of Solvation : $\quad-52.9506956 \mathrm{~kJ} / \mathrm{mol}$

SCF total energy: -1956.4687603 hartrees

\begin{tabular}{lcccc}
\multicolumn{5}{c}{ Cartesian Coordinates (Angstroms) } \\
Atom & X & Y & Z \\
------ & ------------- & \\
1 C C1 & 1.5952030 & 0.7333446 & 0.0441483 \\
2 S S1 & 1.6635447 & 2.4007986 & 0.2631658 \\
3 N N1 & 2.6756253 & -0.0897793 & -0.0166115 \\
4 H H3 & 2.4767442 & -1.0823297 & -0.0879662 \\
5 N N2 & 0.4226245 & 0.0080000 & -0.0880125 \\
6 H H2 & 0.5483018 & -0.9972603 & -0.1664797 \\
7 C C2 & 4.1102989 & 0.2383665 & 0.0647189 \\
8 C C3 & 6.1393058 & 1.2792881 & -1.0261030 \\
9 C C5 & 6.0635616 & 0.9824361 & 1.4896544 \\
10 C C4 & 6.5761466 & 1.8510810 & 0.3311464 \\
11 C C6 & 4.5378219 & 0.8042879 & 1.4322258 \\
12 C C7 & 4.6133093 & 1.1082627 & -1.1021982 \\
13 H H6 & 6.6275112 & 0.3044453 & -1.1799822 \\
14 H H10 & 6.5515906 & -0.0035736 & 1.4436710 \\
15 H H7 & 6.1792177 & 2.8700966 & 0.4394038 \\
16 H H11 & 4.0388394 & 1.7647623 & 1.5909800 \\
17 H H1 & 4.1241710 & 2.0859963 & -1.0598350
\end{tabular}




\begin{tabular}{|c|c|c|c|c|}
\hline \multicolumn{2}{|l|}{$18 \mathrm{H} \mathrm{H} 5$} & 4.5940035 & -0.7433766 & -0.0409491 \\
\hline \multicolumn{2}{|c|}{$19 \mathrm{H} \mathrm{H} 8$} & 6.4792094 & 1.9287265 & -1.8423378 \\
\hline \multicolumn{2}{|l|}{20 H H9 } & 6.3478005 & 1.4232046 & 2.4533071 \\
\hline \multicolumn{2}{|c|}{$21 \mathrm{H} \mathrm{H} 12$} & 7.6698484 & 1.9324407 & 0.3739562 \\
\hline \multicolumn{2}{|c|}{$22 \mathrm{H} \mathrm{H} 13$} & 4.1981217 & 0.1281450 & 2.2272805 \\
\hline \multicolumn{2}{|c|}{$23 \mathrm{H} \mathrm{H} 14$} & 4.3236099 & 0.6421610 & -2.0525812 \\
\hline \multicolumn{2}{|l|}{$24 \mathrm{C} \mathrm{C} 8$} & -0.9277924 & 0.3676463 & 0.0113348 \\
\hline \multicolumn{2}{|l|}{$25 \mathrm{C}$ C9 } & -3.7145773 & 0.8047764 & 0.1521659 \\
\hline \multicolumn{2}{|c|}{$26 \mathrm{C} \mathrm{C} 10$} & -1.8114578 & -0.6789050 & 0.3229922 \\
\hline \multicolumn{2}{|c|}{$27 \mathrm{C}$ C11 } & -1.4557385 & 1.6450832 & -0.2284856 \\
\hline \multicolumn{2}{|c|}{$28 \mathrm{C} \mathrm{C} 12$} & -2.8348374 & 1.8441898 & -0.1474284 \\
\hline \multicolumn{2}{|c|}{$29 \mathrm{C} \mathrm{C} 13$} & -3.1840969 & -0.4646432 & 0.3813974 \\
\hline $30 \mathrm{H} \mathrm{H} 4$ & & -1.4114795 & -1.6671008 & 0.5264415 \\
\hline $31 \mathrm{H} \mathrm{H} 1$ & & -0.7940325 & 2.4654782 & -0.4664050 \\
\hline $32 \mathrm{H} \mathrm{H} 1$ & & -4.7819816 & 0.9788589 & 7793 \\
\hline $33 \mathrm{C} \mathrm{C1}$ & & -3.3828445 & 3.2089138 & -0.4796631 \\
\hline $34 \mathrm{C} \mathrm{C} 1$ & & -4.0805406 & -1.6097604 & 0.7639730 \\
\hline 35 F F1 & & -2.5806387 & 4.1964517 & -0.0352357 \\
\hline $36 \mathrm{~F} \mathrm{~F} 2$ & & -3.5095358 & 3.3751045 & -1.8173948 \\
\hline 37 F F3 & & -4.6081015 & 3.4036318 & 0.0584985 \\
\hline 38 F F4 & & -5.3452318 & -1.4326828 & 0.33 \\
\hline 39 F F5 & & -3.6380001 & -2.7873292 & 0.24 \\
\hline 40 F F6 & & -4.1353108 & -1.7836049 & 2.1033204 \\
\hline $41 \mathrm{O} O 1$ & & 1.2480579 & -2.8525303 & -0.3172280 \\
\hline $42 \mathrm{C} \mathrm{C} 1$ & & 1.2948172 & -4.0713893 & 3710 \\
\hline $43 \mathrm{O} O 2$ & & 0.3576792 & -4.8098861 & -0.9959849 \\
\hline $44 \mathrm{C} \mathrm{C1}$ & & 2.4050764 & -4.9250320 & 0.1589341 \\
\hline $45 \mathrm{H} \mathrm{H} 1$ & & 2.0011934 & -5.6153152 & 0.9067990 \\
\hline $46 \mathrm{C} \mathrm{C} 1$ & & -0.7574220 & -4.1157608 & -1.5961210 \\
\hline $47 \mathrm{H} \mathrm{H} 2$ & & -0.4040796 & -3.2929423 & -2.2209658 \\
\hline $48 \mathrm{H} \mathrm{H} 2$ & & -1.4305995 & -3.7337878 & -0.8253293 \\
\hline $49 \mathrm{H} \mathrm{H} 2$ & & 2.8494155 & -5.5304766 & -0.6372784 \\
\hline $50 \mathrm{H} \mathrm{H} 2$ & & 3.1651870 & -4.2909740 & 0.6157751 \\
\hline $51 \mathrm{H} \mathrm{H} 3$ & & -1.2689827 & -4.8654246 & -2.1989798 \\
\hline 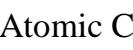 & h & & & \\
\hline & & ostatic $\mathrm{Mu}$ & ken Natural & \\
\hline $1 \mathrm{C} 1$ & & +0.397 & $381+0.282$ & \\
\hline $2 \mathrm{~S} 1$ & $\cdot$ & $-0.384 \quad-0.33$ & $32-0.280$ & \\
\hline $3 \mathrm{~N} 1$ & 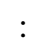 & $-0.505 \quad-0.55$ & $\begin{array}{ll}51 & -0.624\end{array}$ & \\
\hline $4 \mathrm{H} 3$ & : & +0.324 & $288+0.436$ & \\
\hline $5 \mathrm{~N} 2$ & $r^{\circ}$ & -0.535 & $\begin{array}{ll}69 & -0.618\end{array}$ & \\
\hline $6 \mathrm{H} 2$ &  & $+0.372+0.3$ & $315+0.442$ & \\
\hline $7 \mathrm{C} 2$ & : & +0.256 & $073-0.060$ & \\
\hline $8 \mathrm{C} 3$ &. & -0.180 & $81 \quad-0.466$ & \\
\hline $9 \mathrm{C} 5$ & 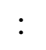 & -0.164 & $81-0.466$ & \\
\hline $10 \mathrm{C} 4$ & : & -0.175 & $76-0.467$ & \\
\hline $11 \mathrm{C} 6$ &  & -0.259 & $63-0.478$ & \\
\hline $12 \mathrm{C} 7$ & 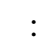 & $-0.221 \quad-0.1$ & $\begin{array}{ll}63 & -0.478\end{array}$ & \\
\hline
\end{tabular}




$\begin{array}{lllll}13 \mathrm{H} 6 & : & +0.087 & +0.088 & +0.225 \\ 14 \mathrm{H} 10 & : & +0.085 & +0.087 & +0.225 \\ 15 \mathrm{H} 7 & : & +0.111 & +0.095 & +0.233 \\ 16 \mathrm{H} 11 & : & +0.112 & +0.122 & +0.254 \\ 17 \mathrm{H} 1 & : & +0.105 & +0.121 & +0.254 \\ 18 \mathrm{H} 5 & : & +0.065 & +0.098 & +0.242 \\ 19 \mathrm{H} 8 & : & +0.088 & +0.092 & +0.246 \\ 20 \mathrm{H} 9 & : & +0.087 & +0.092 & +0.246 \\ 21 \mathrm{H} 12 & : & +0.074 & +0.087 & +0.241 \\ 22 \mathrm{H} 13 & : & +0.083 & +0.085 & +0.240 \\ 23 \mathrm{H} 14 & : & +0.077 & +0.086 & +0.241 \\ 24 \mathrm{C} 8 & : & +0.457 & +0.336 & +0.181 \\ 25 \mathrm{C} 9 & : & -0.326 & -0.119 & -0.214 \\ 26 \mathrm{C} 10 & : & -0.420 & -0.140 & -0.226 \\ 27 \mathrm{C} 11 & : & -0.369 & -0.078 & -0.219 \\ 28 \mathrm{C} 12 & : & +0.192 & -0.068 & -0.151 \\ 29 \mathrm{C} 13 & : & +0.176 & -0.050 & -0.161 \\ 30 \mathrm{H} 4 & : & +0.187 & +0.120 & +0.251 \\ 31 \mathrm{H} 15 & : & +0.171 & +0.137 & +0.284 \\ 32 \mathrm{H} 18 & : & +0.176 & +0.120 & +0.267 \\ 33 \mathrm{C} 14 & : & +0.350 & +0.789 & +1.134 \\ 34 \mathrm{C} 15 & : & +0.370 & +0.786 & +1.135 \\ 35 \mathrm{~F} 1 & : & -0.140 & -0.266 & -0.361 \\ 36 \mathrm{~F} 2 & : & -0.155 & -0.263 & -0.364 \\ 37 \mathrm{~F} 3 & : & -0.147 & -0.273 & -0.367 \\ 38 \mathrm{~F} 4 & : & -0.144 & -0.267 & -0.361 \\ 39 \mathrm{~F} 5 & : & -0.168 & -0.276 & -0.374 \\ 40 \mathrm{~F} 6 & : & -0.147 & -0.259 & -0.361 \\ 41 \mathrm{O} 1 & : & -0.604 & -0.491 & -0.677 \\ 42 \mathrm{C} 16 & : & +0.916 & +0.578 & +0.866 \\ 43 \mathrm{O} 2 & : & -0.307 & -0.430 & -0.533 \\ 44 \mathrm{C} 17 & : & -0.902 & -0.384 & -0.790 \\ 45 \mathrm{H} 19 & : & +0.257 & +0.168 & +0.276 \\ 46 \mathrm{C} 18 & : & -0.331 & -0.113 & -0.342 \\ 47 \mathrm{H} 21 & : & +0.155 & +0.147 & +0.231 \\ 48 \mathrm{H} 26 & : & +0.164 & +0.136 & +0.224 \\ 49 \mathrm{H} 28 & : & +0.255 & +0.166 & +0.275 \\ 50 \mathrm{H} 29 & : & +0.244 & +0.148 & +0.264 \\ 51 \mathrm{H} 30 & : & +0.194 & +0.150 & +0.242\end{array}$


CL

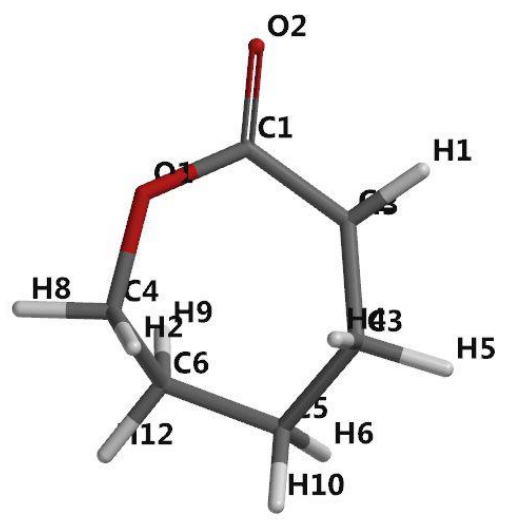

Method: RB3LYP

Basis set: $6-31 \mathrm{G}^{* *}$

Number of shells: 62

Number of basis functions: 170

Multiplicity: 1

Solvation: toluene [SM8]

Free Energy of Solvation : $\quad-27.6012335 \mathrm{~kJ} / \mathrm{mol}$

SCF total energy: $\quad-385.1315410$ hartrees

\begin{tabular}{|c|c|c|c|}
\hline \multirow[b]{2}{*}{ Atom } & \multicolumn{3}{|c|}{ Cartesian Coordinates (Angstroms) } \\
\hline & $\mathrm{X}$ & $\mathrm{Z}$ & \\
\hline $1 \mathrm{C} \mathrm{C} 1$ & 1.3901836 & -0.0473335 & -0.0528234 \\
\hline $2 \mathrm{OO} 1$ & 0.8528370 & 1.1802829 & 0.1803832 \\
\hline $3 \mathrm{C} \mathrm{C} 2$ & 0.5164992 & -1.2873440 & -0.2544354 \\
\hline $4 \mathrm{H} \mathrm{H} 1$ & 1.1796647 & -2.1224822 & -0.0138065 \\
\hline $5 \mathrm{H} \mathrm{H} 3$ & 0.3134986 & -1.3672387 & -1.3324608 \\
\hline $6 \mathrm{C} \mathrm{C} 3$ & -0.7952861 & -1.3869217 & 0.5367320 \\
\hline $7 \mathrm{H} \mathrm{H} 4$ & -0.6210855 & -1.0567782 & 1.5689137 \\
\hline $8 \mathrm{H} \mathrm{H} 5$ & -1.0831465 & -2.4424270 & 0.6083026 \\
\hline $9 \mathrm{C} \mathrm{C} 4$ & -0.5385932 & 1.5050871 & 0.3735628 \\
\hline $10 \mathrm{H} \mathrm{H} 2$ & -0.8013482 & 1.3262948 & 1.4246261 \\
\hline $11 \mathrm{H} \mathrm{H} 8$ & -0.5595467 & 2.5856240 & 0.2101825 \\
\hline $12 \mathrm{C} \mathrm{C} 5$ & -1.9496983 & -0.5885756 & -0.0876377 \\
\hline 13 H H6 & -2.3695512 & -1.1439524 & -0.9364141 \\
\hline $14 \mathrm{H} \mathrm{H} 10$ & -2.7580355 & -0.4966170 & 0.6489899 \\
\hline $15 \mathrm{C} \mathrm{C} 6$ & -1.5229819 & 0.8107349 & -0.5661060 \\
\hline 16 H H9 & -1.0672327 & 0.7657148 & -1.5635231 \\
\hline 17 H H12 & -2.4082469 & 1.4506515 & -0.6689660 \\
\hline $18 \mathrm{O} O 2$ & 2.5939492 & -0.1218670 & -0.1355831 \\
\hline
\end{tabular}

Atomic Charges:

Electrostatic Mulliken Natural

$1 \mathrm{C} 1 \quad$ : $\quad+0.686+0.532+0.830$ 


$$
\begin{array}{lllll}
2 \mathrm{O} 1 & : & -0.396 & -0.465 & -0.561 \\
3 \mathrm{C} 2 & : & -0.291 & -0.260 & -0.588 \\
4 \mathrm{H} 1 & : & +0.102 & +0.136 & +0.277 \\
5 \mathrm{H} 3 & : & +0.131 & +0.142 & +0.264 \\
6 \mathrm{C} 3 & : & -0.090 & -0.196 & -0.478 \\
7 \mathrm{H} 4 & : & +0.085 & +0.109 & +0.239 \\
8 \mathrm{H} 5 & : & +0.071 & +0.111 & +0.256 \\
9 \mathrm{C} 4 & : & +0.049 & +0.041 & -0.121 \\
10 \mathrm{H} 2 & : & +0.097 & +0.119 & +0.221 \\
11 \mathrm{H} 8 & : & +0.095 & +0.127 & +0.246 \\
12 \mathrm{C} 5 & : & -0.181 & -0.179 & -0.470 \\
13 \mathrm{H} 6 & : & +0.088 & +0.103 & +0.242 \\
14 \mathrm{H} 10 & : & +0.080 & +0.104 & +0.243 \\
15 \mathrm{C} 6 & : & -0.205 & -0.214 & -0.510 \\
16 \mathrm{H} 9 & : & +0.117 & +0.116 & +0.245 \\
17 \mathrm{H} 12 & : & +0.091 & +0.119 & +0.260 \\
18 \mathrm{O} 2 & : & -0.529 & -0.448 & -0.594 \\
& & & & \\
\text { CL + TU } & & & &
\end{array}
$$

\section{$\mathbf{C L}+\mathbf{T U}$}

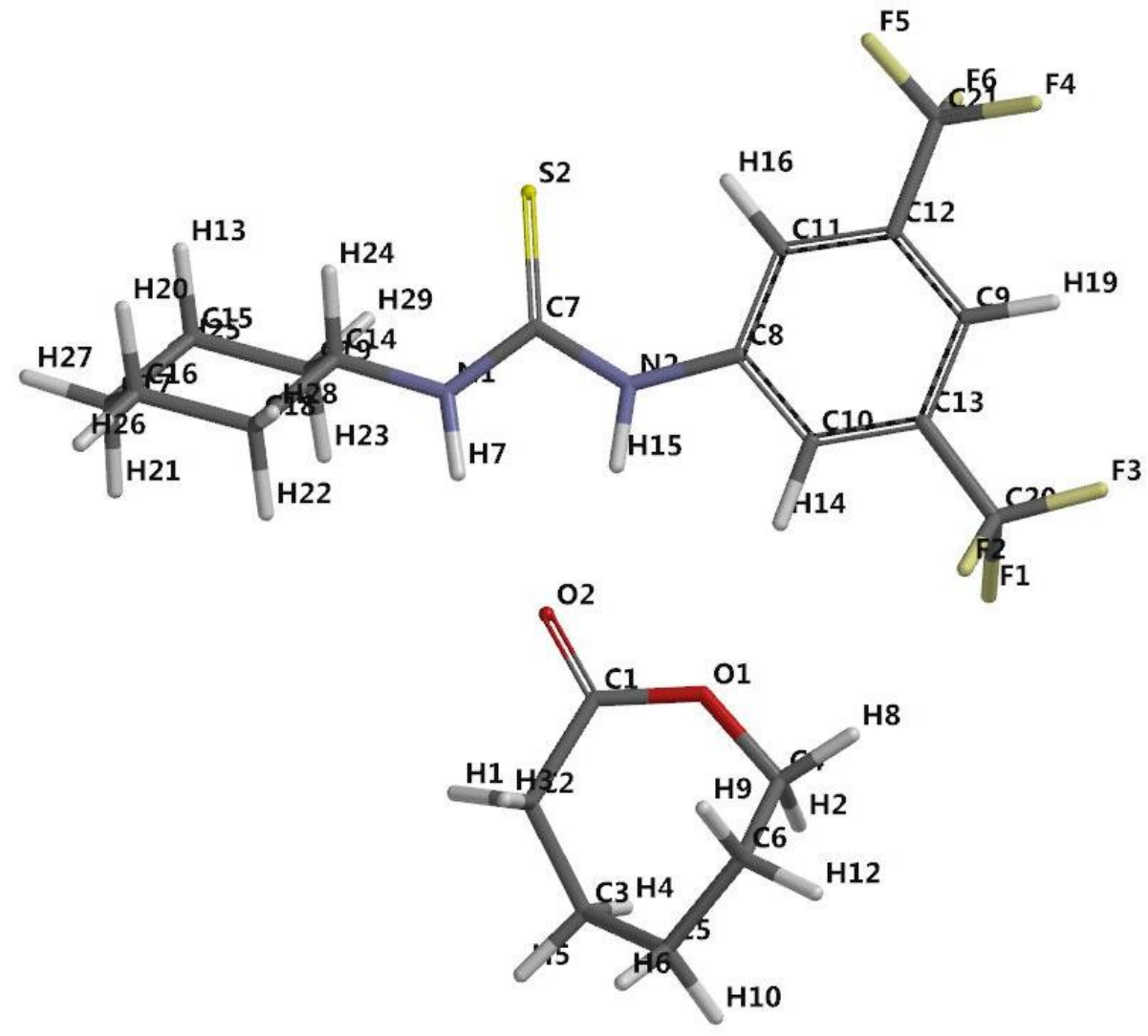

Method: RB3LYP

Basis set: 6-31G**

Number of shells: 207

Number of basis functions: 614

Multiplicity: 1 
Solvation: toluene [SM8]

Free Energy of Solvation : $\quad-67.1539901 \mathrm{~kJ} / \mathrm{mol}$ SCF total energy: -2073.2133692 hartrees

\begin{tabular}{lccc}
\multicolumn{5}{c}{ Cartesian Coordinates (Angstroms) } \\
Atom & X & Y & Z \\
------- & ----------------- & \\
1 C C1 & 1.3556592 & -2.9799822 & -0.0179994 \\
2 O O1 & 0.0286644 & -3.1243347 & 0.1044697 \\
3 C C2 & 2.3106176 & -4.1611860 & -0.1231865 \\
4 H H1 & 3.2615594 & -3.7801995 & 0.2589632 \\
5 H H3 & 2.4720089 & -4.3422279 & -1.1960297 \\
6 C C3 & 1.9192017 & -5.4650853 & 0.5857446 \\
7 H H4 & 1.5484436 & -5.2334024 & 1.5920889 \\
8 H H5 & 2.8264927 & -6.0610485 & 0.7335772 \\
9 C C4 & -0.7482785 & -4.3550525 & 0.1780217 \\
10 H H2 & -0.7687060 & -4.6736679 & 1.2261189 \\
11 H H8 & -1.7477296 & -4.0124178 & -0.0909079 \\
12 C C5 & 0.8847899 & -6.3023945 & -0.1834914 \\
13 H H6 & 1.3779159 & -6.8318410 & -1.0082672 \\
14 H H10 & 0.4895056 & -7.0759512 & 0.4862943 \\
15 C C6 & -0.2781302 & -5.4674085 & -0.7504384 \\
16 H H9 & -0.0121397 & -5.0188759 & -1.7160781 \\
17 H H12 & -1.1361854 & -6.1196172 & -0.9531745 \\
18 H H7 & 2.6211478 & 0.0982237 & -0.1265331 \\
19 N N1 & 2.6090876 & 1.1110760 & -0.1941774 \\
20 C C7 & 1.4041485 & 1.7404043 & -0.1694951 \\
21 N N2 & 0.3644754 & 0.8320089 & -0.1213712 \\
22 H H15 & 0.6625485 & -0.1430287 & -0.0938473 \\
23 C C8 & -1.0278079 & 0.9725106 & -0.0712504 \\
24 C C9 & -3.8568664 & 0.9705203 & 0.0281879 \\
25 C C10 & -1.7506343 & -0.2275133 & 0.0428328 \\
26 C C11 & -1.7426823 & 2.1801530 & -0.1367718 \\
27 C C12 & -3.1370678 & 2.1599565 & -0.0828545 \\
28 C C13 & -3.1410042 & -0.2235349 & 0.0882977 \\
40 H H21 & 6.9869898 & 0.9040785 & 0.9259831
\end{tabular}




$\begin{array}{lccc}\text { 41 H H22 } & 4.9849698 & -0.0819867 & -0.5373812 \\ \text { 42 H H23 } & 4.4769721 & 1.1011058 & 1.7718990 \\ \text { 43 H H24 } & 3.7706515 & 2.7279294 & -0.7178907 \\ \text { 44 H H25 } & 6.1729044 & 2.8623902 & 2.2178518 \\ \text { 45 H H26 } & 7.0099796 & 0.9226648 & -1.5589569 \\ \text { 46 H H27 } & 7.7944804 & 2.3607546 & 0.3519123 \\ \text { 47 H H28 } & 4.5440774 & 0.7672588 & -2.0208638 \\ \text { 48 H H29 } & 3.7034470 & 2.6903546 & 1.7355554 \\ \text { 49 S S2 } & 1.2528590 & 3.4197791 & -0.2053256 \\ \text { 50 C C20 } & -3.8423684 & -1.5470583 & 0.2060571 \\ \text { 51 C C21 } & -3.8765014 & 3.4739292 & -0.0878418 \\ \text { 52 F F1 } & -3.4862190 & -2.2073810 & 1.3363913 \\ \text { 53 F F2 } & -3.5179951 & -2.3800986 & -0.8226034 \\ \text { 54 F F3 } & -5.1834490 & -1.4298682 & 0.2135398 \\ \text { 55 F F4 } & -5.1438299 & 3.3344404 & -0.5417927 \\ \text { 56 F F5 } & -3.2675361 & 4.3937782 & -0.8634014 \\ \text { 57 F F6 } & -3.9615967 & 3.9981387 & 1.1569125 \\ \text { 58 O O2 } & 1.7847186 & -1.8374163 & -0.0697308\end{array}$

Atomic Charges:

\begin{tabular}{|c|c|c|c|c|}
\hline & \multicolumn{3}{|c|}{ Electrostatic Mulliken } & \\
\hline $\mathrm{C} 1$ & & +0.750 & +0.580 & \\
\hline & & & $-0.462-$ & \\
\hline & & & $263-$ & \\
\hline & 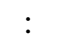 & & +0.150 & \\
\hline & : & & + & \\
\hline & & & $02-$ & \\
\hline & . & & & \\
\hline & . & & 21 & \\
\hline & & & 30 & \\
\hline & $:$ & & & \\
\hline & 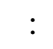 & & 33 & + \\
\hline & . & & -0.1 & \\
\hline & . & & & ) \\
\hline & & & & \\
\hline C &  & & -0.223 & \\
\hline & $\cdot$ & & +0 . & + \\
\hline & & & 32 & 2 \\
\hline & & & +0.297 & +0 \\
\hline & & & -0.552 & -0 . \\
\hline $\mathrm{C} 7$ & . & & .387 & \\
\hline & $\cdot$ & & -0.678 & -0 . \\
\hline & & 2 & +0.306 & $6+0$ \\
\hline & . & & +0.329 & P \\
\hline$+\mathrm{C} 9$ & • & & -0.124 & -0.219 \\
\hline & & & & \\
\hline & & & -0.084 & -0.2 \\
\hline & & +0.16 & -0.072 & -0.15 \\
\hline & & FU. & .050 & -0.16 \\
\hline
\end{tabular}




$\begin{array}{lllll}29 \mathrm{H} 14 & : & +0.177 & +0.146 & +0.261 \\ 30 \mathrm{H} 16 & : & +0.168 & +0.138 & +0.284 \\ 31 \mathrm{H} 19 & : & +0.177 & +0.118 & +0.266 \\ 32 \mathrm{C} 14 & : & +0.383 & +0.085 & -0.066 \\ 33 \mathrm{C} 15 & : & -0.182 & -0.184 & -0.468 \\ 34 \mathrm{C} 16 & : & -0.131 & -0.182 & -0.465 \\ 35 \mathrm{C} 17 & : & -0.197 & -0.172 & -0.467 \\ 36 \mathrm{C} 18 & : & -0.286 & -0.188 & -0.474 \\ 37 \mathrm{C} 19 & : & -0.263 & -0.158 & -0.475 \\ 38 \mathrm{H} 13 & : & +0.100 & +0.097 & +0.233 \\ 39 \mathrm{H} 20 & : & +0.092 & +0.098 & +0.234 \\ 40 \mathrm{H} 21 & : & +0.101 & +0.088 & +0.227 \\ 41 \mathrm{H} 22 & : & +0.101 & +0.090 & +0.226 \\ 42 \mathrm{H} 23 & : & +0.106 & +0.082 & +0.225 \\ 43 \mathrm{H} 24 & : & +0.031 & +0.127 & +0.265 \\ 44 \mathrm{H} 25 & : & +0.090 & +0.091 & +0.245 \\ 45 \mathrm{H} 26 & : & +0.075 & +0.093 & +0.245 \\ 46 \mathrm{H} 27 & : & +0.081 & +0.090 & +0.243 \\ 47 \mathrm{H} 28 & : & +0.091 & +0.103 & +0.250 \\ 48 \mathrm{H} 29 & : & +0.085 & +0.109 & +0.258 \\ 49 \mathrm{~S} 2 & : & -0.413 & -0.342 & -0.291 \\ 50 \mathrm{C} 20 & : & +0.407 & +0.798 & +1.134 \\ 51 \mathrm{C} 21 & : & +0.389 & +0.790 & +1.134 \\ 52 \mathrm{~F} 1 & : & -0.183 & -0.273 & -0.366 \\ 53 \mathrm{~F} 2 & : & -0.179 & -0.275 & -0.371 \\ 54 \mathrm{~F} 3 & : & -0.148 & -0.268 & -0.360 \\ 55 \mathrm{~F} 4 & : & -0.159 & -0.275 & -0.368 \\ 56 \mathrm{~F} 5 & : & -0.152 & -0.268 & -0.362 \\ 57 \mathrm{~F} 6 & : & -0.160 & -0.263 & -0.364 \\ 58 \mathrm{O} 2 & : & -0.641 & -0.480 & -0.656\end{array}$

$\operatorname{tnCL}$

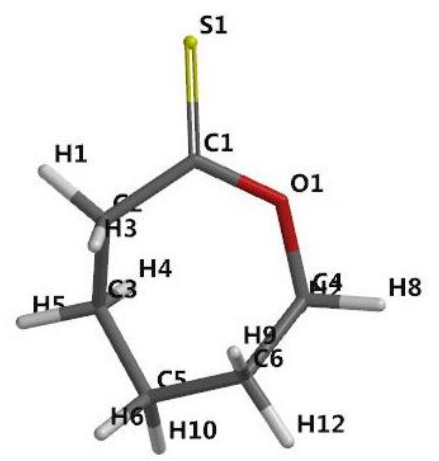

Method: RB3LYP

Basis set: $6-31 \mathrm{G}^{* *}$

Number of shells: 63

Number of basis functions: 174

Multiplicity: 1 
Solvation: toluene [SM8]

Free Energy of Solvation : $\quad-38.5515024 \mathrm{~kJ} / \mathrm{mol}$ SCF total energy: $\quad-708.0828269$ hartrees

\begin{tabular}{|c|c|c|c|}
\hline \multicolumn{4}{|c|}{ Cartesian Coordinates (Angstroms) } \\
\hline Atom & $\mathrm{X}$ & $\mathrm{Z}$ & \\
\hline $1 \mathrm{~S} \mathrm{~S} 1$ & 2.6443549 & -0.0542319 & -0.0384754 \\
\hline $2 \mathrm{C} \mathrm{C} 1$ & 0.9951803 & 0.0078158 & -0.0410194 \\
\hline 3001 & 0.4233157 & 1.2054108 & 0.1334787 \\
\hline $4 \mathrm{C} \mathrm{C} 2$ & 0.1571504 & -1.2365433 & -0.3052132 \\
\hline $5 \mathrm{H} \mathrm{H} 1$ & 0.8236792 & -2.0848949 & -0.1453574 \\
\hline $6 \mathrm{H} \mathrm{H} 3$ & -0.0899162 & -1.2444339 & -1.3779870 \\
\hline $7 \mathrm{C} \mathrm{C} 3$ & -1.1308171 & -1.4050806 & 0.5231224 \\
\hline $8 \mathrm{H} \mathrm{H} 4$ & -0.9387787 & -1.0951792 & 1.5584049 \\
\hline $9 \mathrm{H} \mathrm{H} 5$ & -1.3701154 & -2.4734876 & 0.5755355 \\
\hline $10 \mathrm{C} \mathrm{C} 4$ & -0.9806943 & 1.4938647 & 0.3606821 \\
\hline $11 \mathrm{H} \mathrm{H} 2$ & -1.1941477 & 1.3131823 & 1.4216044 \\
\hline 12 H H8 & -1.0236433 & 2.5718663 & 0.1913628 \\
\hline 13 C C5 & -2.3358146 & -0.6428765 & -0.0499868 \\
\hline 14 H H6 & -2.7731846 & -1.2089190 & -0.8822678 \\
\hline 15 H H10 & -3.1163601 & -0.5745124 & 0.7187103 \\
\hline $16 \mathrm{C} \mathrm{C6}$ & -1.9699699 & 0.7670394 & -0.5442406 \\
\hline 17 H H9 & -1.5474492 & 0.7345369 & -1.5558824 \\
\hline $18 \mathrm{H} \mathrm{H} 12$ & -2.8764956 & 1.3809489 & -0.6164145 \\
\hline
\end{tabular}

Atomic Charges:

Electrostatic Mulliken Natural

$\begin{array}{lllll}1 \mathrm{~S} 1 & : & -0.332 & -0.275 & -0.132 \\ 2 \mathrm{C} 1 & : & +0.292 & +0.220 & +0.241 \\ 3 \mathrm{O} 1 & : & -0.367 & -0.418 & -0.508 \\ 4 \mathrm{C} 2 & : & -0.170 & -0.220 & -0.556 \\ 5 \mathrm{H} 1 & : & +0.040 & +0.145 & +0.283 \\ 6 \mathrm{H} 3 & : & +0.124 & +0.149 & +0.270 \\ 7 \mathrm{C} 3 & : & +0.032 & -0.198 & -0.477 \\ 8 \mathrm{H} 4 & : & +0.067 & +0.113 & +0.241 \\ 9 \mathrm{H} 5 & : & +0.042 & +0.116 & +0.259 \\ 10 \mathrm{C} 4 & : & +0.199 & +0.031 & -0.123 \\ 11 \mathrm{H} 2 & : & +0.073 & +0.134 & +0.230 \\ 12 \mathrm{H} 8 & : & +0.057 & +0.139 & +0.253 \\ 13 \mathrm{C} 5 & : & -0.259 & -0.180 & -0.470 \\ 14 \mathrm{H} 6 & : & +0.097 & +0.106 & +0.244 \\ 15 \mathrm{H} 10 & : & +0.086 & +0.106 & +0.244 \\ 16 \mathrm{C} 6 & : & -0.183 & -0.215 & -0.513 \\ 17 \mathrm{H} 9 & : & +0.122 & +0.122 & +0.249 \\ 18 \mathrm{H} 12 & : & +0.079 & +0.125 & +0.264\end{array}$




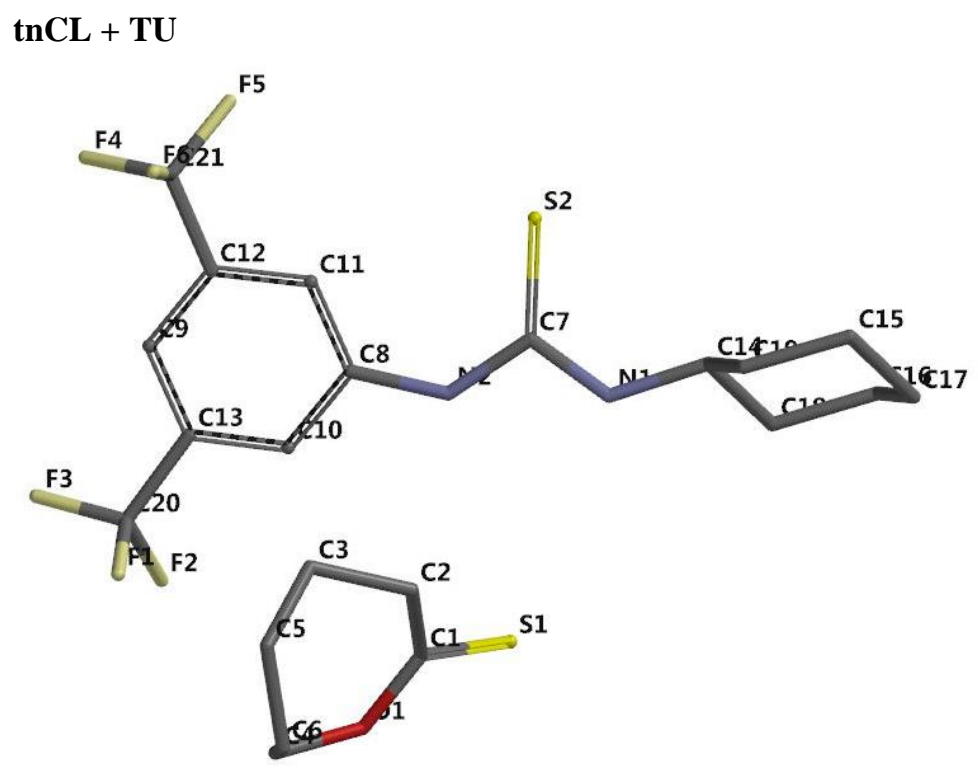

Method: RB3LYP

Basis set: 6-31G**

Number of shells: 208

Number of basis functions: 618

Multiplicity: 1

Solvation: toluene [SM8]

Free Energy of Solvation : $\quad-75.7255567 \mathrm{~kJ} / \mathrm{mol}$

SCF total energy: -2396.1542883 hartrees

\begin{tabular}{|c|c|c|c|}
\hline \multirow[b]{2}{*}{ Atom } & \multicolumn{3}{|c|}{ Cartesian Coordinates (Angstroms) } \\
\hline & $\mathrm{X}$ & $\mathrm{Z}$ & \\
\hline S S1 & 1.3491588 & -2.8471042 & -1.0393901 \\
\hline $\mathrm{C} \mathrm{C} 1$ & 0.1040721 & -3.1533537 & 0.0292555 \\
\hline $\mathrm{O} \mathrm{O} 1$ & -0.7958012 & -4.0384500 & -0.3616225 \\
\hline $\mathrm{C} \mathrm{C} 2$ & 0.0511756 & -2.4934327 & 1.3999643 \\
\hline H H1 & 0.65 & -1.5845935 & 1.3246986 \\
\hline H H3 & 0.6045472 & -3.1526959 & 2.0867197 \\
\hline $\mathrm{C} \mathrm{C} 3$ & -1.3368626 & -2.1729564 & 1.9856477 \\
\hline $8 \mathrm{H} \mathrm{H} 4$ & -1.9809033 & -1.7587246 & 1.2027872 \\
\hline $9 \mathrm{H} \mathrm{H} 5$ & -1.21 & -1.3703357 & 7124 \\
\hline $10 \mathrm{C} \mathrm{C} 4$ & -2.0354421 & -4.4246774 & 0.3145382 \\
\hline $11 \mathrm{H} \mathrm{H} 2$ & -2.7848296 & -3.6668164 & 0.0669271 \\
\hline H H8 & -2.2929019 & -5.3466709 & -0.2093948 \\
\hline $13 \mathrm{C} \mathrm{C5}$ & -2.0192545 & -3.3756158 & 2.6522934 \\
\hline 14 H H6 & -1.5801047 & -3.5568173 & 3.6412218 \\
\hline $5 \mathrm{H} \mathrm{H} 10$ & -3.0747983 & -3.1314246 & 2.8228422 \\
\hline $16 \mathrm{C} \mathrm{C6}$ & -1.9139575 & -4.6614815 & 1.8141225 \\
\hline 17 H H9 & -0.9683074 & -5.1846144 & 2.002368 \\
\hline
\end{tabular}




$\begin{array}{lccc}\text { 18 H H12 } & -2.7059020 & -5.3626838 & 2.1049019 \\ \text { 19 H H7 } & 2.7723736 & -0.5942661 & -0.5216390 \\ \text { 20 N N1 } & 3.0027104 & 0.3921703 & -0.4174054 \\ \text { 21 C C7 } & 1.9764439 & 1.2787815 & -0.3566598 \\ \text { 22 N N2 } & 0.7686086 & 0.6580343 & -0.6383258 \\ \text { 23 H H15 } & 0.8603093 & -0.3036100 & -0.9605748 \\ \text { 24 C C8 } & -0.5651825 & 1.0705614 & -0.5475773 \\ \text { 25 C C9 } & -3.3317144 & 1.6294265 & -0.3730184 \\ \text { 26 C C10 } & -1.5202233 & 0.1699952 & -1.0520267 \\ \text { 27 C C11 } & -1.0126161 & 2.2687228 & 0.0325536 \\ \text { 28 C C12 } & -2.3802859 & 2.5306357 & 0.1041271 \\ \text { 29 C C13 } & -2.8816097 & 0.4426730 & -0.9492600 \\ \text { 30 H H14 } & -1.1936413 & -0.7493962 & -1.5296633 \\ \text { 31 H H16 } & -0.2945796 & 2.9851599 & 0.4043463 \\ \text { 32 H H19 } & -4.3897780 & 1.8503094 & -0.3065686 \\ \text { 33 C C14 } & 4.4232252 & 0.7006500 & -0.2594304 \\ \text { 34 C C15 } & 6.4307145 & 0.6035223 & 1.2931811 \\ \text { 35 C C16 } & 6.7370945 & 0.2762483 & -1.2027721 \\ \text { 36 C C17 } & 7.2441807 & -0.1011085 & 0.1970089 \\ \text { 37 C C18 } & 5.2351315 & -0.0106945 & -1.3544054 \\ \text { 38 C C19 } & 4.9262682 & 0.3248545 & 1.1464580 \\ \text { 39 H H13 } & 6.6045685 & 1.6878997 & 1.2360995 \\ \text { 40 H H20 } & 6.9168594 & 1.3465059 & -1.3787791 \\ \text { 41 H H21 } & 7.1624036 & -1.1902891 & 0.3285190 \\ \text { 42 H H22 } & 5.0633927 & -1.0962285 & -1.2871330 \\ \text { 43 H H23 } & 4.7323393 & -0.7445619 & 1.3204051 \\ \text { 44 H H24 } & 4.5144297 & 1.7831218 & -0.3857656 \\ \text { 45 H H25 } & 6.7723267 & 0.2860841 & 2.2865672 \\ \text { 46 H H26 } & 7.2989400 & -0.2657382 & -1.9735443 \\ \text { 47 H H27 } & 8.3086002 & 0.1469717 & 0.2945992 \\ \text { 48 H H28 } & 4.8769287 & 0.3072722 & -2.3407883 \\ \text { 49 H H29 } & 4.3517505 & 0.8841728 & 1.8937895 \\ \text { 50 S S2 } & 2.1762255 & 2.9129807 & -0.0099106 \\ \text { 51 C C20 } & -3.8603117 & -0.6086166 & -1.3891064 \\ \text { 52 C C21 } & -2.8432360 & 3.7941321 & 0.7858673 \\ \text { 53 F F1 } & -4.0257575 & -1.5570621 & -0.4137172 \\ \text { 54 F F2 } & -3.4443046 & -1.2701417 & -2.4877590 \\ \text { 55 F F3 } & -5.0820655 & -0.1067290 & -1.6420984 \\ \text { 56 F F4 } & -4.0042584 & 4.2465536 & 0.2613097 \\ \text { 57 F F5 } & -1.9368008 & 4.7854054 & 0.6877692 \\ \text { 58 F F6 } & -3.0640469 & 3.5858089 & 2.1059056\end{array}$

Atomic Charges:

Electrostatic Mulliken Natural

$\begin{array}{lllll}1 \mathrm{~S} 1 & : & -0.335 & -0.274 & -0.186 \\ 2 \mathrm{C} 1 & : & +0.324 & +0.222 & +0.284 \\ 3 \mathrm{O} 1 & : & -0.347 & -0.407 & -0.491 \\ 4 \mathrm{C} 2 & : & -0.230 & -0.233 & -0.562 \\ 5 \mathrm{H} 1 & : & +0.072 & +0.150 & +0.283\end{array}$




\begin{tabular}{|c|c|c|c|c|}
\hline & & & & \\
\hline & & & -0.211 & \\
\hline I4 & & +0.063 & & \\
\hline & & & & \\
\hline & & & & \\
\hline I2 & & & & +0 . \\
\hline & & & & \\
\hline & & & -0.184 & -0.4 \\
\hline I6 & & & & \\
\hline & & & & \\
\hline & & & -0.2 & -0 . \\
\hline & & & 77 & \\
\hline 12 & & & & $4+0$ \\
\hline 7 & & & & \\
\hline 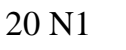 & & & -0.5 & -0 \\
\hline & & & +0 & \\
\hline & & & -0.651 & \\
\hline & & & & \\
\hline & & & & \\
\hline & & & & \\
\hline & & & & \\
\hline & & & & \\
\hline & & & & \\
\hline & & & & 61 \\
\hline & & & & \\
\hline & & & & v \\
\hline & & & & $1+c$ \\
\hline & & + & +0 & $4 \quad-0$. \\
\hline & & & & \\
\hline & & & & \\
\hline & & & & \\
\hline & & & & \\
\hline & & & & 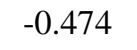 \\
\hline & & & & \\
\hline & & & & \\
\hline & & & 0 & $0+0$ \\
\hline & & & & +0 \\
\hline & &  & $T$ & $9+0$ \\
\hline US & & 6 & 31 & $1+0$. \\
\hline & & & & $3+0.2$ \\
\hline & & 7 & +0 & $5+0.2$ \\
\hline & & & 1 & $1+0$. \\
\hline 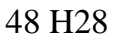 & & +0.060 & +0.103 & $3+0.25$ \\
\hline & & +0.092 & +0.108 & $8+0.2$ \\
\hline & & & -0.326 & -0.272 \\
\hline ת & & & & +1 \\
\hline & & & +0.789 & $9+1.13$ \\
\hline & & & & -0.376 \\
\hline $\mathrm{F} 2$ & & -0.172 & -0.264 & -0.360 \\
\hline
\end{tabular}




$\begin{array}{lllll}55 \text { F3 } & : & -0.149 & -0.263 & -0.358 \\ 56 \text { F4 } & : & -0.150 & -0.272 & -0.366 \\ 57 \text { F5 } & : & -0.145 & -0.265 & -0.360 \\ \text { 58 F6 } & : & -0.166 & -0.263 & -0.364\end{array}$

tCL

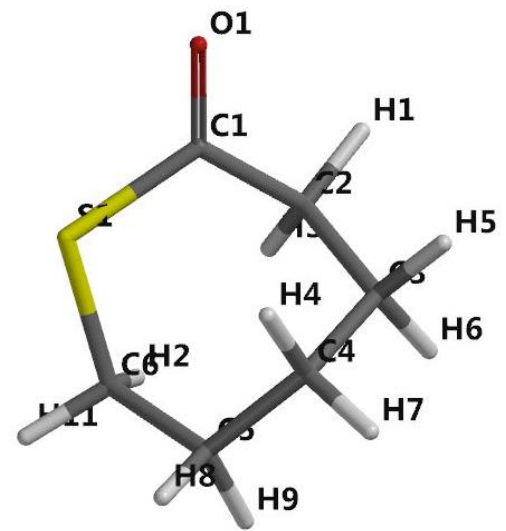

Method: RB3LYP

Basis set: 6-31G**

Number of shells: 63

Number of basis functions: 174

Multiplicity: 1

Solvation: toluene [SM8]

Free Energy of Solvation : $\quad-30.4982395 \mathrm{~kJ} / \mathrm{mol}$ SCF total energy: $\quad-708.1027241$ hartrees

\begin{tabular}{|c|c|c|c|}
\hline \multirow[b]{2}{*}{ Atom } & \multicolumn{3}{|c|}{ Cartesian Coordinates (Angstroms) } \\
\hline & $\mathrm{X}$ & $\mathrm{Z}$ & \\
\hline $\mathrm{O} O 1$ & -2.4407090 & 0.4476469 & 0.4665337 \\
\hline $\mathrm{C} \mathrm{C} 1$ & -1.3644326 & 0.2182883 & -0.0346220 \\
\hline S S1 & -0.6590842 & -1.4456467 & 0.1632378 \\
\hline $\mathrm{C} \mathrm{C} 2$ & -0.5678713 & 1.2364792 & -0.8391372 \\
\hline H H1 & -1.2652899 & 2.0436255 & -1.0823049 \\
\hline $\mathrm{H} \mathrm{H} 3$ & -0.2600530 & 0.7761104 & 2466 \\
\hline $\mathrm{C} \mathrm{C} 3$ & 0.66 & 1.8171488 & -0.0978687 \\
\hline H H5 & 0.3247390 & 2.6441594 & 0.5354685 \\
\hline 9 H H6 & 1.33 & 2.2605906 & -0.8505440 \\
\hline $0 \mathrm{C} \mathrm{C} 4$ & 1.4568502 & 0.8455705 & 0.7952601 \\
\hline $\mathrm{H} \mathrm{H} 4$ & 0.8243155 & 0.5423431 & 1.6384396 \\
\hline $2 \mathrm{H} \mathrm{H} 7$ & 2.2962033 & 1.3981341 & 1.2362081 \\
\hline $3 \mathrm{C} \mathrm{C} 5$ & 2.0192904 & -0.4191545 & 0.1266987 \\
\hline $4 \mathrm{H} \mathrm{H} 8$ & 2.5044339 & -1.0187639 & 0.9063752 \\
\hline $5 \mathrm{H} \mathrm{H} 9$ & 2.8072701 & -0.1458773 & -0.5893921 \\
\hline $6 \mathrm{C} \mathrm{C6}$ & 1.0018437 & -1.3042781 & -0.6216590 \\
\hline $7 \mathrm{H} \mathrm{H} 2$ & 0.8669112 & -0.9785480 & -1.6564981 \\
\hline
\end{tabular}


Atomic Charges:

Electrostatic Mulliken Natural

$\begin{array}{lllll}1 \mathrm{O} 1 & : & -0.417 & -0.425 & -0.547 \\ 2 \mathrm{C} 1 & : & +0.501 & +0.246 & +0.402 \\ 3 \mathrm{~S} 1 & : & -0.202 & +0.083 & +0.211 \\ 4 \mathrm{C} 2 & : & -0.438 & -0.241 & -0.565 \\ 5 \mathrm{H} 1 & : & +0.143 & +0.136 & +0.273 \\ 6 \mathrm{H} 3 & : & +0.160 & +0.133 & +0.255 \\ 7 \mathrm{C} 3 & : & +0.058 & -0.180 & -0.464 \\ 8 \mathrm{H} 5 & : & +0.051 & +0.118 & +0.251 \\ 9 \mathrm{H} 6 & : & +0.047 & +0.112 & +0.245 \\ 10 \mathrm{C} 4 & : & -0.182 & -0.205 & -0.479 \\ 11 \mathrm{H} 4 & : & +0.095 & +0.121 & +0.244 \\ 12 \mathrm{H} 7 & : & +0.061 & +0.105 & +0.253 \\ 13 \mathrm{C} 5 & : & -0.110 & -0.162 & -0.481 \\ 14 \mathrm{H} 8 & : & +0.081 & +0.113 & +0.249 \\ 15 \mathrm{H} 9 & : & +0.072 & +0.111 & +0.245 \\ 16 \mathrm{C} 6 & : & -0.165 & -0.363 & -0.615 \\ 17 \mathrm{H} 2 & : & +0.103 & +0.150 & +0.249 \\ 18 \mathrm{H} 11 & : & +0.141 & +0.149 & +0.272\end{array}$

tCL + TU

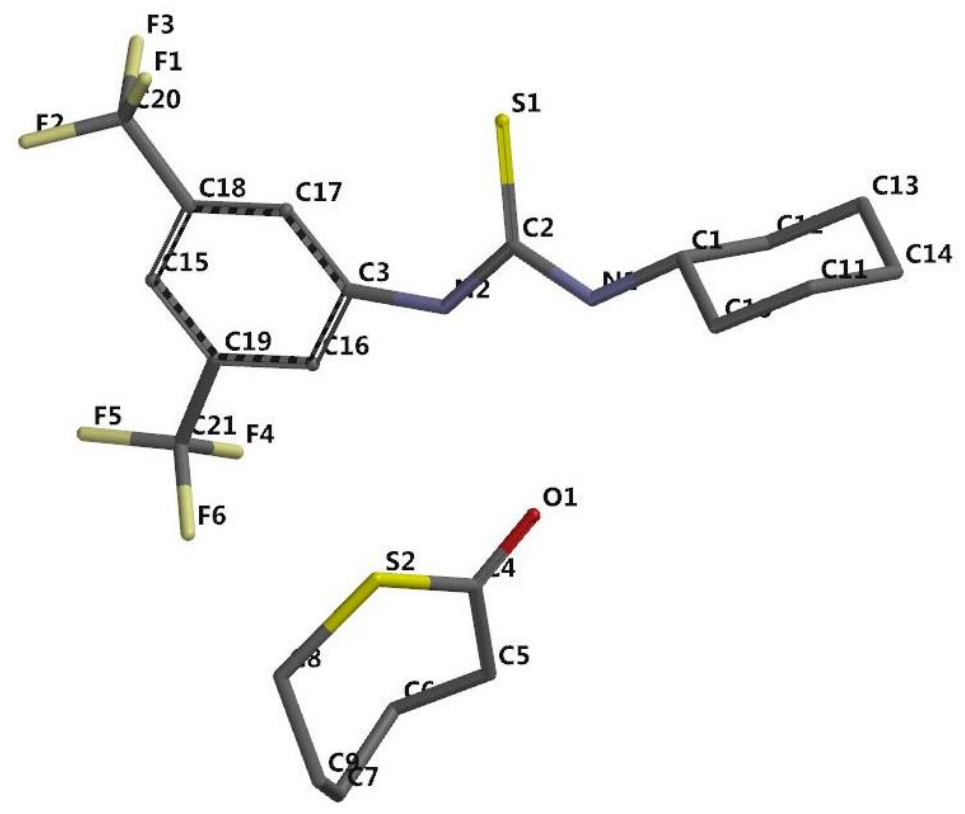

Method: RB3LYP

Basis set: 6-31G**

Number of shells: 208

Number of basis functions: 618

Multiplicity: 1

Solvation: toluene [SM8] 
Free Energy of Solvation : $\quad-69.9213362 \mathrm{~kJ} / \mathrm{mol}$ SCF total energy: -2396.1749852 hartrees

\begin{tabular}{|c|c|c|c|}
\hline \multicolumn{4}{|c|}{ Cartesian Coordinates (Angstroms) } \\
\hline Atom & $X$ & $\mathrm{Z}$ & \\
\hline $1 \mathrm{C} \mathrm{C} 1$ & 4.4731186 & 0.5180586 & 0.0196382 \\
\hline $2 \mathrm{H} \mathrm{H} 2$ & 4.6041948 & 1.5502341 & 0.3587454 \\
\hline $3 \mathrm{~N} \mathrm{~N} 1$ & 3.0312180 & 0.2853340 & -0.0116268 \\
\hline $4 \mathrm{H} \mathrm{H} 3$ & 2.7365844 & -0.6865595 & -0.0390605 \\
\hline $5 \mathrm{C} \mathrm{C} 2$ & 2.0692538 & 1.2378774 & -0.1276174 \\
\hline $6 \mathrm{SS} 1$ & 2.3885880 & 2.8902985 & -0.1574533 \\
\hline $7 \mathrm{~N} \mathrm{~N} 2$ & 0.8164257 & 0.6643585 & -0.2366545 \\
\hline $8 \mathrm{H} \mathrm{H} 5$ & 0.8267138 & -0.3481394 & -0.3399058 \\
\hline 9001 & 1.1349538 & -2.3399877 & 397 \\
\hline $10 \mathrm{C} \mathrm{C} 4$ & 0.2301512 & -3.0407593 & -0.0464579 \\
\hline $11 \mathrm{~S} \mathrm{~S} 2$ & -0.4137692 & -2.5594007 & 1.5500226 \\
\hline $12 \mathrm{C} \mathrm{C} 5$ & -0.2546244 & -4.2581078 & -0.8157840 \\
\hline $13 \mathrm{H} \mathrm{H} 10$ & 0.1812353 & -5.1485753 & -0.3411537 \\
\hline $14 \mathrm{H} \mathrm{H} 11$ & 0.2141627 & -4.1652256 & 1561 \\
\hline $15 \mathrm{C} \mathrm{C} 6$ & -1.7858473 & -4.4070522 & -0.9354245 \\
\hline $16 \mathrm{H} \mathrm{H} 13$ & -2.0102573 & -4.8444159 & 8458 \\
\hline $17 \mathrm{H} \mathrm{H} 14$ & -2.2549797 & -3.4162478 & 8794 \\
\hline $18 \mathrm{C} \mathrm{C} 7$ & -2.4304378 & -5.2860921 & 526 \\
\hline $19 \mathrm{H} \mathrm{H} 15$ & -2.2105830 & -6.3413674 & 9731 \\
\hline $20 \mathrm{H} \mathrm{H} 16$ & -3.5199824 & -5.1787666 & 0.0700652 \\
\hline $21 \mathrm{C} \mathrm{C} 8$ & -1.9755299 & -3.4809374 & 1.9247270 \\
\hline $22 \mathrm{H} \mathrm{H} 9$ & -2.0610836 & -3.3262319 & 3.0049830 \\
\hline $23 \mathrm{H} \mathrm{H} 18$ & -2.8030420 & -2.9467176 & 1.4487483 \\
\hline $24 \mathrm{C}$ C9 & -1.9932265 & -4.9761572 & 1.5898458 \\
\hline $25 \mathrm{H} \mathrm{H} 17$ & -2.6885994 & -5.4714885 & 2.2792491 \\
\hline $26 \mathrm{H} \mathrm{H} 19$ & -1.0062098 & -5.4054045 & 9996 \\
\hline $27 \mathrm{C} \mathrm{C} 10$ & 5.1292800 & -0.4376107 & 3091 \\
\hline $28 \mathrm{H} \mathrm{H} 1$ & 4.9004009 & -1.4778860 & 0.7456754 \\
\hline $29 \mathrm{H} \mathrm{H} 12$ & 4.6934901 & -0.2763367 & 2.0224205 \\
\hline $30 \mathrm{C} \mathrm{C} 11$ & 8336 & -0.2473069 & 1.0684747 \\
\hline $31 \mathrm{H} \mathrm{H} 21$ & 7.0994169 & -0.9673584 & 1.7660856 \\
\hline $32 \mathrm{H} \mathrm{H} 22$ & 6.8798170 & 0.7532006 & 1.4649746 \\
\hline $33 \mathrm{C} \mathrm{C} 12$ & 5.0993587 & 0.3727727 & -1.3790692 \\
\hline 34 H H4 & 4.8667792 & -0.6296586 & -1.7699556 \\
\hline $35 \mathrm{H} \mathrm{H} 24$ & 4.6367138 & 1.0990284 & -2.0566580 \\
\hline $36 \mathrm{C} \mathrm{C} 13$ & 6.6228848 & 0.5652278 & -1.3316673 \\
\hline 37 H H23 & 7.0476424 & 0.4197086 & -2.3327632 \\
\hline 38 H H26 & 6.8477029 & 1.6034436 & -1.0478238 \\
\hline 39 C C14 & 7.2806336 & -0.3906667 & -0.3261487 \\
\hline $40 \mathrm{H} \mathrm{H} 25$ & 7.1569211 & -1.4267938 & -0.6755785 \\
\hline $41 \mathrm{H} \mathrm{H} 28$ & 8.3610942 & -0.2058237 & -0.2753113 \\
\hline $42 \mathrm{C} \mathrm{C} 3$ & -0.4741850 & 1.2027422 & -0.2494049 \\
\hline
\end{tabular}




$\begin{array}{lccc}\text { 43 C C15 } & -3.1869498 & 1.9923376 & -0.1992111 \\ \text { 44 C C16 } & -1.4863032 & 0.3506145 & -0.7159586 \\ \text { 45 C C17 } & -0.8332059 & 2.4732486 & 0.2298223 \\ \text { 46 C C18 } & -2.1754776 & 2.8508162 & 0.2368277 \\ \text { 47 C C19 } & -2.8236031 & 0.7338239 & -0.6723299 \\ \text { 48 H H6 } & -1.2193286 & -0.6261543 & -1.1063270 \\ \text { 49 H H7 } & -0.0695428 & 3.1440754 & 0.5966148 \\ \text { 50 H H27 } & -4.2260473 & 2.2932836 & -0.1605675 \\ \text { 51 C C20 } & -2.5399429 & 4.2374636 & 0.7033079 \\ \text { 52 C C21 } & -3.8544729 & -0.2581946 & -1.1303667 \\ \text { 53 F F1 } & -1.6812562 & 4.6996674 & 1.6348392 \\ \text { 54 F F2 } & -3.7808069 & 4.2712677 & 1.2426064 \\ \text { 55 F F3 } & -2.5329855 & 5.1225335 & -0.3188637 \\ \text { 56 F F4 } & -3.6797434 & -0.6124188 & -2.4228545 \\ \text { 57 F F5 } & -5.1135158 & 0.1944099 & -0.9966566 \\ \text { 58 F F6 } & -3.7741031 & -1.4208735 & -0.4124282\end{array}$

Atomic Charges:

\begin{tabular}{|c|c|c|c|c|}
\hline & \multicolumn{3}{|c|}{ Electrostatic Mu } & \\
\hline $\mathrm{C} 1$ & & +0.449 & +0.077 & \\
\hline & & & +0.131 & \\
\hline & & & -0.539 & -0 . \\
\hline H3 & & & +0.285 & + \\
\hline & & & & \\
\hline & & & -0 . & \\
\hline & & & -0.670 & \\
\hline & & & +0.311 & \\
\hline & & & -0.469 & \\
\hline & & & +0.258 & $3+$ \\
\hline & & & +0 & \\
\hline & & & o & -0 \\
\hline & & & & $6+$ \\
\hline & & & & 6 \\
\hline C & & & -0.2 & -0. \\
\hline & & & & \\
\hline & & & & 6 \\
\hline & & & -0.183 & -0. \\
\hline & & & 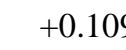 & +0 \\
\hline 16 & & & 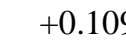 & 0 \\
\hline & & & -0.363 & -0.607 \\
\hline & & & +0 . & -0. \\
\hline 8 & & & & م \\
\hline & $\cdot$ & -0 . & -0.190 & -0.487 \\
\hline & & & & +0.2 \\
\hline & & & +0.12 & +0.24 \\
\hline & & & -0.183 & -0.474 \\
\hline & & & +0.090 & +0.226 \\
\hline & & & & $6+0.25$ \\
\hline & & -0.163 & -0.18 & -0.466 \\
\hline
\end{tabular}




$\begin{array}{lllll}31 \mathrm{H} 21 & : & +0.084 & +0.094 & +0.246 \\ 32 \mathrm{H} 22 & : & +0.100 & +0.098 & +0.234 \\ 33 \mathrm{C} 12 & : & -0.285 & -0.161 & -0.475 \\ 34 \mathrm{H} 4 & : & +0.109 & +0.086 & +0.227 \\ 35 \mathrm{H} 24 & : & +0.089 & +0.108 & +0.255 \\ 36 \mathrm{C} 13 & : & -0.193 & -0.184 & -0.468 \\ 37 \mathrm{H} 23 & : & +0.095 & +0.092 & +0.245 \\ 38 \mathrm{H} 26 & : & +0.104 & +0.098 & +0.234 \\ 39 \mathrm{C} 14 & : & -0.193 & -0.174 & -0.467 \\ 40 \mathrm{H} 25 & : & +0.104 & +0.090 & +0.228 \\ 41 \mathrm{H} 28 & : & +0.083 & +0.090 & +0.244 \\ 42 \mathrm{C} 3 & : & +0.467 & +0.343 & +0.185 \\ 43 \mathrm{C} 15 & : & -0.340 & -0.120 & -0.215 \\ 44 \mathrm{C} 16 & : & -0.482 & -0.133 & -0.221 \\ 45 \mathrm{C} 17 & : & -0.352 & -0.080 & -0.218 \\ 46 \mathrm{C} 18 & : & +0.162 & -0.071 & -0.152 \\ 47 \mathrm{C} 19 & : & +0.242 & -0.047 & -0.163 \\ 48 \mathrm{H} 6 & : & +0.210 & +0.118 & +0.249 \\ 49 \mathrm{H} 7 & : & +0.175 & +0.138 & +0.284 \\ 50 \mathrm{H} 27 & : & +0.174 & +0.120 & +0.267 \\ 51 \mathrm{C} 20 & : & +0.396 & +0.791 & +1.134 \\ 52 \mathrm{C} 21 & : & +0.335 & +0.792 & +1.135 \\ 53 \mathrm{~F} 1 & : & -0.154 & -0.268 & -0.362 \\ 54 \mathrm{~F} 2 & : & -0.164 & -0.274 & -0.368 \\ 55 \mathrm{~F} 3 & : & -0.156 & -0.261 & -0.362 \\ 56 \mathrm{~F} 4 & : & -0.149 & -0.265 & -0.361 \\ 57 \mathrm{~F} 5 & : & -0.127 & -0.265 & -0.358 \\ 58 \mathrm{~F} 6 & : & -0.185 & -0.273 & -0.378\end{array}$




\section{MANUSCRIPT - VI}

Prepared for submission to Macromolecules

Organocatalytic Synthesis of Poly(thionolactone)s: New Materials Abilities from Sulfur-Containing Polylactones

Partha P. Datta, U. L. D. Inush Kalana, Rukshika S. Hewawasam and Matthew K.

Kiesewetter

Chemistry, University of Rhode Island, Kingston, RI, USA

Corresponding Author: $\quad$ Matthew Kiesewetter, Ph.D.

Chemistry

University of Rhode Island

140 Flagg Road

Kingston, RI, 02881, USA

Email address: mkiesewetter@chm.uri.edu 


\begin{abstract}
H-bonding organocatalysts have been implemented for the first time in the ringopening homopolymerization of a range of lactones, including their thiono- derivatives. A good control of the reaction end-products in the form of polymers were synthesized in terms of rate, selectivity and molecular weights. The characteristic thiono-carbonyl motif in the polymers was retained during the process for all the thionated monomers studied. In line with our previous mechanism of action for such lactones, either H-bonding or imidatelike pathways have also been proposed to follow with respect to the type of catalyst systems utilized. Some larger lactones (and thiono-lactones) were also studied during this exploration which also showed excellent control for some of the highly active catalyst systems developed in our group over the years. Kinetic and thermodynamic studies were also performed on the polymerization process of these monomers, corresponding quite well with the expected behavior for the lactone ring sizes. Copolymers were also synthesized for some of the monomers to create blocky polymers, opening up avenues of further research into new materials development.
\end{abstract}




\section{INTRODUCTION}

Ring-opening polymerization (ROP) has come a long way with the advent of organocatalysis in $2001 .^{1}$ The range of monomers that have undergone polymerization by this technique using commercially available and cheaply synthesized catalyst systems have enabled a vast array of polymers to be produced with excellent rate, selectivity and control..$^{2-5}$ As our worldly demand for polyester production increases over the years for biomedical, plastics and microelectronic applications, the need for suitable, fine-tuned materials are also necessitated to meet those requirements. ${ }^{6-10}$ If the pathways to make such materials are following organic catalytic systems, then the industrial viability of these processes are also attractive from a commercial perspective. Thus, research has been focused on expanding the polymer community's understanding of a breadth of monomers with organocatalysis over the past few decades. ${ }^{1,411-14}$ As the suite of monomers have extended over the years, some of the thiono- derivatives of lactones for polyesters have not been studied with organic catalysts which will be the aim of this manuscript.

Huisgen $e t$. al. has looked at a range of lactones with different ring sizes along with their physical properties after ROP in 1950s. ${ }^{15-17}$ Enzymatic catalysis with lipases i.e. Novozym-435 have been conducted since then on many of these larger lactones

(macrolactones). ${ }^{18-35}$ Although it is apparent that dipole moments is one of the principal factors that can alter the physical properties (i.e. melting point and enthalpy of melting) of the polymers from these macrolactones, other factors like monomeric electrophilicity and enzyme-activated monomer intermediate formation could also play significant role in the enzymatic ring-opening polymerizations (eROP) based on previous studies..$^{18}$ Additionally, 
in the hydrolysis of these monomers, eROP can be governed by reaction temperature, solvent and initiator choice, and concentrations of enzymes and water content. ${ }^{18}$

Other metal-based catalysts like tin (II) octoate have also been employed in the ROP of certain macrolactones. ${ }^{13,19,36-41}$ The trans- conformation in those systems were principally responsible for dictating the reduced energy level at ground state as ring strain becomes almost negligible and the cyclic lactones begin to act as open chain esters. ${ }^{18}$ Metal alkoxides have also been implemented in the ROP of some lactones where equilibrium is generally reached rapidly in which the initiation process supersedes propagation and almost full elimination of termination. ${ }^{19}$

We decided to study some of these strained and non-strained lactones and their thiono- counterparts in this following manuscript, namely, $\zeta$-heptalactone (HL), $\zeta$ thionoheptalactone (tnHL), $\eta$-nonalactone (NL), $\eta$-thionononalactone (tnNL), $\omega$ pentadecalactone (PDL), $\omega$-thionopentadecalactone (tnPDL), ethylene brassylate (EB) and thiono-ethylene brassylate (tnEB) as shown in Scheme 6.1. There has been very few literature publications on the 8- and 9-membered lactones where eROP was exercised in the understanding of kinetics and thermodynamics of these macrolides, though copolymers with these monomers have been reported. ${ }^{18,19,38,42-47}$ A growth in research has been observed over the past few years in the production of macrolactones like PDL and EB due to their ductile and tensile enhancement as hydrophobicity augmented with increased alkyl chains. ${ }^{20,37,48-53}$ Hydrolytic degradation was also observed to have enriched for these polymers as metal catalysts i.e. zinc, yttrium, aluminum, magnesium were employed in the ROP for these substrates. ${ }^{40,55-57}$ Although $\delta$-valerolactone, $\varepsilon$-caprolactone and L-lactide are well studied and continued to be under research with organic systems like $\mathrm{N}$ - 
heterocyclic carbenes (NHCs), amidine, guanidine bases, monomers like PDL has not been homo-polymerized with controlled organocatalytic systems yet. ${ }^{1-3,50,58-63}$ Besides conducting copolymerization of various natures for PDL along with a co-monomer, like $\varepsilon$ decalactone (DL), $\varepsilon$-caprolactone (CL) etc., research into the homopolymers of this monomer via organocatalytic approach is very limited..$^{51,64-68}$ Similarly, before our group's efforts into the solvent-free polymerization of $\mathrm{EB},{ }^{54}$ there were only a handful of reports on the organocatalytic ROP of this di-ester motif monomer. ${ }^{48,49}$ In continuation of our previous efforts in other sulfur containing monomers, ${ }^{69,70}$ we have embarked on the comprehension of ROP for these oxygenated monomers in contrast to their sulfur counterparts through this work using organocatalysts as demonstrated in Scheme 6.2. 


\section{EXPERIMENTAL SECTION}

\section{General Considerations}

All chemicals were used as received unless stated otherwise. Hexamethyldisiloxane (HMDO), $\mathrm{P}_{4} \mathrm{~S}_{10}$, cycloheptanone, cyclooctanone, 3-chloroperbenzoic acid ( $m$-CPBA) and 2-tert-butylimino-2-diethylamino-1,3-dimethylperhydro-1,3,2-diazaphosphorine (BEMP) were supplied by Acros Organics. Sodium thiosulfate $\left(\mathrm{Na}_{2} \mathrm{~S}_{2} \mathrm{O}_{3} \cdot 5 \mathrm{H}_{2} \mathrm{O}\right)$ was purchased from Allied Chemical. Sigma-Aldrich provided $\omega$-pentadecalactone. Acetonitrile, potassium carbonate, sodium carbonate, sodium bicarbonate, sodium sulfate, sodium phosphate dibasic, magnesium sulfate, benzyl alcohol, benzoic acid, ethyl acetate, dichloromethane, toluene and hexane were purchased from Fisher Scientific. $\varepsilon^{-}$ Caprolactone (CL) and $\delta$-valerolactone (VL) were supplied by Alfa Aesar and distilled from $\mathrm{CaH}_{2}$ under high vacuum. Acetone- $d_{6}$, chloroform- $d$ and benzene- $d_{6}$ were supplied by Cambridge Isotope Laboratories and distilled from $\mathrm{CaH}_{2}$ under a nitrogen atmosphere. Benzyl alcohol was distilled from $\mathrm{CaH}_{2}$ under high vacuum. Toluene was dried on an Innovated Technologies solvent purification system with alumina columns and nitrogen working gas. 1 [3,5-bis(trifluoromethyl)phenyl]-3-cyclohexyl-thiourea, 2 1,1',1”(nitrilotris(ethane-2,1-diyl))tris(3-(3,5-bis(trifluromethyl)phenyl)urea, 3 1,1'-(propane1,3-diyl)bis(3-(3,5-bis(trifluoromethyl)phenyl)thiourea) and 4 1,1',1'-(nitrilotris(ethane2,1-diyl))tris(3-(3,5-bis(trifluoromethyl)phenyl)thiourea were synthesized and purified according to literature procedures. $^{2,71,72}$ Triclocarban $\quad$ (TCC), $1,8-$ Diazabicyclo[5.4.0]undec-7-ene (DBU), 7-methyl-1,5,7-triazabicyclo[4.4.0]dec-5-ene (MTBD), and 1,5,7-triazabicyclo[4.4.0]dec-5-ene (TBD) were purchased from Tokyo 
Chemical Industry (TCI). All polymerization reactions were performed in an MBRAUN or INERT stainless steel glovebox equipped with a gas purification system under a nitrogen atmosphere using glass vials and magnetic stir bars which were baked overnight at $140^{\circ} \mathrm{C}$. NMR experiments were performed on a Bruker Avance III $300 \mathrm{MHz}$ or $400 \mathrm{MHz}$ spectrometer. The chemical shifts for proton $\left({ }^{1} \mathrm{H}\right)$ and carbon $\left({ }^{13} \mathrm{C}\right)$ NMR were recorded in parts per million (ppm) relative to a residual solvent. Size exclusion chromatography (SEC) was performed at $30^{\circ} \mathrm{C}$ in dichloromethane (DCM) using an Agilent Infinity GPC system equipped with three Agilent PLGel columns $7.5 \mathrm{~mm} \times 300 \mathrm{~mm}(5 \mu \mathrm{m}$ pore sizes: 103,104 , and $105 \AA$ ). . Molecular weight and $M_{w} / M_{n}$ were determined versus polystyrene standards (500 g/mol - $3150 \mathrm{~kg} / \mathrm{mol}$; Polymer Laboratories). Mass spectrometry experiments were performed using a Thermo Electron (San Jose, CA, USA) LTQ Orbitrap XL mass spectrometer affixed with electrospray ionization (ESI) interface in a positive ion mode. Collected mass spectra was averaged for at least 50 scans. Tune conditions for infusion experiments ( $10 \mu \mathrm{L} / \mathrm{min}$ flow, sample concentration $2 \mu \mathrm{g} / \mathrm{mL}$ in $50 / 50 \mathrm{v} / \mathrm{v}$ water/methanol) were as follows: ion spray voltage, $4000 \mathrm{~V}$; capillary temperature, $275^{\circ} \mathrm{C}$; sheath gas $\left(\mathrm{N}_{2}\right.$, arbitrary units), 15; auxiliary gas ( $\mathrm{N}_{2}$, arbitrary units), 2; capillary voltage, $21 \mathrm{~V}$; and tube lens, $90 \mathrm{~V}$; multipole 00 offset, $-4.25 \mathrm{~V}$; lens 0 voltage, - 5.00; multipole 1 offset, - $8.50 \mathrm{~V}$; Multipole RF Amplitude, 400 V; Ion trap's AGC target settings for Full MS was 3.0e4 and FT's 2.0e5 (with 3 and 2 averaged microscans, respectively). Prior to analysis, the instrument was calibrated for positive ions using Pierce LTQ ESI positive ion calibration solution (lot \#PC197784). Differential scanning calorimetry (DSC) curves were obtained on a Shimadzu DSC-60A instruments under $\mathrm{N}_{2}$ calibrated with an indium standard. The heating and cooling curves of DSC were run under a nitrogen atmosphere at a heating rate 
of $\pm 10^{\circ} \mathrm{C} / \mathrm{min}$ in a $40 \mu \mathrm{L}$ aluminum pans. Thermogravimetric analysis (TGA) was performed using a TGA-50 from Shimadzu under a $\mathrm{N}_{2}$ atmosphere at a heating rate of $20^{\circ} \mathrm{C} / \mathrm{min}$ from 25 to $600^{\circ} \mathrm{C}$. MALDI-TOF MS analysis was performed at the University of Akron: reflectron mode with trans-2-[3-(4-tert-butylphenyl)-2-methyl-2propenylidene]-malononitrile (DCTB) matrix with sodium trifluoroacetate (NaTFA) salt.

Synthesis of $\zeta$-Heptalactone (HL)

The procedure to synthesize $\zeta$-heptalactone $(\zeta-\mathrm{HL})$ was adopted from previous literatures with some modifications. ${ }^{73,74}$ Initially, appropriate amount of $m$-CPBA (4.6 g, $18 \mathrm{mmol}$ ) was subjected to a round bottom flask, followed by the addition of dichloromethane $(50 \mathrm{~mL})$ and cycloheptanone $(2.10 \mathrm{~mL}, 27 \mathrm{mmol})$. The reaction mixture was stirred at moderate speed for 5 days after which the reaction was quenched with $10 \%$ $(w / v)$ sodium thiosulfate. The mixture was then washed with sodium bicarbonate followed by extraction with dichloromethane thrice. After drying with sodium sulfate, rotary evaporation was performed to yield a colorless oil. This oil was then purified by silica gel column chromatography with 1:1 mixture of ethyl acetate and hexane. Yield: $2.17 \mathrm{~g}, 95 \%$. Product matched previous literature characterization. ${ }^{73,74}$

Synthesis of $\zeta$-Thionoheptalactone (tnHL)

This procedure for the synthesis of $\zeta$-thionoheptalactone (tnHL) was also adapted from a previous literature study with some modifications. ${ }^{75,76}$ Similar to tnCL synthesis, ${ }^{70}$ $\zeta$-HL (4.04 g, $31.50 \mathrm{mmol}), \mathrm{HMDO}(11.20 \mathrm{~mL}, 52.49 \mathrm{mmol}), \mathrm{P}_{4} \mathrm{~S}_{10}(3.04 \mathrm{~g}, 7.87 \mathrm{mmol})$ and acetonitrile $(35 \mathrm{~mL})$ were refluxed for 2 hours at moderate stirring. The reaction was cooled in an ice-water bath for 30 mins during which quenching with distilled water (2 $\mathrm{mL} / \mathrm{mmol}$ of $\left.\mathrm{P}_{4} \mathrm{~S}_{10}\right)$ and sodium phosphate dibasic $\left(8 \mathrm{mmol} / \mathrm{mmol}\right.$ of $\left.\mathrm{P}_{4} \mathrm{~S}_{10}\right)$ was performed. 
Extraction with ethyl acetate followed thrice. After solvent removal, the yellow-orange oil was purified through a silica gel column chromatography with 3:7 ethyl acetate-to-hexane solvent mixture to give a light yellow solid powders in $42 \%$ yield, $1.89 \mathrm{~g}$. Product was verified with previous literature characterization. ${ }^{71,75,77}$

Synthesis of $\eta$-Nonalactone (NL)

Previous literature procedure was followed for the synthesis of $\eta$-nonalactone $(\eta$ NL) with few modifications. ${ }^{51,78}$ Necessary amount of $m$-CPBA (40.98 g, $\left.237.6 \mathrm{mmol}\right)$ was placed in a round bottom flask with the addition of dichloromethane $(1000 \mathrm{~mL})$ and cyclooctanone $(10 \mathrm{~g}, 79.2 \mathrm{mmol})$ subsequently. The reaction mixture was refluxed at medium speed for 10 days after which the reaction mixture was filtered to remove excess $m$-CPBA. The filtrate was then washed with $10 \%$ sodium thiosulfate, followed by sodium carbonate and brine. Extraction was performed with dichloromethane thrice and magnesium sulfate was used to dry the organic contents. After removal of volatiles, column chromatography was performed in silica gel with 1:10 mixture of ethyl acetate to hexane. Following this purification step, distillation with Kugelrohr was performed for about one hour at $40^{\circ} \mathrm{C}$ and 100 mtorr to yield a colorless oil as the product in $33 \%$ yield $(3.22 \mathrm{~g})$. Product was validated with previous literature characterization. ${ }^{51,78}$

Synthesis of $\eta$-Thionononalactone (tnNL)

The procedure for the synthesis of $\eta$-thionononalactone ( $\mathrm{tnNL}$ ) was adapted from a previous literature study as well with some alterations. ${ }^{77}$ Just like tnCL synthesis, ${ }^{70} \eta$-NL (3.22 g, $22.64 \mathrm{mmol}), \operatorname{HMDO}(8.2 \mathrm{~mL}, 37.73 \mathrm{mmol}), \mathrm{P}_{4} \mathrm{~S}_{10}(2.51 \mathrm{~g}, 5.66 \mathrm{mmol})$ and acetonitrile $(23 \mathrm{~mL})$ were refluxed for about 5 hours at medium stir speed. After that, the reaction mixture was cooled to $0^{\circ} \mathrm{C}$ with stirring for almost 30 mins. Saturated sodium carbonate and distilled water (half the volume of reaction solvent) were also added to 
quench the reaction during this time. Extraction was performed with ethyl acetate thrice, followed by drying the organic phase with magnesium sulfate. After removal of solvent, a silica gel column chromatography was conducted with 1:10 mixture of ethyl acetate and hexane. Subsequently, a Kugelrohr distillation was performed at $60^{\circ} \mathrm{C}$ and 200 mtorr for about 3 hours to generate a light yellow oil in $22 \%$ yield $(666 \mathrm{mg})$. Product was verified with previous literature study subsequently. ${ }^{77}$

Synthesis of $\omega$-Thionopentadecalactone (tnPDL)

As with the other synthesis of thionated lactones, the synthesis of $\omega$ thionopentadecalactone (tnPDL) was also adapted from previous literature study with few alterations. ${ }^{79}$ Just as tnCL synthesis goes,${ }^{70} \omega$-PDL (5.0 g, $\left.20.8 \mathrm{mmol}\right)$, HMDO (7.4 mL, $34.67 \mathrm{mmol}), \mathrm{P}_{4} \mathrm{~S}_{10}(2.31 \mathrm{~g}, 5.20 \mathrm{mmol})$ and $o$-xylene $(21 \mathrm{~mL})$ were refluxed for about 4 hours. Consequently, the mixture was cooled to $0^{\circ} \mathrm{C}$ with stirring for about 30 minutes while saturated sodium carbonate $(6.5 \mathrm{~mL})$ and distilled water $(5.2 \mathrm{~mL})$ were added to quench the reaction. Extraction was performed next with hexane, followed by organic phase drying with magnesium sulfate. After removal of volatiles under reduced pressure, silica gel column chromatography was performed in $1 \%$ ethyl acetate in hexane. Kugerohr distillation was executed next with $120^{\circ} \mathrm{C}$ and $100 \mathrm{mmol}$ for about 3 hours which after solvent removal gave light yellow oil in $42 \%$ yield $2.24 \mathrm{~g}$, Product matched previous literature studies. $^{79}$

Synthesis of Thiono-ethylene brassylate (tnEB)

Curphey's method was followed for the synthesis of thiono-ethylene brassylate (tnEB). ${ }^{75}$ The necessary reagents, ethylene brassylate $(13 \mathrm{~mL}, 50 \mathrm{mmol}), \mathrm{HMDO}(17 \mathrm{~mL}$, $80 \mathrm{mmol}), \mathrm{P}_{4} \mathrm{~S}_{10}(11.11 \mathrm{~g}, 25 \mathrm{mmol})$ and $o$-xylene $(50 \mathrm{~mL})$, were refluxed for about 9 hours after which the reaction mixture was cooled in an ice-water bath for almost an hour after 
quenching the reaction with aqueous sodium carbonate solution and distilled water. Extraction was then executed with dichloromethane thrice. The yellow oil that was obtained after solvent removal was then subjected to silica gel column chromatography with 5:95 ethyl acetate-to-hexane mixture. Then removal of solvent gave the pure form of product in yellow oil with $50 \%$ yield, $7.54 \mathrm{~g}$. HRMS m/z calcd $\left(\mathrm{C}_{15} \mathrm{H}_{27} \mathrm{O}_{2} \mathrm{~S}_{2}{ }^{+}\right) 303.1447$, found 303.1436. ${ }^{1} \mathrm{H} \mathrm{NMR}\left(400 \mathrm{MHz}, \mathrm{CDCl}_{3}\right) \delta 4.72(\mathrm{~s}, 4 \mathrm{H}), 2.75(\mathrm{t}, J=7.2,4 \mathrm{H}), 1.70$ (p, $J=7.1,4 \mathrm{H}), 1.37-1.11(\mathrm{~m}, 12 \mathrm{H}) .{ }^{13} \mathrm{C} \mathrm{NMR}\left(100 \mathrm{MHz}, \mathrm{CDCl}_{3}\right) \delta 25.9,25.9,26.0,26.2$, 26.9, 45.9, 68.3, 223.4. Product spectra are shown below (Figure 6.1, 6.2 respectively). Example Ring-Opening Polymerization of tnHL Just like in a conventional ROP, tnHL $(0.150 \mathrm{~g}, 1.040 \mathrm{mmol})$ was added to a $7 \mathrm{~mL}$ scintillation vial with a stir bar while TCC $(0.016 \mathrm{~g}, 0.052 \mathrm{mmol}), \mathrm{BEMP}(0.014 \mathrm{~g}, 0.052$ $\mathrm{mmol})$ and benzyl alcohol $\left(1.1 \times 10^{-3} \mathrm{~g}, 0.010 \mathrm{mmol}\right)$ were added in another such vial in the glovebox. Benzene- $d_{6}(0.52 \mathrm{~mL}, 2 \mathrm{M}$ in tnHL) was divided equally between the two vials. The contents of the vials were stirred for about 2 mins at moderate speed after which the mixture from tnHL was transferred to the other vial and stirred for another minute. The whole content was then moved into a NMR tube and taken out of the glovebox. Reaction progress was monitored by ${ }^{1} \mathrm{H}$ NMR and after reaching target conversion, the reaction mixture was quenched with benzoic acid ( 2 mol eq. to base). The polymer was then precipitated out of hexane. The supernatant was decanted afterwards and volatiles were removed under reduced pressure to yield the polymer. Yield $94 \% ; M_{w} / M_{n}=14,700 ; M_{n}$ $(\mathrm{GPC})=1.19 .{ }^{1} \mathrm{H}$ and ${ }^{13} \mathrm{C} \mathrm{NMR}$ spectra display characteristic resonances of the polymer with thionoester repeat unit and thiocarbonyl peak at $224 \mathrm{ppm}$ in the ${ }^{13} \mathrm{C}$ spectrum (see Figure $6.3)$. 


\section{Example Ring-Opening Polymerization of tnPDL}

Just like any other ROP of cyclic esters, tnPDL ( $0.25 \mathrm{~g}, 0.976 \mathrm{mmol})$ was charged to a $20 \mathrm{~mL}$ scintillation vial with a stir bar along with toluene $(195 \mu \mathrm{L})$ to make a $5 \mathrm{M}$ solution. Afterwards, TCC (0.015 g, $0.049 \mathrm{mmol})$, BEMP $(0.013 \mathrm{~g}, 0.049 \mathrm{mmol})$ and benzyl alcohol $\left(1.1 \times 10^{-3} \mathrm{~g}, 9.76 \times 10^{-3} \mathrm{mmol}\right)$ were added in the same vial within the glovebox. A quench solution of benzoic acid ( 2 mol eq. to base) was prepared in $1 \mathrm{~mL}$ toluene. Aliquots were taken at various time points with $50 \mu \mathrm{L}$ of reaction mixture and 150 $\mu \mathrm{L}$ of quench solution. Polymer progression was monitored by subjecting the aliquot solution into $\mathrm{CDCl}_{3}$ through ${ }^{1} \mathrm{H}$ NMR. Once desired conversion was observed, the entire reaction solution was quenched with the leftover quench solution and precipitation was obtained by applying hexane to the solution. Volatiles were removed under reduced pressure and high vacuum was applied to obtain final polymer sample for GPC. Yield 91\%; $M_{w} / M_{n}=50,400 ; M_{n(\mathrm{GPC})}=3.78 .{ }^{1} \mathrm{H}$ and ${ }^{13} \mathrm{C}$ NMR spectra display characteristic resonances of the polymer with thionoester repeat unit and thiocarbonyl peak at $224 \mathrm{ppm}$ in the ${ }^{13} \mathrm{C}$ spectrum (see Figure 6.5).

\section{Example Ring-Opening Polymerization of tnEB}

Similar to other ROP reactions, $\operatorname{tnEB}(0.400 \mathrm{~g}, 1.32 \mathrm{mmol})$ was added to a $20 \mathrm{~mL}$ scintillation vial with a stir bar along with toluene $(660 \mu \mathrm{L})$ to make a $2 \mathrm{M}$ solution. Subsequently, TCC $(0.021 \mathrm{~g}, 0.066 \mathrm{mmol})$, BEMP $(0.018 \mathrm{~g}, 0.066 \mathrm{mmol})$ and benzyl alcohol $\left(1.4 \times 10^{-3} \mathrm{~g}, 6.61 \times 10^{-3} \mathrm{mmol}\right)$ were added in the same vial within the glovebox. The vial was then placed in a pre-heated hot plate, set at $80^{\circ} \mathrm{C}$. A stock quench solution was prepared in another vial with benzoic acid at ( 2 mol eq. to base) in toluene. Aliquots ( $\sim 50$ $\mu \mathrm{L})$ were obtained at various time points from the reaction mixture with quench solution 
$(\sim 150 \mu \mathrm{L})$. The aliquots were analyzed by ${ }^{1} \mathrm{H} \mathrm{NMR}$ in $\mathrm{CDCl}_{3}$ to obtain polymer conversion. Polymer precipitation was obtained by hexane and high vacuum was implemented to get rid of volatiles to obtain molecular weights by GPC. Yield $65 \% ; M_{w} / M_{n}=10,600 ; M_{n}$ (GPC) $=1.95 .{ }^{1} \mathrm{H}$ and ${ }^{13} \mathrm{C}$ NMR spectra display characteristic resonances of the polymer with thionoester repeat unit and thiocarbonyl peak at $224 \mathrm{ppm}$ in the ${ }^{13} \mathrm{C}$ spectrum (see Figure $6.6)$.

Determination of Thermodynamic Parameters for tnHL

A polymerization reaction was run with tnHL $(0.100 \mathrm{~g}, 0.693 \mathrm{mmol})$, TBD $\left(4.83 \times 10^{-3} \mathrm{~g}, 0.035 \mathrm{mmol}\right)$ initiated from benzyl alcohol $\left(7.50 \times 10^{-4} \mathrm{~g}, 6.93 \times 10^{-3} \mathrm{mmol}\right)$ in $\mathrm{C}_{6} \mathrm{D}_{6}(1 \mathrm{M}$ in monomer) inside an NMR tube. After determining equilibrium for the reaction at room temperature, ${ }^{1} \mathrm{H}$ NMR were acquired from $298 \mathrm{~K}$ to $333 \mathrm{~K}$ by heating the sample in a variable temperature NMR probe. Data points were taken twice, during heating and cooling. Since both the heating and cooling $[\mathrm{M}]_{\mathrm{eq}}$ values are within error of each other, only the heating values are shown in Figure 6.7. Then the thermodynamic values for the ROP of tnHL were determined from a Van't Hoff plot of the data where the error was calculated from linear regression at 95\% confidence interval (see Figure 6.7). 


\section{RESULTS AND DISCUSSION}

Organocatalyzed ROP of $\mathrm{HL}$ and tnHL

Similar to the tnCL approach previously studied by our group, ${ }^{70}$ we wanted to observe the ROP behavior of the 8-membered lactone with organic catalysts initially. When the oxygenated HL underwent ROP with TCC and MTBD mediated catalysis, 94\% converted polymer was produced in about 6 hours with good control in weight and polydispersity. With a much stronger phosphazene base like BEMP, that reaction reached about $88 \%$ conversion in 4 minutes. Both of these reactions were performed at $2 \mathrm{M}$ concentration of the monomer in deuterated benzene with 1 mol\% loading of benzyl alcohol. The fast nature of TCC/BEMP-catalyzed ROP for this monomer coincided with previous studies performed in our group with $\delta$-valerolactone and $\varepsilon$-caprolactone ${ }^{80} \mathrm{Few}$ other catalysts like $\mathbf{2}$ in conjunction with MTBD or BEMP were also tried. Though known to be quite strong in binding with a cocatalyst pair from a previous study, ${ }^{81} \mathrm{DBU}$ also showed remarkable control in polymer weight and polydispersity with a slower rate of ROP (see Table 6.1). A living characteristic feature was observed with increased monomer evolution to polymer by weight and narrow polydispersity when TCC/MTBD were implemented in the ROP of HL in benzene- $d_{6}$ (see Figure 6.8a). A first-order kinetic rate plot also further demonstrated the living behavior of this system (see Figure 6.9a).

After the successful ROP performed by organocatalysis for HL, the thionated counterpart, tnHL, was underway. As depicted in Table 6.2, TCC/BEMP-catalyzed ROP of tnHL at $2 \mathrm{M}$ concentration in $\mathrm{C}_{6} \mathrm{D}_{6}$ produced polymer faster than TCC/MTBD at $5 \mathrm{~mol} \%$ loading, imitated from $1 \mathrm{~mol} \%$ benzyl alcohol. This is quite contrasting to the ROP 
behavior of CL versus tnCL in terms of the rate. With a mono-thiourea motif, it was shown previously that tnCL could produce polymer faster than CL under the same conditions. ${ }^{70,80}$ Since ureas could facilitate ROP by an imidate-like mechanism, ${ }^{80,82}$ it is quite possible that the rate could change drastically between oxygenated and thionated systems. The application of 2 with MTBD further validated this point as the reaction slowed down significantly, while BEMP-catalyzed one did not proceed at all to the desired polymer. The H-bonding mechanism is possibly weaker with these larger ring systems which might be the cause of slow growth of polymer. A stronger phosphazene base like BEMP may become inhibitory to the polymer growth completely. However, TBD-catalyzed ROP of tnHL was the fastest, demonstrating the dual activation of monomer and initiation of the alcohol at the same time (see Table 6.2). This corroborates quite well with the TCC/base mediated catalysis, if the imidate-like mechanism is believed to be at play. Similar to the HL data, a linear evolution of molecular weight versus conversion portrayed the livingness of this system along with narrow polydispersity (see Figure 6.8b). A first-order kinetics plot also proved this point further (see Figure 6.9b).

Organocatalyzed ROP of NL and tnNL

Just like HL, the 9-membered oxygenated lactone, NL, was screened for optimal conditions of ROP. As before, the fastest organocatalyst systems, TCC and $\mathbf{2}$ were tried with different bases, MTBD, BEMP and DBU. Although a screening of solvents were also performed from tetrahydrofuran, dichloromethane, chloroform- $d$ to benzene, toluene and acetone. Due to solubility preference, acetone was displayed to be the best solvent where TCC/BEMP produced controlled PNL in little over a day (entry-2, Table 6.3). A good control of molecular weight was also observed in both these systems with relatively narrow 
polydispersity. ${ }^{18,19,26,44,45,83}$ Even though 2/BEMP produced PNL in benzene, molecular weight and polydispersity was not better than TCC/BEMP catalyzed ROP in acetone (entry-3, Table 6.3). A living nature of the polymerization system was observed with linear evolution of $\mathrm{M}_{\mathrm{n}}$ versus conversion to polymer (see Figure 6.10a). A first-order kinetic plot also demonstrated the controlled behavior in the ROP of NL (Figure 6.11a).

Since TCC and $\mathbf{2}$ have been shown previously to perform ROP with fast rate from our previous studies, ${ }^{71,80}$ these were again implemented to thionated NL (tnNL). Due to solubility, acetone was again the preferred solvent for ROP of tnNL, especially when catalyzed by TCC. No polymer was produced when 2 was used, either in conjunction BEMP, one of the best performing bases demonstrated before ${ }^{81}$ However, TCC was able to form PtnNL with BEMP (5 mol\% of cocatalyst pair) at a much faster rate than PNL with relatively good control in molecular weight and polydispersity index (see Table 6.4). ${ }^{18,19,45,83}$ The MTBD-catalyzed ROP of the same monomer in comparable conditions only produced about $75 \%$ of PtnNL (Table 6.4). An increase in molecular weight $\left(\mathrm{M}_{\mathrm{n}}\right)$ versus conversion to polymer was observed with steady hold on polydispersity (see Figure 6.10b). A first-order kinetic plot was also indicative of the living trend of tnNL polymerization (Figure 6.11b).

As shown by HL and tnHL, these 9-membered lactone systems also exhibited polymerization by the imidate-like mechanism we had proposed previously. This is particularly exemplified with tnNL where ROP was only possible with TCC, but not $\mathbf{2}$. Hbonding mechanism might not be at play as $\mathbf{3}$ was unable to produce polymer at all for NL (Table 6.4) and no polymerization took place for tnNL in conjunction with 2. Further studies need to be performed, in terms of molecular modeling to understand whether or not 
the cis/trans- isomerism is behind the poor or complete disfavor of H-bonding mechanism of these 9-membered lactones. Although enzymatic catalysis was performed for the oxygenated lactone before, high conversion, molecular weight and narrow polydispersity were always a challenge. Even though copolymers produced of these monomer with other lactones showed good control, complete homopolymerization with organic H-bond donating catalysts were never performed for these monomers. More investigation with binding and computer modeling may help to comprehend the overall mechanism for the ROP of NL and tnNL in the future.

\section{Organocatalyzed ROP of tnPDL}

The sulfur-containing thionated PDL (tnPDL) has not been studied with organic catalytic systems even though the oxygenated PDL is well studied. ${ }^{20,27,38,39,50,84}$ Our approach to the ROP of tnPDL was mainly inspired from Dove's report. ${ }^{50}$ Since it is welldocumented that lactones of larger ring sizes chiefly polymerize at an elevated temperature due to entropic contribution as the driving force of the reaction, ${ }^{85,86}$ we attempted the same scenario with our fast known catalysts, TCC and $\mathbf{2}$ at a much higher monomer concentration ( $5 \mathrm{M})$ than usual. Similar to tnNL, no polymers were produced when $\mathbf{2}$ or $\mathbf{4}$ were used (Table 6.5). TCC was able to generate polymer with almost full conversion while displaying poor handle on polymer dispersity and molecular weight control (Table 6.5). This is expected for macrolactone like this 16-membered lactone which is why copolymerizations are generally performed for this monomer's oxygenated derivative with other lactones. ${ }^{22,23,25,51,64,66,67,87,88}$ Just as tnNL, the ability of TCC to produce PtnPDL and inability of $\mathbf{2}$ or $\mathbf{4}$ to yield any polymer is indicative of a imidate-based mechanism to be in effect. Further studies need to be performed to fully understand the H-bonding pathway for 
this monomer, if there is any. Computer modeling could prove to be very handy for that purpose.

\section{Organocatalyzed ROP of tnEB}

Initially, the ring-opening polymerization of this monomer was attempted in neat conditions. Similar to previous reports on $\mathrm{EB}$, the reactions were performed at $80^{\circ} \mathrm{C}$ within the glovebox. However, the initial conversion data obtained for neat conditions were not fully consistent as dissolution of the catalyst in the monomeric solution only happened after few minutes of heating the overall mixture. Thus, it was best to perform the reaction in solution phase with a high boiling point solvent, like toluene. Based on the results of those ROPs, we can observe that with a $2 \mathrm{M}$ concentration of tnEB in toluene, TCC/BEMP or TCC/MTBD (5 mol\%) does not produce a full $90 \%$ or over of PtnEB, but about high $60 \%$ conversion (see Table 6.6). This is quite consistent with previous ROP results of EB in toluene where $44 \%$ polymeric conversion is reached while neat conditions produced almost full conversion. ${ }^{48,54}$ This is also in correlation with what can be expected of macrolactones of this size where entropy is driving the reaction forward with minimal or negligible contribution from enthalpy toward the polymeric process. ${ }^{89}$ In fact, when we tried to obtain a Van't Hoff plot of the polymerization process of this system, we did not see any enthalpic impact on the reaction and only entropic contribution which was within NMR error.

Thermodynamics of Macrolactones

We had performed ROP of larger lactones (or macrolactones), especially tnPDL and tnEB to validate entropic driving force for these polymerization reactions. In fact, all the macrolactones, both oxygenated or thionated, demonstrated almost zero to very negligible enthalpic contribution while entropy was quite substantial compared to smaller 
lactones, like HL and tnHL. For NL and tnNL, there is a possibility of cis/trans- isomerism formation for the $s$-ester moiety. That could contribute to whether or not polymerization will proceed via H-bonding or imidate-like mechanism. Thermodynamic data suggests that polymerization is mainly governed by entropy as expected. ${ }^{85,89}$ Future studies on the polymerization processes of these monomers ( $\mathrm{NL}$ and $\mathrm{tnNL}$ ) may shed light on the conformational change of the ester functionality, if there is any.

Mechanistic Aspects of ROP

Although this work is still currently undergoing, we can surmise based on the evidences presented to us from various ROP reactions that the TCC-based polymerizations generally follow a imidate-like mechanism while $\mathbf{2}$ proceeds in a traditional H-bond donating pathway (Scheme 6.3). This holds true for both the oxygenated and thionated monomers studied in this project. With the larger macrolactones (tnPDL), the entropic driving force is not enough for H-bond systems to cause polymer formation (see entry-3, Table 6.5), but is sufficient for imidate-mediated mechanism to occur. With tnEB, the transesterification mechanism that usually accompanies larger lactones generally prevents full polymer formation and lower molecular weights with imidate-based systems (Table 6.6). Non-organic catalysts might be able to lead to higher molecular weights for such a dual ester motif containing substrate, but that was not attempted by this research since organocatalysis was the backbone of this project. Future studies on binding interactions may lead to understand the overall mechanism of these macrolactones better. 


\section{CONCLUSION}

The breadth of studies that have been performed on the oxygenated lactones and still undergoing is significant compared to the thionated lactone derivatives. ${ }^{9-11,90,91}$ The lack of studies performed for the thionated counterparts could be due to controlled polymerization catalysts. The growth of $\mathrm{H}$-bond donating catalysts in the last few decades have enabled an array of opportunities for these less explored substrates. It was with this objective that we had performed ROP to produce homopolymers of 8-, 9-, 16-, 17-(di-ester) membered lactones with some of the fast known (thio)urea based catalysts. As predicted, homopolymers were generated in good control of molecular weight and polydispersity for the smaller lactones until $s$-trans moiety of the ester becomes a dominant factor with larger ring size. ${ }^{18,19}$ Moreover, unlike some of the other catalytic systems that produced thionated polymers in the past, our organic catalysts were able to retain thiocarbonyl in the final polymer. ${ }^{92}$ Thermodynamic studies performed for the ROP of these lactones also correlated well with literature. ${ }^{89}$ With these understandings and future experimentations to comprehend the mechanistic aspects of these systems will allow new material production. 


\section{LIST OF REFERENCES}

(1) Nederberg, F.; Connor, E. F.; Möller, M.; Glauser, T.; Hedrick, J. L. Angew. Chem. Int. Ed. Engl. 2001, 40, 2712-2715.

(2) Lohmeijer, B. G. G.; Pratt, R. C.; Leibfarth, F.; Logan, J. W.; Long, D. A.; Dove, A. P.; Nederberg, F.; Choi, J.; Wade, C.; Waymouth, R. M.; Hedrick, J. L. Macromolecules 2006, 39 (25), 8574-8583.

(3) Kiesewetter, M. K.; Shin, E. J.; Hedrick, J. L.; Waymouth, R. M. Macromolecules 2010, 43 (5), 2093-2107.

(4) Dove, A. P.; Pratt, R. C.; Lohmeijer, B. G. G.; Waymouth, R. M.; Hedrick, J. L.; V, S. U. J. Am. Chem. Soc. 2005, 127, 13798-13799.

(5) Pratt, R. C.; Lohmeijer, B. G. G.; Long, D. A.; Lundberg, P. N. P.; Dove, A. P.; Li, H.; Wade, C. G.; Waymouth, R. M.; Hedrick, J. L. Macromolecules 2006, 39 (23), $7863-7871$.

(6) Hedrick, J. L.; Magbitang, T.; Connor, E. F.; Glauser, T.; Volksen, W.; Hawker, C. J.; Lee, V. Y.; Miller, R. D. Chem. - A Eur. J. 2002, 8 (15), 3308-3319.

(7) Zhang, L.; Xiong, C.; Deng, X. J. Appl. Polym. Sci. 1995, 56 (1), 103-112.

(8) Tokiwa, Y.; Calabia, B. P.; Ugwu, C. U.; Aiba, S. Int. J. Mol. Sci. 2009, 10 (9), $3722-3742$.

(9) Labet, M.; Thielemans, W. Chem. Soc. Rev. 2009, 38 (12), 3484.

(10) Auras, R.; Harte, B.; Selke, S. Macromol. Biosci. 2004, 4 (9), 835-864.

(11) Nuyken, O.; Pask, S. D. Polymers (Basel). 2013, 5 (2), 361-403.

(12) Myers, M.; Connor, E. F.; Glauser, T.; McK, A.; Nyce, G.; Hedrick, J. L. J. Polym. Sci. Part A Polym. Chem. 2002, 40 (7), 844-851. 
(13) Connor, E. F.; Nyce, G. W.; Myers, M.; Möck, A.; Hedrick, J. L. J. Am. Chem. Soc. 2002, 124 (6), 914-915.

(14) Shibasaki, Y.; Sanada, H.; Yokoi, M.; Sanda, F.; Endo, T. Macromolecules 2000, 33 (12), 4316-4320.

(15) Huisgen, R.; Ott, H. Tetrahedron 1959, 6 (3), 253-267.

(16) Huisgen, R.; Ott, H. Angew. Chemie 1958, 70, 312.

(17) Huisgen, R. Angew. Chemie 1957, 69, 341-359.

(18) Van Der Mee, L.; Helmich, F.; De Bruijn, R.; Vekemans, J. A. J. M.; Palmans, A. R. A.; Meijer, E. W. Macromolecules 2006, 39 (15), 5021-5027.

(19) Duda, A.; Kowalski, A.; Penczek, S.; Uyama, H.; Kobayashi, S. Macromolecules 2002, 35 (11), 4266-4270.

(20) Focarete, M. L.; Scandola, M.; Kumar, A.; Gross, R. a. J. Polym. Sci. Part B Polym. Phys. 2001, 39, 1721-1729.

(21) Bouyahyi, M.; Pepels, M. P. F.; Heise, A.; Duchateau, R. Macromolecules 2012, $45(8), 3356-3366$.

(22) Uyama, H.; Kikuchi, H.; Takeya, K.; Kobayashi, S. Acta Polym. 1996, 47, 357360.

(23) Kumar, A.; Kalra, B.; Dekhterman, A.; Gross, R. A. Macromolcules 2000, 33, 6303-6309.

(24) Bouyahyi, M.; Duchateau, R. Macromolecules 2014, 47 (2), 517-524.

(25) Jasinska-walc, L.; Hansen, R.; Dudenko, D. D.; Rozanski, A.; Bouyahyi, M.; Wagner, M.; Duchateau, R. Polym. Chem. 2014, 5, 3306-3310.

(26) Namekawa, S.; Suda, S.; Uyama, H.; Kobayashi, S. Int. J. Biol. Macromol. 1999, 
$25(1-3), 145-151$.

(27) Bisht, K. S.; Henderson, L. A.; Gross, R. A.; Kaplan, D. L.; Swift, G. Macromolecules 1997, 30 (9), 2705-2711.

(28) de Geus, M.; van der Meulen, I.; Goderis, B.; van Hecke, K.; Dorschu, M.; van der Werff, H.; Koning, C. E.; Heise, A. Polym. Chem. 2010, 1 (4), 525.

(29) Van Der Meulen, I.; De Geus, M.; Antheunis, H.; Deumens, R.; Joosten, E. A. J.;

Koning, C. E.; Heise, A. Biomacromolecules 2008, 9 (12), 3404-3410.

(30) Claudino, M.; Van Der Meulen, I.; Trey, S.; Jonsson, M.; Heise, A.; Johansson, M. J. Polym. Sci. Part A Polym. Chem. 2012, 50 (1), 16-24.

(31) Ates, Z.; Heise, A. Polym. Chem. 2014, 5 (8), 2936.

(32) Alaaeddine, A.; Thomas, C. M.; Roisnel, T.; Carpentier, J. F. Organometallics 2009, 28 (5), 1469-1475.

(33) Sinenkov, M.; Kirillov, E.; Roisnel, T.; Fukin, G.; Trifonov, A.; Carpentier, J. F. Organometallics 2011, 30 (20), 5509-5523.

(34) Hayes, C. E.; Sarazin, Y.; Katz, M. J.; Carpentier, J. F.; Leznoff, D. B. Organometallics 2013, 32 (5), 1183-1192.

(35) Wang, L.; Poirier, V.; Ghiotto, F.; Bochmann, M.; Cannon, R. D.; Carpentier, J. F.; Sarazin, Y. Macromolecules 2014, 47 (8), 2574-2584.

(36) Nakayama, Y.; Watanabe, N.; Kusaba, K.; Sasaki, K.; Cai, Z.; Shiono, T.; Tsutsumi, C. J. Appl. Polym. Sci. 2011, 121 (4), 2098-2103.

(37) Lebedev, B.; Yevstropov, A. Makromol. Chem. 1984, 1253, 1235-1253.

(38) Zhong, Z.; Dijkstra, P. J.; Feijen, J. Macromol. Chem. Phys. 2000, 201 (12), 1329 1333. 
(39) Jedliriski, Z.; Juzwa, M.; Adarnus, G.; Kowalczuk, M.; Montaudo, M. Macromol. Chem. Phys. 1996, 197, 2923-2929.

(40) Van Der Meulen, I.; Gubbels, E.; Huijser, S.; Sablong, R.; Koning, C. E.; Heise, A.; Duchateau, R. Macromolecules 2011, 44 (11), 4301-4305.

(41) Makiguchi, K.; Yamanaka, T.; Kakuchi, T.; Terada, M.; Satoh, T. Chem. Commun. 2014, 50 (22), 2883-2885.

(42) Nomura, R.; Ueno, A.; Endo, T. Macromolecules 1994, 27 (2), 620-621.

(43) Uyama, H.; Takeya, K.; Hoshi, N.; Kobayashi, S. Macromolecules 1995, 28 (21), 7046-7050.

(44) Namekawa, S.; Uyama, H.; Kobayashi, S. Polymer Journal. 1998, pp 269-271.

(45) Kobayashi, S.; Uyama, H.; Namekawa, S.; Hayakawa, H. Macromolecules 1998, 31 (17), 5655-5659.

(46) Kobayashi, S.; Takeya, K.; Suda, S.; Uyama, H. Macromol. Chem. Phys. 1998, $199(8), 1729-1736$.

(47) Namekawa, S.; Uyama, Hiroshi, K. S. Proc. Japan Acad. 1998, 74 (B), 65-68.

(48) Pascual, A.; Sardón, H.; Ruipérez, F.; Gracia, R.; Sudam, P.; Veloso, A.; Mecerreyes, D. J. Polym. Sci. Part A Polym. Chem. 2014, 53 (4), 552-561.

(49) Pascual, A.; Sardon, H.; Veloso, A.; Ruipérez, F.; Mecerreyes, D. ACS Macro Lett. 2014, 3 (9), 849-853.

(50) Wilson, J. A.; Hopkins, S. A.; Wright, P. M.; Dove, A. P. Polym. Chem. 2014, 5 (8), 2691-2694.

(51) Wilson, J. A.; Hopkins, S. A.; Wright, P. M.; Dove, A. P. Macromolecules 2015, $48(4), 950-958$. 
(52) Gazzano, M.; Focarete, M. L.; Scandola, M.; Gross, R. a. J. Polym. Sci. Part BPolym. Phys. 2003, 41, 1009-1013.

(53) Simpson, N.; Takwa, M.; Hult, K. Macromol. Symp. 2008, 41 (Figure 1), 36133619.

(54) Pothupitiya, J. U.; Dharmaratne, N. U.; Jouaneh, T. M. M.; Fastnacht, K. V.; Coderre, D. N.; Kiesewetter, M. K. Macromolecules 2017, 50 (22), 8948-8954.

(55) Ajellal, N.; Carpentier, J.-F.; Guillaume, C.; Guillaume, S. M.; Helou, M.; Poirier, V.; Sarazin, Y.; Trifonov, A. Dalt. Trans. 2010, 39 (36), 8363.

(56) Guillaume, S. M.; Carpentier, J.-F. Catal. Sci. Technol. 2012, 2 (5), 898.

(57) Fang, H. J.; Lai, P. S.; Chen, J. Y.; Hsu, S. C. N.; Peng, W. De; Ou, S. W.; Lai, Y. C.; Chen, Y. J.; Chung, H.; Chen, Y.; Huang, T. C.; Wu, B. S.; Chen, H. Y. J. Polym. Sci. Part A Polym. Chem. 2012, 50 (13), 2697-2704.

(58) Chuma, A.; Horn, H. W.; Swope, W. C.; Pratt, R. C.; Zhang, L.; Lohmeijer, B. G. G.; Wade, C. G.; Waymouth, R. M.; Hedrick, J. L.; Rice, J. E. J. Am. Chem. Soc. 2008, 130 (21), 6749-6754.

(59) Pratt, R. C.; Lohmeijer, B. G. G.; Long, D. A.; Waymouth, R. M.; Hedrick, J. L. J. Am. Chem. Soc. 2006, 128 (14), 4556-4557.

(60) Kamber, N. E.; Jeong, W.; Waymouth, R. M.; Pratt, R. C.; Lohmeijer, B. G. G.; Hedrick, J. L. Chem. Rev. 2007, 107 (12), 5813-5840.

(61) Helou, M.; Miserque, O.; Brusson, J. M.; Carpentier, J. F.; Guillaume, S. M. Chem. - A Eur. J. 2010, 16 (46), 13805-13813.

(62) Pratt, R. C.; Nederberg, F.; Waymouth, R. M.; Hedrick, J. L. Chem. Commun. 2008, No. 1, 114-116. 
(63) Dove, A. P.; Pratt, R. C.; Lohmeijer, B. G. G.; Culkin, D. A.; Hagberg, E. C.; Nyce, G. W.; Waymouth, R. M.; Hedrick, J. L. Polymer (Guildf). 2006, 47 (11), $4018-4025$.

(64) Jiang, Z.; Azim, H.; Gross, R. A.; Focarete, M. L.; Scandola, M.

Biomacromolecules 2007, 8 (7), 2262-2269.

(65) Mazzocchetti, L.; Scandola, M.; Jiang, Z. Macromolecules 2009, 42 (20), 78117819.

(66) Mazzocchetti, L.; Scandola, M.; Jiang, Z. Eur. Polym. J. 2012, 48 (11), 18831891.

(67) Kumar, A.; Garg, K.; Gross, R. A. Macromolecules 2001, 34 (11), 3527-3533.

(68) Bouyahyi, M.; Pepels, M. P. F.; Heise, A.; Duchateau, R. Macromolecules 2012, 45 (8), 3356-3366.

(69) Bannin, T. J.; Kiesewetter, M. K. Macromolecules 2015, 48 (16), 5481-5486.

(70) Datta, P. P.; Kiesewetter, M. K. Macromolecules 2016, 49 (3), 774-780.

(71) Fastnacht, K. V.; Spink, S. S.; Dharmaratne, N. U.; Pothupitiya, J. U.; Datta, P. P.; Kiesewetter, E. T.; Kiesewetter, M. K. ACS Macro Lett. 2016, 5 (8), 982-986.

(72) Spink, S. S.; Kazakov, O. I.; Kiesewetter, E. T.; Kiesewetter, M. K. Macromolecules 2015, 48 (17), 6127-6131.

(73) Kai, K.; Takeuchi, J.; Kataoka, T.; Yokoyama, M.; Watanabe, N. Tetrahedron 2008, 64 (28), 6760-6769.

(74) Rajabi, M.; Lanfranchi, M.; Campo, F.; Panza, L. Synth. Commun. 2014, 44 (8), 1149-1154.

(75) Curphey, T. J. Tetrahedron Lett. 2002, 43 (3), 371-373. 
(76) Glass, R. S. Sci. Synth. 2005, 22, 85-108.

(77) Hagen, J. P. J. Org. Chem. 1993, 58 (2), 506-508.

(78) Wilson, J. A.; Hopkins, S. A.; Wright, P. M.; Dove, A. P. ACS Macro Lett. 2016, 5 (3), 346-350.

(79) Kraft, P.; Cadalbert, R. Synlett 1997, 5, 600-602.

(80) Dharmaratne, N. U.; Pothupitiya, J. U.; Bannin, T. J.; Kazakov, O. I.; Kiesewetter, M. K. ACS Macro Lett. 2017, 6 (4), 421-425.

(81) Kazakov, O. I.; Datta, P. P.; Isajani, M.; Kiesewetter, E. T.; Kiesewetter, M. K. Macromolecules 2014, 47 (21), 7463-7468.

(82) Lin, B.; Waymouth, R. M. J. Am. Chem. Soc. 2017, 139 (4), 1645-1652.

(83) Kobayashi, S. Macromol. Symp. 2006, 240, 178-185.

(84) Cai, J.; Liu, C.; Cai, M.; Zhu, J.; Zuo, F.; Hsiao, B. S.; Gross, R. A. Polymer (Guildf). 2010, 51 (5), 1088-1099.

(85) Strandman, S.; Gautrot, J. E.; Zhu, X. X. Polym. Chem. 2011, 2, 791-799.

(86) Duda, A.; Kowalski, A. In Handbook of Ring-Opening Polymerization; Dubois, P., Coulembier, O., Raquez, J.-M., Eds.; Wiley-VCH Verlag GmbH \& Co. KGaA, 2009; pp 1-52.

(87) Ceccorulli, G.; Scandola, M.; Kumar, A.; Kalra, B.; Gross, R. A. Biomacromolecules 2005, 6 (2), 902-907.

(88) Zotzmann, J.; Behl, M.; Feng, Y.; Lendlein, A. Adv. Funct. Mater. 2010, 20 (20), $3583-3594$.

(89) Duda, A.; Kowalski, A. In Handbook of Ring-Opening Polymerization; Dubois, P., Coulembier, O., Raquez, J.-M., Eds.; Wiley-VCH Verlag GmbH \& Co. KGaA, 
2009; pp 1-52.

(90) Masutani, K.; Kimura, Y. PLA Synthesis and Polymerization; 2014.

(91) Dorgan, J. R.; Lehermeier, H. J.; Palade, L. I.; Cicero, J. Macromol. Symp. 2001, $175,55-66$.

(92) Sanda, F.; Jirakanjana, D.; Hitomi, M.; Endo, T. Macromolecules 1999, 32 (24), 8010-8014. 

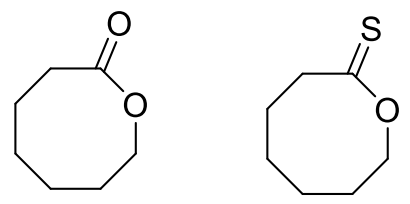

$\mathrm{HL}$

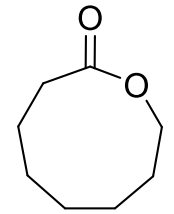

NL

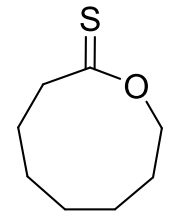

$\operatorname{tnNL}$<smiles>O=C1CCCCCCCCCCCCCOC(=O)C1</smiles><smiles>O=C1CCCCCCCCCCCCCO1</smiles>

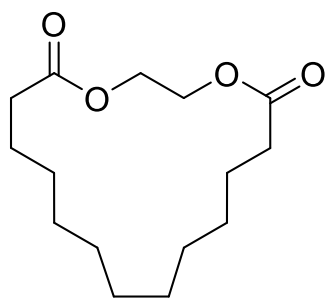

EB



tnEB

Scheme 6.1. Monomers studied for the ROP in this study. 


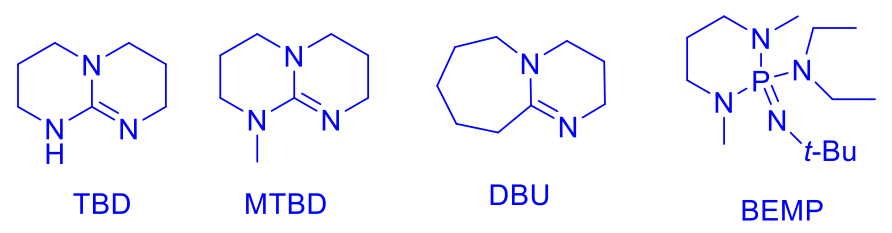<smiles>O=C(Nc1ccc(Cl)cc1)Nc1ccc(Cl)c(Cl)c1</smiles><smiles>FC(F)(F)c1cc(NC(=S)NC2CCCCC2)cc(C(F)(F)F)c1</smiles><smiles>O=C(NCCN(CCNC(=O)Nc1cc(C(F)(F)F)cc(C(F)(F)F)c1)CCNC(=O)Nc1cc(C(F)(F)F)cc(C(F)(F)F)c1)Nc1cc(C(F)(F)F)cc(C(F)(F)F)c1</smiles><smiles>FC(F)(F)c1cc(NC(=S)NCCCNC(=S)Nc2cc(C(F)(F)F)cc(C(F)(F)F)c2)cc(C(F)(F)F)c1</smiles><smiles>FC(F)(F)c1cc(NC(=S)NCCN(CCNC(=S)Nc2cc(C(F)(F)F)cc(C(F)(F)F)c2)CCNC(=S)Nc2cc(C(F)(F)F)cc(C(F)(F)F)c2)cc(C(F)(F)F)c1</smiles>

Scheme 6.2. Bases and (thio)urea cocatalysts screened during this study. 


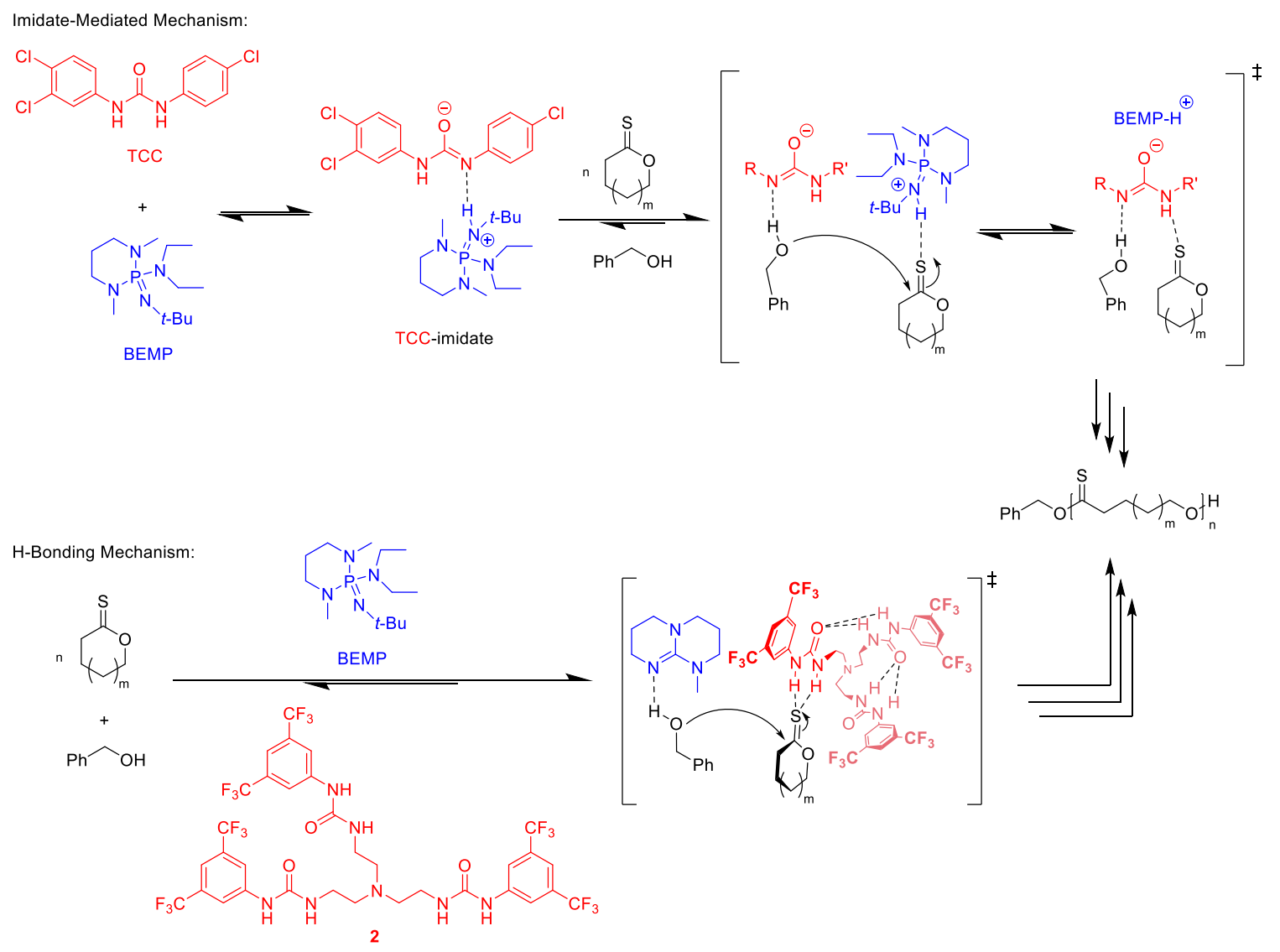

Scheme 6.3. Proposed imidate-mediated and H-bond mediated mechanism for the BEMP catalyzed ROP of cyclic ester monomers where $\mathrm{m}=$ number of methylene units for the different monomers studied as shown in Scheme 6.1. 




Figure 6.1. ${ }^{1} \mathrm{H} \mathrm{NMR}\left(400 \mathrm{MHz}, \mathrm{CDCl}_{3}\right)$ spectrum of tnEB. 




Figure 6.2. ${ }^{13} \mathrm{C}$ NMR $\left(100 \mathrm{MHz}, \mathrm{CDCl}_{3}\right)$ spectrum of $\mathrm{tnEB}$. 


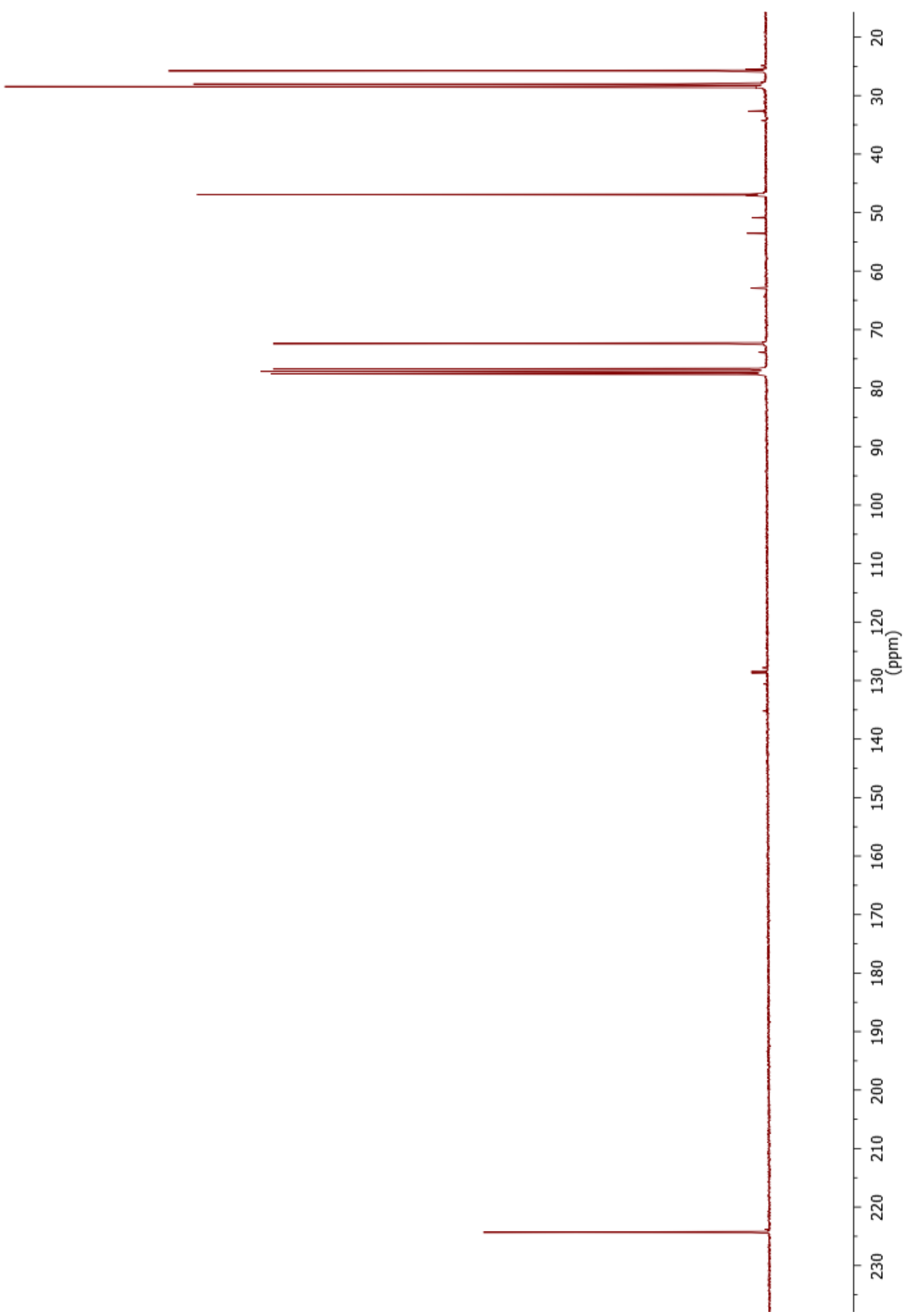

Figure 6.3. ${ }^{13} \mathrm{C}\left(75 \mathrm{MHz}, \mathrm{CDCl}_{3}\right)$ spectrum of homopolymer of PtnHL (2M, $\left.\mathrm{C}_{6} \mathrm{D}_{6}\right)$ initiated from benzyl alcohol (1 mol\%) catalyzed by TCC/BEMP (5 mol\% each), displaying almost no carbonyl peak but thiocarbonyl resonance at $224 \mathrm{ppm}$. 


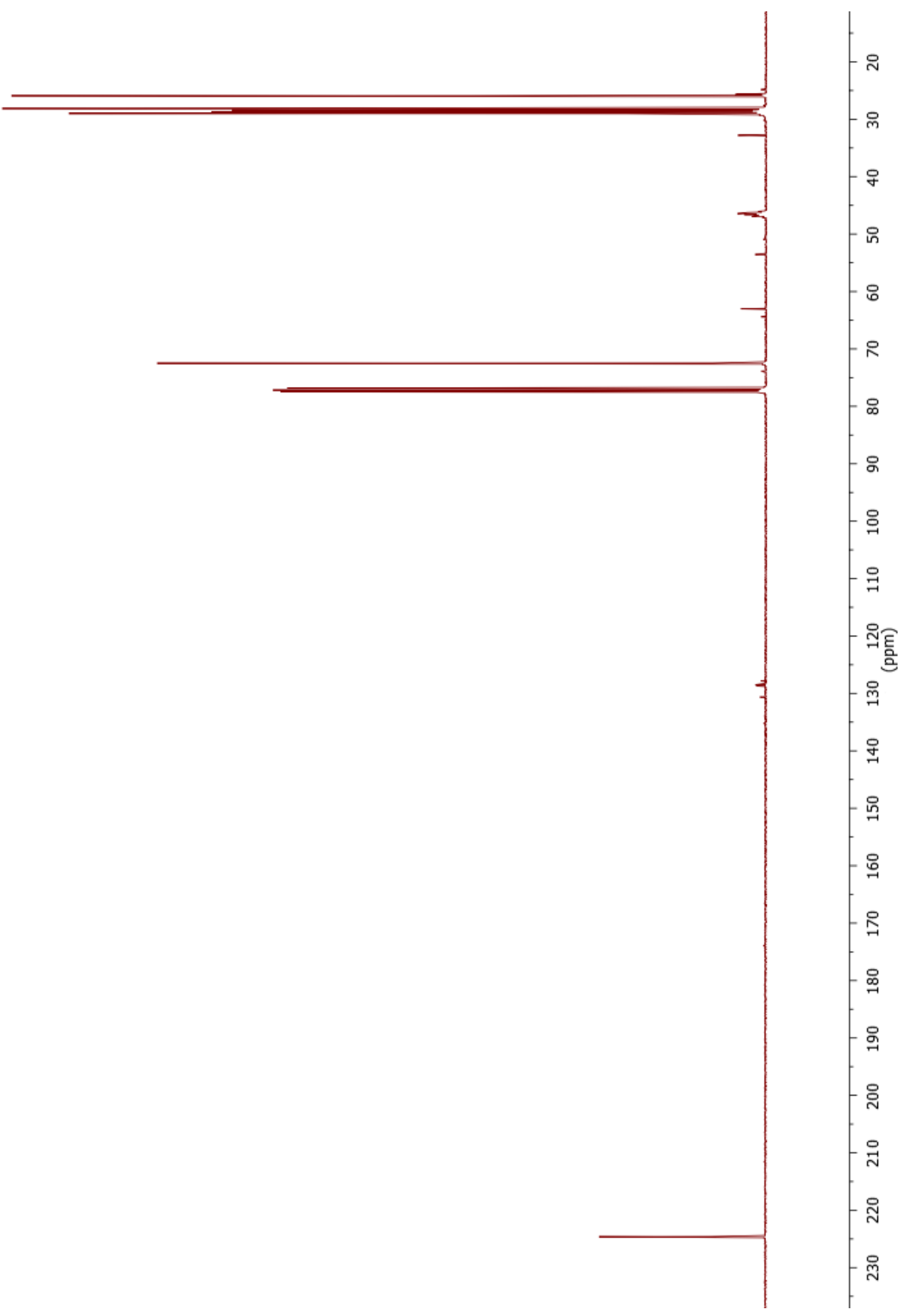

Figure 6.4. ${ }^{13} \mathrm{C}\left(100 \mathrm{MHz}, \mathrm{CDCl}_{3}\right)$ spectrum of homopolymer of PtnNL (2M, acetone- $\left.d_{6}\right)$ initiated from benzyl alcohol (1 mol\%) catalyzed by TCC/BEMP (5 mol\% each), displaying almost no carbonyl peak but thiocarbonyl resonance at $224 \mathrm{ppm}$. 


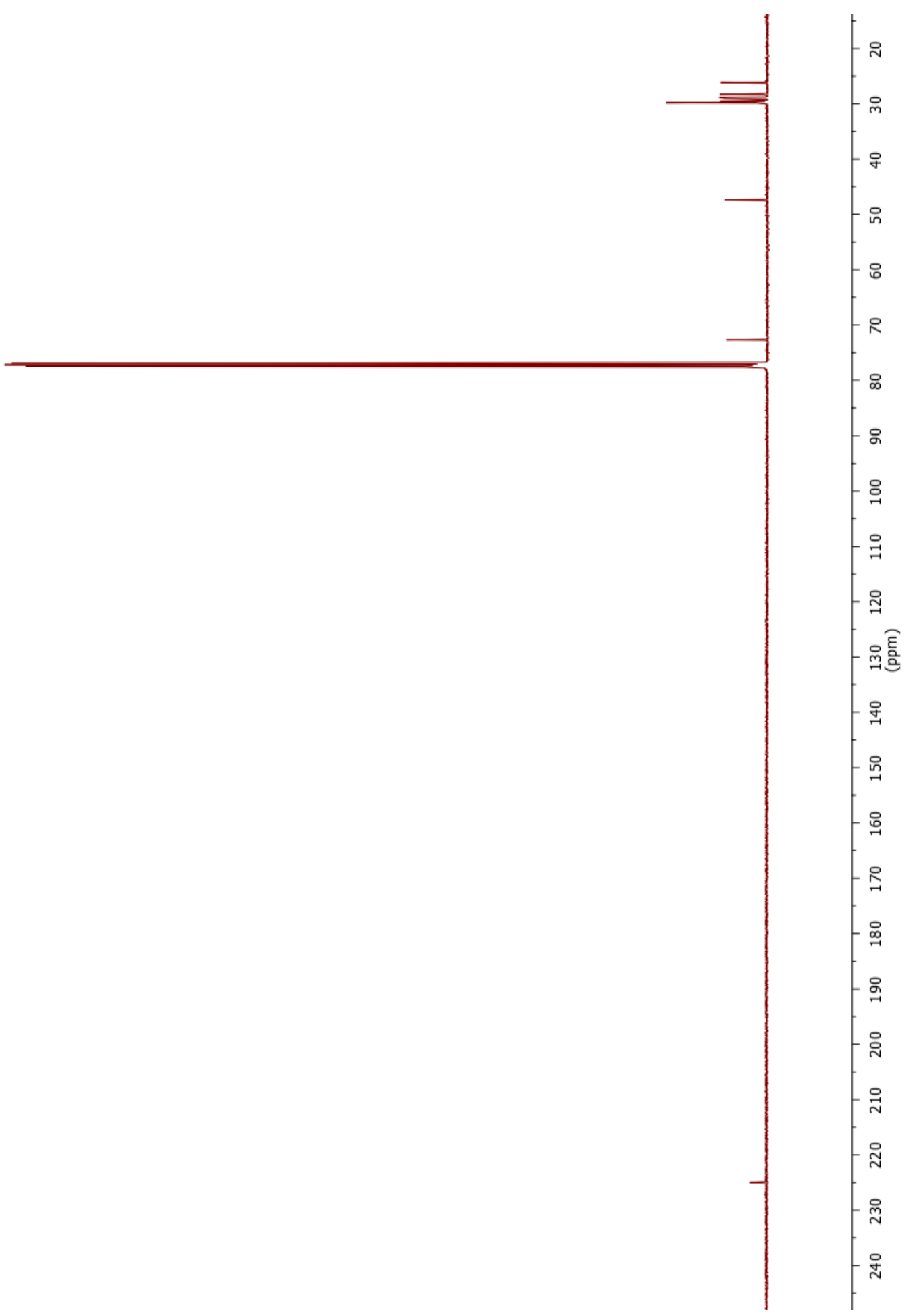

Figure 6.5. ${ }^{13} \mathrm{C}\left(100 \mathrm{MHz}, \mathrm{CDCl}_{3}\right)$ spectrum of homopolymer of PtnPDL (5M, toluene) initiated from benzyl alcohol (1 mol\%) catalyzed by TCC/BEMP (5 mol\% each), displaying almost no carbonyl peak but thiocarbonyl resonance at $224 \mathrm{ppm}$. 


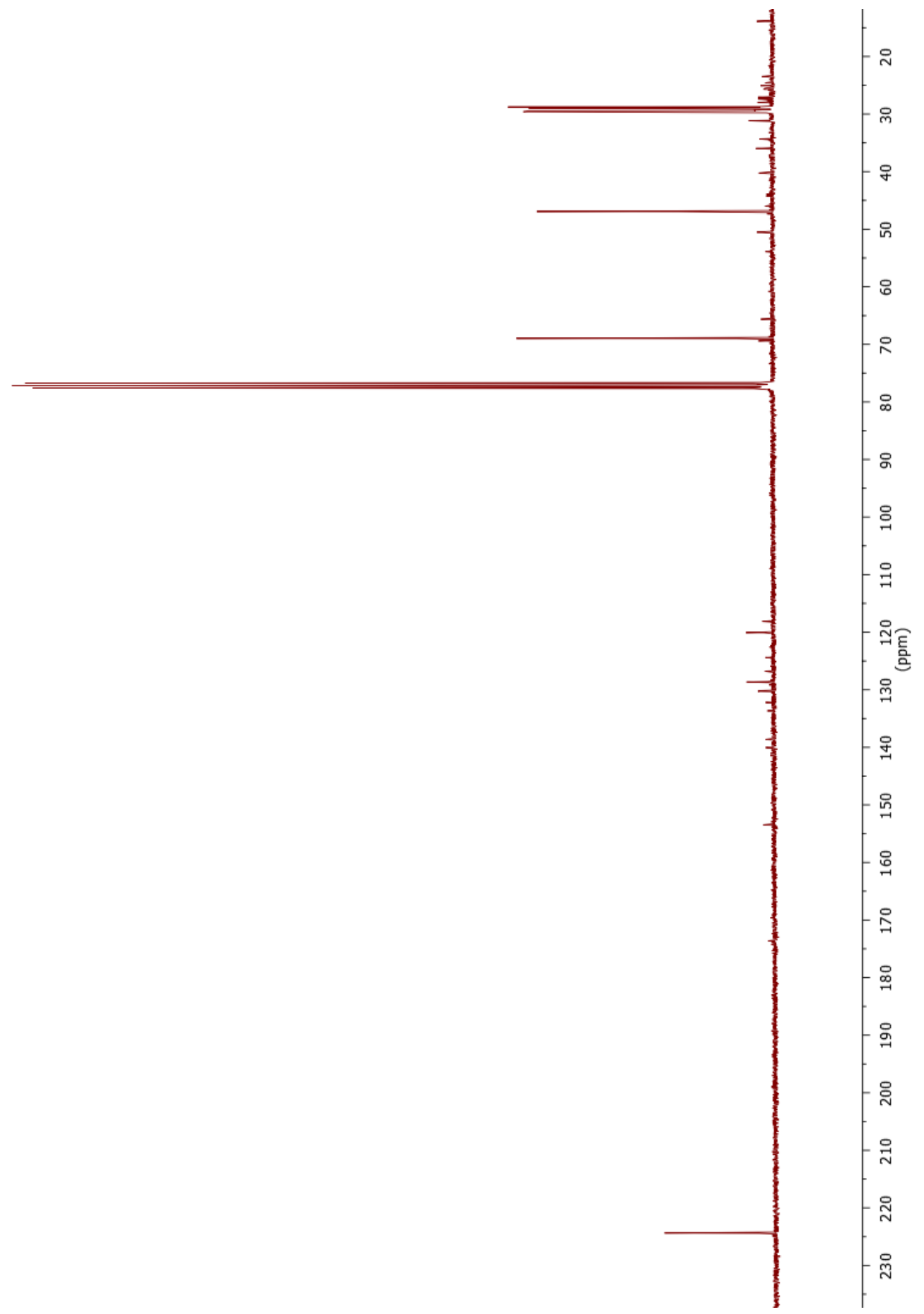

Figure 6.6. ${ }^{13} \mathrm{C}\left(75 \mathrm{MHz}, \mathrm{CDCl}_{3}\right)$ spectrum of homopolymer of PtnEB (2M, toluene) initiated from benzyl alcohol $(1 \mathrm{~mol} \%)$ catalyzed by TCC/BEMP $(5 \mathrm{~mol} \%$ each $)$ at $80^{\circ} \mathrm{C}$, displaying almost no carbonyl peak but thiocarbonyl resonance at $224 \mathrm{ppm}$. 


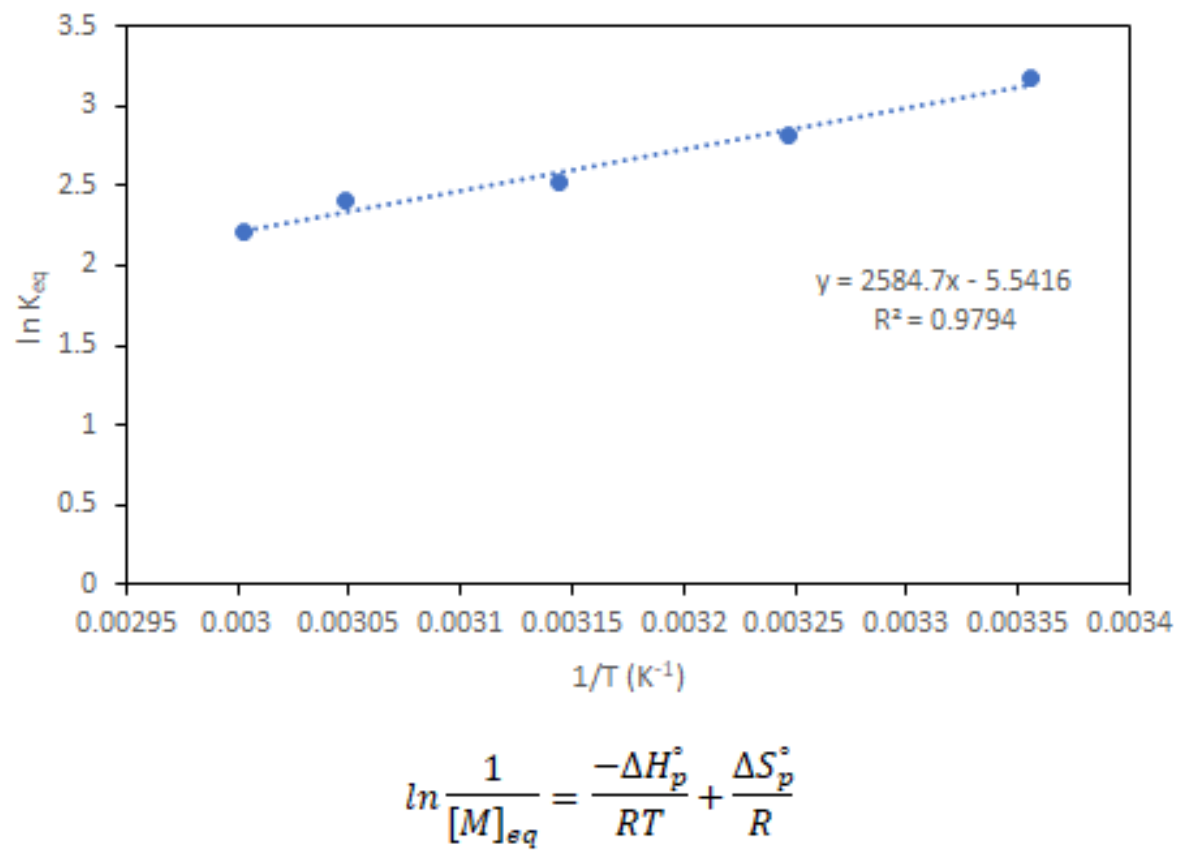

Figure 6.7. Temperature dependent equilibrium constant for the reversible ROP of tnHL $\left(1 \mathrm{M}, \mathrm{C}_{6} \mathrm{D}_{6}\right)$ catalyzed by TBD (5 mol\%) from benzyl alcohol (1 mol\%). 
(a)

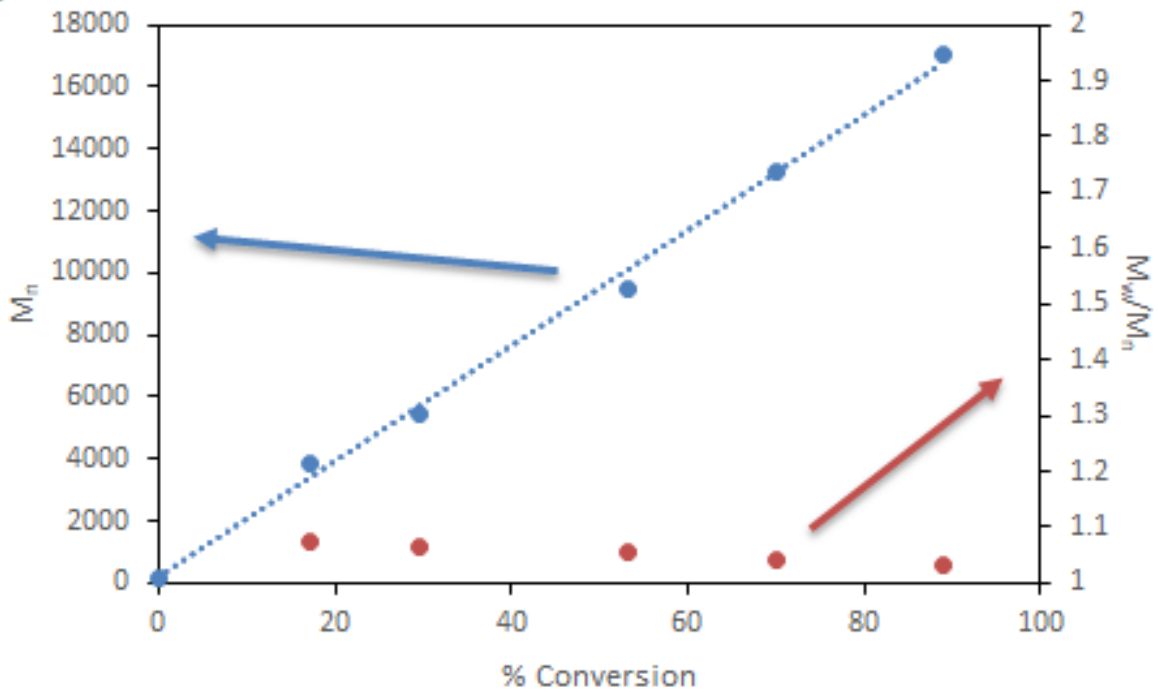

(b)

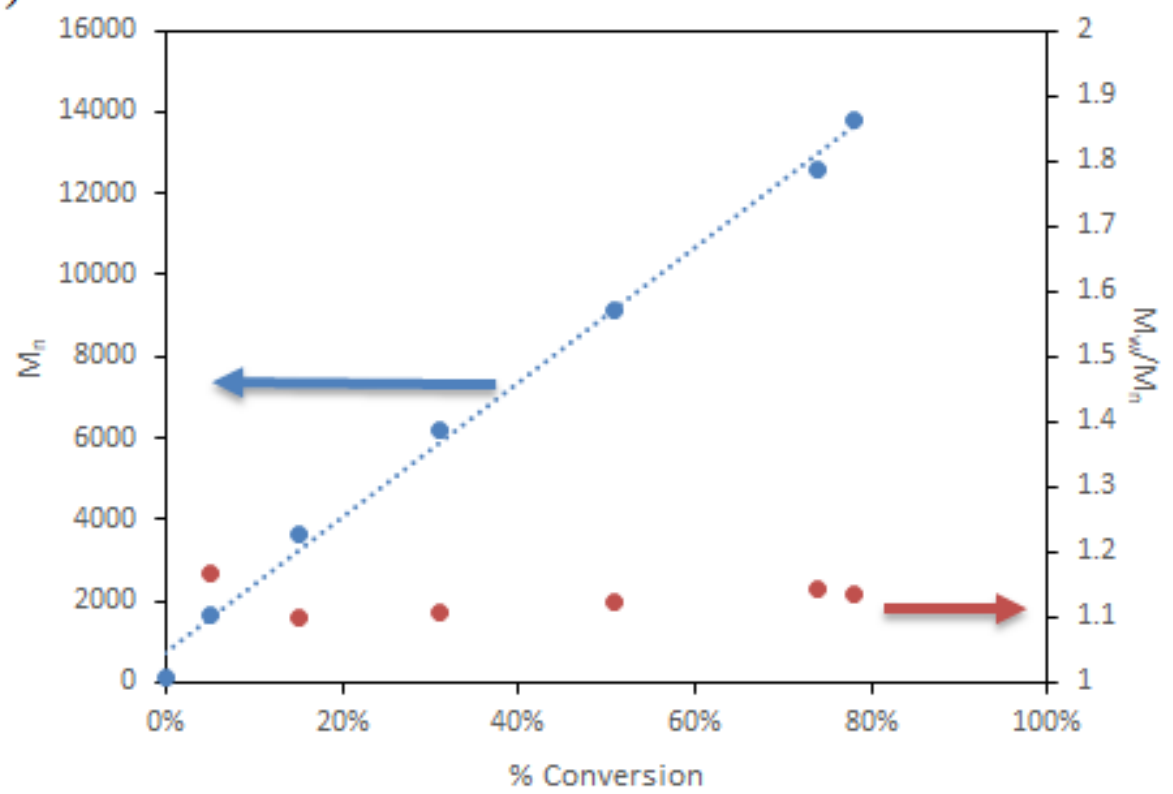

Figure 6.8. Evolution of $\mathrm{M}_{\mathrm{n}}$ vs conversion for the (a) TCC/MTBD (5 mol\% each) catalyzed ROP of $\mathrm{HL}\left(2 \mathrm{M}\right.$ in $\left.\mathrm{C}_{6} \mathrm{D}_{6}\right)$ initiated from benzyl alcohol (1 mol\%); (b) TCC/MTBD (5 mol\% each) catalyzed ROP of tnHL (2M in $\left.\mathrm{C}_{6} \mathrm{H}_{6}\right)$ initiated from benzyl alcohol (1 mol\%). 
(a)

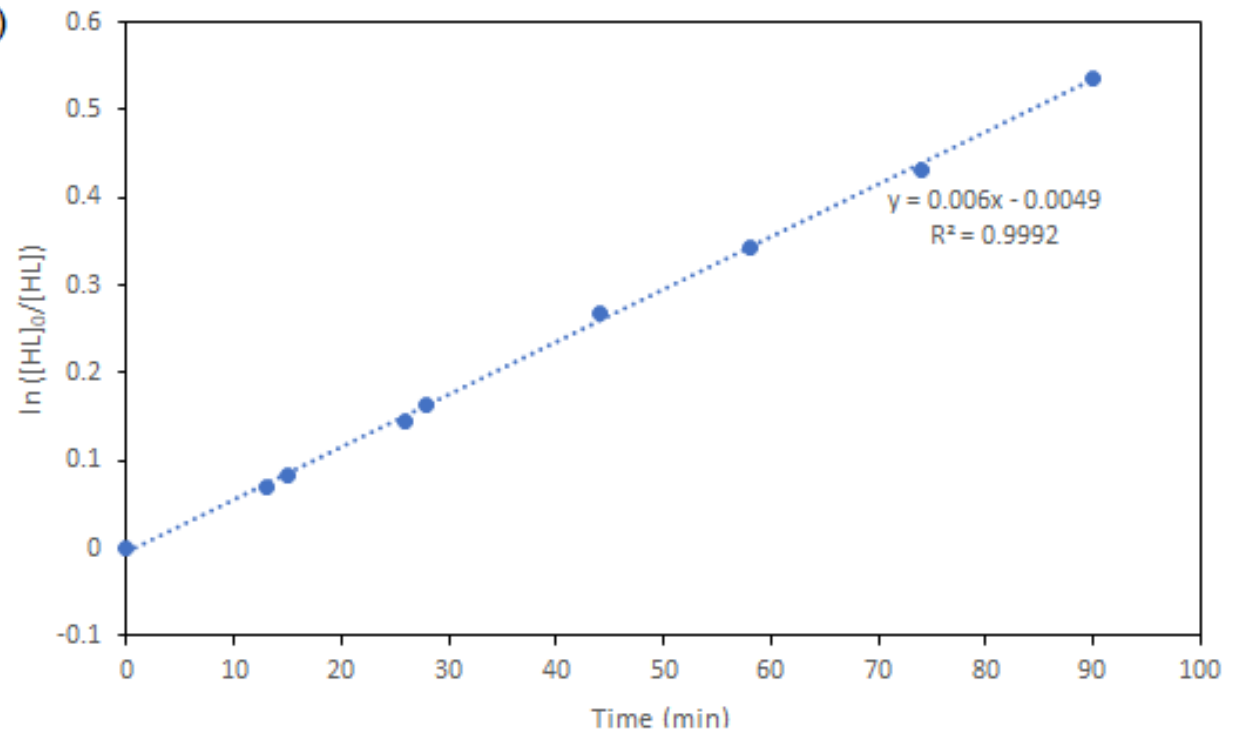

(b)

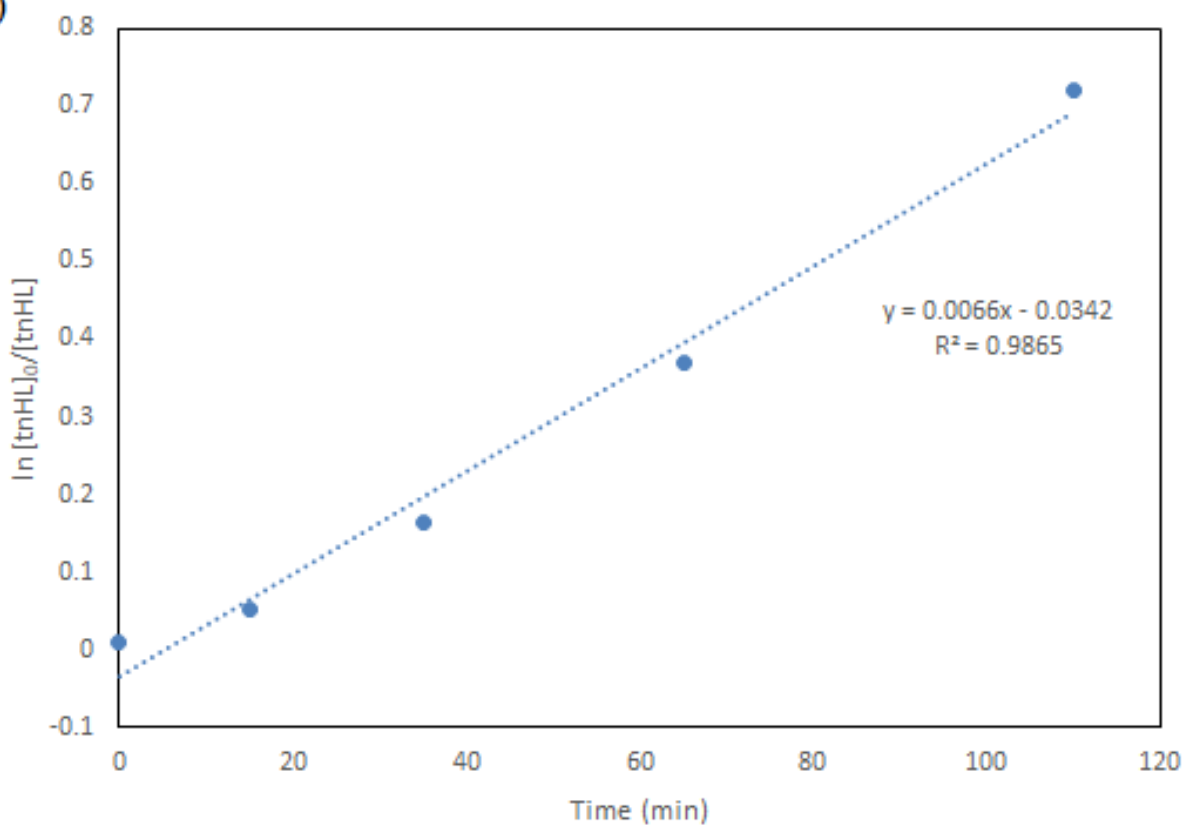

Figure 6.9. First order evolution of (a) [HL] and (b) [tnHL] vs time in the TCC/MTBD catalyzed ROP from benzyl alcohol. 

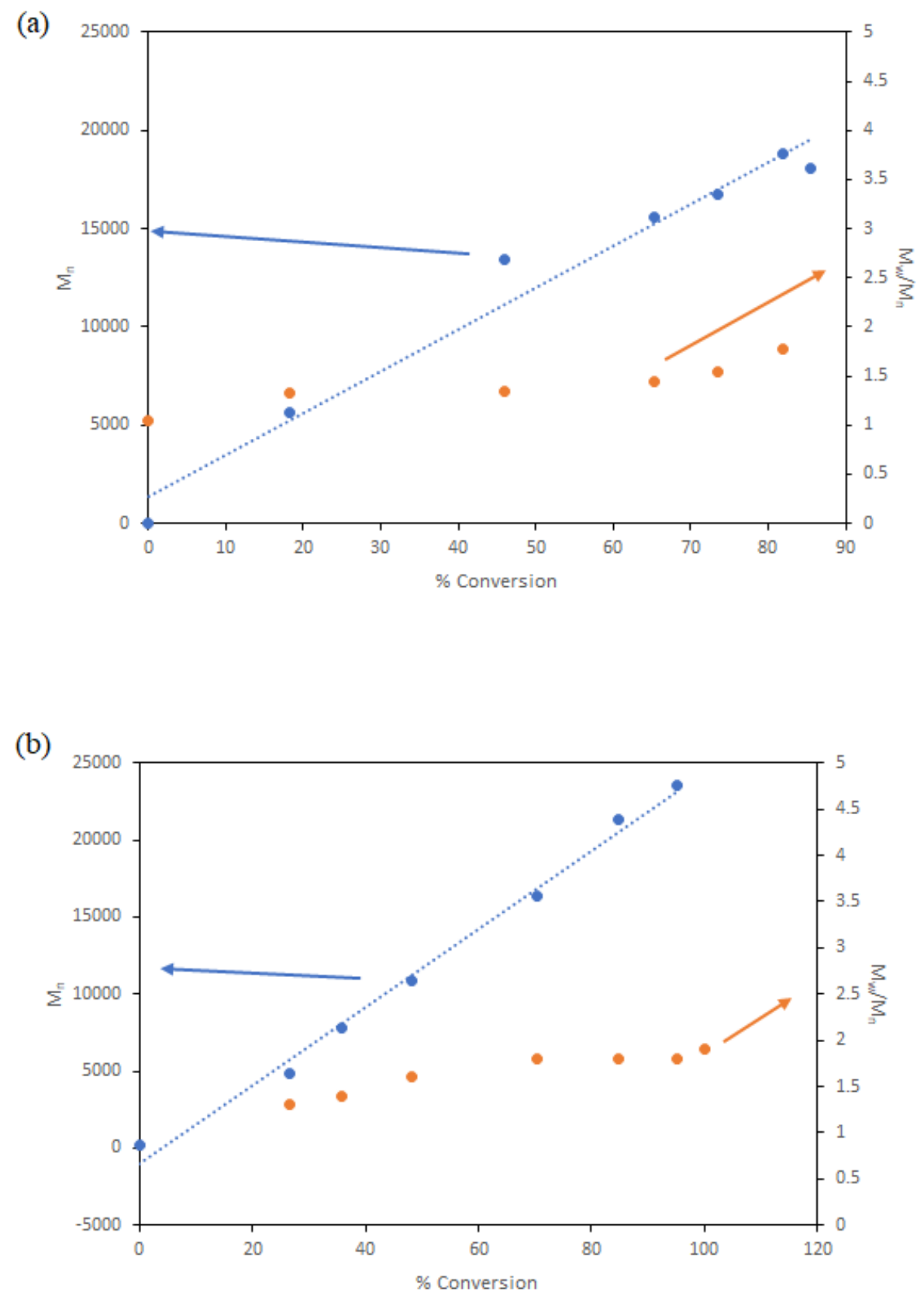

Figure 6.10. Evolution of $\mathrm{M}_{\mathrm{n}}$ vs conversion for the (a) TCC/BEMP (5 mol\% each) catalyzed ROP of NL (2M in acetone- $\left.d_{6}\right)$ initiated from benzyl alcohol (1 mol\%); (b) TCC/BEMP ( $5 \mathrm{~mol} \%$ each) catalyzed ROP of tnNL (2M in acetone- $d_{6}$ ) initiated from benzyl alcohol (1 mol\%). 

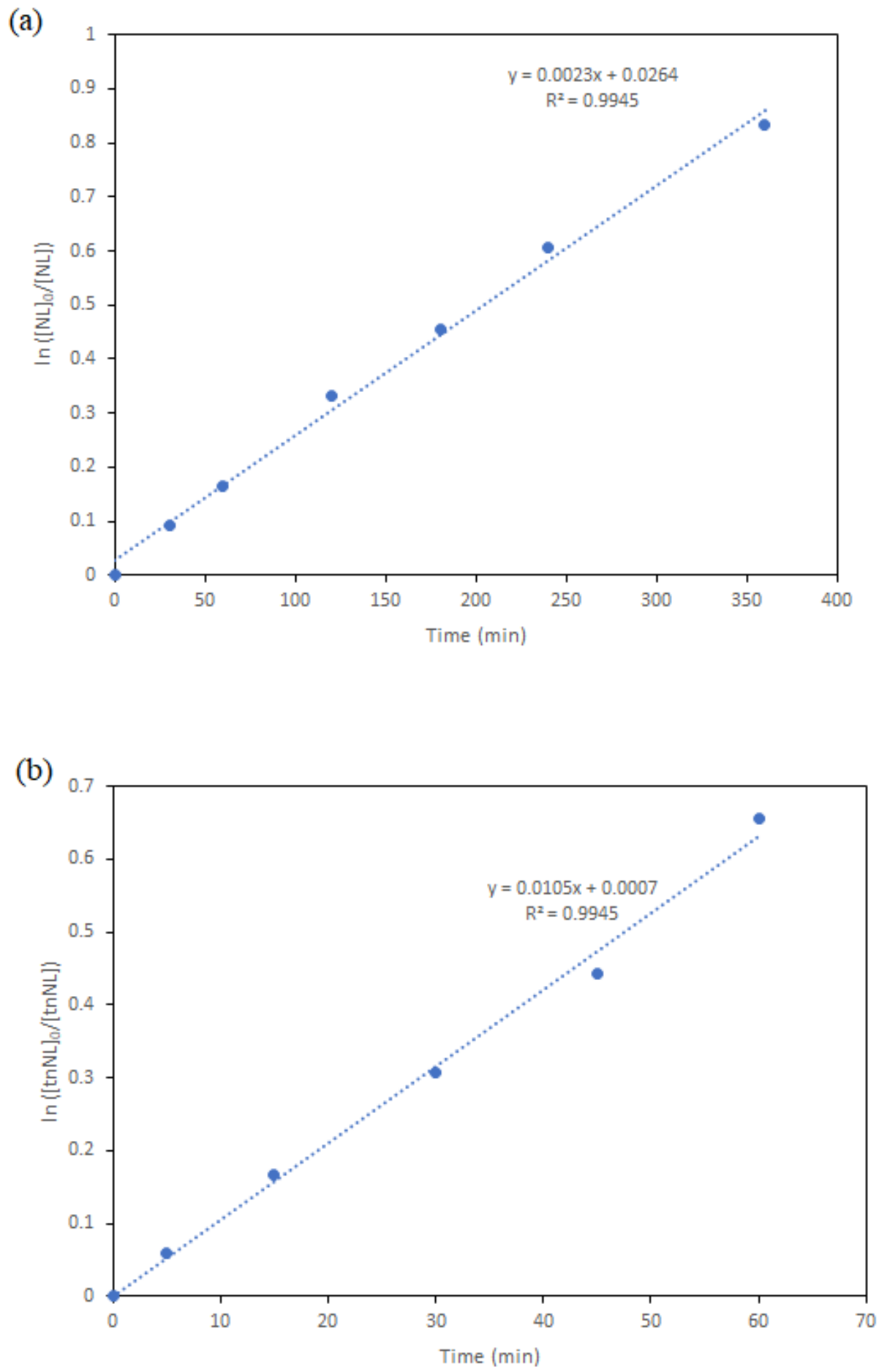

Figure 6.11. First order evolution of (a) $[\mathrm{NL}]$ and (b) [tnNL] vs time in the TCC/BEMP catalyzed ROP from benzyl alcohol. 
n

$\overbrace{}^{\mathrm{O}}+\mathrm{Ph}^{\mathrm{O}}$
Base

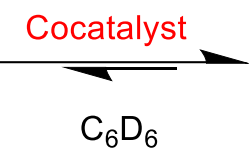

r.t.

\begin{tabular}{|c|c|c|c|c|c|c|}
\hline Entry & Base & Cocatalyst & Conv. $^{\mathrm{b}}(\%)$ & Time & $\mathrm{M}_{\mathrm{n}}{ }^{\mathrm{c}}(\mathrm{g} / \mathrm{mol})$ & $\mathrm{M}_{\mathrm{w}} / \mathrm{M}_{\mathrm{n}}{ }^{\mathrm{c}}$ \\
\hline 1 & BEMP & TCC & 88 & $4 \mathrm{mins}$ & 12,600 & 1.04 \\
\hline 2 & MTBD & TCC & 94 & $6 \mathrm{hrs}$ & 23,800 & 1.02 \\
\hline 3 & DBU & TCC & 89 & $21 \mathrm{hrs}$ & 18,200 & 1.03 \\
\hline $4^{\mathrm{d}}$ & BEMP & $\mathbf{2}$ & 98 & $50 \mathrm{mins}$ & 23,800 & 1.13 \\
\hline $5^{\mathrm{d}}$ & MTBD & $\mathbf{2}$ & 89 & $2 \mathrm{hrs}$ & 24,300 & 1.03 \\
\hline $6^{\mathrm{d}}$ & DBU & $\mathbf{2}$ & 89 & $18 \mathrm{hrs}$ & 17,800 & 1.03 \\
\hline $7^{\mathrm{e}}$ & TBD & - & 93 & $2 \mathrm{hrs}$ & 24,600 & 1.59 \\
\hline
\end{tabular}

Table 6.1. ROP of HL with urea base cocatalyst system.

(a) Reaction conditions: $2 \mathrm{M}(0.78 \mathrm{mmol}, 1 \mathrm{eq}) \mathrm{HL}, 1 \mathrm{~mol} \%$ benzyl alcohol, $5 \mathrm{~mol} \%$ base and cocatalyst and $\mathrm{C}_{6} \mathrm{D}_{6}$. (b) Conversion to polymer was obtained by ${ }^{1} \mathrm{H}$ NMR. (c) Determined by GPC $\left(\mathrm{CH}_{2} \mathrm{Cl}_{2}\right)$ vs polystyrene standards. (d) $1.67 \mathrm{~mol} \%$ base and cocatalyst. (e) 1 mol\% TBD. 
$\mathrm{n}$

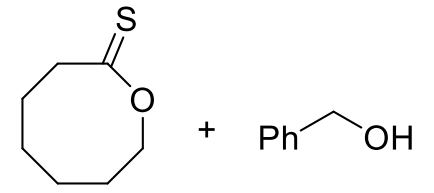

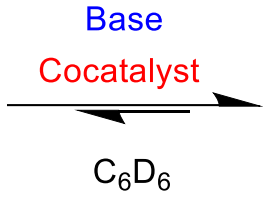

r.t.<smiles>[OH2+]CCCCCCC(=S)OCc1ccccc1</smiles>

$n$

\begin{tabular}{|c|c|c|c|c|c|c|}
\hline Entry & Base & Cocatalyst & Conv. $^{\mathrm{b}}(\%)$ & Time & $\mathrm{M}_{\mathrm{n}}{ }^{\mathrm{c}}(\mathrm{g} / \mathrm{mol})$ & $\mathrm{M}_{\mathrm{w}} / \mathrm{M}_{\mathrm{n}}{ }^{\mathrm{c}}$ \\
\hline 1 & BEMP & TCC & 92 & $38 \mathrm{mins}$ & 14,700 & 1.19 \\
\hline 2 & MTBD & TCC & 92 & $3.5 \mathrm{hrs}$ & 14,900 & 1.20 \\
\hline $3^{\mathrm{d}}$ & BEMP & $\mathbf{2}$ & 0 & $38 \mathrm{hrs}$ & - & - \\
\hline $4^{\mathrm{d}}$ & MTBD & $\mathbf{2}$ & 85 & $12 \mathrm{hrs}$ & 11,700 & 1.19 \\
\hline $5^{\mathrm{e}}$ & TBD & - & 89 & $20 \mathrm{~min}$ & 19,400 & 1.13 \\
\hline
\end{tabular}

Table 6.2. ROP of tnHL with urea base cocatalyst system.

(a) Reaction conditions: 2 M (1.04 mmol, 1 eq) tnHL, 1 mol\% benzyl alcohol, 5 mol\% base and cocatalyst and $\mathrm{C}_{6} \mathrm{D}_{6}$. (b) Conversion to polymer was obtained by ${ }^{1} \mathrm{H}$ NMR. (c) Determined by GPC $\left(\mathrm{CH}_{2} \mathrm{Cl}_{2}\right)$ vs polystyrene standards. (d) $1.67 \mathrm{~mol} \%$ base and cocatalyst. (e) $1 \mathrm{~mol} \%$ TBD. 


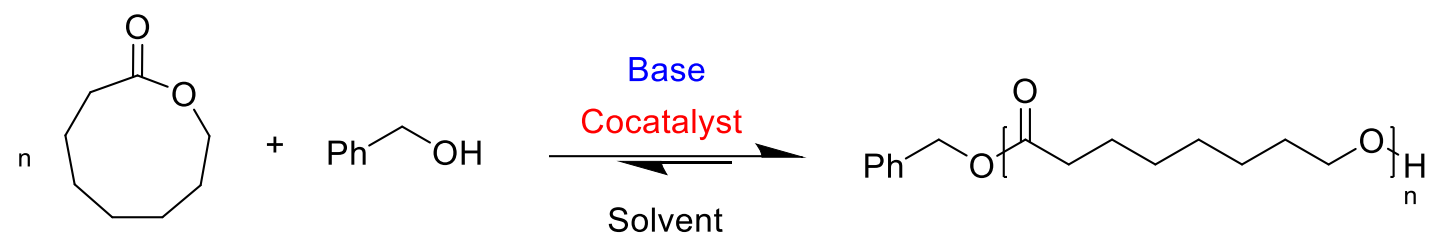

r.t.

\begin{tabular}{|c|c|c|c|c|c|c|}
\hline Entry & Base & Cocatalyst & Conv. $^{\mathrm{b}}(\%)$ & Time & $\mathrm{M}_{\mathrm{n}}{ }^{\mathrm{c}}(\mathrm{g} / \mathrm{mol})$ & $\mathrm{M}_{\mathrm{w}} / \mathrm{M}_{\mathrm{n}}{ }^{\mathrm{c}}$ \\
\hline $1^{\mathrm{e}}$ & MTBD & TCC & 27 & $24 \mathrm{hrs}$ & - & - \\
\hline $2^{\mathrm{e}}$ & BEMP & TCC & 96 & $26 \mathrm{hrs}$ & 18,000 & 1.8 \\
\hline $3^{\mathrm{f}}$ & BEMP & $\mathbf{2}$ & 93 & $10 \mathrm{hrs}$ & 25,500 & 1.4 \\
\hline $4^{\mathrm{e}}$ & DBU & TCC & 0 & $24 \mathrm{hrs}$ & - & - \\
\hline $5^{\mathrm{g}}$ & TBD & - & 85 & 3 days & 12,100 & 1.6 \\
\hline
\end{tabular}

Table 6.3. ROP of NL with (thio)urea base cocatalyst system.

(a) Reaction conditions: 2 M (0.703 mmol, 1 eq) NL, 1 mol\% benzyl alcohol, 5 mol\% base and cocatalyst and solvent. (b) Conversion to polymer was obtained by ${ }^{1} \mathrm{H}$ NMR. (c) Determined by GPC $\left(\mathrm{CH}_{2} \mathrm{Cl}_{2}\right)$ vs polystyrene standards. (d) $1.67 \mathrm{~mol} \%$ base and cocatalyst. (e) Acetone- $d_{6}$. (f) Benzene- $d_{6}$. (g) 1 mol\% TBD. 




r.t.

\begin{tabular}{|c|c|c|c|c|c|c|}
\hline Entry & Base & Cocatalyst & Conv. $^{\mathrm{b}}(\%)$ & Time & $\mathrm{M}_{\mathrm{n}}^{\mathrm{c}}(\mathrm{g} / \mathrm{mol})$ & $\mathrm{M}_{\mathrm{w}} / \mathrm{M}_{\mathrm{n}}{ }^{\mathrm{c}}$ \\
\hline $1^{\mathrm{d}}$ & BEMP & TCC & 95 & $4 \mathrm{hrs}$ & 23,500 & 1.8 \\
\hline $2^{\text {ef }}$ & BEMP & $\mathbf{2}$ & 0 & $24 \mathrm{hrs}$ & - & - \\
\hline $3^{\mathrm{d}}$ & MTBD & TCC & 75 & 2 days & 14,800 & 1.7 \\
\hline
\end{tabular}

Table 6.4. ROP of tnNL with (thio)urea base cocatalyst system.

(a) Reaction conditions: $2 \mathrm{M}(0.632 \mathrm{mmol}, 1 \mathrm{eq}) \mathrm{tnNL}, 1 \mathrm{~mol} \%$ benzyl alcohol, $5 \mathrm{~mol} \%$ base and cocatalyst and solvent. (b) Conversion to polymer was obtained by ${ }^{1} \mathrm{H}$ NMR. (c) Determined by GPC $\left(\mathrm{CH}_{2} \mathrm{Cl}_{2}\right)$ vs polystyrene standards. (d) Acetone- $d_{6}$. (e) Benzene- $d_{6}$. (f) $1.67 \mathrm{~mol} \%$ base and cocatalyst. (g) 1 mol\% TBD. 
$\mathrm{n}$<smiles>OC[Pb]c1ccccc1</smiles>

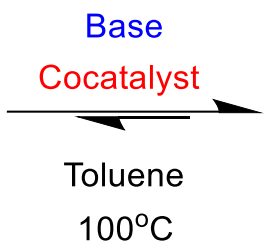<smiles>S=C(CCCCCCO[TlH])OCc1ccccc1</smiles>

\begin{tabular}{|c|c|c|c|c|c|c|}
\hline Entry & Base & Cocatalyst & Conv. $^{\mathrm{b}}(\%)$ & Time & $\mathrm{M}_{\mathrm{n}}{ }^{\mathrm{c}}(\mathrm{g} / \mathrm{mol})$ & $\mathrm{M}_{\mathrm{w}} / \mathrm{M}_{\mathrm{n}}{ }^{\mathrm{c}}$ \\
\hline $1^{\mathrm{d}}$ & TBD & - & 90 & $2 \mathrm{hrs}$ & 29,790 & 1.69 \\
\hline 2 & BEMP & TCC & 90 & $5 \mathrm{hrs}$ & 50,400 & 3.78 \\
\hline $3^{\mathrm{e}}$ & MTBD & $\mathbf{2}$ & - & $24 \mathrm{hrs}$ & - & - \\
\hline $4^{\mathrm{e}}$ & MTBD & $\mathbf{4}$ & - & $24 \mathrm{hrs}$ & - & - \\
\hline
\end{tabular}

Table 6.5. ROP of tnPDL with (thio)urea base cocatalyst system.

(a) Reaction conditions: $5 \mathrm{M}$ (0.975 mmol, $1 \mathrm{eq})$ tnPDL, $1 \mathrm{~mol} \%$ benzyl alcohol, $5 \mathrm{~mol} \%$ base and cocatalyst and solvent at $100^{\circ} \mathrm{C}$. (b) Conversion to polymer was obtained by ${ }^{1} \mathrm{H}$ NMR. (c) Determined by GPC $\left(\mathrm{CH}_{2} \mathrm{Cl}_{2}\right)$ vs polystyrene standards. (d) 5 mol\% TBD. (e) $1.67 \mathrm{~mol} \%$ base and cocatalyst. 


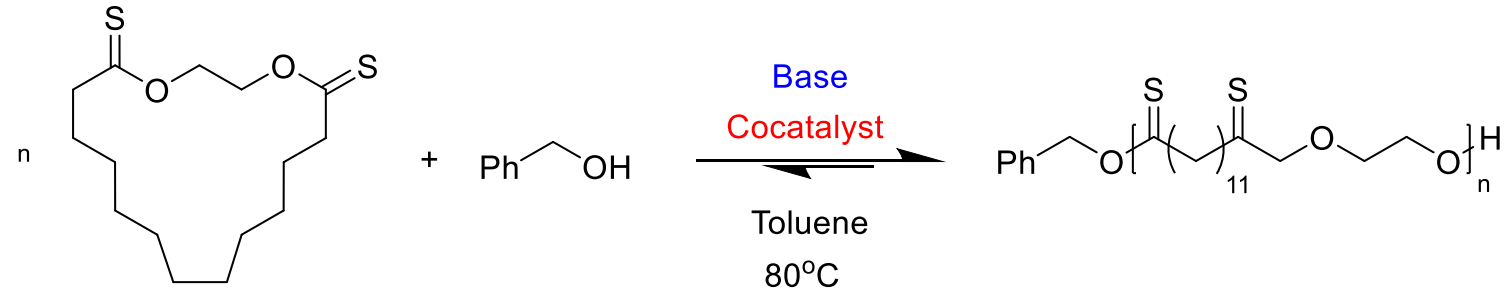

\begin{tabular}{|c|c|c|c|c|c|c|}
\hline Entry & Base & Cocatalyst & Conv. $^{\mathrm{b}}(\%)$ & Time & $\mathrm{M}_{\mathrm{n}}{ }^{\mathrm{c}}(\mathrm{g} / \mathrm{mol})$ & $\mathrm{M}_{\mathrm{w}} / \mathrm{M}_{\mathrm{n}}{ }^{\mathrm{c}}$ \\
\hline 1 & MTBD & TCC & 67 & $8 \mathrm{hrs}$ & 8,900 & 1.84 \\
\hline 2 & BEMP & TCC & 64 & $1 \mathrm{hr}$ & 10,600 & 1.95 \\
\hline $3^{\mathrm{d}}$ & MTBD & $\mathbf{2}$ & 29 & 3.5 days & 2,900 & 1.63 \\
\hline $4^{\mathrm{d}}$ & BEMP & $\mathbf{2}$ & 6 & 3.5 days & - & - \\
\hline
\end{tabular}

Table 6.6. ROP of tnEB with (thio)urea base cocatalyst system.

(a) Reaction conditions: 2 M (1.32 mmol, 1 eq) tnEB, 1 mol\% benzyl alcohol, 5 mol\% base and cocatalyst and solvent at $80^{\circ} \mathrm{C}$. (b) Conversion to polymer was obtained by ${ }^{1} \mathrm{H}$ NMR. (c) Determined by GPC $\left(\mathrm{CH}_{2} \mathrm{Cl}_{2}\right)$ vs polystyrene standards. (d) $1.67 \mathrm{~mol} \%$ base and cocatalyst. 


\section{MANUSCRIPT - VII}

\section{Possibilities with Thionated Monomers and Beyond}

Partha P. Datta and Matthew K. Kiesewetter

Chemistry, University of Rhode Island, Kingston, RI, USA

Corresponding Author: Matthew Kiesewetter, Ph.D.

Chemistry

University of Rhode Island

140 Flagg Road

Kingston, RI, 02881, USA

Email address: mkiesewetter@ chm.uri.edu 


\begin{abstract}
As the worldly demand for polymeric materials increases with a rising global human population, the need for robust, efficient methods will require attention from the scientific community. A segment of that polymeric materials would constitute various polyesters, polyamides, polyurethanes etc. Based on our previous studies of sulfurcontaining polymers of different polyesters, other polymers with sulfur backbone propelled our interest in it. Although few works have been done on these sulfur-containing polymers in the past, the use of organic catalysts to produce the polymers was missing. With the surge in organic catalysis, particularly hydrogen-bond donating, new possibilities have opened to accomplish new polymer synthesis and tune materials to the researchers' desires. It is with this vision that we had set out to produce a slate of new sulfur-containing monomers which underwent ring-opening polymerizations (ROP). Although high molecular weight polymers were not achieved for these new monomers, the facile synthetic approaches to their manufacture poses the opportunity for future developments.
\end{abstract}




\section{INTRODUCTION}

A surge in polymer research was observed when Hedrick et al. reported the first organic catalyst mediated ring-opening polymerization (ROP) of lactide in $2001 .{ }^{1}$ Since that report, the field of organic catalysis, or commonly known as organocatalysis, has seen significant growth in research for fast, efficient, selective catalyst developments. ${ }^{2-4}$ With the progress in catalyst improvements, the scope of monomers kept on expanding over the years. ${ }^{5}$ Though the initial and even current research focuses mainly on a set of lactones and lactides, more studies are underway for extending that capacity. ${ }^{6-11}$ As the demand for better materials in medicine, plastics and microelectronics which is where these polylactones are generally in use continue to amplify, research in the polymeric materials to meet these needs will remain active..$^{12-15}$

In our research group we have gone from relatively slow to some of the fastest, highly active, vastly selective catalysts in ROP over the last few years. ${ }^{16-20}$ This enabled a wide range of monomers to be studied for polymer production in a living, controlled manner. Although more studies are currently underway for understanding the mechanistic aspects of these systems, a Hydrogen-bond mediated or imidate-mediated mode of action is believed to be in play according to the polymer community. This mechanism of action can be tuned based on the substrate or monomer to have a more effective activation process. This has enabled polymer production for some of the previously uncontrolled, nonselective monomers. This report is an extension of further studies performed on a new set of monomers using these H-bonding catalysts. 
Sulfur-containing polymers has prominent appeal in material designs due to the possibility of cross-linking. ${ }^{21}$ Our research group has worked on the controlled, living ROP of $\varepsilon$-thiocaprolactone and $\varepsilon$-thionocaprolactone in the past. ${ }^{22,23}$ These sulfur-containing 7membered rings produced polymers with good control and narrow dispersity when subjected to organic catalysts. The study on some more sulfur-containing monomers was extended with larger ring systems which generated polymers catalyzed for the first time by organocatalysts. ${ }^{24}$ In continuation of broadening that scope of monomers we report some of the attempts made by our group in opening rings of systems other than thionolactones, namely thionolactams and thionolactides. Moreover, the possibility of cross-linking for these thionated monomers was attempted for one of the first thionolactone studied in our group. 


\section{EXPERIMENTAL SECTION}

\section{General Considerations}

All chemicals were used as received unless stated otherwise. Hexamethyldisiloxane (HMDO), $\mathrm{P}_{4} \mathrm{~S}_{10}, \varepsilon$-caprolactam, L-lactide, tetrahydrofuran, $p$-toluenesulfonic acid monohydrate (PTSA), bis(trifluoromethane)sulfonimide (TFMSI), 1,4,7-trimethyl-1,4,7triazacyclononane (TACN), 1,4-benzenedimethanol, potassium tert-butoxide $(t$-BuOK), sodium methoxide (NaOMe) and 2-tert-butylimino-2-diethylamino-1,3-dimethylperhydro1,3,2-diazaphosphorine (BEMP) were supplied by Acros Organics. Potassium carbonate, magnesium sulfate, benzyl alcohol, benzoic acid, trifluoroacetic acid, ethyl acetate, dichloromethane, toluene, o-dichlorobenzene, hexane and 1,4-dioxane were purchased from Fisher Scientific. Sigma-Aldrich provided diphenyl ether, diphenyl phosphate (DPP) and bis(4-nitrophenyl) phosphate (B4NPP). Alfa Aesar delivered tin (II) 2-ethylhexanoate $\left(\mathrm{Sn}(\mathrm{Oct})_{2}\right)$ and sodium ethoxide (NaOEt). Chloroform- $d$ and benzene- $d_{6}$ were supplied by Cambridge Isotope Laboratories and distilled from $\mathrm{CaH}_{2}$ under a nitrogen atmosphere. Benzyl alcohol was distilled from $\mathrm{CaH}_{2}$ under high vacuum. Toluene, dichloromethane and tetrahydrofuran were dried on an Innovated Technologies solvent purification system with alumina columns and nitrogen working gas. 1 [3,5-bis(trifluoromethyl)phenyl]-3cyclohexyl-thiourea and 2 1,1',1"'-(nitrilotris(ethane-2.1-diyl))tris(3-(3,5bis(trifluromethyl)phenyl)urea were synthesized and purified according to literature procedures. $^{6,19}$ Triclocarban (TCC), 1,8-Diazabicyclo[5.4.0]undec-7-ene (DBU), 7methyl-1,5,7-triazabicyclo[4.4.0]dec-5-ene (MTBD), and 1,5,7-triazabicyclo[4.4.0]dec-5ene (TBD) were purchased from Tokyo Chemical Industry (TCI). All polymerization 
reactions were set up in an MBRAUN or INERT stainless steel glovebox equipped with a gas purification system under a nitrogen atmosphere using glass vials and magnetic stir bars which were baked overnight at $140^{\circ} \mathrm{C}$ and then carried out in a hot plate at variable temperatures. NMR experiments were performed on a Bruker Avance III $300 \mathrm{MHz}$ or 400 $\mathrm{MHz}$ spectrometer. The chemical shifts for proton $\left({ }^{1} \mathrm{H}\right)$ and carbon $\left({ }^{13} \mathrm{C}\right)$ NMR were recorded in parts per million (ppm) relative to a residual solvent. Size exclusion chromatography (SEC) was performed at $30^{\circ} \mathrm{C}$ in dichloromethane (DCM) using an Agilent Infinity GPC system equipped with three Agilent PLGel columns $7.5 \mathrm{~mm} \times 300$ mm (5 $\mu$ m pore sizes: 103,104 , and $105 \AA)$. Molecular weight and $M_{w} / M_{n}$ were determined versus polystyrene standards (500 g/mol - $3150 \mathrm{~kg} / \mathrm{mol}$; Polymer Laboratories).

Synthesis of $\varepsilon$-Thionocaprolactam

The procedure to synthesize $\varepsilon$-thionocaprolactam ( $\varepsilon$-tnCLa) was adopted from Curphey's method with some modifications. ${ }^{25}$ Initially, $\mathrm{P}_{4} \mathrm{~S}_{10}(0.98 \mathrm{~g}, 2.2 \mathrm{mmol}), \varepsilon-$ caprolactam $(1.36 \mathrm{~g}, 12 \mathrm{mmol})$ and hexamethyldisiloxane $(4.25 \mathrm{~mL}, 20 \mathrm{mmol})$ in dichloromethane $(12 \mathrm{~mL})$ was added into a reaction vessel. The solution was stirred at moderate speed for about 3 hours after which saturated potassium carbonate solution and distilled water were used to quench the reaction. The solution was kept in an ice-water bath for about 30 mins with stirring at this stage. Extraction was performed few times with dichloromethane, followed by washing with brine. Magnesium sulfate was used to dry the reaction solution afterwards with subsequent filtration for obtaining the product in solvent. After removal of the solvent, a silica-gel flash column was run with dichloromethane to remove some leftover crude mixture from the synthesis. Recrystallization was carried out 
using diethyl ether which gave the pure product in solid crystals form. Yield: $1.15 \mathrm{~g}, 74 \%$. Product was verified from previous literature characterization. ${ }^{25}$

Synthesis of L-Thionolactide (tnLA)

Curphey's method as adopted for the synthesis of L-thionolactide (tnLA). ${ }^{25}$ The necessary reagents, L-lactide $(2 \mathrm{~g}, 13.9 \mathrm{mmol})$, hexamethyldisiloxane $(4.9 \mathrm{~mL}, 23.1$ $\mathrm{mmol}), \mathrm{P}_{4} \mathrm{~S}_{10}(3.10 \mathrm{~g}, 6.9 \mathrm{mmol})$ and toluene $(15 \mathrm{~mL})$, were refluxed for about 1.5 hours. Then the reaction mixture was cooled in an ice-water bath for almost an hour after quenching the reaction with aqueous potassium carbonate solution and distilled water. Extraction was then executed with ethyl acetate few times. Drying with magnesium sulfate followed. Then removal of the solvent was performed followed by further purification by column chromatography using $100 \%$ dichloromethane through silica-gel. Sublimation was carried out with heating at $\sim 90^{\circ} \mathrm{C}$ and high-vacuum at $\sim 100$ mtorr for 1-2 hours. Product was obtained as semi-solid yellow-orange powder in $9 \%$ yield, $0.22 \mathrm{~g} .{ }^{1} \mathrm{H}$ NMR $(400 \mathrm{MHz}$, $\left.\mathrm{CDCl}_{3}\right) \delta 4.72(\mathrm{~s}, 4 \mathrm{H}), 2.75(\mathrm{t}, J=7.2,4 \mathrm{H}), 1.70(\mathrm{p}, J=7.1,4 \mathrm{H}), 1.37-1.11(\mathrm{~m}, 12 \mathrm{H}) .{ }^{13} \mathrm{C}$ NMR (100 MHz, $\left.\mathrm{CDCl}_{3}\right) \delta 25.9,25.9,26.0,26.2,26.9,45.9,68.3,223.4$. Product spectra are shown below (Figure 7.1 and 7.2, respectively). Product was validated with a previous literature characterization. ${ }^{26}$

\section{Example Ring-Opening Polymerization of tnCLa}

Just like in a conventional ROP, tnCLa $(0.250 \mathrm{~g}, 2.21 \mathrm{mmol})$ and $p$-toluenesulfonic acid $(0.021 \mathrm{~g}, 0.11 \mathrm{mmol})$ were added to a $20 \mathrm{~mL}$ scintillation vial with a stir bar in the glovebox. The contents of the vials were stirred for about a minute at moderate speed after which the vial was taken out of the glovebox and placed in an oil bath already at $200^{\circ} \mathrm{C}$ within a hot plate with medium stirring. Reaction was concluded by taking the vial out of 
the hot plate and submerging it into a liquid nitrogen bath which formed white solid residues on the sides of the glass vial. The contents of the vial was then dissolved in trifluoroacetic acid (TFA) and placed into a NMR tube with a $\mathrm{C}_{6} \mathrm{D}_{6}$ sealed capillary to obtain ${ }^{1} \mathrm{H}$ spectrum. No polymer was observed by ${ }^{1} \mathrm{H}$ NMR or high molecular weight on GPC.

\section{Example Ring-Opening Polymerization of tnLA}

Similar to any typical ROP, tnLA $(0.100 \mathrm{~g}, 0.567 \mathrm{mmol})$ was added to a $20 \mathrm{~mL}$ scintillation vial with a stir bar along with $t$-BuOK $(6.40 \mathrm{mg}, 0.057 \mathrm{mmol})$ in toluene to make a $1 \mathrm{M}$ solution within the glovebox. The vial was then placed in a pre-heated hot plate within the glovebox set at $100^{\circ} \mathrm{C}$. The reaction mixture was then stirred until all the catalysts dissolved in the monomer solution. Aliquots $(\sim 50 \mu \mathrm{L})$ were then taken from the reaction vial at various time intervals and dissolved in about $400 \mu \mathrm{L}$ of $\mathrm{CDCl}_{3}$ for ${ }^{1} \mathrm{H} \mathrm{NMR}$ to determine conversion. No polymer was observed by ${ }^{1} \mathrm{H}$ NMR for about a day or high weight distribution by GPC.

Example Block Copolymerization of tnCL and LA

A copolymerization reaction was run to make triblock substances. First, tnCL (0.5 $\mathrm{g}, 3.84 \mathrm{mmol}$ ) was placed in a $7 \mathrm{~mL}$ scintillation vial with a stir bar. In another similar

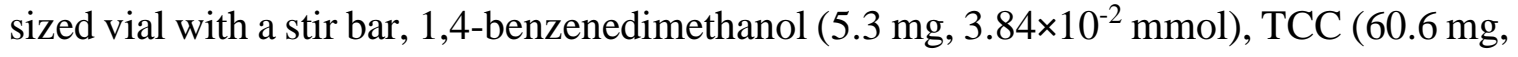
$0.19 \mathrm{mmol})$ and BEMP $(55.5 \mu \mathrm{L}, 0.19 \mathrm{mmol})$ were added. Benzene $(1.92 \mathrm{~mL})$ was added equally to the two vials and stirred at moderate speed for a minute. The contents of tnCL vial was withdrawn and transferred to the other vial and mixed completely. About $500 \mu \mathrm{L}$ of that mixed solution was then transferred into a NMR tube with a sealed $\mathrm{C}_{6} \mathrm{D}_{6}$ capillary. NMR $\left({ }^{1} \mathrm{H}\right)$ was obtained until reaching about $90 \%$ conversion after which benzoic acid (2 
mol eq. to base) was added to the overall contents of the reaction mixture. Precipitation from hexane was performed on the polymer afterwards. Dialysis was conducted on the polymer in methanol with 6-8 $\mathrm{kDa}$ bags after dissolving the polymer in dichloromethane for 2 days. The purified polymer was then subjected to high-vacuum to remove any leftover solvents. This pure PtnCL was then taken into the glovebox. Just like before, the scintillation vial containing PtnCL was charged with L-LA (0.138 g, $0.96 \mathrm{mmol})$ and a stir bar. In another similar sized vial, $1\left(17.8 \mathrm{mg}, 4.79 \times 10^{-2} \mathrm{mmol}\right)$, TACN $\left(9.3 \mu \mathrm{L}, 4.79 \times 10^{-2}\right.$ mmol $)$ and $\mathrm{CDCl}_{3}(960 \mu \mathrm{L})$ were added with a stir bar to make an overall concentration of $1 \mathrm{M}$ with respect to the total monomers. After obtaining full homogeneity within few minutes, the second vial's contents were transferred to the monomer vial and stirred for few more minute. Only about $500 \mu \mathrm{L}$ of solution was withdrawn from the overall solution to be placed into a NMR tube. ${ }^{1} \mathrm{H}$ NMR spectra were obtained until monomer conversion reached almost $90 \%$ for L-LA. The overall reaction solution was then quenched with benzoic acid ( 2 mol eq. to base) and subsequently precipitated from hexane. Dialysis was performed again in a similar fashion as before for 2 days after which high-vacuum was applied to eliminate any trace amount of solvents. Final ${ }^{1} \mathrm{H}$ and ${ }^{13} \mathrm{C}$ spectra were obtained for the copolymer sample (shown below) along with GPC for molecular weights. Yield $91 \% ; M_{w} / M_{n}=1.21 ; M_{n}(\mathrm{GPC})=17,800 .{ }^{1} \mathrm{H}$ and ${ }^{13} \mathrm{C}$ NMR spectra display characteristic resonances of the polymer with thiocarbonyl and carbonyl peak at 224 and $170 \mathrm{ppm}$ in the ${ }^{13} \mathrm{C}$ spectrum respectively (see Figure 7.3 ). 


\section{RESULTS AND DISCUSSION}

\section{Ring-Opening Polymerization Attempts of tnCLa}

Similar to other thionated monomers that have undergone ROP, ${ }^{23,24}$ tnCLa (Scheme 7.1) was initially attempted to be opened for polymer in a parallel manner. This monomer was tried to be opened by TBD (5 mol\%) with an initiator like benzyl alcohol (1 mol\%) in various solvents $\left(\mathrm{CDCl}_{3}, \mathrm{C}_{6} \mathrm{D}_{6}\right.$, dichloromethane, THF, toluene, o-dichlorobenzene) at room temperature first. Since solubility was one of the obstacles for many of these solvents, no polymer production was observed for almost two days with chloroform- $d$, dichloromethane and THF even with complete dissolution. Since full monomer solubility was possible with these solvents, chloroform was applied at high temperature $\left(50^{\circ} \mathrm{C}\right)$ in a similar way as before. After almost 2 days of monitoring, about $13 \%$ conversion to polymer was achieved (Table 7.1). Inspired by our group's recently developed 2, this was also applied for $\mathrm{ROP}$ of tnCLa in chloroform and $\mathrm{THF}$ at $50^{\circ} \mathrm{C}$ with similar initiator and catalyst loading. No polymer was observed for any of these systems for up to 2 days (Table 7.1).

A recent publication demonstrated the use of organic acids for the ROP of $\varepsilon$ caprolactam. ${ }^{27} \mathrm{We}$ attempted similar approaches for tnCLa with a range of organic acids already available in our lab (Scheme 7.2). Even with the use of 10 mol\% loading of these acid catalysts with $1 \mathrm{~mol} \%$ benzyl alcohol at $100^{\circ} \mathrm{C}$ in chloroform- $d$, no polymeric conversion was noticed for up to 2 days. It was then the attempt with high boiling point solvents to try opening up this cyclic amide. Thus, diphenyl ether, 1,4-dioxane and toluene were tried with organic bases and $\mathbf{2}$ with no avail (Table 7.1). 
Based on that publication, ${ }^{27}$ those organic acids were successfully able to produce polyamides at a much higher temperature. Taking a leaf out of their work, we attempted to open tnCLa at $180-200^{\circ} \mathrm{C}$ using those acid catalysts (Scheme 7.2). Though PTSA yielded a 17\% conversion after 3 days without solvent and initiator, the molecular weights were nothing but oligomeric peaks of short polymer chains supposedly (entry 11, Table 7.1). Some alkoxides were also implemented to attempt ROP of this monomer, but no polymerization were observed for almost 24 hours at elevated temperature (Table 7.1). These results also varied when they were conducted in different media, like using sand bath versus silicon oil bath or aluminum bead bath. Though a thermocouple was placed in all these cases, thermometer gave different reading than what was targeted for in the hot plate dial. Oil bath seemed to be quite consistent in desired temperature to the actual one, but fluctuations was still evident on humid and rainy days. Another factor that could have contributed to inconsistency in these polymer characterization is the method of determining their conversions. As the publication demonstrated ${ }^{27}$ reaction for poly( $\varepsilon$-caprolactam) was quenched with liquid nitrogen followed by dissolution of the product in TFA- $d$. Due to the unavailability of TFA- $d$, we had to apply a sealed $\mathrm{C}_{6} \mathrm{D}_{6}$ capillary which may not give us an accurate conversion data if TFA is degrading poly(e-thionocaprolactam), for instance. More studies are currently undergoing that can help to understand lactam based polymerization systems with organocatalysis and how to proceed for ROP in a controlled fashion for monomers like this.

Ring-Opening Polymerization Attempts of tnLA

Based on some preliminary unpublished work within our lab, we had observed that the ROP of L-tnLA (Scheme 7.1) does not occur with organic H-bonding catalysts. After 
attempting that for tnLA polymer even at elevated temperature, no high conversion or molecular weight polymer were observed at all. Thus, some of the common alkoxides and metal oxides were implemented to try ROP of this system. Since the yield is not very significant either and traces of epimerization was present in the NMR spectra (both ${ }^{1} \mathrm{H}$ and ${ }^{13} \mathrm{C}$, Figure 7.1, 7.2 respectively), ROP did not seem to have occurred. From the various metal catalysts utilized for the ring-opening, none showed any growth of polymer within one day of reaction at elevated temperature (Table 7.2). Further studies are currently undergoing to develop facile synthetic technique for the monomer production while molecular modeling may help in understanding the viability of polymerization for this substrate.

Triblock Copolymerization of tnCL with L-LA

Motivated by our previous results of copolymerization of thionated lactones (tnCL) to commercially known lactones ( $\delta$-valerolactone) ${ }^{23}$ we wanted to look at the other forms of copolymers that could be produced using these thionated systems. Since the statistical random copolymers of PtnCL-co-PVL showed an increased flexible nature from the homopolymers of PtnCL, we wondered if other ester motifs would help in making rubbery texture for the copolymers. With that thought, we started to look for crystalline-based polymers that can be easily synthesized. Due to a plethora of studies on a known crystalline polymer like PLLA, ${ }^{28}$ we decided to incorporate this as a block into the system with PtnCL. Our target was to produce a triblock copolymer (ABA-type) with crystalline-amorphouscrystalline moieties, or in other words, PLLA-PtnCL-PLLA. In order to be able to have such a triblock system, the initiator had to be different from our previous studies of copolymerizations (benzyl alcohol). We decided to go with 1,4-benzenedimethanol as the 
initiator where we hoped to initially form the B-block for the middle part followed by double A-block incorporation afterwards (Scheme 7.1).

Just as the thought process was envisaged, we carried out the polymerizations as planned. The B-block (tnCL) underwent homopolymerization with 1,4-benzenedimethanol at first. The pure form of PtnCL was then re-introduced to ROP with L-LA, hoping that the PtnCL block would act as a macroinitiator. Organic catalysts were utilized for carrying out the ROPs for these systems. A slate of copolymers of varying ratios of ABA blocks were produced in similar manner (see Table 7.3). As hypothesized, a general trend of enhanced flexibility in the polymer texture was observed with decreased PLLA content in the copolymer content physically. Further studies are currently in progress with our collaborator to understand the physical and mechanical properties of these materials.

Cross-Linking Abilities of Thionated Systems

Due to previous literature studies on Sulfur-containing polymers to create networks within themselves via cross-linking, ${ }^{21}$ we wanted to look at that possibility with our thionated monomers as well. This was performed by dissolving a sample of PtnCL in dichloromethane first, followed by addition of equal volume of commercial bleach solution (containing mostly sodium hypochlorite). After stirring the mixture for about 2 days, a formation of thickened solid-like material was obtained. Following filtration to remove the solvent, the material that was attained was quite hard in its physical state. In fact, no common organic solvents were able to dissolve the substance which made it quite difficult to obtain NMR, GPC or any other analytical tools to understand the material. Due to the inability for solvents to dissolve the material, it was quite plausible that cross-linking might have happened. Further studies on such polymers are currently underway in our lab at this 
time with the assistance from our collaborator to characterize the phenomenon. Once fully comprehended, it could open up the possibility of a whole array of materials which might be useful from a commercial perspective. 


\section{CONCLUSION}

With the growth in H-bonding catalysts in the last decade or two, a whole set of prospects came about in synthesizing polymers of various kinds. Although oxygenated cyclic esters were primarily the focus of research initially, other cyclic esters, particularly thionated amides and lactides were not studied with in-depth analysis. With the emergence of very fast, selective H-bonding catalysts, some of these newly synthesized monomers described above could be studied. That is what the above study attempted to do with $\varepsilon$ thionocaprolactam and L-thionolactide. Although the preliminary data suggests failure in producing well-controlled, living ROP for these systems using H-bonding catalysts, attempts to polymerize these substrates using other catalysts and conditions are currently being investigated.

Additionally, some new materials were manufactured from the previously studied $\varepsilon$-thionocaprolactone, ${ }^{23}$ with regards to copolymers and cross-linked materials. The copolymers do exhibit some rubbery features from a physical texture point of view, but future studies need to be conducted to understand these materials from a mechanical perspective. Moreover, cross-linking with these poly( $\varepsilon$-thionocaprolactone $)$ could open up new avenues of research as more understanding of the flexible polymers could be useful in plastics and rubber applications. Though it is too early for these paths to develop, proper methods with engineering mindset could shape this to be a vast domain to explore for future polymer chemists. 


\section{LIST OF REFERENCES}

(1) Nederberg, F.; Connor, E. F.; Möller, M.; Glauser, T.; Hedrick, J. L. Angew. Chem. Int. Ed. Engl. 2001, 40, 2712-2715.

(2) Kiesewetter, M. K.; Shin, E. J.; Hedrick, J. L.; Waymouth, R. M. Macromolecules 2010, 43 (5), 2093-2107.

(3) Dove, A. P.; Pratt, R. C.; Lohmeijer, B. G. G.; Waymouth, R. M.; Hedrick, J. L.; V, S. U. J. Am. Chem. Soc. 2005, 127, 13798-13799.

(4) Kamber, N. E.; Jeong, W.; Waymouth, R. M.; Pratt, R. C.; Lohmeijer, B. G. G.; Hedrick, J. L. Chem. Rev. 2007, 107 (12), 5813-5840.

(5) Thomas, C.; Bibal, B. Green Chem. 2014, 16 (4), 1687-1699.

(6) Lohmeijer, B. G. G.; Pratt, R. C.; Leibfarth, F.; Logan, J. W.; Long, D. A.; Dove, A. P.; Nederberg, F.; Choi, J.; Wade, C.; Waymouth, R. M.; Hedrick, J. L. Macromolecules 2006, 39 (25), 8574-8583.

(7) Zhang, L.; Nederberg, F.; Pratt, R. C.; Waymouth, R. M.; Hedrick, J. L.; Wade, C. G. Macromolecules 2007, 40 (12), 4154-4158.

(8) Pratt, R. C.; Lohmeijer, B. G. G.; Long, D. A.; Lundberg, P. N. P.; Dove, A. P.; Li, H.; Wade, C. G.; Waymouth, R. M.; Hedrick, J. L. Macromolecules 2006, 39 (23), $7863-7871$.

(9) Lohmeijer, B. G. G.; Dubois, G.; Leibfart, F.; Pratt, R. C.; Nederberg, F.; Nelson, A.; Waymouth, R. M.; Wade, C.; Hedrick, J. L. Org. Lett. 2006, 8 (21), 46834686.

(10) Pratt, R. C.; Lohmeijer, B. G. G.; Long, D. A.; Waymouth, R. M.; Hedrick, J. L. J. 
Am. Chem. Soc. 2006, 128 (14), 4556-4557.

(11) Thomas, C.; Peruch, F.; Bibal, B. RSC Adv. 2012, 2 (33), 12851.

(12) Wang, S.; Cai, Q.; Bei, J. Macromol. Symp. 2003, 195 (8), 263-268.

(13) Hedrick, J. L.; Magbitang, T.; Connor, E. F.; Glauser, T.; Volksen, W.; Hawker, C. J.; Lee, V. Y.; Miller, R. D. Chem. - A Eur. J. 2002, 8 (15), 3308-3319.

(14) Dorgan, J. R.; Lehermeier, H. J.; Palade, L. I.; Cicero, J. Macromol. Symp. 2001, $175,55-66$.

(15) Sinha Ray, S.; Okamoto, M. Macromol. Rapid Commun. 2003, 24 (14), 815-840.

(16) Kazakov, O. I.; Datta, P. P.; Isajani, M.; Kiesewetter, E. T.; Kiesewetter, M. K. Macromolecules 2014, 47, 7463-7468.

(17) Kazakov, O. I.; Kiesewetter, M. K. Macromolecules 2015, 48 (17), 6121-6126.

(18) Spink, S. S.; Kazakov, O. I.; Kiesewetter, E. T.; Kiesewetter, M. K. Macromolecules 2015, 48 (17), 6127-6131.

(19) Fastnacht, K. V.; Spink, S. S.; Dharmaratne, N. U.; Pothupitiya, J. U.; Datta, P. P.; Kiesewetter, E. T.; Kiesewetter, M. K. ACS Macro Lett. 2016, 5 (8), 982-986.

(20) Dharmaratne, N. U.; Pothupitiya, J. U.; Bannin, T. J.; Kazakov, O. I.; Kiesewetter, M. K. ACS Macro Lett. 2017, 6 (4), 421-425.

(21) Krejsa, M. R.; Koenig, J. L. Rubber Chem. Technol. 1993, 66 (3), 376-410.

(22) Bannin, T. J.; Kiesewetter, M. K. Macromolecules 2015, 48 (16), 5481-5486.

(23) Datta, P. P.; Kiesewetter, M. K. Macromolecules 2016, 49 (3), 774-780.

(24) Datta, P. P.; Kalana, U. L. D. I.; Hewawasam, R. S.; Kiesewetter, M. K. Unpubl. results.

(25) Curphey, T. J. J. Org. Chem. 2002, 67 (18), 6461-6473. 
(26) Mangalum, A.; Boadi, F.; Masand, S. A.; Lalancette, R. A.; Pietrangelo, A. RSC Adv. 2016, 6 (78), 74250-74253.

(27) Sanchez-Sanchez, A.; Basterretxea, A.; Mantione, D.; Etxeberria, A.; Elizetxea, C.; de la Calle, A.; García-Arrieta, S.; Sardon, H.; Mecerreyes, D. J. Polym. Sci. Part A Polym. Chem. 2016, 54 (15), 2394-2402.

(28) Auras, R.; Harte, B.; Selke, S. Macromol. Biosci. 2004, 4 (9), 835-864. 


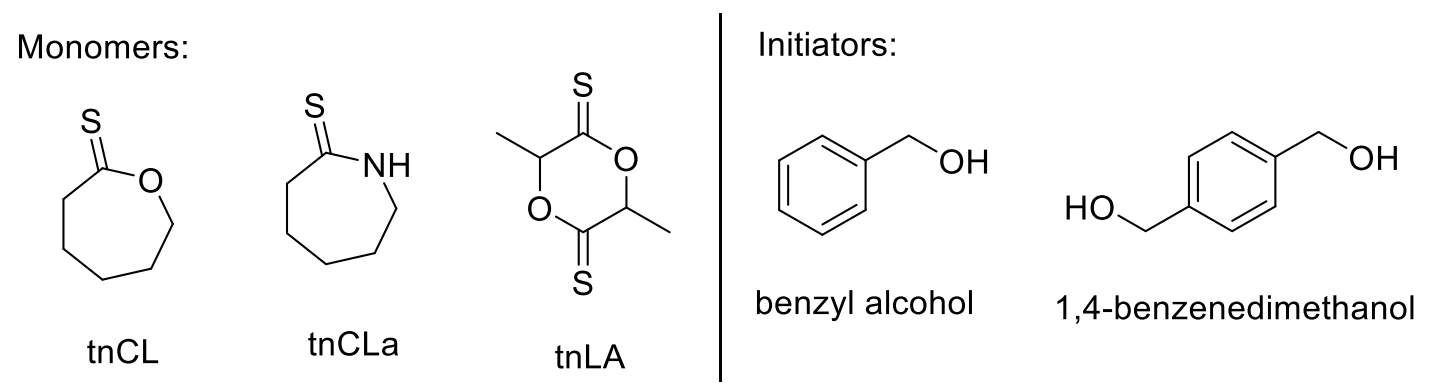

Scheme 7.1. Monomers and initiators studied in this project. 

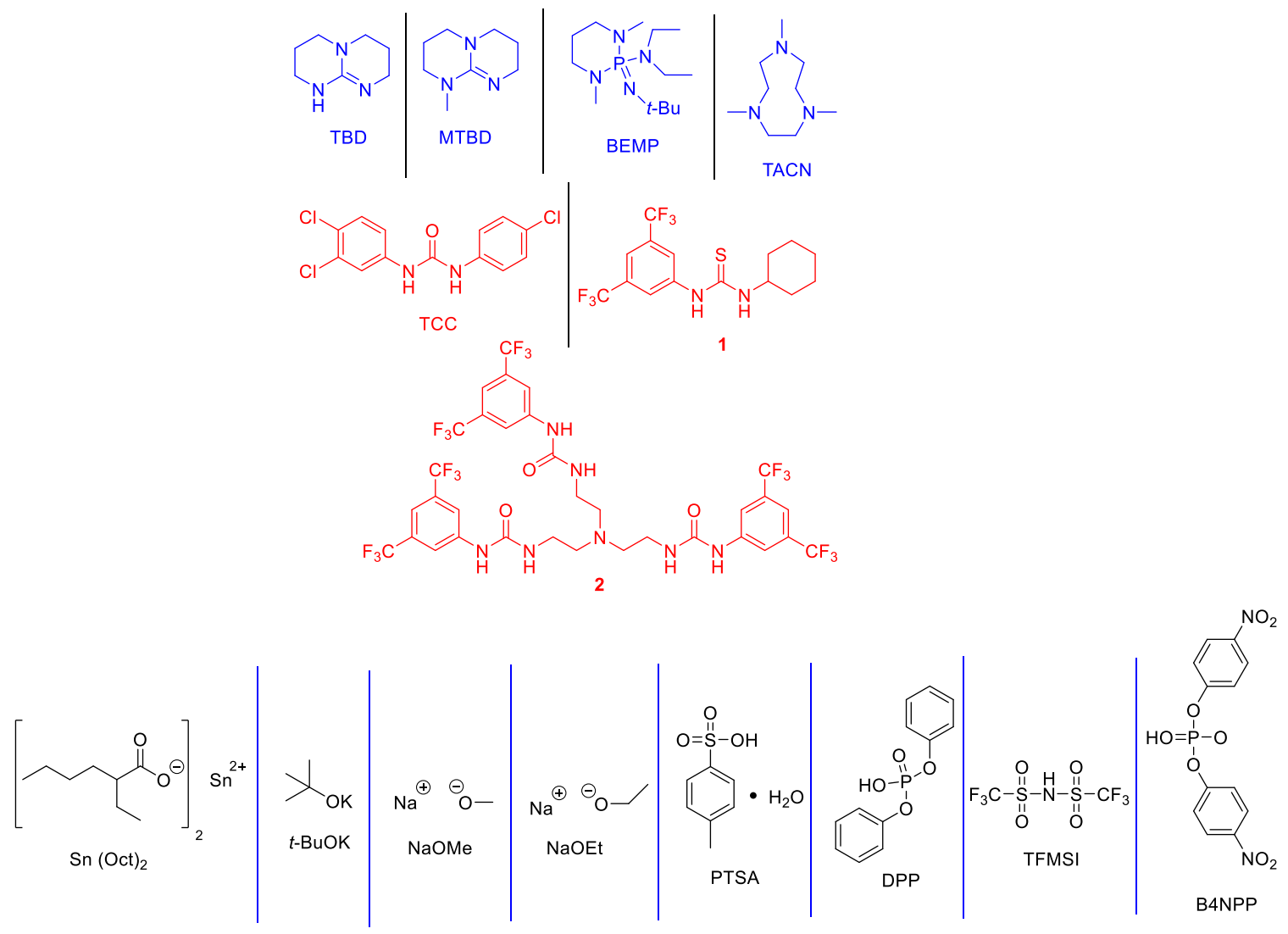

Scheme 7.2. Acid/Base and (co)catalysts studied in the attempt for ROP of cyclic ester monomers shown above (Scheme 7.1). 


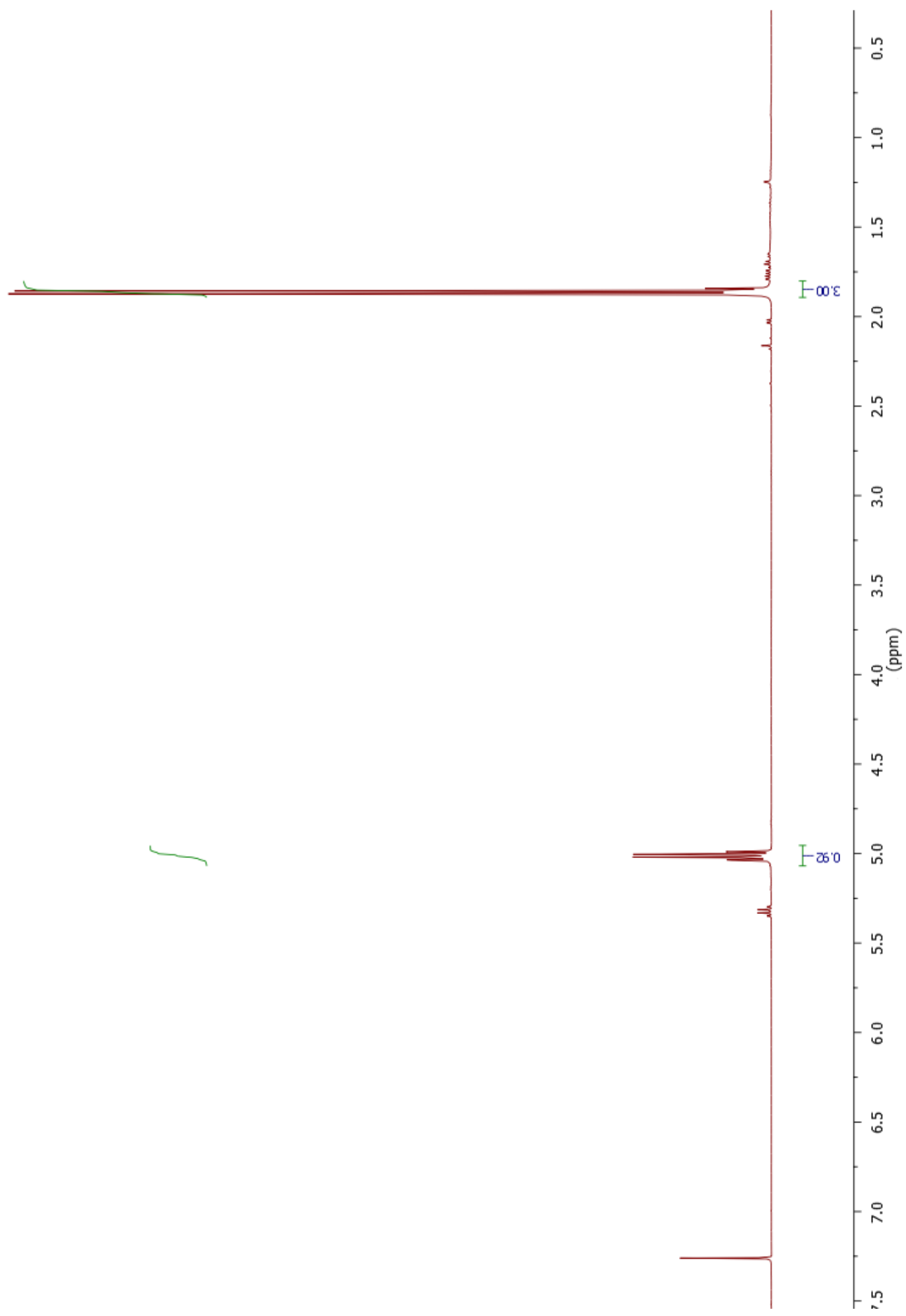

Figure 7.1. ${ }^{1} \mathrm{H} \mathrm{NMR}\left(400 \mathrm{MHz}, \mathrm{CDCl}_{3}\right)$ of L-tnLA monomer. 


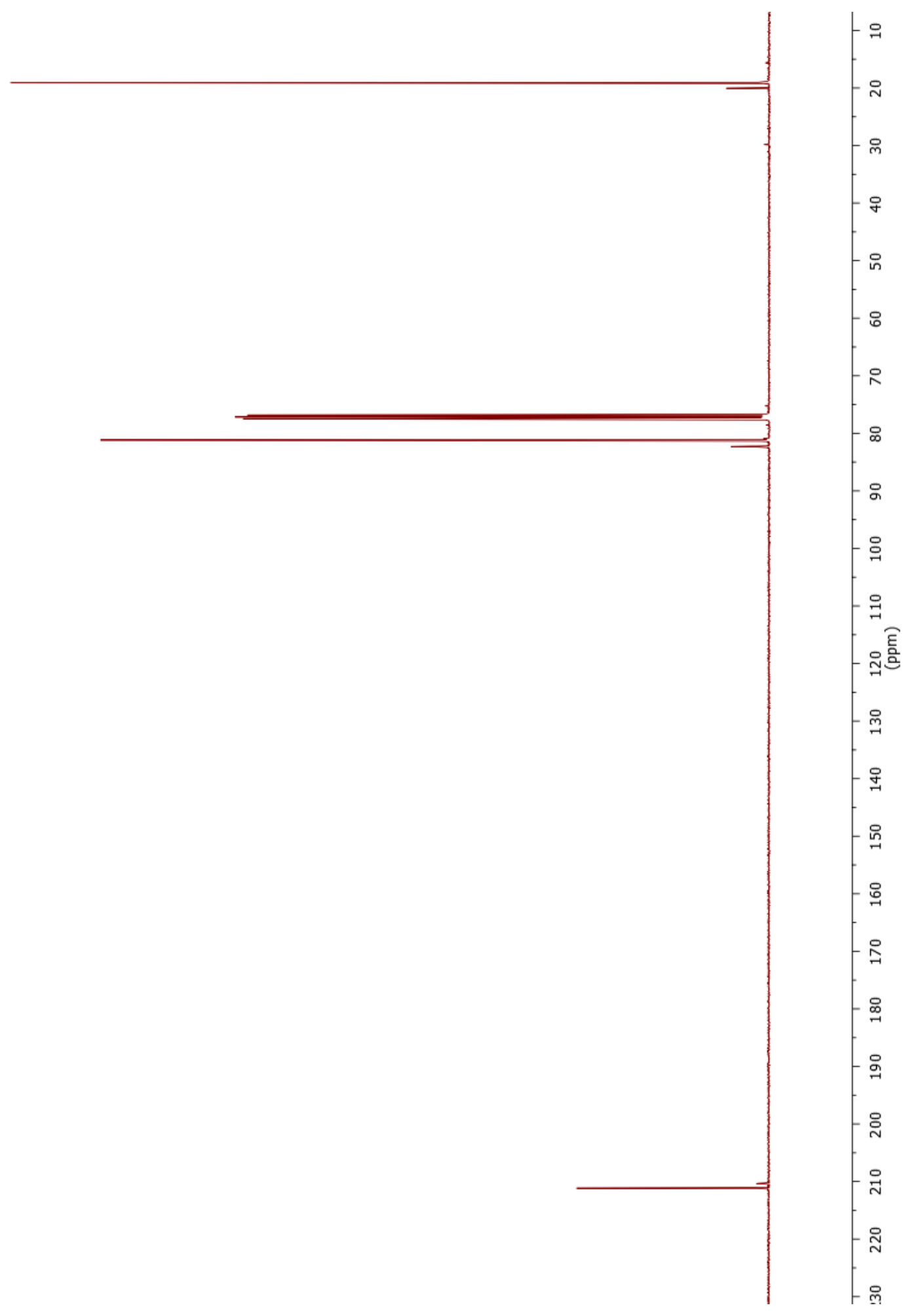

Figure 7.2. ${ }^{13} \mathrm{C} \mathrm{NMR}\left(100 \mathrm{MHz}, \mathrm{CDCl}_{3}\right)$ of L-tnLA monomer. 


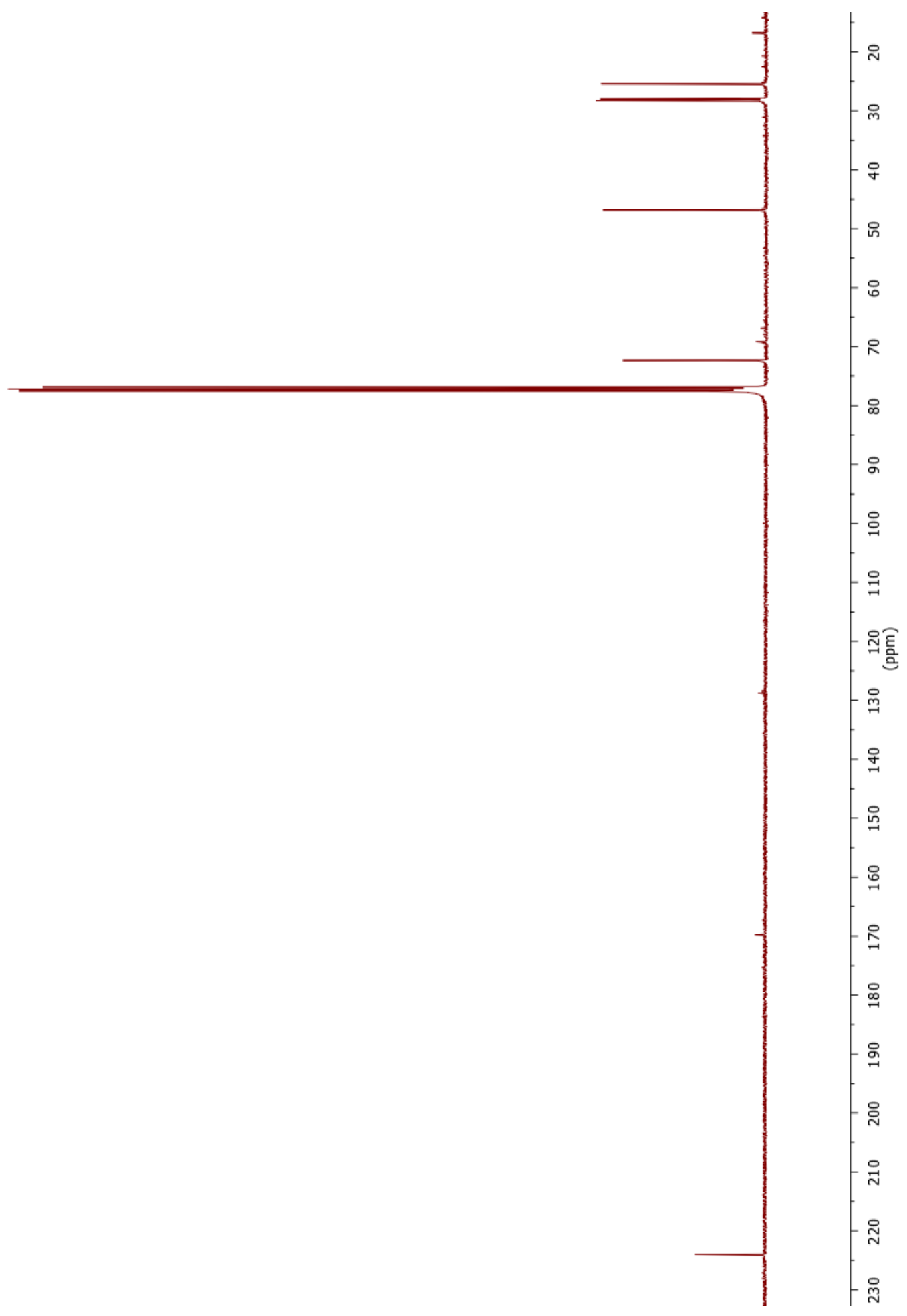

Figure 7.3. ${ }^{13} \mathrm{C}$ NMR (100 MHz, $\left.\mathrm{CDCl}_{3}\right)$ spectrum of P(LLA-co-tnCL-co-LLA) $(0.25: 1: 0.25)$. 


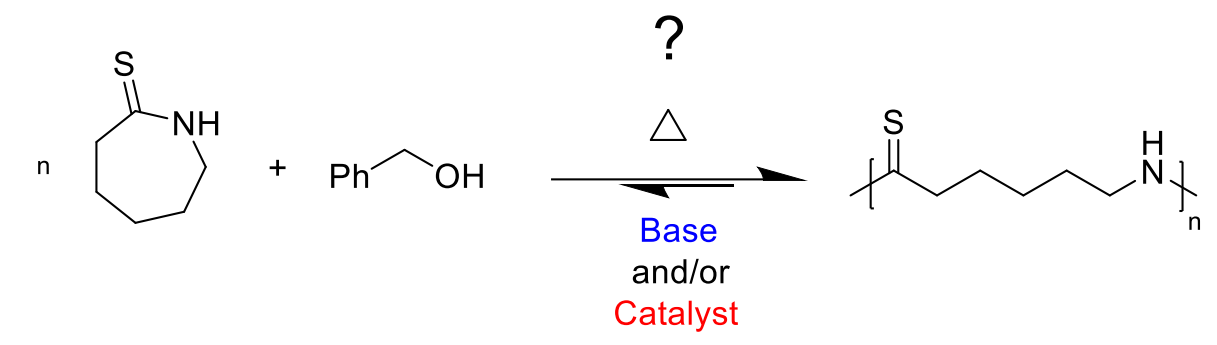

\begin{tabular}{|c|c|c|c|c|c|}
\hline Entry & Acid/Base & Cocatalyst & Solvent & Time & Conv. $^{\text {b }}(\%)$ \\
\hline $1^{\mathrm{c}}$ & MTBD & $\mathbf{2}$ & $\mathrm{CHCl}_{3}$ & 2 days & 0 \\
\hline $2^{\mathrm{d}}$ & TBD & - & $\mathrm{CDCl}_{3}$ & 2 days & 13 \\
\hline $3^{\mathrm{e}}$ & MTBD & $\mathbf{2}$ & $\mathrm{THF}$ & 2 days & 0 \\
\hline $4^{\mathrm{d}}$ & DPP & - & $\mathrm{CDCl}_{3}$ & 2 days & 0 \\
\hline $5^{\mathrm{d}}$ & PTSA & - & $\mathrm{CDCl}_{3}$ & 2 days & 0 \\
\hline $6^{\mathrm{d}}$ & B4NPP & - & $\mathrm{CDCl}_{3}$ & 2 days & 0 \\
\hline $7^{\mathrm{d}}$ & TFMSI & - & $\mathrm{CDCl}_{3}$ & 2 days & 0 \\
\hline $8^{\mathrm{ce}}$ & MTBD & $\mathbf{2}$ & $1,4-$ dioxane & 2 days & 0 \\
\hline $9^{\text {ce }}$ & MTBD & $\mathbf{2}$ & diphenyl ether & 2 days & 0 \\
\hline $10^{\mathrm{ce}}$ & MTBD & $\mathbf{2}$ & toluene & 2 days & 0 \\
\hline $11^{\mathrm{fg}}$ & PTSA & - & - & 2 days & 17 \\
\hline $12^{\mathrm{h}}$ & - & $\mathrm{NaOMe}$ & toluene & 1 day & 0 \\
\hline $13^{\mathrm{h}}$ & - & $\mathrm{NaOEt}$ & toluene & 1 day & 0 \\
\hline $14^{\mathrm{h}}$ & - & $t$-BuOK & toluene & 1 day & 0 \\
\hline
\end{tabular}

Table 7.1. ROP Attempts of tnCLa with various catalysts.

(a) Reaction conditions: unless stated otherwise, all reactions were performed at $2 \mathrm{M}$ with benzyl alcohol (1 mol\%) in room temperature at $5 \mathrm{~mol} \%$ acid/base and cocatalyst loading. (b) Conversion to polymer was obtained by ${ }^{1} \mathrm{H}$ NMR. (c) $1.67 \mathrm{~mol} \% \mathrm{acid} / \mathrm{base}$ and cocatalyst loading. (d) Reaction at $50^{\circ} \mathrm{C}$. (e) Reaction at $100^{\circ} \mathrm{C}$. (f) Reaction at $180^{\circ} \mathrm{C}$. (g) No benzyl alcohol was applied. (h) $10 \mathrm{~mol} \%$ catalyst loading at $1 \mathrm{M}$ solution. 
n<smiles>CC1OC(=S)C(C)OC1=S</smiles>
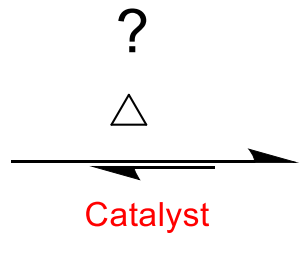



\begin{tabular}{|c|c|c|c|}
\hline Entry & Catalyst & Time & Conv. $^{\mathrm{b}}(\%)$ \\
\hline $1^{\mathrm{c}}$ & $\mathrm{Sn}(\mathrm{Oct})_{2}$ & $24 \mathrm{hrs}$ & 0 \\
\hline $2^{\mathrm{d}}$ & $t$-BuOK & $24 \mathrm{hrs}$ & 0 \\
\hline
\end{tabular}

Table 7.2. ROP Attempts of L-tnLA with different catalysts.

(a) Reaction conditions: unless stated otherwise, all reactions were performed at $2 \mathrm{M}$ with benzyl alcohol ( $1 \mathrm{~mol} \%)$ in toluene at $100^{\circ} \mathrm{C}$ with $1 \mathrm{~mol} \%$ catalyst loading. (b) Conversion to polymer was obtained by ${ }^{1} \mathrm{H}$ NMR. (d) No benzyl alcohol applied with 10 mol\% catalyst loading at $1 \mathrm{M}$. 
n<smiles>S=C1CCCCCC1</smiles>

B

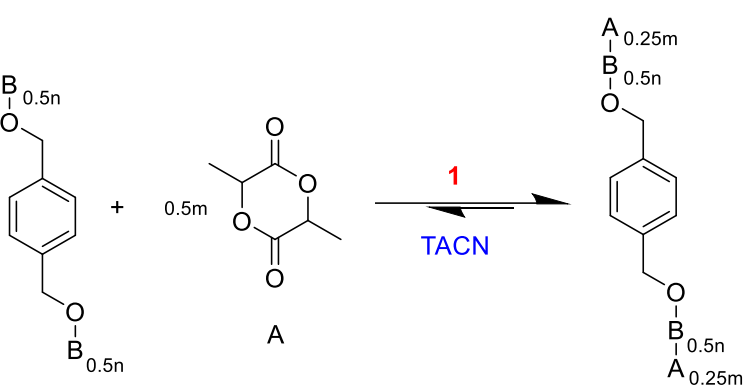

\begin{tabular}{|c|c|c|c|c|c|}
\hline Entry & tnCL (\% feed) & LA (\% feed) & Conv. $^{\mathrm{b}}(\%)$ & $\mathrm{M}_{\mathrm{n}}{ }^{\mathrm{c}}(\mathrm{g} / \mathrm{mol})$ & $\mathrm{M}_{\mathrm{w}} / \mathrm{M}_{\mathrm{n}}{ }^{\mathrm{c}}$ \\
\hline 1 & 67 & 33 & $90: 94$ & 17,800 & 1.21 \\
\hline 2 & 80 & 20 & $89: 92$ & 19,300 & 1.19 \\
\hline 3 & 89 & 11 & $92: 83$ & 20,100 & 1.33 \\
\hline
\end{tabular}

Table 7.3. ABA triblock copolymers of tnCL and L-LA with different monomer feeds.

(a) Reaction conditions: $2 \mathrm{M}$ in $\mathrm{C}_{6} \mathrm{D}_{6}$ for the $\mathrm{ROP}$ of B-block (tnCL) with 5 mol\%

TCC/BEMP loading with 1 mol\% initiator (1,4-benzenedimethanol); $1 \mathrm{M}$ in $\mathrm{CDCl}_{3}$ for the ROP of A-block (L-LA) with 5 mol\% 1/TACN loading. (b) Conversion to polymer obtained by ${ }^{1} \mathrm{H}$ NMR. (c) Determined by $\mathrm{GPC}\left(\mathrm{CH}_{2} \mathrm{Cl}_{2}\right)$ vs polystyrene standards. 


\author{
MANUSCRIPT - VIII \\ Prepared for submission to publish in Journal of Chemical Education
}

\title{
Stilbene Synthesis by Olefin Metathesis Reaction
}

Timothy J. Bannin, Partha P. Datta, Elizabeth T. Kiesewetter and Matthew K. Kiesewetter Chemistry, University of Rhode Island, Kingston, RI, USA

Corresponding Author: $\quad$ Matthew Kiesewetter, Ph.D.

Chemistry

University of Rhode Island

140 Flagg Road

Kingston, RI, 02881, USA

Email address: mkiesewetter@chm.uri.edu 


\begin{abstract}
In this experiment, students are asked to compare catalytic-cross metathesis and the Wittig reaction within the confines of 'Green' chemistry and atom economy. Students synthesize stilbene from styrene using Grubbs second generation catalyst. Products are minimally characterized by IR spectroscopy and melting point, but using ${ }^{1} \mathrm{H}$ NMR spectroscopy is preferred. Students find that the Wittig reaction is selective for cis-stilbene while the metathesis reaction produces all trans-stilbene. Students determine the cis/trans selectivity, turnover number (TON) and maximum turnover frequency (TOF) of the reaction. The experiment is conducted alongside the synthesis of stilbene using Wittig chemistry from a published procedure.
\end{abstract}




\section{INTRODUCTION}

The 2005 Nobel Prize in Chemistry went to Robert Grubbs, Yves Chauvin and Richard Schrock for the development of the metathesis reaction in organic synthesis. ${ }^{1}$ Development of metathesis chemistry continues apace, with new catalysts and abilities being reported more than a decade after the Nobel Prize. ${ }^{2}$ Indeed, the reaction has revolutionized several branches of chemistry and found applications in polymer, medicinal and organic chemistry. ${ }^{3-5}$ The olefin metathesis reaction is an intra- or inter-molecular rearrangement reaction where one or more carbon-carbon double bonds are broken and reformed. Intramolecular metathesis is generally called ring-closing metathesis, while intermolecular reactions are cross-metathesis or, sometimes, homo-cross-metathesis to emphasize the use of only one reagent. Polymers can also be constructed via metathesis using acyclic diene metathesis (ADMET) or ring-opening metathesis polymerization (ROMP) methods. The process must be catalyzed, and olefin metathesis catalysts contain a metal center ${ }^{6,7}$ - usually Ru or Mo - although organocatalytic methods for carbonylolefin metathesis have been reported. ${ }^{8}$ In an uncontrolled olefin metathesis reaction, a random mixture of products is generated. The development of advanced (asymmetric) catalysts and inherent (substrate driven) kinetic or thermodynamic control often provides fewer products. In the present experiment, the sole metathesis partner, styrene, conspires to substantially reduce the complexity of the reaction products, giving trans-stilbene as the only non-volatile product, Scheme 8.1.

The Wittig reaction, a classic means of preparing olefins, serves as a natural foil for the metathesis experiment. In the Wittig reaction, an aldehyde or ketone is reacted with a 
phosphonium salt in the presence of base to yield an olefin, Scheme 8.2. Besides being a widely known organic reaction that undergraduates normally learn during sophomore organic chemistry, the Wittig reaction is robust. A host of phosphonium salts is available with which to make a massive diversity of alkene products. These reactions can be performed on large or small scale, are often high yielding and can easily be performed by student chemists. ${ }^{9}$ The Wittig Reaction also has a Nobel Prize in Chemistry. ${ }^{10}$ This reaction also is a hallmark example of a non- 'Green' reaction, ${ }^{11}$ and it displays poor atom economy, ${ }^{12}$ meaning a considerable fraction of reagent mass is waste product, the triphenylphosphine oxide, which must be separated from the desired products. In contrast, metathesis catalysts are often used catalytically and then constitute a very small fraction of the reagent mass. Metathesis catalysts are also operative in a variety of solvents and can be used heterogeneously, which facilitates catalyst removal and recycling. ${ }^{13}$

In our Advanced Organic Laboratory course, students are asked in two consecutive laboratory experiments to synthesize stilbene, first using Wittig chemistry and second by the cross-metathesis of styrene. The Wittig synthesis of stilbene, ${ }^{9}$ which reacts benzaldehyde with benzyltriphenylphosphonium chloride in the presence of base, is selective for the cis-product ( $60 \%$ cis-stilbene). This selectivity contrasts markedly with that of metathesis reaction, which produces entirely trans-stilbene. This notable difference starts the students on a journey of 'unpacking' the differences, virtues and deficits of the two methods. 


\section{EXPERIMENTAL SECTION}

This experiment was accomplished in an advanced organic chemistry course with 16 students in a section. Conducting the experiment with larger numbers of students (e.g. a non-majors sophomore organic course) is feasible, but the cost of Grubbs 2 reagent should be considered. Lab sections met twice in a week for 3-hour sessions. The experiment is performed over two lab sessions. On the first day, students are asked to follow a procedure to make stilbene without a partner. The metathesis experiment can easily be finished in a 3-hour lab period. On day two, students were asked to form a hypothesis and work in small groups to build a series of data to reach a conclusion. In the lab report, students are asked to compare and contrast the synthesis of stilbene with metathesis versus the Wittig reaction, performed as the previous experiment. The published Wittig procedure requires a single 3-hour lab period to complete. ${ }^{9}$

In this experiment, we employ a Ru-centered catalyst (Grubbs 2nd generation catalyst) (1,3-Bis(2,4,6-trimethylphenyl)-2imidazolidinylidene)dichloro(phenylmethylene)(tricyclohexyl-phosphine)ruthenium _ which will perform the selective metathesis of styrene to make a single detectable product, trans-stilbene. ${ }^{6,14}$ In this transformation, the diastereoselectivity of the reaction is entirely substrate driven, producing the thermodynamic ratio of stilbene, $\sim 100 \%$ trans-stilbene.

Experimental Procedure

Since commercial styrene contains an inhibitor from the manufacturer which may disrupt the metathesis reaction, we removed the inhibitor in bulk before the lab period began. This was achieved by stirring a mixture of $3 \mathrm{~g}$ of alumina for every $20 \mathrm{~mL}$ of 
styrene for 5 minutes. Then the slurry was gravimetrically filtered through a qualitative filter paper to obtain pure styrene. The students can perform the purification individually on a reduced scale. Then a $20 \mathrm{~mL}$ scintillation vial was charged with a magnetic stir bar, Grubbs 2 (14.80 mg, $0.017 \mathrm{mmol})$ and dichloromethane $(10 \mathrm{~mL})$. Next, styrene $(0.2 \mathrm{~mL}$, $1.74 \mathrm{mmol}$ ) was added to the vial. The scintillation vial was then fitted with a polymer cone or foil backed cap and placed on a stir plate to stir for about 1 hour. After 1 hour, the solvent was removed under reduced pressure.

A miniature silica column was prepared. First, a pipette $(8 \times 142 \mathrm{~mm})$ was plugged with a piece of cotton or glass wool on one end. The pipet was then filled with dry silica from $1 / 2$ to $3 / 4$ of its volume. The crude product was dissolved in about $0.5 \mathrm{~mL}$ of dichloromethane. The silica plug was then wetted with hexanes and subsequently flushed with this solution of product in hexanes. An additional $20-25 \mathrm{~mL}$ of hexanes was used to flush the contents of the product through the silica. The solvent was then removed of volatiles in vacuo and ${ }^{1} \mathrm{H}-\mathrm{NMR}$, IR and a melting point was obtained. Students use chemical shift in the ${ }^{1} \mathrm{H}$ NMR spectrum to identify cis- versus trans-stilbene, but melting point can also be used to identify which diastereomer is made.

\section{Hazards}

All synthesized products and intermediates should be handled with caution. Avoid contact with skin and in the event of accidental exposure, wash the afflicted area with copious amounts of water. Styrene is flammable, may cause skin irritation, is a serious eye irritant, a suspected carcinogen and suspected of damaging fertility or the unborn child. Grubbs 2 is a flammable solid. $\mathrm{CH}_{2} \mathrm{Cl}_{2}$ can cause skin irritation, is a serious eye irritant, may cause respiratory irritation, may cause drowsiness/dizziness, suspected of causing 
cancer, if swallowed it may cause damage to the liver, blood and if inhaled it may cause damage to the central nervous system. Hexane is highly flammable, may be fatal if swallowed and enters the airways, can cause skin irritation, may cause drowsiness/dizziness, is suspected of damaging fertility or the unborn child, may cause damage to the nervous system and is toxic to aquatic life. $\mathrm{CDCl}_{3}$ is harmful if swallowed, causes skin and serious eye irritation, toxic if inhaled, suspected of causing cancer and of damaging fertility or the unborn child and can cause damage to organs. Appropriate personal protective equipment should be used at all times, and the reagents should only be handled in a well-ventilated fume hood. 


\section{RESULTS AND DISCUSSION}

This laboratory experiment was designed with two goals in mind: to give students experience with popular and versatile metathesis chemistry and to contrast this chemistry with the complementary Wittig reaction, which students performed previously in the semester from a published procedure. ${ }^{9}$ The reactions are perfect foils: the Wittig is cisselective while metathesis makes all trans-stilbene; the Wittig requires stoichiometric reagents while metathesis is catalytic; both reactions require purification to remove catalyst or phosphine oxide, but different methods of purification are required. This experiment also employs common and advanced organic chemistry concepts and techniques that students will find useful in industrial or academic setting: rotary evaporation, filtration, flash chromatography on small scale, spectroscopic identification, thermodynamic versus kinetic selectivity, properties of diastereomers and catalysis.

The purification of the reaction is facile. Students generally obtained about 80$90 \%$ yield after the column chromatography purification. Because the reaction is so selective, melting point can also be used to identify the isomer (m.p. cis-stilbene $=-5{ }^{\circ} \mathrm{C}$, m.p. trans-stilbene $\left.=122-126{ }^{\circ} \mathrm{C}\right),{ }^{15,16}$ and students find values of $\sim 120-124{ }^{\circ} \mathrm{C}$. This and IR spectroscopy provide reasonable proof of compound identity and purity; however, we asked students to use ${ }^{1} \mathrm{H}$ NMR spectroscopy to identify the product. The chemical literature indicates that the olefinic resonances for trans-stilbene $(\sim 7.15 \mathrm{ppm})$ appear markedly downfield of those for cis-stilbene ( 6.57 ppm) in the ${ }^{1} \mathrm{H}$ NMR spectrum. ${ }^{17}$ Further, close examination of the 6.1-8.0 ppm region of their spectrum reveals no spectroscopic indication of cis-product, indicating perfect diastereoselectivity. The reaction is under 
thermodynamic control and produces a minor amount $(0.2 \%)$ of the cis-isomer, but this small amount cannot be detected by ${ }^{1} \mathrm{H}$ NMR or melting point analysis. We were not equipped in our lab; however, HPLC could be performed to detect cis-stilbene; a very small amount is expected to be present.

On the second day of experimentation, students are asked to form a hypothesis and work in teams to come to a conclusion. Common variations included testing the turnover number and frequency limits of the reaction (within a lab period). These values are bookended by raising and lowering the catalyst concentration and conducting the workup (quenching the reaction) at various time points. Students measured turnover numbers (TON) of about $67-92$ and turnover frequency (TOF) of about $0.96-1.5 \mathrm{~min}^{-1}$. Students were also able to construct a crude first order plot (styrene) by quenching identical reactions at different time points and determining conversion by ${ }^{1} \mathrm{H}$ NMR. Quenching the reaction at various time points allowed some students to observe that the cis/trans ratio does not change as a function of conversion. From this, they concluded that the reaction was under thermodynamic control. Some students asked if the stabilizer slows down the reaction; the students were not able to discern a difference in TON or TOF with or without stabilizer in the styrene.

Students were graded based on the purity of their product (NMR and melting point) in addition to the post lab questions. The main thrust of the questions is to get the students to compare Wittig and metathesis methodologies. The obvious differences in cis/trans ratios between the methods was universally identified. After going to the literature (or conducting cis/trans ratio versus reaction time experiments), most students identified that 
the metathesis reaction was under thermodynamic control and the Wittig exhibits a kinetic preference for the cis- isomer. 


\section{CONCLUSION}

This laboratory experiment is not, at its core, about stilbene or metathesis, but rather it is about introducing the students to the unclear nature behind the concepts of Green chemistry ${ }^{18}$ and atom economy ${ }^{12}$ by comparing two robust and complementary synthetic approaches. Students were able to understand the concept of atom economy by stating that the metathesis reaction produced less reagent waste product than the Wittig. However, some students insist the Wittig is more utilitarian due to the facile nature of separation in that lab experiment. To us, there is no clear answer as to which process is 'Greener' or less wasteful (atom economic plus purification waste), but some students were able to present nuanced arguments for both sides. We feel that being able to see the big picture - even if it does not contain any clear answer(s) - is a primary goal of comparing these two reactions. 


\section{LIST OF REFERENCES}

(1) Grubbs, R. H. Angew. Chem. Int. Ed. 2006, 45, 3760-3765.

(2) Montgomery, T. P.; Johns, A. M.; Grubbs, R. H. Catalysts 2017, 7 (3), 87.

(3) Sinclair, F.; Alkattan, M.; Prunet, J.; Shaver, M. P. Polym. Chem. 2017, 8 (22), $3385-3398$.

(4) Liu, M.; Mountford, S. J.; Richardson, R. R.; Groenen, M.; Holliday, N. D.; Thompson, P. E. J. Med. Chem. 2016, 59 (13), 6059-6069.

(5) Grandner, J. M.; Shao, H.; Grubbs, R. H.; Liu, P.; Houk, K. N. J. Org. Chem. 2017, 82 (19), 10595-10600.

(6) Chatterjee, A. K.; Choi, T. L.; Sanders, D. P.; Grubbs, R. H. J. Am. Chem. Soc. 2003, 125 (37), 11360-11370.

(7) Nguyen, T.; Koh, M. J.; Shen, X.; Romiti, F.; Schrock, R. R.; Hoveyda, A. H. Science (80-. ). 2016, 352 (6285), 569-575.

(8) Griffith, A. K.; Vanos, C. M.; Lambert, T. H. J. Am. Chem. Soc. 2012, 134 (45), $18581-18584$.

(9) Warner, J. C.; Anastas, P. T.; Anselme, J.-P. J. Chem. Educ. 1985, 62 (4), 346.

(10) Gericke, D. Fortschr. Med. 1979, 97 (43), 1958-1964.

(11) Noyori, R. Chem. Commun. 2005, No. 14, 1807-1811.

(12) Trost, B. M. Angew. Chemie Int. Ed. English 1995, 34 (3), 259-281.

(13) Lwin, S.; Wachs, I. E. ACS Catal. 2014, 4 (8), 2505-2520.

(14) Sabila, P. Univers. J. Chem. 2015, 3 (3), 87-90.

(15) Thermo Fisher Scientific and Acros Organics. Cis-stilbene, (97\%); Safety Data Sheet; Fair Lawn, NJ, 2018. 
(16) Thermo Fisher Scientific and Acros Organics. Trans-stilbene, (97\%); Safety Data Shee; Fair Lawn, NJ, 2018.

(17) Wheeler, O. H.; de Pabon, H. N. B. J. Org. Chem. 1965, 30 (5), 1473-1477.

(18) Doxsee, K. M.; Hutchison, J. E. Green Organic Chemistry: Strategies, Tools and Laboratory Experiments; Thomson Learning Custom Publishing, 2002. 


\section{MATERIALS AND METHODS}

Styrene was purchased from Acros Organics, Grubbs $2^{\text {nd }}$ generation catalyst from SigmaAldrich, ACS reagent grade dichloromethane and hexane from Fisher Scientific. Silica gel (60Å/200-425 mesh) was purchased from Silicycle. $\mathrm{CDCl}_{3}$ was purchased from Cambridge Isotopes Laboratories. NMR experiments were performed on a Bruker Avance III $300 \mathrm{MHz}$ spectrometer in $\mathrm{CDCl}_{3}$. IR spectra were obtained on a Thermo Nicolet 380 FT-IR equipped with a Smart Orbit attachment. Melting points were obtained on a Stuart SMP10 melting point apparatus.

Required Reagents (CAS Number)

1. (1,3-Bis(2,4,6-trimethylphenyl)-2imidazolidinylidene)dichloro(phenylmethylene)(tricyclohexyl-phosphine)ruthenium (Grubbs Catalyst $2^{\text {nd }}$ Generation, CAS 246047-72-3)

2. styrene (CAS 100-42-5)

3. dichloromethane (CAS 75-09-2)

4. hexane (CAS 110-54-3)

5. silica gel (60尺̊/200-425 mesh, CAS 7631-86-9)

6. $\mathrm{CDCl}_{3}$ (CAS 865-49-6)

Apparatus and Lab Materials

Students will each require:

1. $20 \mathrm{~mL}$ scintillation vial with a polypropylene screw cap

2. magnetic stir bar $(0.5 \times 0.125$ in. $)$ 
3. glass/cotton wool

4. volumetric pipette bulb

5. $8 \times 142 \mathrm{~mm}$ glass pipettes

6. 9-inch Pasteur pipettes

7. 3 cc pipette bulb

8. 3-pronged clamp

9. clamp stand with base

10. $3 \times 3$ inch weighing paper (for loading silica into pipette)

11. vial-to-rotavap adapter (we use a 24/40 septa, $1-1 \frac{1 / 4}{4}$ in $22 \mathrm{G}$ needle)

12. magnetic stir plate

$\underline{\text { Student need access to shared: }}$

1. Rotary evaporator

2. IR spectrometer

3. Melting point apparatus

4. (optional) ${ }^{1} \mathrm{H}$ NMR spectrometer

\section{Design of Experiment}

This experiment can fill one or two 3-hour lab periods. The main experiment, designed to take one day, takes the student through the synthesis of stilbene. The second day is freeform, and the students are encouraged to pair with one or more students to gather additional information about the reaction. 
Common kinetics-type experiments on the second day include: Finding the order of the reaction in a reagent by collecting conversion versus time data (the order in Grubbs 2 requires at least two observed rate constants $\left(\mathrm{k}_{\mathrm{obs}}\right)$ from the first order plot of [styrene] vs time), determining the turnover number and limits thereof for the reaction. The reaction is first order in [Grubbs 2] $]_{\mathrm{o}}$ and first order in [styrene $]_{\mathrm{o}}$.

Other experiments include varying the reagents. Students can also attempt the reaction with Grubbs catalyst, $1^{\text {st }}$ Generation (Grubbs 1), but this catalyst produces no conversion even at high catalyst loadings. This is related to the olefin type. ${ }^{1,2}$ Students can also run the reaction in the presence of inhibitor; no change in the reaction versus the uninhibited reaction is observed. 


\section{NOTES TO INSTRUCTORS}

Removal of Inhibitor. Styrene from a chemical supplier contains an inhibitor. The inhibitor may not be disruptive to the metathesis reaction, but it was removed prior to the lab period by stirring a mixture of $3 \mathrm{~g}$ of alumina for every $20 \mathrm{~mL}$ of styrene for $5 \mathrm{~min}$. Then, the slurry was filtered gravimetrically through qualitative filter paper. Uninhibited styrene will undergo auto polymerization over several days; this inhibitor-free styrene should be disposed of after the lab period, and the glassware cleaned.

Solvent Removal from a Vial by Rotovap. To remove solvent by rotovap from a 20 $\mathrm{mL}$ scintillation vial requires a specialized adapter (Chemglass CG-1318-10 Glass Rotary Evaporator Vial Adapter, 24/40 Joint). However, we employ 24/40 septa and needles which are usually readily available in an organic chemistry lab. To attach the vial to the rotovap, the septa must be inverted so the opening of the vial fits into the $40 \mathrm{~mm}$ side of the septa. Then, insert the needle through the $24 \mathrm{~mm}$ side which fits as a slip joint on a 14/20 bump trap or 14/20 adapter.

Metathesis Reaction. Our students ran reactions in disposable $20 \mathrm{~mL}$ scintillation vials, but a conventional $10 \mathrm{~mL}$ round bottom flask is acceptable. The students should notice a dark purple color upon the addition of the Grubbs 2 catalyst. The Grubbs 2 catalyst can be dispensed in a stock solution of $\mathrm{CH}_{2} \mathrm{Cl}_{2}$, but this stock solution has a finite lifetime. Students were asked to syringe styrene directly from the dispensing area (in a hood) and transport the capped syringe back to their workspace. This greatly minimized exposure to styrene, which has a potent odor.

Purification by Silica Gel Chromatography. Our students purified their stilbene with a microscale, Pasteur pipette silica gel column. A glass wool/cotton plug was loaded 
into the column ( 8 x $142 \mathrm{~mm}$ glass pipette) using a 9 inch Pasteur pipette push rod, see Figure $8.1 \mathrm{~A}$ in the student handout section. Then silica was loaded into the $8 \mathrm{x} 142 \mathrm{~mm}$ pipette using weigh paper folded in half diagonally. A volumetric pipette bulb was used to force hexanes through the column with slight, constant pressure. The silica bed can crack if abrupt pressure changes are applied. A traditional silica gel column can also be employed, but once practiced, we find that the pipette column becomes a favorite tool for easy separations. Instructors may wish to check the setup for the column prior to elution of the product, depending on the class size. Students achieved the best and most facile separations when the product was loaded onto the column in a minimal volume of $\mathrm{CH}_{2} \mathrm{Cl}_{2}$ $(<0.5 \mathrm{~mL})$ and eluted with hexanes $(\sim 25 \mathrm{~mL})$. Students should be reminded to load the product solution entirely onto the silica before eluting with hexanes. Our students typically get an isolated yield of $\sim 70-90 \%$. Students who do not obtain a yield of at least $60 \%$ may be able to flush their column with more hexanes to obtain residual product on the silica gel.

Identification of cis- versus trans-stilbene. Students will observe that the metathesis reaction produces $\sim 100 \%$ trans-stilbene. The cis/trans ratio is most conveniently determined from ${ }^{1} \mathrm{H}$ NMR, where the chemical shift of the ethylene resonance is isomer-dependent: cis-stilbene at $6.60 \mathrm{ppm}$ and trans-stilbene at $7.15 \mathrm{ppm}^{3}$ With Grubbs 2, the metathesis reaction should produce the approximate thermodynamic ratio of products. For stilbene, the thermodynamic ratio is $\sim 0.2 \%$ cis-isomer, $\mathrm{K}_{\mathrm{eq}}=0.002, \Delta \mathrm{G}^{\mathrm{o}}=$ $3.7 \mathrm{kcal} / \mathrm{mol}$. In our experimentation, we do not observe any $c i s$-stilbene in the ${ }^{1} \mathrm{H}$ NMR spectrum. Alternatively, the melting points of the two isomers are drastically different (cism.p. $=-5^{\circ} \mathrm{C}$ and trans- m.p. $\left.=122-126^{\circ} \mathrm{C}\right) .{ }^{4,5}$ Potential post-lab questions are apparent: 


\section{EXAMPLE POST LAB QUESTIONS AND ANSWERS}

We select 4-5 of the questions below as post lab questions to be answered in the lab report.

1. What are the advantages of synthesizing stilbene with metathesis vs Wittig? Disadvantages?

Answer: Wittig processes confer high selectivity for the cis-isomer, which can be difficult to access using metathesis. Wittig reagents and methods are robust, structurally diverse and are often easy to separate from the product, but they must be used stoichiometrically. Metathesis catalysts are highly functional group tolerant, readily available and general (i.e. one can apply a SINGLE metathesis catalyst to many syntheses, but a new Wittig reagent is needed for every product). The Grubbs reagents (we use the common term 'catalyst' in this document are really pre-catalysts or initiators ${ }^{1}$ are usually applied catalytically which minimized waste. However, the metathesis products can re-enter the catalytic cycle, eroding yield and stereocontrol (if present), depending on what type of olefin describe the

product and reagent. ${ }^{1}$ Stilbene is a Type II olefin with respect to Grubbs $1^{\text {st }}$ generation catalyst, ${ }^{1}$ and it will not readily undergo subsequent metathesis. However, if the product is symmetric (as with stilbene), these processes are not evident even if they occur

2. What is the cis/trans ratio produced by metathesis and how does it compare to the Wittig reaction?

Answer: According to Warner et al., the Wittig reaction produced cis- and transstilbene in a $60: 40$ ratio while the present metathesis reaction produces $\sim 100 \%$ 
trans- product. $^{6}$ The Wittig reaction is selective for $c i s$-product while Grubbs 2 is not selective.

3. Draw the catalytic cycle that produces stilbene. Where is the stereochemistry set (i.e. at what point does the product become cis- or trans-?



Answer: A full answer will include the catalytic cycle above which shows the generation of the active catalyst (middle to top), formation of the ruthenium metallocycle butane (right) where the stereochemistry of the product is set, and the regeneration of the catalytically productive ruthenium benzylidene (top) via evolution of an equivalent of ethylene (left).

4. A properly-designed catalyst can produce non-thermodynamic distributions of products (i.e. a kinetic or Curtin-Hammett distribution of products). Is this metathesis reaction thermodynamically or kinetically controlled? Can you design an experiment to test your answer? 
Answer: The product distribution in the present metathesis reaction is under thermodynamic control; the achiral catalyst imparts no kinetic preference for one isomer over another. However, asymmetric metathesis catalysts are available.

To test the possibility of thermodynamic versus kinetic control, a group of students can perform reactions where the reactions are stopped at different intervals: from 20 min up to days. Students will observe only trans-product at all time points. If the reaction were under measurable kinetic control, cis/trans ratio would be a function of reaction time. This requires stilbene to undergo metathesis, which as a Type II olefin, ${ }^{1}$ it does so only sparingly.

5. Is this catalyst a good choice for olefin metathesis? (hint: take a look at your TON and TOF). Hit the literature, what other catalyst might you suggest for metathesis? Answer: Grubbs-type catalysts are widely used because they are long-lived (decent TON) and tolerant to a wide variety of functional groups and reaction conditions. ${ }^{1,7}$ A host of metathesis catalysts is available. Various specialized catalysts are available for rapid initiation, ${ }^{8}$ ring-closing metathesis, ${ }^{9}$ and densely-functionalized substrates. ${ }^{10}$ Catalysts employing other metals, particularly molybdenum, are capable of effecting rapid and selective metathesis reactions. ${ }^{11}$

6. If you produced the thermodynamic ratio of stilbene (trans-stilbene $\Leftrightarrow$ cis-stilbene; $\left.\mathrm{K}_{\mathrm{eq}}=0.002\right)$, why is none observed in the ${ }^{1} \mathrm{H}$ NMR?

Answer: The thermodynamic ratio suggests $0.2 \%$ cis- product $\left(\mathrm{K}_{\mathrm{eq}}=0.002=(100-\right.$ $\mathrm{x}) / \mathrm{x} ; \mathrm{x}=99.8$ ). This value is far below the detection limits of NMR spectroscopy.

7. What factors influence cis/trans ratios? 
Answer: Catalysts and reagents (e.g. Wittig) can be stereoselective, but the Grubbs 2-catalyzed formation of stilbene from styrene is not. This experiment produces the thermodynamic ratio of products. This ratio is determined by the relative stability of the two products where the bulky phenyl rings strongly favor a transisomer for steric reasons. ${ }^{1}$

8. Why is the cis/trans ratio of stilbene so small? For comparison, the thermodynamic distribution of isomers for 2-butene is about $30 \%$ cis-isomer. $^{12}$

Answer: The phenyl rings in stilbene are much bulkier than the methyl groups in 2-butene, which makes the reaction far more selective for the trans-product in the case of stilbene versus 2-butene. The effect is augmented because the phenyl rings in stilbene prefer to be coplanar for $\pi$-delocalization.

9. Convert cis/trans ratio into $\mathrm{K}_{\mathrm{eq}}$ and/or $\Delta \mathrm{G}^{\mathrm{o}}$.

Answer: The values are $\sim 0.2 \%$ cis-isomer, $\mathrm{K}_{\mathrm{eq}}=0.002, \Delta \mathrm{G}^{\mathrm{o}}=3.7 \mathrm{kcal} / \mathrm{mol}$ which can be found using the standard equations:

$$
\begin{gathered}
K_{e q}=\frac{[\text { trans }- \text { stilbene }]_{e q}}{[\text { cis }- \text { stilbene }]_{e q}} \\
\Delta G^{0}=-R T \ln K_{e q}
\end{gathered}
$$


n

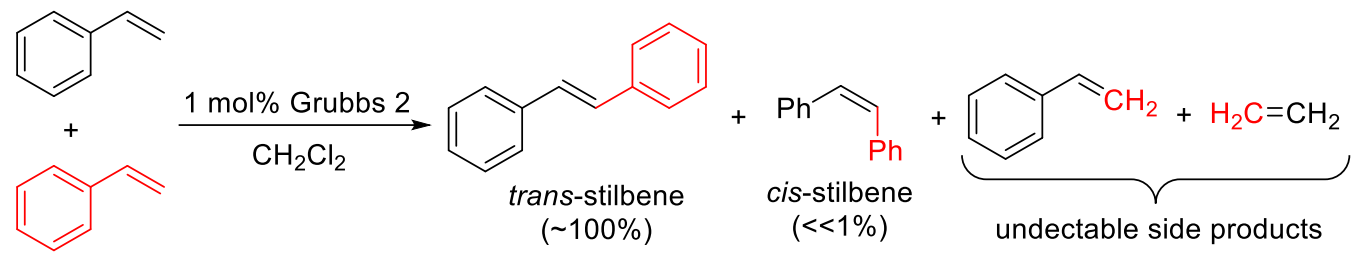

Scheme 8.1. The homo-cross-metathesis reaction of stilbene produces only one nonvolatile product. Other products are undetectable (unproductive metathesis products), boil off (ethylene) or thermodynamically disfavored (cis-stilbene). Stilbene does not re-enter the catalytic cycle. 


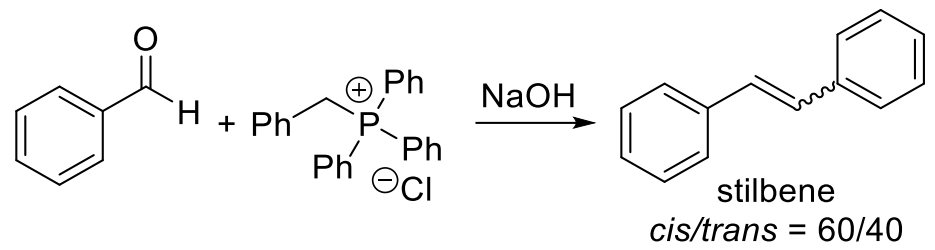

Scheme 8.2. Example Wittig reaction to synthesize stilbene. 


\section{Characterization Data and Spectra of Stilbene Products}

trans-stilbene<smiles>C(=C/c1ccccc1)\c1ccccc1</smiles>

${ }^{1} \mathrm{H}-\mathrm{NMR}\left(300 \mathrm{MHz}, \mathrm{CDCl}_{3}\right): \delta 7.57-7.47(\mathrm{~d}, J=7.6 \mathrm{~Hz}, 2 \mathrm{H}), 7.43-7.32(\mathrm{t}, J=14.8,7.9,7.0 \mathrm{~Hz}$, $2 \mathrm{H}), 7.32-7.21(\mathrm{t}, J=14.8,8.5,6.0 \mathrm{~Hz}, 1 \mathrm{H}), 7.15-7.09(\mathrm{~s}, 1 \mathrm{H})$.

IR: $A=3058.69 \mathrm{~cm}^{-1}(=\mathrm{C}-\mathrm{H}) ; \mathrm{B}=3020.13 \mathrm{~cm}^{-1}(=\mathrm{C}-\mathrm{H}) ; \mathrm{C}=1596.85 \mathrm{~cm}^{-1}, 1577.56 \mathrm{~cm}^{-1}(\mathrm{C}=\mathrm{C}$, aromatic); $\mathrm{D}=1494.64 \mathrm{~cm}^{-1}\left(\mathrm{C}=\mathrm{C}\right.$, aromatic); $\mathrm{E}=1450.28 \mathrm{~cm}^{-1}(\mathrm{C}=\mathrm{C}$, aromatic) (see spectra below)

MP: $122-125^{\circ} \mathrm{C}$.

Yield: About 92\% (determined by NMR).

cis-stilbene (for comparison, none observed)<smiles>C(=C\c1ccccc1)\c1ccccc1</smiles>

${ }^{1} \mathrm{H}$ NMR $\left(89.56 \mathrm{MHz}, \mathrm{CDCl}_{3}\right):^{13} \delta 7.38-6.98(\mathrm{~m}, 10 \mathrm{H}), 6.57(\mathrm{~s}, 2 \mathrm{H})$

$M P:{ }^{4}-5{ }^{\circ} \mathrm{C}$ 







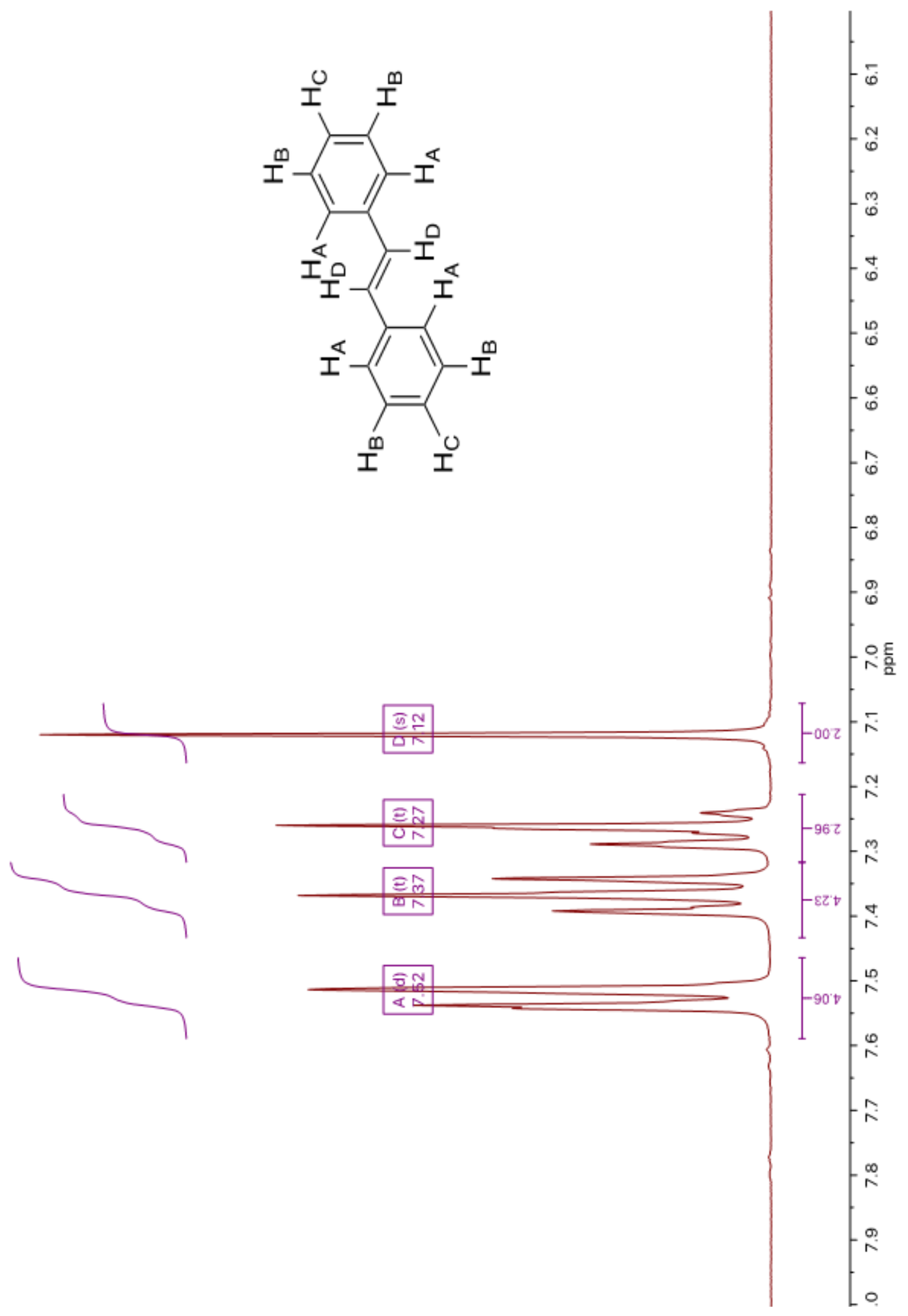




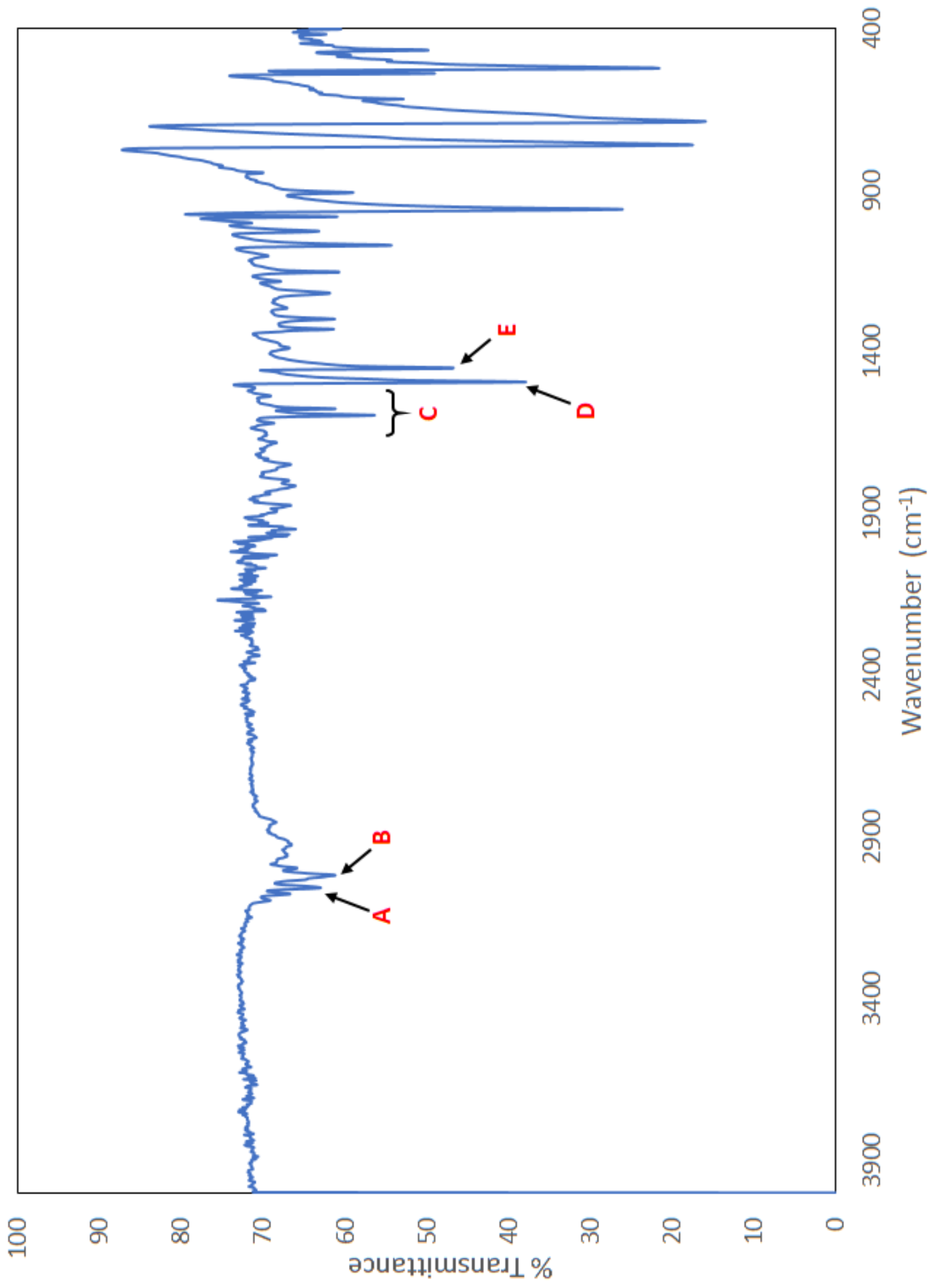




\section{REFERENCES FOR SUPPLEMENTAL INFORMATION}

1. Chatterjee, A. K.; Choi, T. -L.; Sanders, D. P.; Grubbs, R. H. A General Model for Selectivity in Olefin Cross Metathesis. J. Am. Chem. Soc. 2003, 125 (37), 1136011370.

2. Sabila, P. Styrene Cross-metathesis Using Low Catalyst Concentrations. Univers. J. Chem. 2015, 3 (3), 87-90.

3. Wheeler, O. H.; Battle de Pabon, H. N. Synthesis of Stilbenes. A Comparative Study. J. Org. Chem. 1965, 30 (5), 1473-1477.

4. Fisher Scientific and Acros Organics. Cis-Stilbene, (97\%); Safety Data Sheet; Fair Lawn, NJ, 2018.

5. Fisher Scientific and Acros Organics. Trans-Stilbene, (97\%); Safety Data Sheet; Fair Lawn, NJ, 2018.

6. Warner, J. C.; Anastas, P. T.; Anselme, J. -P. The Wittig Reaction in the Undergraduate Organic Laboratory. J. Chem. Ed. 1985, 62 (4), 346.

7. Dinger, M. B.; Mol, J. C. High Turnover Numbers with Ruthenium-Based Metathesis Catalysts. Adv. Synth. Catal. 2002, 344 (6-7), 671-677.

8. Romero, P. E.; Piers, W. E.; McDonald, R. Rapidly Initiating Ruthenium OlefinMetathesis Catalysts. Angew. Chem. 2004, 116 (45), 6287-6291.

9. Stewart, I. C.; Ung, T.; Pletnev, A. A.; Berlin, J. M.; Grubbs, R. H.; Schrodi, Y. Highly Efficient Ruthenium Catalysts for the Formation of Tetrasubstituted Olefins via Ring-Closing Metathesis. Org. Lett. 2007, 9 (8), 1589-1592. 
10. Nguyen, S. T.; Grubbs, R. H. Syntheses and Activities of New Single-Component, Ruthenium-Based Olefin Metathesis Catalysts. J. Am. Chem. Soc. 1993, 115 (21), 9858-9859.

11. Nguyen, T.; Koh, M. J.; Shen, X.; Romiti, F.; Schrock, R. R.; Hoveyda, A. H. Kinetically controlled $E$-selective catalytic olefin metathesis. Science, 2016, 352 (6285), 569-575.

12. Scott, R. B.; Ferguson, W. J.; Brickwedde, F. G. Thermodynamic Properties of cis2-Butene from $15^{\circ}$ to $1500^{\circ}$ K. J. Res. Natl. Bur. Stand. 1944, 33 (1), 1-20.

13. Spectral Database for Organic Compounds (SDBS). SDBS- ${ }^{1} \mathrm{H}$ NMRSDBS No. 7295HSP-04-012 cis-stilbene. http://sdbs.db.aist.go.jp/sdbs/cgibin/direct_frame_top.cgi (accessed Jan 25, 2018). 


\section{Example Grading Rubric}

1. Descriptive Title/Purpose (5 pts): Title should be concise yet describe the experiment completely. A sentence or two should be devoted to the purpose of the experiment.

2. Reaction Scheme (10 pts): A reaction scheme should be provided after the title and purpose. The scheme should pertain to this specific experiment including reagents, reaction conditions and product. A complete mechanism for the reaction should follow with proper arrow pushing and formal charges.

3. Data Analysis and Characterization (20 pts): All the spectra should be provided, including IR and NMR data. These should be properly labeled with assignments of relevant peaks. A table or lists of peaks could be used for this instance.

4. Yield (5 pts): Theoretical and percent yield should be provided with all step-by-step calculations.

5. Post-Lab Questions (30 pts): All the questions should be answered fully but succinctly. If drawings or mechanisms can help in the answer, they should be provided. 
6. Lab Notebook (20 pts): Students should provide signed (by TA or instructor) carbon copies of their lab notebook where they should have a completed data table and any relevant observations.

7. Lab Technique/citizenship (10 pts): The lab should be returned to the condition in which you found it. Violations that are not attributable will be assessed to the whole class. Improper handling or use of equipment/chemicals will also cause deduction in points.

TOTAL $/ 100$ 


\section{STILBENE SYNTHESIS BY OLEFIN METATHESIS}

\section{STUDENT HANDOUT}

In a previous laboratory experiment, ${ }^{1}$ stilbene was synthesized via a Wittig reaction. The Wittig reaction is robust and widely-used in industrial and academic research labs. It is also a hallmark counterexample of a 'Green' ${ }^{2}$ process, and the reaction exhibits poor atom economy. ${ }^{3}$ That is, the mass of product divided by mass of 'wasted' Wittig reagent byproduct is low and can be less than unity, depending on the reaction. ${ }^{1}$ Catalytic methods offer an alternative. The primary advantage of a catalytic approach is the ability to generate many moles of product for each mole of catalyst (i.e. a good catalyst will have a high turnover number, $\mathrm{TON}=$ mols substrate/mols catalyst $)$ and keep waste to a minimum. The multitude of synthetic possibilities and advantages rendered by tuning ligand structure - to change regiochemistry, stereochemistry, rate, and substrate scope - makes catalysis an attractive field of research. Stoichiometric (e.g. Wittig) and catalytic (e.g. metathesis) reactions have concomitant benefits and drawbacks. An overarching goal of the two stilbene synthesis experiments is to directly compare and contrast the two approaches.

Catalysts for olefin metathesis, particularly ruthenium $(\mathrm{Ru})$-containing catalysts, have revolutionized synthetic chemistry. ${ }^{4}$ These catalysts have impacted pharmaceutical, ${ }^{5}$ natural products ${ }^{6}$ and polymer chemistry. ${ }^{7}$ The development of olefin metathesis catalysts was awarded the 2005 Nobel Prize in Chemistry. ${ }^{8}$ In this experiment, you will be using a Ru olefin metathesis catalyst - commonly called Grubbs Catalyst, Second Generation or 'Grubbs 2' - to perform the homodimerization (or cross-metathesis) of styrene. The diastereoselectivity (cis/trans selectivity) of the metathesis transformation is different than the Wittig process. ${ }^{4}$ 
2<smiles>c1ccccc1</smiles>

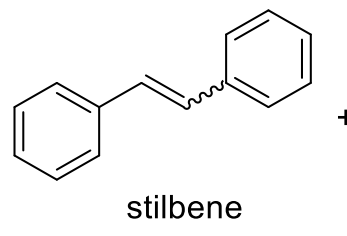

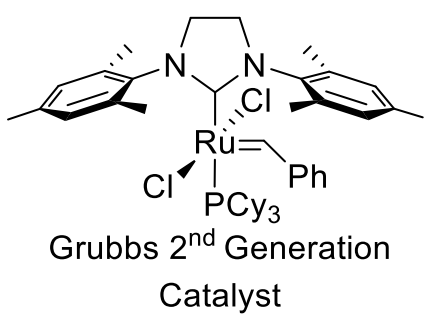

Scheme 1.

1. Warner, J. C.; Anastas, P. T.; Anselme, J. -P. The Wittig Reaction in the Undergraduate Organic Laboratory. J. Chem. Ed. 1985, 62 (4), 346.

2. Noyori, R. Pursuing practical elegance in chemical synthesis. Chem. Commun. 2005, 14, 1807-1811.

3. Trost, B. M. Atom Economy - A Challenge for Organic Synthesis: Homogeneous Catalysis Leads the Way. Angew. Chem. Int. Ed. Engl. 1995, 34 (3), 259-281.

4. Chatterjee, A. K.; Choi, T. -L.; Sanders, D. P.; Grubbs, R. H. A General Model for Selectivity in Olefin Cross Metathesis. J. Am. Chem. Soc. 2003, 125 (37), 1136011370.

5. Liu, M.; Mountford, S. J.; Richardson, R. R.; Groenen, M.; Holliday, N. D.; Thompson, P. E. Optically Pure, Structural, and Fluorescent Analogues of a Dimeric $\mathrm{Y}_{4}$ Receptor Agonist Derived by an Olefin Metathesis Approach. J. Med. Chem. 2016, 59 (13), 6059-6069.

6. Lad, N. P.; Kulkarni, S.; Sharma, R.; Mascarenhas, M.; Kulkarni, M. R.; Pandit, S. S. Piperlongumine derived cyclic sulfonamides (sultams): Synthesis and in vitro exploration for therapeutic potential against HeLa cancer cell lines. Eur. J. Med. Chem. 2017, 126, 870-878. 
7. Sinclair, F.; Alkattan, M.; Prunet, J.; Shaver, M. P. Olefin Cross Metathesis and Ring-Closing Metathesis in Polymer Chemistry. Polym. Chem. 2017, 8 (22), 33853398.

8. Grubbs, R. H. Olefin-Metathesis Catalysts for the Preparation of Molecules and Materials (Nobel Lecture). Angew. Chem. Int. Ed. 2006, 45 (23), 3760-3765. 


\section{EXPERIMENTAL}

\section{DAY 1}

Charge a $20 \mathrm{~mL}$ scintillation vial with a magnetic stir bar, Grubbs 2 (14.80 mg, $0.017 \mathrm{mmol})$ and dichloromethane $\left(\mathrm{CH}_{2} \mathrm{Cl}_{2}\right)(10 \mathrm{~mL}, 0.157 \mathrm{mmol})$. Next, add the styrene* $(0.2 \mathrm{~mL}, 1.74 \mathrm{mmol})$, fit the scintillation vial with a polymer cone or foil backed cap and place on stir plate. Let the reaction stir for 1 hour, and then remove the solvent in vacuo.

Prepare a mini silica column, Figure 8.1. First, loosely plug one end of a pipet ( 8 x $142 \mathrm{~mm}$ ) with a piece of cotton or glass wool. Next, fill pipet with silica to a height of $\sim 5 \mathrm{~cm}$. Add the hexanes mobile phase to the top of the mini column and use a volumetric pipette bulb to push the mobile phase onto the column. Use gradual pressure changes to move the solvent without cracking the silica gel stationary phase; this can take practice and patience.

Re-dissolve the vial contents in minimal $\mathrm{CH}_{2} \mathrm{Cl}_{2}(0.5-1.0 \mathrm{~mL})$. Pipet this solution onto the silica plug, trying not to disturb the wet silica. After loading the reaction solution onto the column, flush the plug with excess hexanes $(\sim 25 \mathrm{~mL})$ to remove the stilbene, collecting in a $100 \mathrm{~mL}$ round bottom flask. A shorter column $(\sim 2 \mathrm{~cm})$ can be eluted with a smaller amount $(\sim 10 \mathrm{~mL})$ of hexanes, but loading in minimal $\mathrm{CH}_{2} \mathrm{Cl}_{2}$ is critical. Remove the solvent in vacuo and collect ${ }^{1} \mathrm{H}$ NMR, IR spectra and melting point. Determine the cis/trans- ratio of the product, turnover number (TON) and maximum turnover frequency (TOF) of the reaction.

*Styrene from a chemical supplier contains an inhibitor. The inhibitor may not be disruptive to the metathesis reaction, but it was removed prior to the lab period. The inhibitor was removed by stirring for 5 min a mixture of $3 \mathrm{~g}$ of alumina for every $20 \mathrm{~mL}$ 
of styrene and gravimetrically filtering the slurry through qualitative filter paper. The instructor may do this prior to the laboratory session for the whole class.

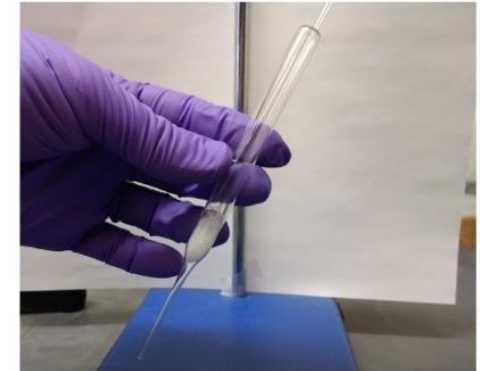

A

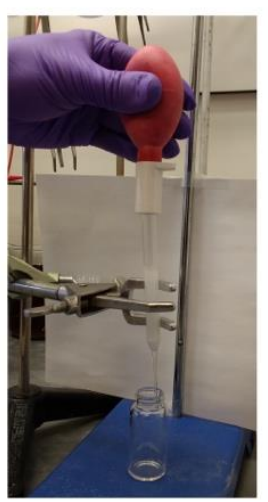

D

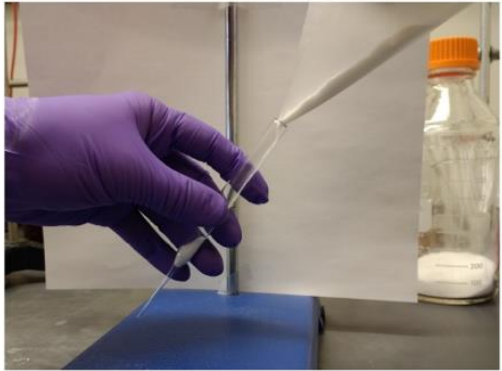

B

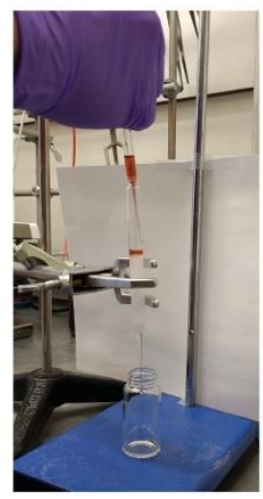

$E$

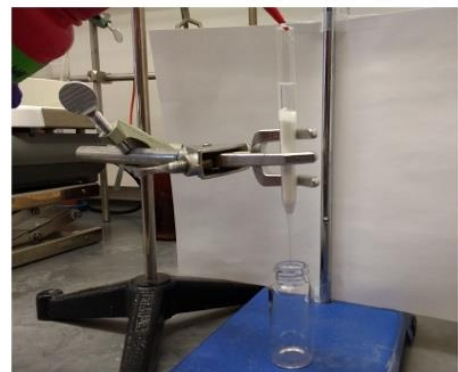

C

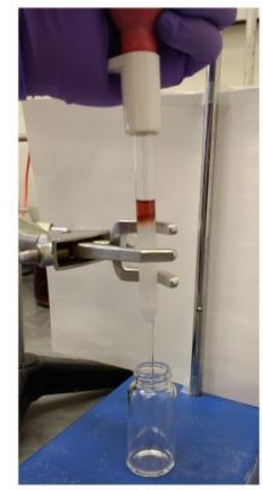

$\mathrm{F}$

Figure 8.1: (a) Insertion of a glass wool into a glass pipette $(8 \times 142 \mathrm{~mm})$ with a 9 inch Pasteur pipette; (b) Loading of dry silica into the glass pipette with weigh paper; (c) Wetting of the silica with hexanes; (d) Application of pressure with a volumetric pipette bulb to elute the solvent; (e) Loading the product mixture on to the wet silica column; (f) Elution of product with firm, constant pressure from pipette bulb.

\section{DAY 2}

Form a hypothesis, design a modification of the experiment and reach a conclusion supported by your data. Possible modifications may be to test the turnover limits of the reaction by reducing the catalyst loading, or by changing the reaction time, temperature 
and/or concentration of reagents. A more complex study of reaction conditions may be screened if you pair with your presentation partner(s) to design your experiments.

\section{Questions}

1. What are the advantages of synthesizing stilbene with metathesis vs Wittig? Disadvantages?

2. What is the cis/trans ratio produced by metathesis and how does it compare to the Wittig reaction?

3. Draw the catalytic cycle that produces stilbene. Where is the stereochemistry set (i.e. at what point does the product become cis- or trans-?

4. A properly-designed catalyst can produce non-thermodynamic distributions of products (i.e. a kinetic or Curtin-Hammett distribution of products). Is this metathesis reaction thermodynamically or kinetically controlled? Can you design an experiment to test your answer?

5. Is this catalyst a good choice for olefin metathesis? (hint: take a look at your TON and TOF). Hit the literature, what other catalyst might you suggest for the metathesis of styrene? 